\title{
EXPERIMENT DATA REPORT FOR SEMISCALE MOD-1 TEST S-28-6 (STEAM GENERATOR TUBE RUPTURE TEST)
}

\author{
MORRIS L. PATTON KENNETH E. SACKETT \\ CHERYL E. COPPIN
}

November 1977

\section{EG\&G Idaho, Inc.}

\section{IDAHO NATIONAL ENGINEERING LABORATORY}

\section{DEPARTMENT OF ENERGY}

IDAHO OPERATIONS OFFICE UNDER CONTRACT EY-76-C-07-1570 


\section{DISCLAIMER}

This report was prepared as an account of work sponsored by an agency of the United States Government. Neither the United States Government nor any agency Thereof, nor any of their employees, makes any warranty, express or implied, or assumes any legal liability or responsibility for the accuracy, completeness, or usefulness of any information, apparatus, product, or process disclosed, or represents that its use would not infringe privately owned rights. Reference herein to any specific commercial product, process, or service by trade name, trademark, manufacturer, or otherwise does not necessarily constitute or imply its endorsement, recommendation, or favoring by the United States Government or any agency thereof. The views and opinions of authors expressed herein do not necessarily state or reflect those of the United States Government or any agency thereof. 


\section{DISCLAIMER}

Portions of this document may be illegible in electronic image products. Images are produced from the best available original document. 
Printed in the United States of America Available from

National Technical Information Service

U.S. Department of Commerce

5285 Port Royal Road

Springfield, Virginia 22161

Price: Printed Copy $\$ 10.75$; Microfiche $\$ 3.00$

\section{NOTICE}

This report was prepared as an account of work sponsored by the United States Government. Neither the United States nor the Department of Energy, nor any of their employees, nor any of their contractors, subcontractors, or their employees, makes any warranty, express or implied, or assumes any legal liability or responsibility for the accuracy, completeness or usefulness of any information, apparatus, product or process disclosed, or represents that its use would not infring privately owned rights.

"The NRC will make available data tapes and operational computer codes on research programs dealing with postulated loss-of-coolant accidents in light water reactors. Persons requesting this information must reimburse the NRC contractors for their expenses in preparing copies of the data tapes and the operational computer codes. Requests should be submitted to the Research Applications Branch, Office of Nuclear Regulatory Research, Nuclear Regulatory Commission, Washington, D.C. 20555." 
TREE-NUREG-1153

\section{EXPERIMENT DATA REPORT FOR SEMISCALE MOD-1}

TEST S-28-6

(STEAM GENERATOR TUBE RUPTURE TEST)
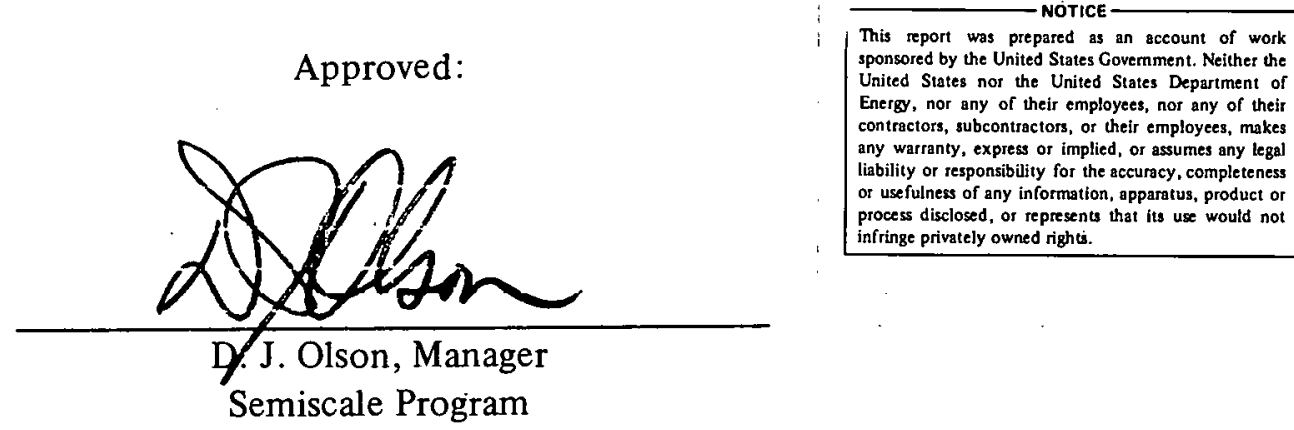

process disclosed, or represents
infringe privately owned righti.

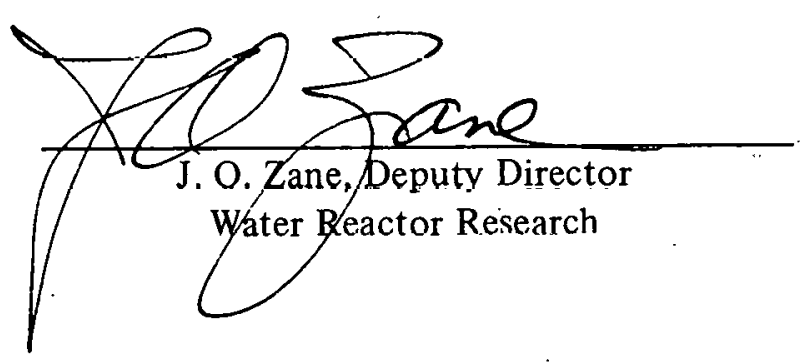


EXPERIMENTT DATA REPORT FOR SEMISCALE MOD-1

TEST S-28-6

(STEAM GENERATOR TUBE RUPTURE TEST)

\author{
By \\ Morris L. Patton \\ Kenneth E. Sackett \\ Cheryl E. Coppin
}

EG\&G IDAHO, INC.

November 1977

PREPARED FOR THE

U.S. NUCLEAR REGULATORY COMMISSION

AND THE

DEPARTMENT OF ENERGY

IDAHO OPERATIONS OFFICE

UNDER CONTRACT NO. EY-76-C-07-1570 


\begin{abstract}
Recorded test data are presented for Test S-28-6 of the Semiscale Mod-1 steam generator tube rupture test series. These tests are among several Semiscale Mod-1 experiments conducted to investigate the thermal and hydraulic phenomena accompanying a hypothesized loss-of-coolant accident in a pressurized water reactor (PWR) system.

Test S-28-6 was conducted from initial conditions of $15770 \mathrm{kPa}$ and $557 \mathrm{~K}$ to investigate the response of the Semiscale Mod-1 system to a depressurization and reflood transient following a simulated double-ended offset shear of the broken loop cold leg piping. During the test, cooling water was injected into the cold leg of the inlact and broken loops to simulate emergency core coolant injection in a PWR. Sixteen steam generator tube ruptures were simulated by a controlled injection from a heated accumulator into the intact loop hot leg.

The purpose of this report is to make available the uninterpreted data from Test S-28-6 for future data analysis and test reporting activities. The data, presented in the form of graphs in engineering units, have been analyzed only to the extent necessary to ensure that they are reasonable and consisteril.
\end{abstract}




\section{SUMMARY}

Test S-28-6 was performed as part of the Semiscale Mod-1 portion of the Semiscale Program conducted by EG\&G Idaho, Inc. for the United States Government. This test is part of the steam generator tube rupture test series (Test Series 28) performed to investigate the response of the Mod-1 system to steam generator tube ruptures during a hypothesized loss-of-coolant accident (LOCA). The test objective specific to Test S-28-6 was to refine the definition of the lower limit set by Test S-28-2 and to investigate the range of steam generator tube ruptures over which high peak cladding temperatures can occur. Hardware configuration and test parameters were selected to yield a system response that simulates the response of a pressurized water reactor during a hypothesized LOCA with subsequent refill and reflood.

'l'est S-28-6 utilized the Semiscale Mod-1 system equipped with a pressure vessel with a 40-rod electrically heated core; an intact loop with pump, steam generator, and pressurizer; a broken loop with simulated pump, simulated steam generator, and rupture assemblies; and a pressure suppression system with header, pressure suppression tank; and heated steam supply system. High and low pressure coolant injection pumps and a coolant injection accumulator were provided for each system loop. An additional injection accumulator was provided for the intact loop hot leg. The in tact loop hot leg injection flow rate was set to simulate the rupture of 16 steam generator tubes. In addition, for Test S-28-6, four heater rods were intentionally unpowered to simulate the effects of control rod guide tubes and the power in three heater rods was increased to produce a slightly peaked power profile.

The test was conducted from initial conditions of $15770 \mathrm{kPa}$ and $557 \mathrm{~K}$ (at the intact loop cold leg vessel inlet) with a simulated full size (200\%) double-ended offset shear of the cold leg broken loop piping at an initial core power level of $1.42 \mathrm{MW}$, and an initial core inlet flow rate of $9.19 \mathrm{l} / \mathrm{s}$. The instantaneous offset shear of the broken loop cold leg piping was simulated by simultaneous (within $10 \mathrm{~ms}$ ) actuation of the rupture assemblies. After initiation of blowdown, power to the heated core was reduced to simulate the predicted heat flux response of nuclear fuel rods during a. LOCA. Blowdown was accompanied by simulated emergency core coolant injected into the cold legs of the intact and broken loops and followed by injection into the intact loop hot leg to simulate steam generator tube ruptures.

Test S-28-6 was generally conducted as specified. Conditions which did not conform to the specified test configuration were considered acceptable for analysis purposes within the test objectives. The instrumentation used generally functioned as intended. All 219 measurements taken produced usable data. 


\section{CONTENTS}

ABSTRACT $\ldots \ldots \ldots \ldots \ldots \ldots$ ii

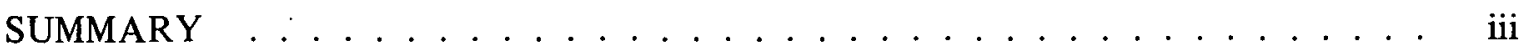

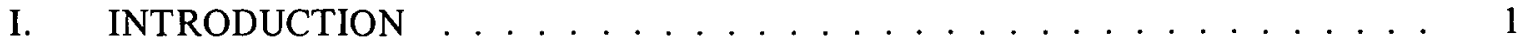

II. SYSTEM, PROCEDURES, CONDITIONS, AND.

EVENTS FOR TEST S-28-6 . . . . . . . . . . . . . . . . 2

1. SYSTEM CONFIGURATION AND
TEST $\mathrm{P} R$ ROCEDURES $\ldots \ldots \ldots \ldots \ldots$

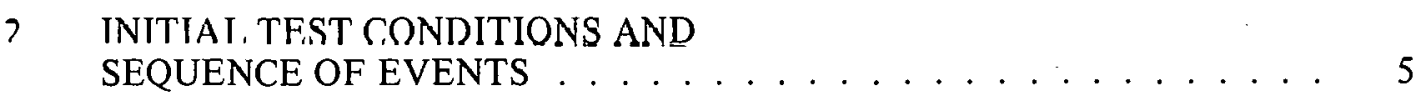

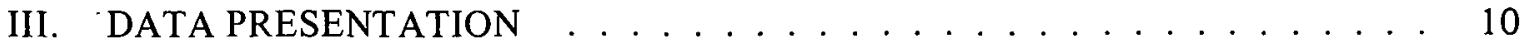

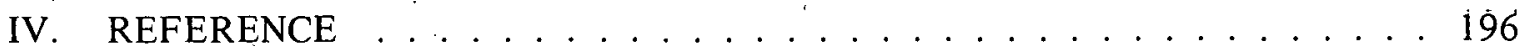

APPENDIX A - DATA ACQUISITION SYSTEM.CAPABILITIES . . . . . . . . 197

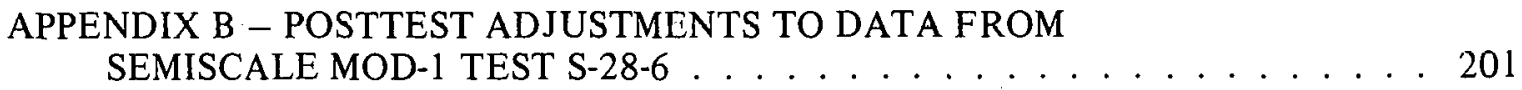

1. PRESSURE MEASUREMENTS .................. 203

2. DIFFERENTIAL PRESSURF MEASUREMENTS . . . . . . . . . . 204

3. MOMENTUM FLUX MEASUREMENTS . . . . . . . . . . . . . . . 206

4: DENSITY MEASUREMENTS . . . . . . . . . . . . . 2U8

APPENDIX C - SELECTED DATA WITH ESTIMATED TOTAL ERROR

BANDS FROM SEMISCALE MOD-1 TEST S-28-6. . . . . . . . . . . . . 213

FIGURES

1. Semiscale Mod-1 system for cold leg break

configuration - isometric . . . . . . . . . . . . . 3

2. Semiscale Mod-1 system for cold leg break

configuration - schematic .................. 4

3. Semiscale Mod-1 system and instrumentation

for cold leg break configuration - isometric . . . . . . . . . . . . . . . . 11

4. Semiscale Mod-1 system and instrumentation

for cold leg break configuration - schematic 
5. Semiscale Mod-1 pressure vessel - cross

section showing instrumentation . . . . . . . . . . . . . 13

6. Semiscale Mod-1 pressure vessel - isometric

showing instrumentation . . . . . . . . . . . . . . . . . . 14

7. Semiscale Mod-1 pressure vessel - penetrations

and instrumentation . . . . . . . . . . . . . . 15

8. Semiscale Mod-1 heated core - plan view . . . . . . . . . . . 16

9. Fluid temperature in intact loop hot leg

(TFU-1 and RBU-2), from -20 to $600 \mathrm{~s} \ldots \ldots . . . \ldots 28$

10. Fluid temperature in in tact loop hot leg

(TFU-1 and RBU-2), from -6 to $42 \mathrm{~s} \ldots \ldots \ldots$. . . . . . . . . . 28

11. Fluid temperature in intact loop hot leg (TFU-6),

from -20 to $600 \mathrm{~s} \ldots \ldots \ldots$. . . . . . . . . . . . 29

12. Fluid temperature in intact loop hot leg (TFU-6),

from -6 to $42 \mathrm{~s} \ldots \ldots \ldots \ldots$. . . . . . . . . . . . 29

13. Fluid temperature in intact loop cold leg

(TFU-7 and TFU-10), from -20 to $600 \mathrm{~s} \ldots \ldots . \ldots 30$

14. Fluid temperature in intact loop cold leg

(TFU-7 and TFU-10), from -6 to $42 \mathrm{~s}$

15. Fluid temperature in in tact loop cold leg

(RBU-14A and TFU-14B), from -20 to $600 \mathrm{~s} \ldots \ldots . \ldots 31$

16. Fluid temperature in intact loop cold leg

(RBU-14A and TFU-14B), from -6 to $42 \mathrm{~s} \ldots \ldots . \ldots 31$

17. Fluid temperature in intact loop cold leg (TFU-15),

from -20 to $600 \mathrm{~s} \ldots \ldots \ldots . \ldots \ldots{ }_{32}$

18. Fluid temperature in intact loop cold leg (TFU-15),

from -6 to $42 \mathrm{~s} \ldots \ldots \ldots . \ldots \ldots 2$

19. Fluid temperature in broken loop, vessel

side (TFB-20 and TFB-23), from -20 to $600 \mathrm{~s} \ldots \ldots . . . \ldots 33$

20. Fluid temperature in broken loop, vessel

side (TFB-20 and TFB-23), from -6 to $42 \mathrm{~s}$

21. Fluid temperature in broken loop, pump side

(TFB-30, TFB-37, and TFB-42), from -20 to $600 \mathrm{~s} \ldots \ldots . . . . . . .34$

22. Fluid temperature in broken loop, pump side

(TFB-30, TFB-37, and TFB-42), from -6 to $42 \mathrm{~s} \ldots \ldots . . \ldots . . . . . .34$ 
23. Fluid temperature in inlet annulus (TFV-ANN-4A and TFV-ANN-4M), from -20 to $600 \mathrm{~s} \ldots \ldots . \ldots 35$

24. Fluid temperature in inlet annulus (TFV-ANN-4A and TFV-ANN-4M), from -6 to $42 \mathrm{~s} \ldots \ldots \ldots 35$

25. Fluid temperature in downcomer annulus (TFV-ANN-35A, TFV-ANN-70A, TFV-ANN-115A, and TFV-ANN-156A), from $-2 U$ to $6 U$ UU $s$

26. Fluid temperature in downcomer annulus (TFV-ANN-35A, TFV-ANN-70A, TFV-ANN-1 15A, and TFV-ANN-156A), from -6 to $42 \mathrm{~s}$.

27. Fluid temperature in upper plenum (TFV-UP+13), fromil -20 to $600 \mathrm{~s}$

28. Fluid tempcrature in upper plenum (TFV-UP+13), from -6 to $42 \mathrm{~s} \ldots \ldots \ldots \ldots$

29. Fluid temperature in lower plenum (TFV-LP-2, TFV-LP-4, and IFV-LP-7), from -20 to $600 \mathrm{~s} \ldots \ldots . \ldots 38$

30. Fluid temperature in lower plenum (TFV-LP-2, TFV-LP-4, and TFV-LP-7), from -6 to $42 \mathrm{~s}$

31. Fluid temperature in core inlet (TFV-CORE-IN), from -20 to $600 \mathrm{~s} \ldots \ldots \ldots \ldots$

32. Fluid temperature in core inlet (TFV-CORE-IN), from 6 to $42 \mathrm{~s} \ldots \ldots \ldots \ldots \ldots$

33. Fluid temperature in core, Grid Spacer 5 (TFG-5CD-45), from -20 to $600 \mathrm{~s}$

34. Fluid temperature in core, Grid Spacer 5 (TFG-5CD-45), from -6 to $42 \mathrm{~s}$

35. Fluid temperature in core, Grid Spacer 6 (TFG-6CD-45), from -20 to $600 \mathrm{~s}$

36. Fluid temperature in core, Grid Spacer 6 (TFG-6CD-45), from -6 to $42 \mathrm{~s}$

37. Fluid temperature in core, Grid Spacer 10 (TFG-10AB-45), from -20 to $600 \mathrm{~s}$

38. Fluid temperature in core, Grid Spacer 10 (TFG-10AB-45), from -6 to $42 \mathrm{~s}$

39. Fluid temperature in in tact loop coolant injection line (TFU-ECC-14), from -20 to $600 \mathrm{~s}$ 
40. Fluid temperature in intact loop coolant

injection line (TFU-ECC-14), from -6 to $42 \mathrm{~s} \ldots \ldots 43$

41. Fluid temperature in broken loop coolant

injection line (TFB-ECC-42), from -20 to $600 \mathrm{~s} \ldots \ldots . . . . . . . .44$

42. Fluid temperature in broken loop coolant

injection line (TFB-ECC-42), from -6 to $42 \mathrm{~s} \ldots \ldots . . . . . . . .444$

43. Fluid temperature in steam generator, feedwater

line (TFU-SGFW), from -20 to $600 \mathrm{~s} \ldots \ldots \ldots$. . . . . . . . 45

44. Fluid temperature in steam generator, feedwater

line (TFU-SGFW), from -6 to $42 \mathrm{~s} \ldots \ldots \ldots$. . . . . . . . . . 45

45. Fluid temperature in steam generator, steam dome

(TFU-SGSD), from -20 to $600 \mathrm{~s} \ldots \ldots \ldots$. . . . . . . . . . 46

46. Fluid temperature in steam generator, steam dome

(TFU-SGSD), from -6 to $42 \mathrm{~s} \ldots \ldots \ldots \ldots$

47. Fluid temperature in steam generator, secondary

side (TFU-SG1, TFU-SG2, and TFU-SG3),

from -20 to $600 \mathrm{~s}$

48. Fluid temperature in steam generator, secondary

side (TFU-SG1, TFU-SG2, and TFU-SG3),

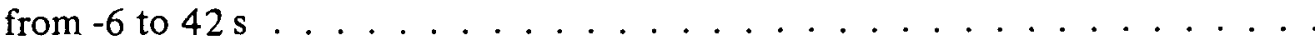

49. Fluid temperature in steam generator rupture system accumulator

(TFU-SGS3), from -20 to $600 \mathrm{~s} \ldots \ldots \ldots$

50. Fluid temperature in steam generator rupture system accumulator

(TFU-SGS3), from -6 to $42 \mathrm{~s}$

51. Fluid temperature in steam generator rupture system injection line

(TFU-SGS), from -20 to $600 \mathrm{~s} \ldots \ldots \ldots \ldots$

52. Fluid temperature in steam generator nupture system injection line

(TFU-SGS), from -6 to $42 \mathrm{~s}$

53. Fluid temperature in steam generator rupture system injection line drain

(TFU-SGS-D), from -20 to $600 \mathrm{~s} \ldots \ldots \ldots \ldots$

54. Fluid temperature in steam generator rupture system injection line drain

(TFU-SGS-D), from -6 to $42 \mathrm{~s} \ldots \ldots \ldots \ldots$

55. Fluid temperature in pressurizer surge line (TFU-PRIZE),

from -20 to $600 \mathrm{~s}$

56. Fluid temperature in pressurizer surge line (TFU-PRIZE),

from -6 to $42 \mathrm{~s}$ 
57. Fluid temperature in pressure suppression tank

(TF-PSS-33 and TF-PSS-130), from -20 to $600 \mathrm{~s}$

58. Fluid temperature in pressure suppression tank

(TF-PSS-33 and TF-PSS-130), from -6 to $42 \mathrm{~s} \ldots \ldots . \ldots 52$

59. Material temperature in intact loop (TMU-1 T16),

from -30 to $600 \mathrm{~s} \ldots \ldots \ldots . \ldots . \ldots . \ldots . \ldots$

60. Material temperature in intact loop (TMU-1T16),

from -6 to $42 \mathrm{~s} \ldots \ldots \ldots \ldots$. . . . . . . . . . . . . . 53

61. Material temperature in broken loop (TMB-20B16),

from -20 to $600 \mathrm{~s} \ldots \ldots \ldots \ldots$. . . . . . . . . . . . . . 54

62. Material temperature in broken loop (TMB-20B16),

from -6 to $42 \mathrm{~s} \ldots \ldots \ldots \ldots \ldots$

63. Material temperature in vessel filler (TMV-FI-115A),

from -20 to $600 \mathrm{~s} \ldots \ldots \ldots \ldots$. . . . . . . . . . . . . . . . . .

64. Material tcmperature in vessel filler (TMV-FI-115A),

from -6 to $42 \mathrm{~s} \ldots \ldots \ldots \ldots \ldots$

65. Material temperature in vessel filler insulator

(TMV-FO-156A), from -20 to $600 \mathrm{~s} \ldots \ldots \ldots 56$

66. Material temperature in vessel filler insulator

(TMV-FO-1 56A), from -6 to $42 \mathrm{~s} \ldots \ldots \ldots$. . . . . . . . . . 56

67. Material temperature in vessel filler insulator

(TIV-FO-35A and TIV-FO-115A), from -20 to $600 \mathrm{~s} \ldots \ldots . . . . . .57$

68. Material temperature in vessel filler insulator

(TIV-FO-35A and TIV-FO-115A), from -6 to $42 \mathrm{~s} \ldots \ldots . . . . . . .57$

69. Material temperature in core barrel inner diameter

(TMV-CI-70A), from -20 to $600 \mathrm{~s} \ldots \ldots \ldots$. . . . . . . . . 58

70. Material temperature in core barrel inner diameter

(TMV-CI-70A), from -6 to $42 \mathrm{~s} \ldots \ldots \ldots \ldots$. . . . . . . . . 58

71. Material temperature in core barrel outer diameter

(TMV-CO-70A and TMV-CO-115A), from -20 to $600 \mathrm{~s} \ldots \ldots$. . . . . . . 59

72. Material temperature in core barrel outer diameter

(TMV-CO-70A and TMV-CO-115A), from -6 to $42 \mathrm{~s}$

73. Material temperature in core housing filler (TMV-HF-115W,

TMV-HF-127W, and TMV-HF-138W), from -20 to $600 \mathrm{~s}$

74. Material temperature in core housing filler (TMV-HF-115W,

TMV-HF-127W, and TMV-HF-138W), from -6 to $42 \mathrm{~s}$ 
75. Material temperature in steam generator (TMU-SG1, TMU-SG2,

and TMU-SG3), from -20 to $600 \mathrm{~s} \ldots \ldots 61$

76. Material temperature in steam generator (TMU-SG1, TMU-SG2, and TMU-SG3), from -6 to $42 \mathrm{~s} \ldots \ldots \ldots 1$

77. Core heater temperature, Rod D-4 (TH-D4-14 and TH-D4-29), from -20 to $600 \mathrm{~s} \ldots \ldots \ldots 62$

78. Core heater temperature, Rod D-4 (TH-D4-14 and TH-D4-29), from -6 to $42 \mathrm{~s} \ldots \ldots \ldots$. . . . . . . . . . . 62

79. Core heater temperature, Rod D-5 (TH-D5-29), from -20 to $600 \mathrm{~s}$

80. Core heater temperature, Rod D-5 (TH-D5-29), from -6 to $42 \mathrm{~s} \ldots \ldots \ldots \ldots$

81. Core heater temperature, Rod E-4 (TH-E4-09, TH-E4-27, and TH-E4-55), from -20 to 600 s

82. Core heater temperature, Rod E-4 (TH-E4-09, TH-E4-27, and TH-E4-55), from -6 to $42 \mathrm{~s} \ldots \ldots \ldots$

83. Core heater temperature, Rod E-5 (TH-E5-20 and TH-E5-25), from -20 to $600 \mathrm{~s}$

84. Core heater temperature, Rod E-5 (TH-E5-20 and TH-E5-25), from -6 to $42 \mathrm{~s}$

85. Core heater temperature, Rod A-4 (TH-A4-09, TH-A4-29, and TH-A4-39), from -20 to $600 \mathrm{~s} \ldots \ldots 66$

86. Core heater temperature, Rod A-4 (TH-A4-09, TH-A4-29, and TH-A4-39), from -6 to $42 \mathrm{~s}$

87. Core heater temperature, Rod A-5 (TH-A5-29 and

TH-A5-45), from -20 to $600 \mathrm{~s}$

88. Core heater temperature, Rod A-5 (TH-A5-29 and TH-A5-45), from -6 to $42 \mathrm{~s}$

89. Core heater temperature, Rod B-3 (TH-B3-32),

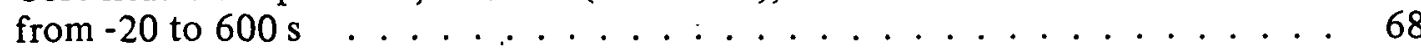

90. Core heater temperature, Rod B-3 (TH-B3-32), from -6 to $42 \mathrm{~s}$

91. Core heater temperature, Rod B-5 (TH-B5-29 and TH-B5-33), from -20 to $600 \mathrm{~s} \ldots \ldots \ldots$

92. Core heater temperature, Rod B-5 (TH-B5-29 and TH-B5-33), from -6 to $42 \mathrm{~s}$ 
93. Core heater temperature, Rod B-6 (TH-B6-29),

from -20 to $600 \mathrm{~s} \ldots \ldots \ldots \ldots$. . . . . . . . . . . . . . . . . . . . . .

94. Core heater temperature, Rod B-6 (TH B6-29),

from -6 to $42 \mathrm{~s} \ldots \ldots \ldots \ldots$. . . . . . . . . . . . . . . . . . . . . . . .

95. Core heater temperature, Rod C-2 (TH-C2-38),

from -20 to $600 \mathrm{~s} \ldots \ldots \ldots \ldots$. . . . . . . . . . . . . . . . . . .

96. Core heater temperature, Rod C-2 (TH-C2-38),

from -6 to $42 \mathrm{~s} \ldots \ldots \ldots \ldots$. . . . . . . . . . . . . 71

97. Core heater temperature, Rod C-4 (TH-C4-20,

TH-C4-26, and TH-C4-53), from -20 to $600 \mathrm{~s} \ldots \ldots . \ldots . . \ldots 72$

98. Core heater temperature, Rod C-4 (TH-C4-20,

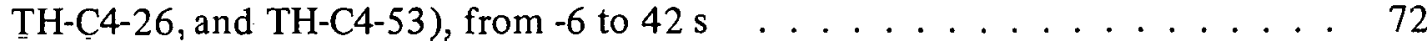

99. Core heater temperature, Rod C-5 (TH-C5-28),

from -20 to $600 \mathrm{~s} \ldots \ldots \ldots$. . . . . . . . . . . . 73

100. Core heater temperature, Rod C-5 (TH-C5-28),

from -6 to $42 \mathrm{~s} \ldots \ldots \ldots 73$

101. Core heater temperature, Rod C-6 (TH-C6-53),

from -20 to $600 \mathrm{~s} \ldots \ldots \ldots \ldots$. . . . . . . . . . . . . . . . . . . . . .

102. Core heater temperature, Rod C-6 ('I'H-C'6-53),

from -6 to $42 \mathrm{~s} \ldots \ldots \ldots \ldots$. . . . . . . . . . . . . . . . . . . .

103. Core heater temperature, Rod C-7 (TH-C7-07

and $\mathrm{TH}-\mathrm{C} 7 \mathrm{-15}$ ), from -20 to $600 \mathrm{~s} \ldots \ldots \ldots 75$

104. Core heater temperature, Rod C-7 (TH-C7-07

and TII-C7-15), from -6 to $42 \mathrm{~s} \ldots \ldots \ldots \ldots$. . . . . . . . . . . .

105. Core heater temperature, Rod D-1 (TH-D1-21),

from -20 to $600 \mathrm{~s} \ldots \ldots \ldots \ldots$. . . . . . . . . . . . . . . . . . . . .

106. Core heater temperature, Rod D-1 ('IH-D1-21),

from -6 to $42 \mathrm{~s} \ldots \ldots \ldots \ldots$. . . . . . . . . . . . . . 76

107. Core heater temperature, Rod D-2 (TH-D2-14 and

TH-D2-61), from -20 to $600 \mathrm{~s}$

108. Core heater temperature, Rod D-2 (TH-D2-14 and

TH-D2-61), from -6 to $42 \mathrm{~s}$

109. Core heater temperature, Rod D-3 (TH-D3-29 and

TH-D3-39), from -20 to $600 \mathrm{~s}$

110. Core heater temperature, Rod D-3 (TH-D3-29 and

TH-D3-39), from -6 to $42 \mathrm{~s}$ 
111. Core heater temperature, Rod D-6 (TH-D6-25),

from -20 to $600 \mathrm{~s}$

112. Core heater temperature, Rod D-6 (TH-D6-25),

from -6 to $42 \mathrm{~s} \ldots \ldots \ldots \ldots \ldots \ldots$

113. Core heater temperature, Rod D-7 (TH-D7-20),

from -20 to 600

114. Core heater temperature, Rod D-7 (TH-D7-20),

from -6 to $42 \mathrm{~s} \ldots \ldots \ldots \ldots \ldots$

115. Core heater temperature, Rod D-8 (TH-D8-26), from -20 to $600 \mathrm{~s}$

116. Core heater temperature, Rod D-8 (TH-D8-26),

from -6 to $42 \mathrm{~s} \ldots \ldots \ldots \ldots \ldots$

117. Core heater temperature, Rod E-1 (TH-E1-33), from -20 to $600 \mathrm{~s}$

118. Core heater temperature, Rod E-1 (TH-E1-33), from -6 to $42 \mathrm{~s}$

119. Core heater temperature, Rod E-2 (TH-E2-33), from -20 to $600 \mathrm{~s}$

120. Core heater temperature, Rod E-2 (TH-E2-33),

121. Core heater temperature, Rod E-3 (TH-E3-05, TH-E3-20, and TH-E3-24), from -20 to $600 \mathrm{~s}$

122. Core heater temperature, Rod E-3 (TH-E3-05, TH-E3-20, and TH-E3-24), from -6 to $42 \mathrm{~s}$

123. Core heater temperature, Rod E-6 (TH-E6-nR,

TH-E6-28, and TH-E6-37), from -20 to $600 \mathrm{~s}$

124. Core heater temperature, Rod E-6 (TH-E6-08, TH-E6-28, and TH-E6-37), from -6 to $42 \mathrm{~s}$

125. Core heater temperature, Rod E-7 (TH-E7-44), from -20 to $600 \mathrm{~s}$

126. Core heater temperature, Rod E-7 (TH-E7-44), from -6 to $42 \mathrm{~s}$

127. Core heater temperature, Rod E-8 (TH-E8-14 and TH-E8-29), from -20 to $600 \mathrm{~s}$

128. Core heater temperature, Rod E-8 (TH-E8-14 and TH-E8-29), from -6 to $42 \mathrm{~s}$. 
129. Core heater temperature, Rod F-2 (TH-F2-07,

TH-F2-22, and TH-F2-25), from -20 to $600 \mathrm{~s} \ldots \ldots \ldots 8$

130. Core heater temperature, Rod F-2 (TH-F2-07, TH-F2-22, and TH-F2-25), from -6'to $42 \mathrm{~s}$

131. Core heater temperature, Rod F-4 (TH-F4-14, TH-F4-29, and TH-F4-44), from -20 to $600 \mathrm{~s}$

132. Core heater temperature, Rod F-4 (TH-F4-14, TH-F4-29, and TH-F4-44), from -6 to $42 \mathrm{~s}$

133. Core heater temperature, Rod F-5 (TH-F5-20, TH-F5-26, TH-F5-33, and $(1 \mathrm{H}-\mathrm{F} 5-53$ ), from -20 to $600 \mathrm{~s}$

134 Tnre, heater temperature, Rod F-5 (TH-F5-20, TH-F5-26, TH-F5-33, and TH-H5-53), from -6 to $42 \mathrm{~s} \ldots \ldots . . . . . .90$

135. Core heater temperature, Rod $\mathrm{G}-3$ ( $\mathrm{IH}-\mathrm{H}-3-13)$, from -20 to $600 \mathrm{~s}$

136. Core heater temperature, Rod G-3.(TH-G3-13), from -6 to $42 \mathrm{~s} \ldots \ldots \ldots$. . . . . . . . . . . . 91

137. Core heater temperature, Rod G-4 (TH-G4-29,

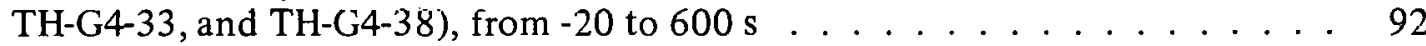

138. Core heater temperature, Rod G-4 (TII $=\mathrm{G} 4-29$, TH-G4-33, and TH-G4-38), from -6 to $42 \mathrm{~s}$

139. Core heater temperature, Rod G-5 (TH-G5-14 and TH-G5-24), from -20 to $600 \mathrm{~s} \ldots \ldots$. . . . . . . . . . . 93

140. Core heater temperature, Rod G-5 (TH-G5-14 and TH-G5-24), from -6 to $42 \mathrm{~s} \ldots \ldots . \ldots . \ldots 93$

141. Core heater temperature, Rod H-5 (TH-H5-32), from $-? n$ tn $600 \mathrm{~s}$

142. Core heater temperature, Rod H-5 (TH-H5-32), from -6 to $42 \mathrm{~s}$

143. Pressure in intact loop (PU-13 and PU-15L), from -20 to $600 \mathrm{~s}$

144. Pressure in intact loop (PU-13 and PU-15L), from -6 to $42 \mathrm{~s}$

145. Pressure in broken loop, vessel side (PB-21 and PB-23), from -20 to $600 \mathrm{~s}$

146. Pressure in broken loop, vessel side (PB-21 and PB-23), from -6 to $42 \mathrm{~s}$ 
147. Pressure in broken loop, pump side (PB-37 and PB-42); from -20 to $600 \mathrm{~s}$

148. Pressure in broken loop, pump side (PB-37 and PB-42), from -6 to $42 \mathrm{~s}$

149. Pressure in broken loop, pump-side nozzle (PB-HN1), from -20 to $600 \mathrm{~s}$

150. Pressure in broken loop, pump-side nozzle (PB-HN1), from -6 to 42

151. Pressure in vessel (PV-UP+10 and PV-LP-166), from -20 to $600 \mathrm{~s}$

152. Pressure in vessel (PV-UP+10 and PV-LP-166), from -6 to $42 \mathrm{~s}$

153. Pressure in intact loop accumulator (PU-ACC1), from -20 to $600 \mathrm{~s}$ 100

154. Pressure in intact loop accumulator (PU-ACC1), from -6 to $42 \mathrm{~s}$

155. Pressure in broken loop accumulator (PB-ACC2), from -20 to $600 \mathrm{~s}$

156. Pressure in broken loop accumulator (PB-ACC2), from -6 to $42 \mathrm{~s}$

157. Pressure in steam generator, secondary side (PU-SGSD), from -20 to $600 \mathrm{~s} \ldots \ldots \ldots 102$

158. Pressure in steam generator, secondary side (PU-SGSD), from -6 to $42 \mathrm{~s}$

159. Pressure in steam generator, tuhe rupture simulation accumulator (PU-SGS3), from -20 to $600 \mathrm{~s}$ 103

160. Pressure in steam generator, tube rupture simulation accumulator (PU-SGS3), from -6 to $42 \mathrm{~s}$

161. Pressure in pressurizer (PU-PRIZE), from -20 to $600 \mathrm{~s}$

162. Pressure in pressurizer (PU-PRIZE), from -6 to $42 \mathrm{~s}$. 104

163. Pressure in pressure suppression tank (P-PSS), from -20 to $600 \mathrm{~s}$

164. Pressure in pressure suppression tank (P-PSS), from -6 to $42 \mathrm{~s}$ 
165. Differential pressure in intact loop (DPU-3-7), from -20 to $600 \mathrm{~s}$

166. Differential pressure in intact loop (DPU-3-7),

from -6 to $42 \mathrm{~s} \ldots \ldots \ldots \ldots$. . . . . . . . . . . . . . . . . . . . . .

167. Differential pressure in intact loop (DPU-6-SGIP), from -20 to $600 \mathrm{~s} \ldots \ldots \ldots \ldots$. . . . . . . . . . . 107

168. Differential pressure in intact loop (DPU-6-SGIP), from -6 to $42 \mathrm{~s}$

169. Differential pressure in intact loop (DPU-SGOP-7), fromil -20 to $600 \mathrm{~s}$

170. Differential pressure in in tact loop (DPU-SGOP-7), from -6 to $42 \mathrm{~s} \ldots \ldots \ldots \ldots$. . . . . . . . . . . . . 108

171. Differential pressure in intact loop (DPU-7-1U), from -20 to $600 \mathrm{~s}$

172. Differential pressure in intact loop (DPU-7-10), from -6 to $42 \mathrm{~s} \ldots \ldots \ldots \ldots$. . . . . . . . . . . 109

173. Differential pressure in intact loop (DPU-12-10), from -20 to $600 \mathrm{~s}$

174. Differential pressure in intact loop (DPU-12-10), from 6 to $12 \varepsilon \ldots \ldots \ldots \ldots \ldots$

175. Differential pressure in intact loop, low range (DPÜ-12-10L), from - 20 to $600 \mathrm{~s}$

176. Differential pressure in in tact loop, low range (DPU-12-10L), from -6 to $42 \mathrm{~s}$

177. Differential pressure in intact loop (DPU-12-15), from $-2010600 \mathrm{~s}$

178. Differential pressure in intact loop (DPU-12-15), from -6 to 4.2 . . . . . . . . . . . . . . . . 112

179. Differential pressure in in tact loop (DPU-15-3), from -20 to $600 \mathrm{~s}$

180. Differential pressure in intact loop (DPU-15-3), from -6 to $42 \mathrm{~s}$

181. Differential pressure in intact loop, low rangè (DPU-15-3L), from -20 to $600 \mathrm{~s}$

182. Differential pressure in in tact loop, low range (DPU-15-3L), from -6 to $42 \mathrm{~s}$ 
183. Differential pressure in pressurizer (DPU-PRESLL),

from -20 to $600 \mathrm{~s}$

184. Differential pressure in pressurizer (DPU-PRESLL),

from -6 to $42 \mathrm{~s}$

185. Differential pressure in intact loop (DPU-PR-4),

from -20 to $600 \mathrm{~s}$

186. Differential pressure in intact loop (DPU-PR-4),

from -6 to $42 \mathrm{~s} \ldots \ldots \ldots \ldots \ldots$. . . . . . . . . . . . . . . . . . . .

187. Differential pressure in broken loop (DPB-21-IANN),

from -20 to $600 \mathrm{~s}$

188. Differential pressure in broken loop (DPB-21-IANN),

from -6 to $42 \mathrm{~s}$

189. Differential pressure in broken loop (DPB-23-CN1), from -20 to $600 \mathrm{~s}$

190. Differential pressure in broken loop (DPB-23-CN1), from -6 to $42 \mathrm{~s}$

191. Differential pressure in broken loop (DPB-CN1-24), from -20 to $600 \mathrm{~s}$

192. Differential pressure in broken loop (DPB-CN1-24), from -6 to $42 \mathrm{~s}$

193. Differential pressure in broken loop (DPB-30-43), from -20 to $600 \mathrm{~s}$

194. Differential pressure in broken loop (DPB-30-43), from -6 to $42 \mathrm{~s} \ldots \ldots \ldots \ldots$. . . . . . . . . . . . 120

195. Differential pressure in broken loop (DPB-32U*36L), from -20 to $600 \mathrm{~s}$

196. Differential pressure in broken loop (DPB-32U-36L), from -6 to $42 \mathrm{~s}$

197. Differential pressure in broken loop (DPB-36L-37), from -20 to $600 \mathrm{~s}$

198. Differential pressure in broken loop (DPB-36L-37), from -6 to $42 \mathrm{~s}$

199. Differential pressure in broken loop (DPB-38-40), from -20 to $600 \mathrm{~s}$

200. Differential pressure in broken lnop (DPB-38-40), from -6 to $42 \mathrm{~s}$ 
201. Differential pressure in broken loop (DPB-40-42),

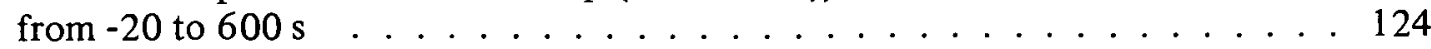

202. Differential pressure in broken loop (DPB-40-42),

from -6 to $42 \mathrm{~s} \ldots \ldots \ldots \ldots \ldots$. . . . . . . . . . . . . . 124

203. Differential pressure in broken loop (DPB-42-HN1),

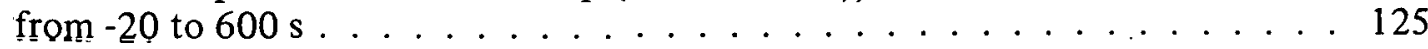

204. Differential pressure in broken loop (DPB-42-HN1),

from -6 to $42 \mathrm{~s}$

205. Differential pressure in broken loop (DPB-HN1-43),

from -20 to $600 \mathrm{~s} \ldots \ldots \ldots \ldots \ldots \ldots$

206. Differential pressure in broken loop (DPB-HN1-43),

from -6 to $42 \mathrm{~s} \ldots \ldots \ldots \ldots \ldots \ldots$

207. Differential pressure in vessel (DPV-UP-IANN),

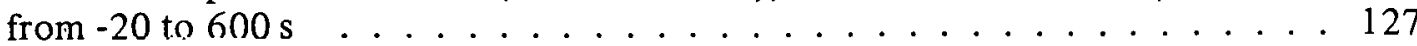

208. Differential pressure in vessel (DPV-UP-IANN),

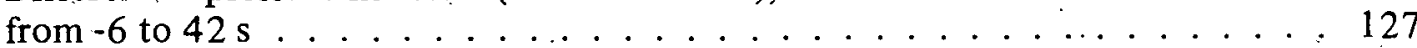

209. Differential pressure in vessel (DPV-O-9GQ),

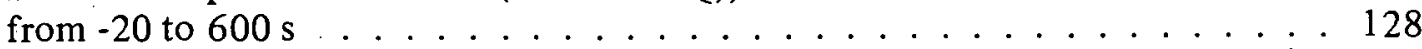

210. Differential pressure in vessel (DPV-U-YG(U),

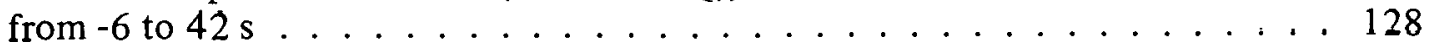

211. Differcntial pressure in vessel (DPV-9-26QQ),

from -20 to $600 \mathrm{~s}$

212. Differential pressure in vessel (DPV-9-26QQ),

from -6 to 42 s

213. Differential pressure in vessel (DPV-9-166QQ),

frnm 20 to $600 \varepsilon \ldots \ldots \ldots \ldots$. . . . . . . . . . . . . . . . . . . .

214. Diflerential pressurc in vessel (DPV-9-166QQ),

from -6 to $42 \mathrm{~s} \ldots \ldots \ldots \ldots$. . . . . . . . . . . . . . . . . . . . . . . . . .

215. Differential pressure in vessel (DPV-26-55QM),

from -20 to $600 \mathrm{~s}$

216. Differential pressure in vessel (DPV-26-55QM),

from -6 to $42 \mathrm{~s}$

217. Differential pressure in vessel (DPV-55-110MM),

from -20 to $600 \mathrm{~s}$

218. Differential pressure in vessel (DPV-55-110MM),

from -6 to $42 \mathrm{~s}$ 
219. Differential pressure in vessel (DP.V-110-156MQ), from -20 to $600 \mathrm{~s} \ldots \ldots \ldots \ldots$. . . . . . . . . . . . . . . . . . . . . . . .

220. Differential pressure in vessel (DPV-110-156MQ), from -6 to $42 \mathrm{~s} \ldots \ldots \ldots \ldots \ldots \ldots \ldots \ldots$

221. Differential pressure in vessel (DPV-156-173QQ), from -20 to $600 \mathrm{~s} \ldots \ldots \ldots \ldots \ldots$

222. Differential pressure in vessel (DPV-156-173QQ), from 6 to $42 \mathrm{~s} \ldots \ldots \ldots \ldots \ldots \ldots \ldots \ldots$

223. Differential pressure in vessel (DPV-166Q+10),

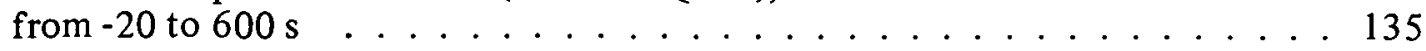

224. Differential pressure in vessel (DPV-166Q+10), from -6 to $42 \mathrm{~s} \ldots \ldots \ldots \ldots \ldots \ldots$. . . . . . . . . . . . . . . . . . . . . . .

225. Differential pressure in intact loop accumulator (DPU-ACC1-TB), from -20 to $600 \mathrm{~s} \ldots \ldots \ldots \ldots \ldots$

226. Differential pressure in intact loop accumulator (DPU-ACC1-TB), from -6 to $42 \mathrm{~s} \ldots \ldots \ldots \ldots \ldots$

227. Differential pressure in broken loop accumulator (DPB-ACC2-TB), from -20 to $600 \mathrm{~s} \ldots \ldots \ldots 137$

228. Differential pressure in broken loop accumulator (DPB-ACC2-TB), from -6 to $42 \mathrm{~s} \ldots \ldots \ldots \ldots \ldots \ldots$

229. Differential pressure in steam generator secondary (DPU-SG-SEC), from -20 to $600 \mathrm{~s} \ldots \ldots \ldots \ldots . \ldots \ldots$

230. Differential pressure in steam generator secondary (DPU-SG-SEC), from -6 to $42 \mathrm{~s} \ldots \ldots \ldots \ldots \ldots \ldots$

231. Differential pressure across steam generator outlet orifice (DPU-SG-DISC), from -20 to $600 \mathrm{~s} \ldots \ldots \ldots . . . \ldots 139$

232. Differential pressure across steam generator outlet orifice (DPU-SG-DISC), from -6 to $42 \mathrm{~s} \ldots \ldots \ldots \ldots$

233. Differential pressure between simulated rupture injection line and Spool 6 (DPU-SGS-6), from -20 to $600 \mathrm{~s} \ldots \ldots \ldots . . . \ldots 140$

234. Differential pressure between simulated rupture injection line and Spool 6 (DPU-SGS-6), from -6 to $42 \mathrm{~s} \ldots \ldots \ldots \ldots$

235. Differential pressure in intact loop tube rupture simulation accumulator (DPU-SGS3-TB), from -20 to $600 \mathrm{~s}$

236. Differential pressure in intact loop tube rupture simulation accumulator (DPU-SGS3-TB), from -6 to $42 \mathrm{~s}$ 
237. Differential pressure in pressure suppression tank

(DP-PSS-TB), from -20 to $600 \mathrm{~s} \ldots \ldots \ldots$. . . . . . . . . 142

238. Differential pressure in pressure suppression tank

(DP-PSS-TB), from -6 to $42 \mathrm{~s} \ldots \ldots \ldots \ldots$. . . . . . . . . . 142

239. Volumetric flow in intact loop (FTU-1 and FTU-9),

from -20 to $600 \mathrm{~s} \ldots \ldots \ldots \ldots . \ldots \ldots 14 \ldots \ldots$

240. Volumetric flow in intact loop (FTU-1 and FTU-9),

from -6 to $42 \mathrm{~s} \ldots \ldots \ldots \ldots$. . . . . . . . . . . . . . . . . . . . . . . . . .

241. Volumetric flow in intact loop (FTU-13 and FTU-15),

from -20 to $600 \mathrm{~s} \ldots \ldots \ldots \ldots . \ldots \ldots$. . . . . . . . . . . . . . . . . . . . . .

242. Volumetrir flow in intact loop (FTU-13 and FTU-15),

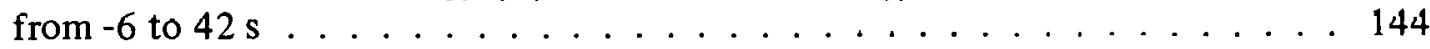

243. Volumetric flow in broken loop (FTB-21),

from -20 to $600 \mathrm{~s}$

244. Volumetric flow in broken loop (FTB-21),

from -6 to $42 \mathrm{~s} \ldots \ldots \ldots \ldots \ldots$. . . . . . . . . . . . . . . . . . . . . . .

245. Volumetric flow in broken loop (FTB-30 and

FTB-37), from -20 to $600 \mathrm{~s} \ldots \ldots \ldots \ldots$. . . . . . . . . 146

246. Volumetric flow in broken loop (FTB-30 and

FI'B-37), from -6 lu $42 \mathrm{~s} \ldots \ldots \ldots \ldots$. . . . . . . . . . . . . . . . . .

247. Volumetric flow in core entrance (FTV-CORE-IN),

from -20 to $600 \mathrm{~s} \ldots \ldots \ldots \ldots$. . . . . . . . . . . . . . . . . . .

248. Volumetric flow in core entrance. (FTV-CORE-IN),

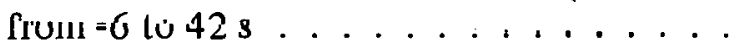

249. Volumetric flow in intact loop high pressure injection line (FTU-HPIS), from -20 to $600 \mathrm{~s} \ldots \ldots \ldots . \ldots . \ldots 148$

250: Volumetric flow in in tact loop high pressure injection line (FTU-HPIS), from -6 to $42 \mathrm{~s} \ldots \ldots \ldots$. . . . . . . . . 148

251. Volumetric flow in broken loop high pressure injection line (FTB-HPIS), from -20 to $600 \mathrm{~s}$

252. Volumetric flow in broken loop high pressure injection line (FTB-HPIS), from -6 to $42 \mathrm{~s}$.

253. Volumetric flow in intact loop low pressure injection line (FTU-LPIS), from -20 to $600 \mathrm{~s}$

254. Volumetric flow in intact loop low pressure injection line (FTU-LPIS), from -6 to $42 \mathrm{~s}$. 
255. Volumetric flow in broken loop low pressure injection line (FTB-LPIS), from -20 to $600 \mathrm{~s}$

256. Volumetric flow in broken loop low pressure injection line (FTB-LPIS), from -6 to $42 \mathrm{~s}$

257. Volumetric flow in intact loop accumulator discharge line (FTU-ACC1), from -20 to $600 \mathrm{~s}$

258. Volumetric flow in in tact loop accumulator dischargé line (FTU-ACC1), from -6 to $42 \mathrm{~s}$

259. Volumetric flow in broken loop accumulator discharge line (FTB-ACC2), from -20 to $600 \mathrm{~s}$

260. Volumetric flow in broken loop accumulator discharge line (FTB-ACC2), from -6 to $42 \mathrm{~s}$

261. Volumetric flow from intact loop steam generator tube rupture simulation accumulator (FTU-SGS), from -20 to $600 \mathrm{~s} \ldots \ldots . . . . .154$

262. Volumetric flow from intact loop steam generator tube rupture simulation accumulator (FTU-SGS), from -6 to $42 \mathrm{~s} \ldots \ldots$. . . . . . . 154

263. Volumetric flow from pressurizer (FTU-PRIZE), from -20 to $600 \mathrm{~s}$

264. Volumetric flow from pressurizer (FTU-PRIZE), from -6 to $42 \mathrm{~s}$

265. Fluid velocity in vessel (FTV-40A and FTV-40M), from -20 to $600 \mathrm{~s}$

266. Fluid velocity in vessel (FTV-40A and FTV-40M), from -6 to $42 \mathrm{~s}$

267. Momentum flux in intact loop (FDU-1), frnm -6 to 42 s

268. Momentum flux in intact loop (FDU-5), from -6 to $42 \mathrm{~s} \ldots \ldots \ldots$. . . .

269. Momentum flux in intact loop (FDU-10), from -6 to $42 \mathrm{~s} \ldots \ldots \ldots \ldots \ldots$. . . . . . . . . . . . . . . .

270. Momentum flux in intact loop (FDU-13), frnm -6 to $42 \mathrm{~s} \ldots \ldots \ldots \ldots \ldots \ldots$

271. Momentum flux in broken loop (FDB-21), from -6 to $42 \mathrm{~s} \ldots \ldots \ldots \ldots \ldots$

272. Momentum flux in broken loop (FDB-23), from -6 to $42 \mathrm{~s}$ 
273. Momentum flux in broken loop (FDB-30),

from -6 to $42 \mathrm{~s} \ldots \ldots \ldots \ldots$. . . . . . . . . . . . . . . . . . . . .

274. Momentum flux in broken loop (FDB-37),

from -6 to $4.2 \mathrm{~s} \ldots \ldots \ldots \ldots$. . . . . . . . . . . . . . . . . . .

275: Momentum flux in broken loop (FDB-42),

from -6 to $42 \mathrm{~s} \ldots \ldots \ldots \ldots \ldots 16 \ldots \ldots \ldots$

276. Momentum flux in core entrance (FDV-CORE-IN),

from -20 to $600 \mathrm{~s} \ldots \ldots \ldots$. . . . . . . . . . . . 162

277 Mnmentum flux in core entrance (FDV-CORE-IN),

from -6 to $42 \mathrm{~s} \ldots \ldots \ldots \ldots$. . . . . . . . . . . . . . . . . . . 62

278. Density in intact loop (GU-1T and GU-1B),

from -20 to $600 \mathrm{~s} \ldots \ldots \ldots \ldots \ldots . \ldots \ldots$

279. Density in in tact loop (GU-1T and GU-1B),

from -6 to $42 \mathrm{~s} \ldots \ldots \ldots \ldots \ldots$

280. Density in intact loop (GU-1C), from

-20 to 600 s . . . . . . . . . . . . . . 164

281. Density in intact loop (GU-1C); from

-6 to $42 \mathrm{~s} \ldots \ldots \ldots \ldots$. . . . . . . . . . . . . . . 164

282. Density in intact loop (GU-5V R and GU-1UVK),

from -20 to $600 \mathrm{~s} \ldots \ldots \ldots \ldots$. . . . . . . . . . . . . . . . . . . 65

283. Density in intact loop (GU-5VR and GU-10VR),

from -6 to $42 \mathrm{~s} \ldots \ldots \ldots \ldots 5$

284.. Density in intact loop (GU-13VR),

from -20 to $600 \mathrm{~s} \ldots \ldots \ldots \ldots . \ldots \ldots$

285. Density in intact loop (GU-13VK),

fron $=6$ to $42.8 \ldots \ldots \ldots 6$

286. Density in intact loop (GU-15T and GU-15B),

from -20 to $600 \mathrm{~s} \ldots \ldots \ldots \ldots$. . . . . . . . . . . . . . . . . . . .

287. Density in intnot. lnop (GU-15T and GIJ-1 5R),

from -6 to $42 \mathrm{~s} \ldots \ldots \ldots \ldots \ldots \ldots$. . . . . . . . . . . . . . . . . . .

288. Density in intact loop (GU-15C),

from -20 to $600 \mathrm{~s} \ldots \ldots \ldots \ldots$. . . . . . . . . . . . . . . . . . .

289. Density in intact loop (GU-15C),

from -6 to $42 \mathrm{~s} \ldots \ldots \ldots \ldots \ldots$. . . . . . . . . . . . 168

290. Density in broken loop (GB-21T and

GB-21 B), from -20 to $600 \mathrm{~s} \ldots \ldots \ldots 9^{\circ}$ 
291. Density in broken loop (GB-21T and

GB-21 B), from -6 to $42 \mathrm{~s}$

292. Density in broken loop (GB-21C),

from -20 to $600 \mathrm{~s}$

293. Density in broken loop (GB-21C),

from -6 to 42

294. Density in broken loop (GB-23VR),

from -20 to $600 \mathrm{~s}$

295. Density in broken loop (GB-23VR),

from -6 to $42 \mathrm{~s}$

296. Density in broken loop (GB-30T and GB-30B),

from -20 to $600 \mathrm{~s}$

297. Density in broken loop (GB-30T and GB-30B),

from -6 to $42 \mathrm{~s}$

298. Density in broken loop (GB-30C),

from -20 to $600 \mathrm{~s}$

299. Density in broken loop (GB-30C),

from -6 to $42 \mathrm{~s}$

300. Density in broken loop (GB-37HZ),

from -20 to $600 \mathrm{~s}$

301. Density in broken loop (GB-37HZ),

from -6 to $42 \mathrm{~s}$

302. Density in broken loop (GB-42VR),

from -20 to $600 \mathrm{~s}$

303. Density in broken loop (GR-42VR), from -6 to $42 \mathrm{~s}$

304. Density in vessel (GV-COR-150HZ), from -20 to $600 \mathrm{~s}$

305. Density in vessel (GV-COR-150HZ), from -6 to $42 \mathrm{~s}$

306. Density in vessel (GVLP-165HZ and GVLP-172HZ), from -20 to $600 \mathrm{~s}$

307. Density in vessel (GVLP-165HZ and GVLP-172HZ), from -6 to $42 \mathrm{~s}$

308. Density in pressurizer (GU-PRIZE), from -20 to $600 \mathrm{~s}$ 
309. Density in pressurizer (GU-PRIZE),

from -6 to $42 \mathrm{~s} \ldots \ldots \ldots \ldots \ldots$. . . . . . . . . . . . . . . . . . . . .

310. Mass flow in intact loop (FDU-1,

GU-1C), from -6 to $42 \mathrm{~s}$

311. Mass flow in intact loop (FTU-1,

GU-1C), from -6 to 42 s

312. Mass flow in intact loop (FDU-5,

GU-5VR), from -6 to $42 \mathrm{~s}$

313. Mass flow in intact loop (FTU-9,

GU-10VR), from -6 to $42 \mathrm{~s}$

314. Mass flow in intact loop (FDU-10,

GU-10VR), from -6 to $42 \mathrm{~s}$

315. Mass flow in intact loop (FDU-13,

GU-13VR), from -6 to $42 \mathrm{~s}$

316: Mass flow in intact loop (FTU-13,

$\mathrm{GU}-13 \mathrm{VR})$, from $=6$ to $42 \mathrm{~s}$

317. Mass flow in intact loop (FTU-15,

GU-1 5C), from -6 to $42 \mathrm{~s}$

318. Mass flow in broken loop (FDB-21,

GB-21C), from -6 to $42 \mathrm{~s}$

319. Mass flow in broken loop (FTB-21,

GB-21C), from -6 to $42 \mathrm{~s}$

320. Mass flow in broken loop (FDB-23,

('B-23VK), trom -6 to $42 \mathrm{~s}$

321. Mass flow in broken loop (FDB-30, GB-3@C), from -6 to $42 \mathrm{~s}$

322. Mass flow in broken loop (FTB-30,

GB-30C), from $=6$ to $42 \mathrm{~s}$.

323. Mass flow in hroken loop (FDB $=37$,

GB-37HZ); from -6 to $42 \mathrm{~s}$.

324. Mass flow in broken loop (FTB-37,

GB-37HZ), from -6 to $42 \mathrm{~s}$.

325. Mass flow in broken loop (FDB-42,

GB-42VR), from -6 to $42 \mathrm{~s}$

326. Mass flow in vessel (FDV-CORE-IN,

GV-COR-150HZ), from -20 to $600 \mathrm{~s}$ 
327. Mass flow in vessel (FDV-CORE-IN,

GV-COR-1 50HZ), from -6 to $42 \mathrm{~s}$

328. Mass flow in vessel (FTV-CORE-IN,

GV-COR-150HZ), from -20 to $600 \mathrm{~s} \ldots \ldots$. . . . . . . . . 190

329. Mass flow in vessel (FTV-CORE-IN, GV-COR-150HZ), from -6 to $42 \mathrm{~s}$

330. Mass flow in pressurizer (FTU-PRIZE,

GU-PRIZE), from -6 to $42 \mathrm{~s} \ldots \ldots \ldots \ldots$. . . . . . . . . . . . . .

331. Core heater rod total power (PWRCOR T-1

and PWRCOR T-2), from -20 to $600 \mathrm{~s}$

332. Core heater rod total power (PWRCOR T-1

and PWRCOR T-2), from -6 to $42 \mathrm{~s} \ldots \ldots \ldots . \ldots 192$

333. Core heater voltage (VOLTCOR-T),

from -20 to $600 \mathrm{~s}$

334. Core heater voltage (VOLTCOR-T),

from -6 to $42 \mathrm{~s}$

335. Core heater total current (AMPCOR-T),

from -20 to $600 \mathrm{~s}$

336. Core heater total current (AMPCOR-T),

from -6 to $42 \mathrm{~s}$

337. Primary pump speed (PUMPU-RPM),

from -20 to $600 \mathrm{~s}$

338. Primary pump speed (PUMPU-RPM),

from -6 to $42 \mathrm{~s}$

B-1. Geometry used for processing of density data

obtained trom two-beam gamma densitometers

C-1. Fluid temperature in broken

loop (TFB-23)

C-2. Fluid temperature in downcomer annulus (TFV-ANN-35A)

C-3. Fluid temperature in lower plenum ('IFV-LP-2)

C-4. Fluid temperature in lower plenum (TFV-LP-4)

C-5. Fluid temperature in lower plenunin (TFV-LP-7) 
C-6. Fluid temperature in core, Grid Spacer 5

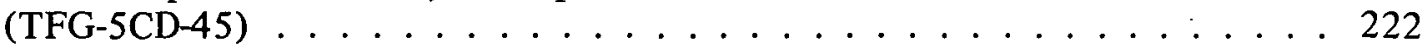

C-7. Fluid temperature in steam generator, secondary side (TFU-SG3) . . . . . . . . . . . . . . . . 223

C-8. Fluid temperature in pressurizer surge line (TFU-PRIZE) . . . . . . . . . . . . . . . 223

C-9. Material temperature in in tact loop (TMU-1T16) . . . . . . . . . . . . . . . . 224

C.-10. Material temperature in vessel filler (TMV-CI-70A) . . . . . . . . . . . . . . . . 224

C-11. Corc heator tomporature, Rod E-4 (TH-E4-09) . . . . . . . . . . . . . . . . . 225

C-12. Core heater temperature, Rod E1(TH-E1-27) . . . . . . . . . . . . . . 225

C-13. Core heater temperature, Rod E-4 (TH-E4-55) . . . . . . . . . . . . . . . . . 226

C-14. Pressure in intact loop, Spool 13 (PU-13)

C-1 5. Pressure in broken loop, Spool 23 (PB-23)

C-16. Differential pressure in intact loop (DPU-6-SGIP)

C-17. Differential pressure in intact loop (DP Ü-SGOP-7)

C-18. Differential pressure in intact lự̆ (DPU-7-10) 228

C-19. Differential pressure in intact loop (DPU-12-10) . . . . . . . . . . . . . . . . 229

$\mathrm{C}=20$. Differcntial pressure in intact loop $($ DPU-12-15) . . . . . . . . . . . . . . . . . . . 229

C-21. Volumetric flow in intact loop (FTU-1)

C-22. Volumetric flow in intact loop (FTU-15) 230

C-23. Volumetric flow in intact loop high pressure injection line (FTU-HPIS) 
C-24. Volumetric flow in in tact loop

low pressure injection line (FTU-LPIS) . . . . . . . . . . . 231

C-25. Volumetric flow in intact loop accumulator discharge line (FTU-ACC1) . . . . . . . . . . . . 232

C-26. Fluid velocity in vessel

(FTV-40A)

C-27. Fluid velocity in vessel (FTV-40M)

C-28. Density in intact loop (GU-1T)

C-29. Density in intact loop

(GU-1B)

C-30. Density in intact loop

(GU-1C)

C-31. Density in intact loop

(GU-10VR)

C-32. Density in intact loop

(GU-15T)

C-33. Density in intact loop

(GU-15B)

C-34. Density in intact loop

(GU-15C)

C-35. Density in broken loop

(GB-23VR)

C-36. Density in vessel

(GV-COR-1 50HZ)

C-37. Density in vessel

(GVLP-165HZ)

C-38. Density in vessel

(GVLP-172HZ)

C-39. Mass flow in intact loop

(FTU-1, GU-1C)

C-40. Mass flow in intact loop (FDU-5, GU-5VR)

C-41. Mass flow in intact loop

(FTU-13, GU-13VK) 
C-42. Mass flow in intact loop

(FTU-15, GU-15C) . . . . . . . . . . . . . . . . 240

C-43. Mass flow in broken loop

(FDB-21, GB-21C) . . . . . . . . . . . . . . . 241

$\mathrm{C}-44$. Mass flow in broken loop

$($ FTB-21, GB-21C) . . . . . . . . . . . . . . . 241

$\mathrm{C}-45$. Mass flow in vessel

(FTV-CORE-IN, GV-COR-150HZ) . . . . . . . . . . . . . . 242

\section{TABLES}

I. Conditions at Blowdown Initiation $\ldots \ldots \ldots \ldots \ldots$

II. Primary Coolant Temperature Distribution

Prior to Rupture ...................... . . 7

III. Water Chemistry Prior to Blowdown ............... 8

IV. Sequence of Events During Test S-28-6 . . . . . . . . . . . . 9

V. Data Presentation for Semiscale Mod-1 Test S-28-6 . . . . . . . . . . 17

B-I. Constants for Pressure Measuremment

Corrections (Test S-28-6) . . . . . . . . . . . . . . . . . . . . 204

B-II. Constants for Differential Pressure

Measurement Correclions (Test S-28-6) . . . . . . . . . . . 206

B-III. Constants for Momentum Flux

Measurement Corrections (Test S-28-6) . . . . . . . . . . . . 207

B-IV. Constants for Density Measurement Conversions

to Engineering Units (Test $\mathrm{S}-28-6) \ldots \ldots$. . . . . . . . . . . . 209

C-I. Random Error Variance (Test S-28-6) . . . . . . . . . . . . 215

C-II. General Measurement Engineering Error

Sources and Frror Values (Test S-28-6) . . . . . . . . . . . . . . . 243

C-III. Time Periods when Flow Regime Errors

were Applied (Test S-28-6) f . . . . . . . . . . . . . . . . . . . . . . . . 248 


\section{EXPERIMENT DATA REPORT FOR SEMISCALE MOD-1}

TEST S-28-6

(STEAM GENERATOR TUBE RUPTURE TEST)

\section{INTRODUCTION}

The Semiscale Mod-1 experiments represent the current phase of the Semiscale Program conducted by EG\&G Idaho, Inc. for the United States Government. The program, which is sponsored by the Nuclear Regulatory Commission through the Department of Energy $[\mathrm{a}]$, is part of the overall program designed to investigate the response of a pressurized water reactor (PWR) system to a hypothesized loss-of-coolant accident (LOCA). The underlying objectives of the Semiscale Program are to quantify the physical processes controlling system behavior during a LOCA and to provide an experimental data base for assessing reactor safety evaluation models. The Semiscale Mod-1 Program has the further objective of providing support to other experimental programs in the form of instrumentation assessment, optimization of test series, selection of test parameters, and evaluation of test results.

Test S-28-6 was conducted July 21, 1977 in the Semiscale Mod-1 system as part of the steam generator tube rupture test series (Test Series 28). This series was designed to obtain thermal-hydraulic response data from blowdown, refill, and reflood transients in a simulated nuclear reactor with a heated core. The specific objective of Test S-28-6 was to refine the definition of the lower limit set by Test S-28-2 and to investigate the range of steam generator tube ruptures over which high peak cladding temperatures can occur. Hardware configuration and test parameters were selected to yield a system response that simulates the response of a PWR to a hypothesized LOCA with subsequent refill and reflood.

The purpose of this report is to present the test data in an uninterpreted but readily usable form for use by the nuclear community in advance of detailed analysis and interpretation. Section II briefly describes the system configuration, procedures, initial test conditions, and events that are applicable to Test S-28-6; Section III presents the data graphs and provides comments and supporting information necessary for in terpretation of the data. A description of the overall Semiscale Program and test series, a more detailed description of the Semiscale Mod-1 system, and a description of the measurement and data processing techniques and uncertainties can be found in Reference 1.

[a] This test was conducted under the Energy Research and Development Administration. 


\section{SYSTEM, PROCEDURES, CONDITIONS, AND EVENTS FOR TEST S-28-6}

The following system configuration, procedures, initial test conditions, and events are specific to Test S-28-6 as indicated.

\section{SYSTEM CONFIGURA'TIUN ANU 'IESI' YKÜEEUURES}

The Semiscale Mod-1 system used for this test consisted of a pressure vessel with internals, including a 40-rod core with 36 electrically heated rods; an in tact loop with steam generator, pump, and pressurizer; a bioken luop with simulated stcam generator, simulated pump, and two rupture assemblies; coolant injection accumulators tor the cold legs of the intact and broken loops, and a heated accumulator for the intact loop hot leg; high and low pressure injection pumps for each of the system loops; and a pressure suppression system with a suppression tank, header, and heated steam supply system. For Test S-28-6, the volume of the lower plenum was reduced to $0.014980 \mathrm{~m}^{3}$ by the addition of a metal filler piece. Semiscale Mod-1 experimental system configuration information is described further in Reference 1. Figures 1 and 2 show the system configuration for Test S-28-6.

For Test S-28-6, 33 rods of the 40-rod electrically heated core were operated at a peak power density of approximately $37.73 \mathrm{~kW} / \mathrm{m}$, three rods (Rods D-4, E-4, and E-5) were operated at a peak power density of $39.70 \mathrm{~kW} / \mathrm{m}$ to yield a slightly peaked power profile, and four rods (Rods $C-3, D-5, F-3$, and $\Gamma \cdot 6$ ) were unpowered to simulate the effect of control rod guide tubes. The resulting total core power was approximately $1.42 \mathrm{MW}$.

In preparation for the test, the accumulators for the intact and broken loop cold legs were filled with treated demineralized water, drained to the specified initial levels, and pressurized with nitrogen to $4223 \mathrm{kPa}$. The steam generator rupture system accumulator for the intact loop hot leg was filled with treated demineralized water, drained to the specified initial level, heated to $531 \mathrm{~K}$, and pressurized to $8225 \mathrm{kPa}$. The system was filled with treated demineralized water and vented at strategic points to ensure a liquid full system. Prior to warmup the system was pressurized to check for leakage, system instrumentation was checked, and transducer readings were initialized. Warmup to initial test conditions was accomplished with the heaters in the vessel core. Heatup of the broken loop piping was accomplished with bypass lines which served to allow circulation through the broken loop. During warmup, the purification and sampling systems were valved into the primary system to maintain water chemistry requirements and to provide a water sample at system conditions for subsequent analysis. At 56-K-temperature intervals during warmup, detector readings were sampled to allow the integrity of the measurement instrumentation and the operability of the data acquisition system to be checked.

Prior to the initial core power level being established, the pressure suppression system

was pressurized to $241 \mathrm{kPa}$ with saturated steam from the steam supply system. After the core power was increased to $1.42 \mathrm{MW}$, initial test conditions were held for $540 \mathrm{~s}$ to establish 


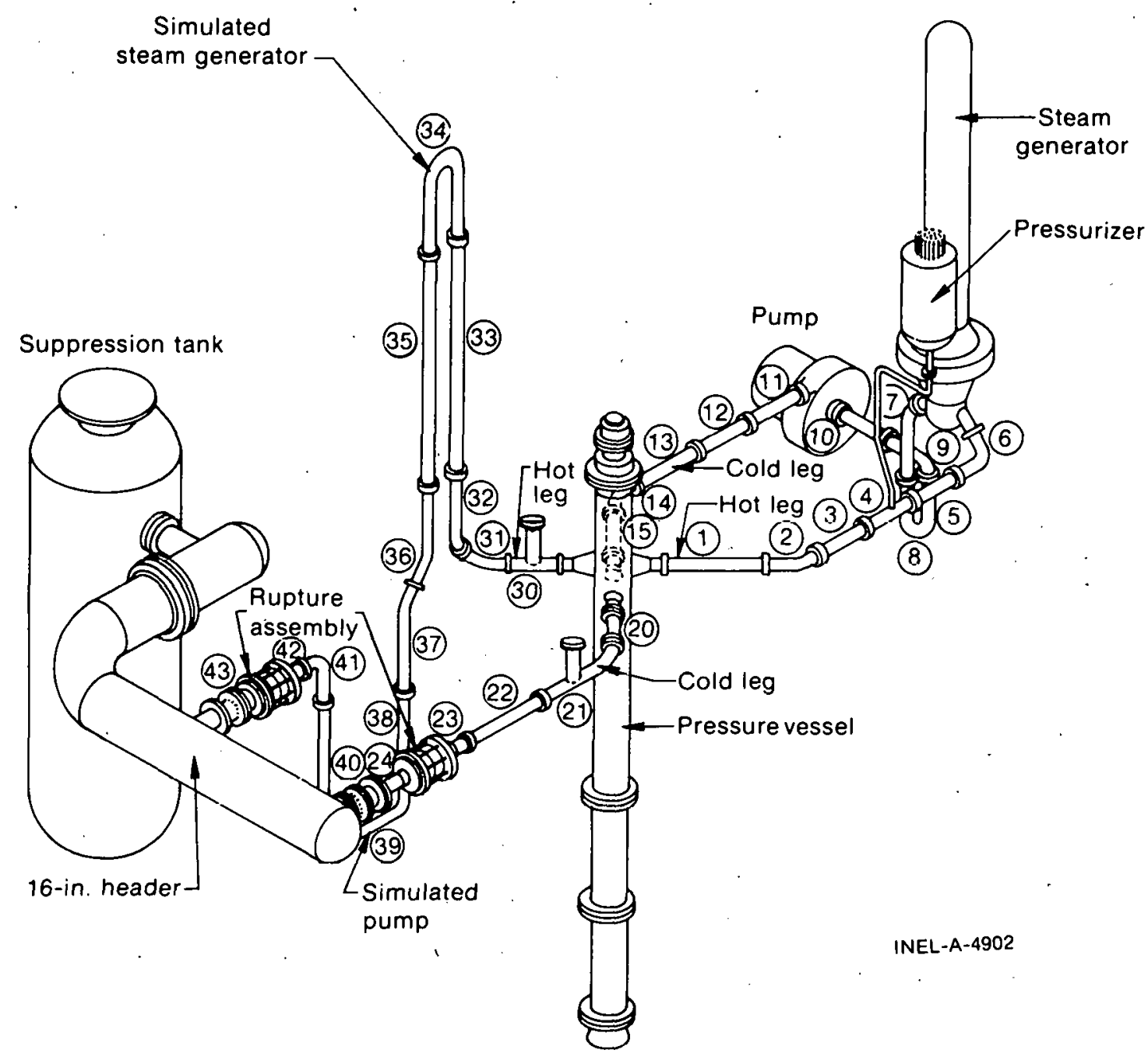

Fig. I Semiscale Mod-1 system for cold leg break configuration -: isometric.

equilibriun in the systemin. At the end of this period all auxiliary systems including the bypass lines were isolated to prevent blowdown through those systems.

The system was successfully subjected to a simulated double-ended cold leg break through two rupture assemblies and two blowdown nozzles, each having a break area of $0.000243 \mathrm{~m}^{2}$. Pressure to operate the rupture assemblies and initiate blowdown was taken from an accumulator system filled with water and pressurized to $15600 \mathrm{kPa}$ with gaseous nitrogen. Immediately (within $0.02 \mathrm{~s}$ ) after initiation of blowdown, the lines to the accumulator were again isolated. 'The effluent was ejected from the primary system in to the pressure suppression system which was vented to maintain a constant pressure of $241 \mathrm{kPa}$. At blowdown, power to the primary coolant circulation pump was reduced and the pump was allowed to coast down to a speed of $1475 \mathrm{rpm}$ which was maintained for the duration of the test. During the blowdown transient, power to the electrically heated core was automatically controlled to simulate the thermal response of nuclear heated fuel rods. 


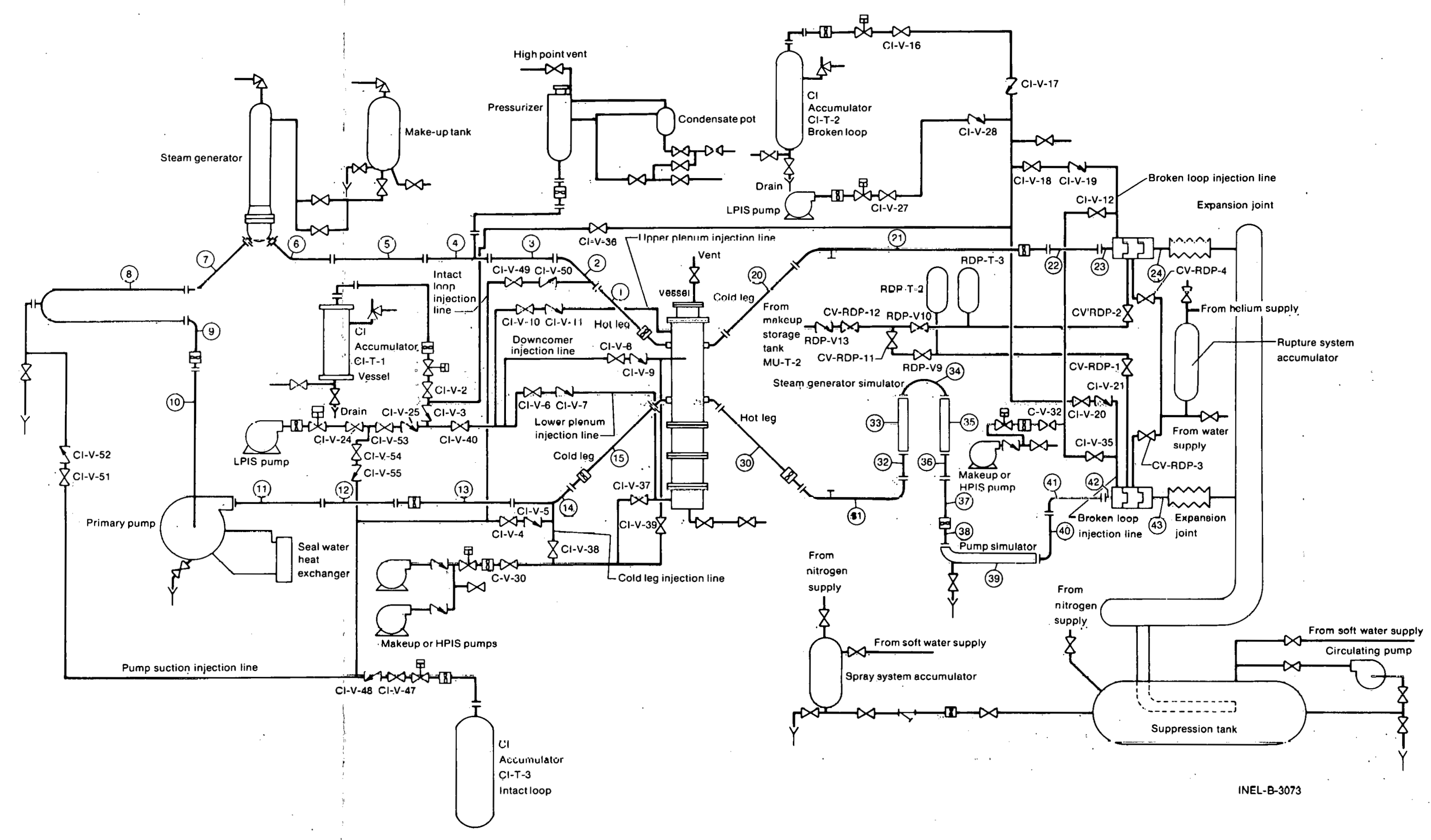

Fig. 2 Semiscale Mod-1 system for cold leg break configuration -schematic. 
For Test S-28-6, the coolant injection systems were arranged to discharge into both system loops at the cold leg injection points (Spool 14 and Spool 42). The high pressure coolant injection pumps were started immediately after initiation of blowdown $(0 \mathrm{~s})$ and continued to the end of the test $(640 \mathrm{~s})$. Low pressure coolant injection was initiated $26 \mathrm{~s}$ after blowdown at a system pressure of $1103 \mathrm{kPa}$ and continued until test termination $(640 \mathrm{~s})$. Coolant injection from the intact loop accumulator (CI-T-1) started $17.0 \mathrm{~s}$ after blowdown, and nitrogen discharge began $65 \mathrm{~s}$ after rupture and was terminated $90 \mathrm{~s}$ after blowdown. The broken loop accumulator (CI-T-2) began injection $3.8 \mathrm{~s}$ after blowdown, nitrogen discharge began $32 \mathrm{~s}$ after rupture and was terminated $67 \mathrm{~s}$ after blowdown. The steam generator rupture system accumulator (CI-T-3) began injection at $40 \mathrm{~s}$ and continued until test termination.

\section{INITIAL TEST CONDITIONS AND SEQUENCE OF EVENTS}

Conditions in the Semiscale Mod-1 system at initiation of blowdown are given in Tables I and II; the primary system water chemistry prior to blowdown is given in Table III; and the sequence of events relative to rupture is given in Table IV. 


\section{TABLE I}

CONDITIONS AT BLOWDOWN INITIATION

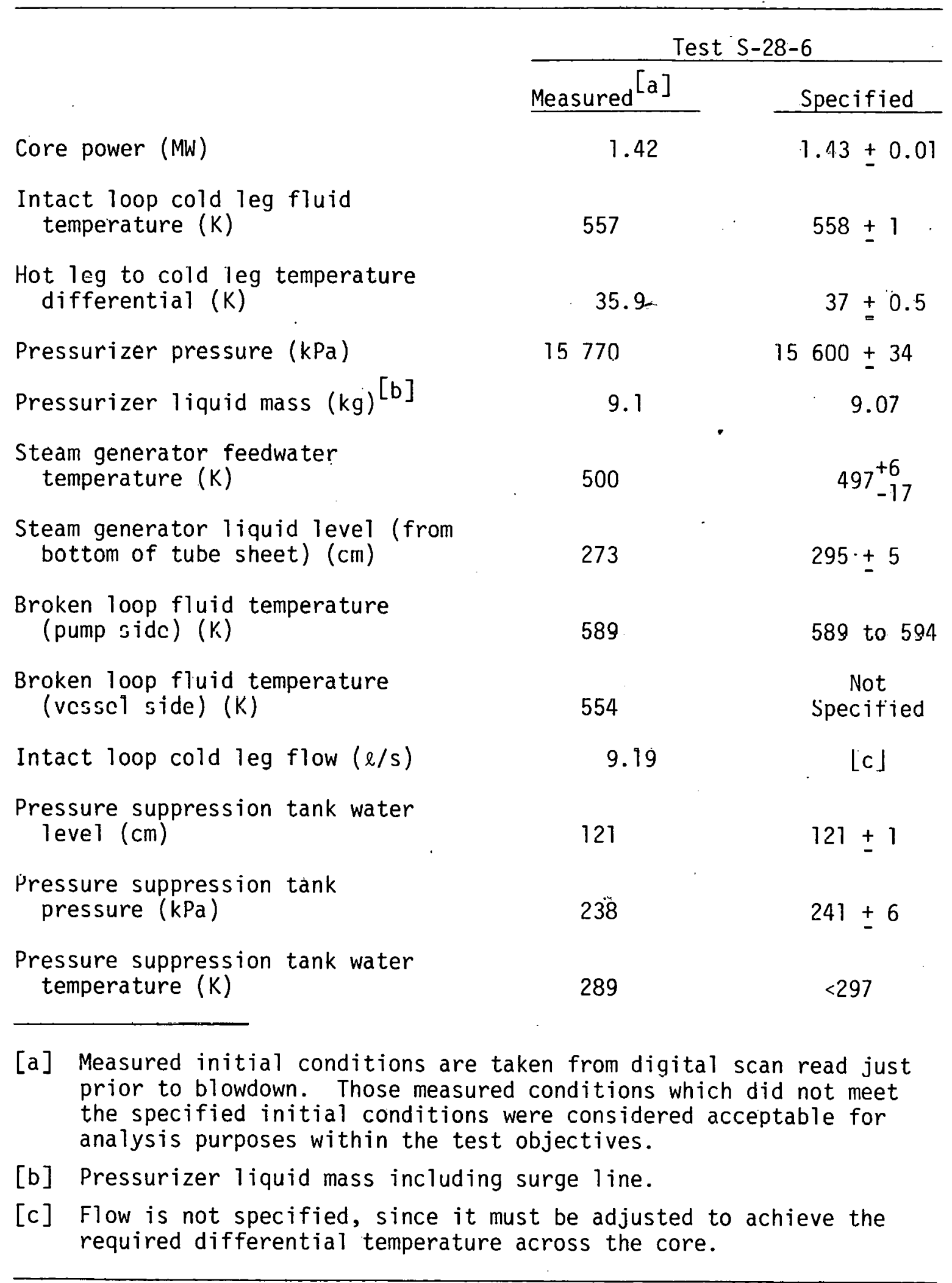




\section{TABLE II}

PRIMARY COOLANT TEMPERATURE DISTRIBUTION PRIOR TO RUPTURE[a]

\begin{tabular}{llc}
\hline & \multicolumn{1}{c}{ Test S-28-6 } \\
\cline { 2 - 2 } $\begin{array}{l}\text { Vessel lower plenum (upper portion } \\
\text { above filler block) }\end{array}$ & Detector & Temperature (K) \\
Intact loop hot leg (near vessel) & TFV-LP-7 & 557 \\
Intact loop cold leg (near pump inlet) & RBU-2 & 593 \\
Intact loop cold leg (near vessel) & RBU-10 & 556 \\
Broken loop cold leg (near nozzle) & TFB-23 & 554 \\
Broken loop hot leg (near vessel) & TFB-30 & 589 \\
Broken loop cold leg (near nozzle) & TFB-42 & 589 \\
\hline [a] Data taken from final digital scan 0.5 S before blowdown. \\
\hline
\end{tabular}




\section{TABLE III}

WATER CHEMISTRY PRIOR TO BLOWDOWN ${ }^{[a]}$

Test S-28-6

$\mathrm{pH}$

9.90

Conductivity $(\mu \mathrm{S} / \mathrm{cm})$

100.0

Lithium $(\mu \mathrm{g} / \mathrm{m} \ell)$

4.4

Chlorides ( $\mu \mathrm{g} / \mathrm{ml})$

0.84

Fluorides $(\mu \mathrm{g} / \mathrm{m} \Omega)^{[b]}$

$-0.5$

Oxygen $(\mathrm{cc} / \ell)$

2.2

Total gas $(\mathrm{cc} / \mathrm{l})$

48.0

Suspended solids $(\mu \mathrm{g} / \mathrm{m} \ell)$

1.75

[a] Water sample taken at a system pressure of approximately $15603 \mathrm{kPa}$ and a system temperature of approximately $555 \mathrm{~K}$ (cold leg).

[b] Present analytical methods prevent accurate determination of fluorides at concentrations of less than $0.4 \mu \mathrm{g} / \mathrm{ml}$. 
TABLE IV

SEQUENCE OF EVENTS DURING TEST S-28-6 ${ }^{\text {[a] }}$

\section{Event}

Core power level established

Bypass lines valved out of system

Blowdown initiated

Pump power reduced

High pressure injection system pumps started ${ }^{[b]}$

ECC accumulators valved in

Steam generator feedwater and discharge valves closed

Core power decay transient started

Low pressure injection system pumps

$$
\text { started }{ }^{[\mathrm{b}]}
$$

Core power tripped off $[\mathrm{c}]$
Time Relative to Rupture (s) $-584$ $-2.5$ 0 0 0

[a] A time-controlled sequencer was used to control critical events during the test.

[b] Injection from ECC accumulators and high and low pressure injection system pumps does not start until system pressure drops below accumulator or pump pressure, respectively.

[c] Core power tripped manually at termination of test. 


\section{DATA PRESENTATION}

The data from Semiscale Mod-1 Test S-28-6 are presented with brief comment. Processing analysis has been performed only to the extent necessary to obtain appropriate engineering units and to ensure that the data are reasonable and consistent. In all cases, in converting transducer output to engineering units, a homogeneous fluid was assumed. Further interpretation and analysis should consider that sudden decompression processes such as those occurring during blowdown may have subjected the measurement devices to nonhomogeneous fluid conditions.

The performance of the system during Test S-28-6 was monitored by 219 detectors. The data obtained were recorded on both digital and analog data acquisition systems. The analog system was used to provide redundant data. The long-term data ( -32 to $640 \mathrm{~s})$ presented in this report were recorded at an effective sample rate of 1.369 points per second. Long-term plots are given for -20 to $600 \mathrm{~s}$ rather than for the full 640 -s recording time to provide better resolution. Short-term data and plots ( -6 to $42 \mathrm{~s}$ ) were recorded at an effective sample rate of 19.17 points per second.

The data are presented in some instances in the form of composite graphs to facilitate comparison of the values of given variables at several locations. The scales selected for the graphs do not reflect the obtainable resolution of the data. (The data processing techniques are described further in Reference 1 and Appendix A).

Figures 3 through 8 and Table $V$ provide supporting information for interpretation of the data graphs shown in Figures 9 through 338 and provide relative locations of all detectors used during Test S-28-6. Table V groups the measurements according to measurement type; identifies the specific measurement location and range of the detector and actual recording range of the data acquisition system; provides brief comments regarding the data; and references the measurements and comments to the corresponding figure. Figures 9 through 338 present all the blowdown and reflood data obtained. Time zero on the graphs is the time of rupture initiation. Appendix A provides information explaining the data acquisition system capabilities. Appendix $\bar{B}$ provides information explaining posttest data processing for data conversion into engineering units and dala adjustments. Presented in Appendix $C$ is an analysis of selected data which provide a guide to the uncertainty associated with data measurements in the Semiscale Mod-1 system. 


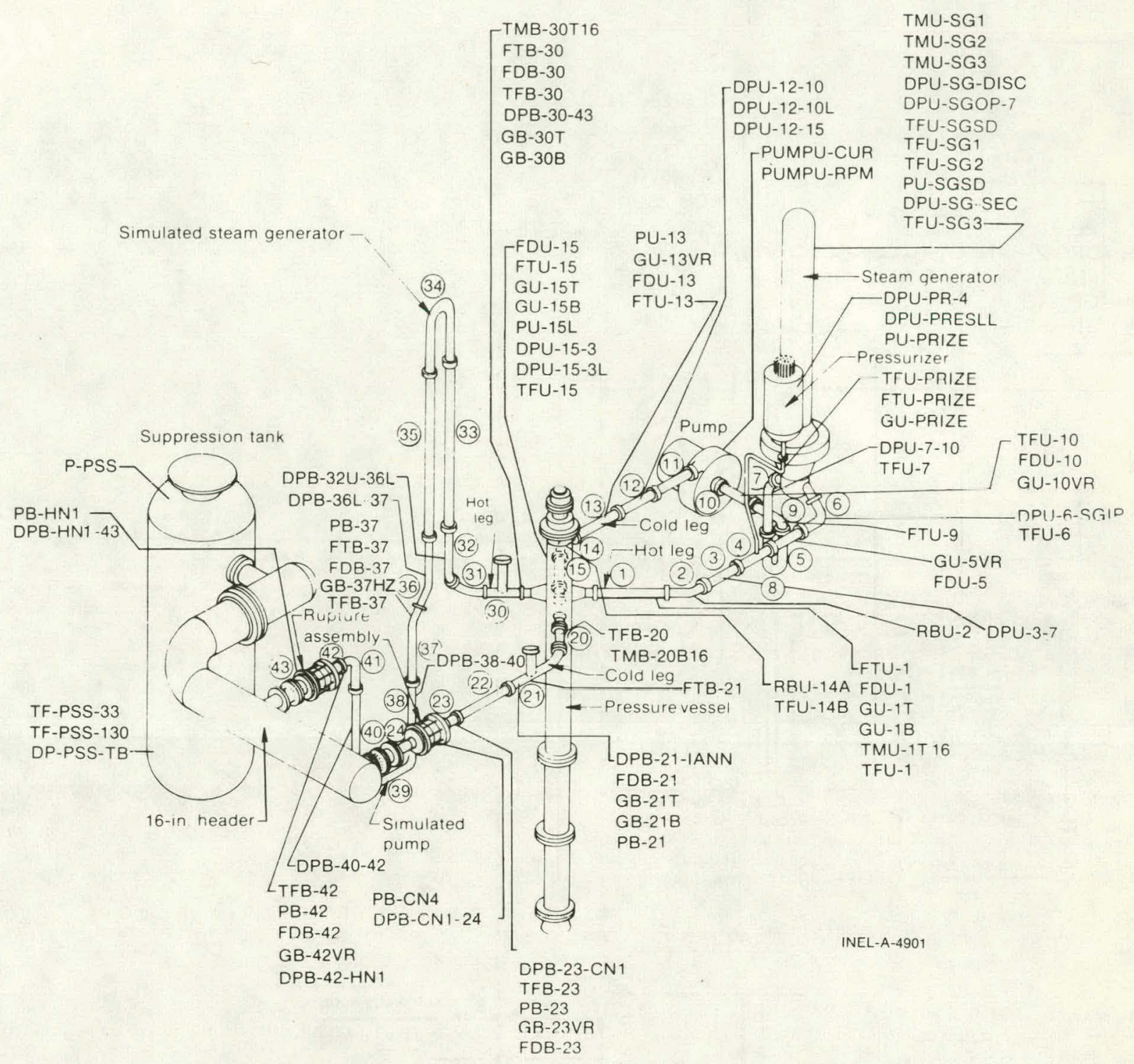

Fig. 3 Semiscale Mod-1 system and instrumentation for cold leg break configuration -- isometric. 


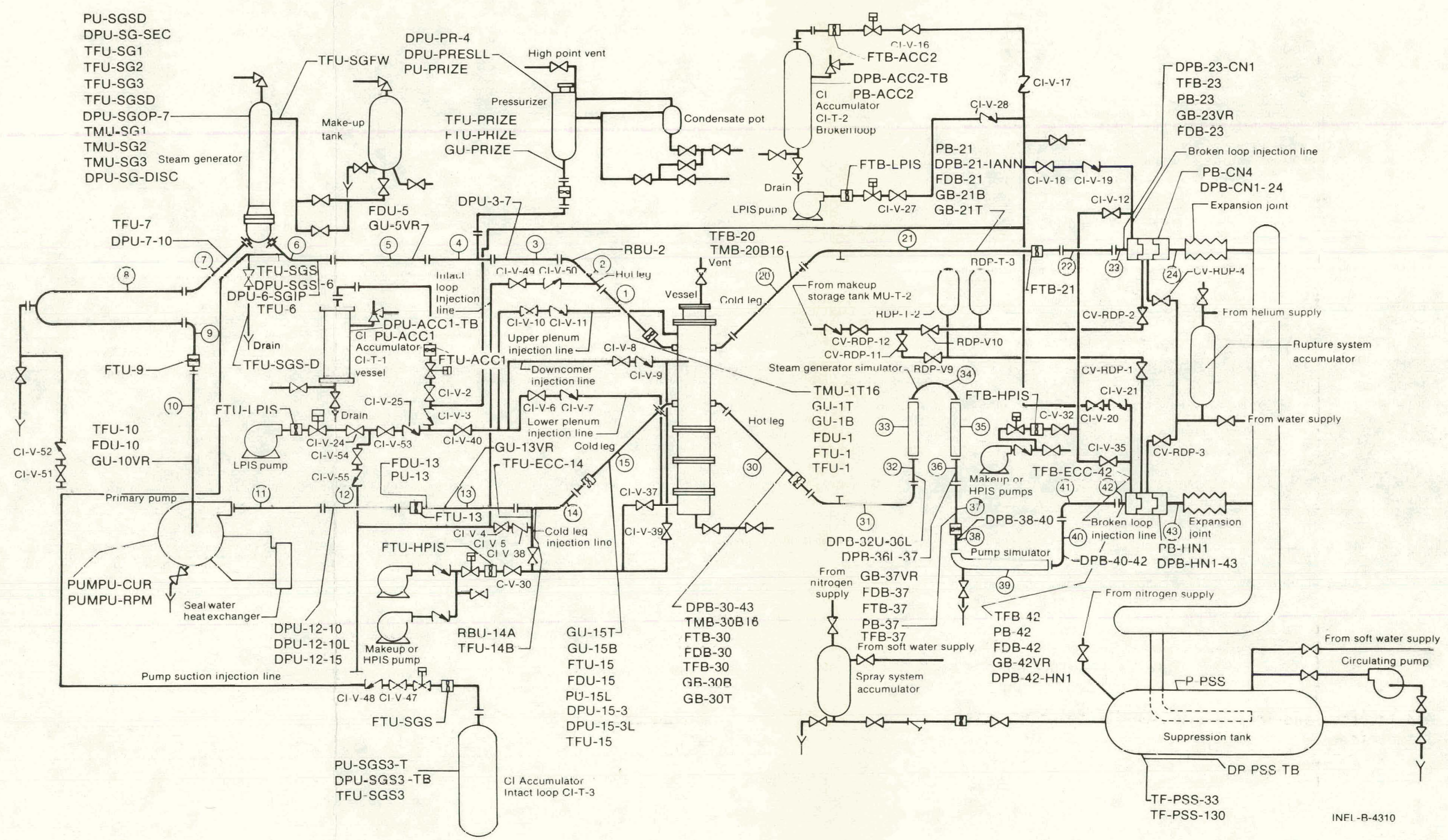

Fig. 4 Semiscale Mod-1 system and instrumentation for cold leg break configuration -- schematic. 


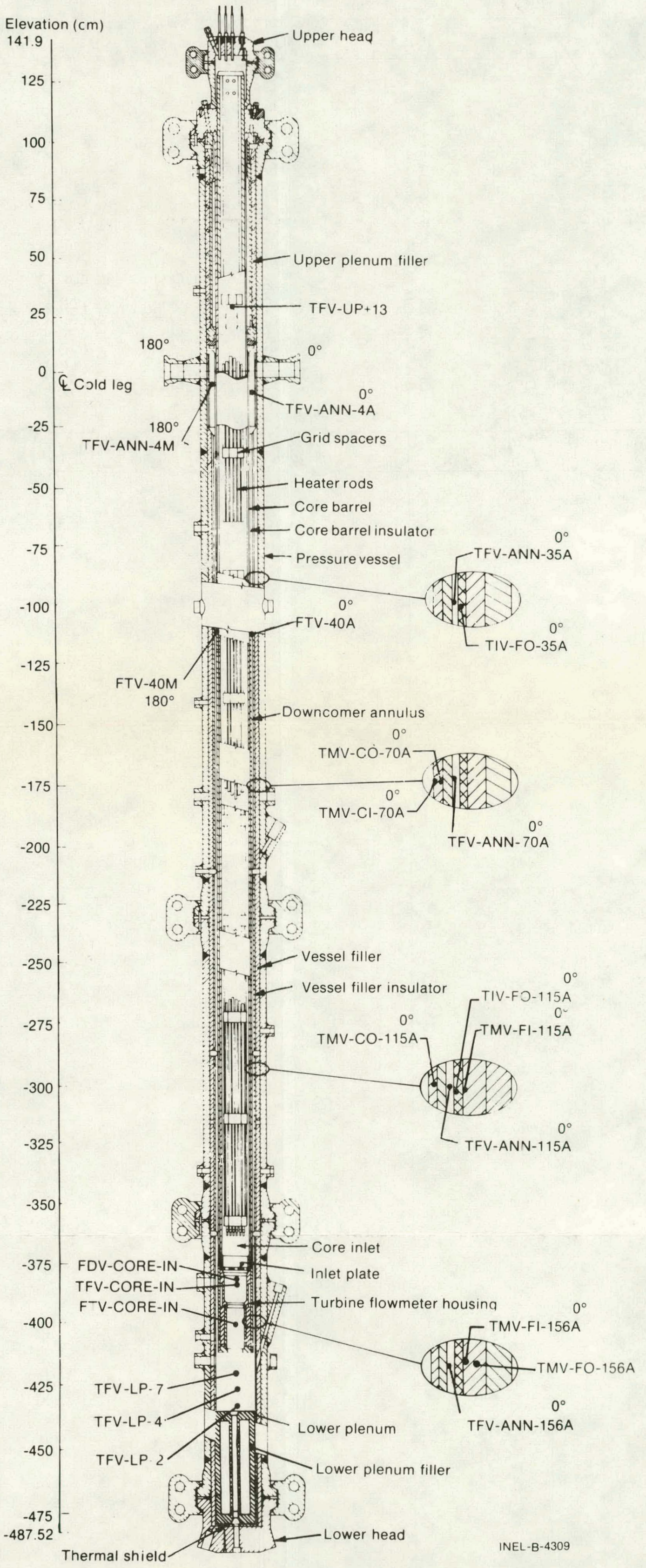

Fig. 5 Semiscale Mod-1 pressure vessel -- cross section showing instrumentation. 


\section{Distance from} cold leg $\&(\mathrm{~cm})$

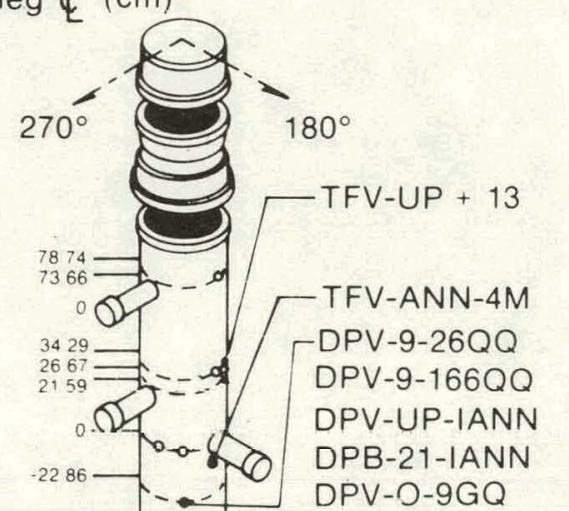

$\begin{array}{r}r_{1}, r_{n} n \\ -6795 \\ \hline\end{array}$
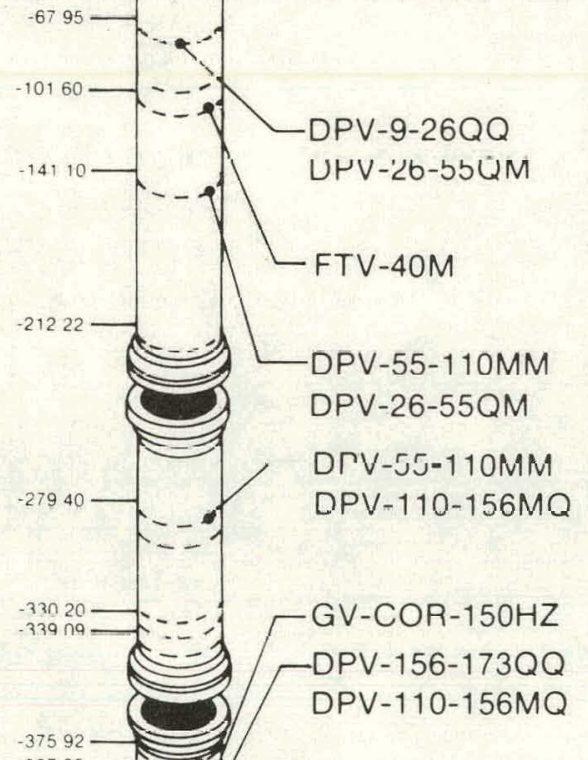

$-37592$

-30795
-39624

$-39624$

$.40894-G V L P-165 \mathrm{HZ}$

$-419104-\cdots$

$-43688=-2=4$ CiVI P-172H

-44958 - 428

45720 DPV-156-173QQ

$48,52-1+3$

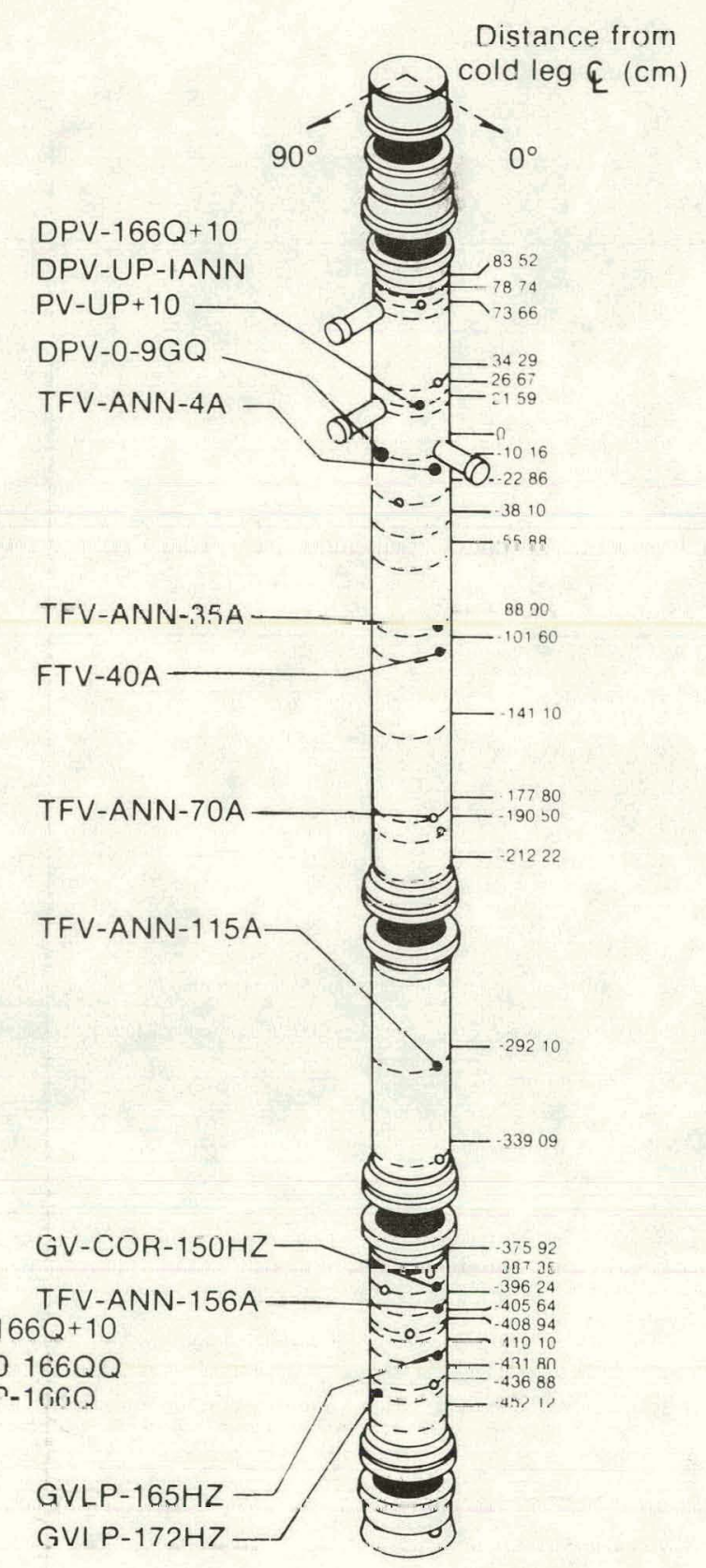

INEL-A-4307

Fig. 6 Semiscale Mod-1 pressure vessel -- isometric showing instrumentation. 


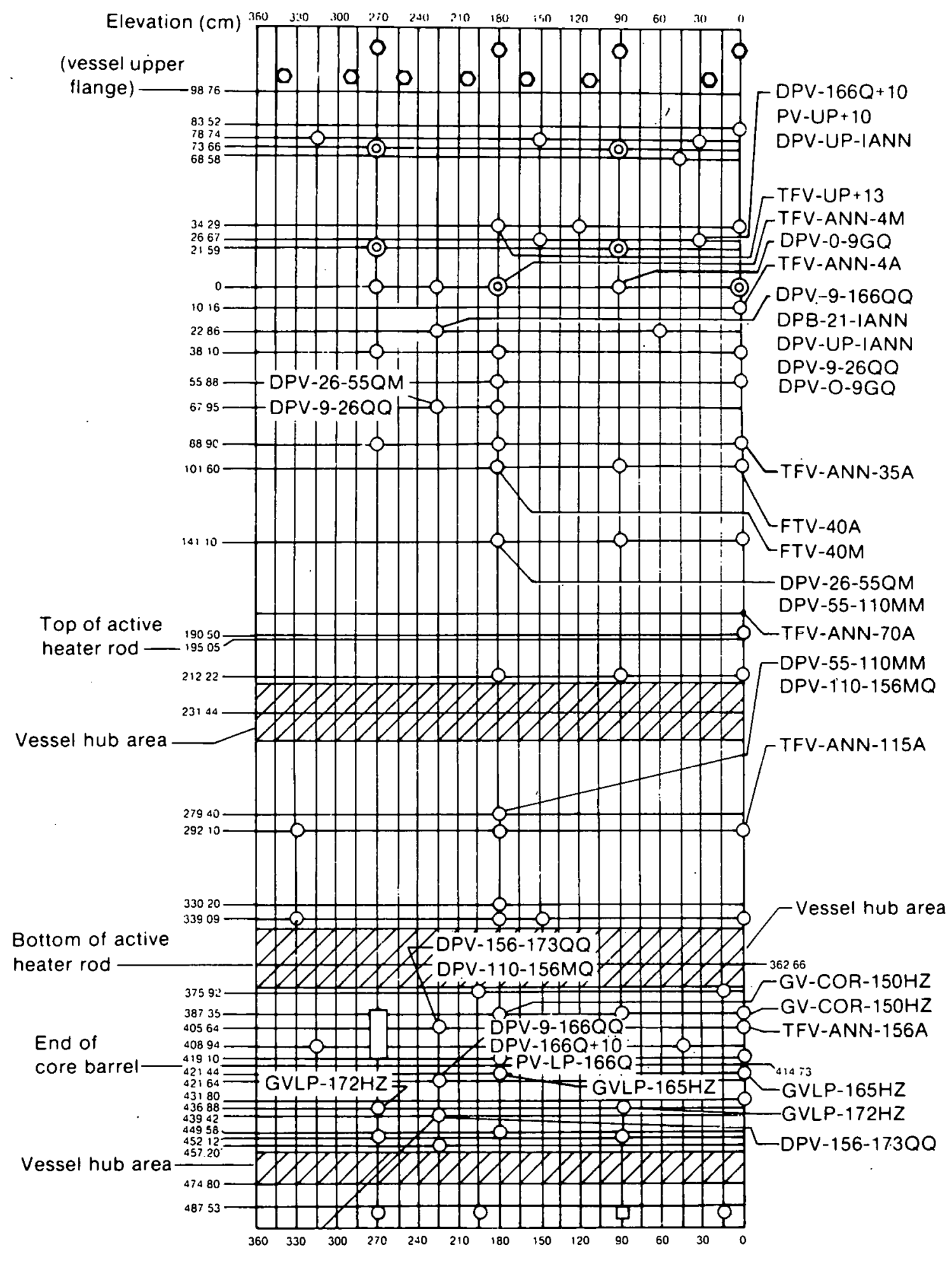

INEL-A-4306

Fig. 7 Semiscale Mod-1 pressure vessel -- penetrations and instrumentation. 
$\begin{array}{lllllllll}\text { A } & \text { B } & \text { C } & \text { D } & \text { E } & \text { F } & \text { G } & \text { H }\end{array}$

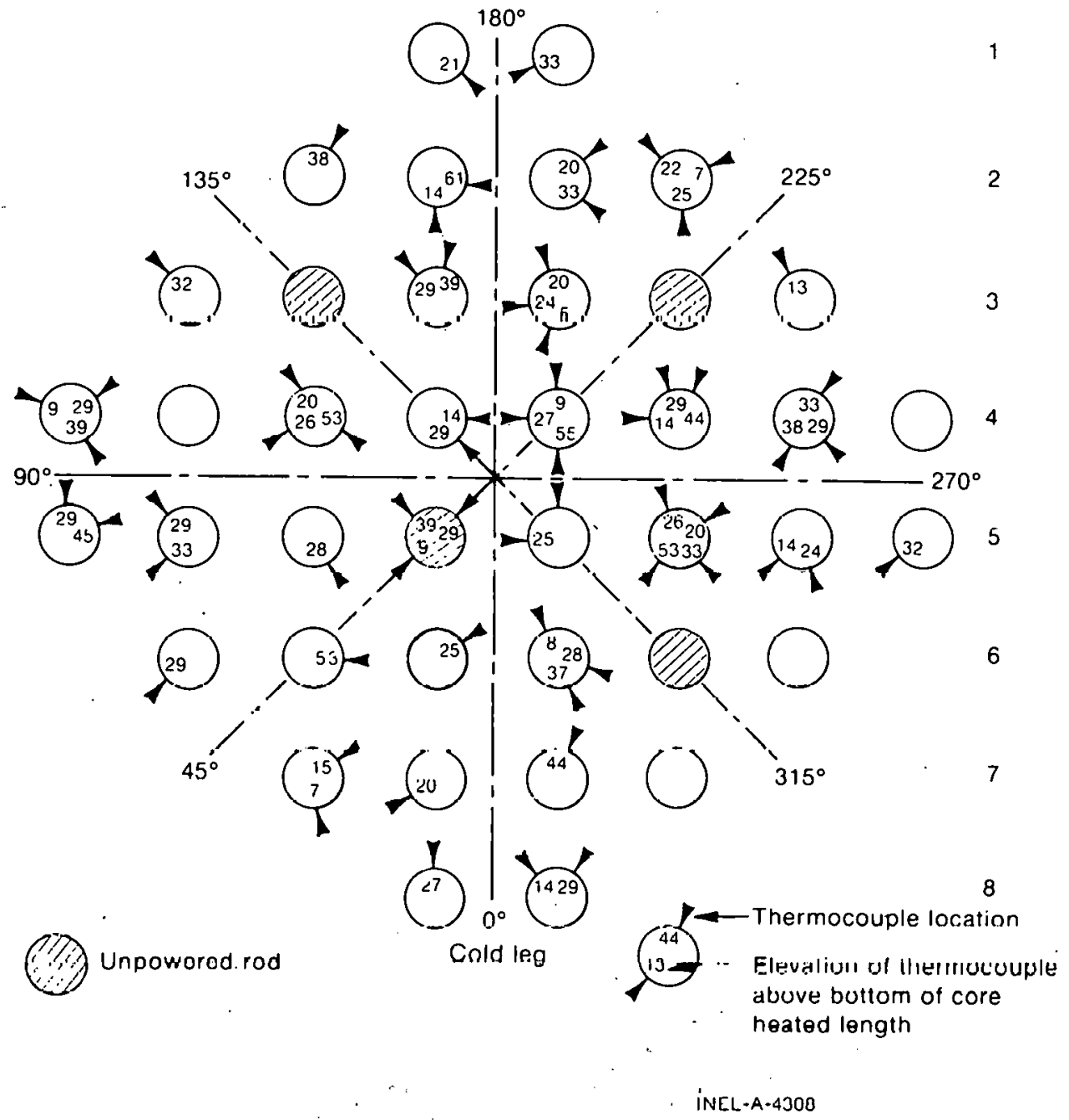

Fig. 8 Semiscale Mod-1 heated core -- plan view. 
TABLE V

DATA PRESENTATION FOR SEHISCALE 1100-1 TEST S-28-6

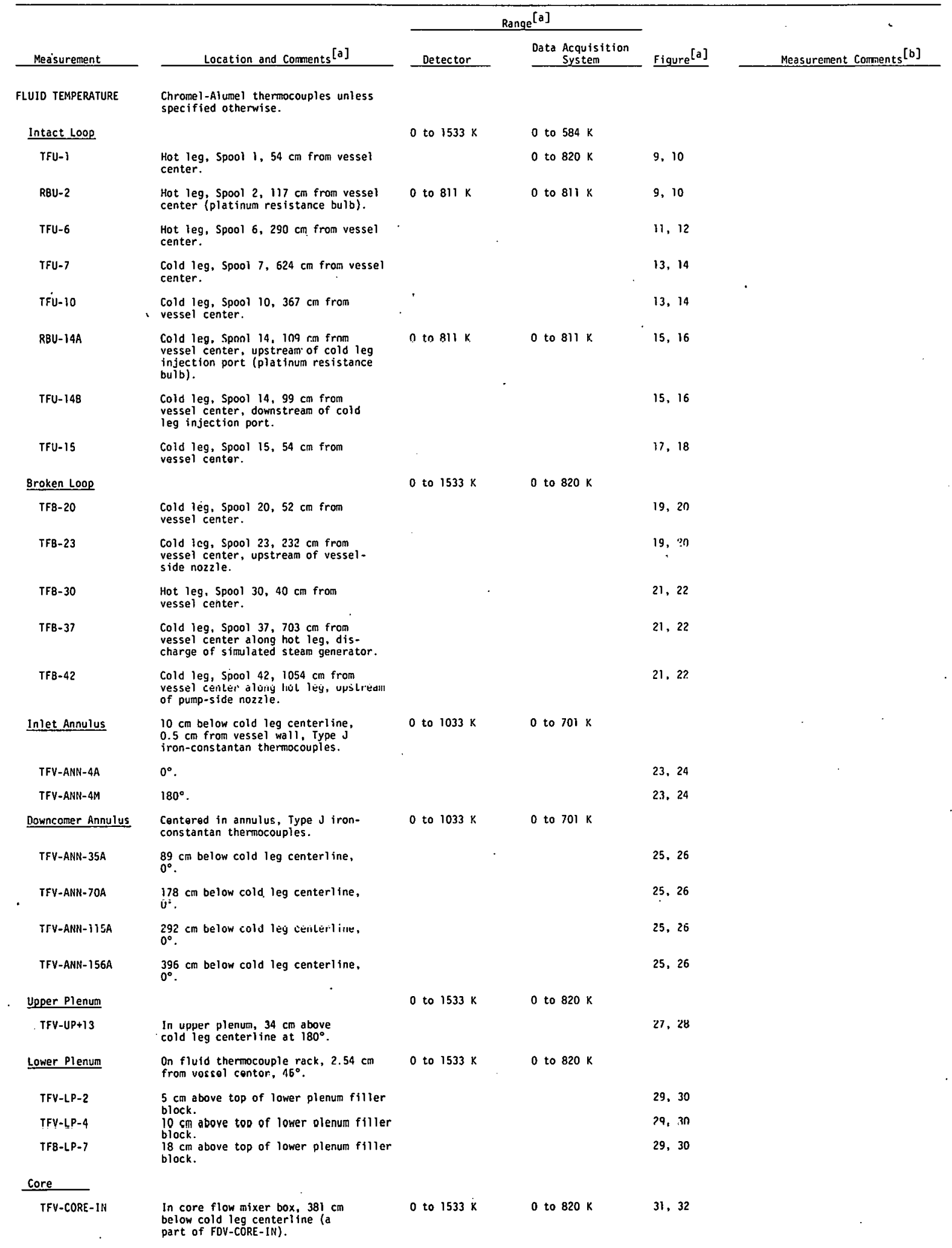


TABLE V (cont inued)

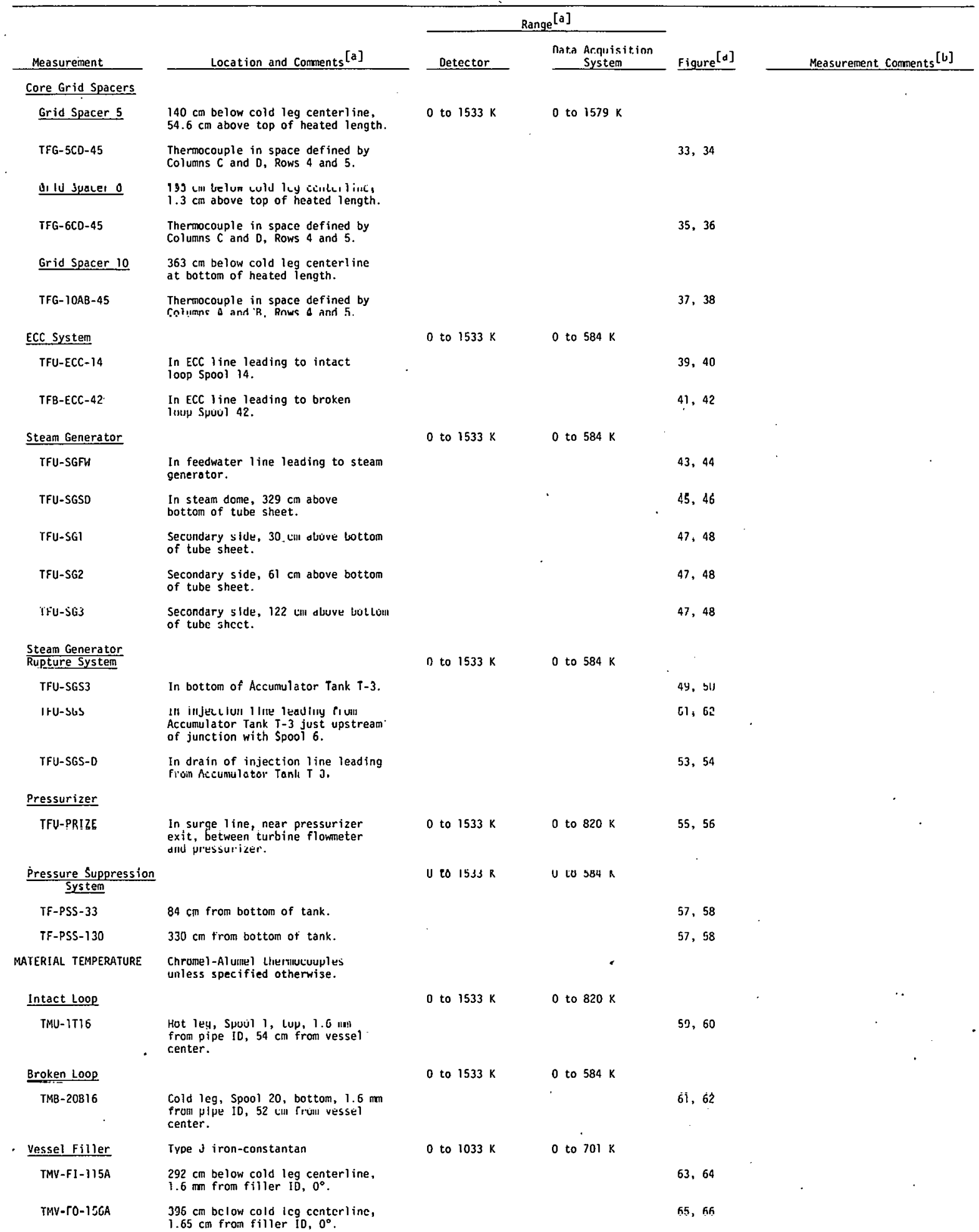


TABLE V (continued)

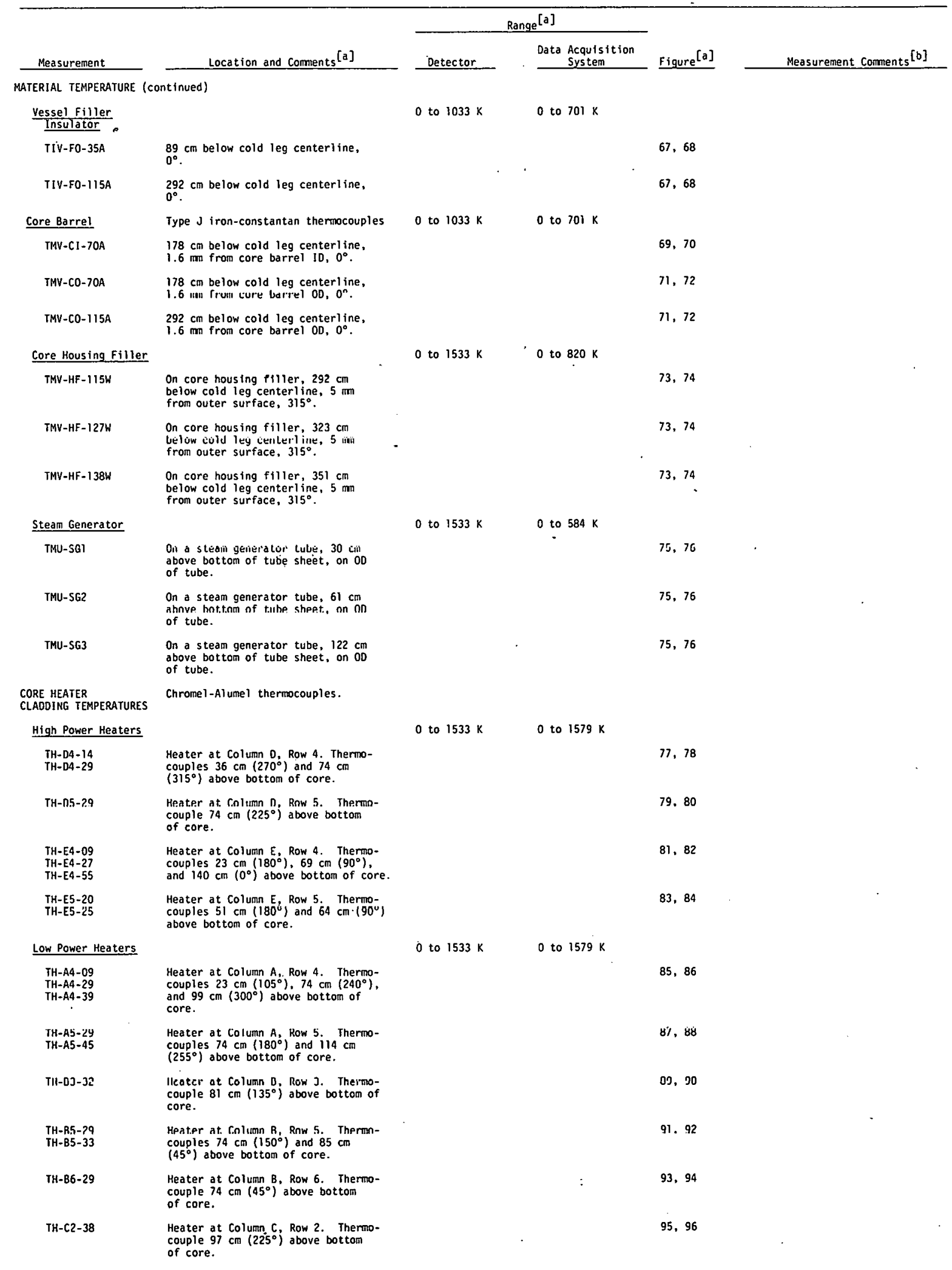




\begin{tabular}{|c|c|c|c|c|c|c|}
\hline \multirow[b]{2}{*}{ Measurement } & \multirow[b]{2}{*}{ Location and comments ${ }^{[a]}$} & \multicolumn{2}{|c|}{ Range $^{[a]}$} & \multirow{3}{*}{$\underline{\text { Figure }}^{[a]}$} & & \multirow[b]{2}{*}{ Measurement Comments ${ }^{[b]}$} \\
\hline & & Detector & $\begin{array}{c}\text { Uata Acquisition } \\
\text { System }\end{array}$ & & & \\
\hline \multicolumn{6}{|l|}{$\begin{array}{l}\text { Low Power Heaters } \\
\text { (continued) }\end{array}$} & \\
\hline $\begin{array}{l}\text { TH-C4-20 } \\
\text { TH-C4-26 } \\
\text { TH-C4-53 }\end{array}$ & $\begin{array}{l}\text { Heater at Column c, Row } 4 \text {. Thermo- } \\
\text { couples } 51 \mathrm{~cm}\left(150^{\circ}\right), 66 \mathrm{~cm}\left(75^{\circ}\right) \text {. } \\
\text { and } 135 \mathrm{~cm}\left(300^{\circ}\right) \text { above bottom } \\
\text { of core. }\end{array}$ & & & 97,98 & & \\
\hline TII sE 38 & 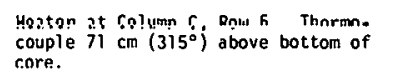 & & & 99. 10ก & & . \\
\hline TH-C6-53 & $\begin{array}{l}\text { Heater at column } C_{C} \text {, Row } 6 \text {. Thermo- } \\
\text { couple } 135 \mathrm{~cm}\left(270^{\circ}\right) \text { above bottom } \\
\text { of core. }\end{array}$ & & & $101, .302$ & & \\
\hline $\begin{array}{l}\text { TH-C7-07 } \\
\text { TH-C7-15 }\end{array}$ & $\begin{array}{l}\text { Heater at column C. Row } 7 \text {. Thermo- } \\
\text { couples } 18 \mathrm{~cm}\left(345^{\circ}\right) \text { and } 38 \mathrm{~cm}\left(255^{\circ}\right) \\
\text { above bot tom of core. }\end{array}$ & & & 103,104 & & \\
\hline TH-DI-21 & $\begin{array}{l}\text { Heater at column D, Row 1. Thermo- } \\
\text { coupie } 53 \mathrm{~cm}\left(330^{\circ}\right) \text { above bottom of } \\
\text { core. }\end{array}$ & & & 105,106 & . & \\
\hline $\begin{array}{l}T H-02-14 \\
T H-02-61\end{array}$ & $\begin{array}{l}\text { Heater at coiumn o, Row } 2 \text {. Thermo- } \\
\text { couples } 36 \mathrm{~cm}\left(0^{\circ}\right) \text { and } 155 \mathrm{~cm}\left(270^{\circ}\right) \\
\text { above bottom of core. }\end{array}$ & & & 107,108 & & \\
\hline $\begin{array}{l}14-43-29 \\
T H-03-39\end{array}$ & $\begin{array}{l}\text { Heater at culumin }{ }^{0} \text { fuw } 3 \text {. Thermo- } \\
\text { couples } 74 \mathrm{~cm}\left(150^{\circ}\right) \text { and } 9 g \mathrm{~cm} \\
\left(210^{\circ}\right) \text { above bottom of core. }\end{array}$ & & & IUy, เiu & & \\
\hline$\tau H-06-25$ & 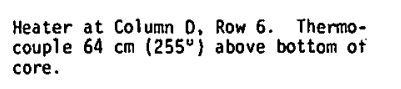 & & & 111,112 & & \\
\hline TH-07-20 & $\begin{array}{l}\text { Heater at Column o, Row } 7 \text {. Thermo- } \\
\text { couple } 51 \mathrm{~cm}\left(60^{\circ}\right)^{\circ} \text { above butluil of } \\
\text { core. }\end{array}$ & & & 113,114 & & \\
\hline Tाн-08-26 & $\begin{array}{l}\text { Heater at column } D_{0} \text { Row 8. Thermo- } \\
\text { couple } 69 \mathrm{~cm}\left(180^{\circ}\right) \text { above bottom of } \\
\text { core. }\end{array}$ & & $\cdot$ & 115,116 & & \\
\hline$T H-E T-33$ & $\begin{array}{l}\text { Heater at calumn E, Row 1. Thermo- } \\
\text { couple } 84 \mathrm{~cm}\left(60^{\circ}\right)^{2} \text { above bottom of } \\
\text { core. }\end{array}$ & & & $117, .118$ & & \\
\hline TH-E2-33 & $\begin{array}{l}\text { Heater at cóliuinith to bow ce. Ineprmo- } \\
\text { couple } 84 \mathrm{~cm}\left(315^{\circ}\right) \text { above bottom of } \\
\text { corc. }\end{array}$ & & & 119,180 & & \\
\hline $\begin{array}{l}T H-E 3-05 \\
T H-E 3-20 \\
T H-E 3-24\end{array}$ & $\begin{array}{l}\text { Heater at column E, Row } 3 \text {. Thermo- } \\
\text { couples } 13 \mathrm{~cm}\left(15^{\circ}\right) \text {, } 51 \mathrm{~cm}\left(165^{\circ}\right) \text {, } \\
\text { and } 61 \mathrm{~cm}\left(75^{\circ}\right) \text { above bottom of } \\
\text { coro. }\end{array}$ & & & 121,122 & & \\
\hline $\begin{array}{l}\text { TH-E6-08 } \\
\text { TH-E6-28 } \\
\text { TH-E6-37 }\end{array}$ & $\begin{array}{l}\text { Heater at Column E, Row 6. Thermo- } \\
\text { couples } 20 \mathrm{~cm}\left(10^{\circ}\right), 71 \mathrm{~cm}\left(285^{\circ}\right) \text {, } \\
\text { and } 94 \mathrm{~cm}\left(330^{\circ}\right) \text { above bottom } \\
\text { of corre. }\end{array}$ & & & 123.124 & & \\
\hline$T H-E 7-44$ & 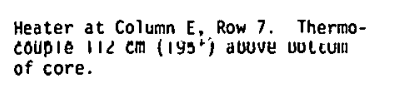 & & & 125,126 & & \\
\hline $\begin{array}{l}\text { TH-E8-14 } \\
\text { TH-EB-2乌 }\end{array}$ & 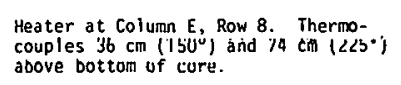 & & & 127,128 & & \\
\hline $\begin{array}{l}T H-F 2-07 \\
T H-F 2-22 \\
T H-F 2-25\end{array}$ & $\begin{array}{l}\text { Heater at Column } F_{i} \text {, Row } 2 . \text { Thermo- } \\
\text { couples } 18 \mathrm{~cm}\left(255^{\circ}\right), 56 \mathrm{~cm}\left(105^{\circ}\right) \text {, } \\
\text { and } 64 \mathrm{~cm}\left(0^{\circ}\right) \text { above bottom of core. }\end{array}$ & & & 129,130 & & \\
\hline $\begin{array}{l}T H-F 4-14 \\
T H-F 4-29 \\
T H-F 4-44\end{array}$ & $\begin{array}{l}\text { Heater at column F, Row } 4 \text {. Thermo- } \\
\text { couples } 36 \mathrm{~cm}\left(90^{\circ}\right), 74 \mathrm{~cm}\left(165^{\circ}\right) \text {, } \\
\text { and } 112 \mathrm{~cm}\left(210^{\circ}\right) \text { above bottom of } \\
\text { core. }\end{array}$ & & & $|3|, 132$ & . & \\
\hline $\begin{array}{l}T H-F 5-20 \\
T H=P G-2 \pi \\
T H-F 5-33 \\
T H-F 5-53\end{array}$ & 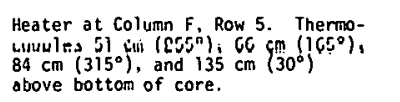 & & & 133,134 & & . \\
\hline TH-63-13 & $\begin{array}{l}\text { Heater at Column G, Row 3. Therno- } \\
\text { couples } 33 \mathrm{cmm}\left(150^{\circ}\right) \text { above bottom of } \\
\text { corc. }\end{array}$ & & & 135,136 & & \\
\hline $\begin{array}{l}T H-64-29 \\
T H-64-33 \\
\mid H-6,1-30\end{array}$ & 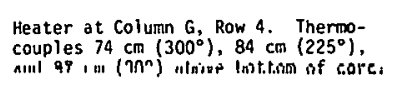 & & & $13 \%, 138$ & & \\
\hline $\begin{array}{l}\text { TH- } 65-14 \\
\text { TH- } 65-24\end{array}$ & $\begin{array}{l}\text { Heater at column G, Row } 5 \text {. Thermo- } \\
\text { couples } 36 \mathrm{~cm}\left(45^{\circ}\right) \text { and bi } \mathrm{cm}\left(330^{\circ}\right) \\
\text { above bottom of core. }\end{array}$ & & & 139,140 & & \\
\hline TH-H5-32 & $\begin{array}{l}\text { Heater at Column H, Row 5. Thermo- } \\
\text { couple } 81 \mathrm{~cm}\left(45^{\circ}\right) \text { above bottom of } \\
\text { core. }\end{array}$ & & & 141,142 & & \\
\hline
\end{tabular}


TABLE V (continued)

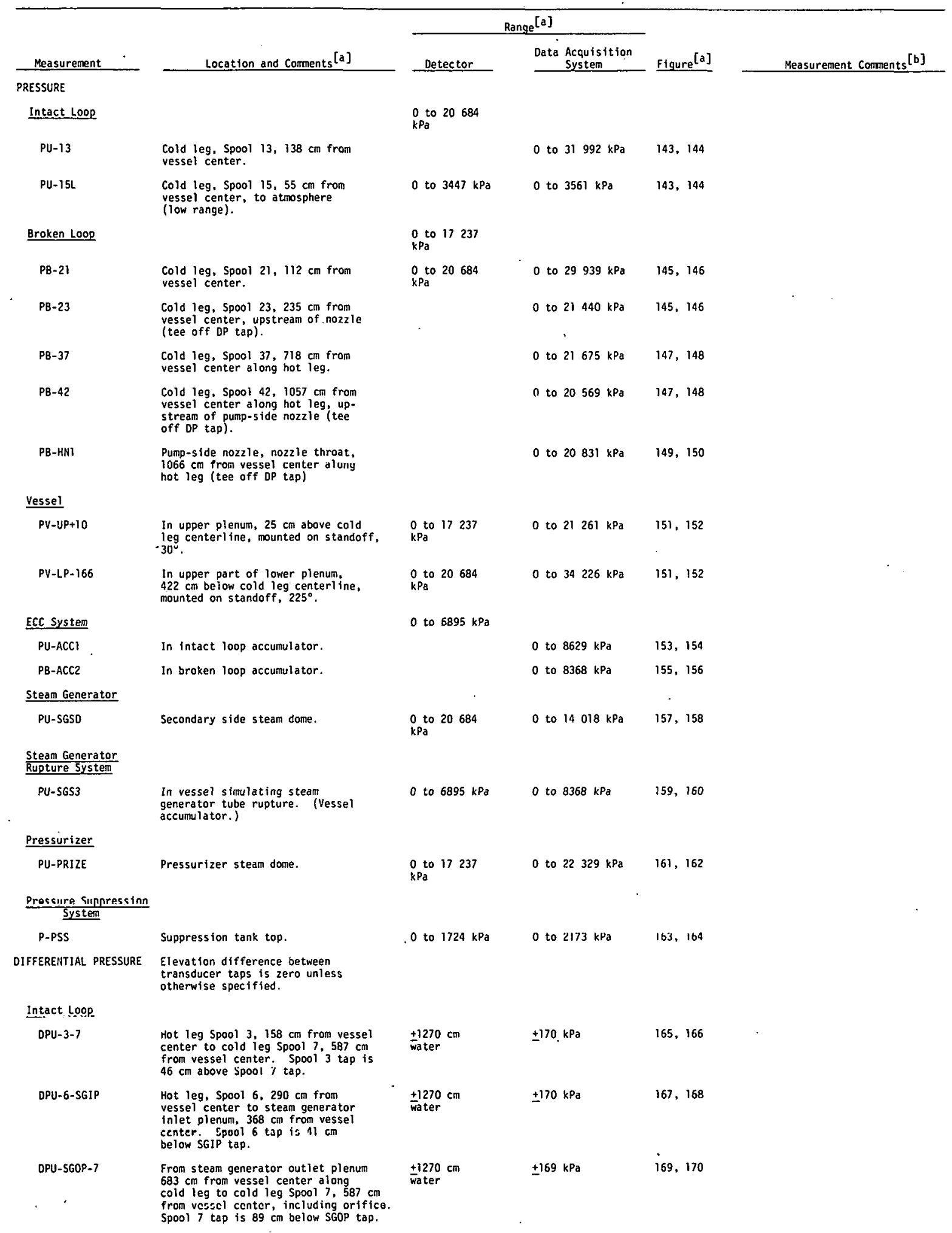




\begin{tabular}{|c|c|c|c|c|c|c|}
\hline \multirow{2}{*}{\multicolumn{2}{|c|}{ Measurement }} & \multirow[b]{2}{*}{ Location and Comments $[\mathrm{d}]$} & \multicolumn{2}{|c|}{ \pm Range $^{[\mathrm{a}]}$} & \multirow{3}{*}{ Figure $^{[a]}$} & \multirow[b]{2}{*}{ Measurement Corments ${ }^{[\mathrm{b}]}$} \\
\hline & & & Detector & $\begin{array}{l}\text { Data Acquisition } \\
\text { System } \\
\end{array}$ & & \\
\hline \multicolumn{6}{|c|}{ Intact Loop (continued) } & \\
\hline OPU $-7-10$ & & $\begin{array}{l}\text { Steam generator outlet to pump } \\
\text { inlet, coid leg Spool } 7,587 \mathrm{~cm} \\
\text { from vessel center, to cold } 1 \text { eg } \\
\text { Spool } 10,359 \mathrm{~cm} \text { from vessel } \\
\text { center. }\end{array}$ & $\frac{+127 \mathrm{~cm}}{\text { water }}$ & $\pm 17.2 \mathrm{kPa}$ & 171,172 & \\
\hline DPU-12-10 & & 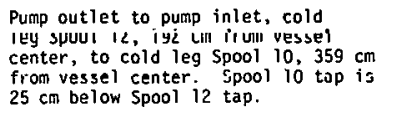 & $\pm 345 \mathrm{kPa}$ & $\pm 343 \mathrm{kPa}$ & 173,174 & \\
\hline DPU-12-10L & & $\begin{array}{l}\text { Pump outlet to pump inlet, cold } \\
\text { leg Spool i2, } 192 \mathrm{~cm} \text { from vessel } \\
\text { center, to cold leg Spool } 10,359 \mathrm{~cm} \\
\text { from vessel center. Spool 10 tap is } \\
25 \mathrm{~cm} \text { below Spool i2 tap (low range). }\end{array}$ & $\begin{array}{l}+254 \mathrm{~cm} \\
\text { water }\end{array}$ & $\pm 33.2 \mathrm{kPa}$ & 175,176 & $\begin{array}{l}\text { Data acquisition system } \\
\text { saturated to } t=7 \mathrm{~s} \text {. }\end{array}$ \\
\hline$D P U-12-15$ & & $\begin{array}{l}\text { culd leg spool } 12,192 \mathrm{~cm} \text { from } \\
\text { vessel center, to cold leg Spool } \\
15,55 \mathrm{~cm} \text { from vessel center. }\end{array}$ & $\begin{array}{l}+254 \mathrm{~cm} \\
\text { water }\end{array}$ & $\pm 33.5 \mathrm{kPa}$ & 177,178 & $\because$ \\
\hline$D Y U-15-3$ & 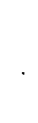 & $\begin{array}{l}\text { Cold leg to hot leg; cold ley Spuul } \\
15,55 \mathrm{~cm} \text { from vessel center, to } \\
\text { hot leg Spool } 3 \text {, } 150 \mathrm{~cm} \text { from vessel } \\
\text { conter. Spool is tap is } 7 ? \text { r.m } \\
\text { below Spool } 3 \text { tap. }\end{array}$ & $\underset{\text { water }}{+1270 \mathrm{col}}$ & $\pm 109 \mathrm{kPa}$ & 179,180 & 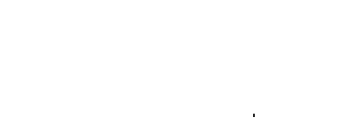 \\
\hline DPU-15-3L & ' & $\begin{array}{l}\text { Cold leg to hot leg, cold leg Spool } \\
15,55 \mathrm{~cm} \text { from vessel center, to } \\
\text { hot leg Spool } 3,158 \mathrm{~cm} \text { from vessel } \\
\text { center. sponl is t.ap is } 22 \mathrm{~cm} \text { below } \\
\text { Spool } 3 \text { tap (low range). }\end{array}$ & $\underset{\text { water }}{+254 \mathrm{~cm}}$ & $\pm 33.8 \mathrm{kPa}$ & 181,182 & $\begin{array}{l}\text { Data acquisition system } \\
\text { saturated to } t=0 \mathrm{~s} \text {. }\end{array}$ \\
\hline DPU-PRESLL & & $\begin{array}{l}\text { Pressurizer walter level. Eleva- } \\
\text { tion ditterence between taps } 1 \mathrm{~s} \\
135 \mathrm{~cm} \text {. Lower. tap is } 9 \mathrm{~cm} \text { above } \\
\text { pressurizer exit. }\end{array}$ & $\frac{1121 \mathrm{~cm}}{\text { water }}$ & $\pm 17.1 \mathrm{kra}$ & 183,181 & * \\
\hline OPU-PR-4 & & $\begin{array}{l}\text { Pressurizer bottom to Spool } 4 \text {. Eleva- } \\
\text { tion difference between taps is } \\
15 / \mathrm{cm} \text {. Spool \& tap is } 140 \mathrm{~cm} \text { below } \\
\text { pressurizer exit. }\end{array}$ & $\pm 3447 \mathrm{kPa}$ & $\pm 3456 \mathrm{kPa}$ & 185,186 & $\begin{array}{l}\text { Daca acquisition system } \\
\text { saturated from } t=0 \text { to } t=11 \mathrm{~s} \text {. }\end{array}$ \\
\hline \multicolumn{7}{|l|}{ Broken Loop } \\
\hline$n P R=? 1$. IANN & & $\begin{array}{l}\text { Cold leg snnol } 21.112 \text { cm from } \\
\text { vessel center, to vessel iniet } \\
\text { annulus, } 23 \mathrm{~cm} \text { below cold leg } \\
\text { centerine st } 225^{\circ} \text {. Inlet anulus } \\
\text { tap is } 23 \mathrm{~cm} \text { below spool } 21 \text { tap. }\end{array}$ & $\begin{array}{l}+254 \mathrm{~cm} \\
\text { water }\end{array}$ & $+33.6 \mathrm{kPa}$ & 187,188 & \\
\hline DPB-23-CN1 & & 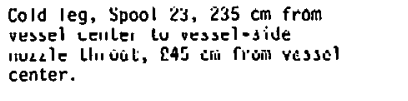 & $\pm 10342 \mathrm{kPa}$ & $\pm 13843 \mathrm{kPd}$ & 189,190 & \\
\hline DPB-CN1-24 & & $\begin{array}{l}\text { Vessel-side nozzle throat, } 245 \mathrm{~cm} \\
\text { from vessel center to Spool } 24 \text {, } \\
264 \mathrm{~cm} \text { from vessel center. }\end{array}$ & $\pm 3447 \mathrm{kPa}$ & $\pm 6961 \mathrm{kPa}$ & 191,192 & . \\
\hline$D P B-30-43$ & & $\begin{array}{l}\text { Across entire broken loop hot leg } \\
\text { including rupture assembly; hot leg } \\
\text { Spool } 30,45 \mathrm{~cm} \text { from vessel to rnld } \\
\text { leg Spool } 43 \text {, } 1086 \mathrm{~cm} \text { from vessel } \\
\text { center along hot lea. }\end{array}$ & $\pm 3447 \mathrm{kPa}$ & $\pm 6973 \mathrm{kPa}$ & 193,194 & $\cdot$ \\
\hline$D P B-3211-361$. & & 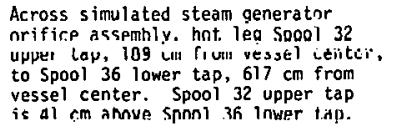 & $\pm 13 / y \mathrm{kPa}$ & $+1388 \mathrm{kPa}$ & 195,196 & \\
\hline DPB-36L-37 & & 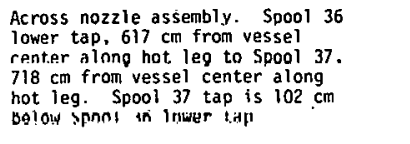 & $\pm 345 \mathrm{kPa}$ & $\$ 346 \mathrm{kPa}$ & 197,198 & . \\
\hline UPB-38-40 & & $\begin{array}{l}\text { Across simulated pump, culd ley } \\
\text { Spool } 38.776 \mathrm{~cm} \text { from vessel center } \\
\text { along hot leg. to cold leg Spool } 40 \text {, } \\
929 \mathrm{~cm} \text { from vessel center along hot } \\
\text { leg. }\end{array}$ & $\pm 6895 \mathrm{kPa}$ & $\pm 9227 \mathrm{kPa}$ & 199,200 & \\
\hline DPB- $40-42$ & & $\begin{array}{l}\text { Across el bow leading to spool up- } \\
\text { stream of pump-side nozzle. Cold } \\
\text { leg tpool } 40 \text {. U2u sm rrom vorcoi } \\
\text { center a long hot leg, to Spool } 42 \text {, } \\
\text { lo57 cm from vessel center along } \\
\text { hot leg. Spool } 40 \text { tap is } 102 \mathrm{~cm} \\
\text { below Spool } 42 \text { tap. }\end{array}$ & $\stackrel{+127 \mathrm{~cm}}{\text { water }}$ & $\pm 16.2 \mathrm{kPa}$ & 201,202 & $\begin{array}{l}\text { Data acquisition system } \\
\text { saturated from } t=0 \text { to } t=1 \mathrm{~s} \text {. }\end{array}$ \\
\hline
\end{tabular}


TABLE V (continued)

\begin{tabular}{|c|c|c|c|c|c|c|c|}
\hline \multirow[b]{2}{*}{ Measurement } & \multirow[b]{2}{*}{ Location and Comments ${ }^{[\mathrm{a}]}$} & \multicolumn{3}{|c|}{ Range $^{[a]}$} & \multirow[b]{2}{*}{ Figure $^{[\mathrm{a}]}$} & & \multirow[b]{2}{*}{ Measurement Comments ${ }^{[b]}$} \\
\hline & & Detector & & $\begin{array}{l}\text { Data Acquisition } \\
\text { System } \\
\end{array}$ & & & \\
\hline \multicolumn{8}{|c|}{ Broken Loop (continued) } \\
\hline DPB-42-HN1 & $\begin{array}{l}\text { Spool } 42 \text { upstream of pump-side } \\
\text { nozzle, } 1057 \mathrm{~cm} \text { from vessel center } \\
\text { along hot leg to nozzle throat. } \\
\text { lo66 cm from vessel center along } \\
\text { hot leg. }\end{array}$ & $\pm 10342 \mathrm{kPa}$ & & $\pm 13843 \mathrm{kPa}$ & 203,204 & - & \\
\hline OPB-HN1-43 & $\begin{array}{l}\text { Pump-side nozzle, nozzle throat } \\
1066 \mathrm{~cm} \text { from vessel center along } \\
\text { hot leg to } 5 \text { pool } 43,1086 \mathrm{~cm} \text { from } \\
\text { vessel center along hot leg. }\end{array}$ & $\pm 3447 \mathrm{kPa}$ & & $\pm 6932 \mathrm{kPa}$ & 205,206 & & \\
\hline \multicolumn{8}{|l|}{ Vessel } \\
\hline DPV-UP-IANN & $\begin{array}{l}\text { Upper plenum, } 27 \mathrm{~cm} \text { above cold } \\
\text { leg centerline at } 30^{\circ} \text { to iniet } \\
\text { anumlus, } 23 \text { cm beluw culd leg center- } \\
\text { line at } 225^{\circ} \text {. Elevation difference } \\
\text { between taps is } 48 \mathrm{~cm} \text {. }\end{array}$ & $\begin{array}{l} \pm 762 \mathrm{~cm} \\
\text { water }\end{array}$ & & $\pm 102 \mathrm{kPa}$ & 207,208 & & \\
\hline OPV $-0-960$ & $\begin{array}{l}\text { Inlet annulus cold leg centerline at } \\
90^{\circ} \text {, to } 23 \mathrm{~cm} \text { below cold leg center- } \\
\text { line at } 225^{\circ} \text { Elevation difference } \\
\text { between taps is } 23 \mathrm{~cm} \text {. }\end{array}$ & $\frac{ \pm 127 \mathrm{~cm}}{\text { water }}$ & & $\pm 18.4 \mathrm{kPa}$ & 209,210 & & \\
\hline DPV-9-2600 & $\begin{array}{l}\text { Inlet annulus, } 23 \mathrm{~cm} \text { below cold leg } \\
\text { centerline at } 225^{\circ} \text {, to downcomer gap, } \\
66 \mathrm{~cm} \text { below coid leg centerline at } \\
225^{n} \text {. Elevation difference between } \\
\text { taps is } 43 \mathrm{~cm} \text {. }\end{array}$ & $\begin{array}{l} \pm 127 \mathrm{~cm} \\
\text { water }\end{array}$ & & $\pm 16.8 \mathrm{kPa}$ & 211,212 & & . \\
\hline$D P V-9-166 Q Q$ & $\begin{array}{l}\text { Inlet annulus, } 23 \mathrm{~cm} \text { below cold leg } \\
\text { centerliné at } 225^{\circ} \text { to lower plenum, } \\
422 \mathrm{~cm} \text { below cold ieg centerline } \\
\text { at } 225^{\circ} \text {. Elevation difference be- } \\
\text { tween taps is } 399 \mathrm{~cm} \text {. }\end{array}$ & $\begin{array}{l}+1270 \mathrm{~cm} \\
\text { water }\end{array}$ & . & $\pm 167 \mathrm{kPa}$ & 213,214 & & - \\
\hline DPV-26-55OM & $\begin{array}{l}\text { Aciuss part of duwncumier, } 66 \mathrm{~cm} \\
\left(225^{\circ}\right) \text { to } 140 \mathrm{~cm}\left(180^{\circ}\right) \text {, below cold } \\
\text { leg centerl ine. Elevation difference } \\
\text { between taps is } 74 \mathrm{~cm} \text {. }\end{array}$ & $\begin{array}{l}+127 \mathrm{cml} \\
\text { water }\end{array}$ & & $\pm 21.2 \mathrm{kPd}$ & 215,216 & & \\
\hline DPV-55-110MM & $\begin{array}{l}\text { Arrnss nart of dnwncamer, } 140 \mathrm{~cm} \\
\left(180^{\circ}\right) \text { to } 279 \mathrm{~cm}\left(180^{\circ}\right) \text {, below } \\
\text { cold leg centerl ine. Elevation } \\
\text { difference between taps is } 140 \mathrm{~cm} \text {. }\end{array}$ & $\begin{array}{l} \pm 254 \mathrm{~cm} \\
\text { water }\end{array}$ & & $\pm 33.8 \mathrm{kPa}$ & 217,218 & & \\
\hline DPV-110-156MQ & $\begin{array}{l}\text { Across part of downcomer, } 279 \mathrm{~cm} \\
\left(180^{\circ}\right) \text { to } 396 \mathrm{rm}\left(225^{\circ}\right) \text {, hel how r.nld } \\
\text { leg centeri ine. Elevation difference } \\
\text { between taps is } 117 \mathrm{~cm} \text {. }\end{array}$ & $\begin{array}{l}+254 \mathrm{~cm} \\
\text { wà tẹr }\end{array}$ & & $\pm 33.7 \mathrm{kPa}$ & 219,220 & & - \\
\hline DPV-156-173QQ & $\begin{array}{l}\text { Across part of lower plenum, } 396 \mathrm{~cm} \\
\left(225^{\circ}\right) \text { to } 439 \mathrm{~cm}\left(225^{\circ}\right) \text {, below cold } \\
\text { leg centerl ine. El evation difference } \\
\text { between taps is } 43 \mathrm{~cm} \text {. }\end{array}$ & $\begin{array}{l}+51 \mathrm{~cm} \\
\text { water }\end{array}$ & & $\pm 6.47 \mathrm{kPa}$ & 221,222 & & \\
\hline DPV- $1660+10$ & $\begin{array}{l}\text { Lower plenum, } 422 \mathrm{~cm} \text { below cold leg } \\
\text { centerline at. } 275^{\circ} \text { ton. upper plenum, } \\
27 \mathrm{~cm} \text { above cold leg centerline at } \\
30^{\circ} \text {. Clevation difference between } \\
\text { taps is } 395 \mathrm{~cm} \text {. }\end{array}$ & $\underset{\text { wat.er }}{+762 \mathrm{~cm}}$ & & $\pm 101 \mathrm{kPa}$ & 223,224 & & \\
\hline \multicolumn{8}{|l|}{ ECC SYSTEM } \\
\hline OPU-ACCI-TB & $\begin{array}{l}\text { Top to bottom of intact loop } \\
\text { accumulator. Elevation difference. } \\
\text { between taps is } 274 \mathrm{~cm} \text {. }\end{array}$ & $\begin{array}{l}+762 \mathrm{~cm} \\
\text { water }\end{array}$ & & $\pm 104 \mathrm{kPa}$ & 225,226 & & \\
\hline DPB-ACC2-TB & $\begin{array}{l}\text { Top to botrom of broken loop } \\
\text { accumulator. Elevation difference } \\
\text { between taps is } 213 \mathrm{~cm} \text {. }\end{array}$ & $\begin{array}{l}+127 \mathrm{~cm} \\
\text { water }\end{array}$ & & $\pm 17.35 \mathrm{kra}^{\prime}$ & $2 z \%, 2 z 8$ & & $\therefore$ \\
\hline \multicolumn{8}{|l|}{ Steam Generator } \\
\hline DPU-SG-SEC & $\begin{array}{l}\text { Secondary side, differential pressure } \\
\text { Lajs al } 114 \text { cill and } 320 \text { cm dbuve } \\
\text { bottom of tube sheet. Elevation } \\
\text { difference between taps is } 206 \mathrm{~cm} \text {. }\end{array}$ & $\begin{array}{l}+254 \mathrm{~cm} \\
\text { waler }\end{array}$ & & $\pm 33.7 \mathrm{kPa}$ & 229,230 & & \\
\hline DPU-SG-DISC & 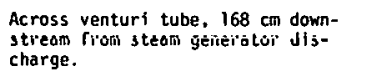 & $\begin{array}{l}+1270 \mathrm{~cm} \\
\text { ind tei }\end{array}$ & - & $\pm 170 \times 9 a$ & 231,232 & & \\
\hline \multicolumn{8}{|l|}{$\begin{array}{l}\text { Steam Generator } \\
\text { Ruptiure Syctipm }\end{array}$} \\
\hline DPU-SGS-6 & $\begin{array}{l}\text { Fram instrumented spool piece } \\
\text { in simulated tube rupture injection } \\
\text { line to hot leg Spool } 6,318 \mathrm{~cm} \text { from } \\
\text { vessel center. }\end{array}$ & $\pm 6895 \mathrm{kPa}$ & & $\pm 9230 \mathrm{kPa}$ & 233,234 & & . \\
\hline OPU-SGS3-TB & $\begin{array}{l}\text { Top to bottom of steam generator } \\
\text { rupture system accumulator tank. }\end{array}$ & $\begin{array}{l}+1270 \mathrm{~cm} \\
\text { water }\end{array}$ & & $\pm 108 \mathrm{kPa}$ & 235,236 & & . \\
\hline
\end{tabular}




\section{IABLE $\vee$ (continued)}

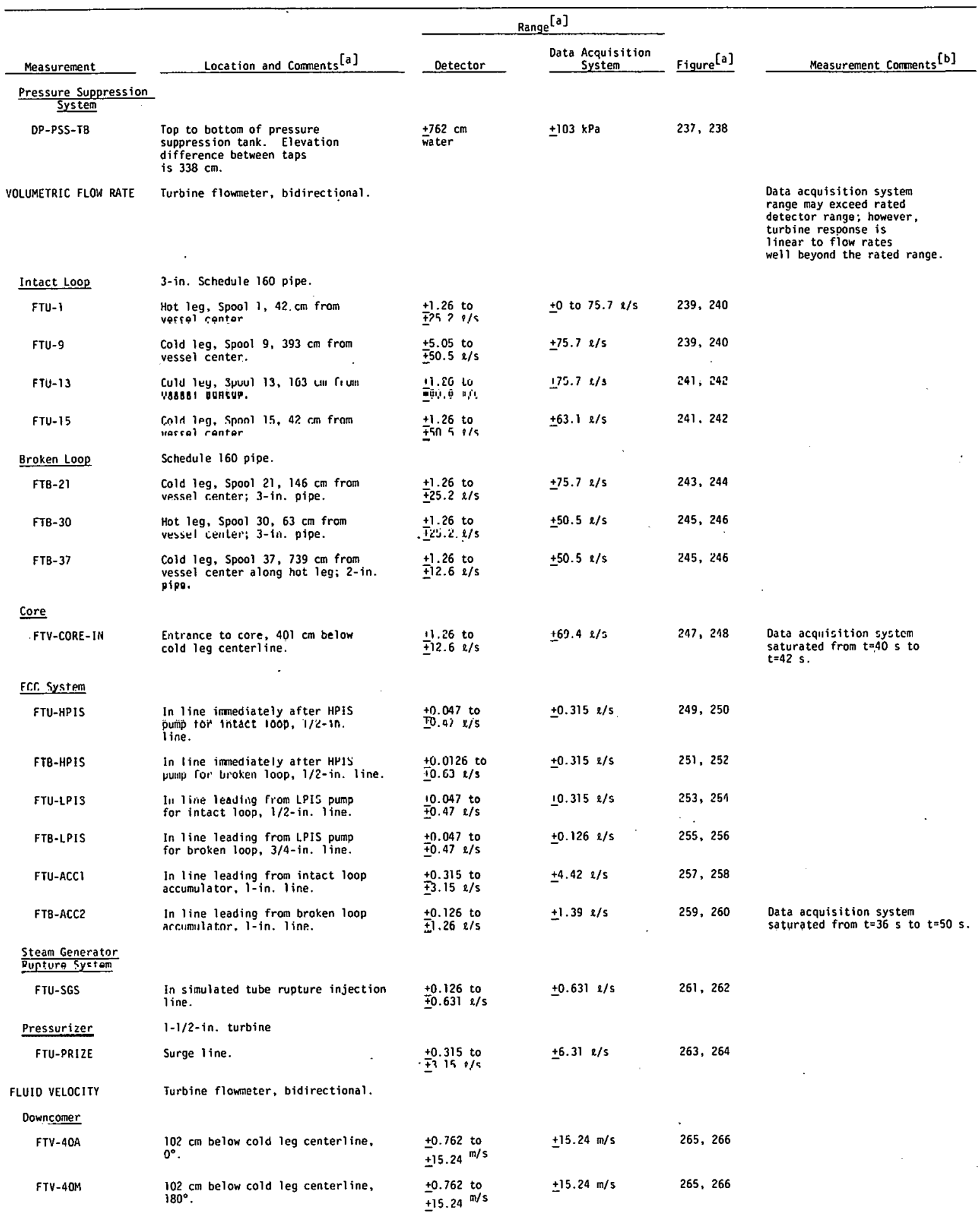


TABLE V (continued)

\begin{tabular}{|c|c|c|c|c|c|}
\hline \multirow{2}{*}{ Measurement } & \multicolumn{3}{|c|}{ Range $[\mathrm{a}]$} & \multirow[b]{2}{*}{ Figure $^{[\mathrm{a}]}$} & \multirow[b]{2}{*}{ Measurement Comments $[\mathrm{b}]$} \\
\hline & Location and Comments $[\mathrm{a}]$ & Detector & $\begin{array}{l}\text { Data Acquisition } \\
\text { System } \\
\end{array}$ & & \\
\hline MOMENTUM FLUX & Drag disc, bidirectional. & & & & $\begin{array}{l}\text { Momentum flux reported oniy } \\
\text { for }-6 \text { to } 425 \text {, except as noted. } \\
\text { Drag disc data may exhibit } \\
\text { significant temperature } \\
\text { dependence. orag disc data } \\
\text { should be used only for short- } \\
\text { term transient response. }\end{array}$ \\
\hline Intact loop & 3-in. pipe. & & & & \\
\hline FOU-1 & $\begin{array}{l}\text { Hot leg, Spool l, } 60 \mathrm{~cm} \text { from } \\
\text { vessel center; tárget size } \\
2.22 \mathrm{~cm} \text {. }\end{array}$ & $\begin{array}{l} \pm 298 \text { to } \\
\mathrm{kg} / \mathrm{m}-\mathrm{s}^{2}\end{array}$ & $\begin{array}{l} \pm 83739 \\
\mathrm{~kg} / \mathrm{m}-\mathrm{s}^{2}\end{array}$ & 267 & . \\
\hline FDU -5 & $\begin{array}{l}\text { Hot leg, Spool } 5,256 \mathrm{~cm} \text { from } \\
\text { vessel center; target size } \\
2.54 \mathrm{~cm} \text {. }\end{array}$ & $\begin{array}{l} \pm 1.49 \text { to } \pm 2976 \\
\mathrm{~kg} / \mathrm{m}-\mathrm{s}^{2}\end{array}$ & $\frac{+6994}{\mathrm{~kg} / \mathrm{m}-\mathrm{s}^{2}}$ & 268 & \\
\hline FDU- 10 & $\begin{array}{l}\text { Cold leg, Spool } 10,349 \mathrm{~cm} \text { from } \\
\text { vessel center; target size } 2.22 \mathrm{~cm} .\end{array}$ & $\frac{ \pm 298 \text { to }}{\mathrm{kg} / \mathrm{m}-\mathrm{s}^{2}} \pm 154769$ & $\begin{array}{l} \pm 35344 \\
\mathrm{~kg} / \mathrm{m}-\mathrm{s}^{2}\end{array}$ & 269 & \\
\hline FDU-13 & $\begin{array}{l}\text { Cold leg, Spool 13, } 138 \mathrm{~cm} \text { from } \\
\text { vessel center; target stze } 2.22 \mathrm{~cm} \text {. }\end{array}$ & $\begin{array}{l} \pm 2 y 8 \text { to }+2 Z i z<5 \\
\mathrm{~kg} / \mathrm{m}-\mathrm{s}^{2}\end{array}$ & $\frac{+28528}{k g / m-s}$ & $2 \%$ & . \\
\hline Broken Loop & . & & & & \\
\hline FDB-21 & $\begin{array}{l}\text { Cold leg, Spool } 21,134 \mathrm{~cm} \text { from } \\
\text { vessel center, } 3-\mathrm{in} \text {. pipe; target } \\
\text { size } 1.67 \mathrm{~cm} \text {. }\end{array}$ & $\begin{array}{l} \pm 298 \text { to } \pm 104915 \\
\mathrm{~kg} / \mathrm{m}-\mathrm{s}^{2}\end{array}$ & $\begin{array}{l} \pm 66774 \\
\mathrm{~kg} / \mathrm{m}-\mathrm{s}^{2}\end{array}$ & 271 & \\
\hline$F D B-23$ & $\begin{array}{l}\text { Cold leg, spool } 23,238 \mathrm{~cm} \text { from } \\
\text { vessel center, upstream of vessel } \\
\text { side nozzle, 2-in. pipe; target } \\
\text { size } 1.03 \mathrm{~cm} \text {. }\end{array}$ & $\begin{array}{l} \pm 298 \text { to } \pm 180067 \\
\mathrm{~kg} / \mathrm{m}-\mathrm{s}^{2}\end{array}$ & $\begin{array}{l} \pm 155319 \\
\mathrm{~kg} / \mathrm{m}-\mathrm{s}^{2}\end{array}$ & 272 & $\begin{array}{l}\text { Doto acquisition system } \\
\text { saturated from } t=0 \text { to } t=2 \mathrm{~s} \text {. }\end{array}$ \\
\hline FOB-30 & $\begin{array}{l}\text { Hot leg, Spool } 30,52 \mathrm{~cm} \text { from } \\
\text { vessel center, 3-in. pipe; target } \\
\text { size l.67 col. }\end{array}$ & $\underset{k 9 / m-s^{2}}{ \pm 298 \text { to } \pm 89290}$ & $\begin{array}{l} \pm 67280 \\
\mathrm{~kg} / \mathrm{m}-\mathrm{s}^{2}\end{array}$ & 273 & \\
\hline FDB-37 & $\begin{array}{l}\text { Cold leg, Spool } 37,725 \mathrm{~cm} \text { from } \\
\text { vessel center along hot. leg, steam } \\
\text { generator outlet, vertical pipe, } \\
\text { 2-in. pipe; target size } 1.03 \mathrm{~cm} \text {. }\end{array}$ & $\begin{array}{l} \pm 298 \text { to } \\
\mathrm{kg} / \mathrm{m}-\mathrm{s}^{2}\end{array}$ & $\begin{array}{l} \pm 374272 \\
\mathrm{~kg} / \mathrm{m}-\mathrm{s}^{2}\end{array}$ & 274 & \\
\hline$F D B-42$ & $\begin{array}{l}\text { Cold leg, Spool } 42,1057 \mathrm{~cm} \text { from } \\
\text { vessel center along hot leg, up- } \\
\text { stream of pump-side nozzle, down- } \\
\text { stream of injection point, } 2-\text { in. } \\
\text { pipe; target size } 1.03 \mathrm{~cm} \text {. }\end{array}$ & $\begin{array}{l} \pm 298 \text { to } \pm 172627 \\
\mathrm{~kg} / \mathrm{m}-\mathrm{s}^{2}\end{array}$ & $\begin{array}{l} \pm 165320 \\
\mathrm{~kg} / \mathrm{m}-\mathrm{s}^{2}\end{array}$ & 275 & \\
\hline \multicolumn{6}{|l|}{ Yessel } \\
\hline FOV-CORE-IN & $\begin{array}{l}\text { In core flow mixer box, } 381 \mathrm{~cm} \\
\text { below cold leg centerline; target } \\
\text { size } 2.54 \mathrm{~cm} \text {. }\end{array}$ & $\begin{array}{l} \pm 1.44 \text { to } \pm 2977 \\
\mathrm{~kg} / \mathrm{m}-\mathrm{s}^{2}\end{array}$ & $\frac{+1725}{\mathrm{~kg} / \mathrm{m}-\mathrm{s}^{2}}$ & 276,277 & \\
\hline \multicolumn{6}{|l|}{ DENSITY } \\
\hline Intact Loop & . & $\begin{array}{l}1.6 \text { to } 1600 \\
\mathrm{~kg} / \mathrm{m}^{3}\end{array}$ & $\begin{array}{l}0 \text { to } 1600 \\
\mathrm{~kg} / \mathrm{m}^{3}\end{array}$ & & \\
\hline $\begin{array}{l}\text { GU-1T } \\
\text { GU-1B } \\
\text { GU-IC }\end{array}$ & $\begin{array}{l}\text { Hot leg, Spool } 1,77 \mathrm{~cm} \text { from } \\
\text { vessel center. T (top) ranges } \\
270 \text { to } 360^{\circ} .8 \text { (bot tom) ranges } \\
30 \text { to } 330^{\circ} \text {. C, mathematical } \\
\text { composite of } T \text { and } B \text {. }\end{array}$ & & & $\begin{array}{l}278,279 \\
280,281\end{array}$ & \\
\hline GU-5VR & $\begin{array}{l}\text { Hot leg, Spool } 5,246 \mathrm{~cm} \mathrm{from} \\
\text { vessel center. vertical. }\end{array}$ & & & 282,283 & \\
\hline GU-IOVR & $\begin{array}{l}\text { Cold leg, Spool } 10,359 \mathrm{~cm} \text { from } \\
\text { vessel center, vertical. }\end{array}$ & & & 282,283 & \\
\hline GU-13VR & $\begin{array}{l}\text { Cold leg, Spool } 13,142 \mathrm{~cm} \text { from } \\
\text { vessel center, vertical. }\end{array}$ & & & 284,285 & \\
\hline $\begin{array}{l}\text { GU-15T } \\
\text { GU-15B } \\
\text { GU-15C }\end{array}$ & $\begin{array}{l}\text { Cold } l \mathrm{cg} \text {, Spool } 15,77 \mathrm{~cm} \text { from vessel } \\
\text { center. } T \text { (top) ranges } 270 \text { to } 360^{\circ} \text {. } \\
B \text { (bottam) ranges } 30 \text { to } 330^{\circ} \text {. } C \text {, } \\
\text { mathematical composite of } T \text { and } 8 \text {. }\end{array}$ & $\cdot$ & & $\begin{array}{l}286,287 \\
288,289\end{array}$ & \\
\hline Broken Loop & & $\begin{array}{l}1.6 \text { to } 1600 \\
\mathrm{~kg} / \mathrm{m}^{3}\end{array}$ & $\begin{array}{l}0 \text { to } 1600 \\
\mathrm{~kg} / \mathrm{m}^{3}\end{array}$ & & \\
\hline $\begin{array}{l}\text { GB-211 } \\
G B-21 B \\
G B-21 C\end{array}$ & $\begin{array}{l}\text { Coid ieg, jpooi } 21 \text {, } 123 \mathrm{~cm} \text { from. } \\
\text { vessel center. T (top) ranges } \\
270 \text { to } 360^{\circ} \text {. B (bottem) ranges } \\
30 \text { to } 330^{\circ} \text { C, mathematical } \\
\text { composite of } T \text { and } B \text {. }\end{array}$ & . & & $\begin{array}{l}290,291 \\
292,293\end{array}$ & \\
\hline$G B-23 V R$ & $\begin{array}{l}\text { Cold leg, Spool } 23,235 \mathrm{~cm} \text { from } \\
\text { vessel center, vertical. }\end{array}$ & & & 294,295 & \\
\hline
\end{tabular}


TABLE $\vee$ (continued)

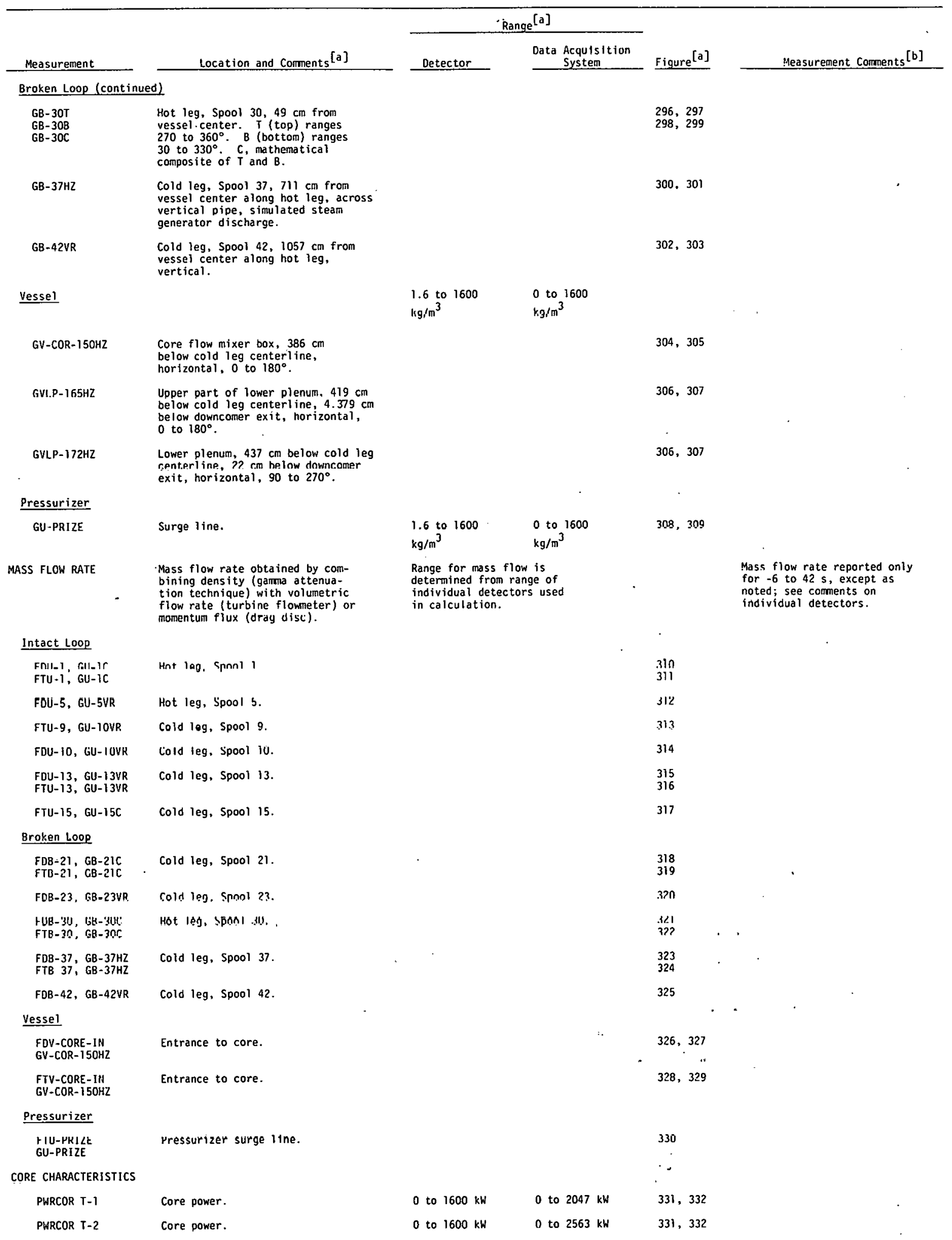


IABLE V (continued)

\begin{tabular}{|c|c|c|c|c|c|}
\hline \multirow[b]{2}{*}{ Measurement } & \multirow[b]{2}{*}{ Location and Comments [a] } & \multicolumn{2}{|c|}{ Range $[a]$} & \multirow[b]{2}{*}{ Figure ${ }^{[a]}$} & \multirow[b]{2}{*}{ Measurement Coments ${ }^{[\mathrm{b}]}$} \\
\hline & & Detector & $\begin{array}{l}\text { Data Acquisition } \\
\text { System }\end{array}$ & & \\
\hline \multicolumn{6}{|c|}{ CORE CHARACTERISIICS (cont inued) } \\
\hline VOLTCOR-T & Core vol tage. & & 0 to $200 \mathrm{Vdc}$ & 333,334 & \\
\hline AMPCOR-T & Core current. & 0 to $10000 \mathrm{~A}$ & 0 to $9645 \mathrm{~A}$ & 335,335 & \\
\hline \multicolumn{6}{|c|}{ PUMP CHARACTERISTICS } \\
\hline PUMPU-RPM & Pump speed. & 0 to $3600 \mathrm{rpm}$ & 0 to $3600 \mathrm{rpm}$ & 337.338 & \\
\hline \multicolumn{6}{|c|}{$\begin{array}{l}\text { [a] Statements at the beginning of a measurement category regarding location and comments, range, and figure apply to all subsequent measurements } \\
\text { within the given category unless specified otherwise. }\end{array}$} \\
\hline \multicolumn{6}{|c|}{$\begin{array}{l}\text { subjected to overrange condit t } \\
\text { suring chacteristics when the }\end{array}$} \\
\hline
\end{tabular}




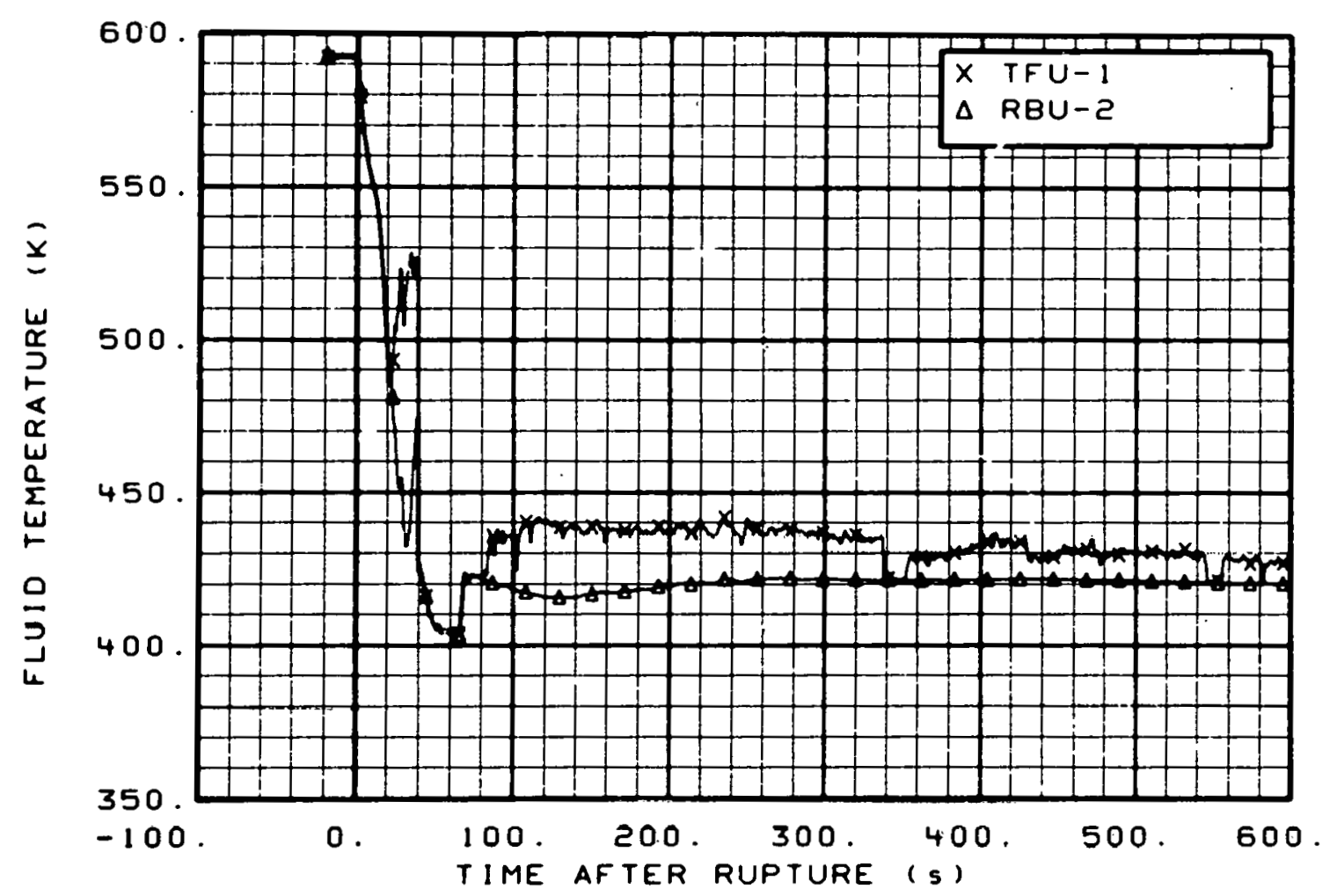

Fig. 9 Fluid temperature in intact loop hot leg (TFU- 1 and RBU-2), from -20 to $600 \mathrm{~s}$.

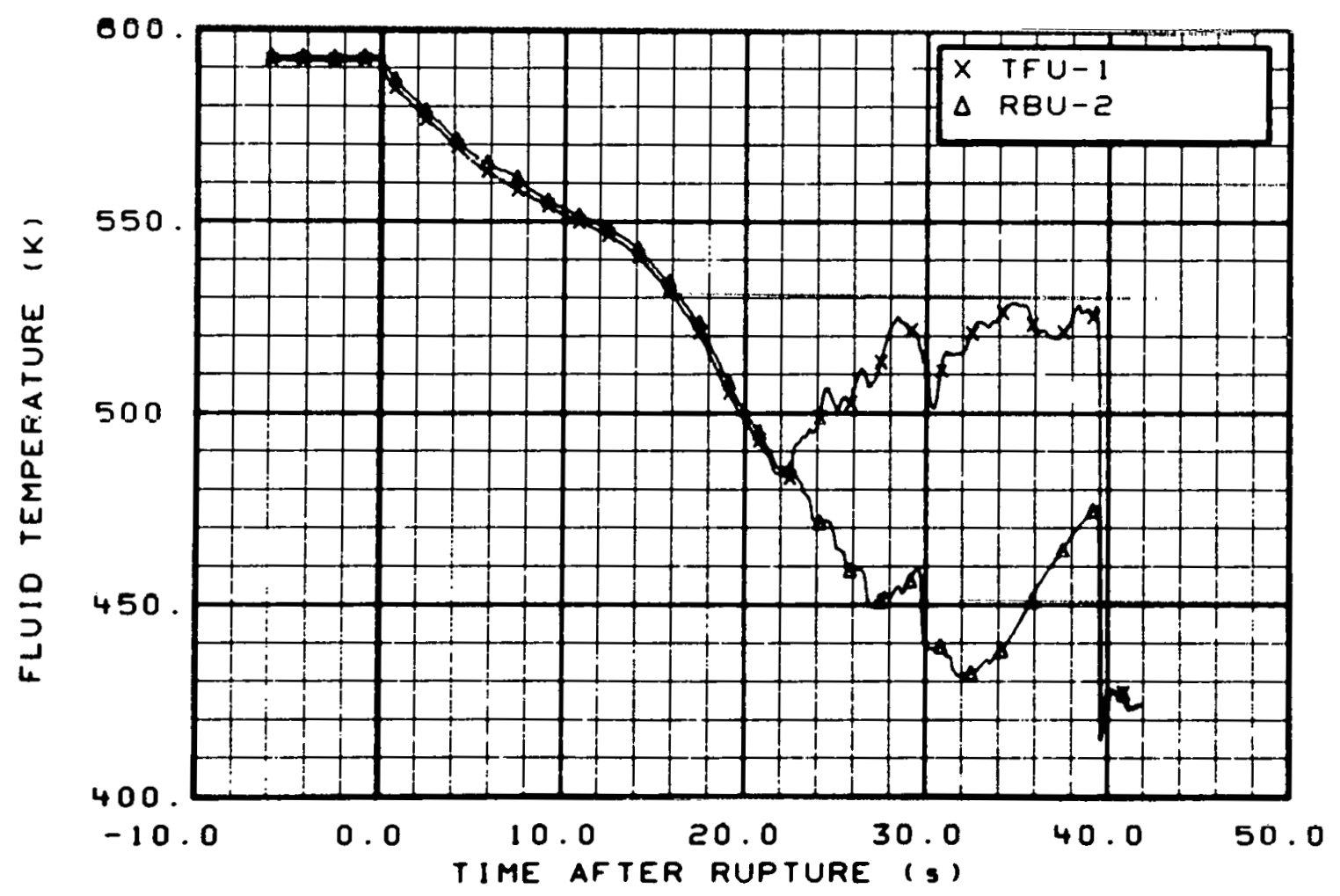

Fig. 10 Fluid temperature in intact loop hot leg (TFU-1 and RBU-2), from -6 to $42 \mathrm{~s}$. 


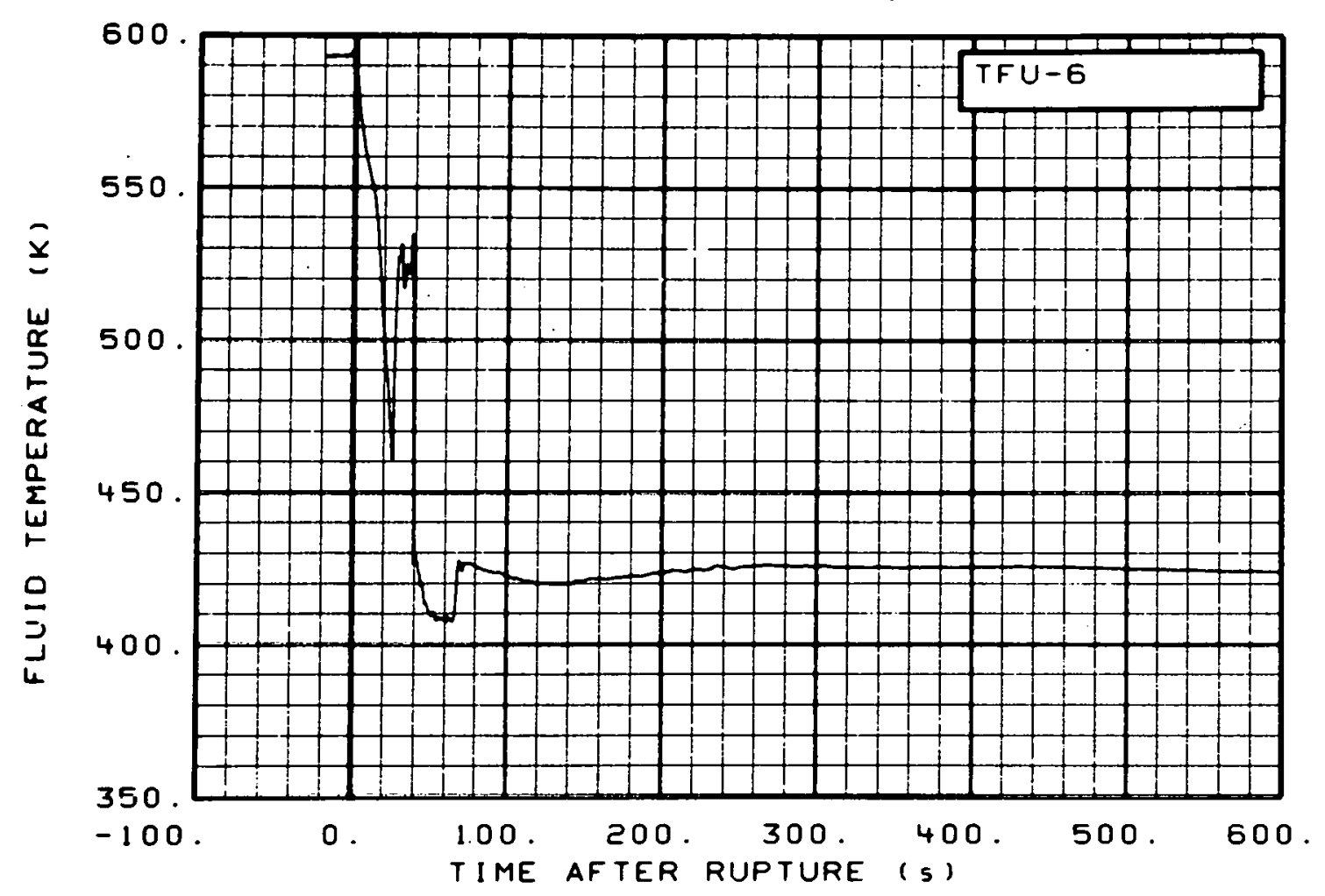

Fig. 11 Fluid temperature in intact loop hot leg (TFU-6), from -20 to $600 \mathrm{~s}$.

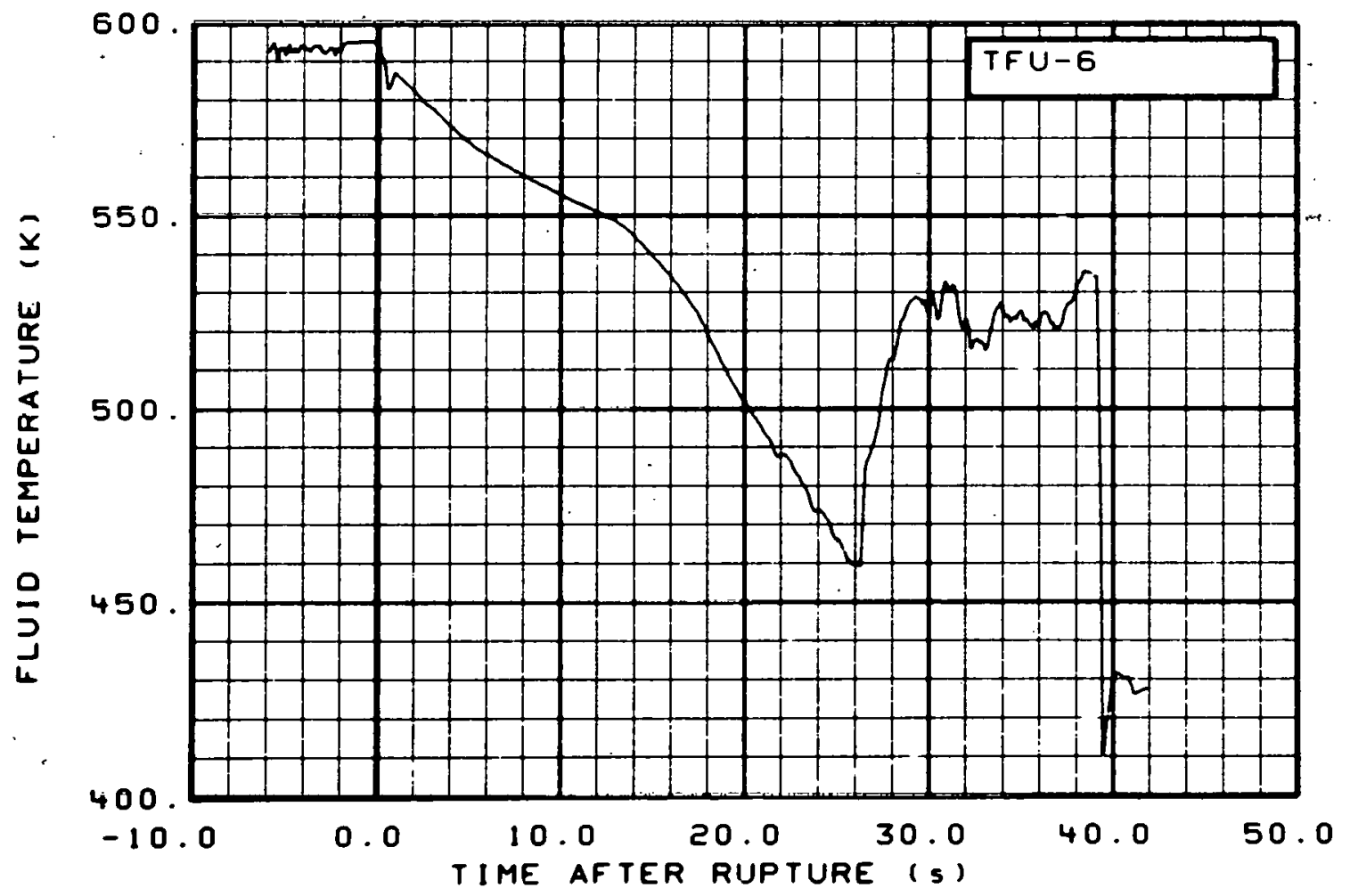

Fig. 12 Fluid temperature in intact loop hot leg (TFU-6), from -6 to $42 \mathrm{~s}$. 


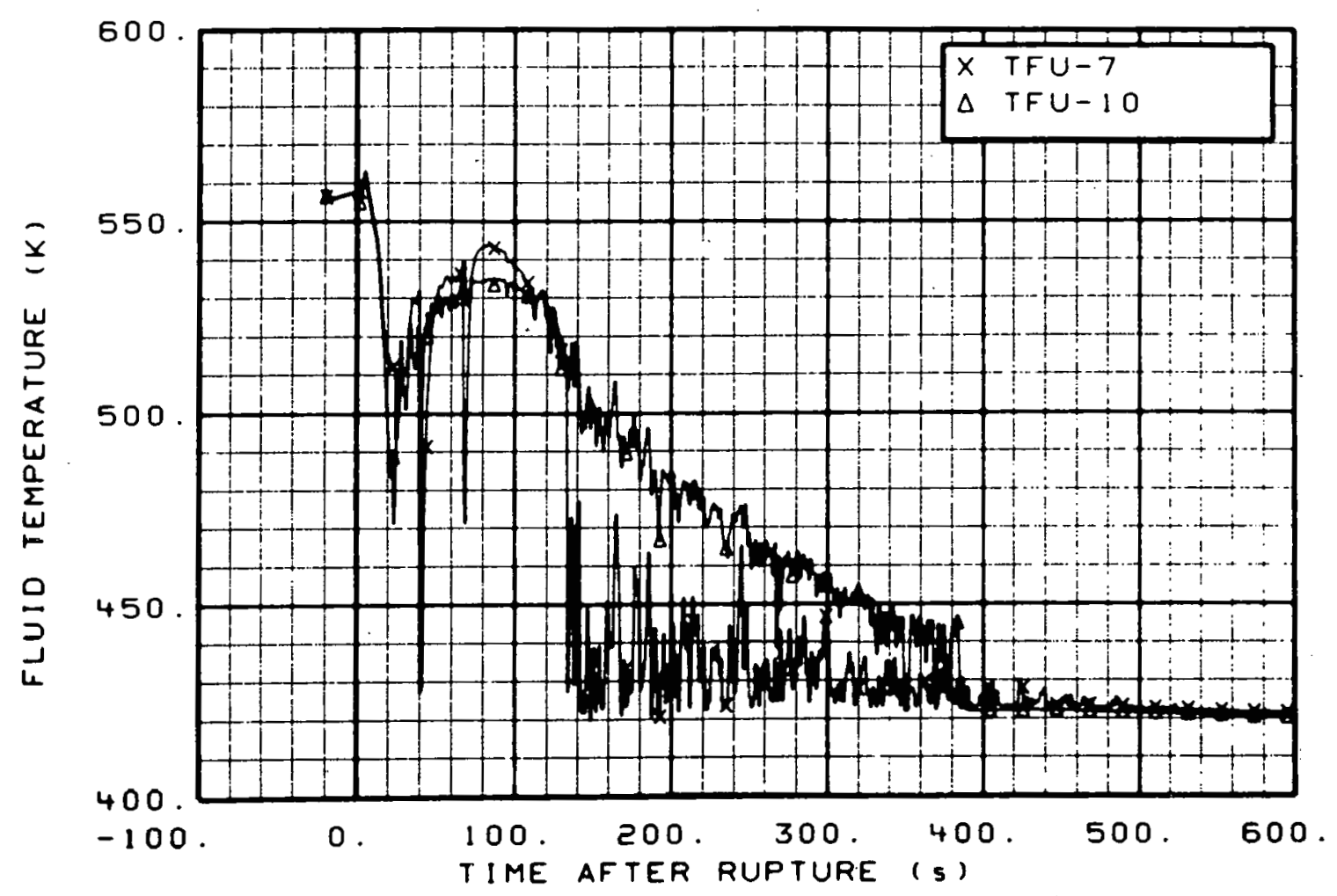

Fig. 13 Fluid temperature in intact loop cold leg (TFU-7 and TFU-10), from -20 to $600 \mathrm{~s}$.

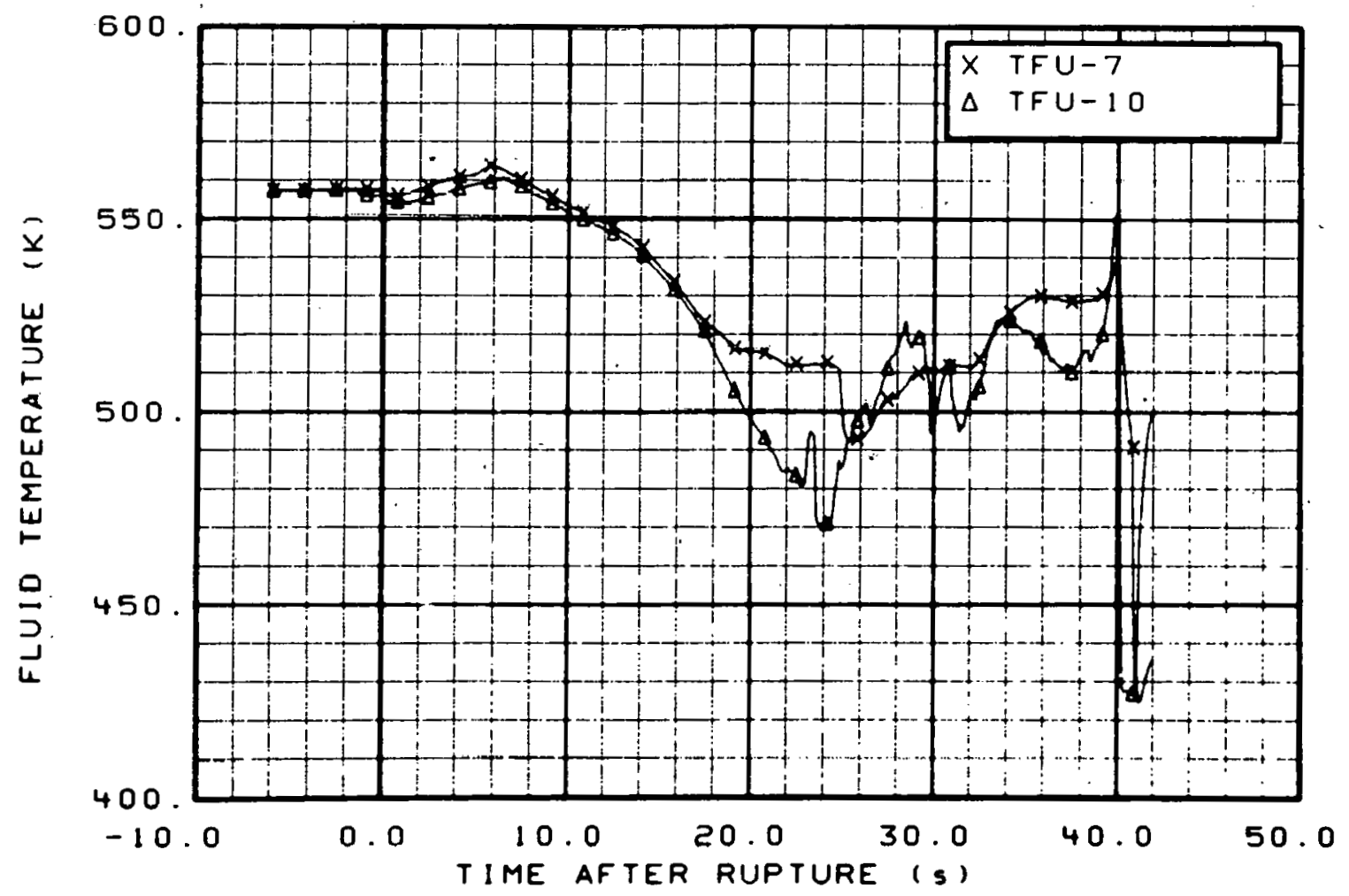

Fig. 14 Fluid temperature in intact loop cold leg (TFU-7 and TFU-10), from -6 to $42 \mathrm{~s}$. 


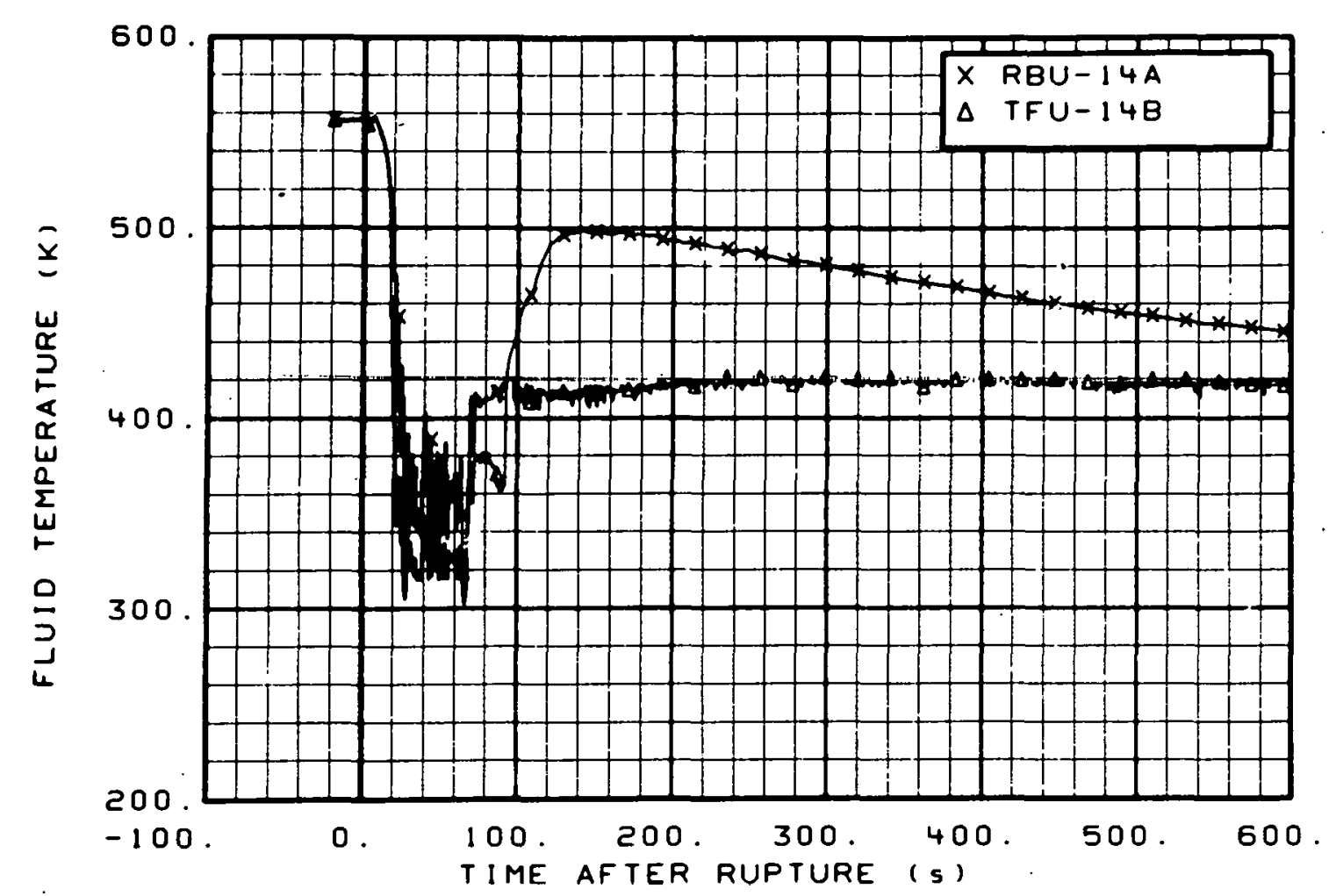

Fig. 15 Fluid temperature in intact loop cold leg (RBU-14A and TFU-14B), from -20 to $600 \mathrm{~s}$.

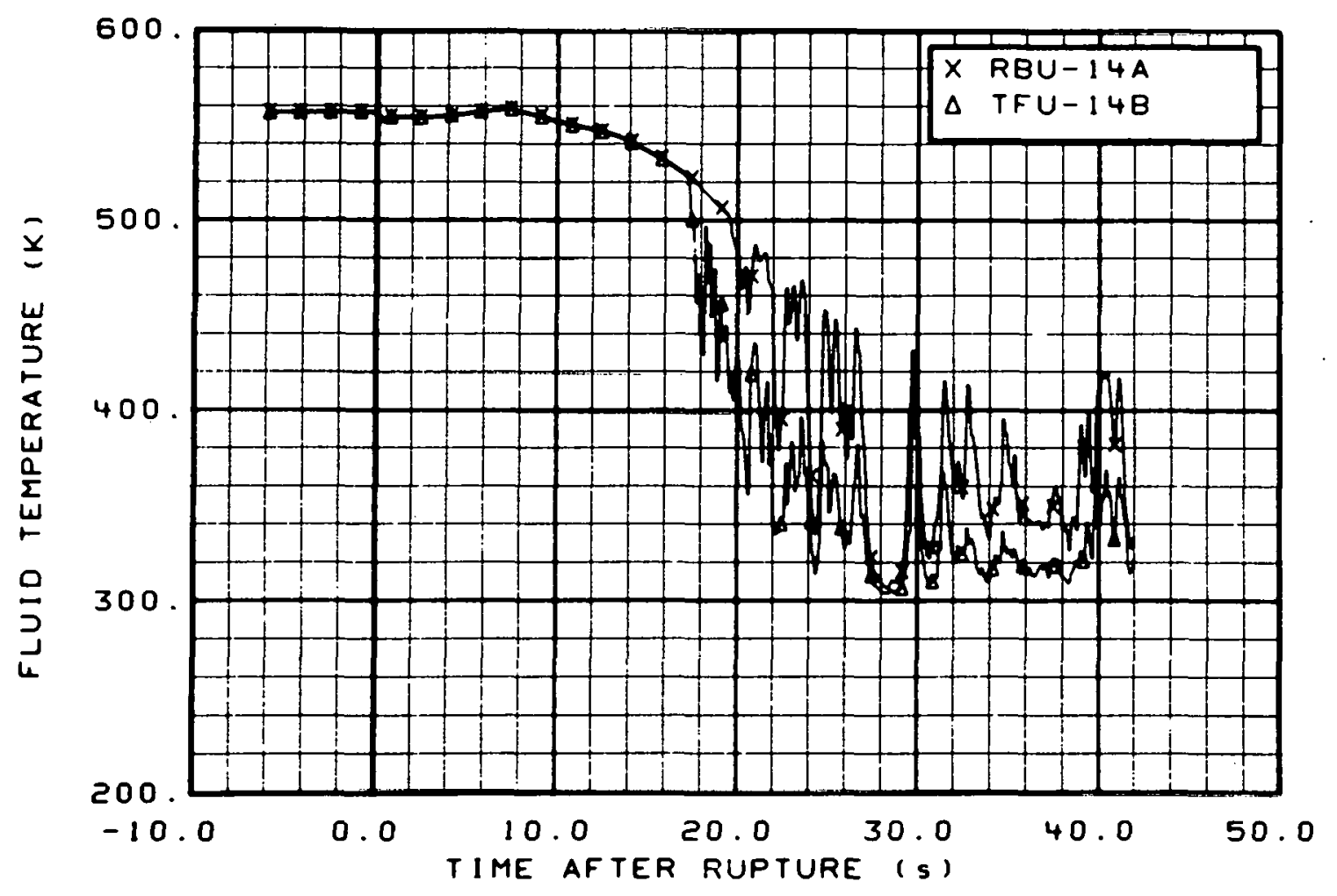

Fig. 16 Fluid temperature in intact loop cold leg (RBU-14A and TFU-14B), from -6 to $42 \mathrm{~s}$. 


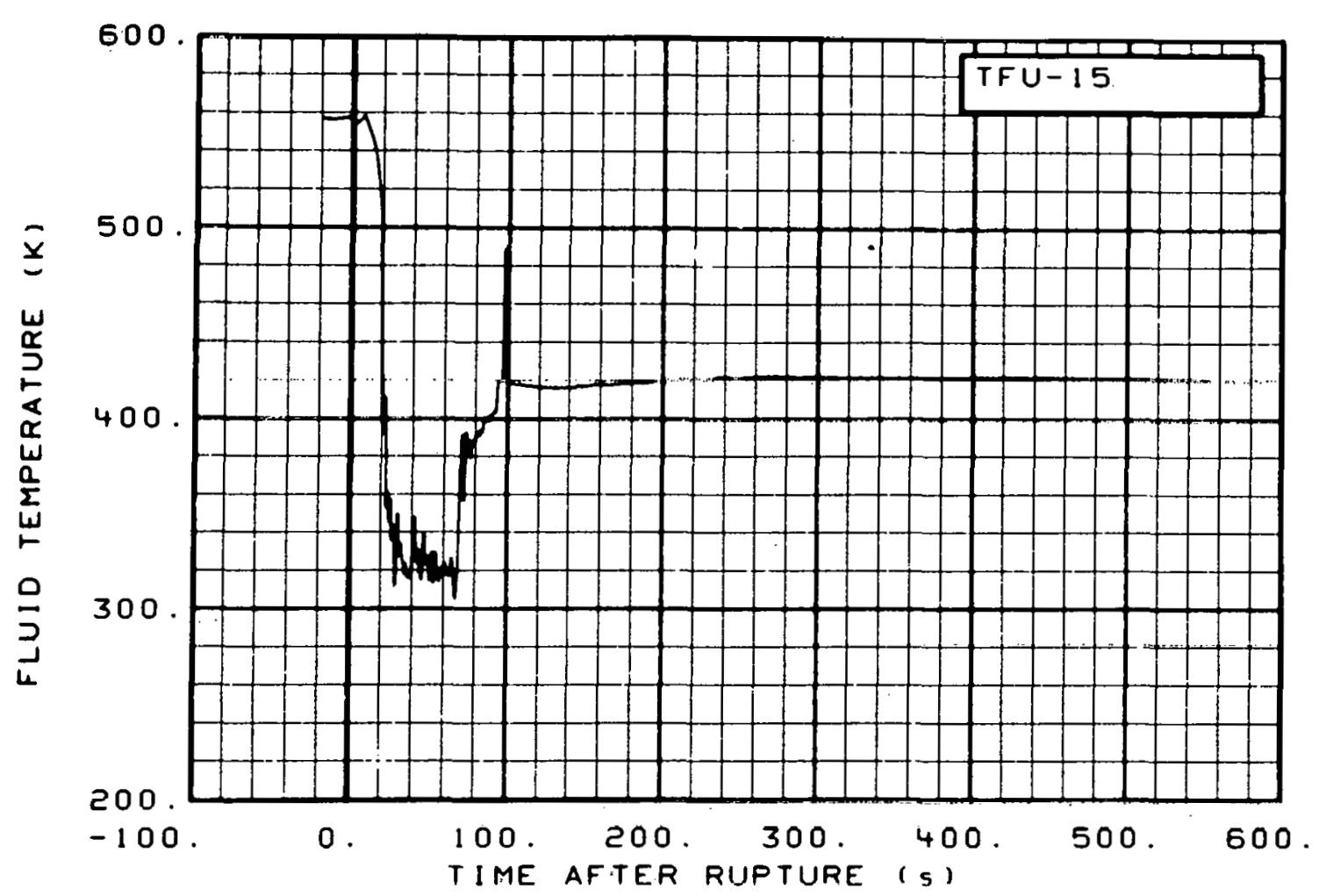

Fig. 17 Fluid temperature in intact loop cold leg (TFU-15), from -20 to $600 \mathrm{~s}$.

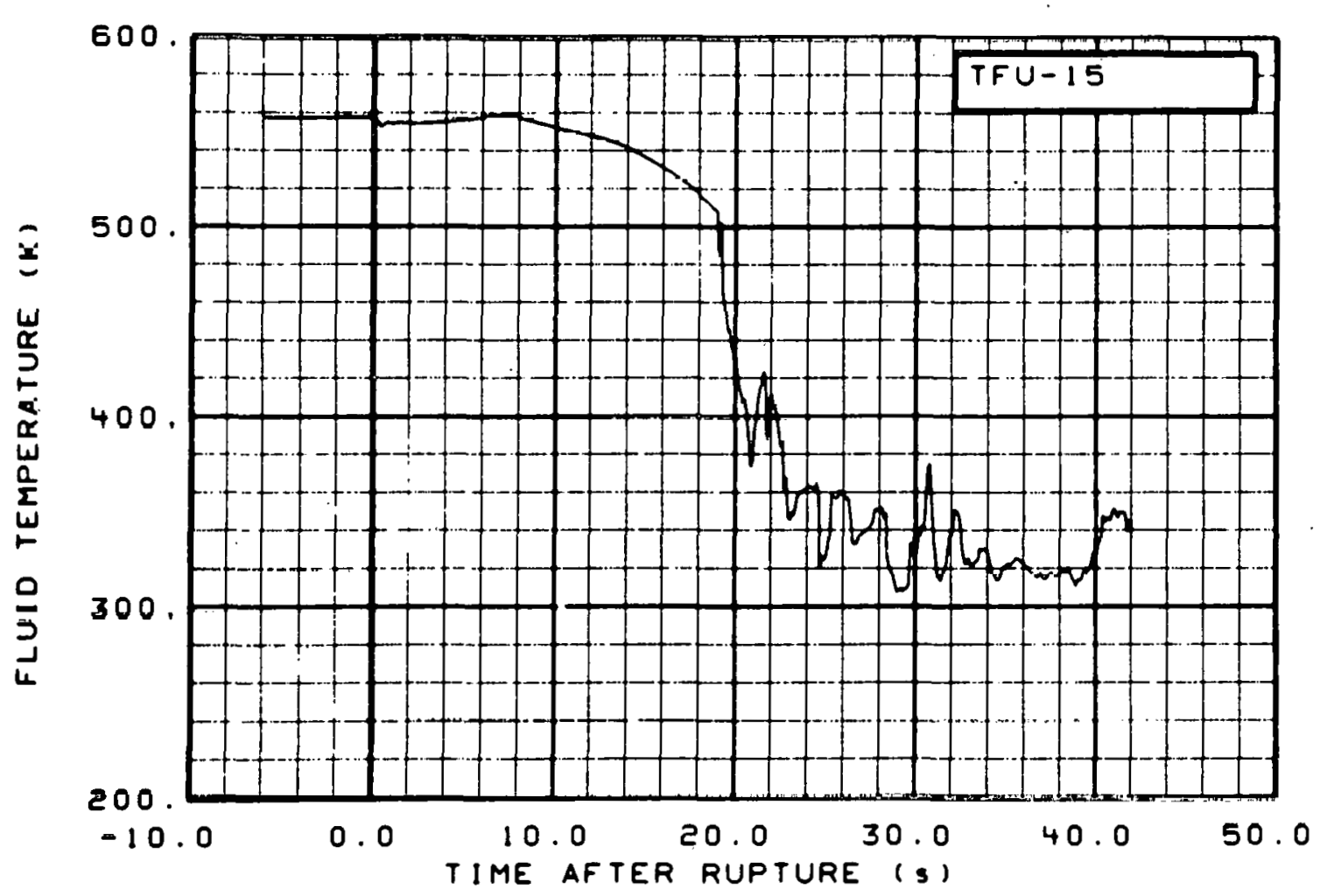

Fig. 18 Fluid temperature in intact loop cold leg (TFU-15), from -6 to $42 \mathrm{~s}$. 


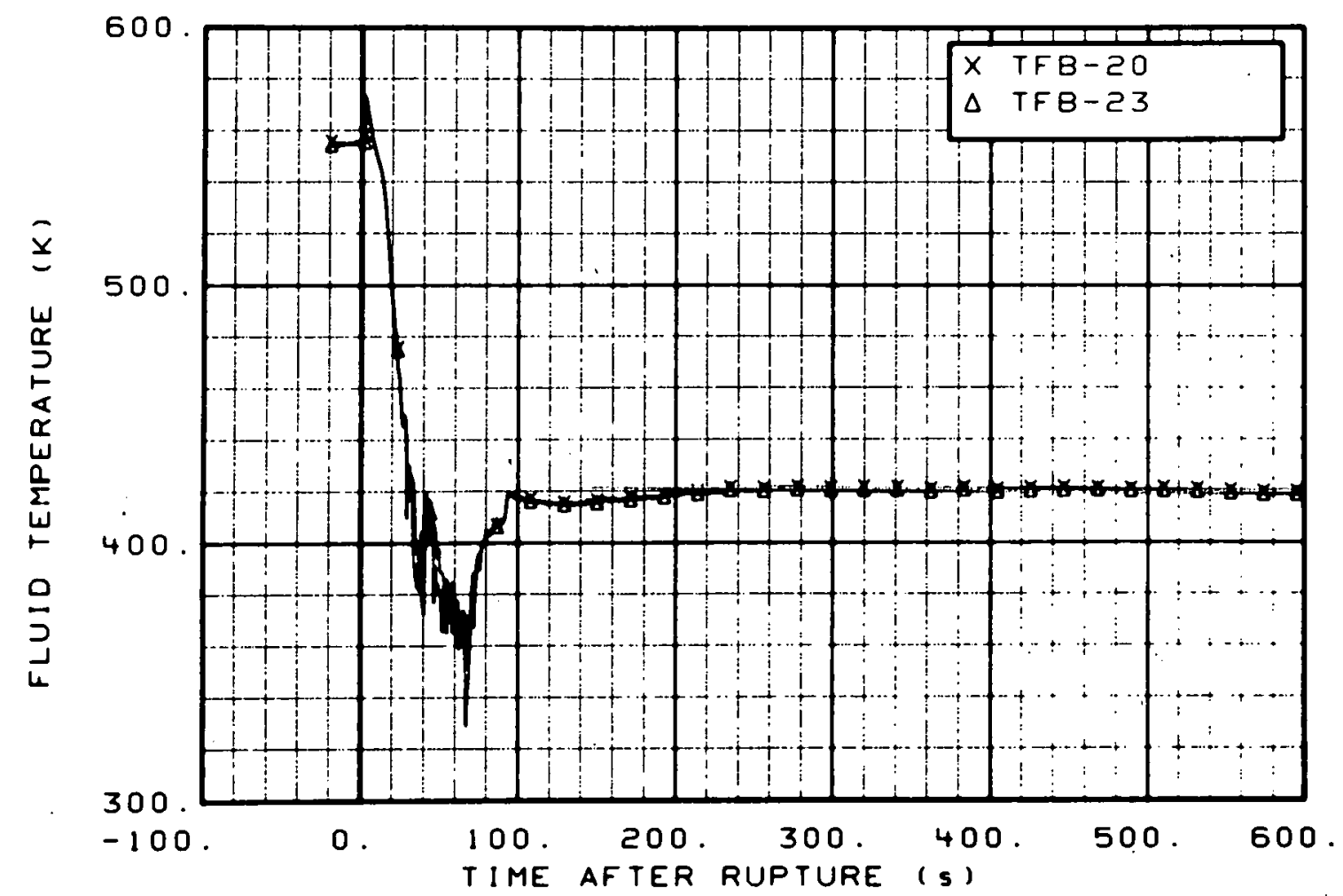

Fig. 19 Fluid temperature in broken loop, vessel side (TFB-20 and TFB-23), from -20 to $600 \mathrm{~s}$.

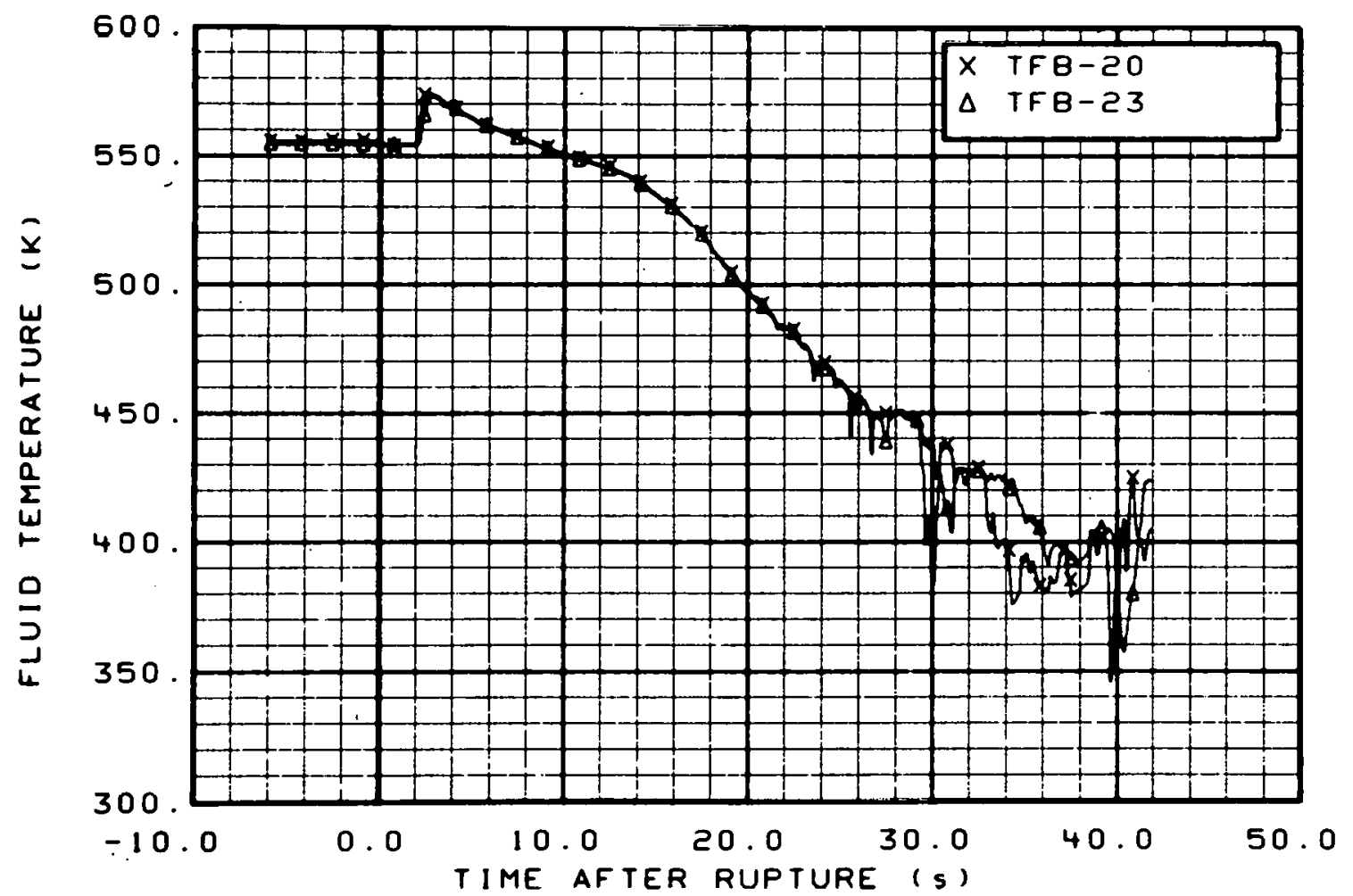

Fig. 20 Fluid temperature in broken loop, vessel side (TFB-20 and TFB-23), from -6 to $42 \mathrm{~s}$. 


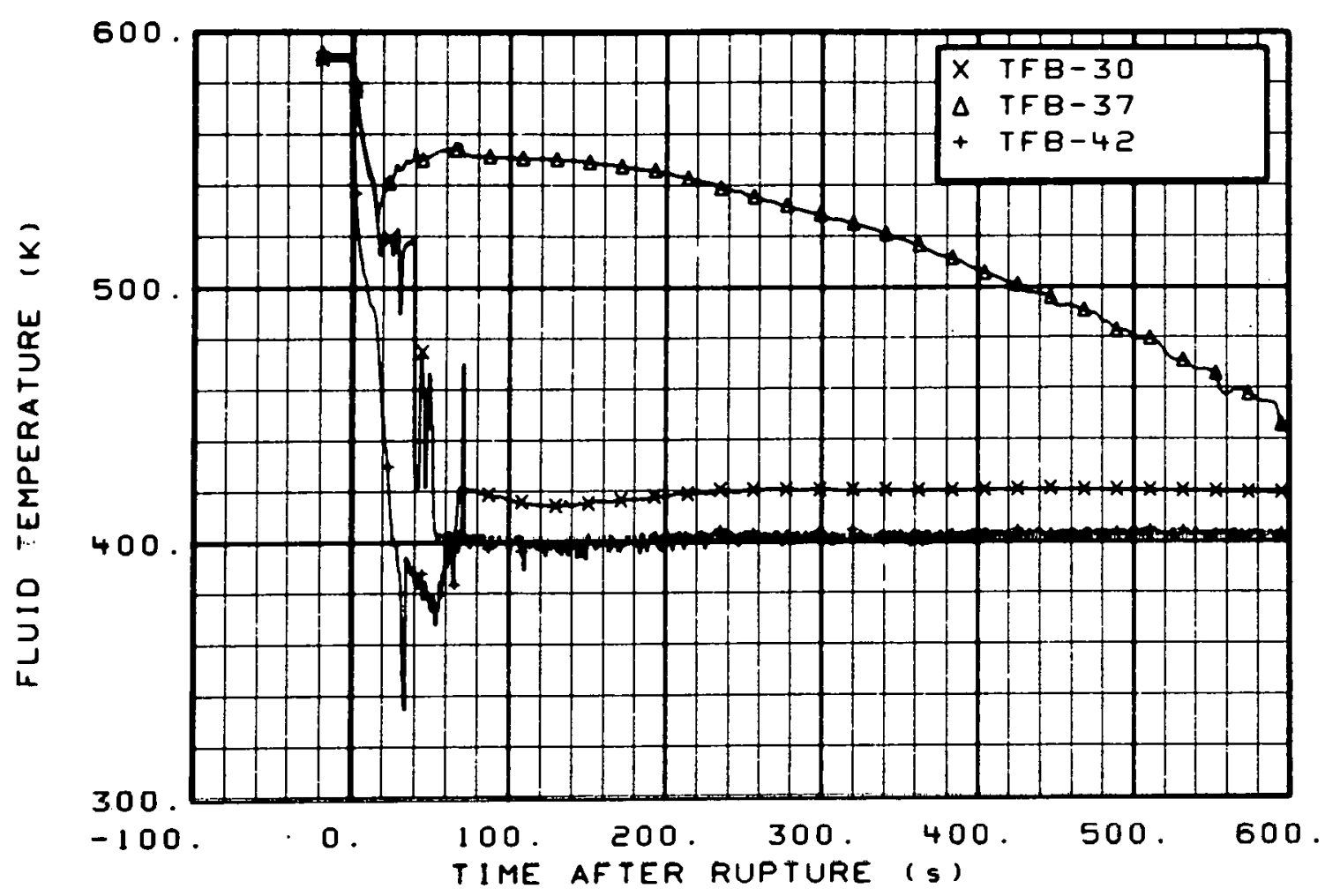

Fig. 21 Fluid temperature in broken loop, pump side (TFB-30, TFB-37, and TFB-42), from -20 to $600 \mathrm{~s}$.

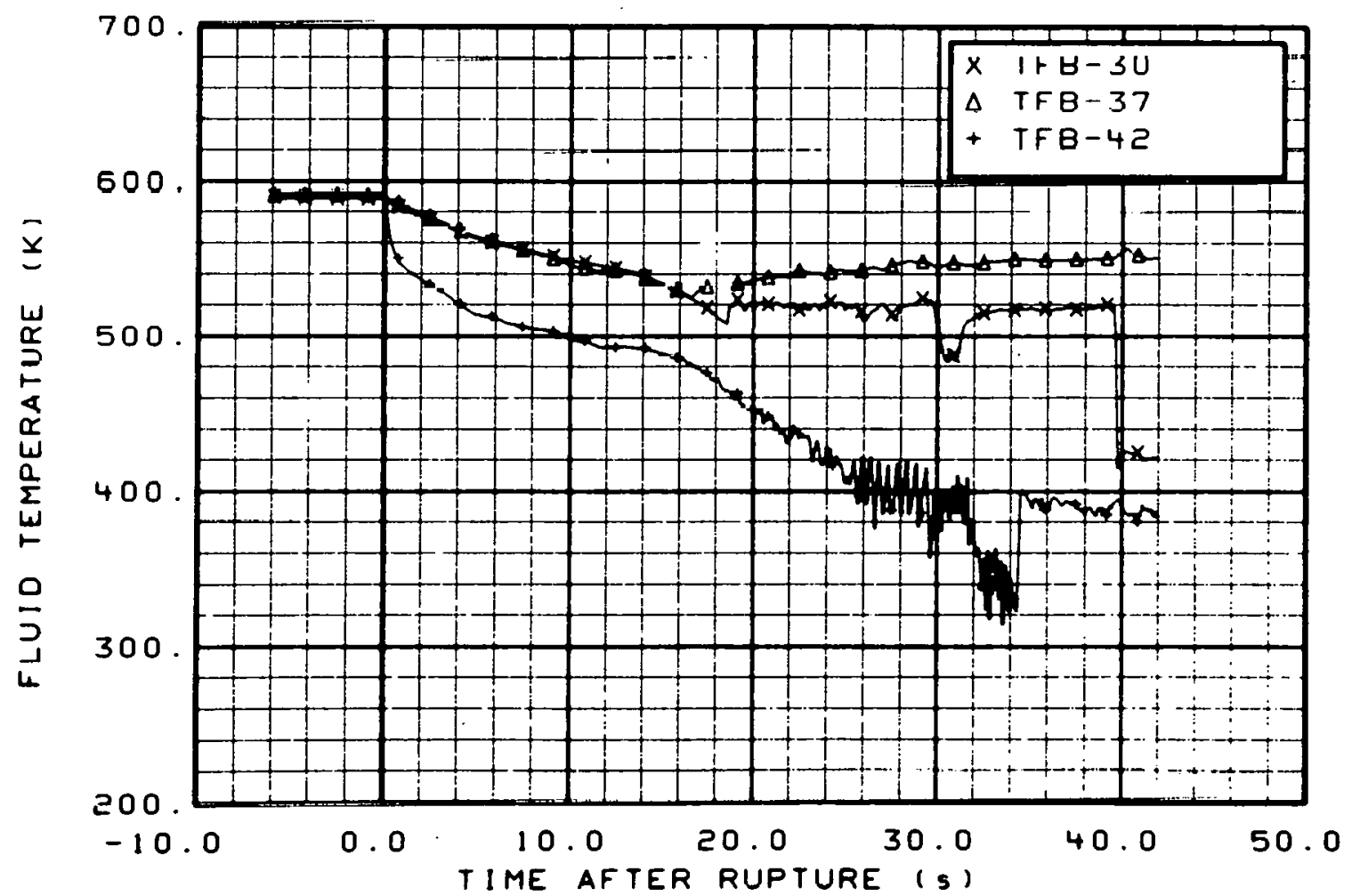

Fig. 22 Fluid temperature in broken loop, pump side (TFB-30, TFB-37, and TFB-42), from -6 to $42 \mathrm{~s}$. 


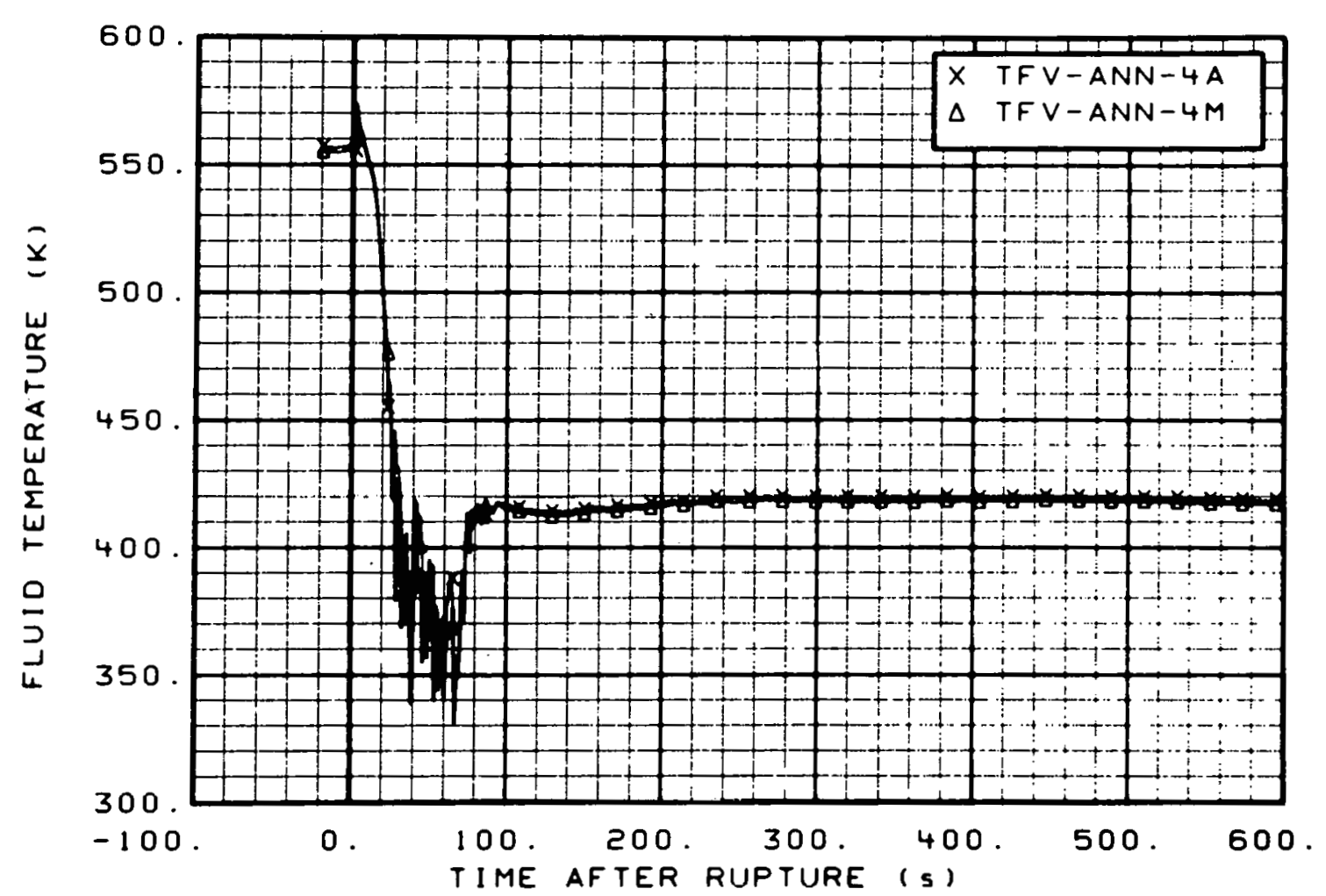

Fig. 23 Fluid temperature in inlet annulus (TFV-ANN-4A and TFV-ANN-4M), from -20 to $600 \mathrm{~s}$.

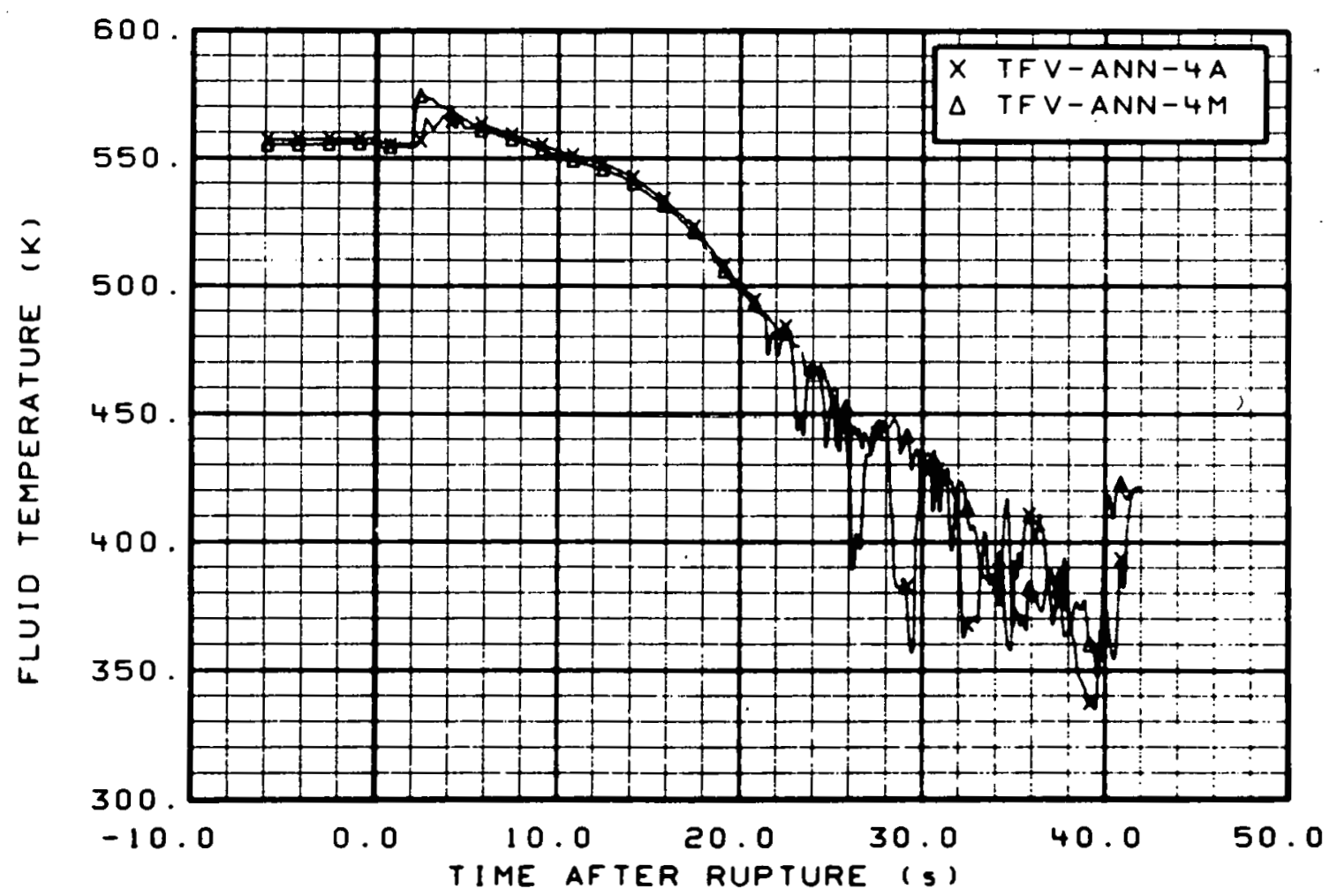

Fig. 24 Fluid temperature in inlet annulus (TFV-ANN-4A and TFV-ANN-4M), from -6 to $42 \mathrm{~s}$. 


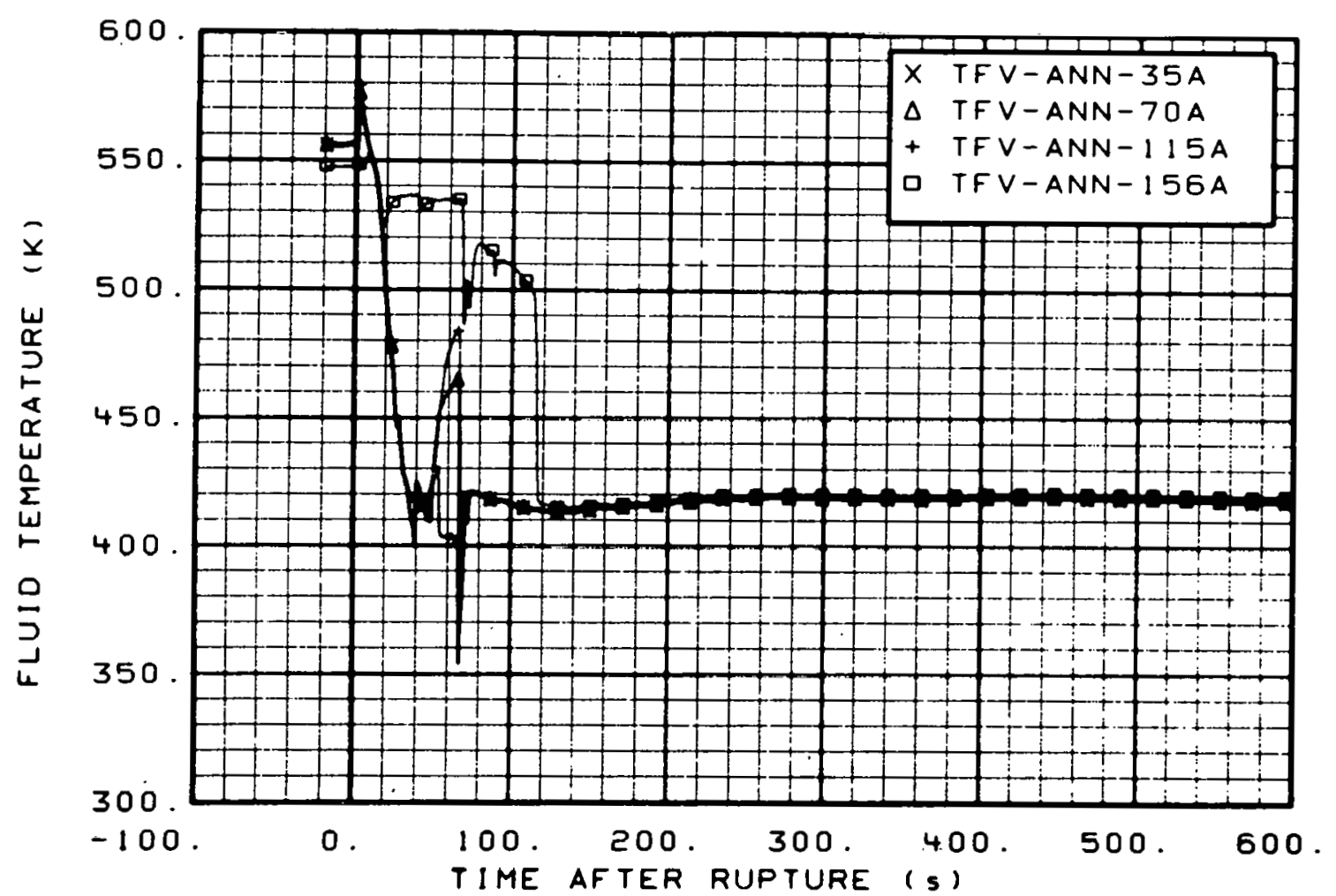

Fig. 25 Fluid temperature in downcomer annulus (TFV-ANN-35A, TFV-ANN-70A, TFV-ANN-115A, and TFV-ANN-156A), from -20 to $600 \mathrm{~s}$.

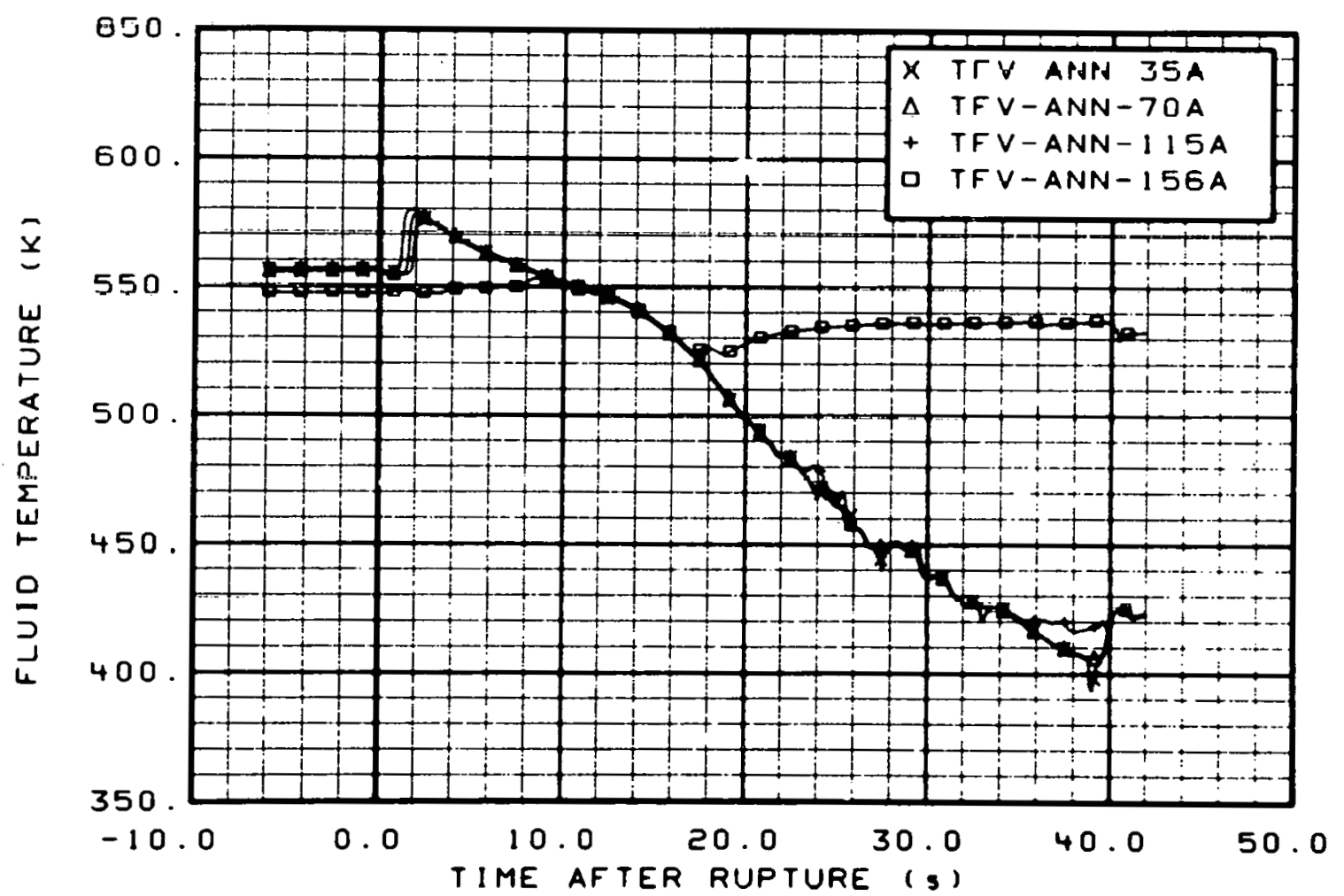

Fig. 26 Fluid temperature in downcomer annulus (TFV-ANN-35A, TFV-ANN-70A, TFV-ANN-115A, and TFV-ANN-156A), from -6 to $42 \mathrm{~s}$. 


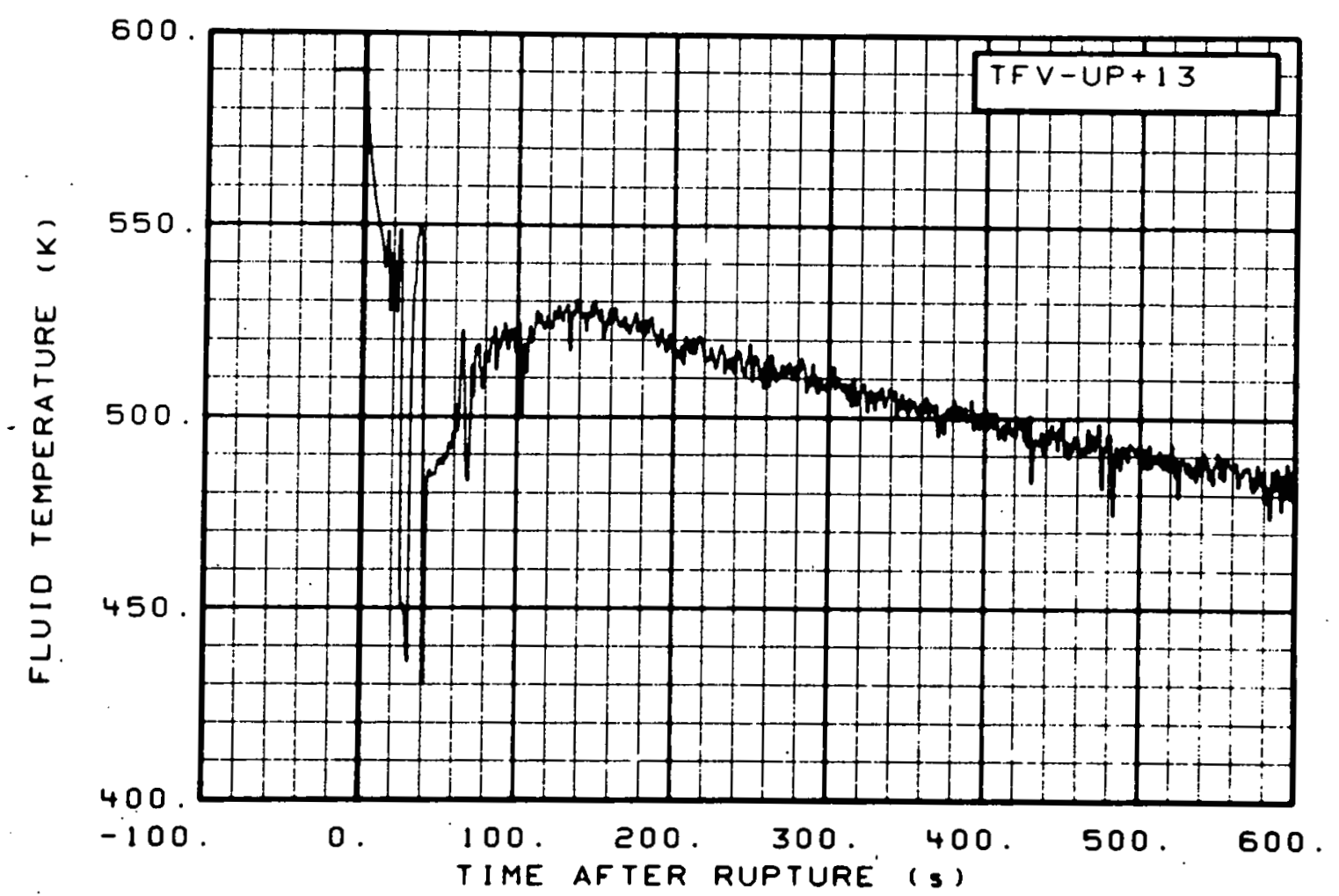

Fig. 27 Fluid temperature in upper plenum (TFV-UP+13), from -20 to $600 \mathrm{~s}$.

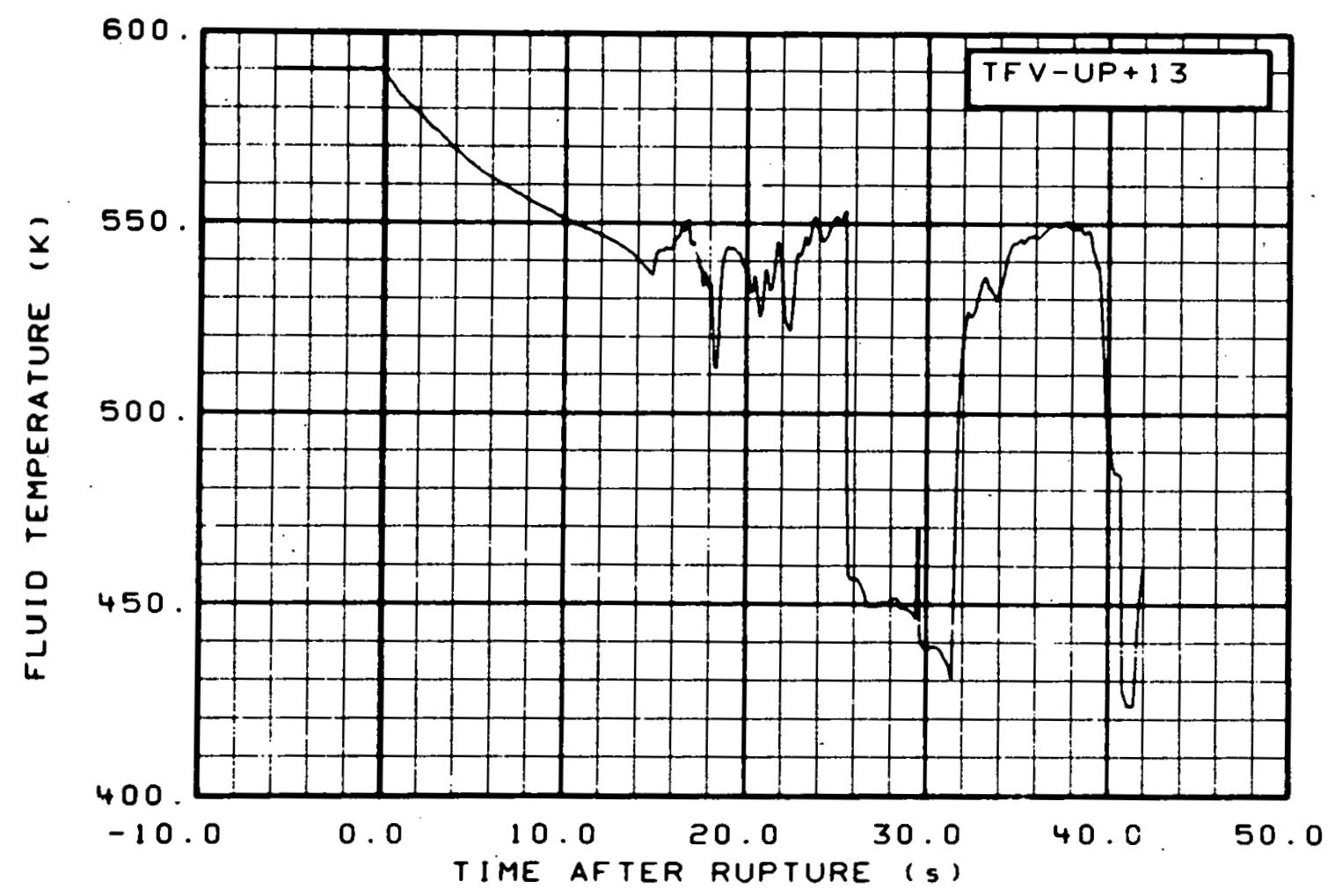

Fig. 28 Fluid temperature in upper plenum (TFV-UP+13), from -6 to $42 \mathrm{~s}$. 


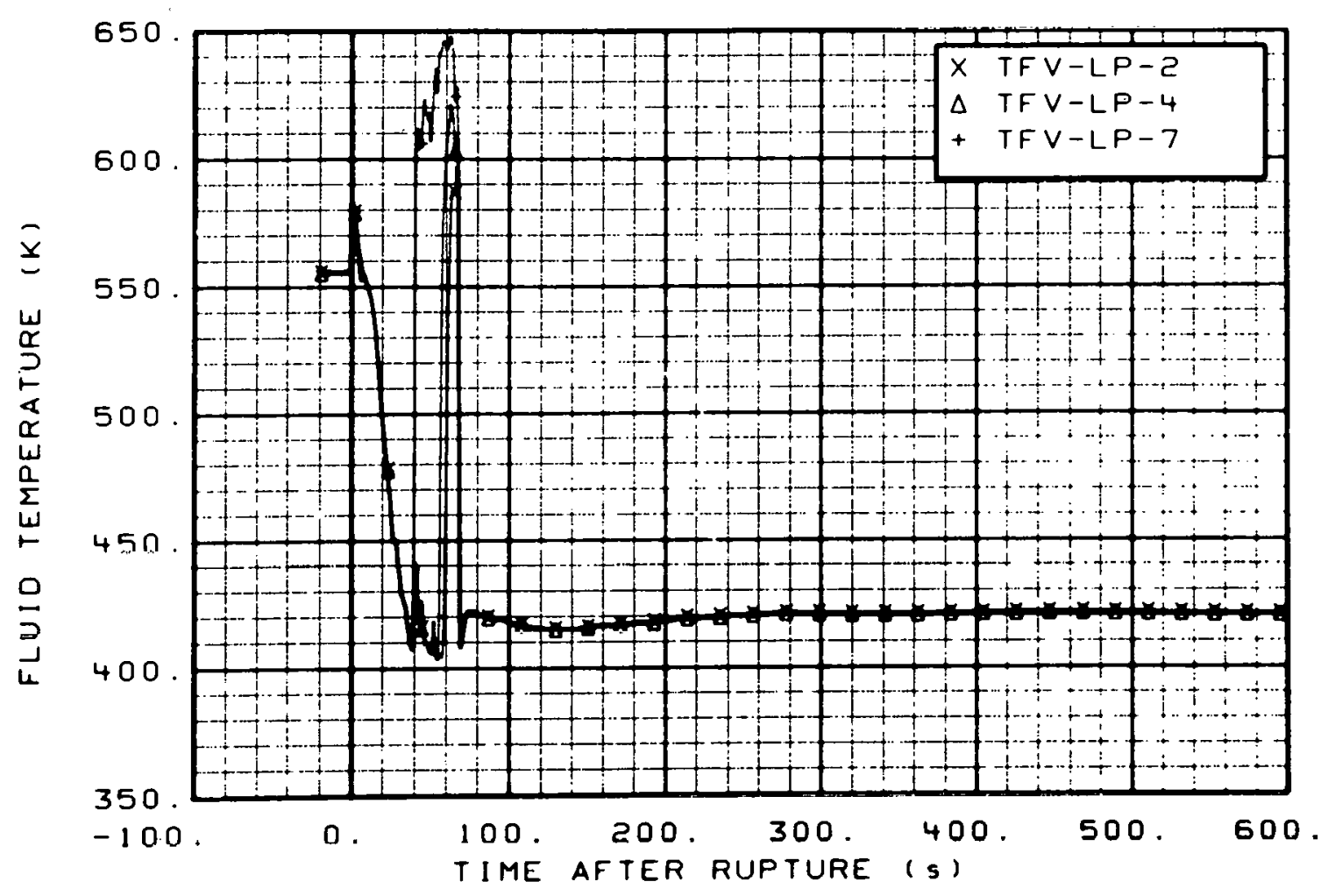

Fig. 29 Fluid temperature in lower plenum (TFV-LP-2, TFV-LP-4, and TFV-LP-7), from -20 to $600 \mathrm{~s}$.

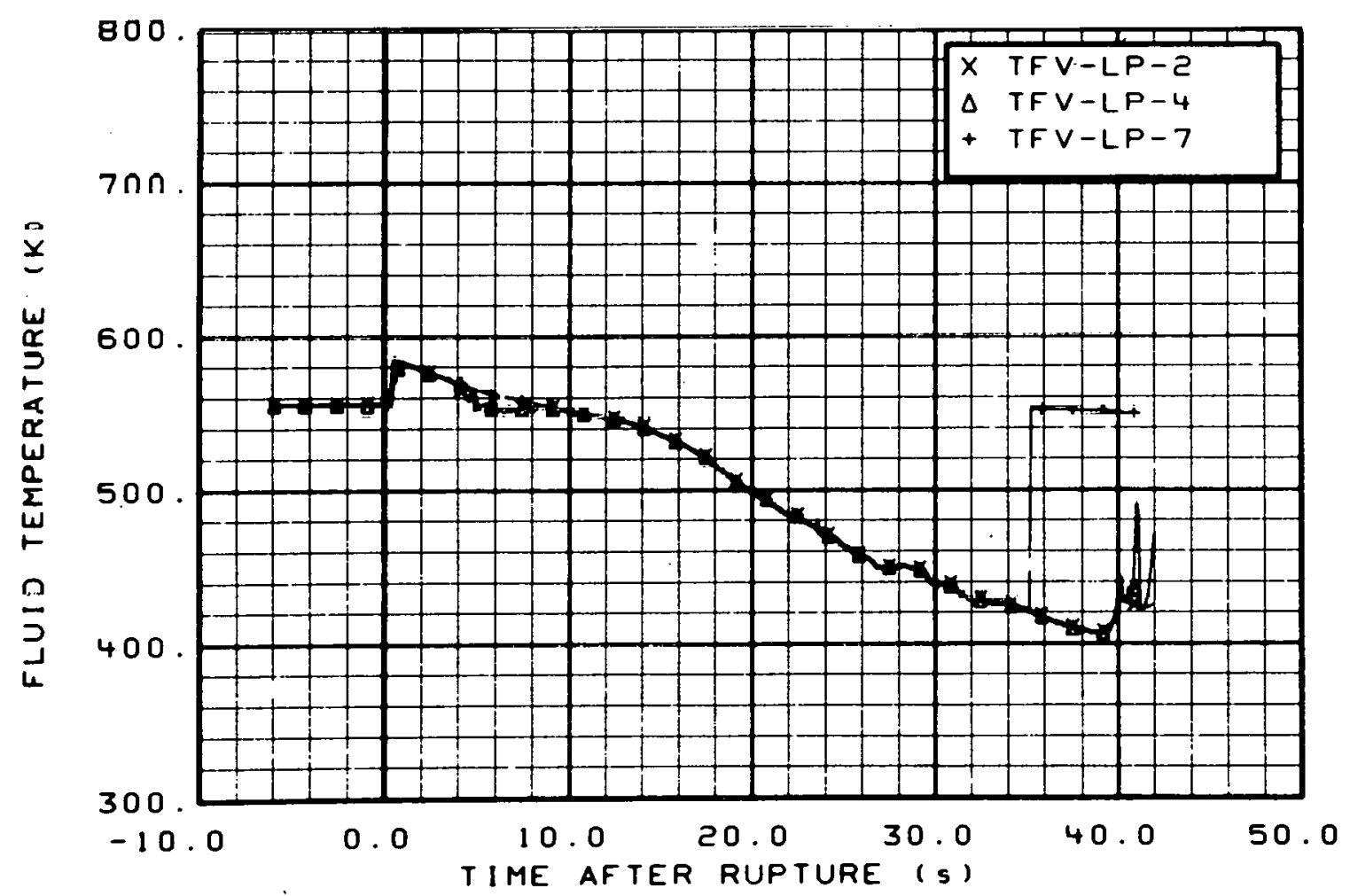

Fig. 30 Fluid temperature in lower plenum (TFV-LP-2, TFV-LP-4, and TFV-LP-7), from -6 to $42 \mathrm{~s}$. 


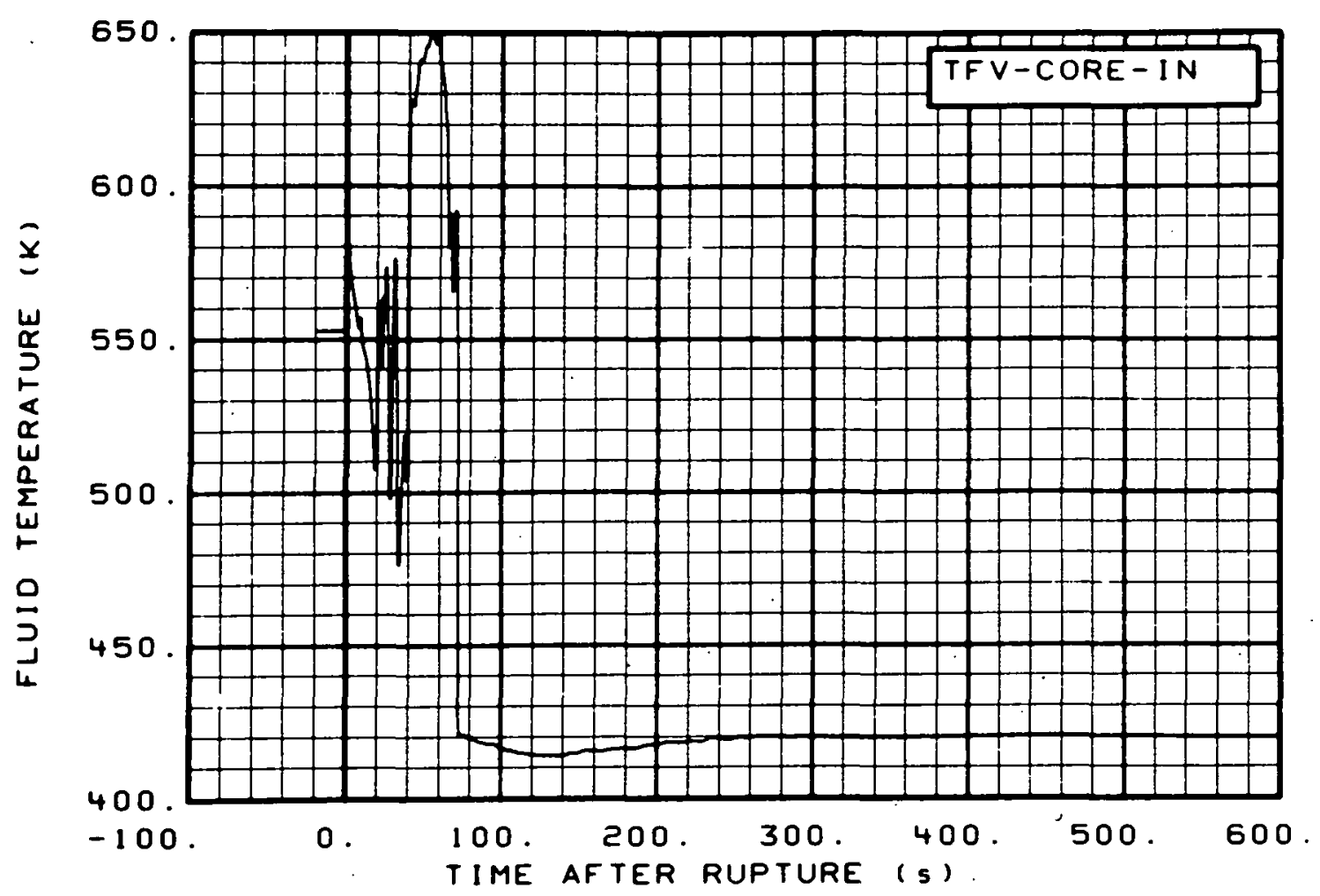

Fig. 31 Fluid temperature in core inlet (TFV-CORE-IN), from -20 to $600 \mathrm{~s}$.

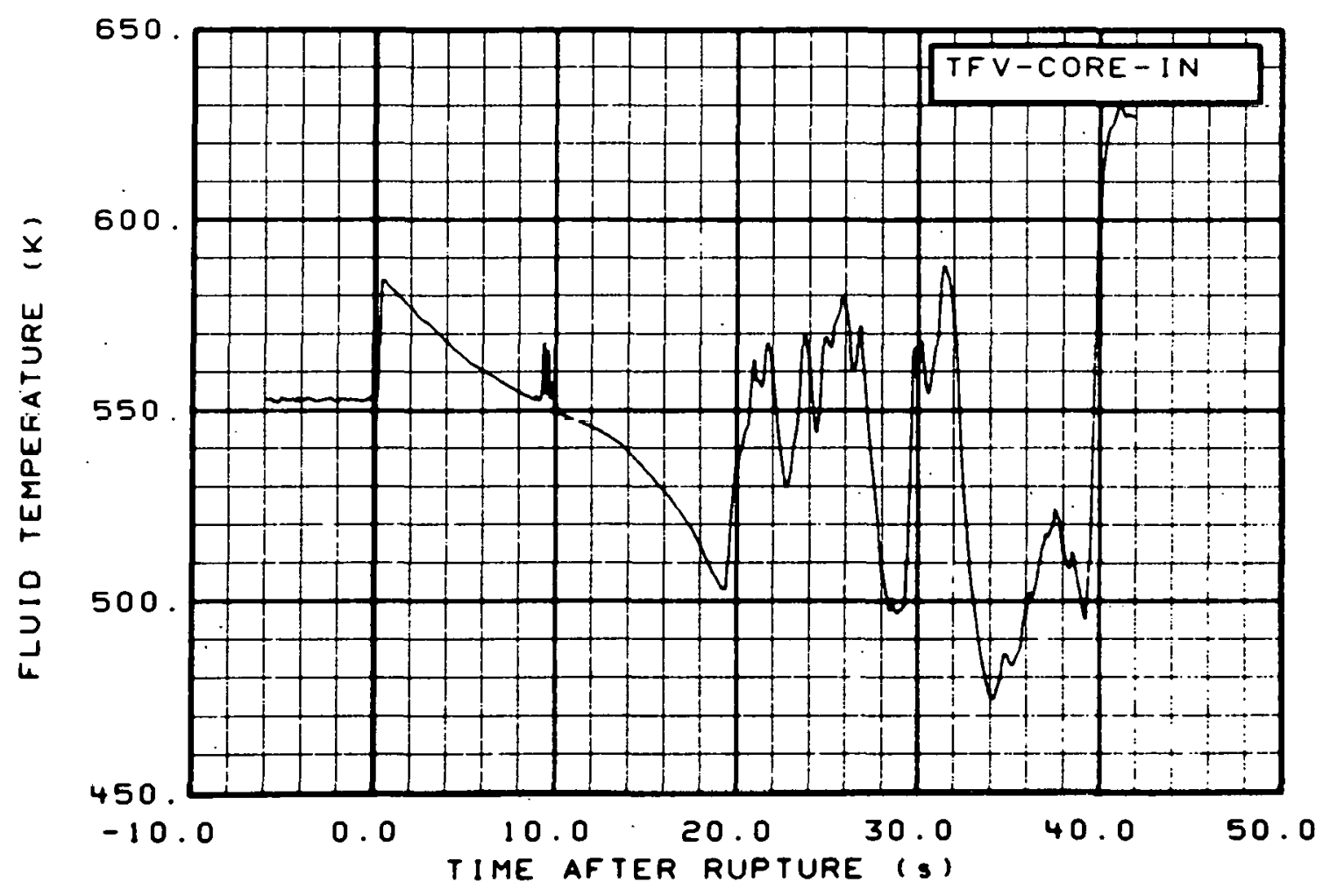

Fig. 32 Fluid temperature in core inlet (TFV-CORE-IN), from -6 to $42 \mathrm{~s}$. 


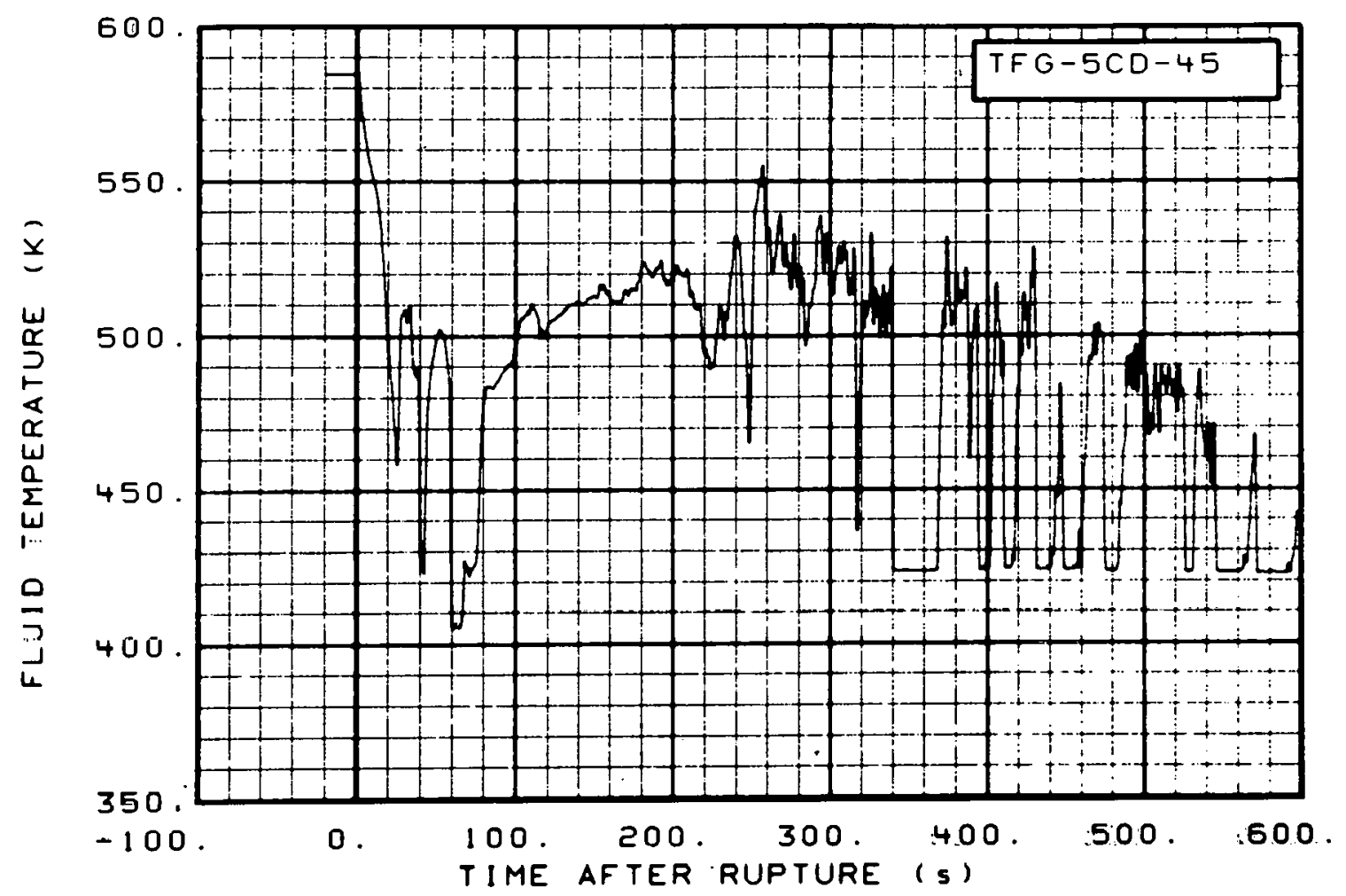

Fig. 33 Fluid temperature in core, Grid Spacer 5 (TFG-5CD-45), from -20 to $600 \mathrm{~s}$.

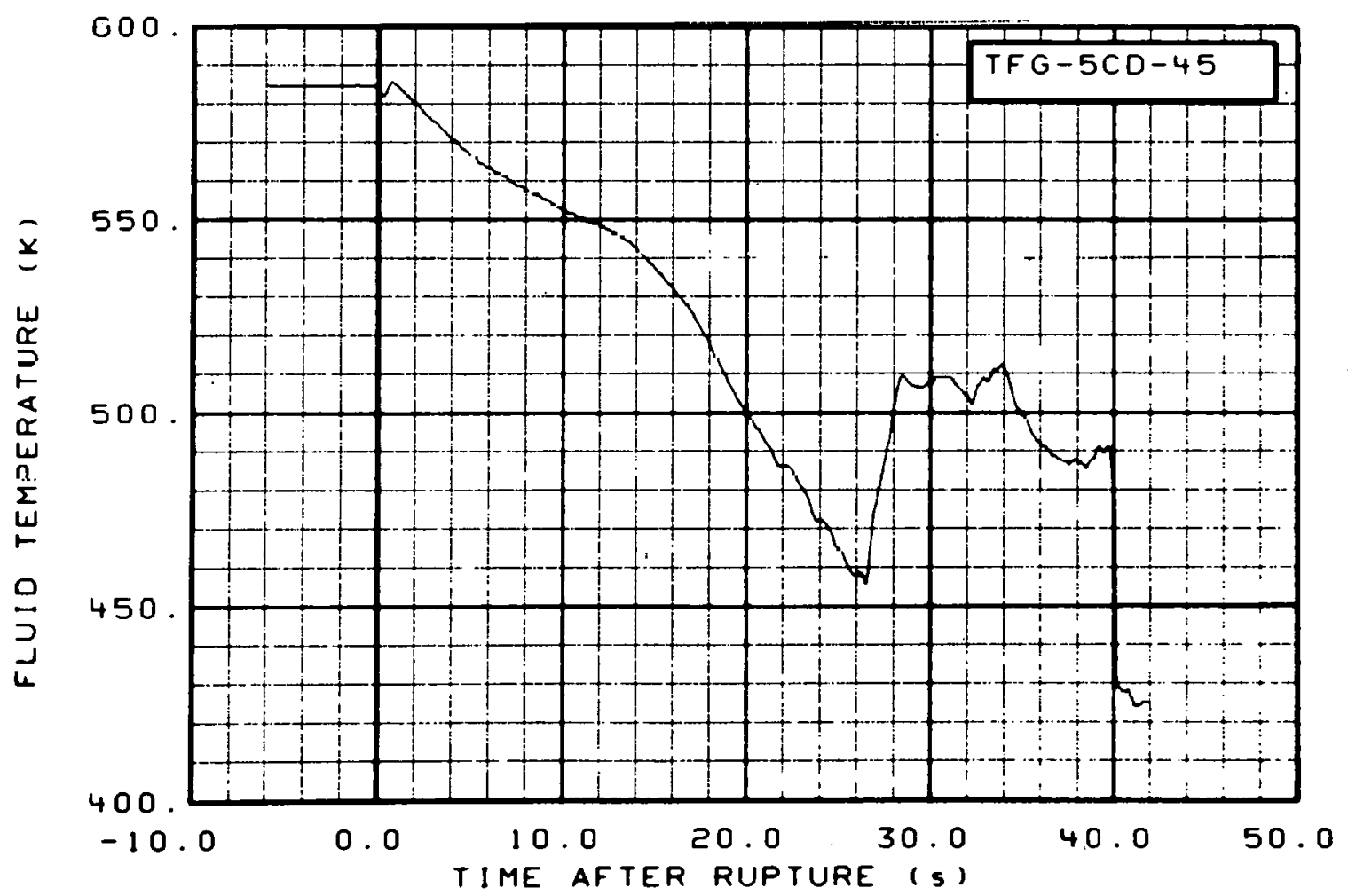

Fig. 34 Fluid temperature in core, Grid Spacer 5 (TFG-5CD-45), from -6 to $42 \mathrm{~s}$. 


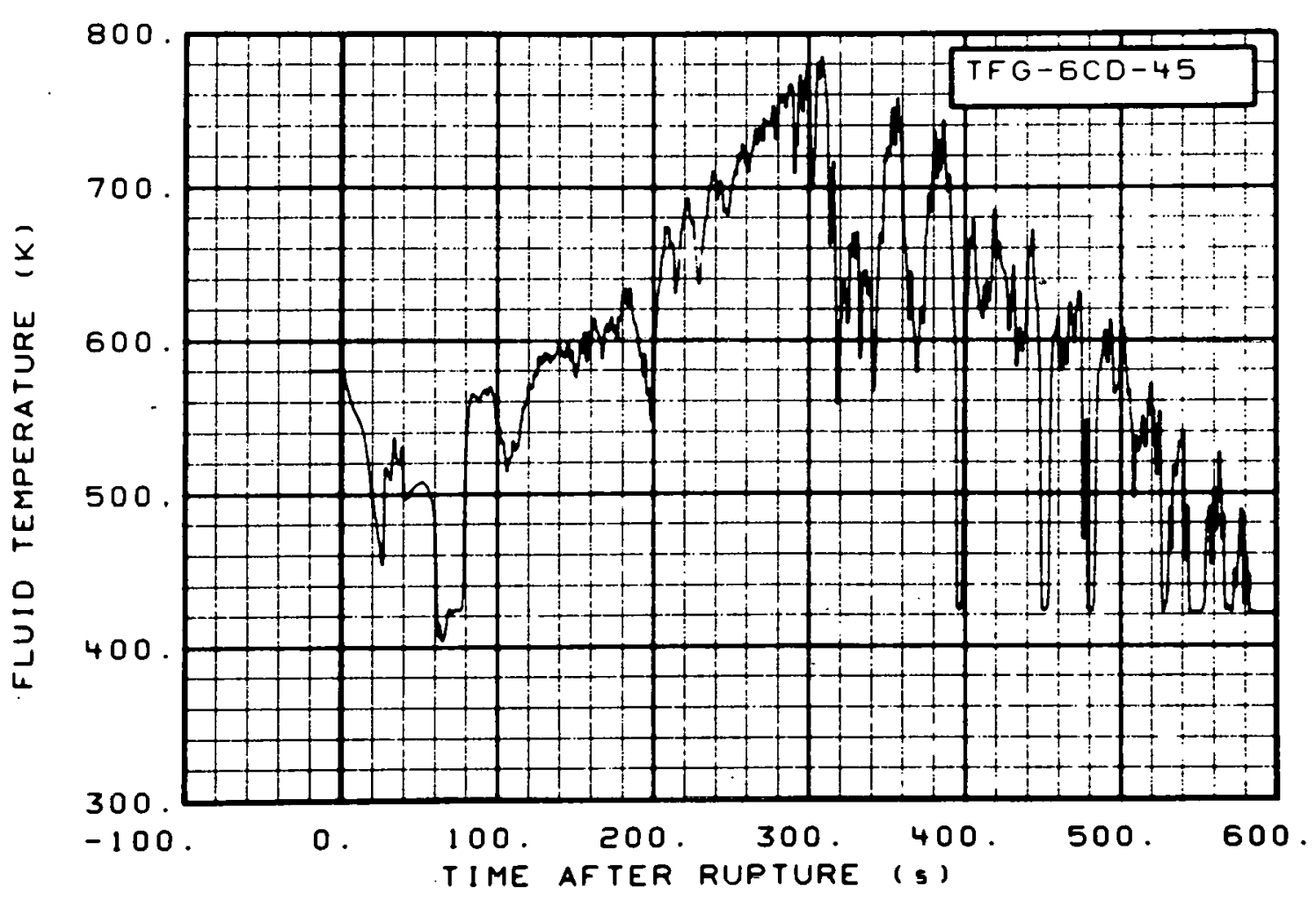

Fig. 35 Fluid temperature in core, Grid Spacer 6 (TFG-6CD-45), from -20 to $600 \mathrm{~s}$.

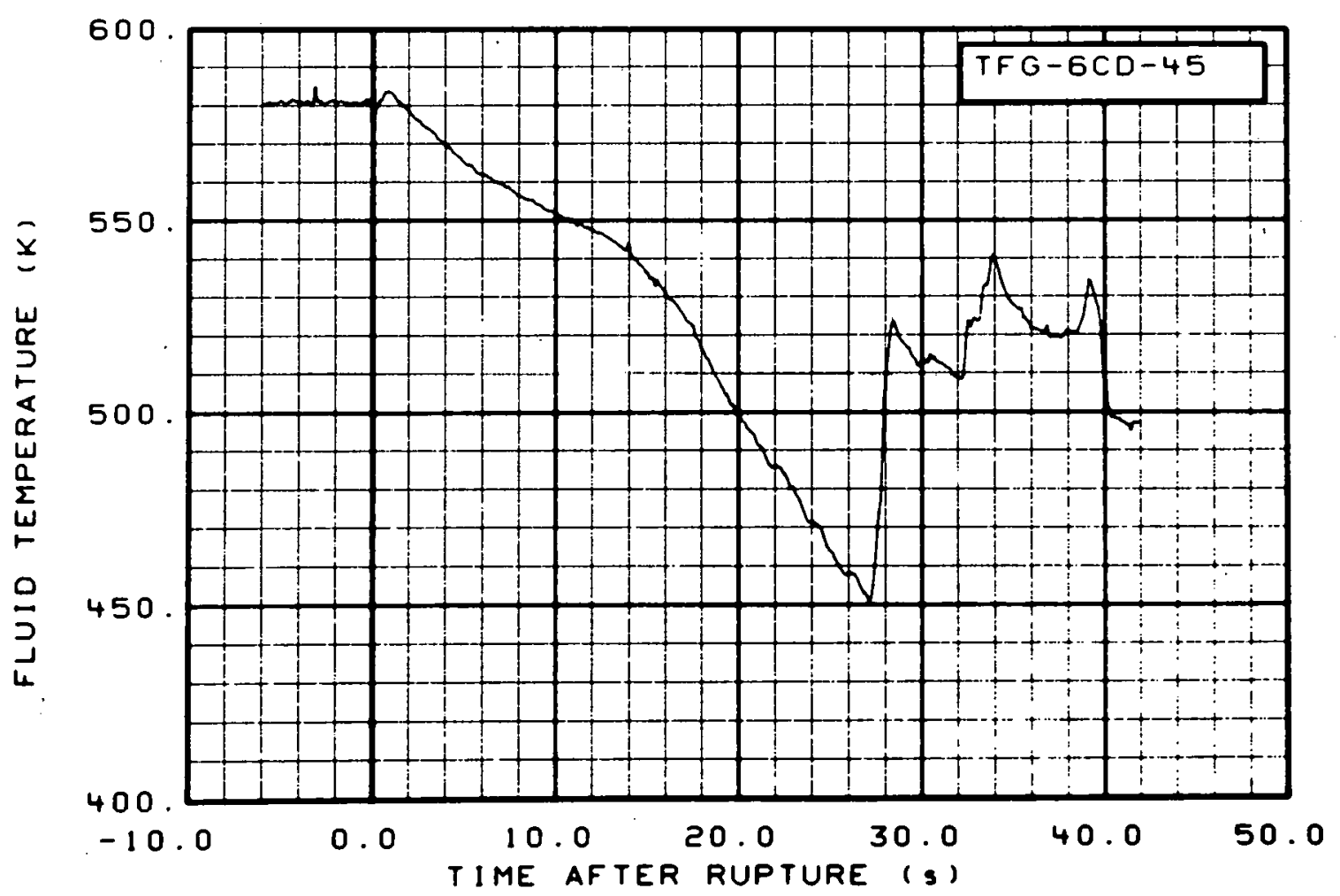

Fig. 36 Fluid temperature in core, Grid Spacer 6 (TFG-6CD-45), from -6 to $42 \mathrm{~s}$. 


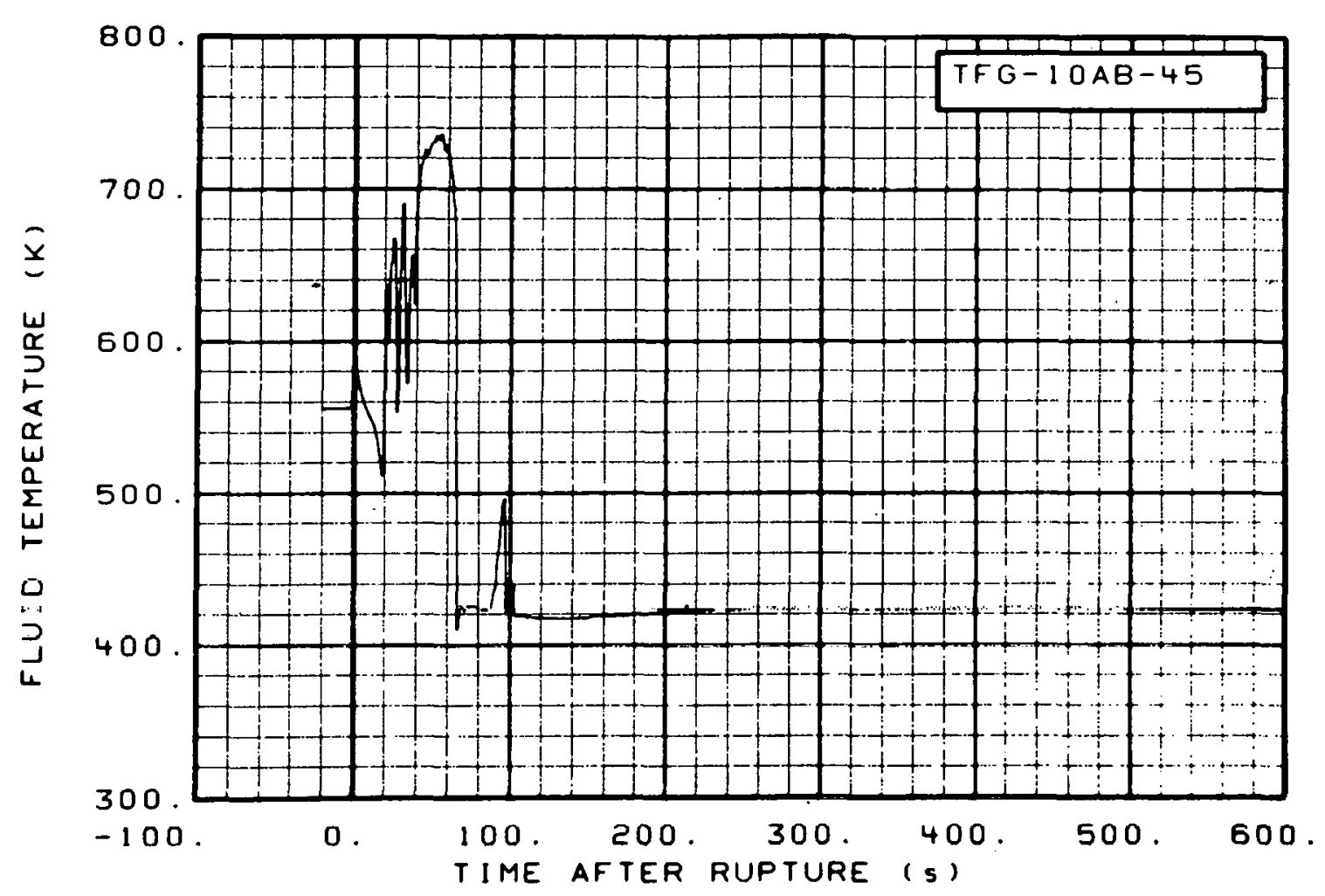

Fig. 37 Fluid temperature in core, Grid Spacer 10 (TFG-10AB-45), from -20 to $600 \mathrm{~s}$.

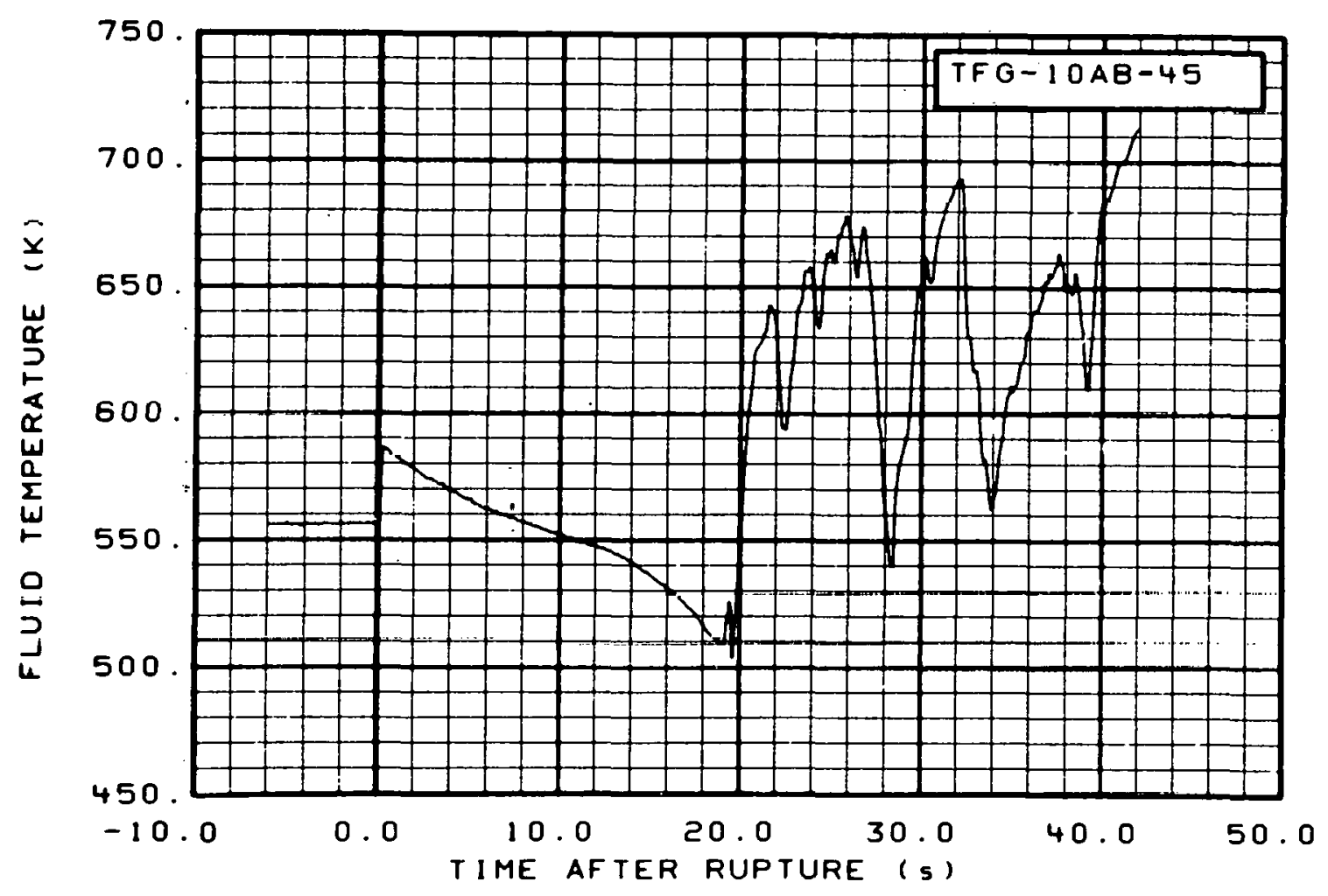

Fig. 38 Fluid temperature in core, Grid Spacer 10 (TFG-10AB-45), from -6 to $42 \mathrm{~s}$. 


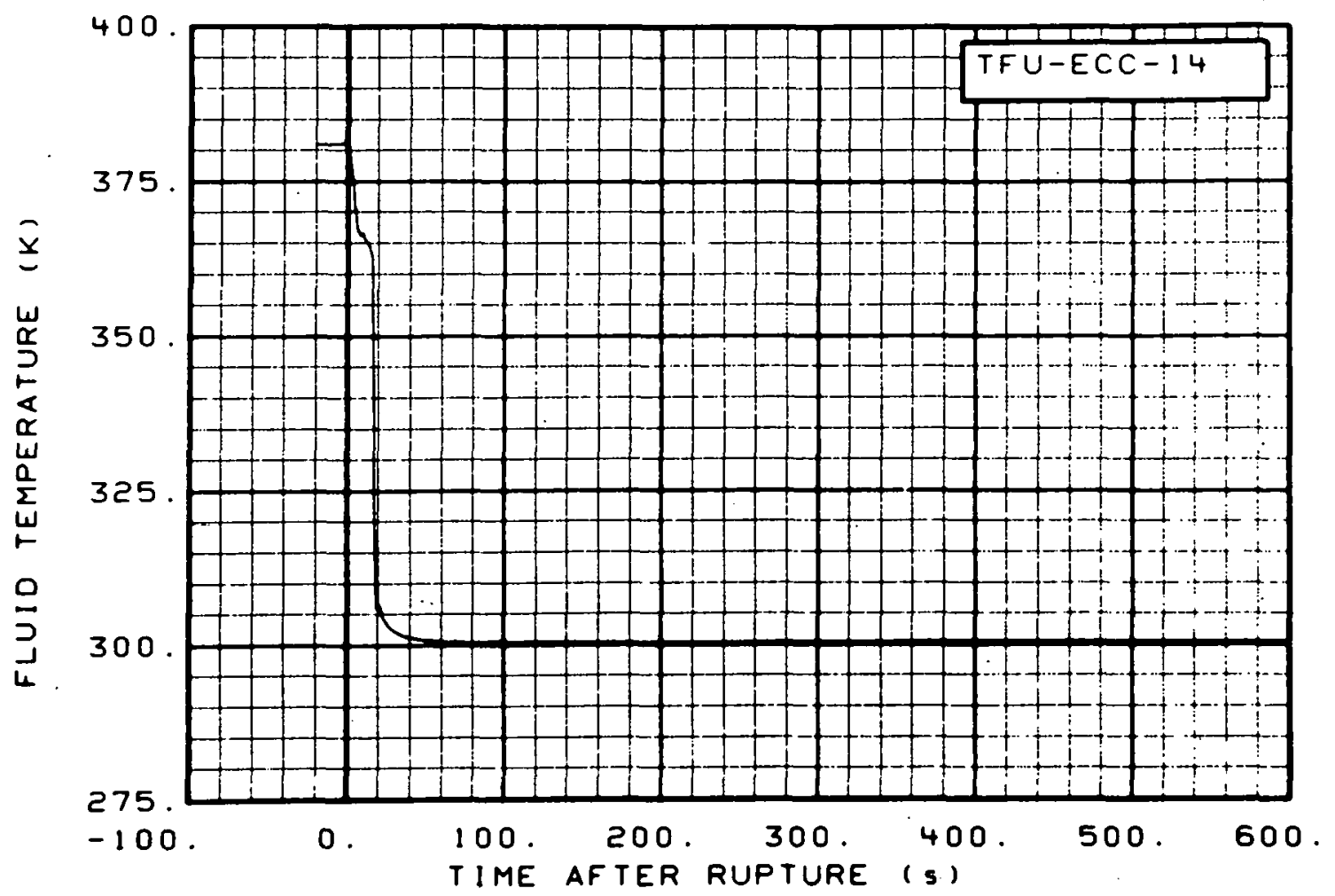

Fig. 39 Fluid temperature in intact loop coolant injection line (TFU-ECC-14), from -20 to $600 \mathrm{~s}$.

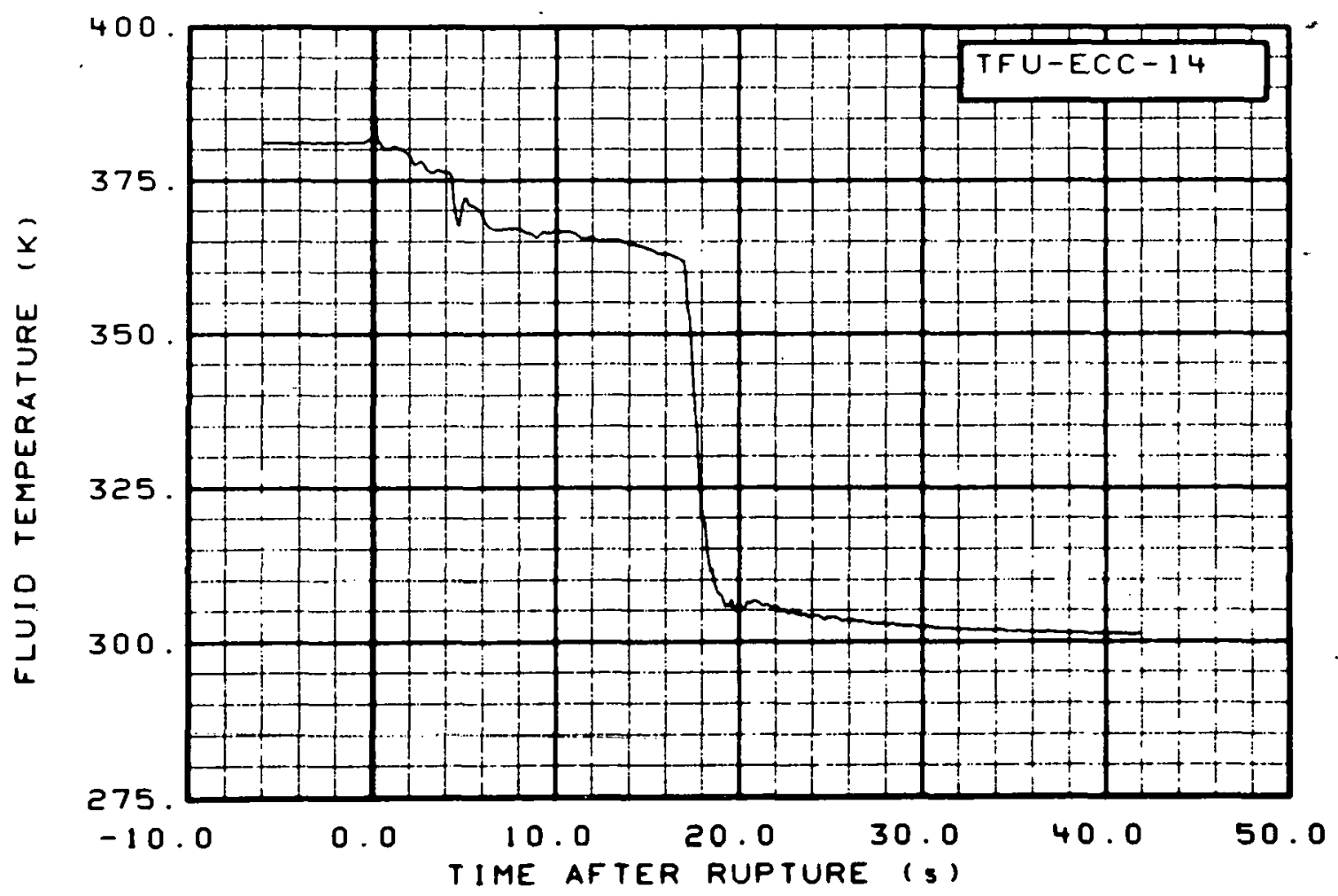

Fig. 40 Fluid temperature in intact loop coolant injection line (TFU-ECC14), from -6 to $42 \mathrm{~s}$. 


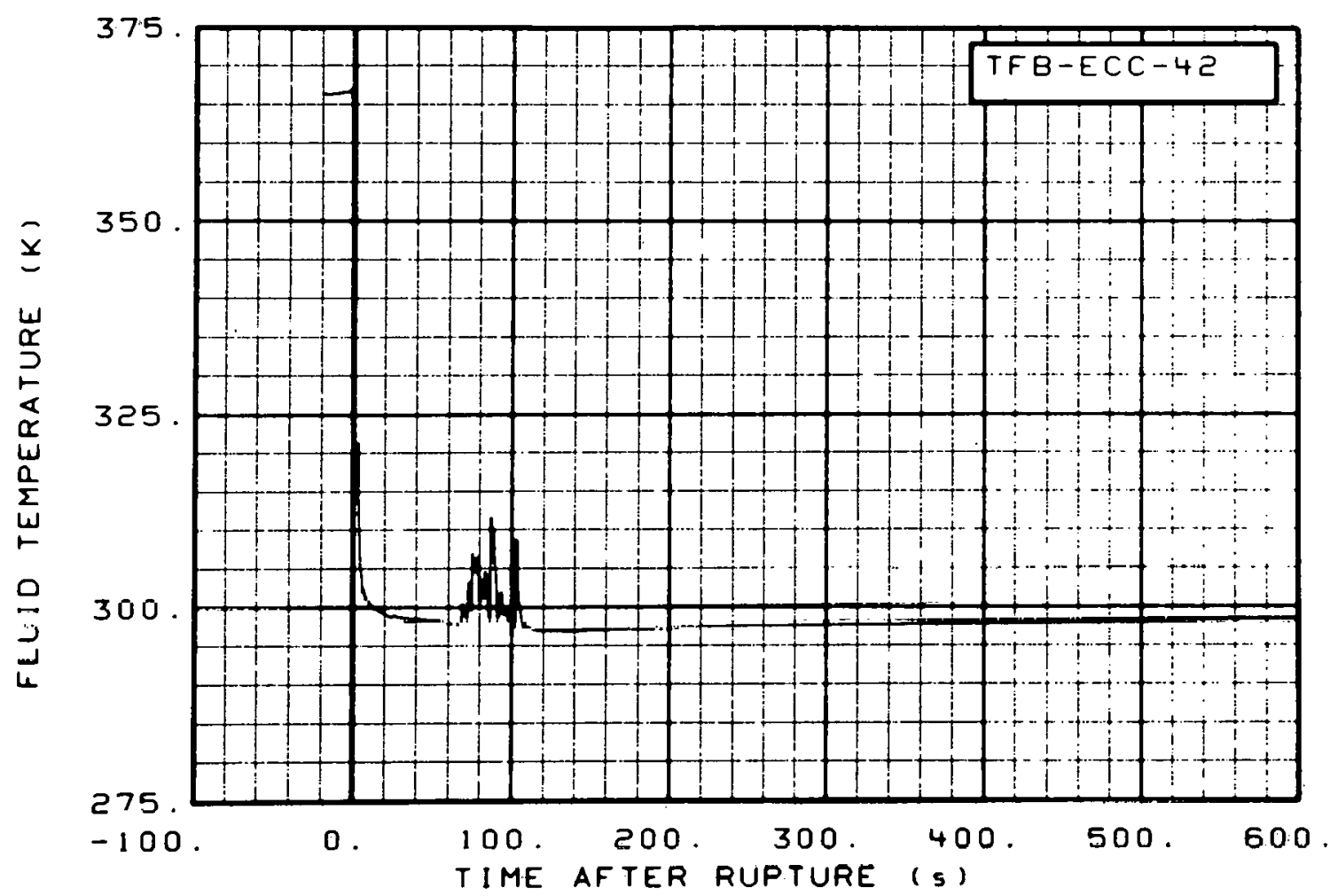

Fig. 41 Fluid temperature in broken loop coolant injection line (TFB-ECC42), from -20 to $600 \mathrm{~s}$.

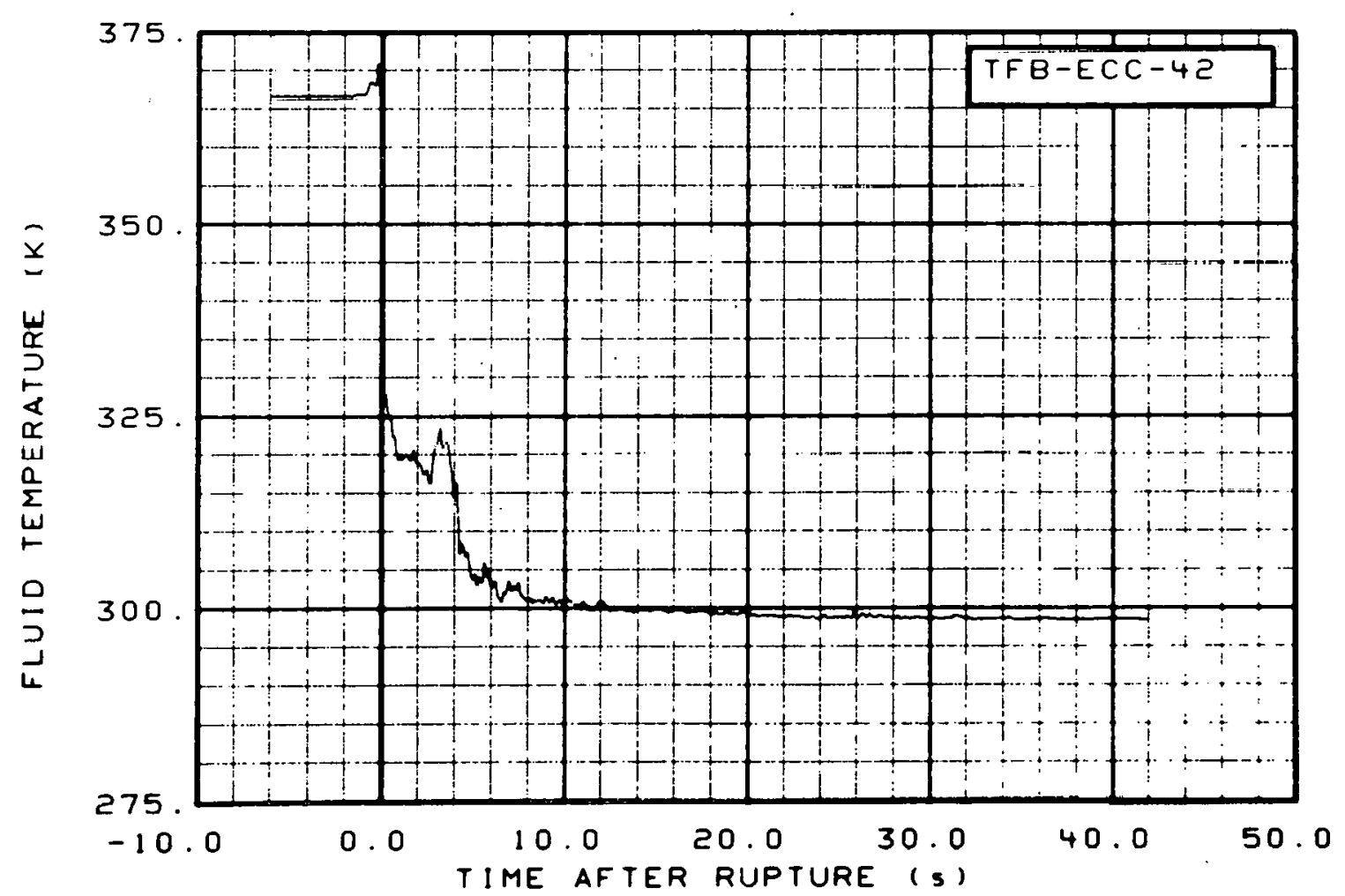

Fig. 42 Fluid temperature in broken loop coolant injection line (TFB-ECC42), from -6 to $42 \mathrm{~s}$. 


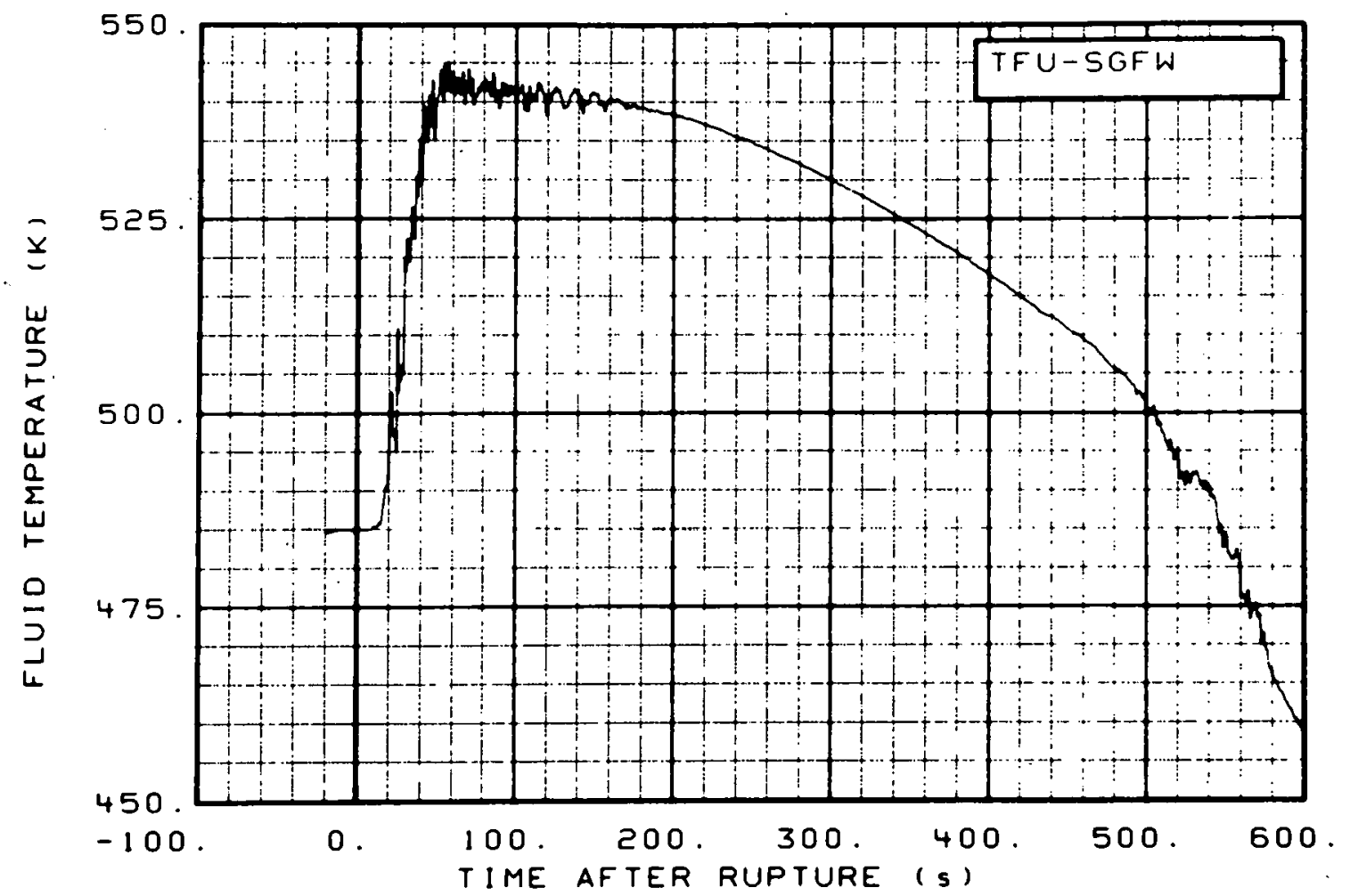

Fig. 43 Fluid temperature in steam generator, feedwater line (TFU-SGFW), from -20 to $600 \mathrm{~s}$.

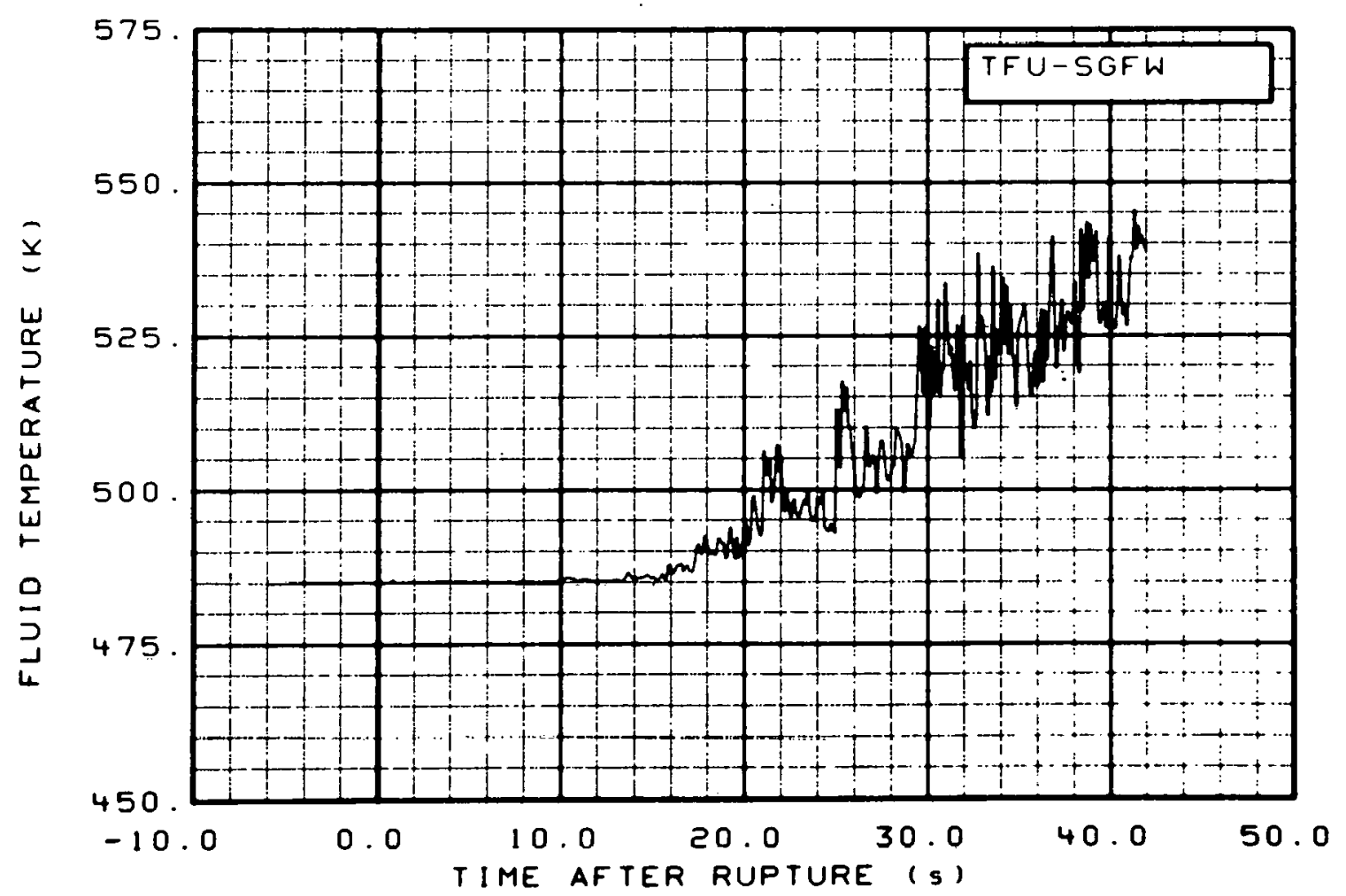

Fig. 44 Fluid temperature in steam generator, feedwater line (TFU-SGFW), from -6 to $42 \mathrm{~s}$. 


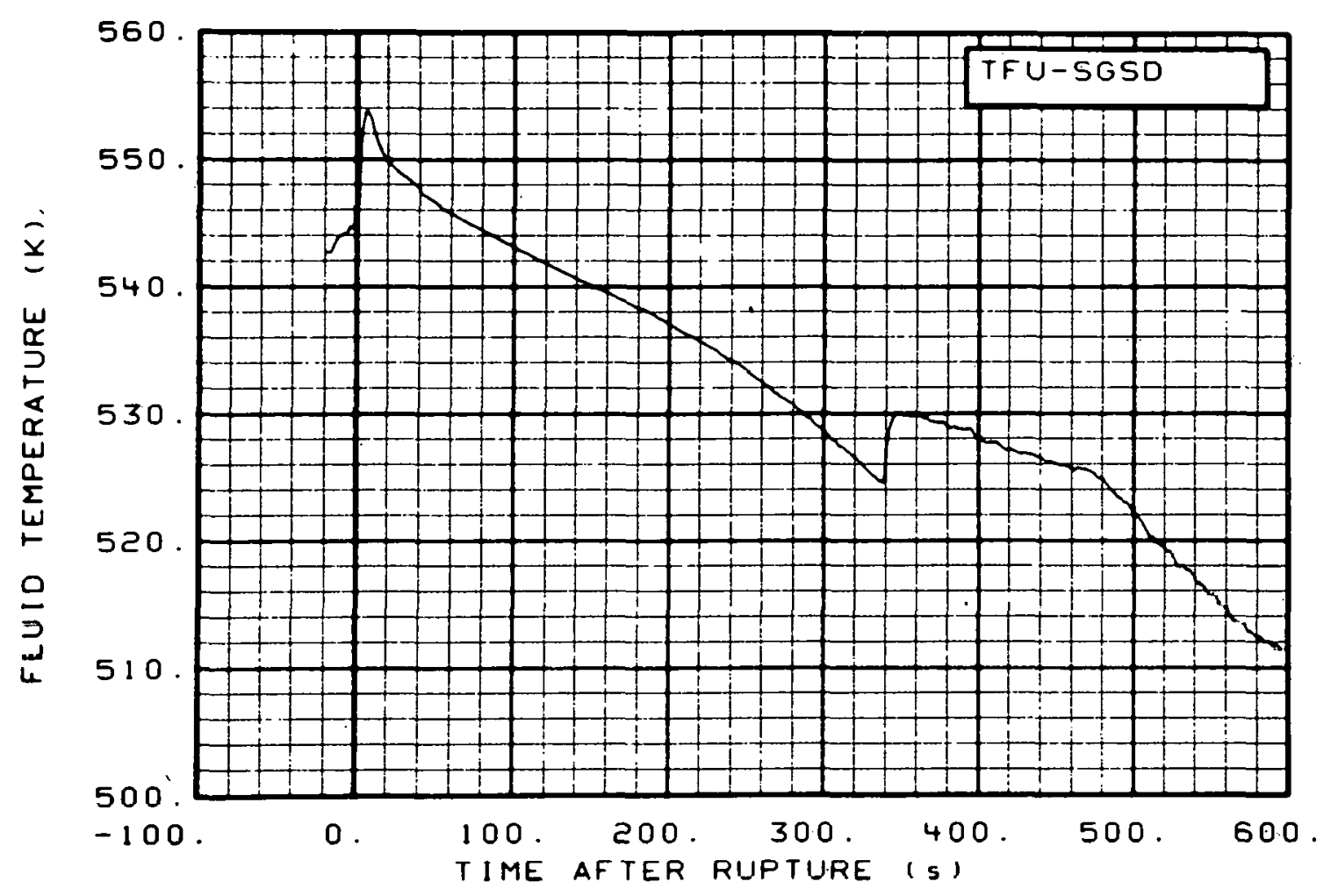

Fig. 45 Fluid temperature in steam generator, steam dome (TFU-SGSD), from -20 to $600 \mathrm{~s}$.

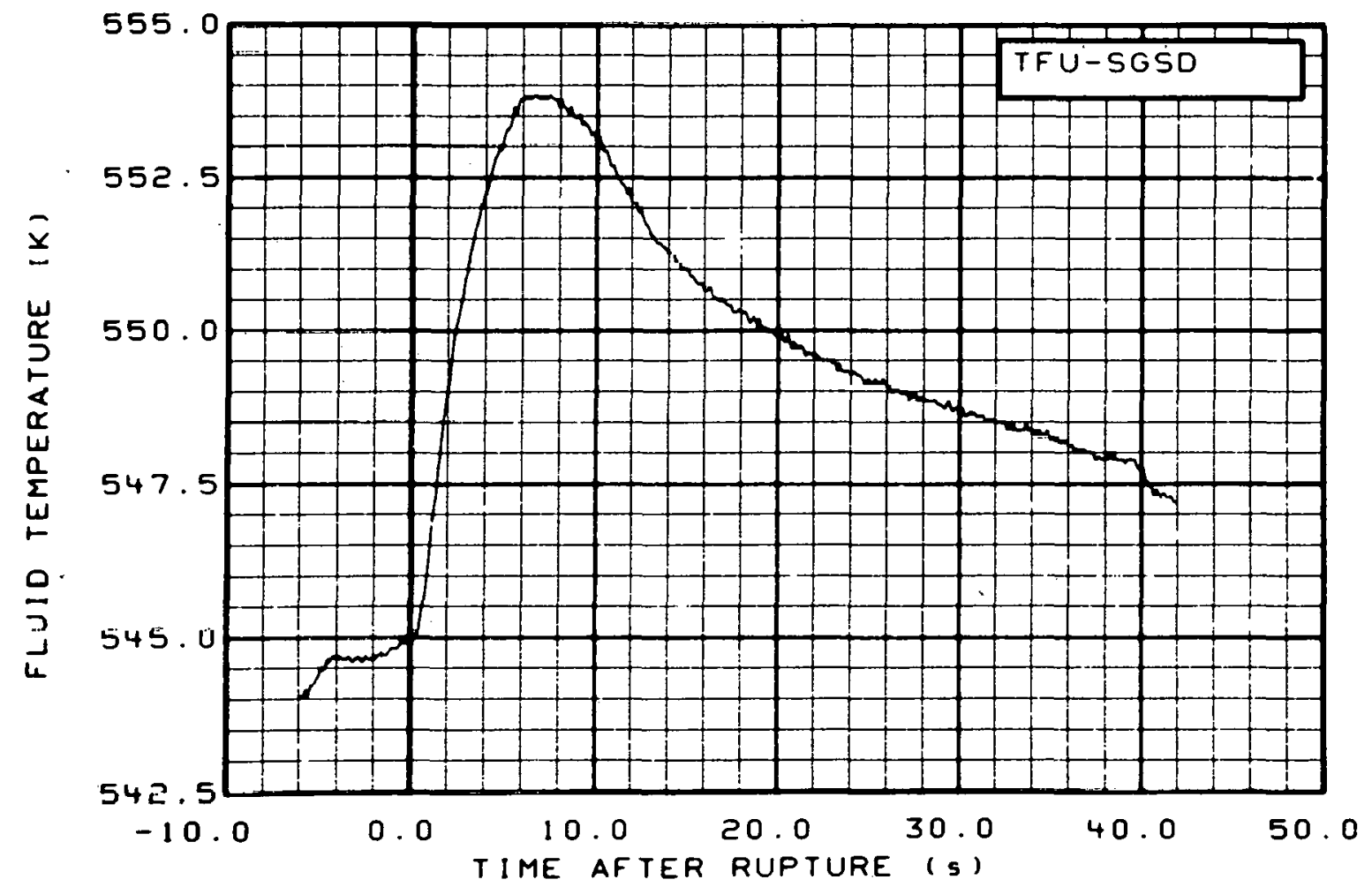

Fig. 46 Fluid temperature in steam generator, steam dome (TFU-SGSD), from -6 to $42 \mathrm{~s}$. 


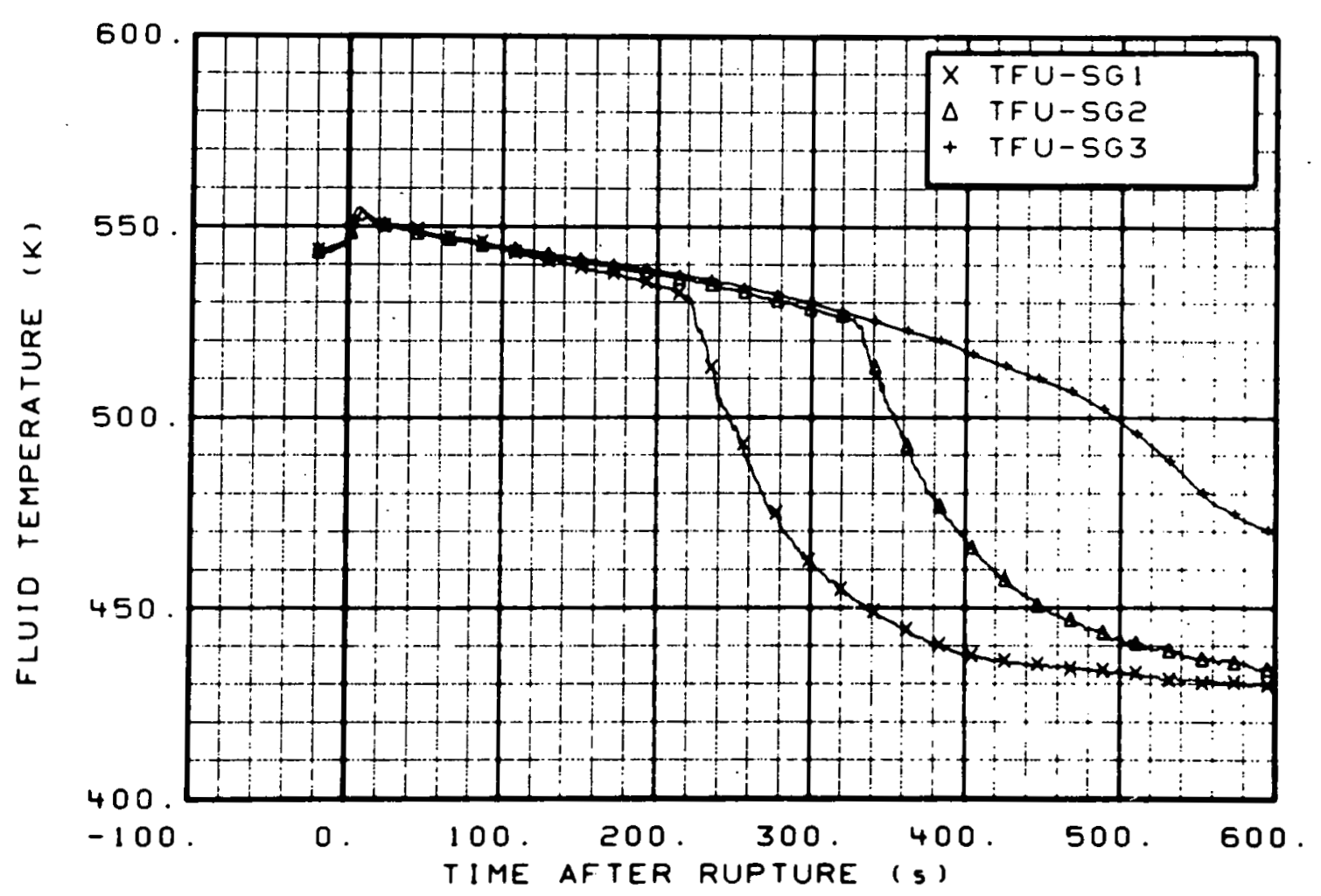

Fig. 47 Fluid temperature in steam generator, secondary side (TFU-SG1, TFU-SG2, and TFU-SG3), from -20 to $600 \mathrm{~s}$.

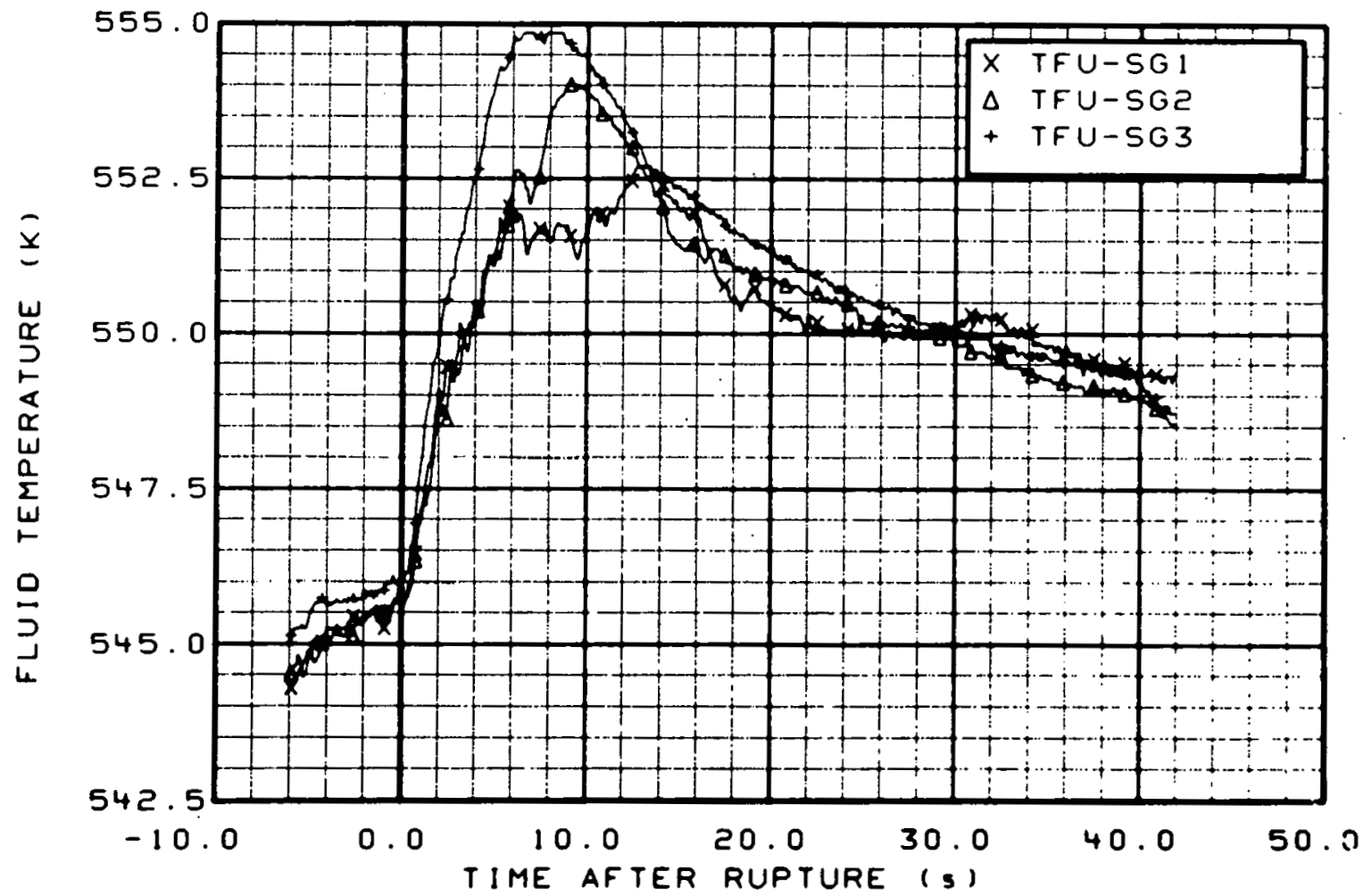

Fig. 48 Fluid temperature in steam generator, secondary side (TFU-SGI, $T F U-S G 2$, and TF(I-SG.3), from -6 to $42 \mathrm{~s}$. 


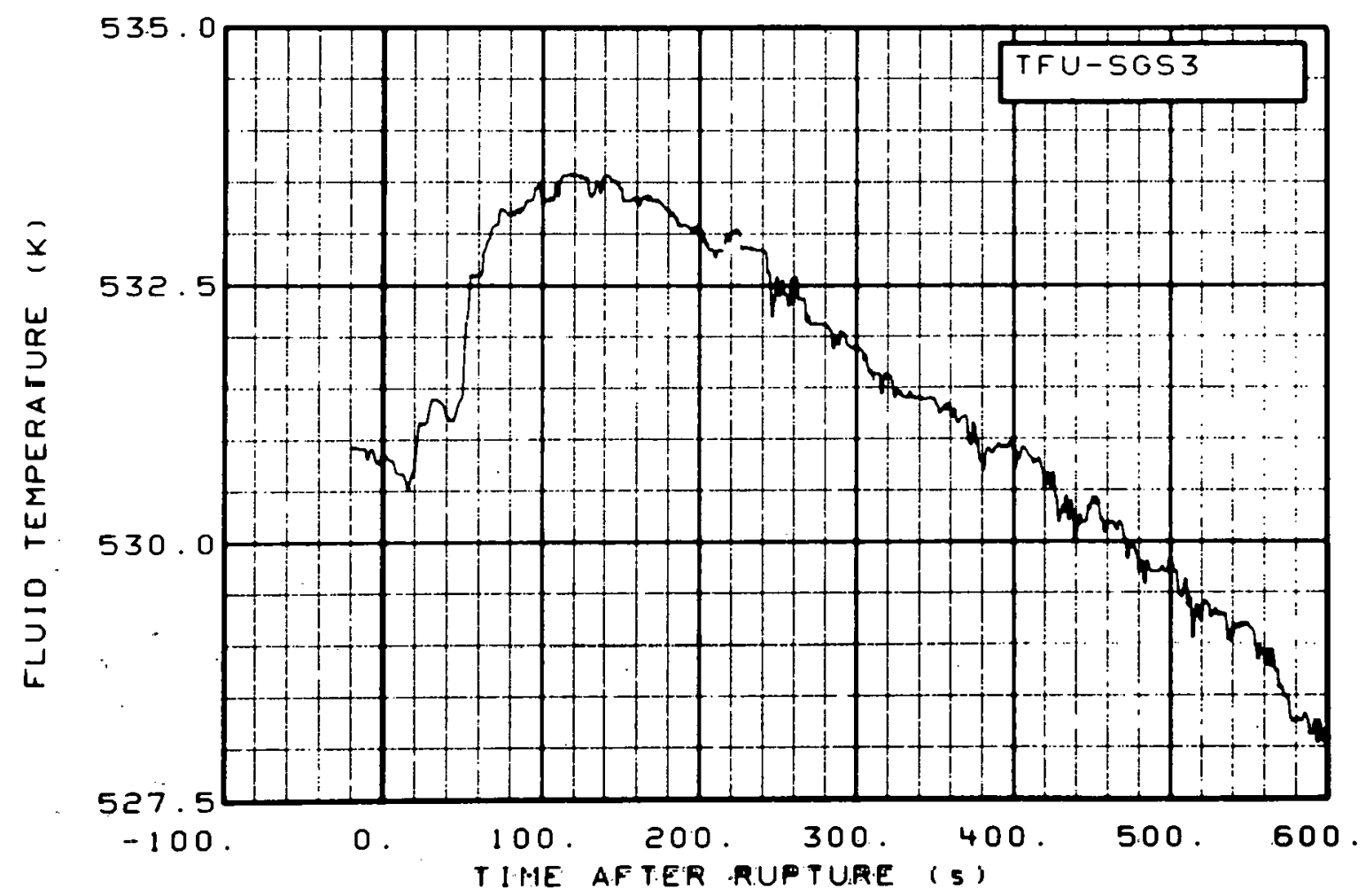

Fig. 49 Fluid temperature in steam generator rupture system accumulator (TFU-SGS3), from -20 to $600 \mathrm{~s}$.

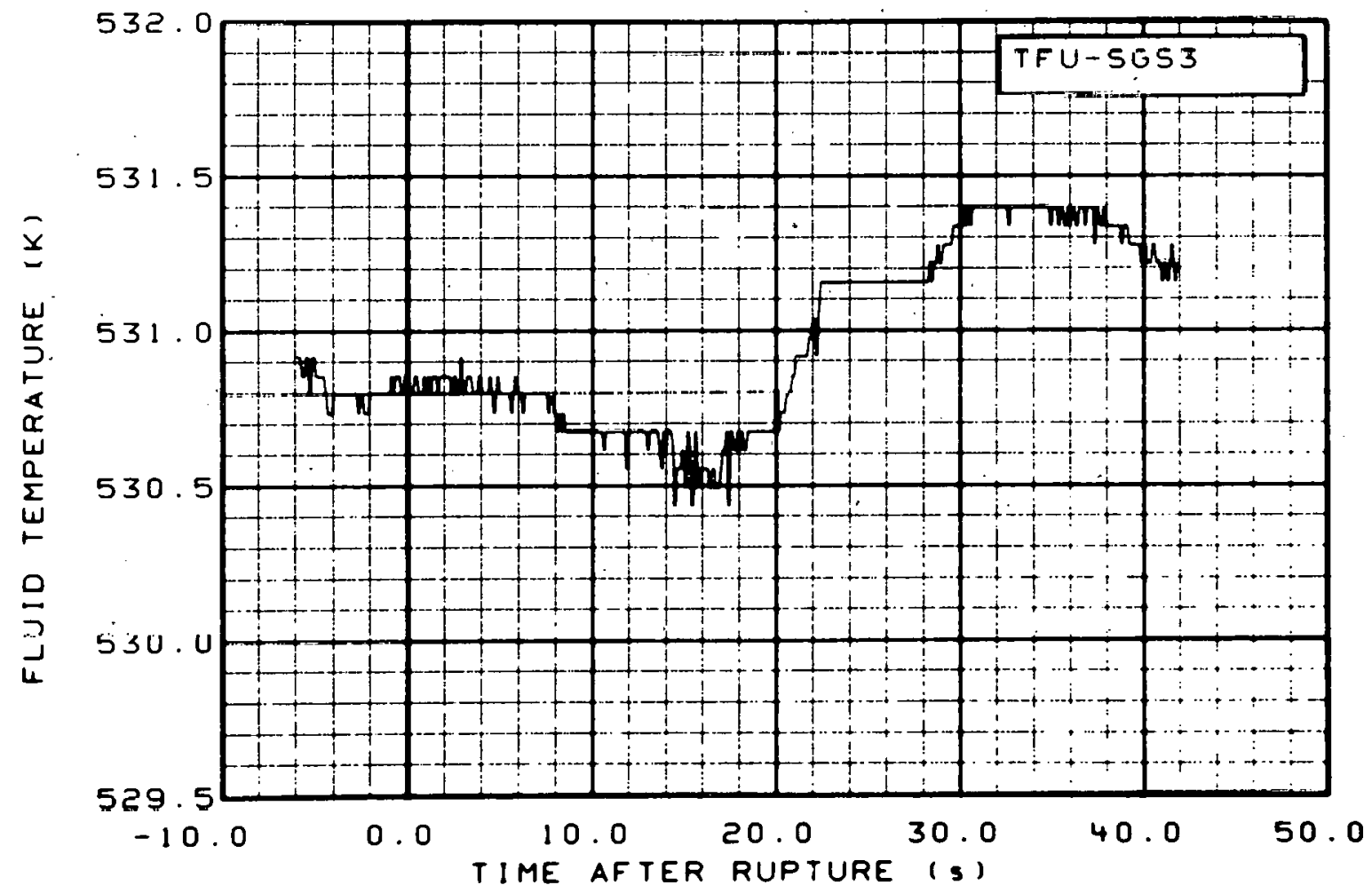

Fig. 50 Fluid temperature in steam generator rupture system accumulator (TFU-SGS3), from -6 to $42 \mathrm{~s}$. 


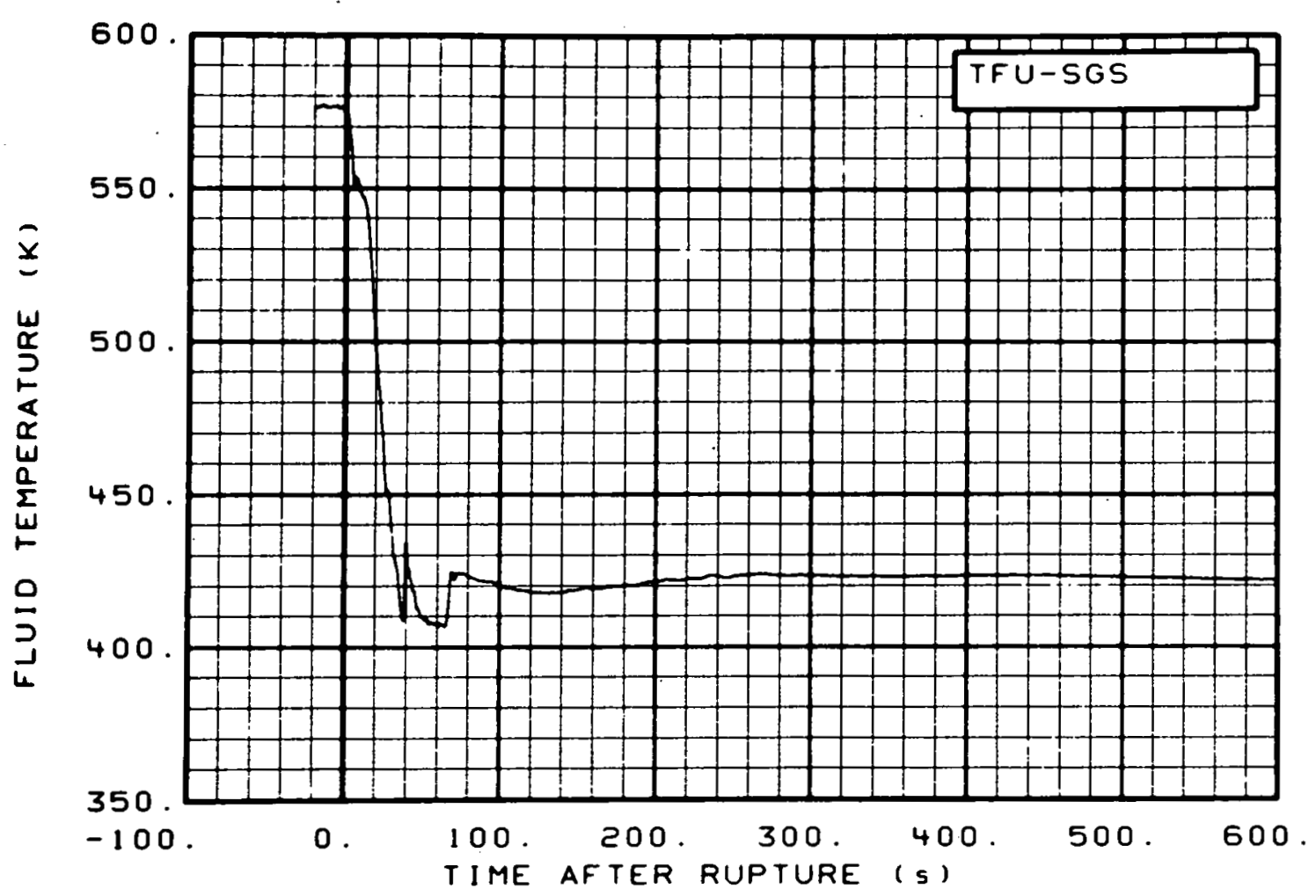

Fig. 51 Fluid temperature in steam generator rupture system injection line (TFU-SGS), from -20 to $600 \mathrm{~s}$.

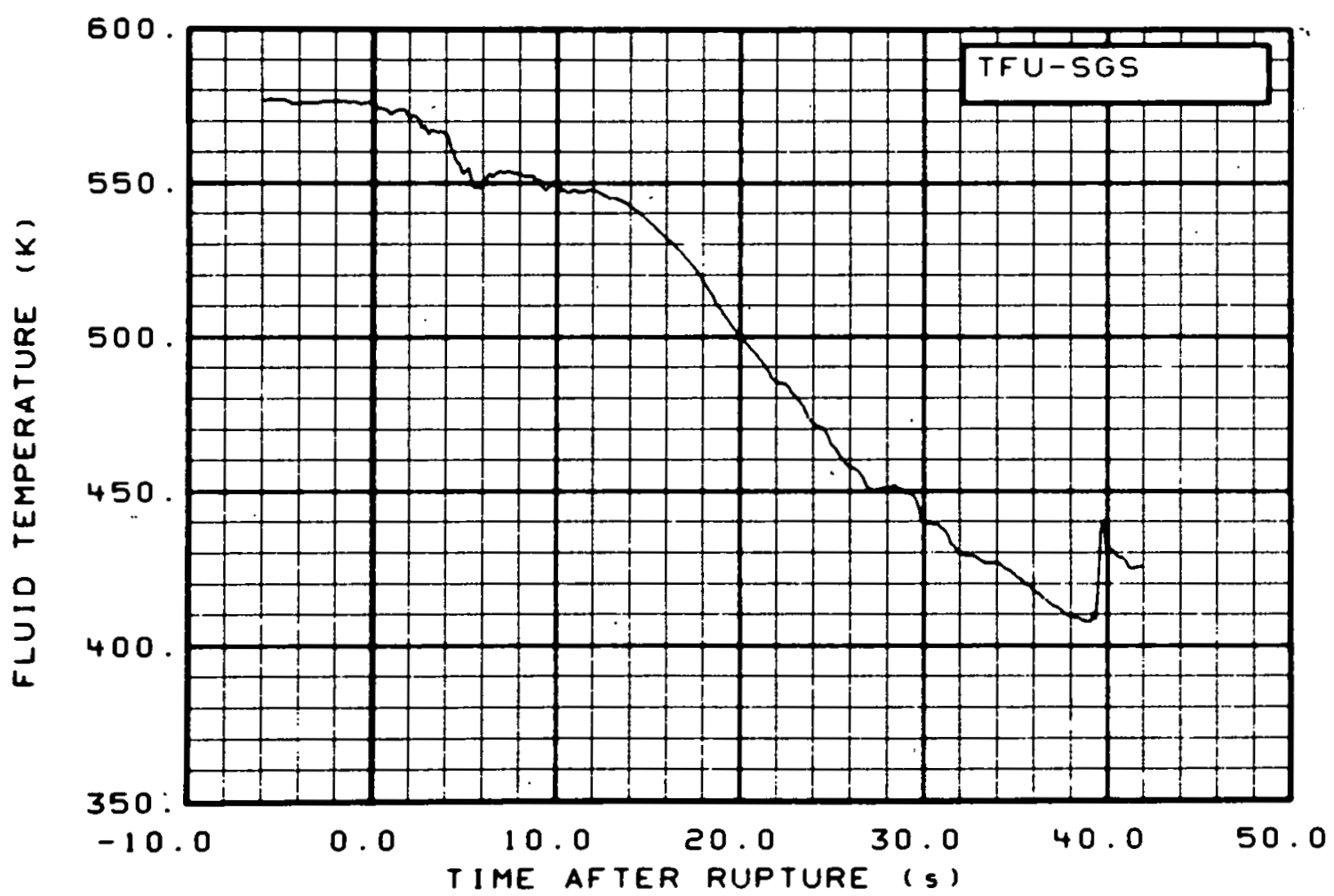

Fig. 52 Fluid temperature in steam generator rupture system injection line (TFU-SGS), from -6 to $42 \mathrm{~s}$. 


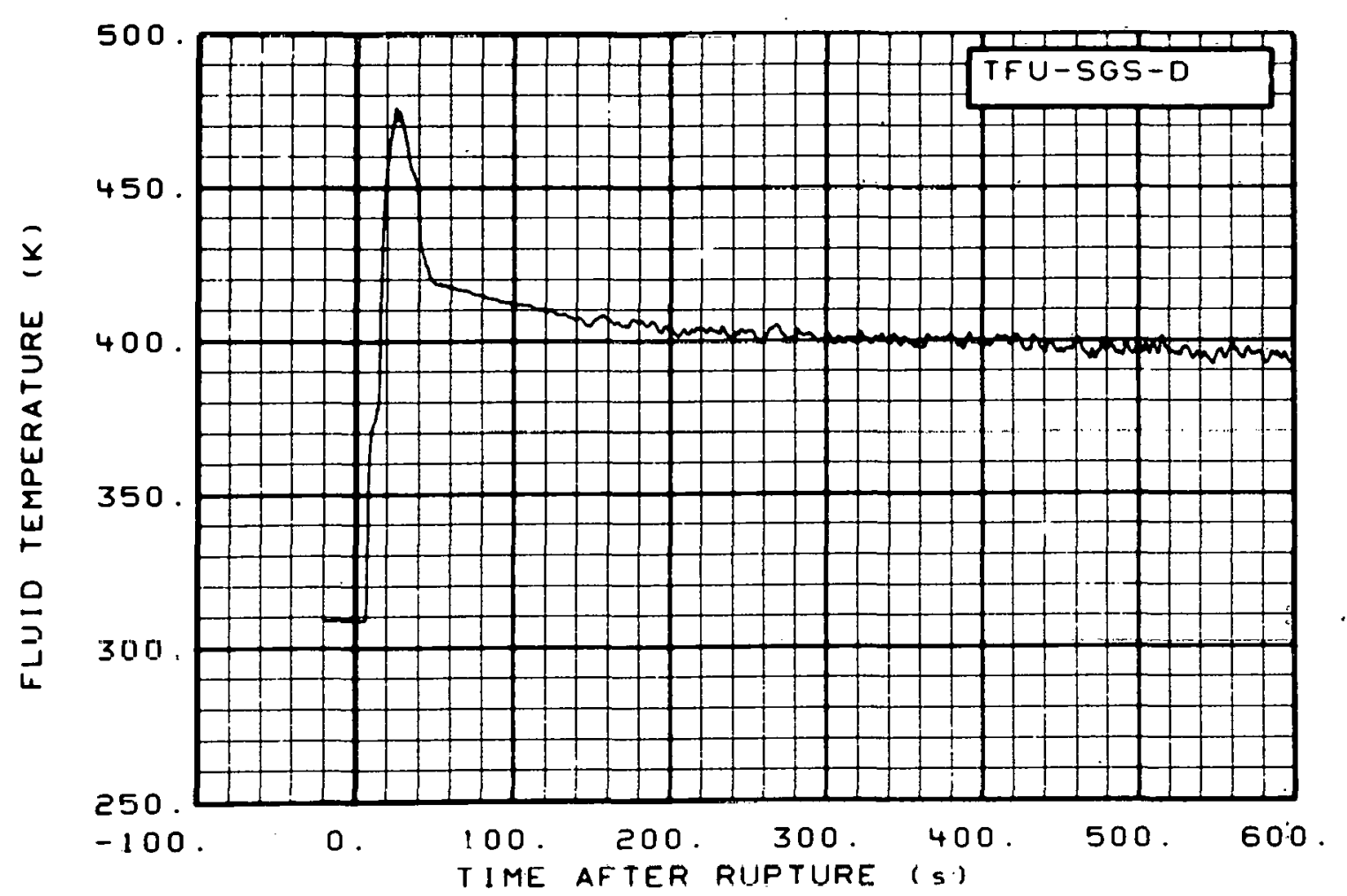

Fig. 53 Fluid temperature in steam generator rupture system injection line drain (TFU-SGS-D), from -20 to $600 \mathrm{~s}$.

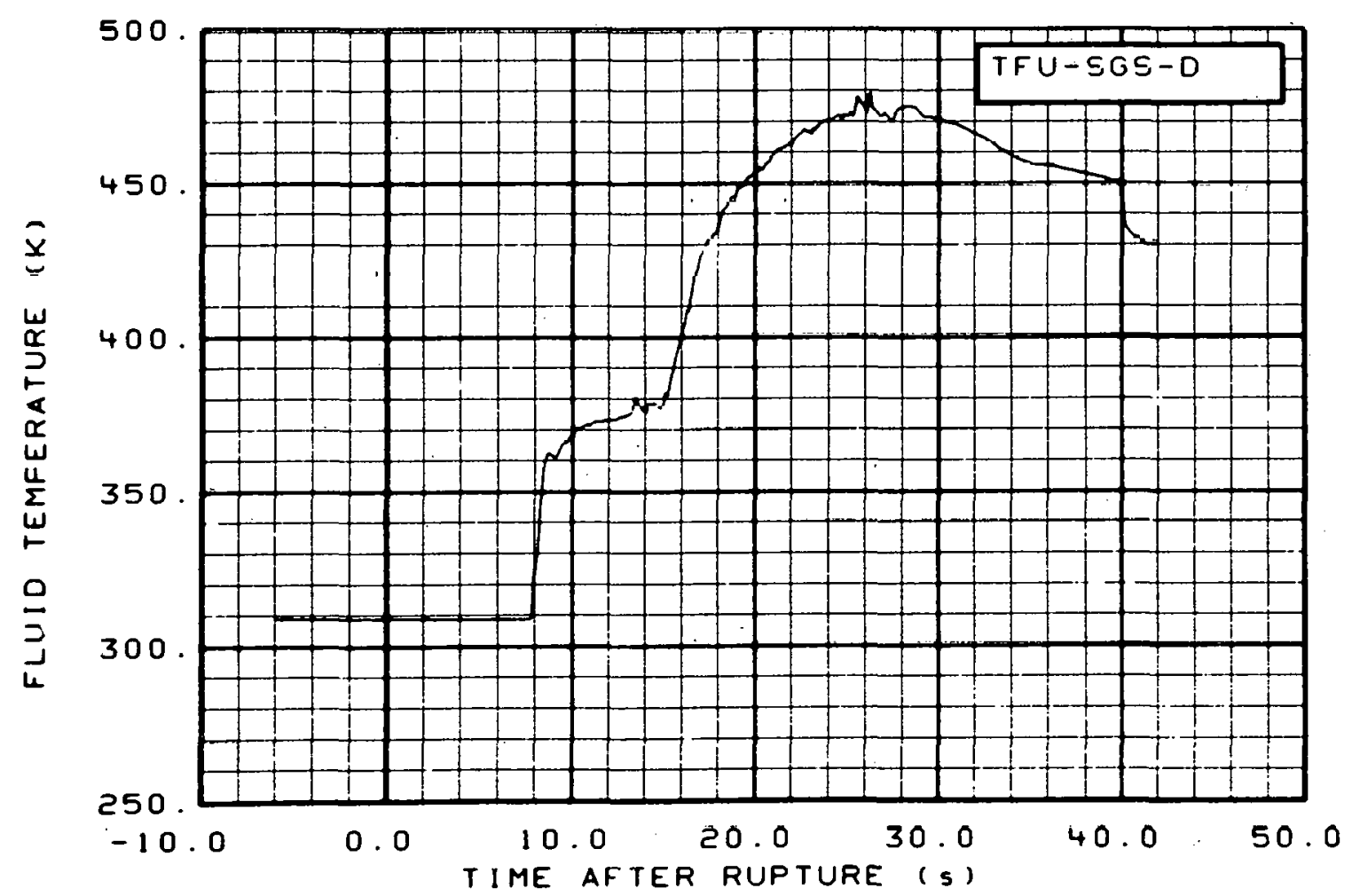

Fig. 54 Fluid temperature in steam generator rupture system injection line drain (TFU-SGS-D), from -6 to $42 \mathrm{~s}$. 


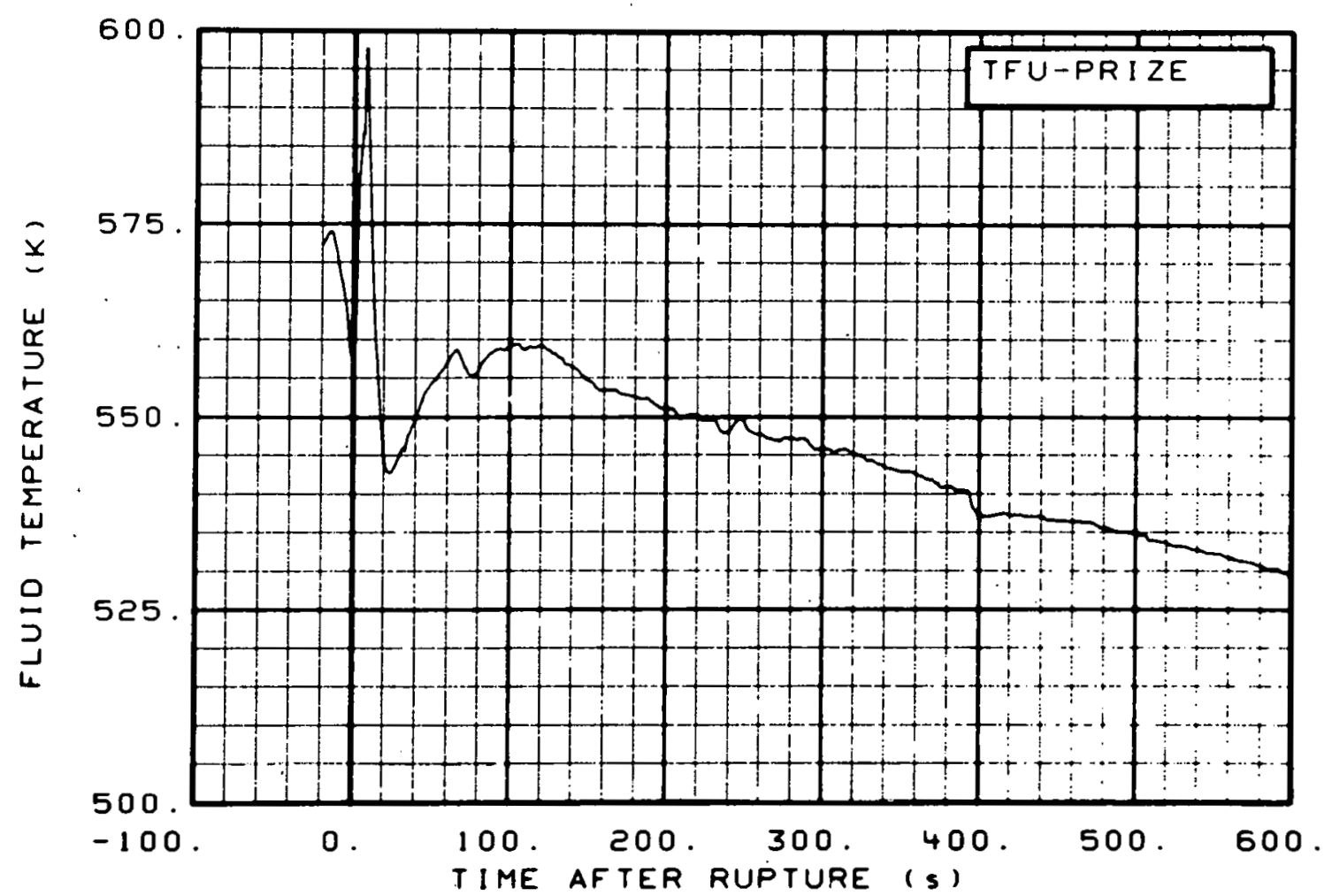

Fig. 55 Fluid temperature in pressurizer surge line (TFU-PRIZE), from -20 : to $600 \mathrm{~s}$.

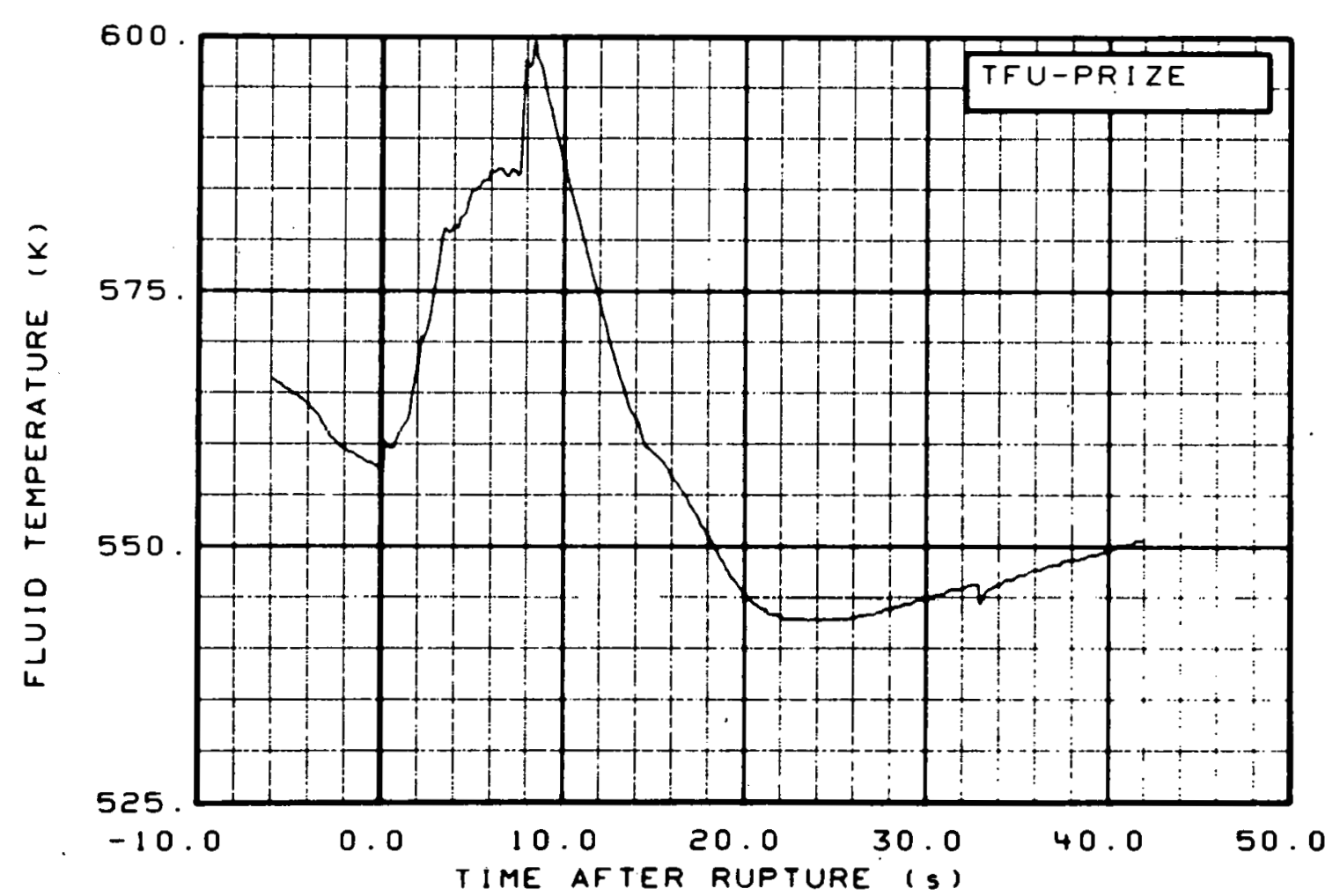

Fig. 56 Fluid temperature in pressurizer surge line (TFU-PRIZE), from -6 to $42 \mathrm{~s}$. 


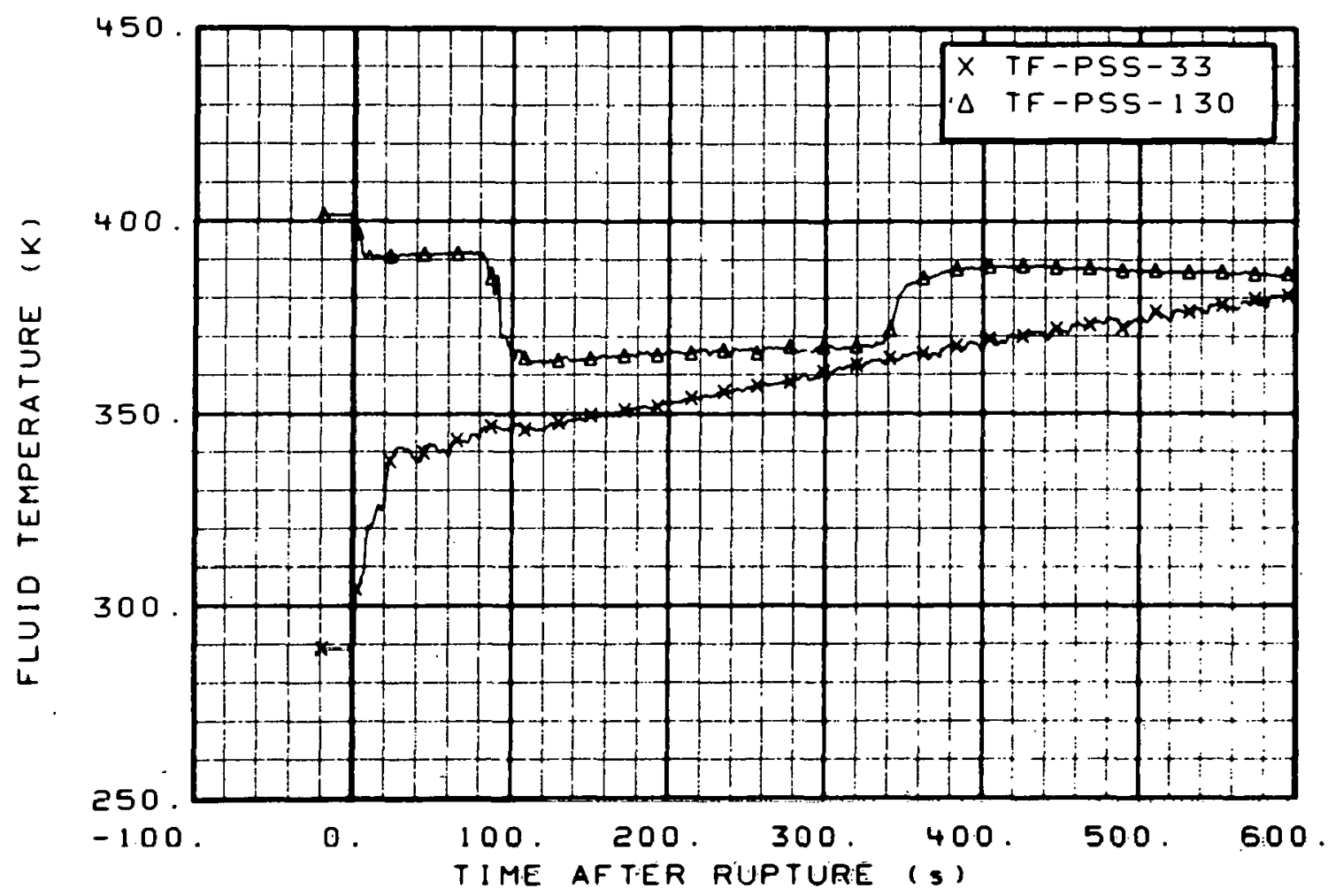

Fig. 57 Fluid temperature in pressure suppression tank (TF-PSS-33 and TF-PSS-130), from -20 to $600 \mathrm{~s}$.

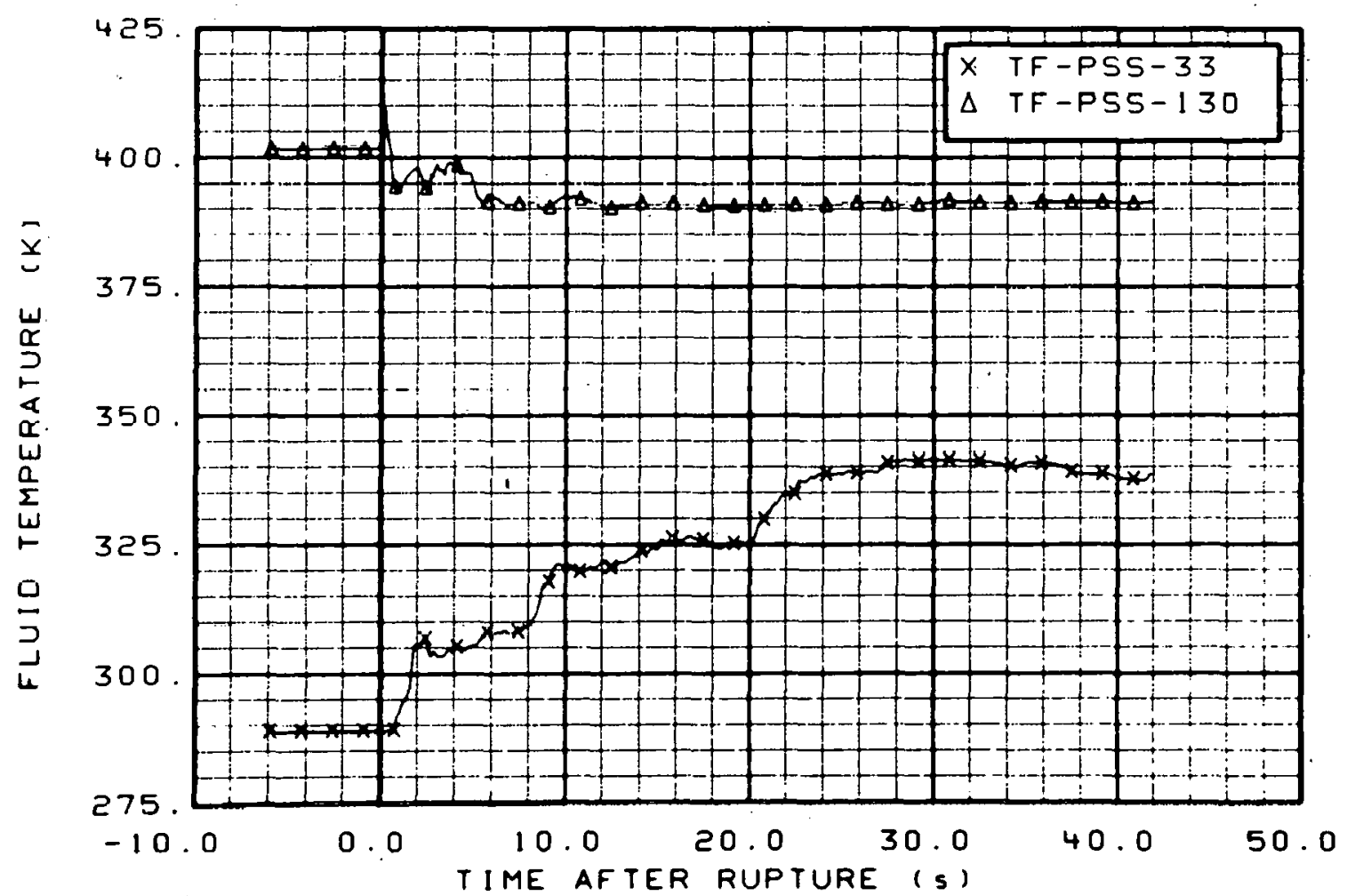

Fig. 58 Fluid temperature in pressure suppression tank (TF-PSS-33 and TF-PSS-130), from -6 to $42 \mathrm{~s}$. 


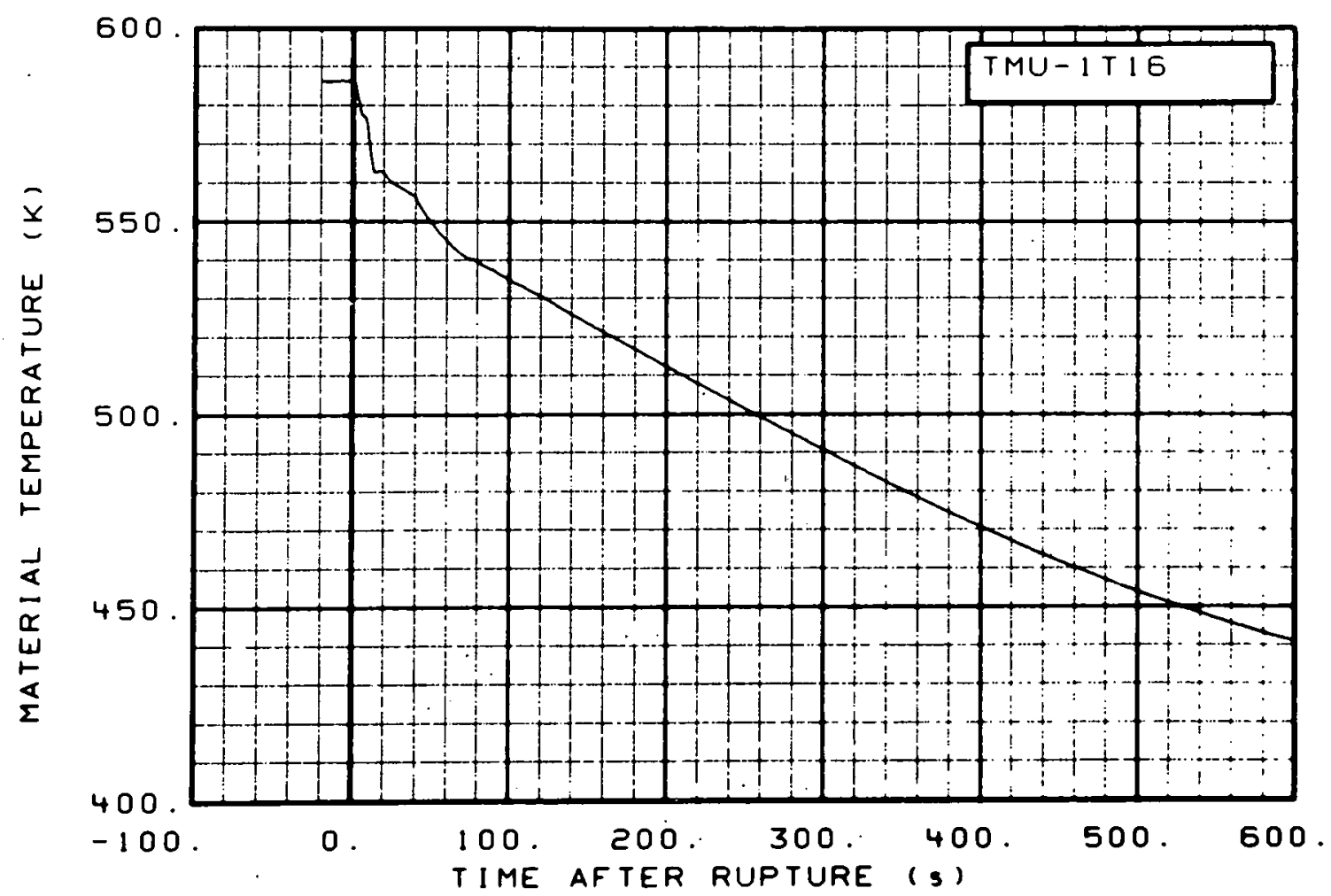

Fig. 59 Material temperature in intact loop (TMU-1T16), from -20 to $600 \mathrm{~s}$.

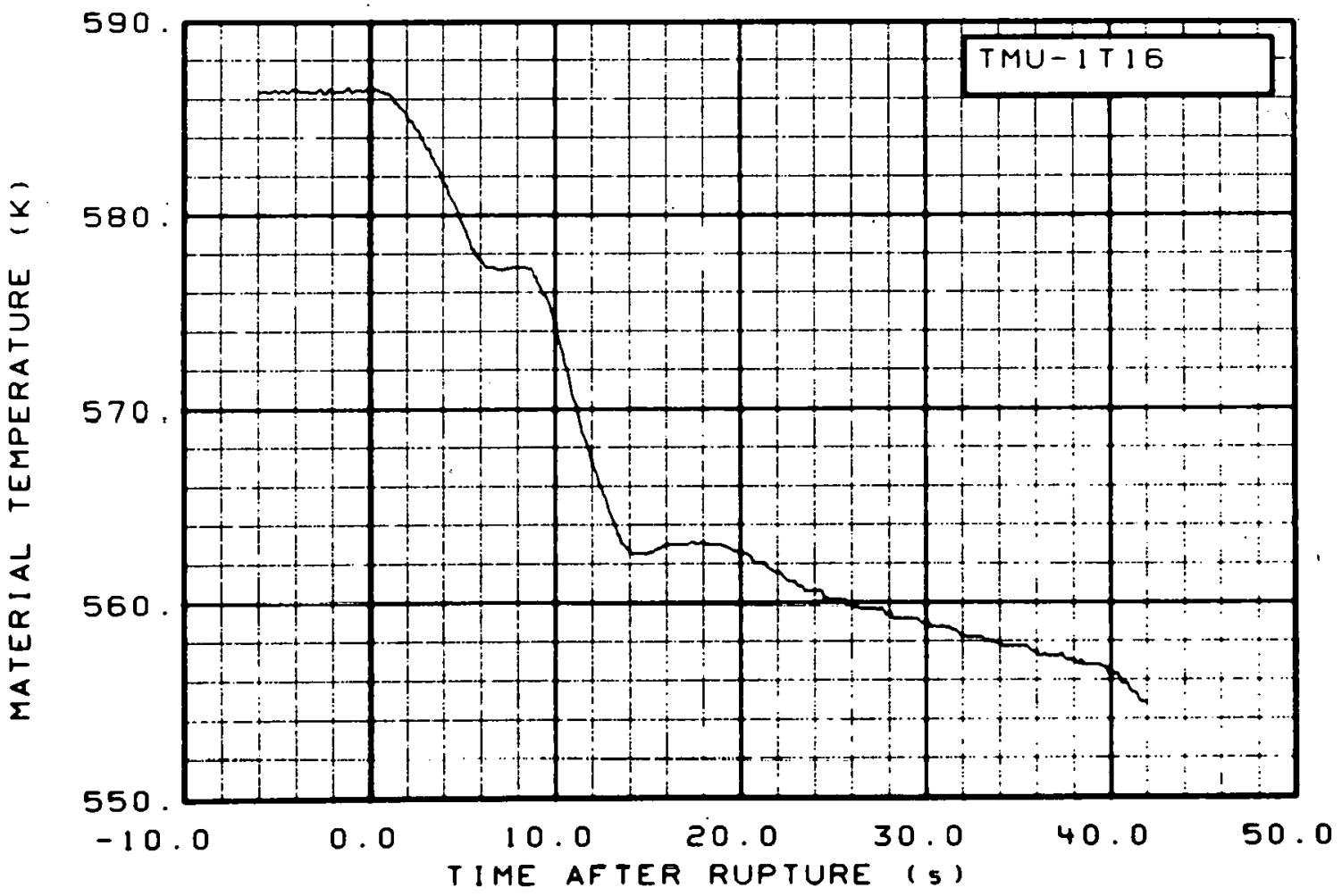

Fig. 60 Material temperature in intact loop (TMU-1T16), from -6 to $42 \mathrm{~s}$. 


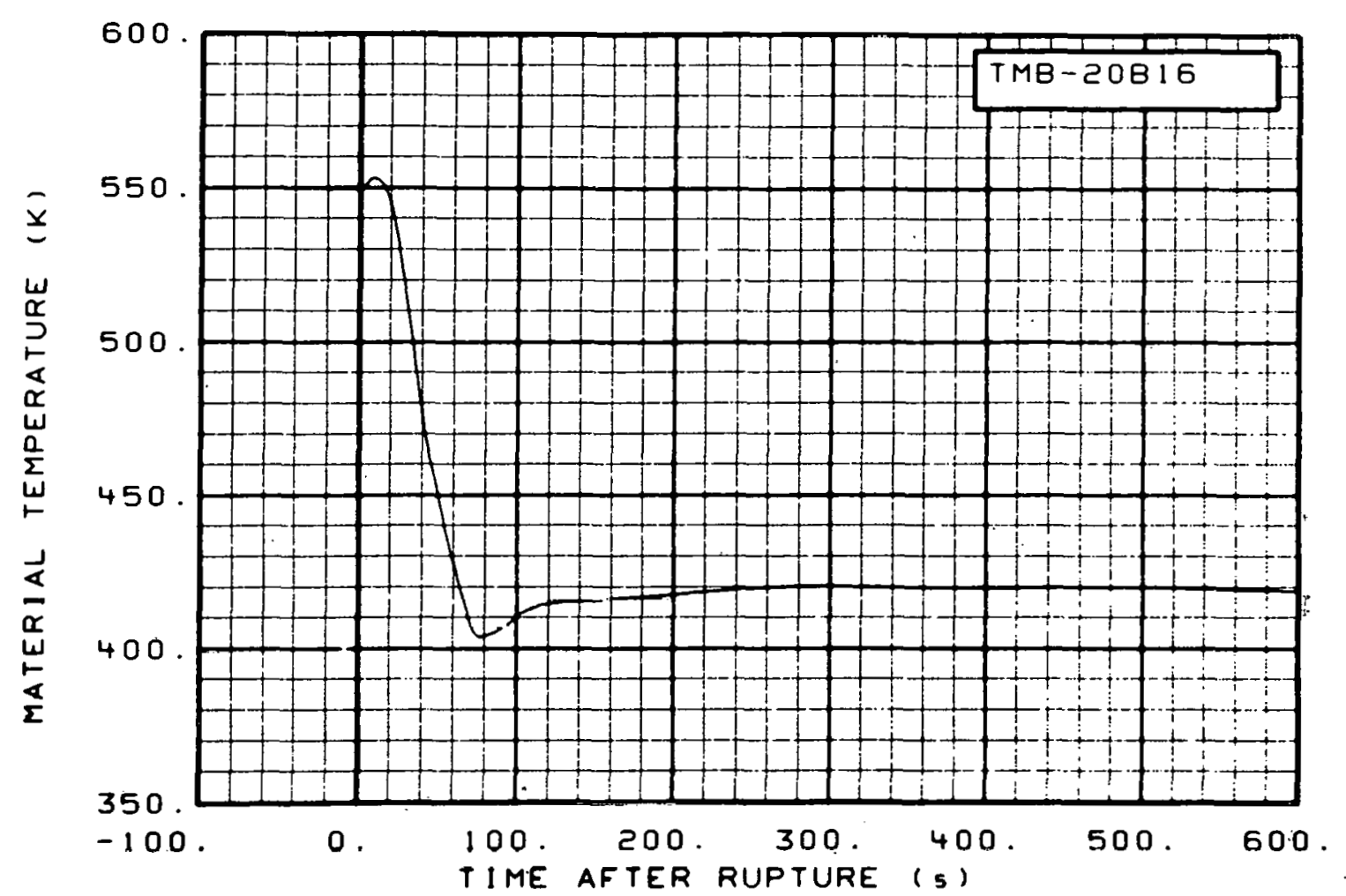

Fig. 61 Material temperature in broken 10op (TMB-20B16), from -20 to $600 \mathrm{~s}$.

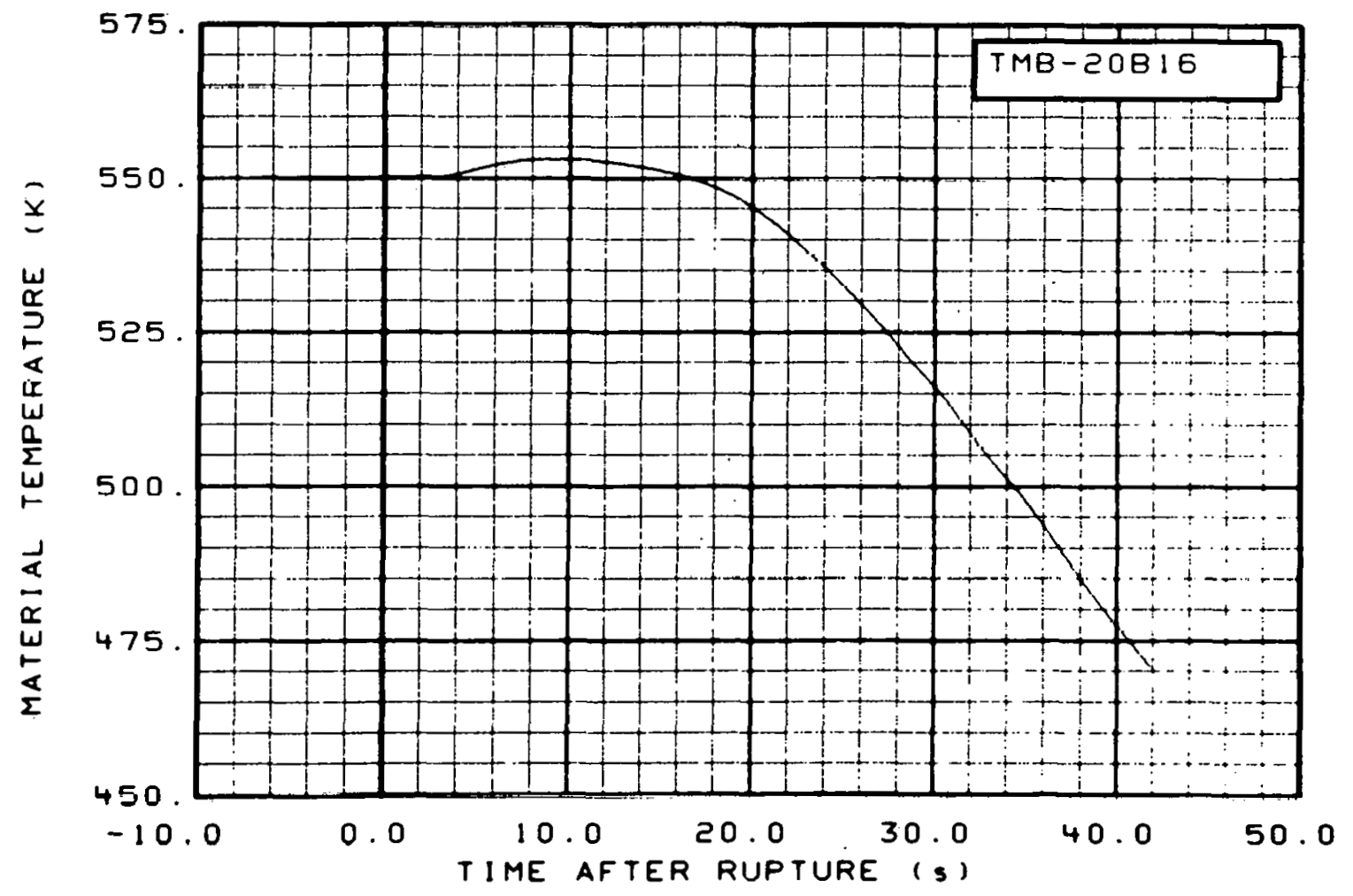

Fig. 62 Material temperature in broken loop (TMB-20B16), from -6 to $42 \mathrm{~s}$. 


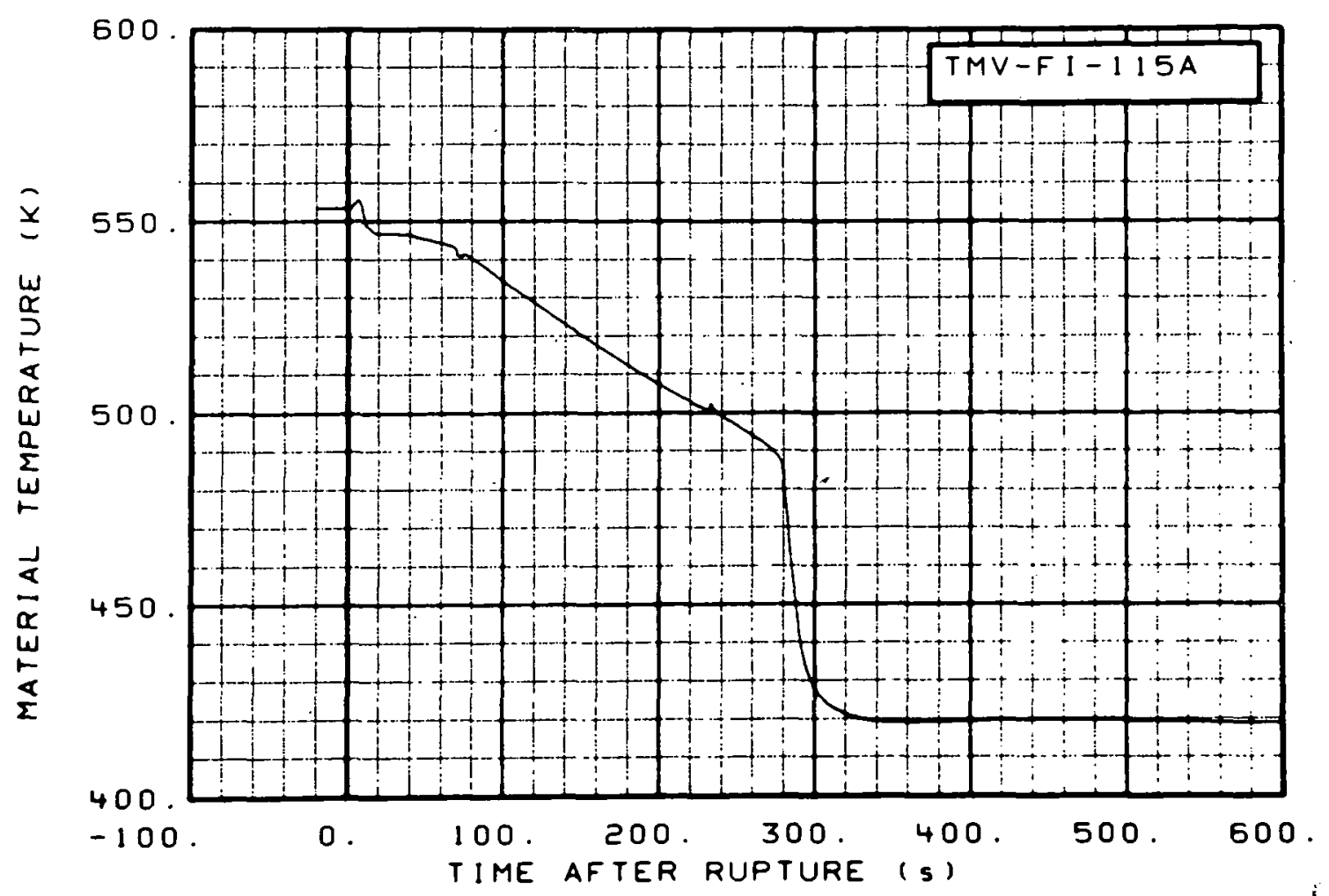

Fig. 63 Material temperature in vessel filler (TMV-FI-115A), from -20." to $600 \mathrm{~s}$.

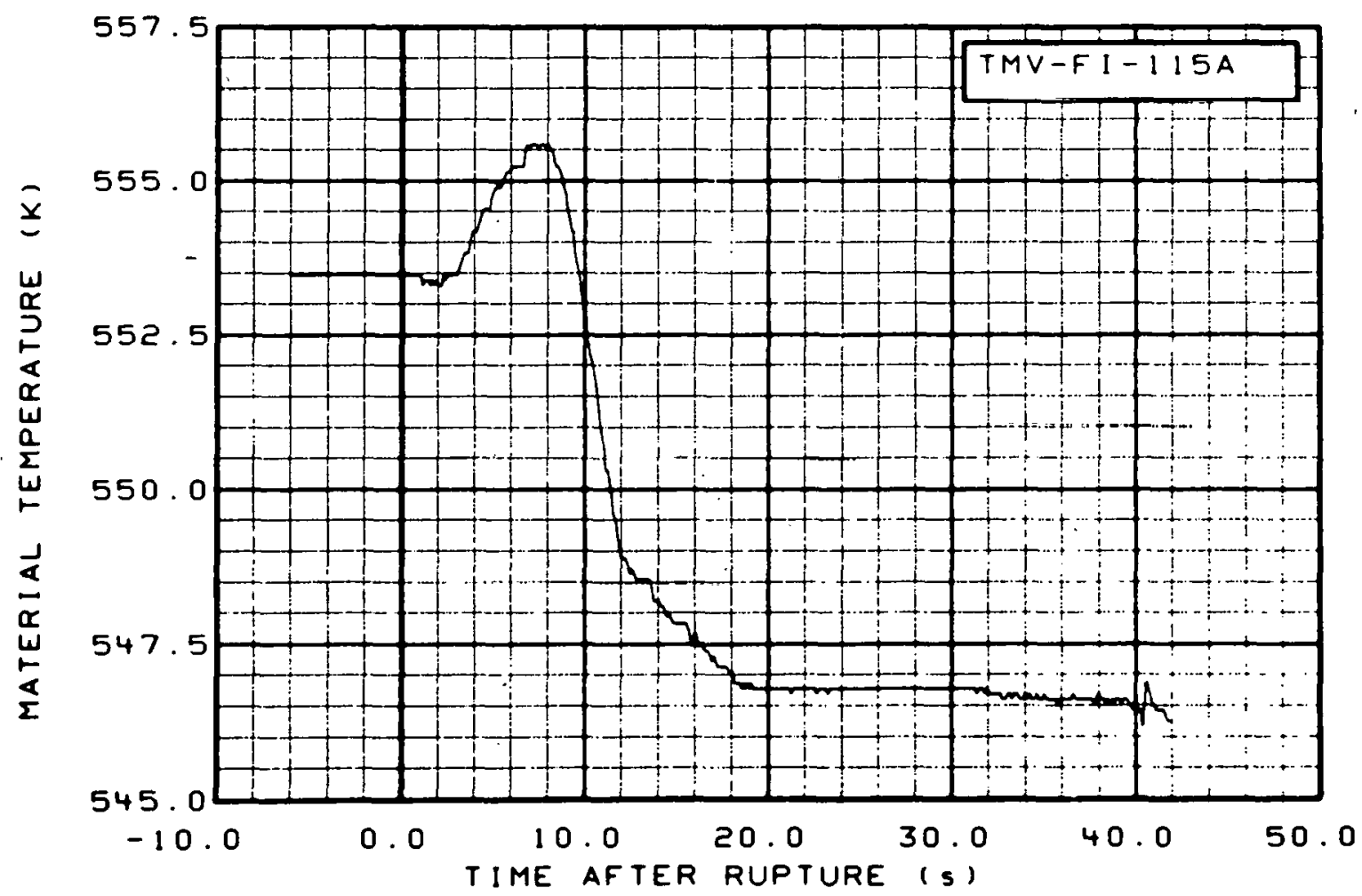

Fig. 64 Material temperature in vessel filler (TMV-FI-115A), from -6 to $42 \mathrm{~s}$. 


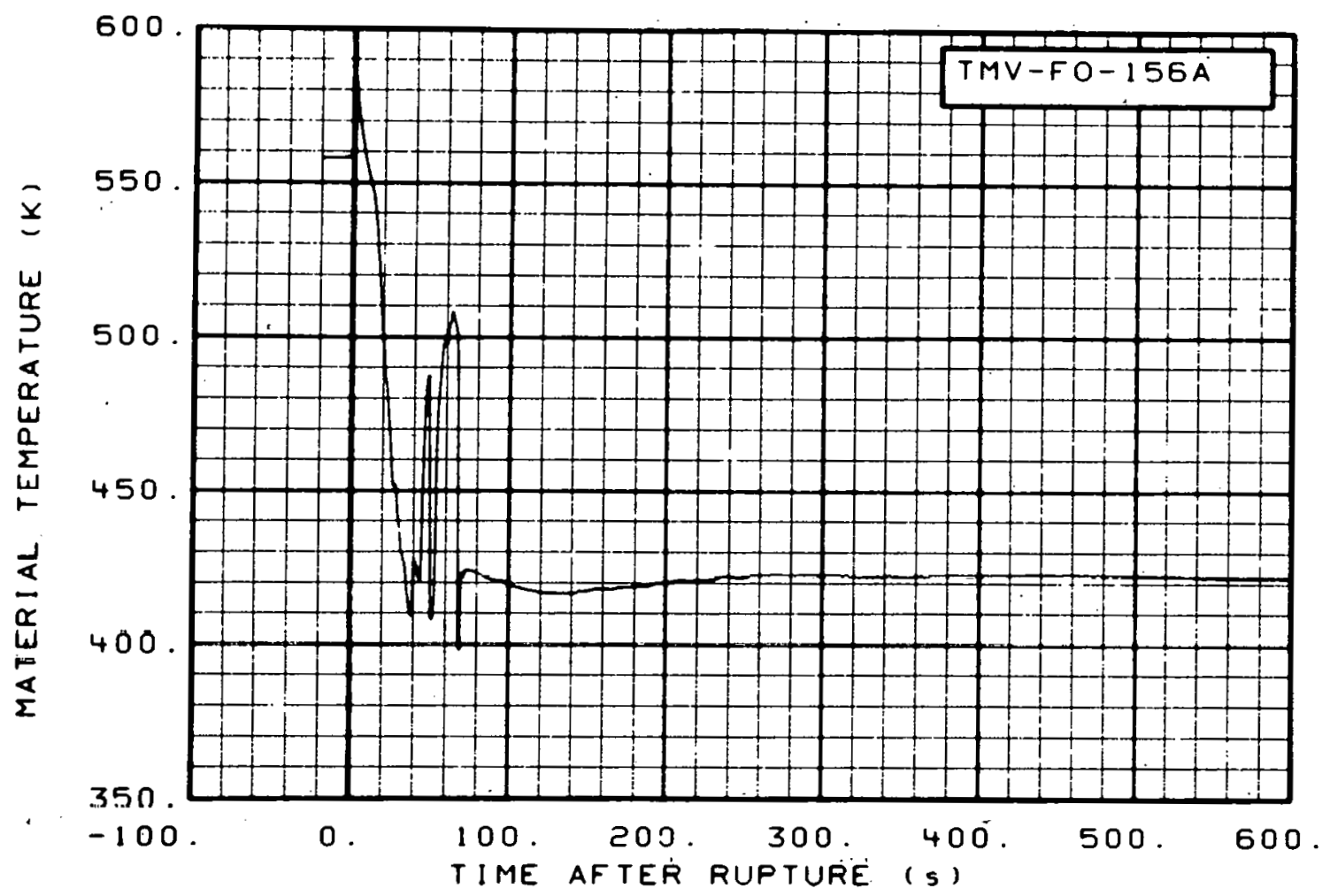

Fig. 65 Material temperature in vessel filler insulator (TMV-FO-156A), from -20 to $600 \mathrm{~s}$.

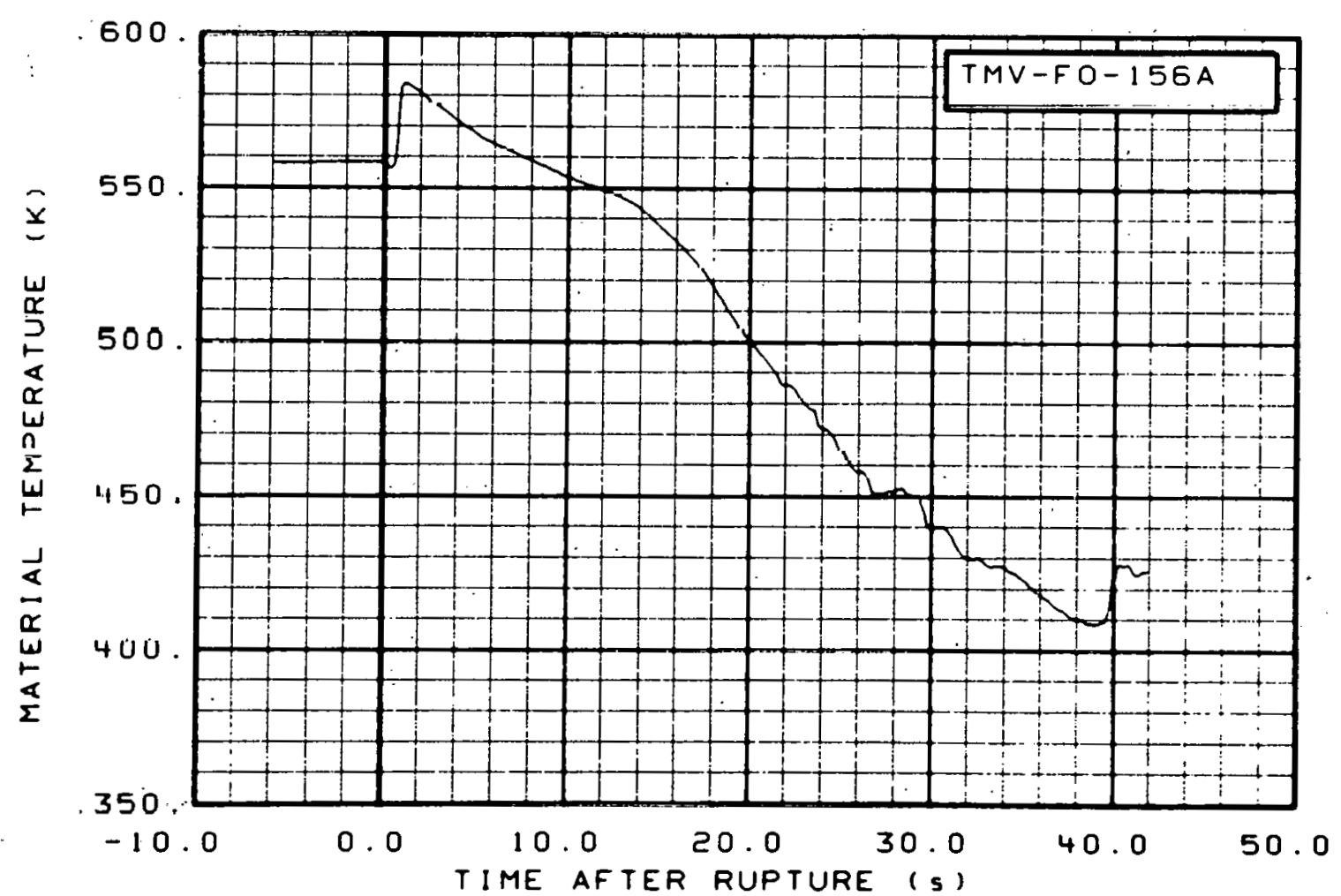

Fig. 66 Material temperature in vessel filler insulator (TMV-F0-156A), from -6 to $42 \mathrm{~s}$. 


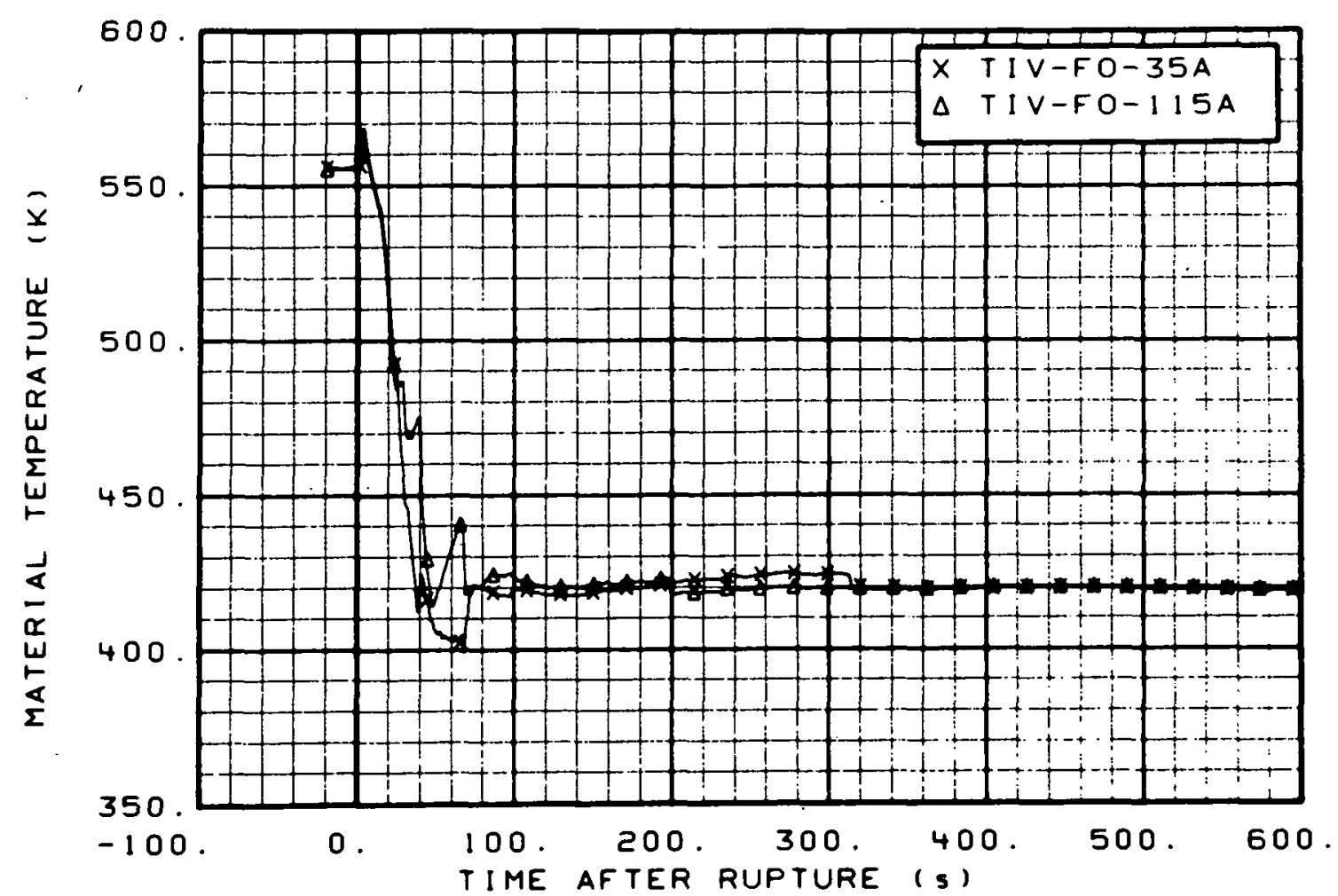

Fig. 67 Material temperature in vessel filler insulator (TIV-FO-35A and TIV-F0-115A), from -20 to $600 \mathrm{~s}$.

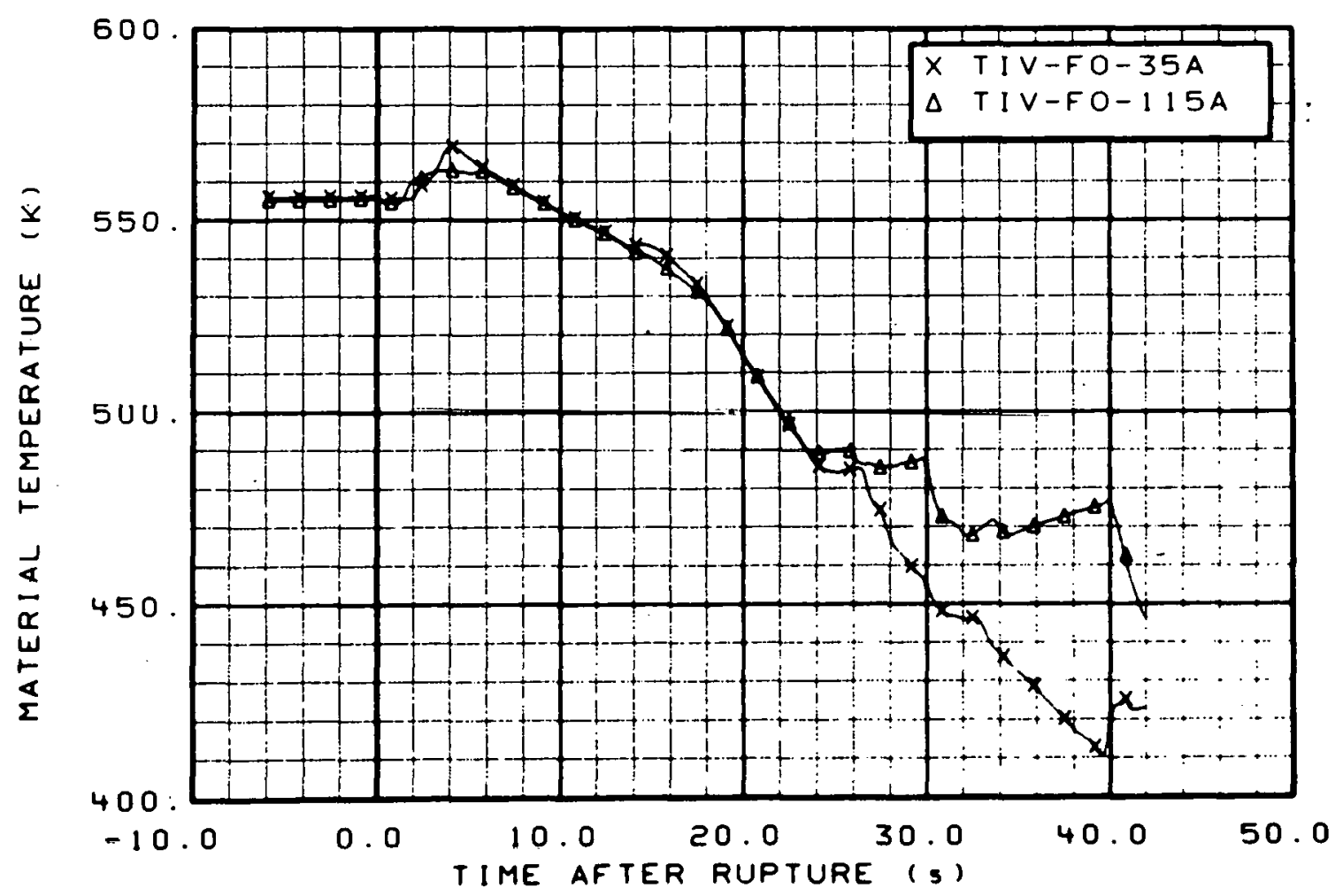

Fig. 68 Material temperature in vessel filler insulator (TIV-F0-35A and TIV-F0-115A), from -6 to 4? s. 


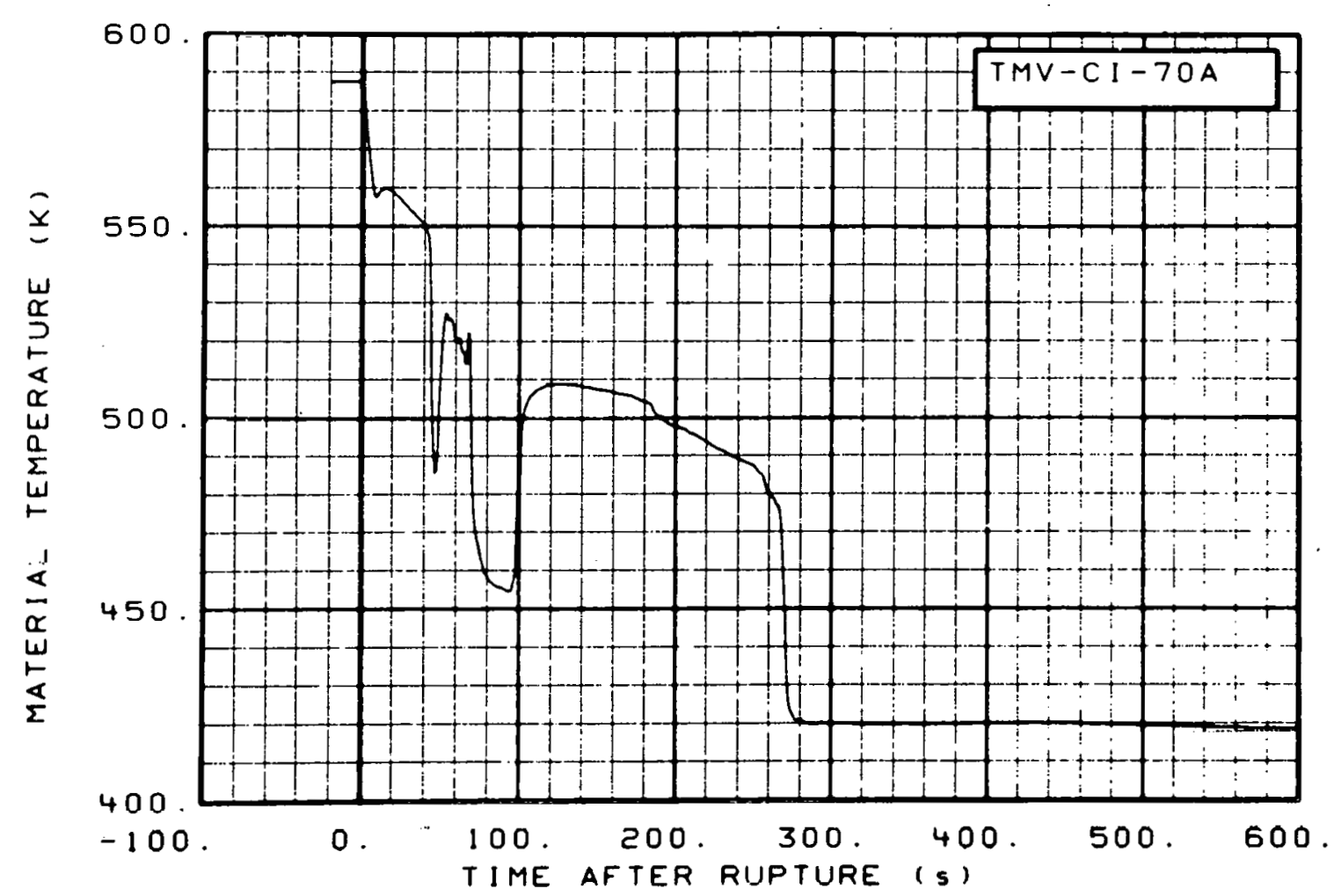

Fig. 69 Material temperature in core barrel inner diameter (TMV-CI-70A), from -20 to $600 \mathrm{~s}$.

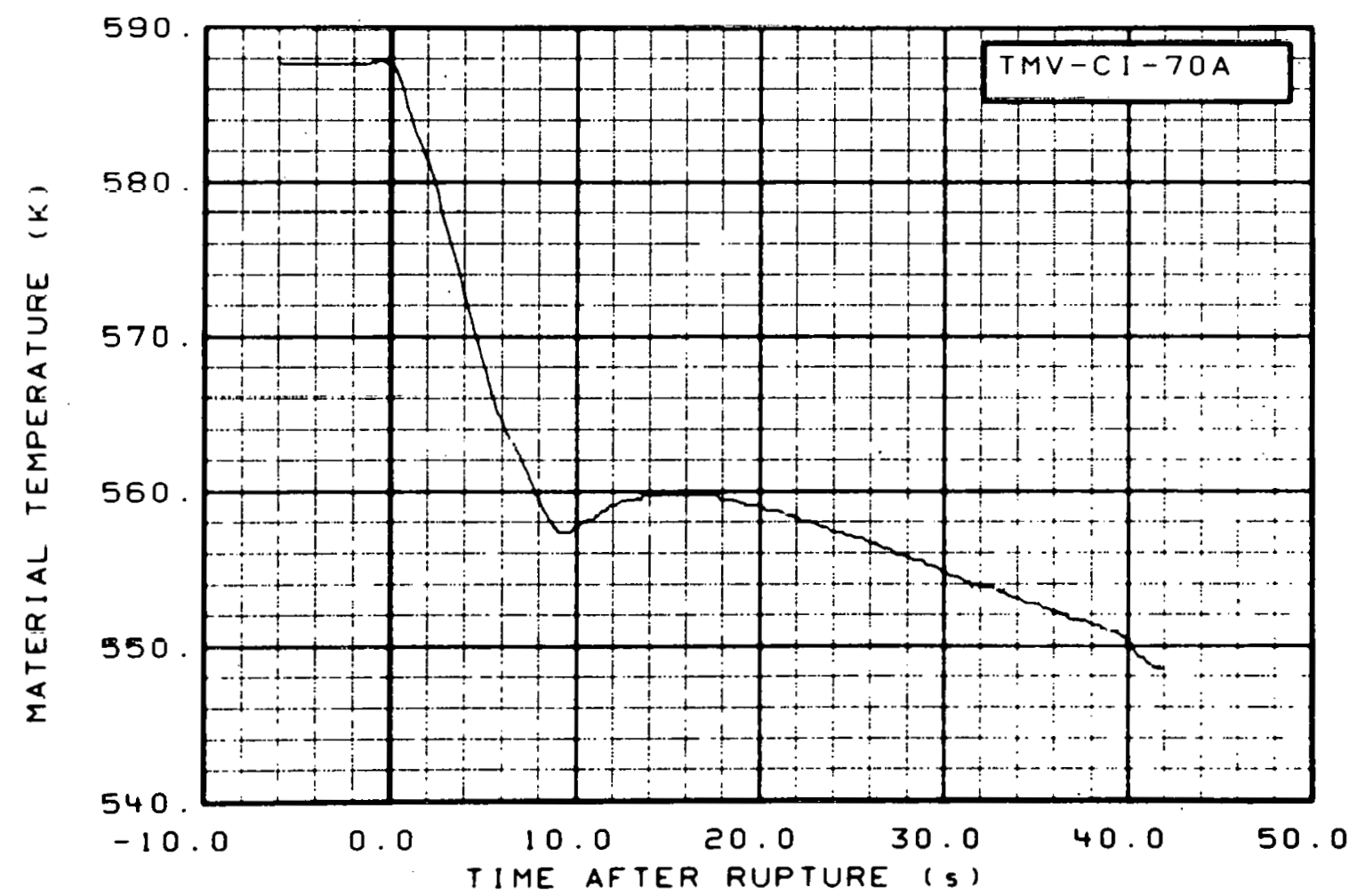

Fig. 70 Material temperature in core barrel inner diameter (TMV-CI-70A), from -6 to $42 \mathrm{~s}$. 


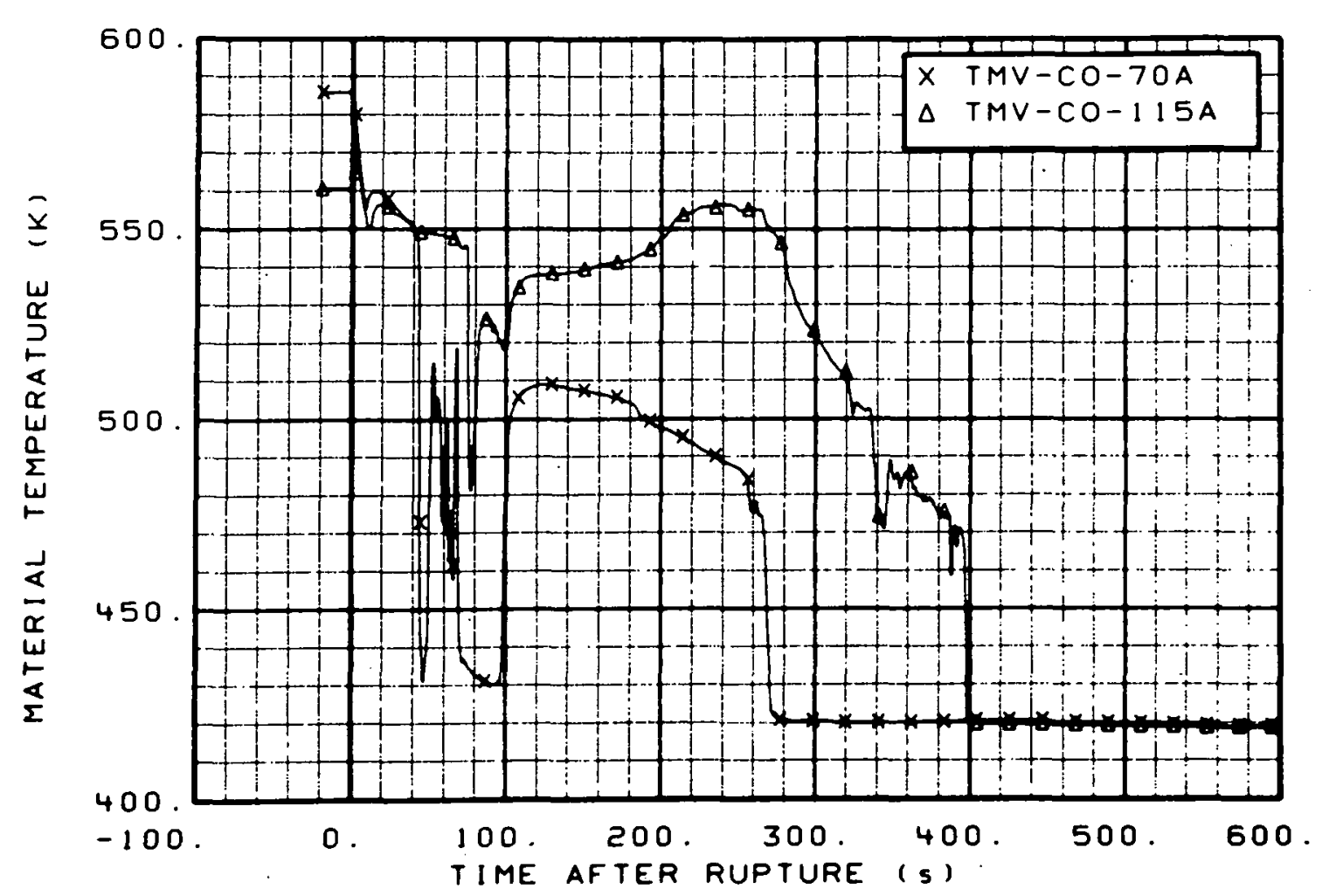

Fig. 71 Material temperature in core barrel outer diameter (TMV-CO-70A: and TMV-CO-115A), from -20 to $600 \mathrm{~s}$.

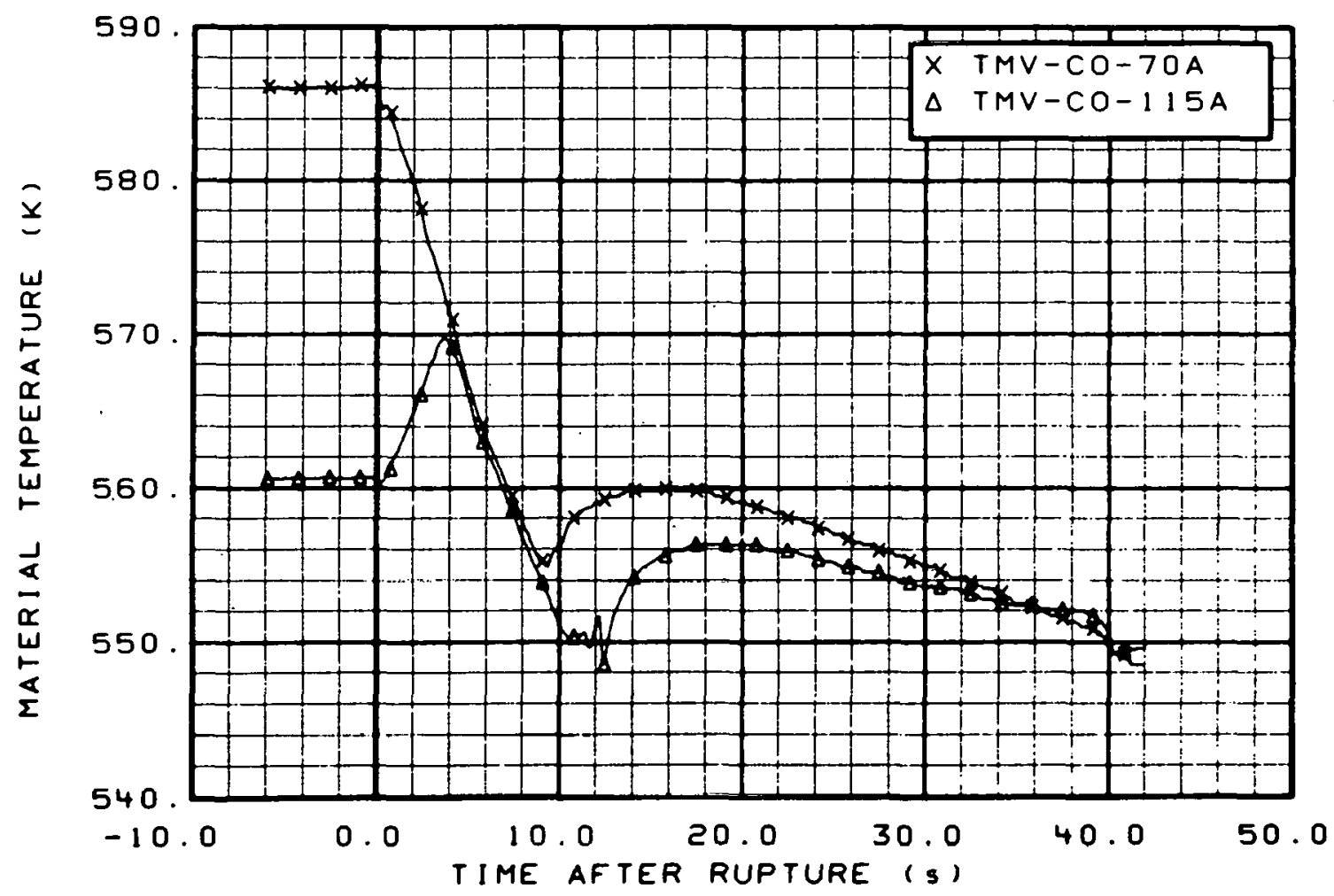

Fig. 72 Material temperature in core barrel outer diameter (TMV-CO-70A and TMV-C0-115A), from -6 to $42 \mathrm{~s}$. 


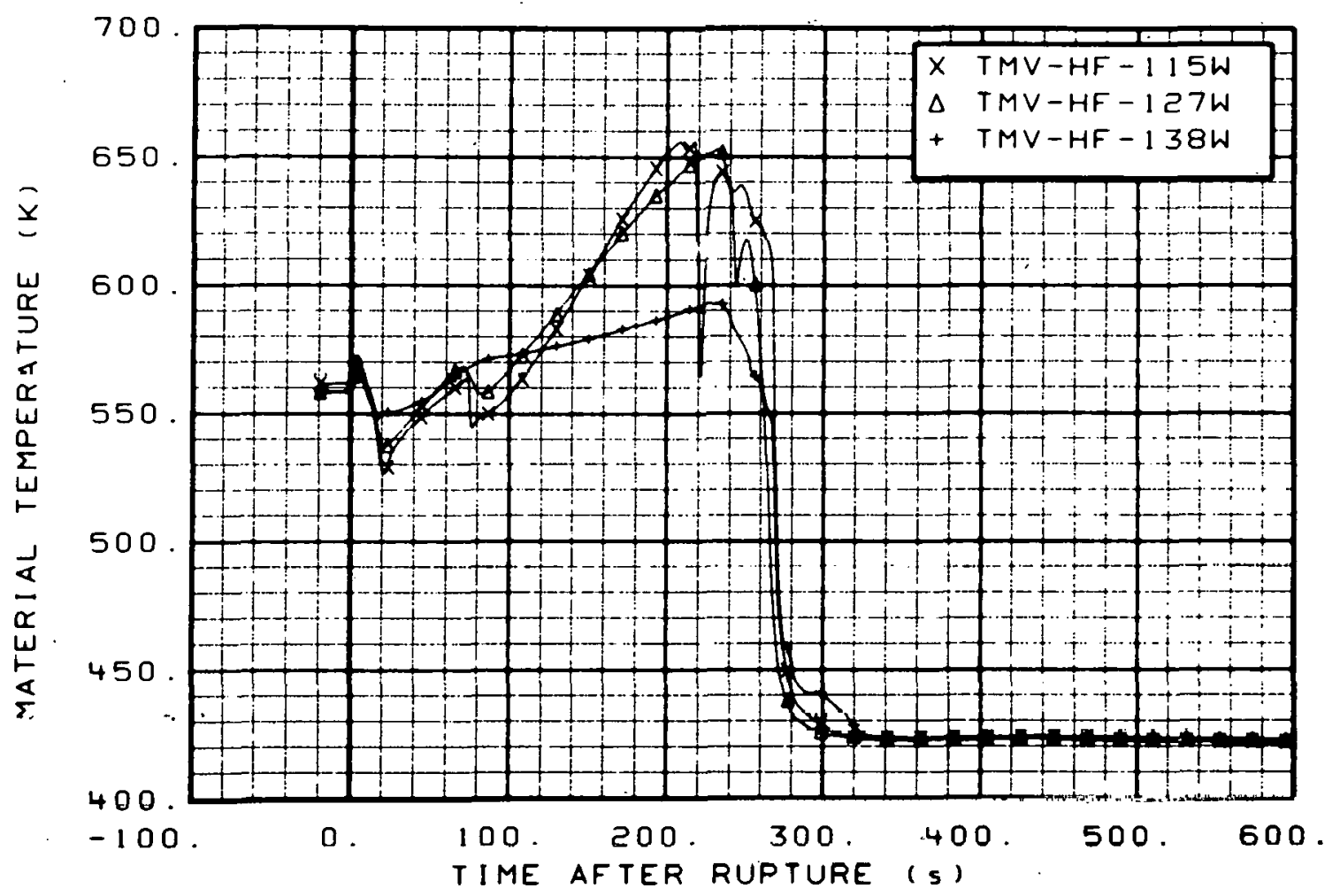

Fig. 73 Material temperature in core housing filler (TMV-HF-115W, TMV-HF-127W, and TMV-HF-138W), from -20 to $600 \mathrm{~s}$.

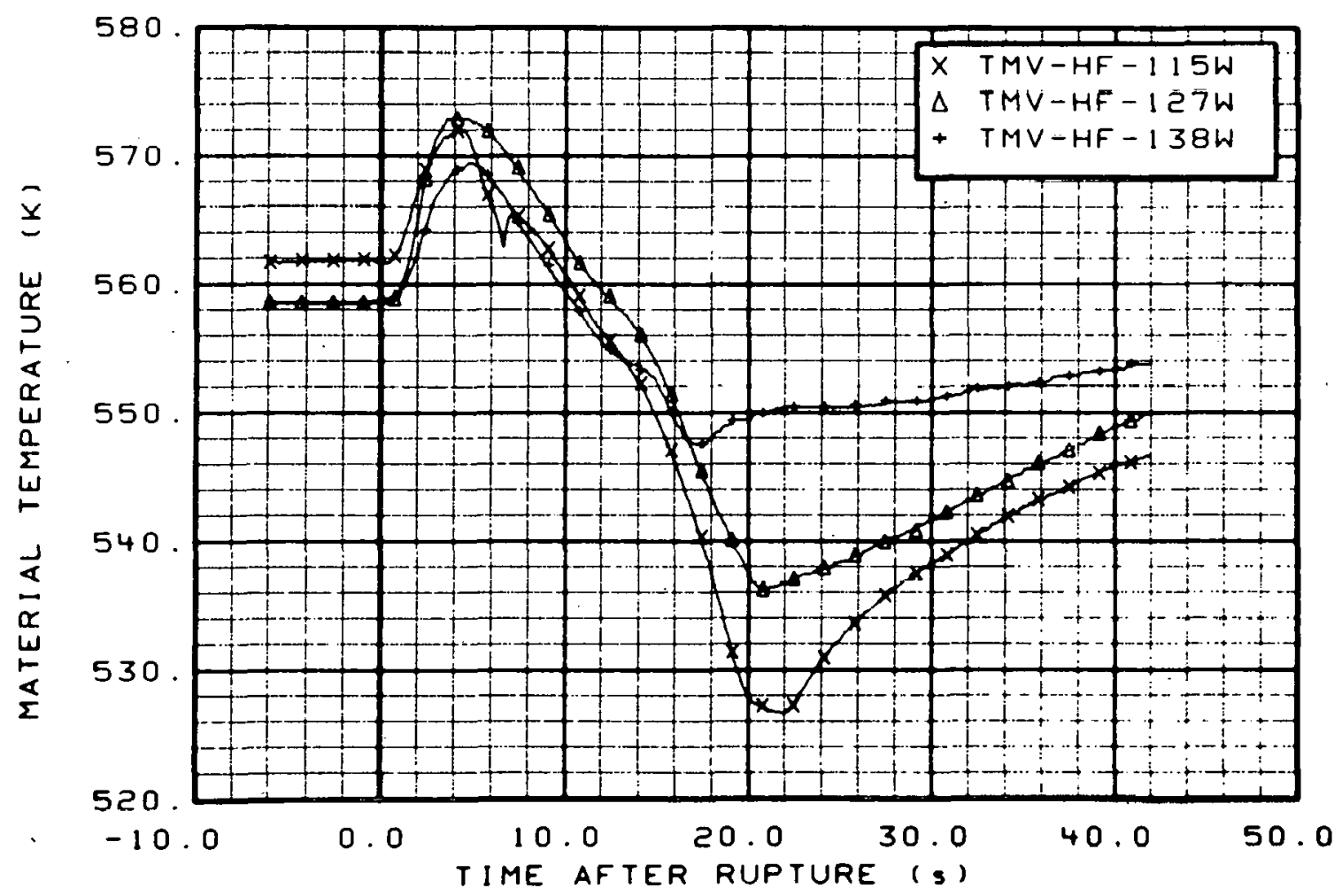

Fig. 74 Material temperature in core housing filler (TMV-HF-115W, TMV-HF-127W, and TMV-HF-138W), from -6 to $42 \mathrm{~s}$. 


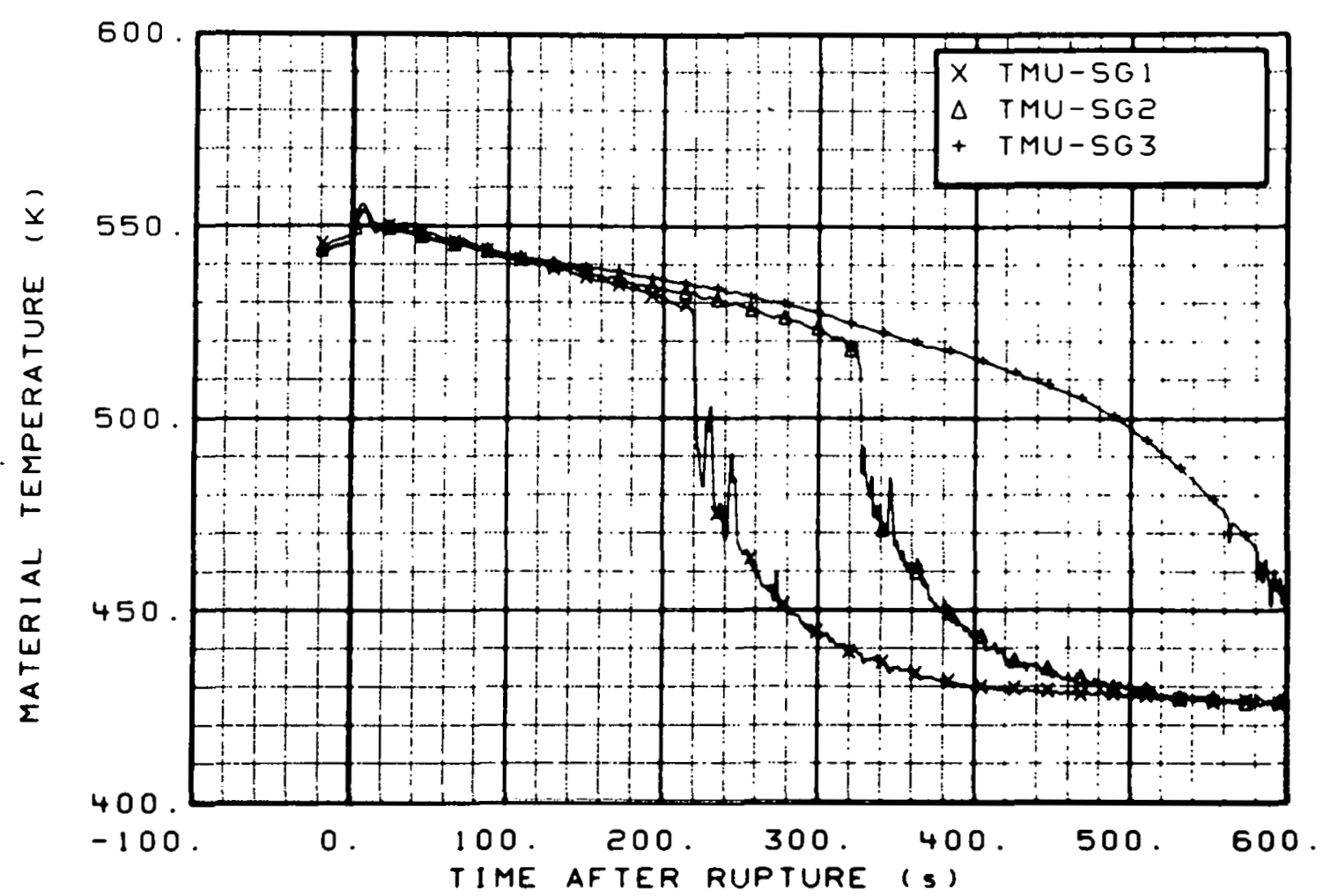

Fig. 75 Material temperature in steam generator (TMU-SG1, TMU-SG2, and TMU-SG3), from -20 to $600 \mathrm{~s}$.

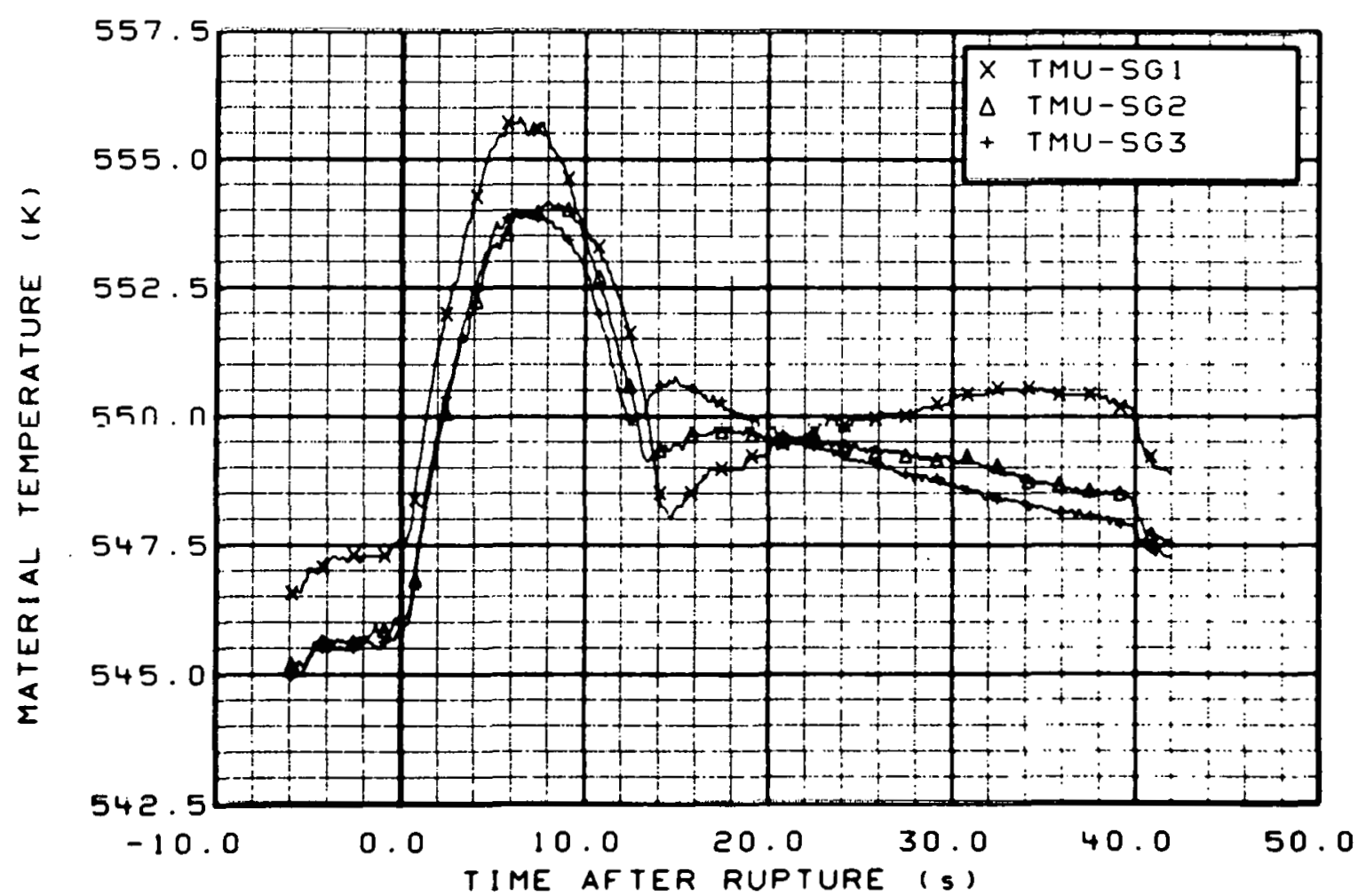

Fig. 76 Material temperature in steam generator (TMU-SG1, TMU-SG2, and TMU-SG3), from -6 to $42 \mathrm{~s}$. 


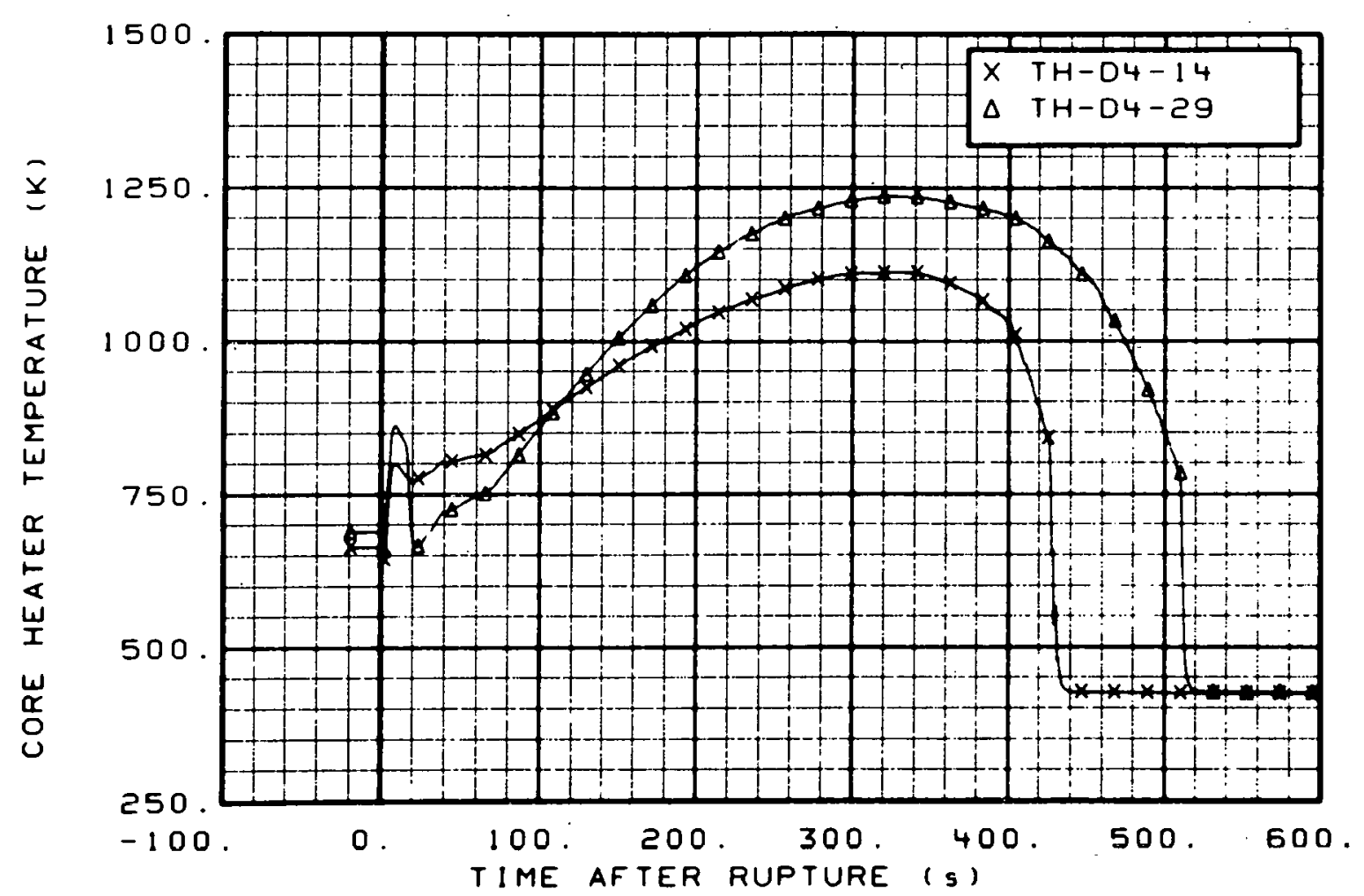

Fig. 77 Core heater temperature, Rod D-4 (TH-D4-14 and TH-D4-29), from -20 to $600 \mathrm{~s}$.

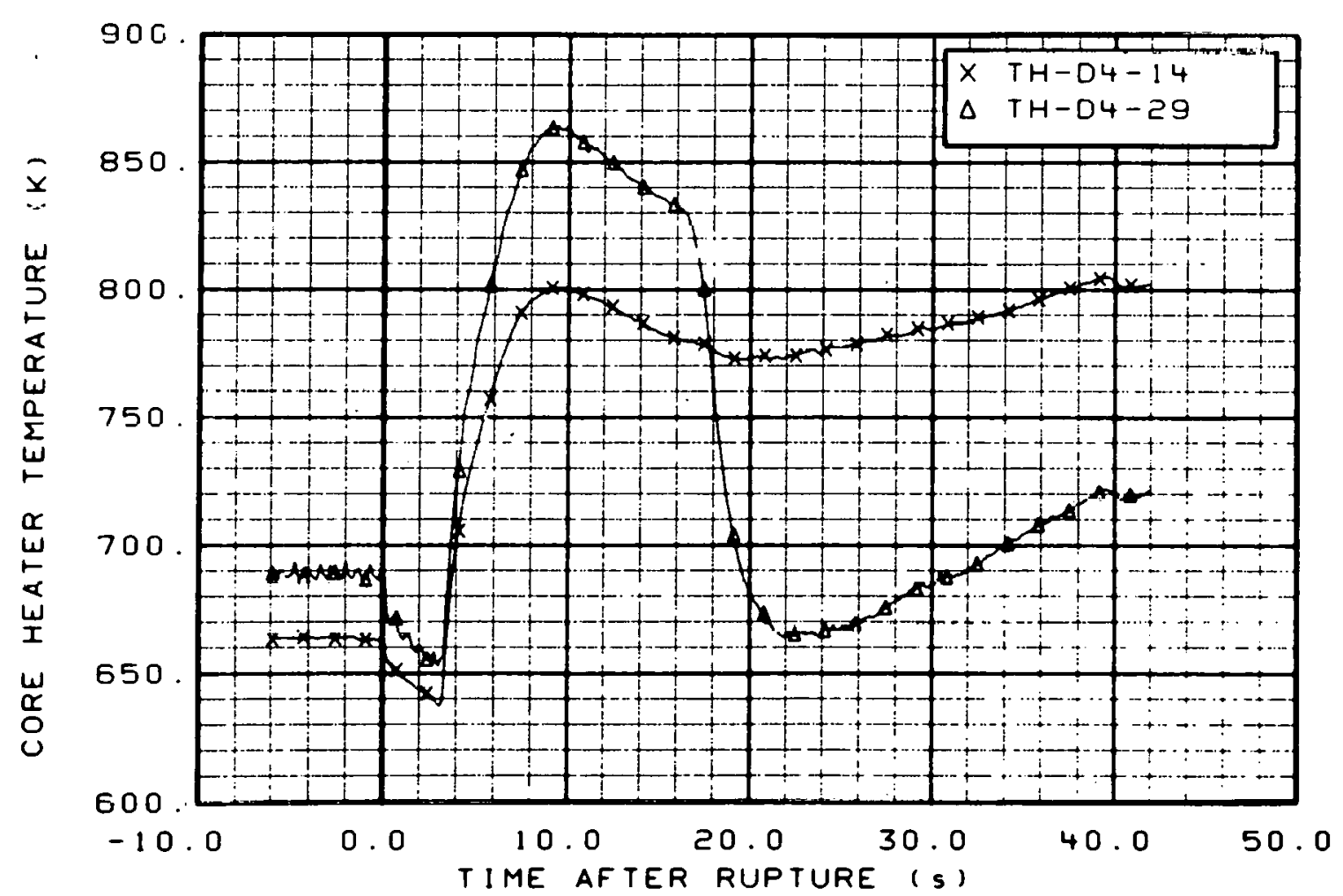

Fig. 78 Core heater temperature, Rod D-4 (TH-D4-14 and TH-D4-29), from -6 to $42 \mathrm{~s}$. 


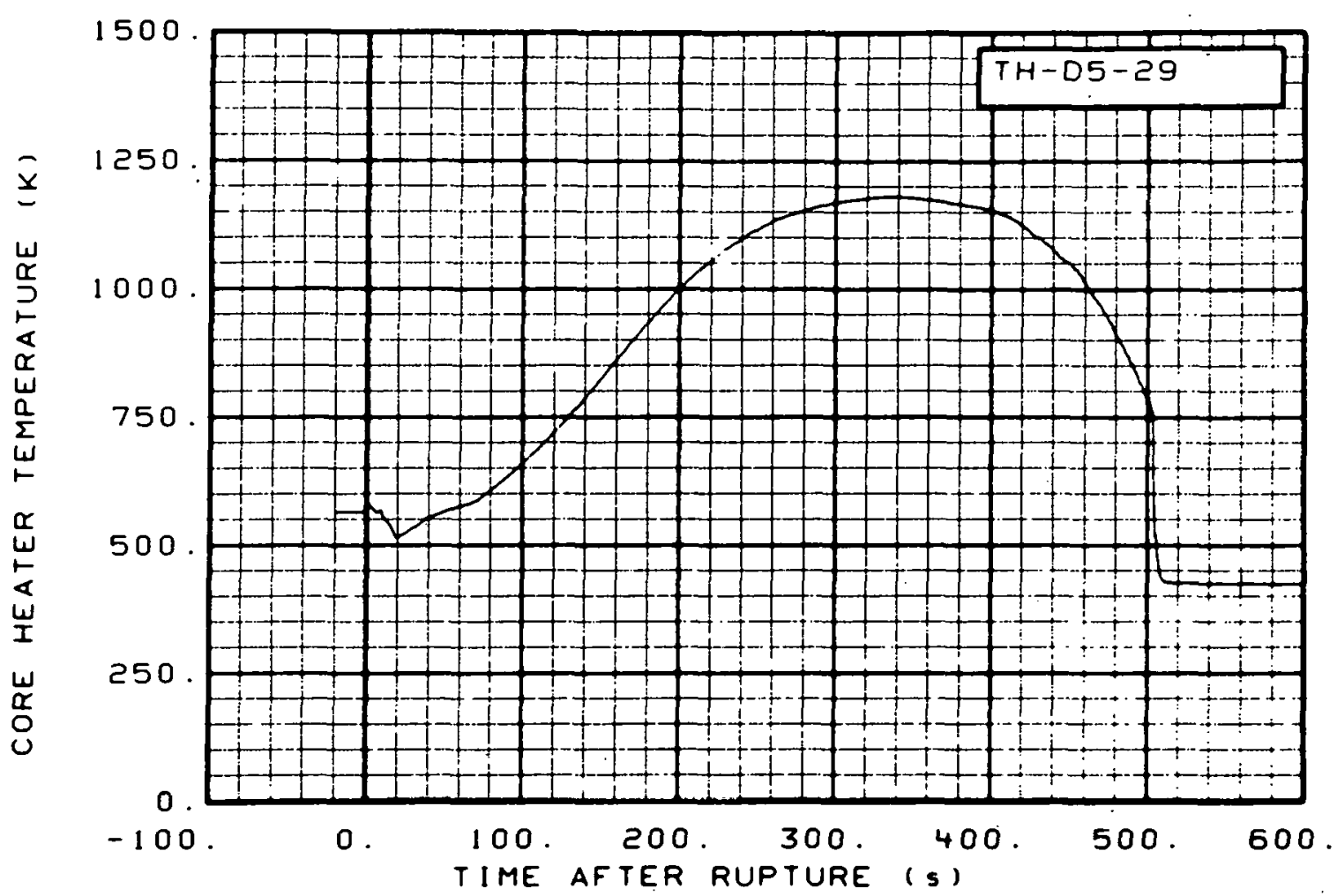

Fig. 79 Core heater temperature, Rod D-5 (TH-D5-29), from -20 to $600 \mathrm{~s}$.

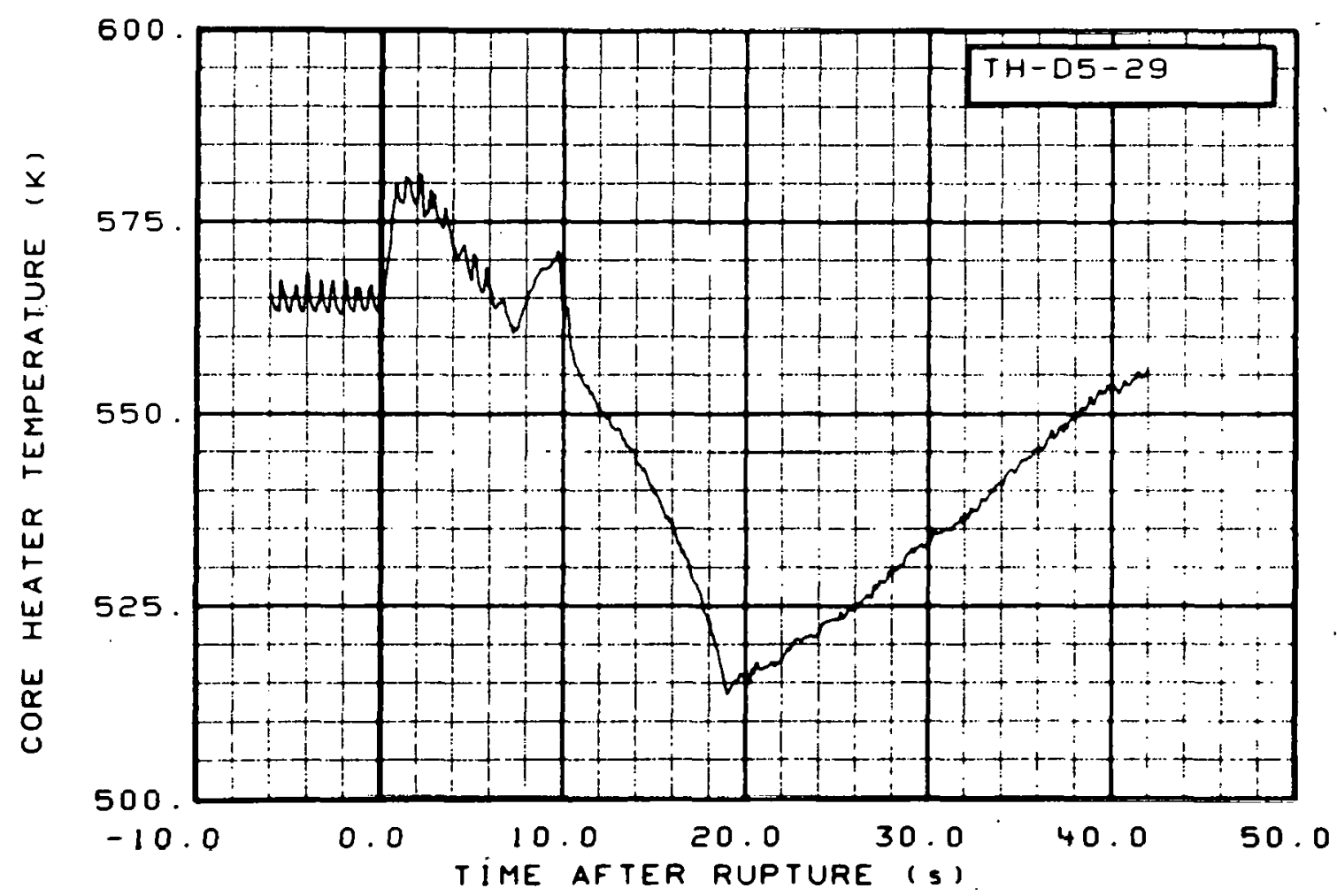

Fig. 80 Core heater temperature, Rod D-5 (TH-D5-29), from -6 to $42 \mathrm{~s}$. 


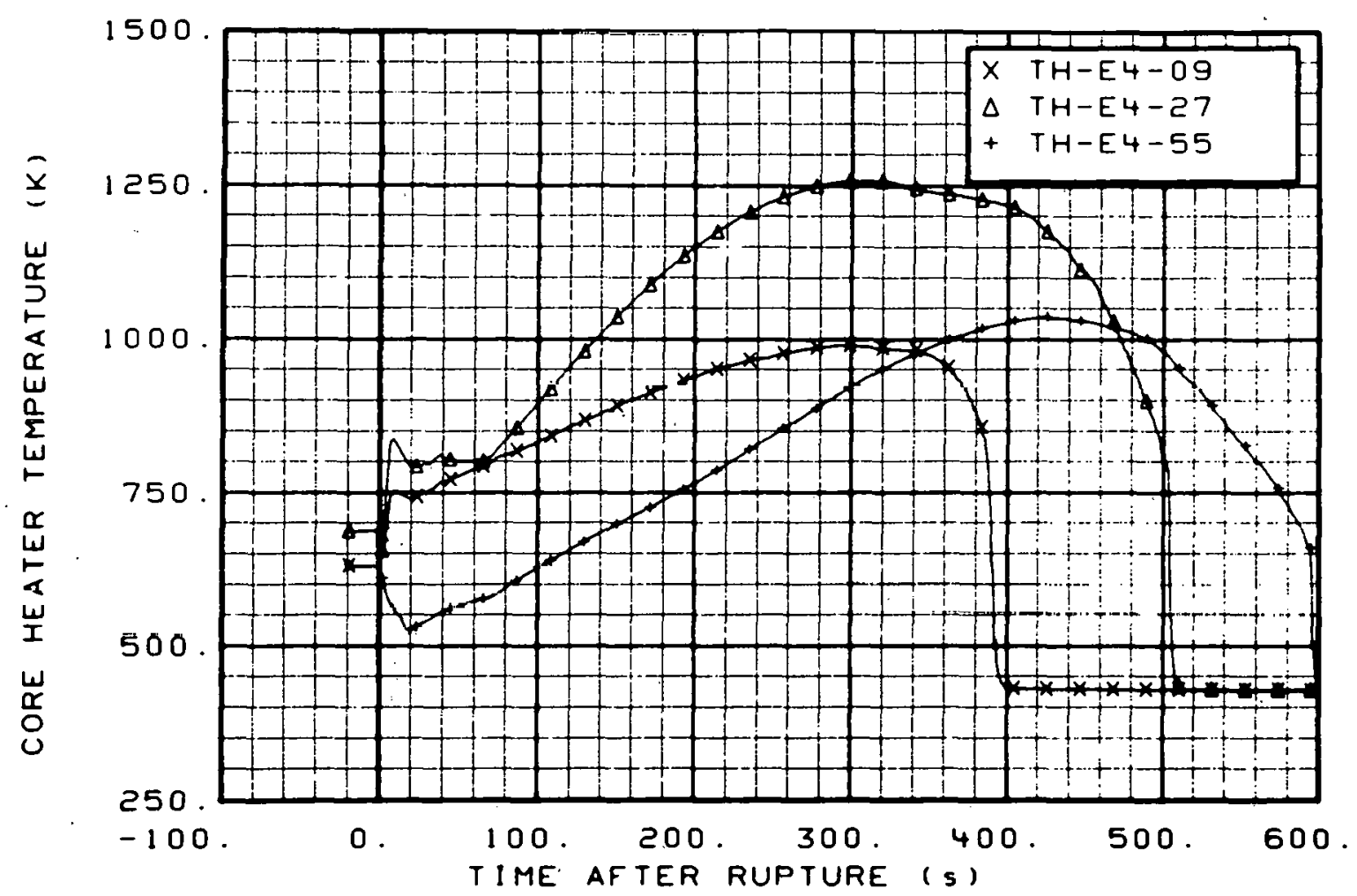

Fig. 81 Core heater temperature, Rod E-4 (TH-E4-09, TH-E4-27, and TH-E4-55), from -20 to $600 \mathrm{~s}$.

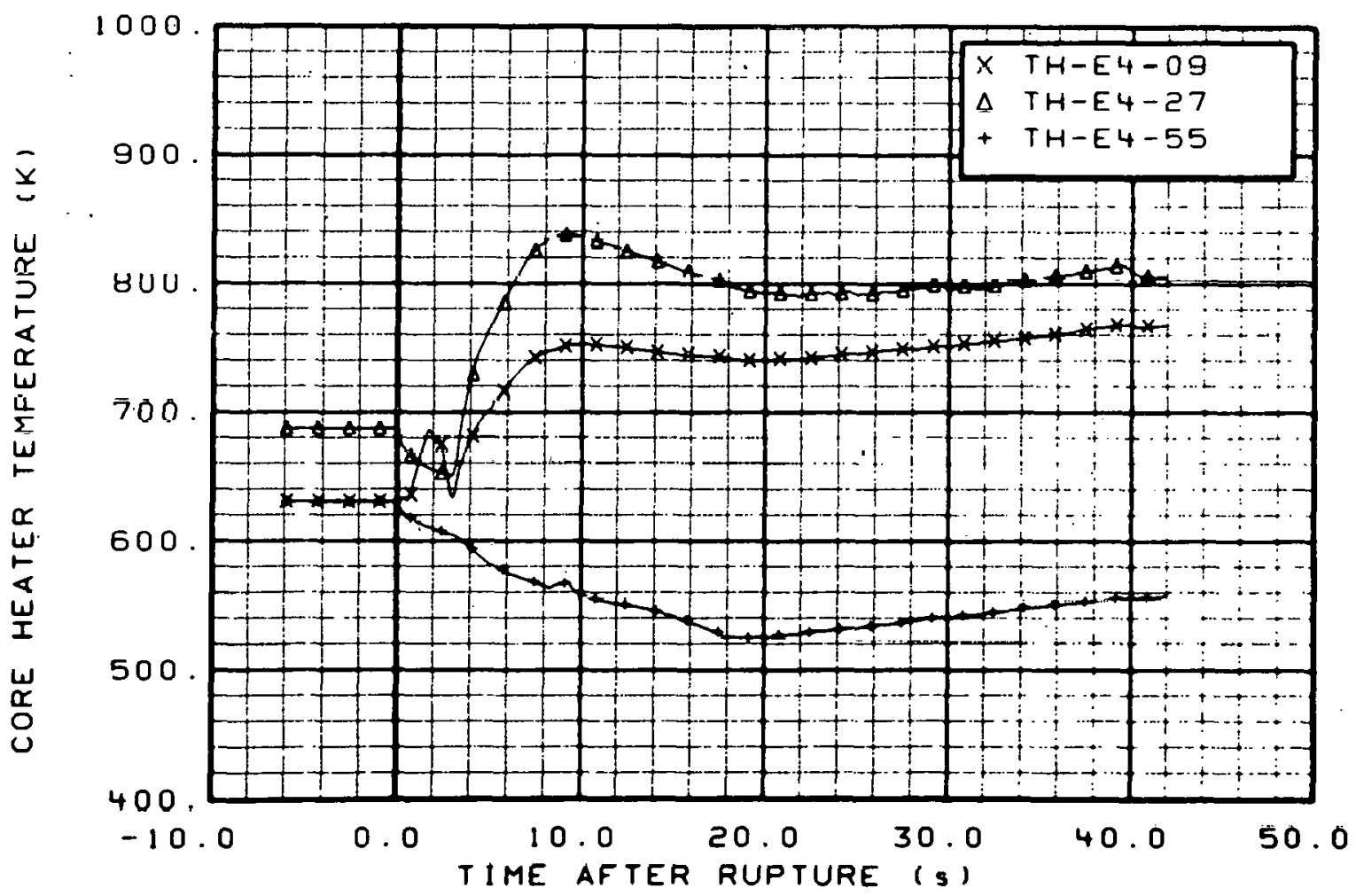

Fig. 82 Core heater temperature, Rod E-4 (TH-E4-09, TH-E4-27, and. TH-E4-55), from -6 to $42 \mathrm{~s}$. 


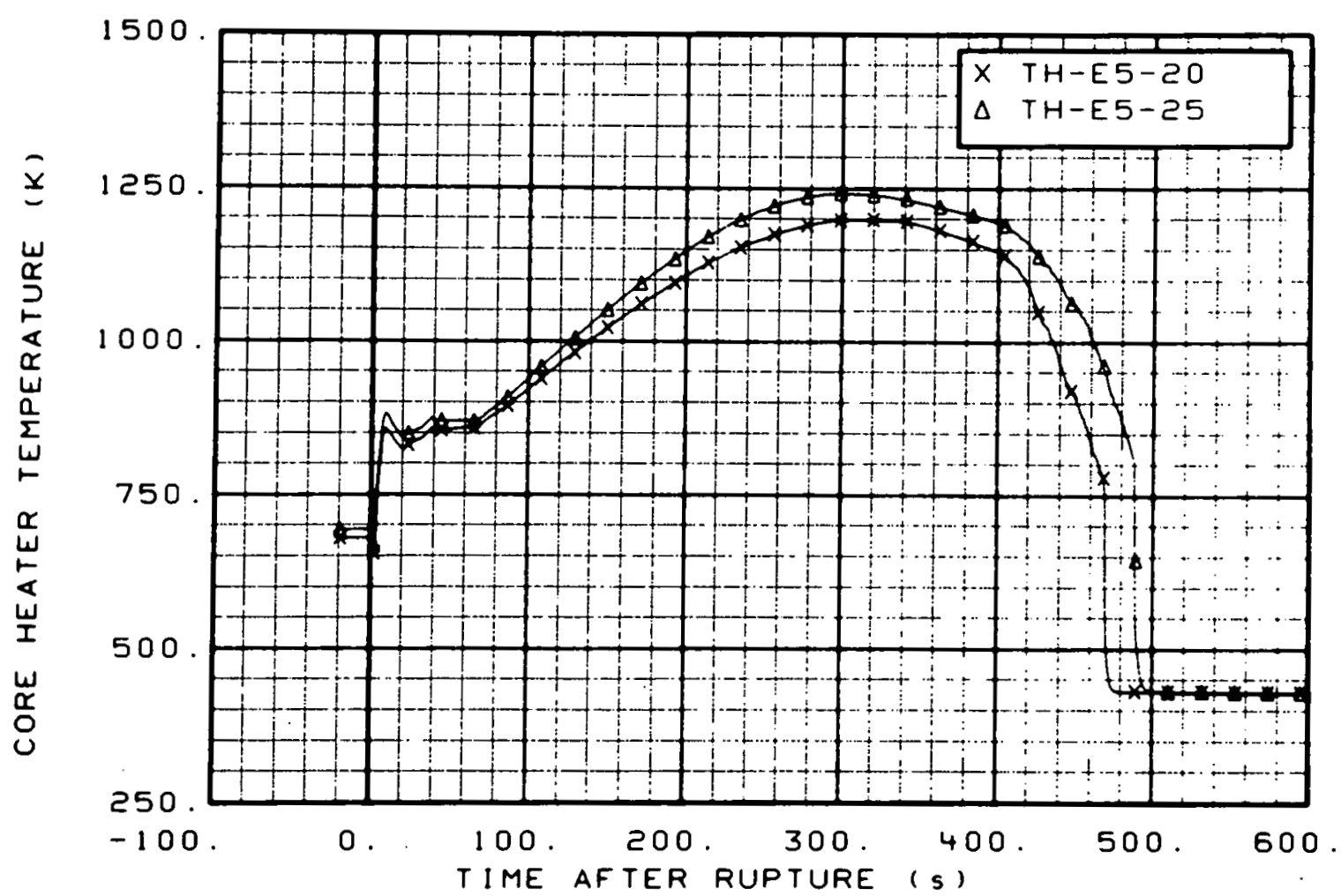

Fig. 83 Core heater temperature, Rod E-5 (TH-E5-20 and TH-E5-25), from -20 to $600 \mathrm{~s}$.

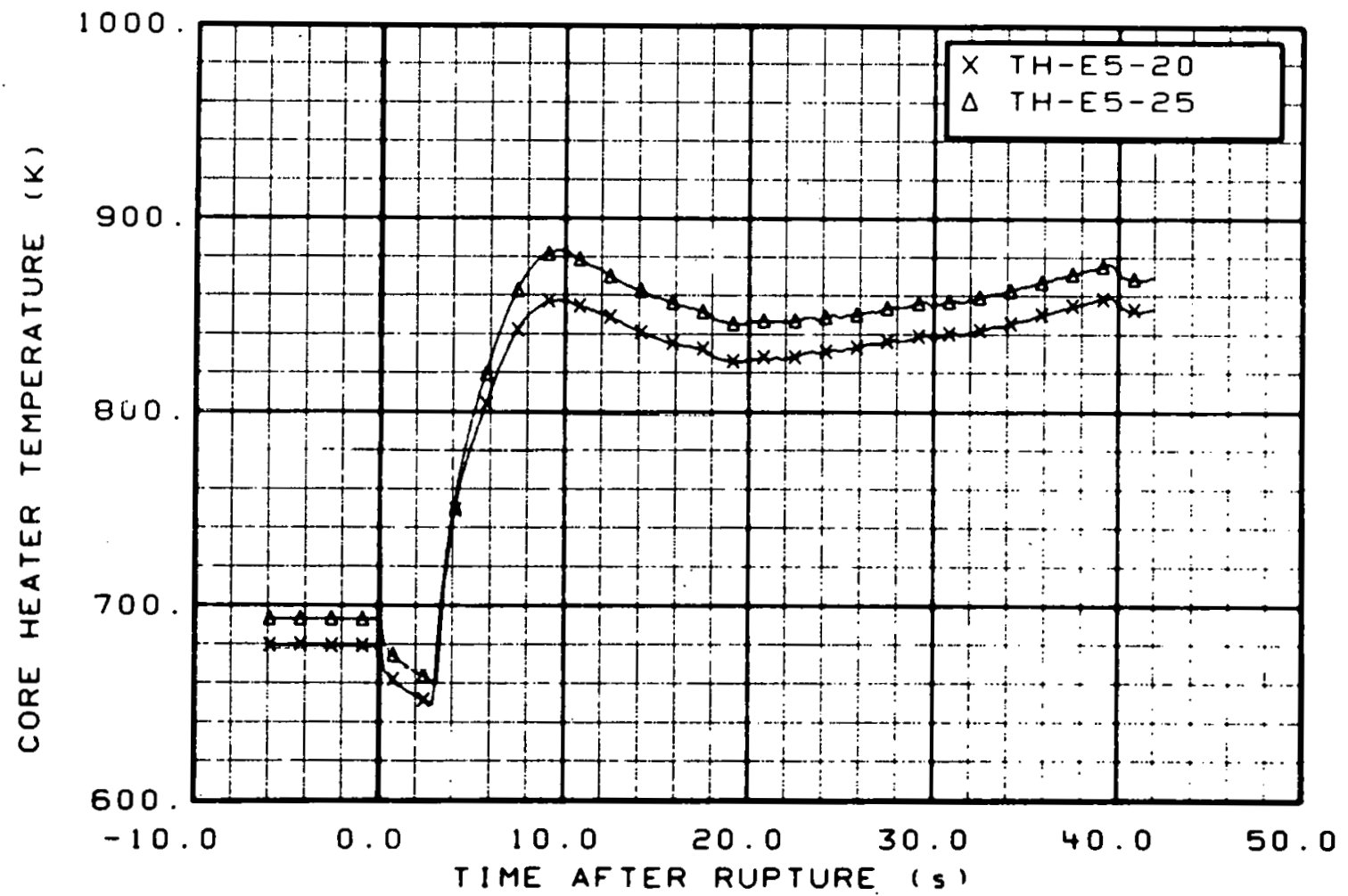

Fig. 84 Core heater temperature, Rod E-5 (TH-E5-20 and TH-E5-25), from -6 to $42 \mathrm{~s}$. 


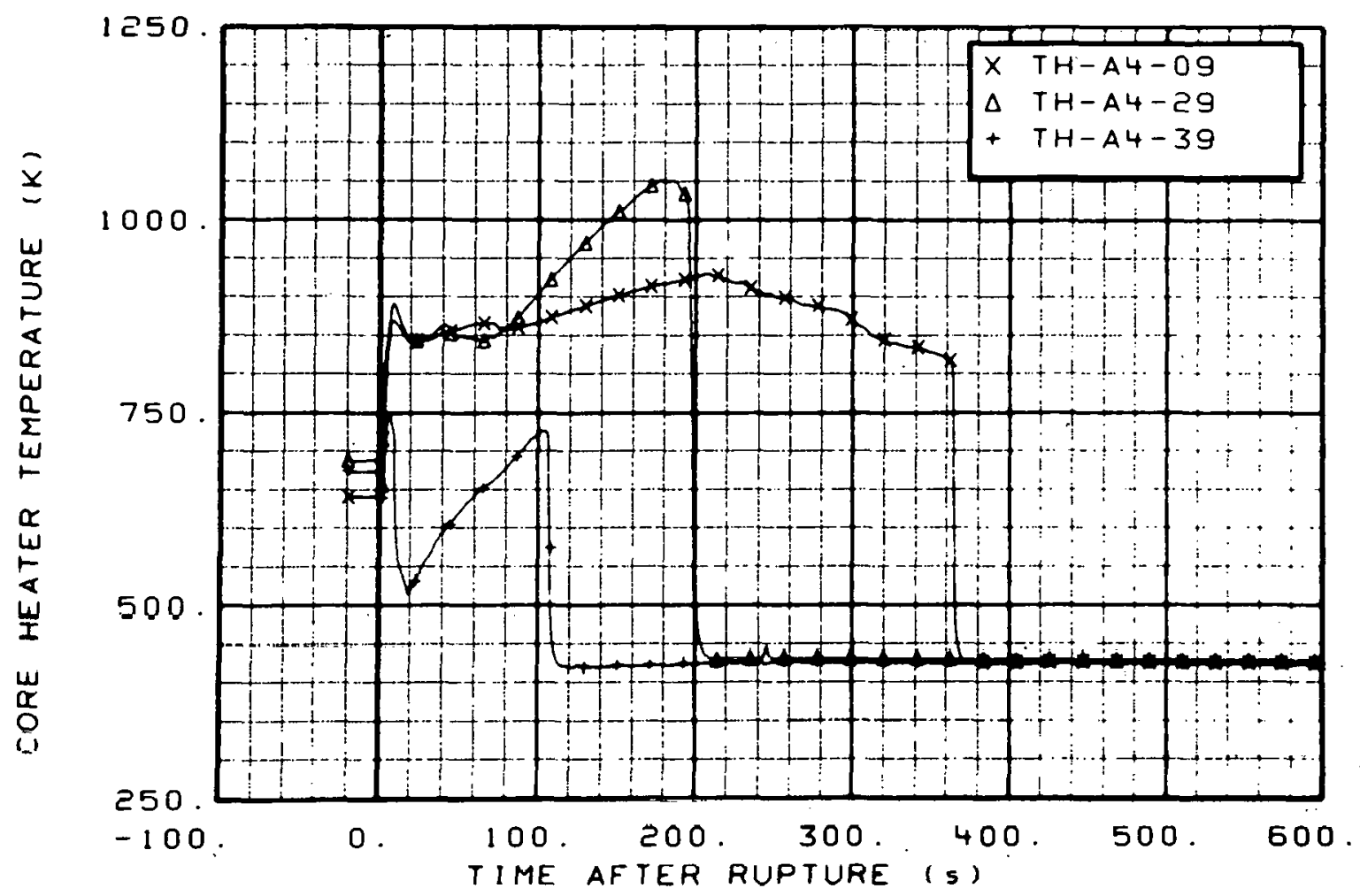

Fig. 85 Core heater temperature, Rod A-4 (TH-A4-09, TH-A4-29, and TH-A4-39), from -20 to $600 \mathrm{~s}$.

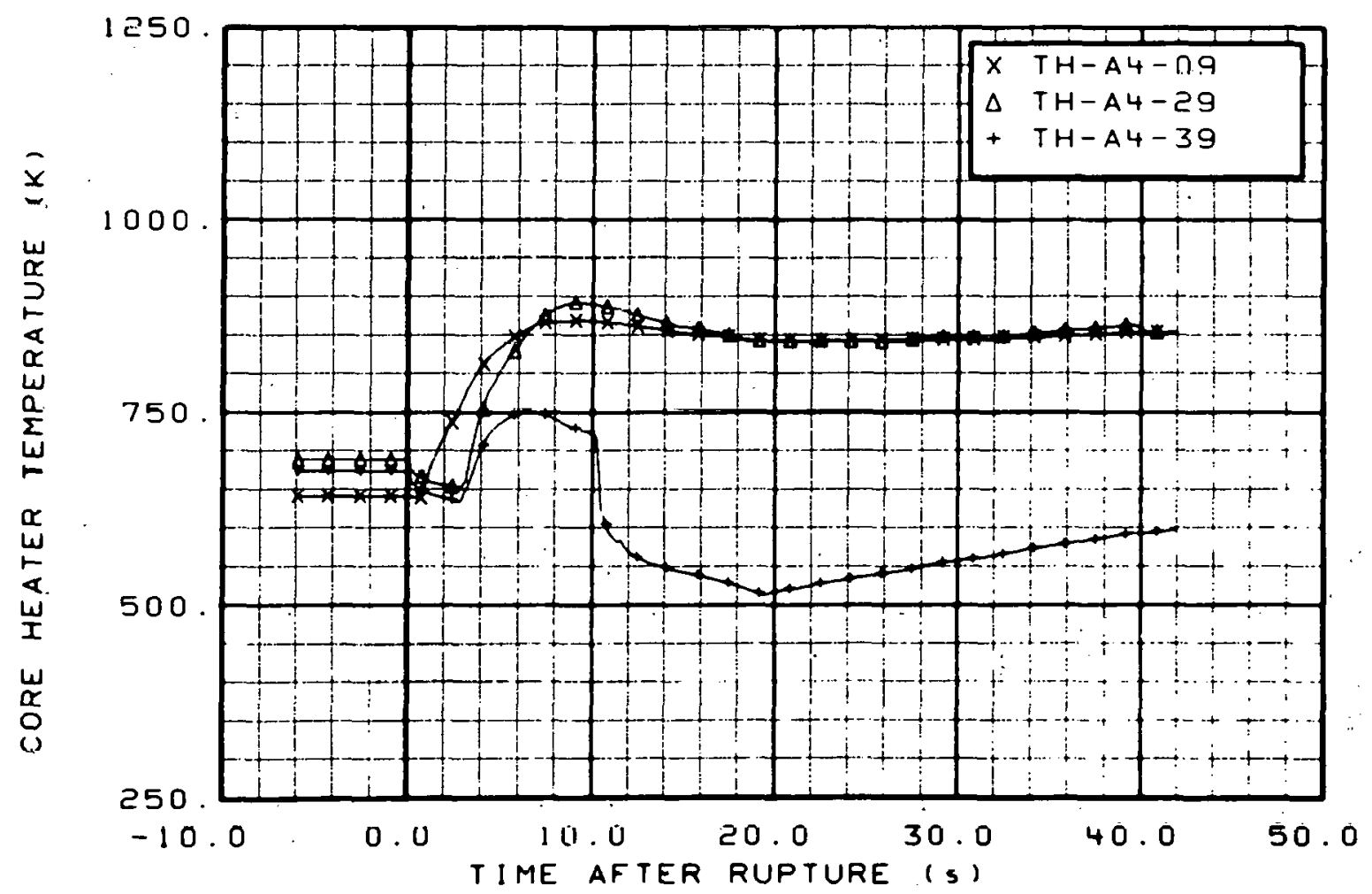

Fig. 86 Core heater temperature, Rod A-4 (TH-A4-09, TH-A4-29, and TH-A4-39), from -6 to $42 \mathrm{~s}$. 


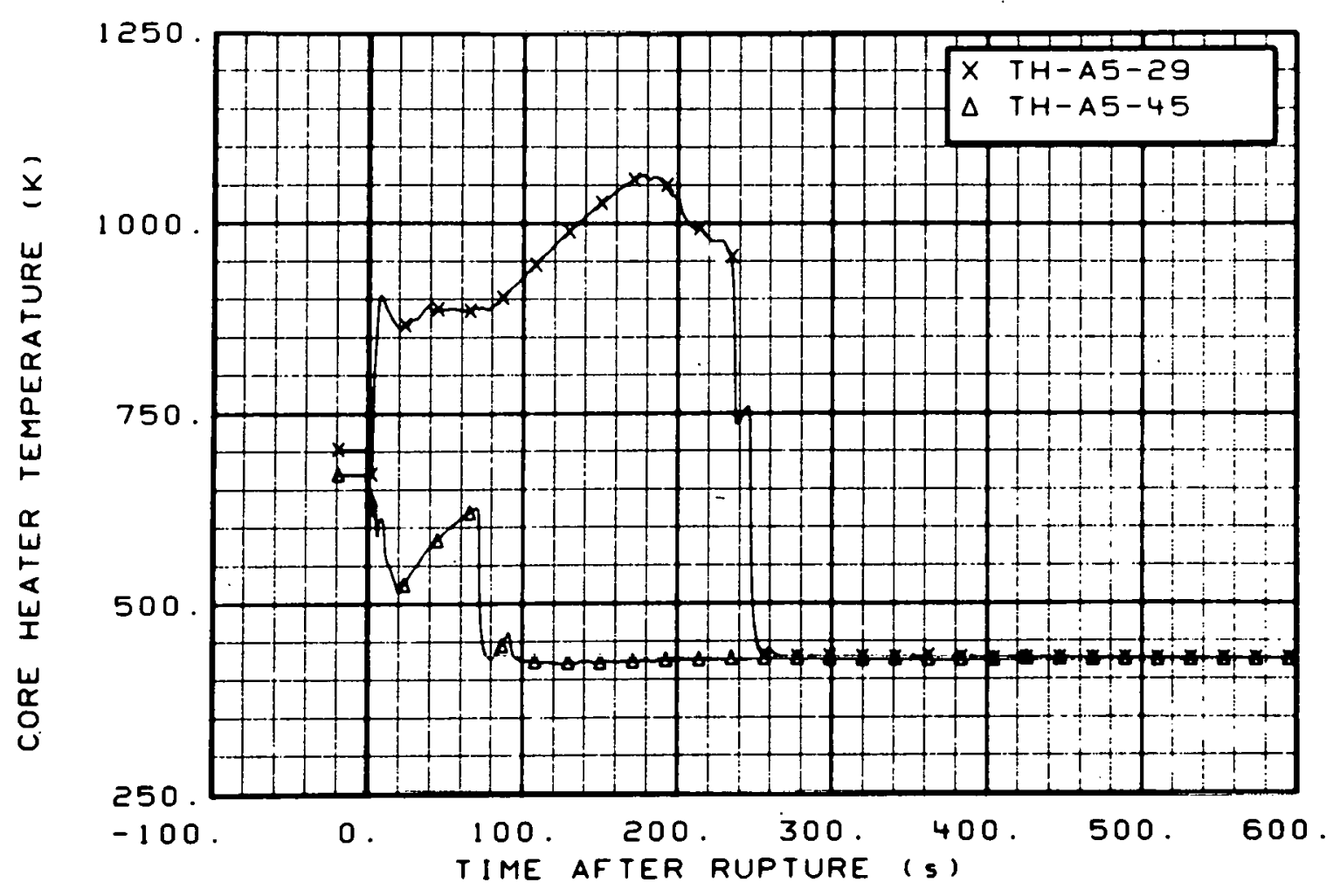

Fig. 87 Core heater temperature, Rod A-5 (TH-A5-29 and TH-A5-45), from -20 to $600 \mathrm{~s}$.

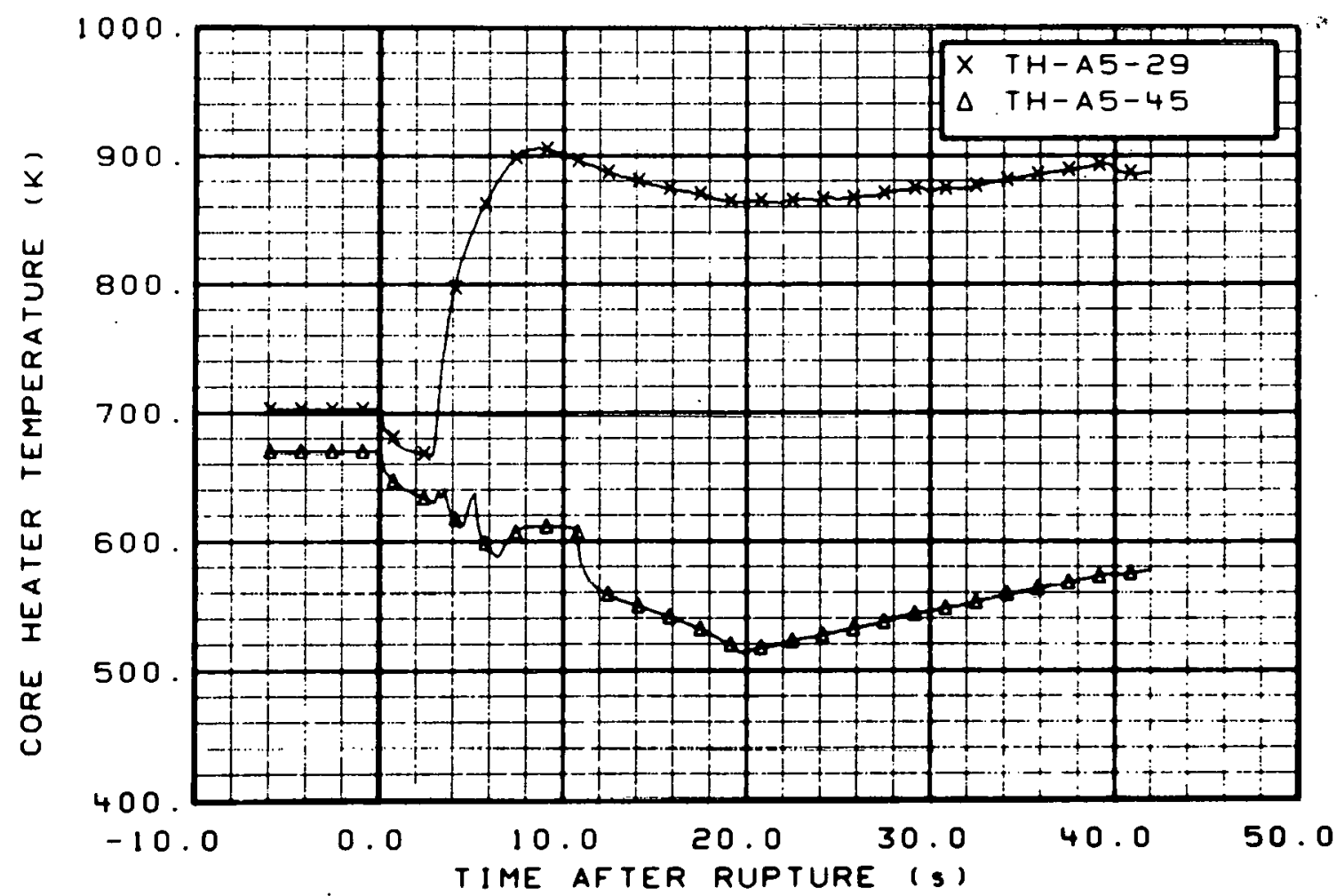

Fig. 88 Core heater temperature, Rod A-5 (TH-A5-29 and TH-A5-45), from -6 to $42 \mathrm{~s}$. 


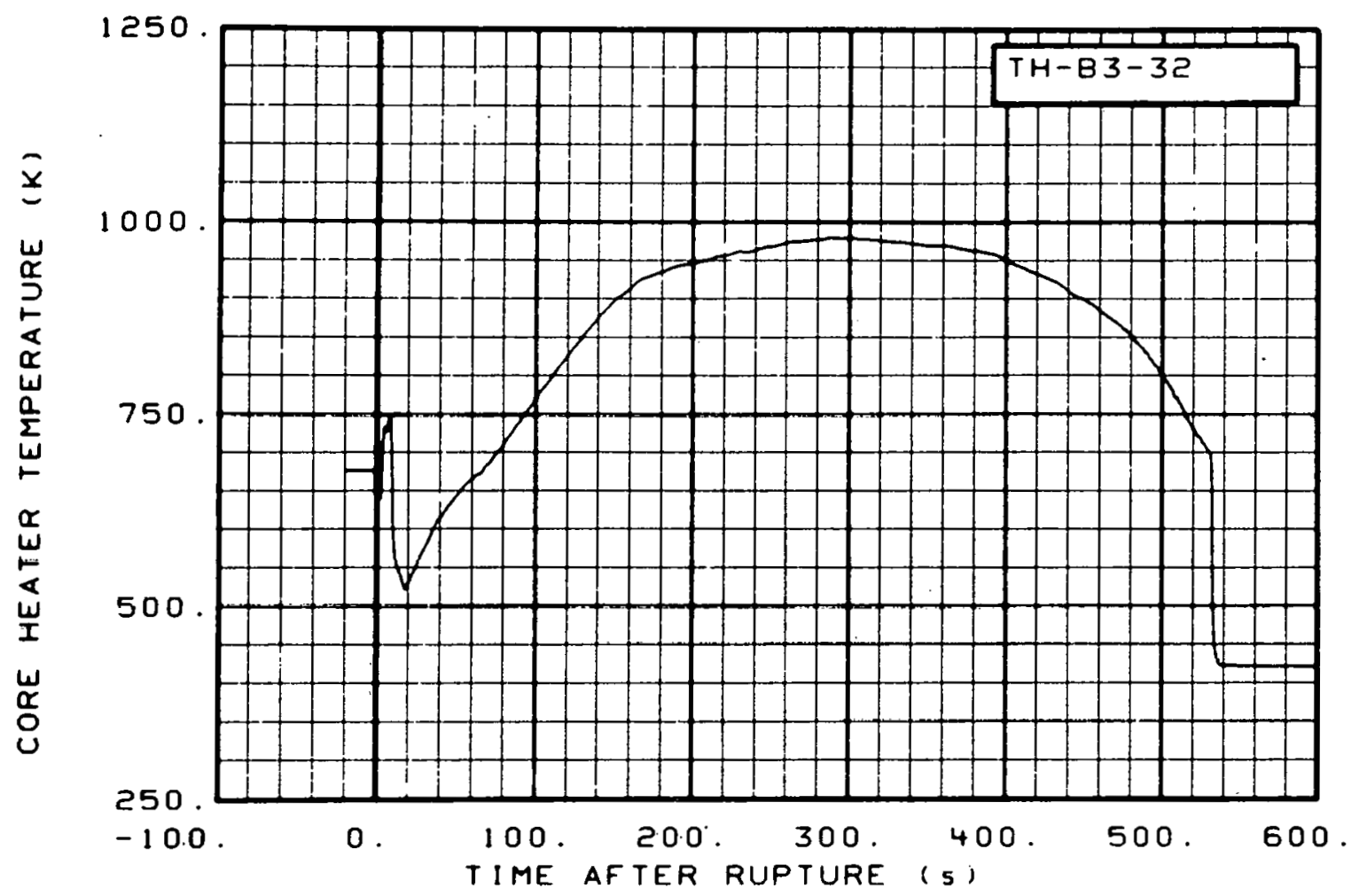

Fig. 89 Core heater temperature, Rod B-3 (TH-B3-32), from -20 to $600 \mathrm{~s}$.

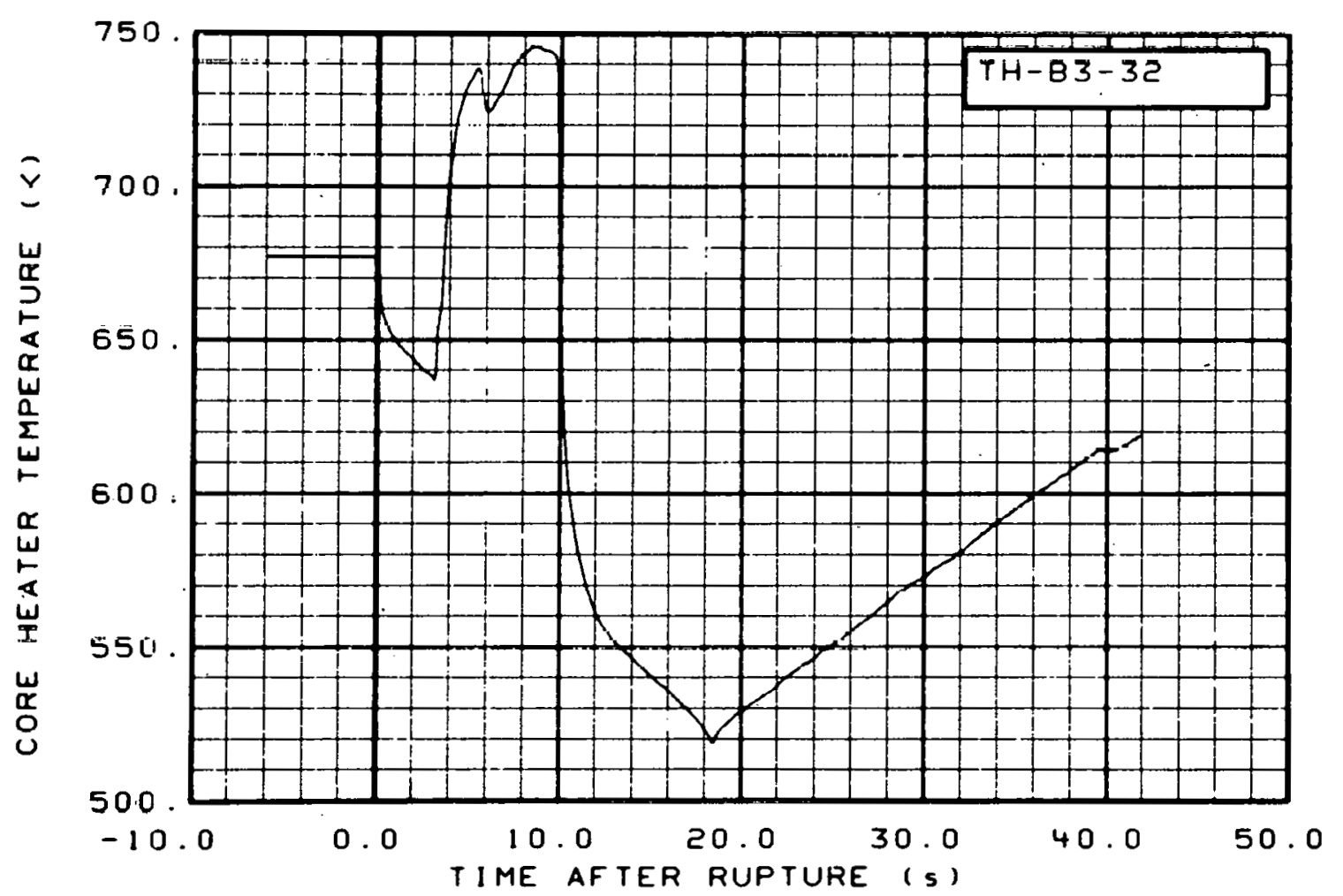

Fig. 90 Core heater temperature, Rod B-3 (TH-B3-32), from -6 to $42 \mathrm{~s}$. 


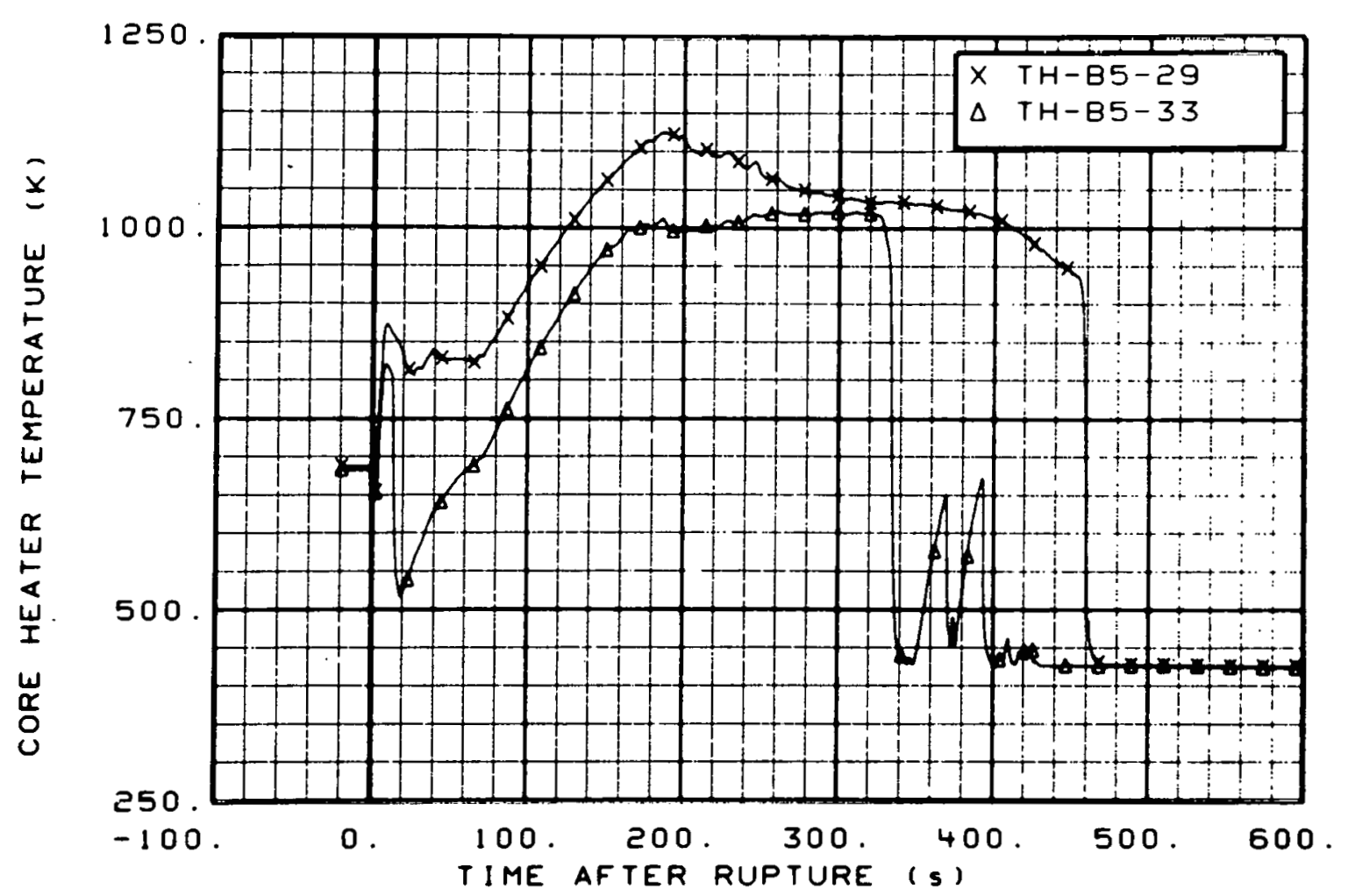

Fig. 91 Core heater temperature, Rod B-5 (TH-B5-29 and TH-B5-33), from -20 to $600 \mathrm{~s}$.

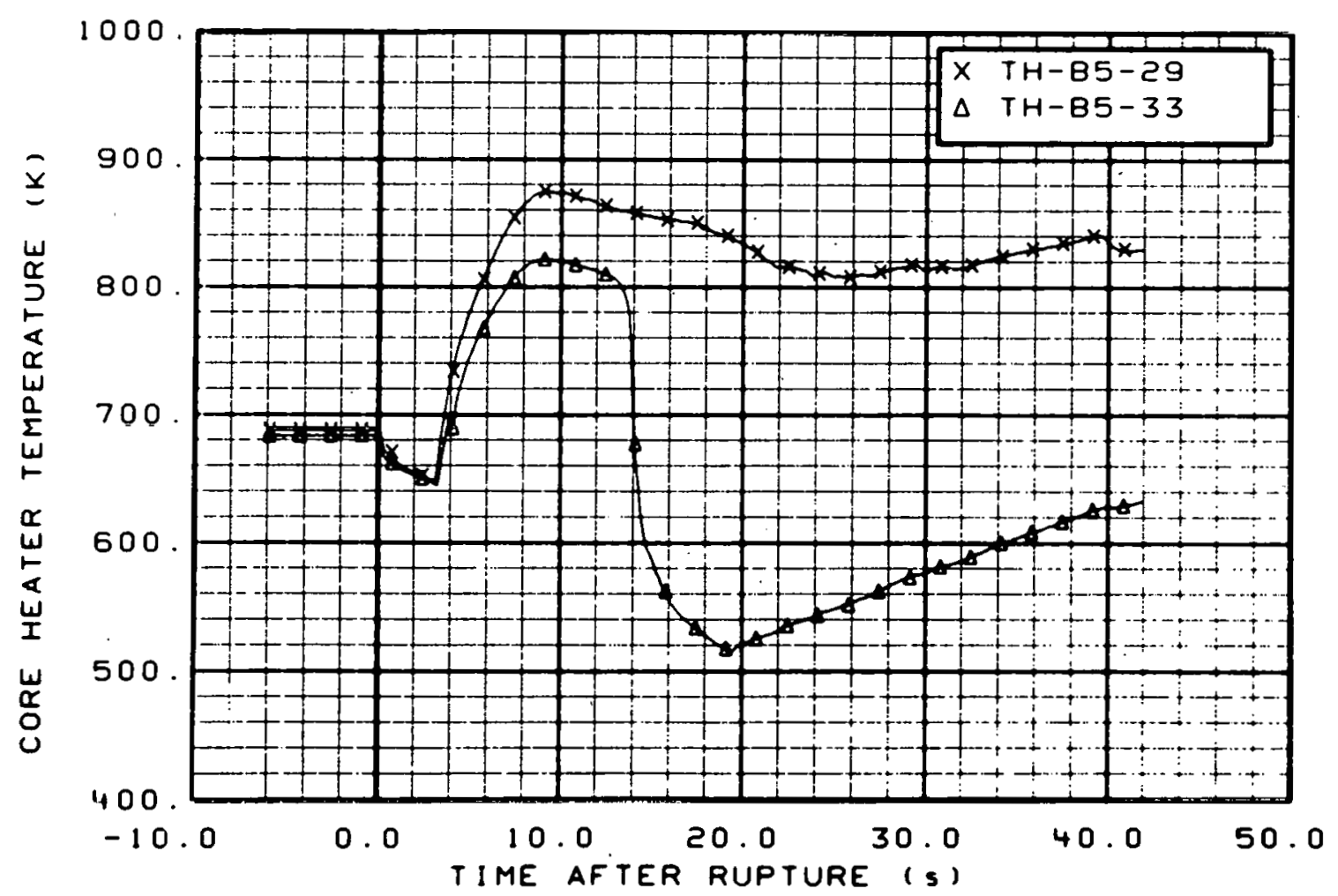

Fig. 92 Core heater temperature, Rod B-5 (TH-B5-29 and TH-B5-33), from -6 to $42 \mathrm{~s}$. 


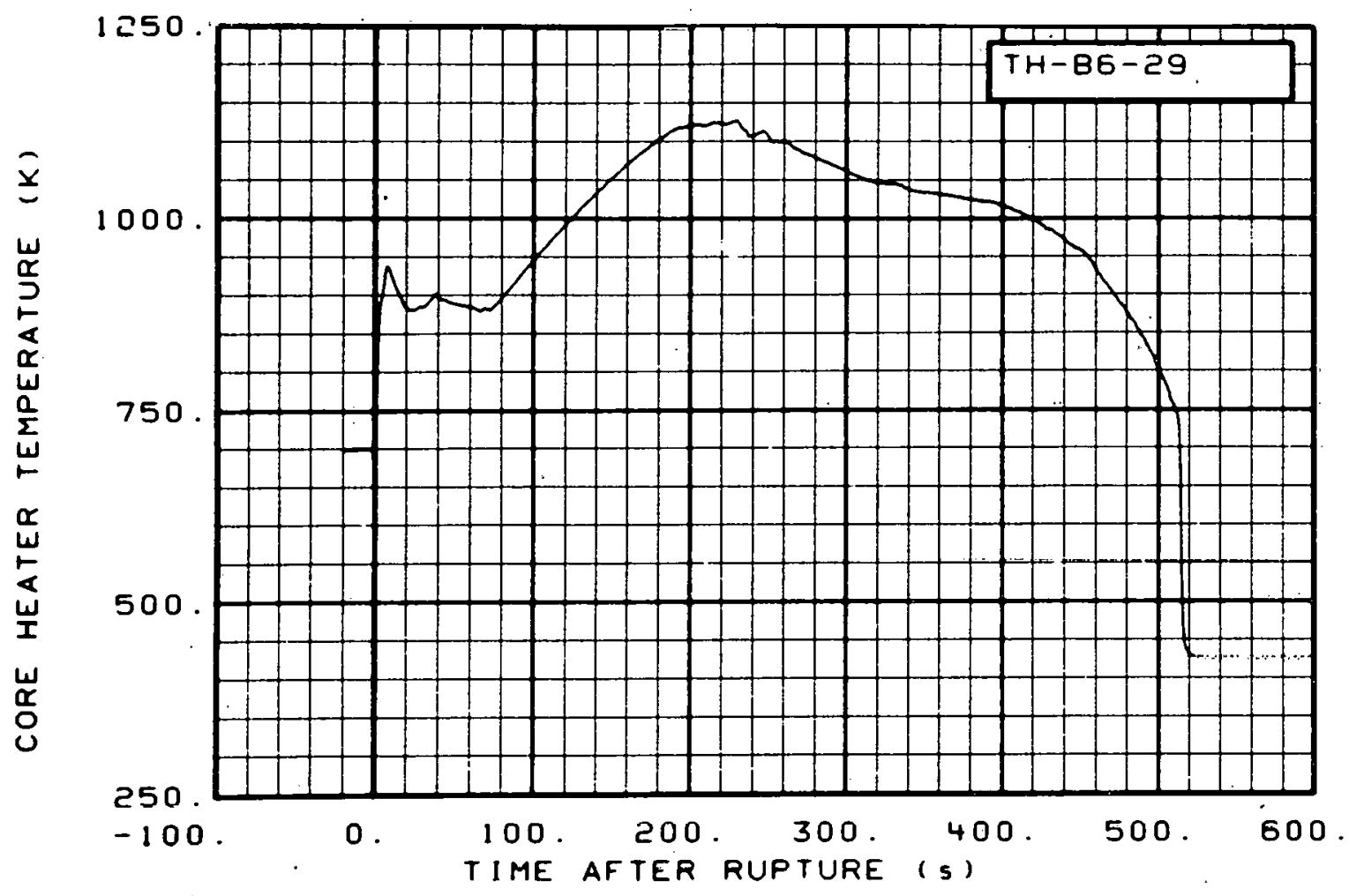

Fig. 93 Core heater temperature, Rod B-6 (TH-B6-29), from -20 to $600 \mathrm{~s}$.

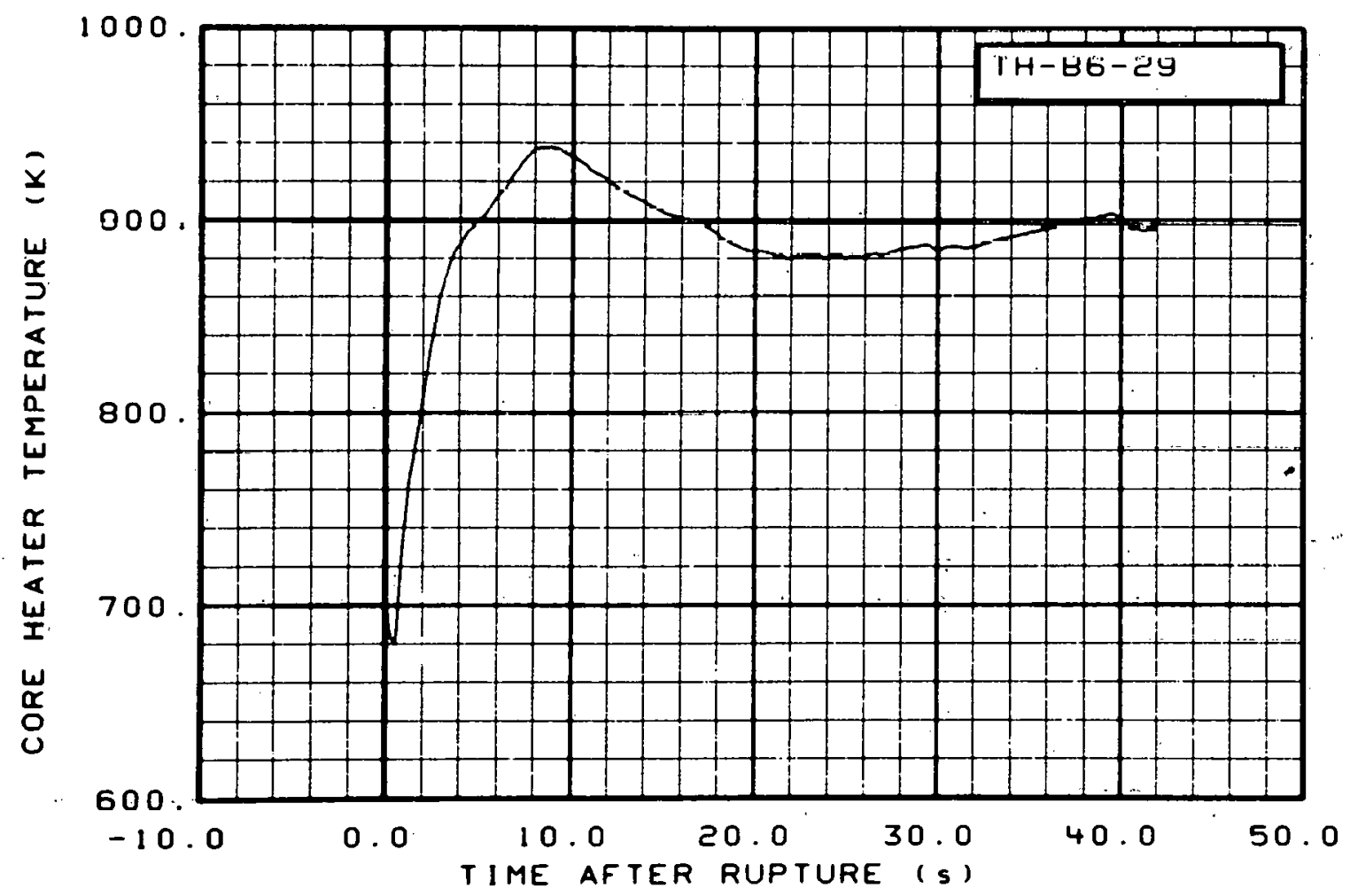

Fig. 94 Core heater tempera.ture, Rod B-6 (TH-B6-29), from -6 to $42 \mathrm{~s}$. 


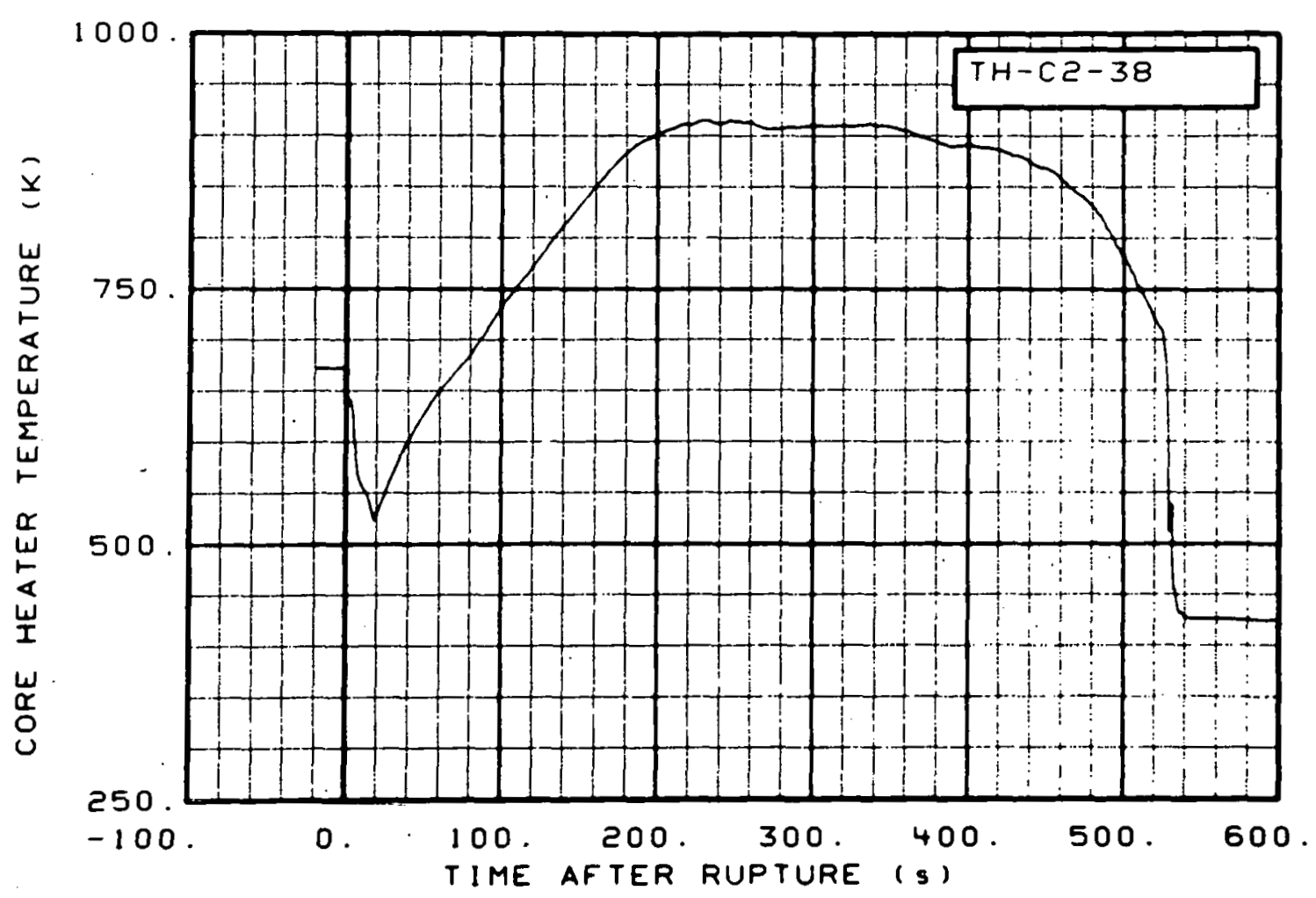

Fig. 95 Core heater temperature, Rod $\mathrm{C}-2$ (TH-C2-38), from -20 to $600 \mathrm{~s}$.

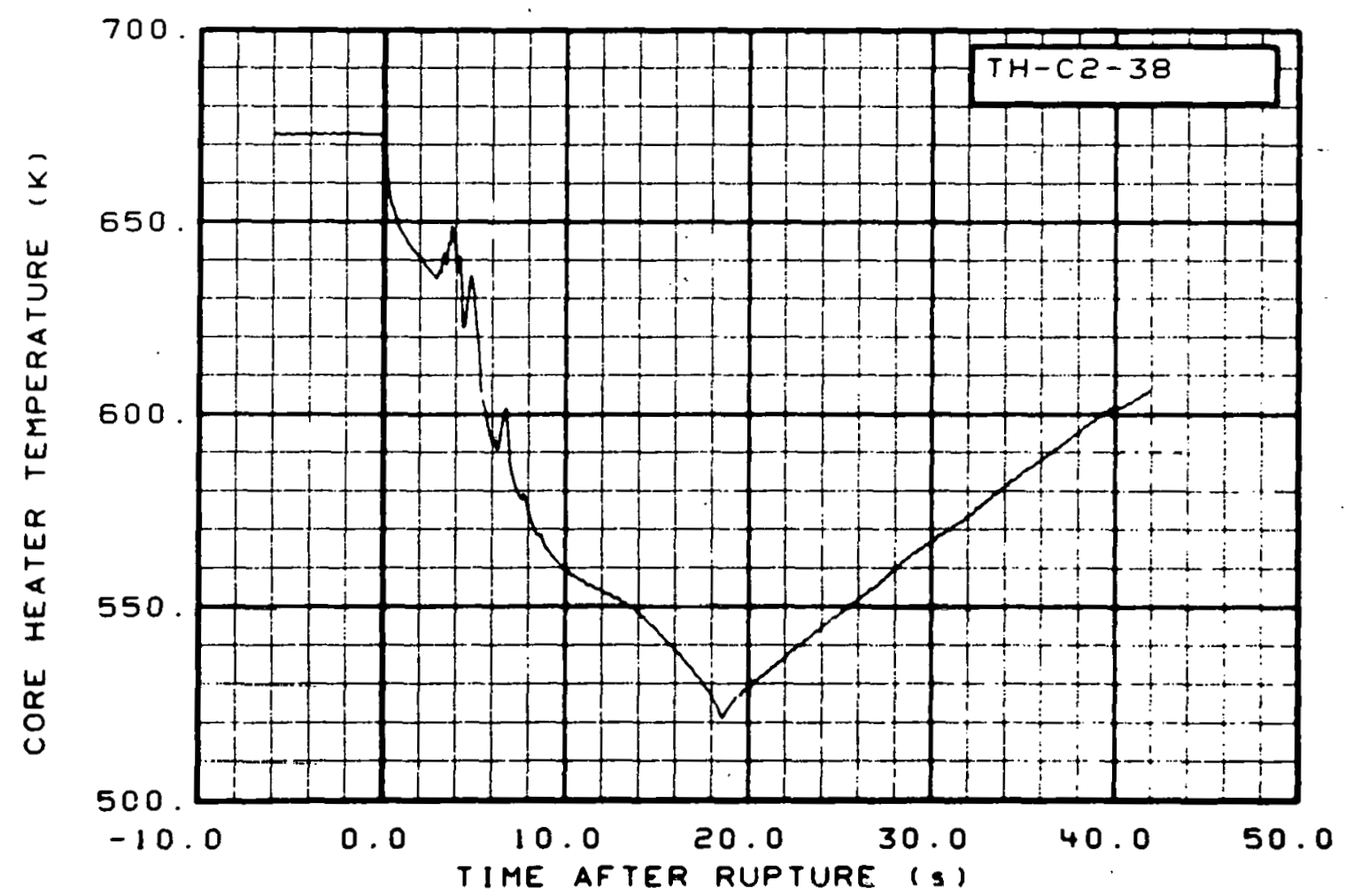

Fig. 96 Core heater temperature, Rod C-2 (TH-C2-38), from -6 to $42 \mathrm{~s}$. 


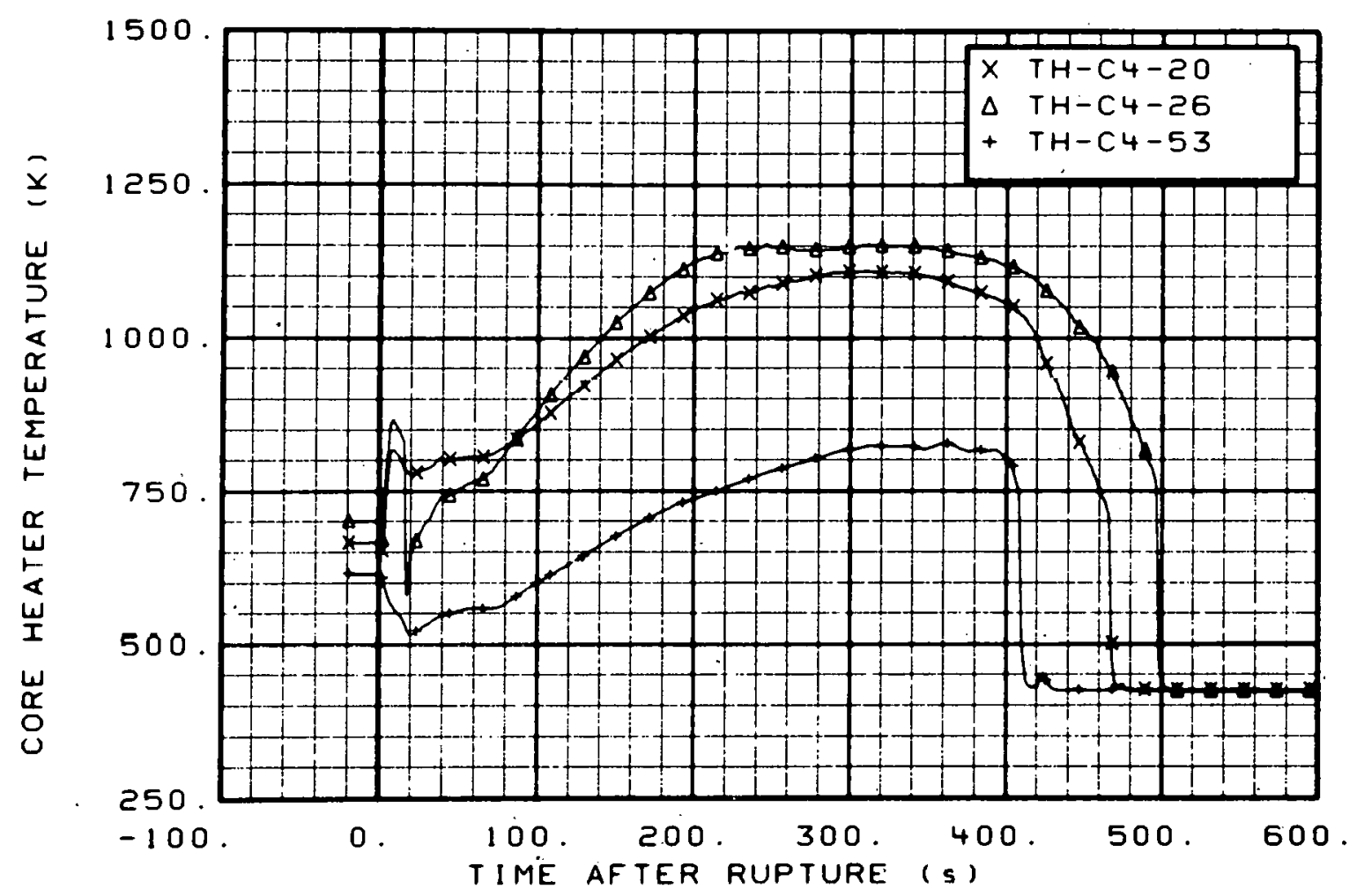

Fig. 97 Core heater temperature, Rod C-4 (TH-C4-20, TH-C4-26, and $\mathrm{TH}-\mathrm{C} 4-53$ ), from -20 to $600 \mathrm{~s}$.

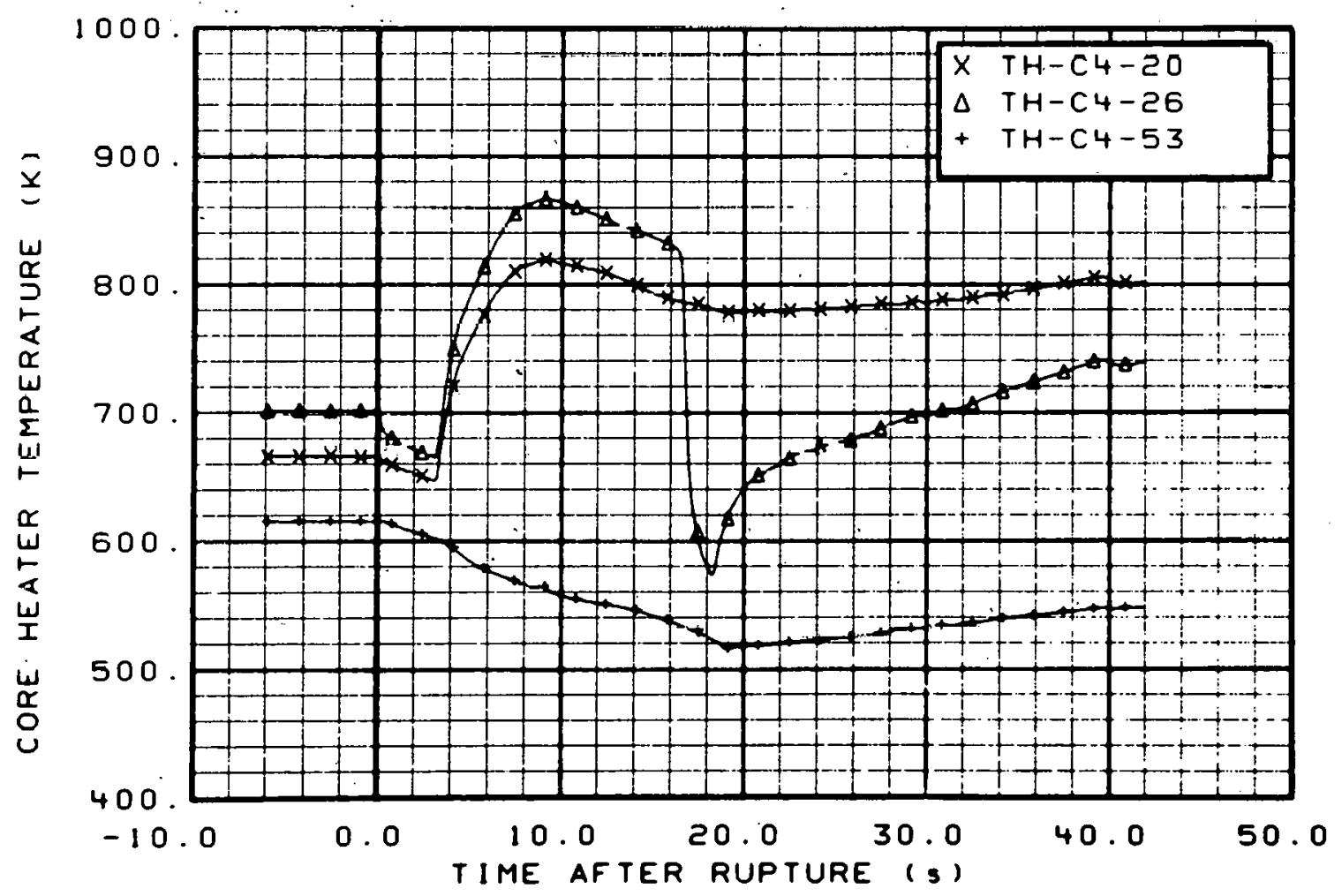

Fig. 98 Core heater temperature, Rod C-4 (TH-C4-20, TH-C4-26, and TH-C4-53), from -6 to $42 \mathrm{~s}$. 


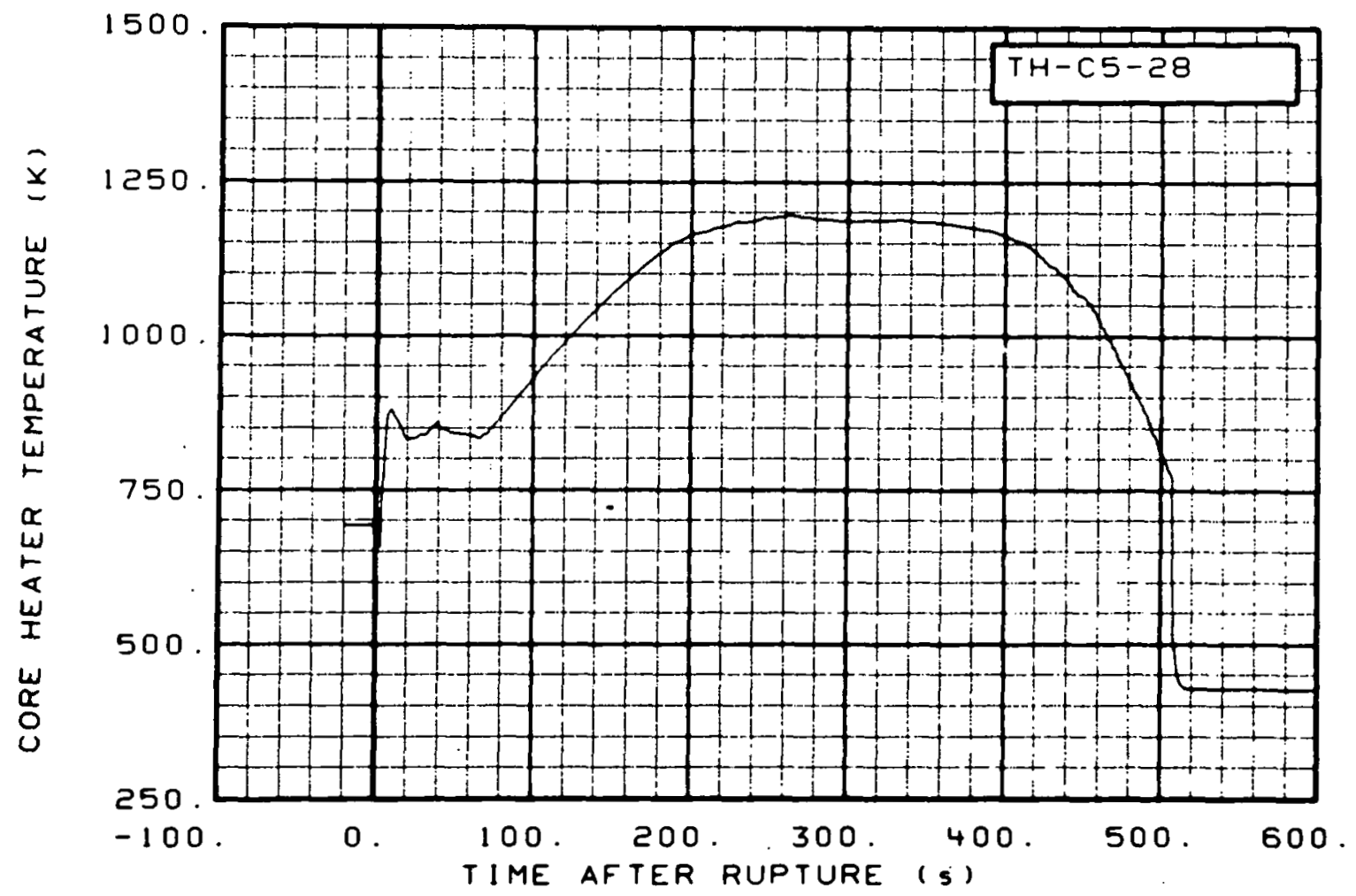

Fig. 99 Core heater temperature, Rod C-5 (TH-C5-28), from -20 to $600 \mathrm{~s}$,

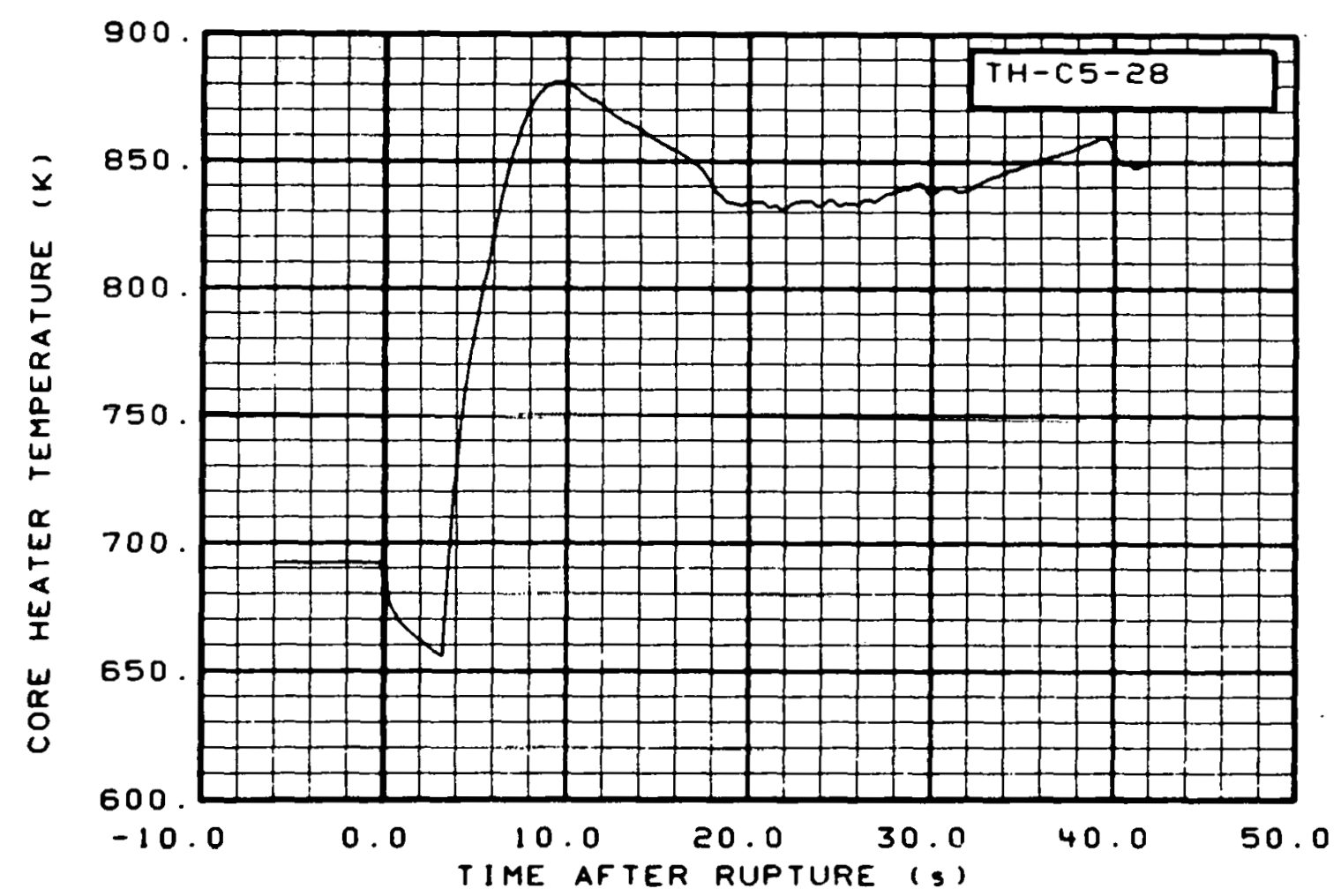

Fig. 100 Core heater temperature, Rod C-5 (TH-C5-28), from -6 to $42 \mathrm{~s}$. 


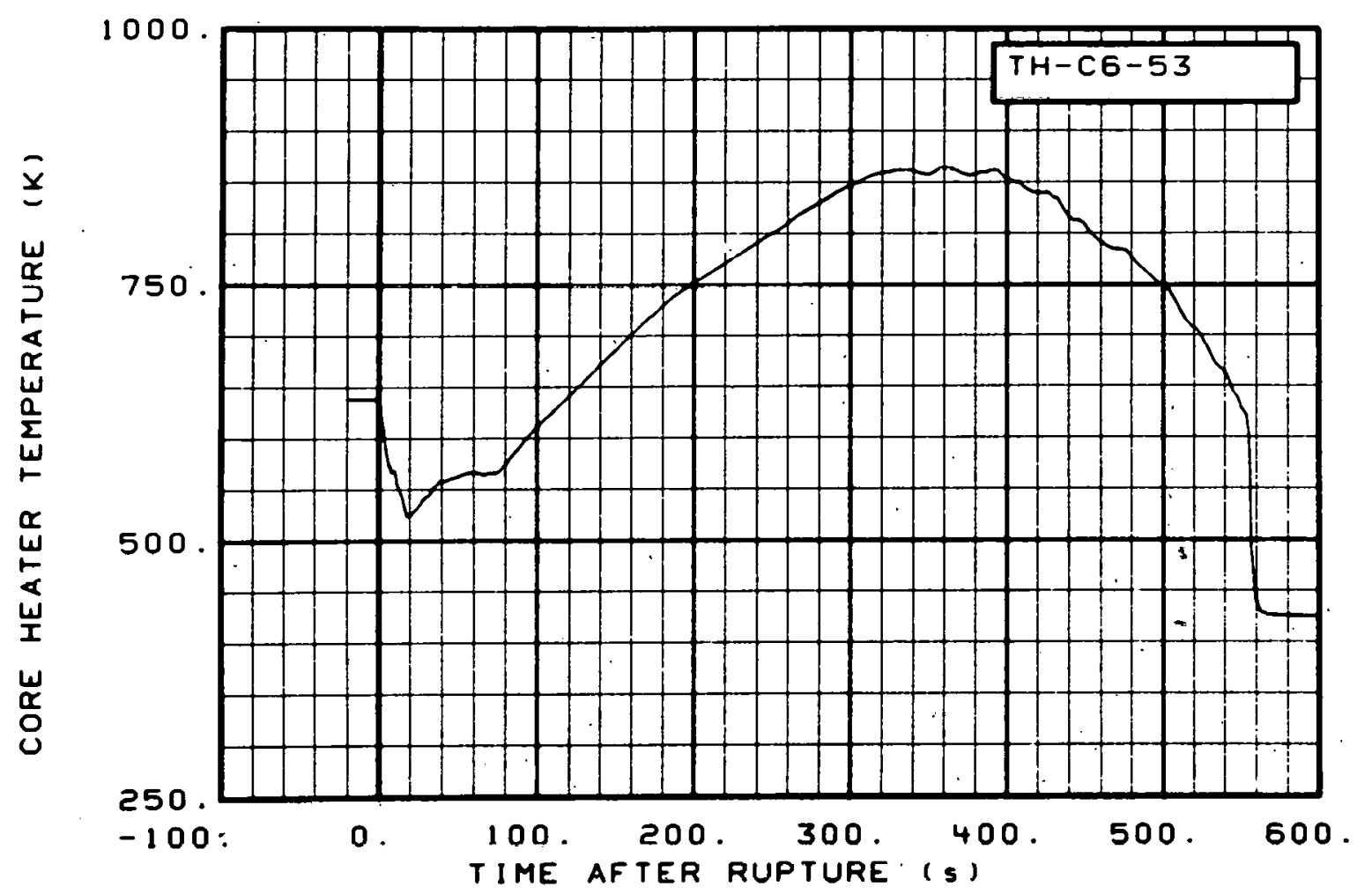

Fig. 101 Core heater temperature, Rod C-6 (TH-C6-53), from -20 to $600 \mathrm{~s}$.

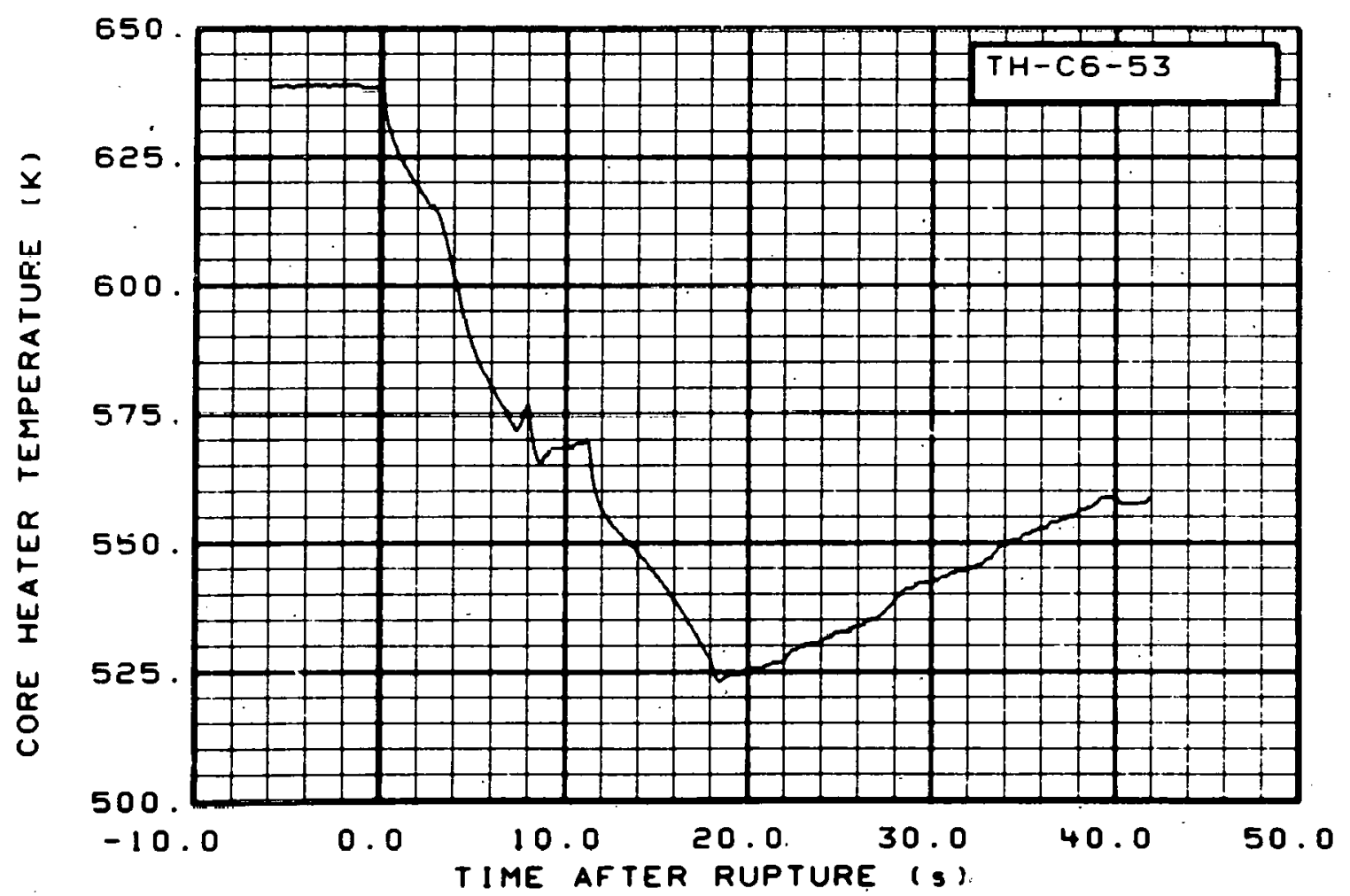

Fig. 102 Core heater temperature, Rod C-6 (TH-C6-53), from -6 to $42 \mathrm{~s}$. 


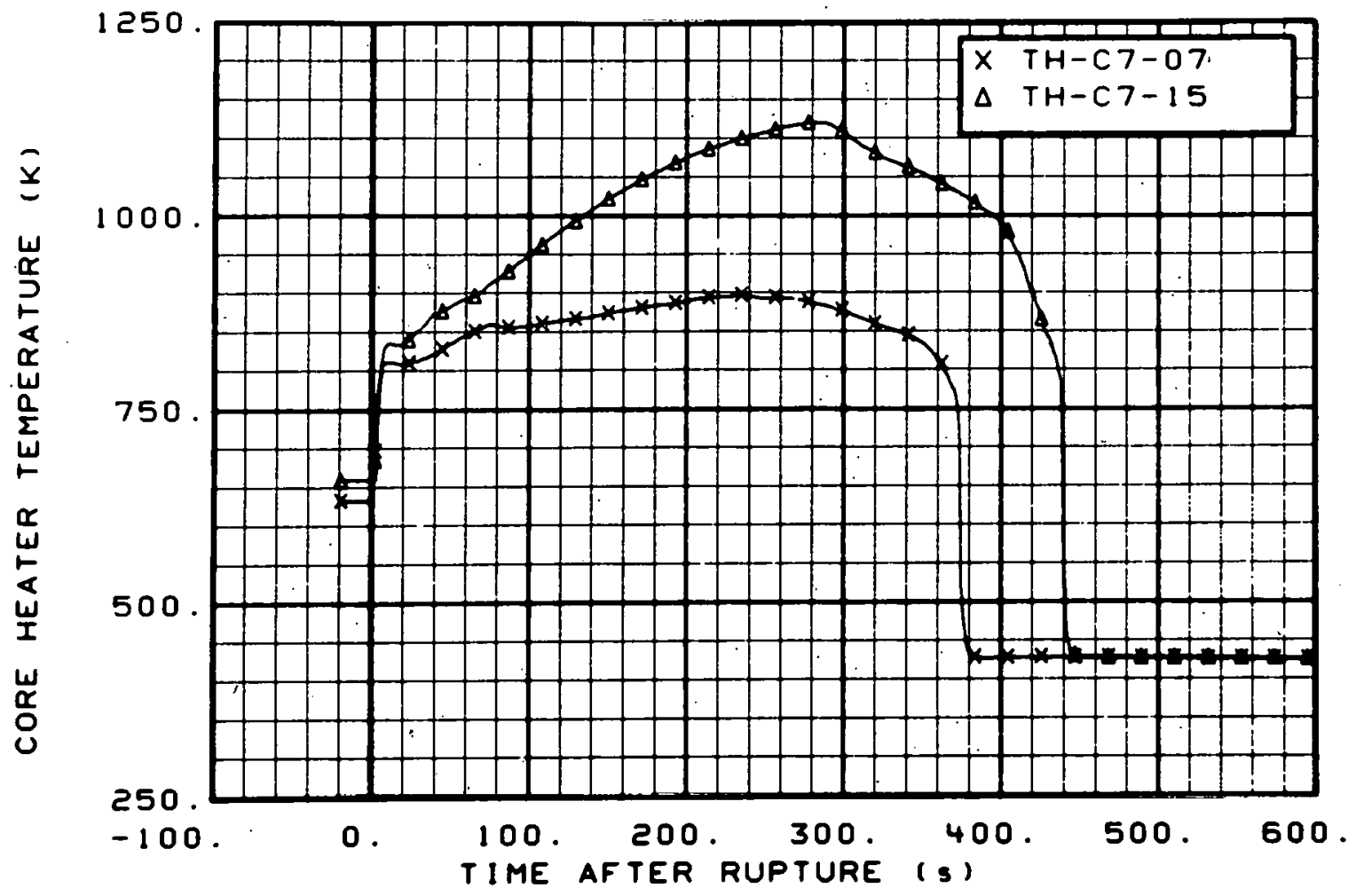

Fig. 103 Core heater temperature, Rod $\mathrm{C}-7$ (TH-C7-07 and TH-C7-15), from -20 to $600 \mathrm{~s}$.

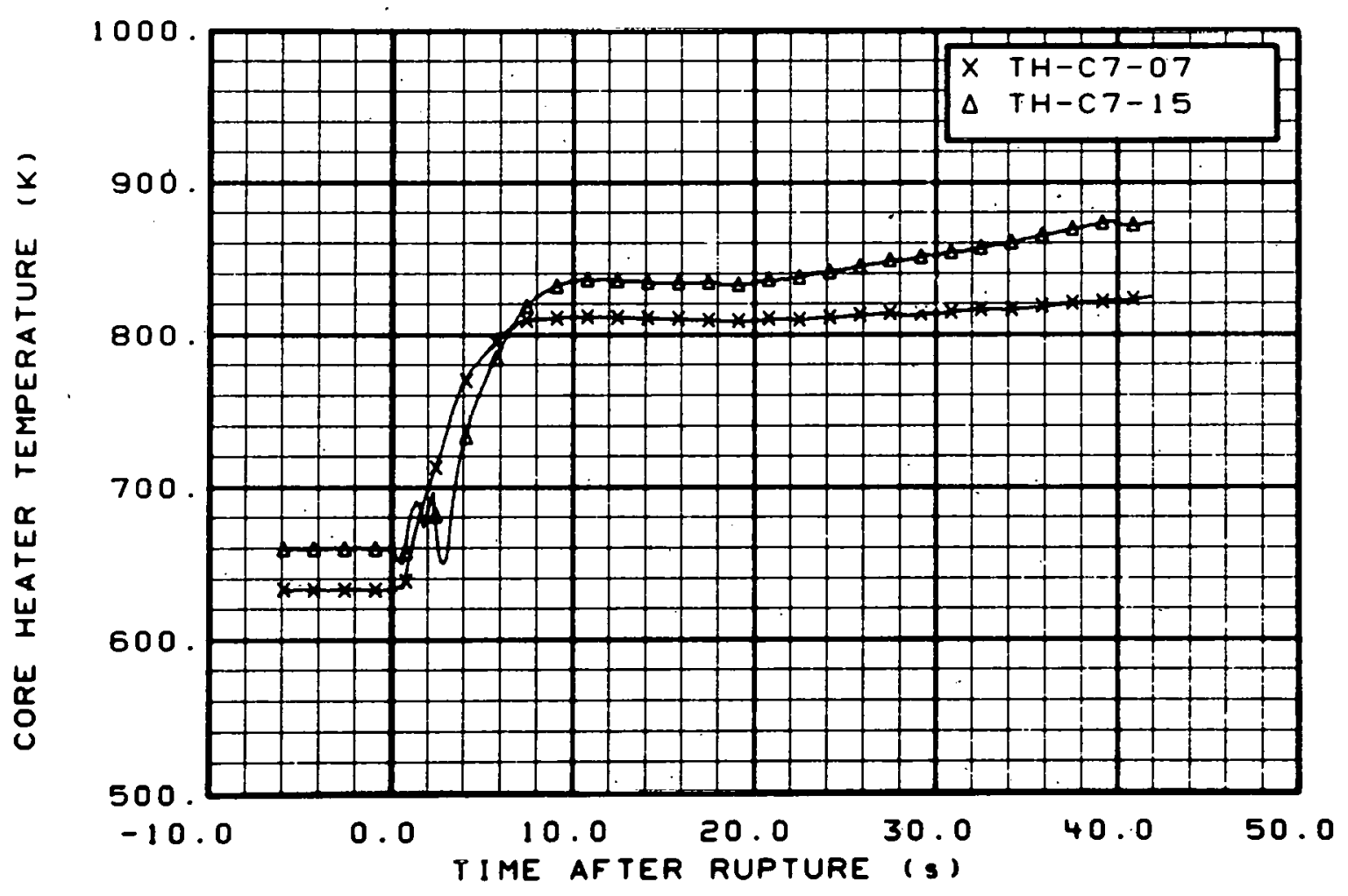

Fig. 104 Core heater temperature, Rod $\mathrm{C}-7$ ( $\mathrm{TH}-\mathrm{C} 7-07$ and $\mathrm{TH}-\mathrm{C} 7-15)$, from -6 to 4 ? $s$. 


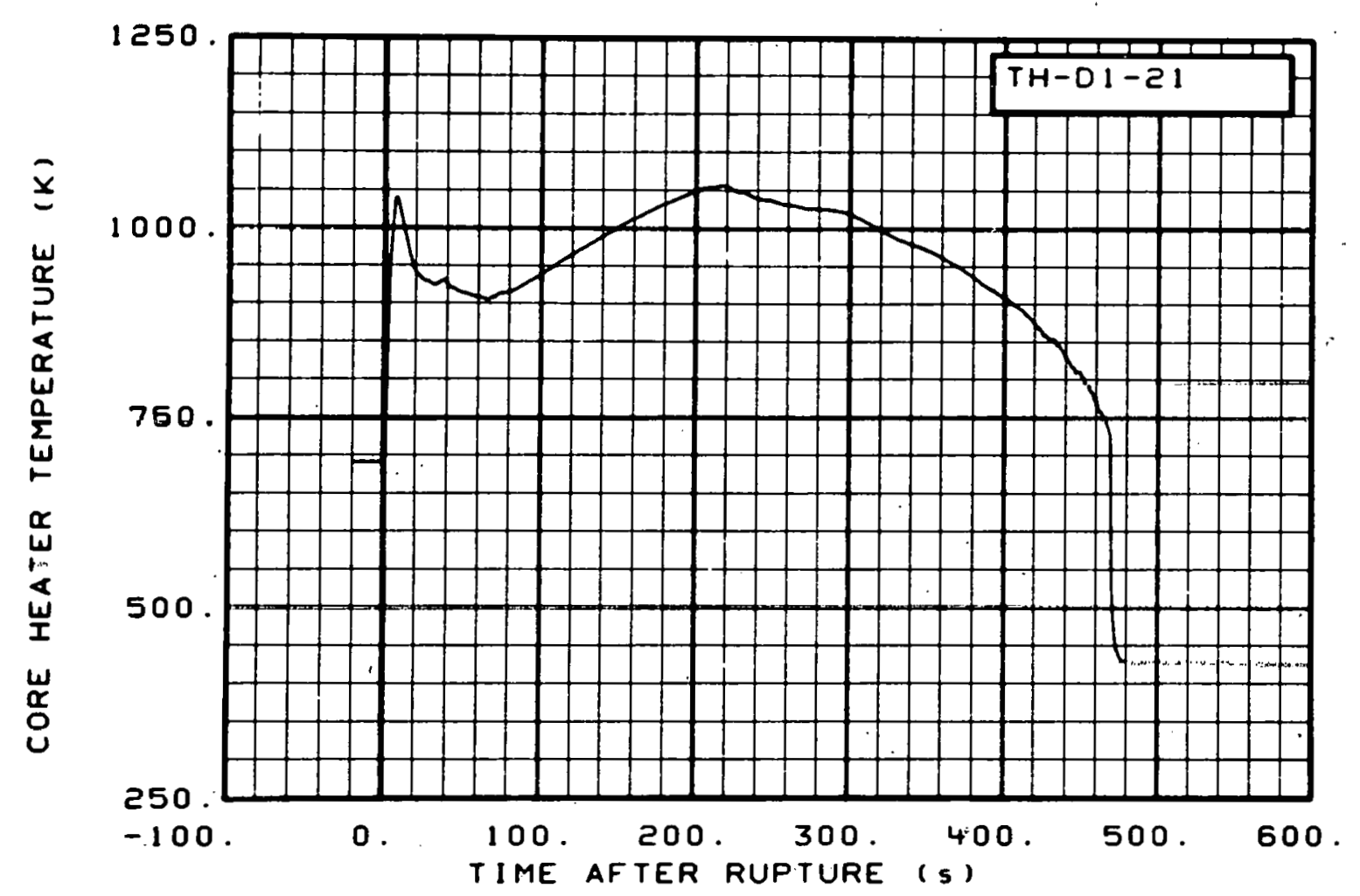

Fig. 105 Core heater temperature, Rod D-1 (TH-DT-21), from -20 to $600 \mathrm{~s}$.

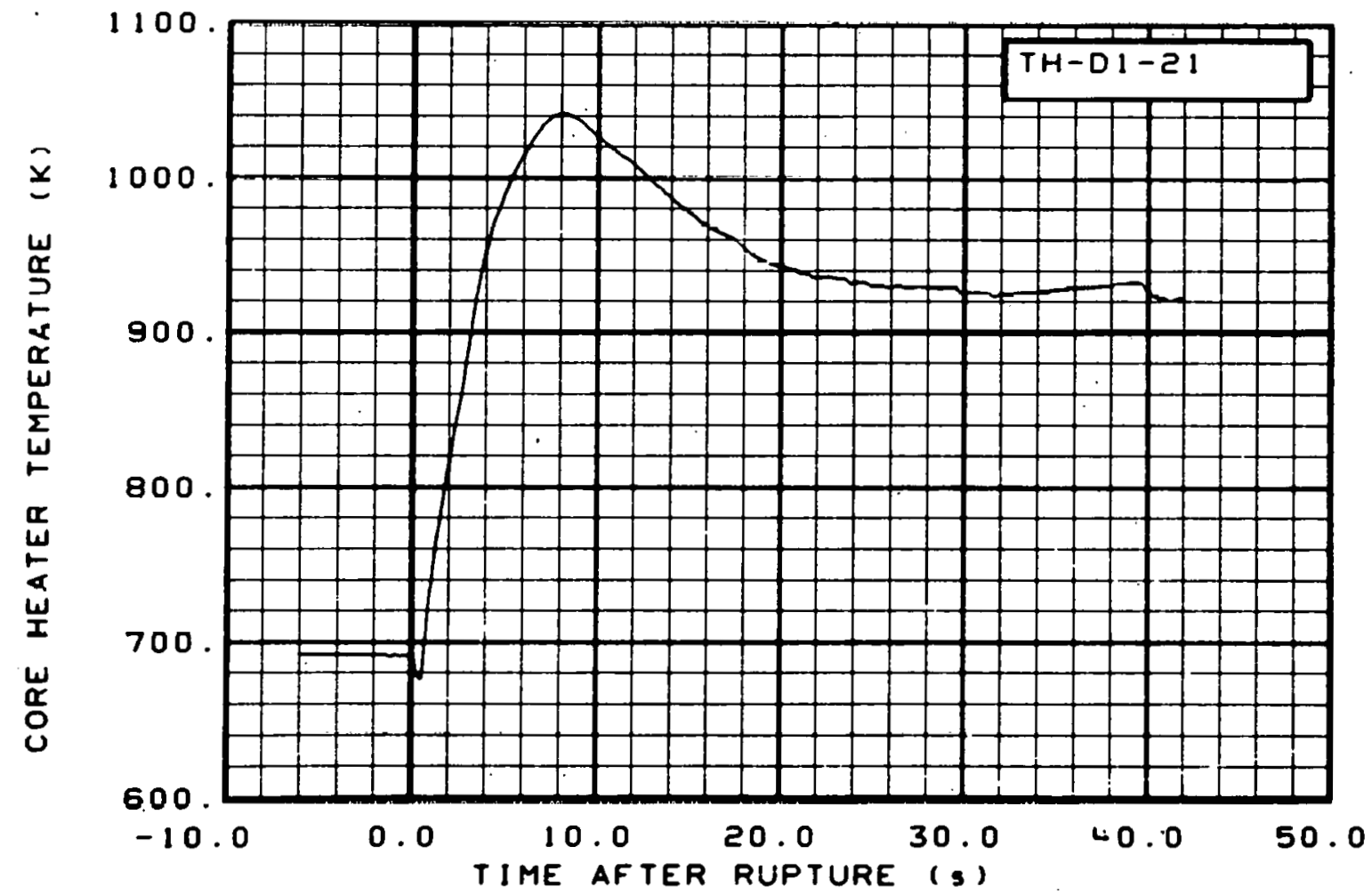

Fig. 106 Core heater temperature, Rod D-1 (TH-DT-21), from -6 to $42 \mathrm{~s}$. 


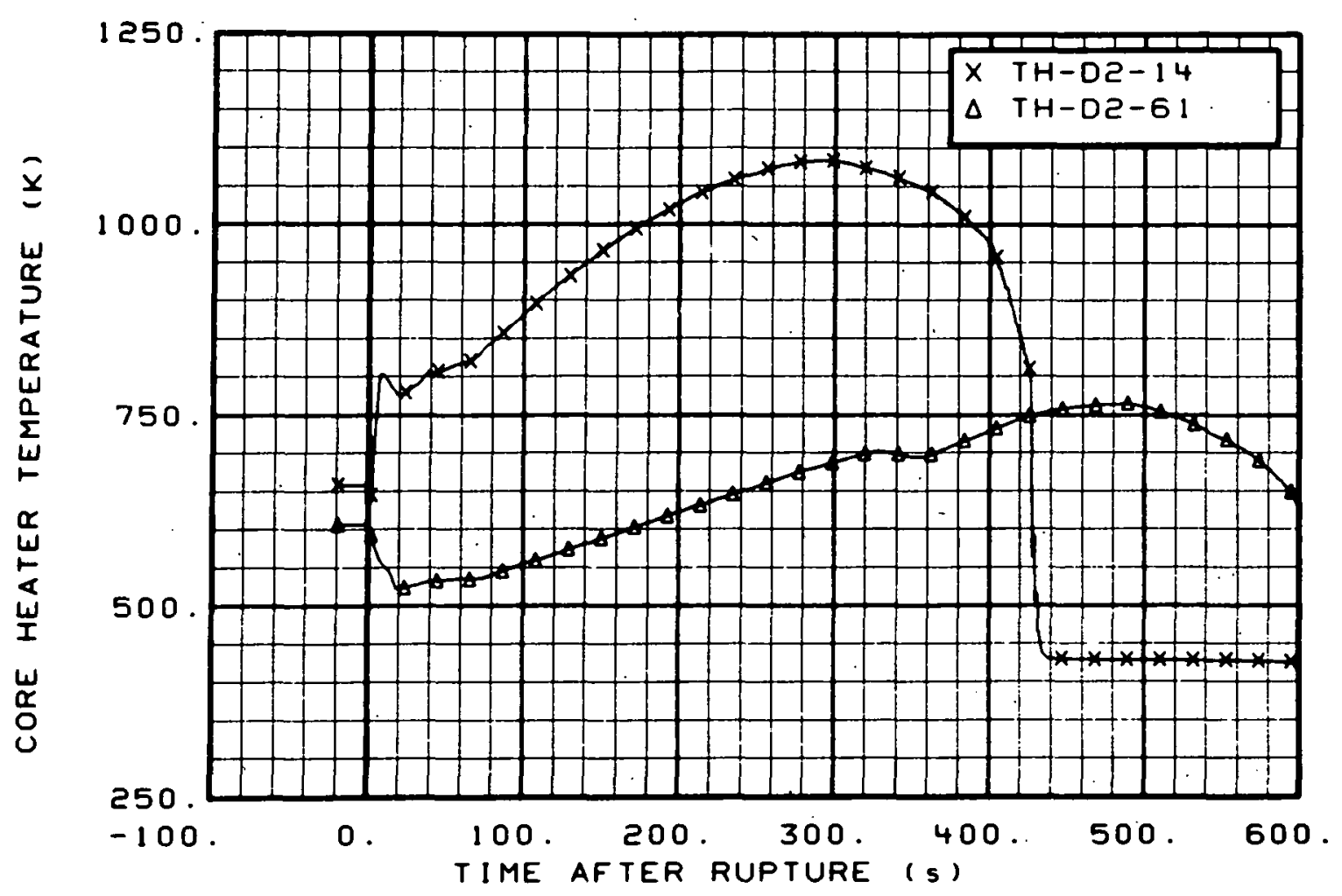

Fig. 107 Core heater temperature, Rod D-2 (TH-D2-14 and TH-D2-61), from -20 to $600 \mathrm{~s}$.

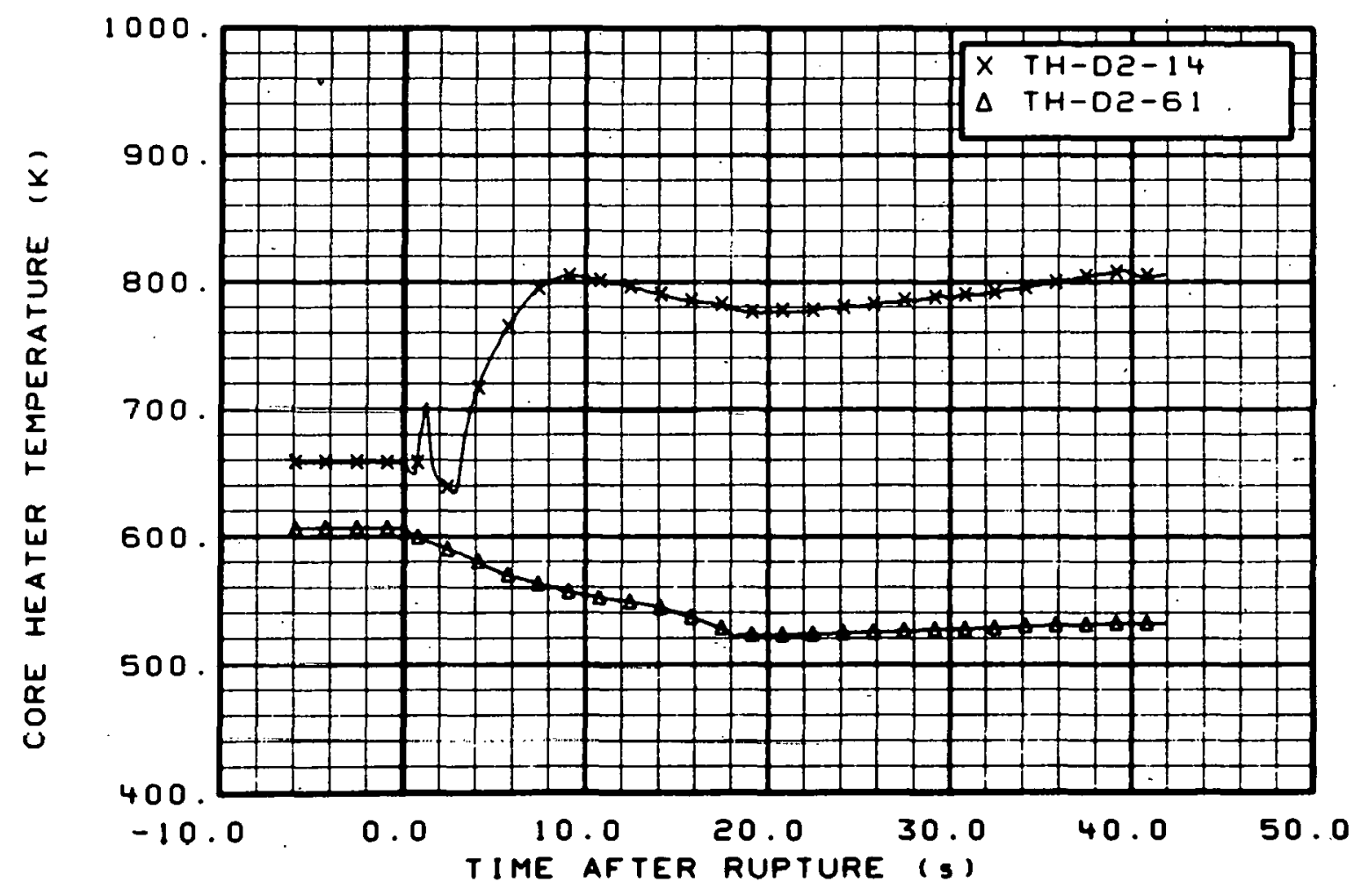

Fig. 108 Core heater temperature, Rod D-2 (TH-D2-14 and TH-D2-61), from -6 to $42 \mathrm{~s}$. 


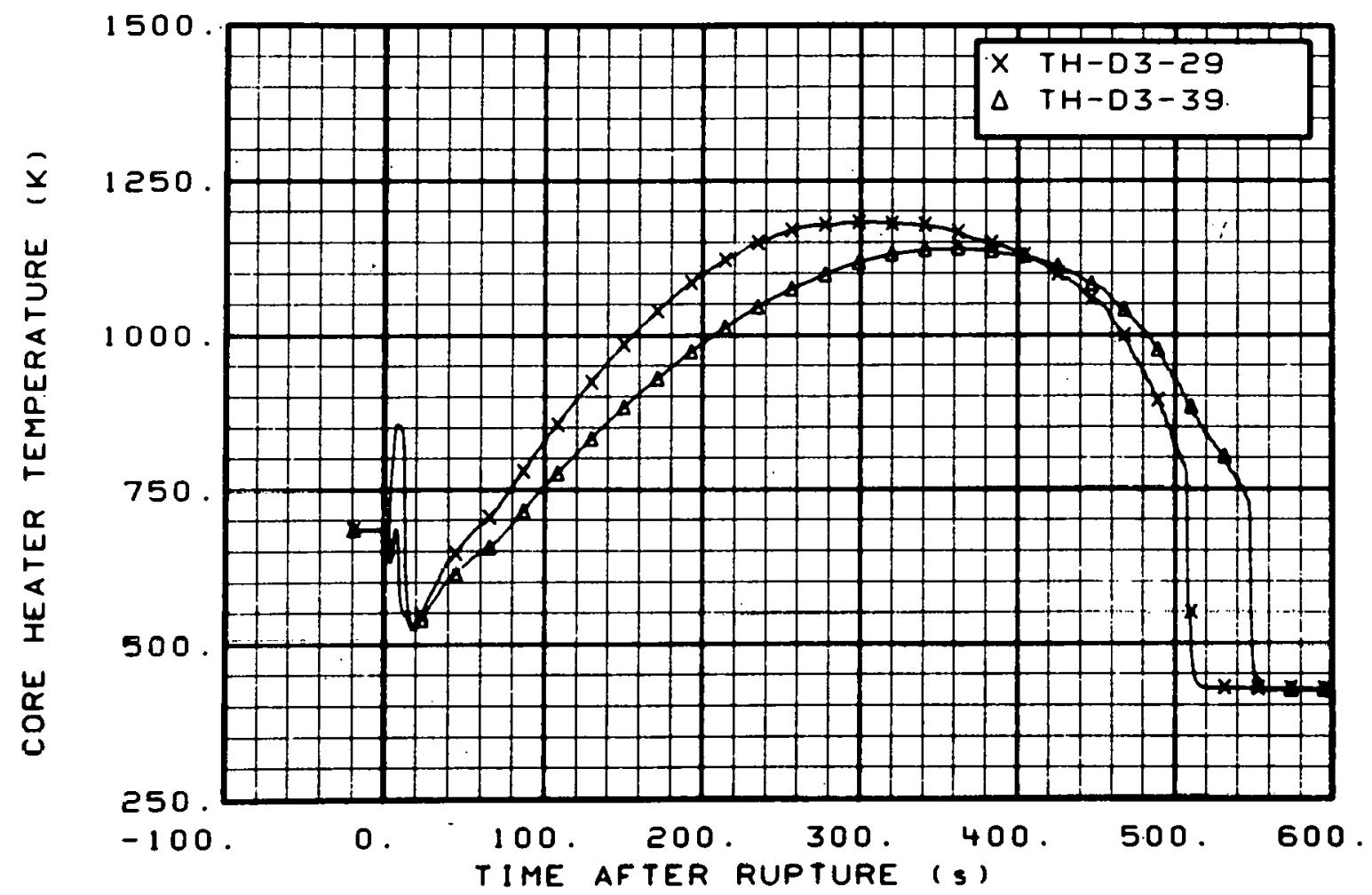

Fig. 109 Core heater temperature, Rod D-3 (TH-D3-29 and TH-D3-39), from -20 to $600 \mathrm{~s}$.

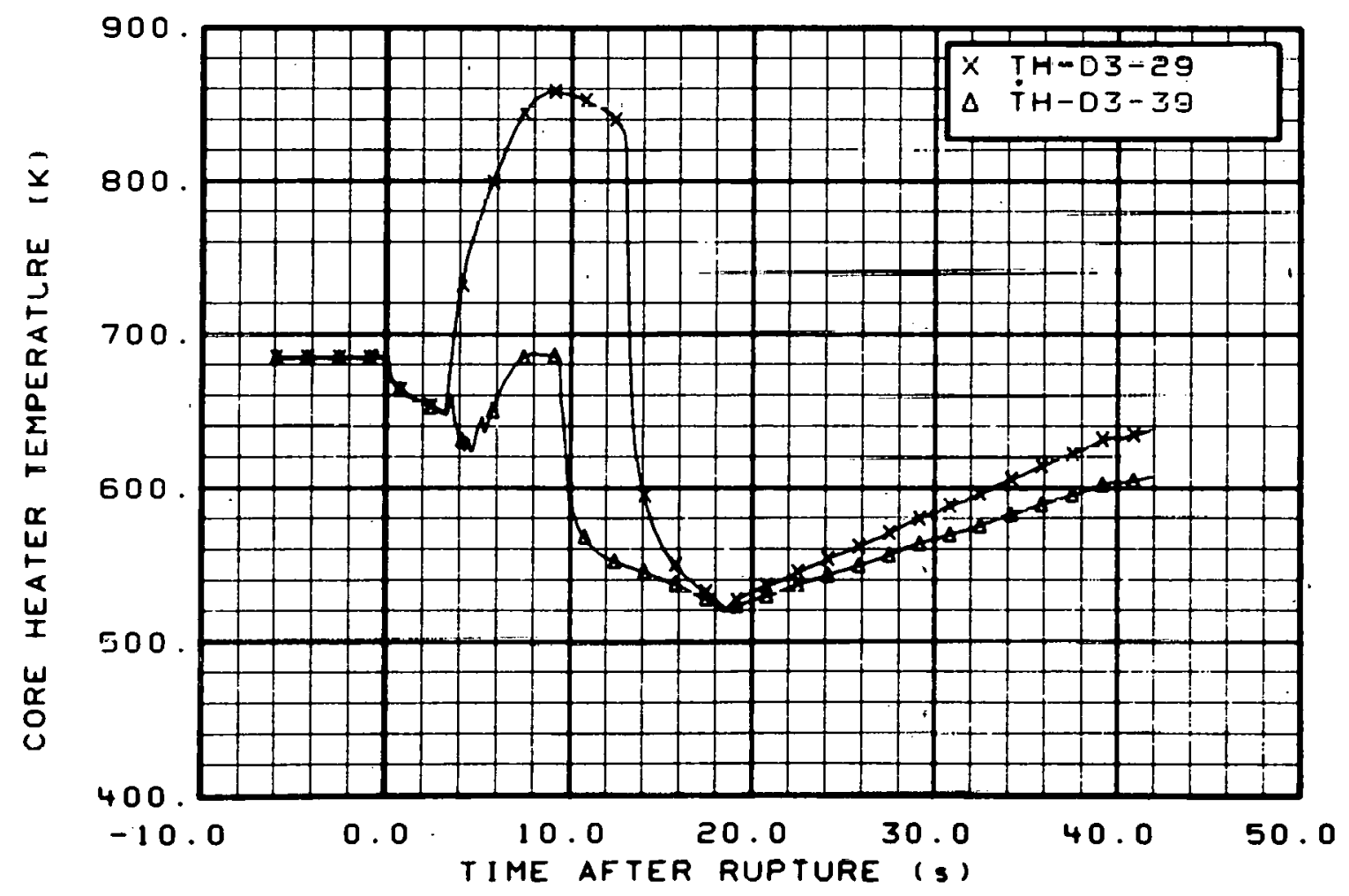

Fig. 110 Core heater temperature, Rod D-3 (TH-D3-29 and TH-D3-39), from -6 to $42 \mathrm{~s}$. 


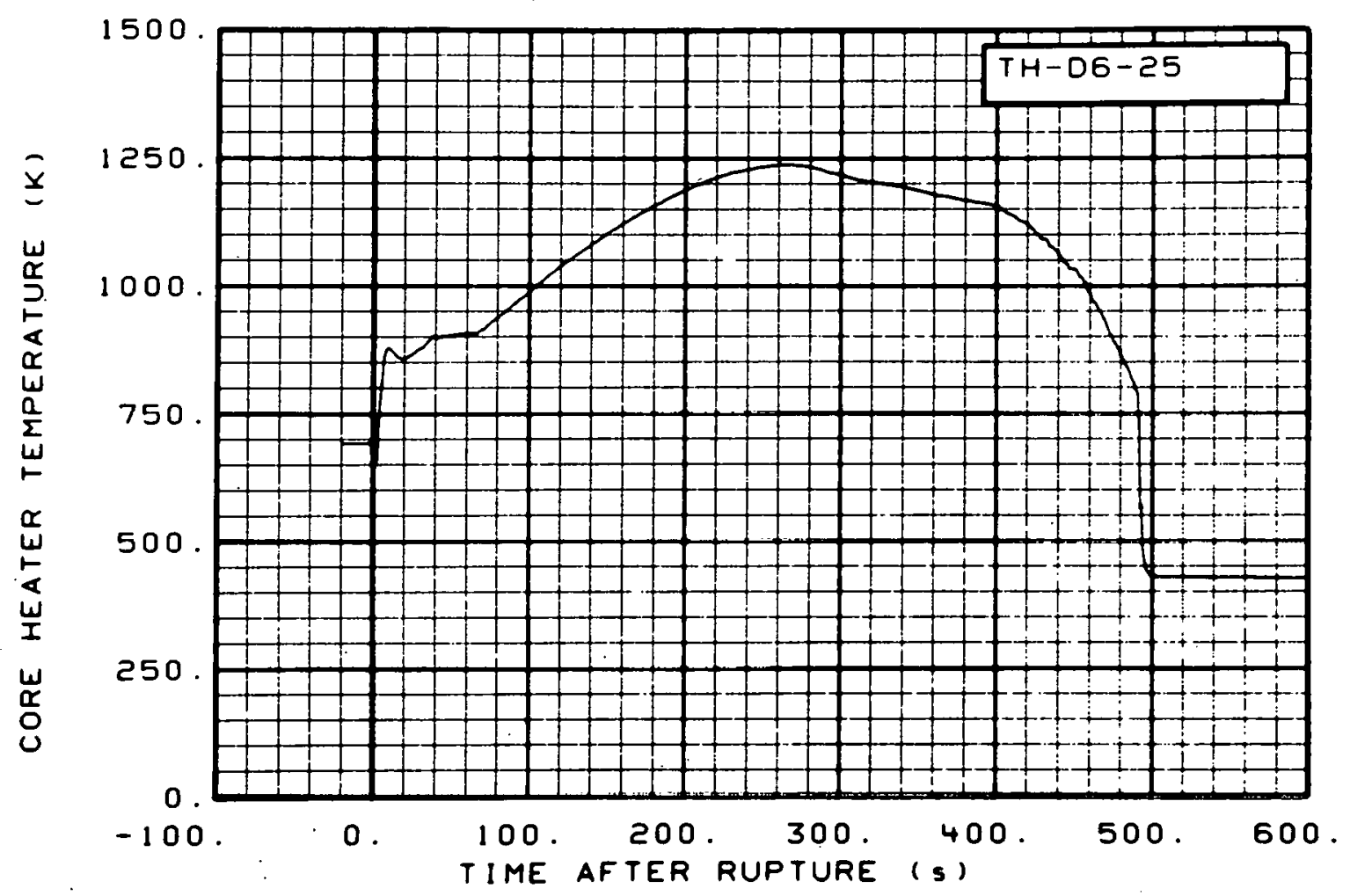

Fig. 111 Core heater temperature, Rod D-6 (TH-D6-25), from -20 to $600 \mathrm{~s}$.

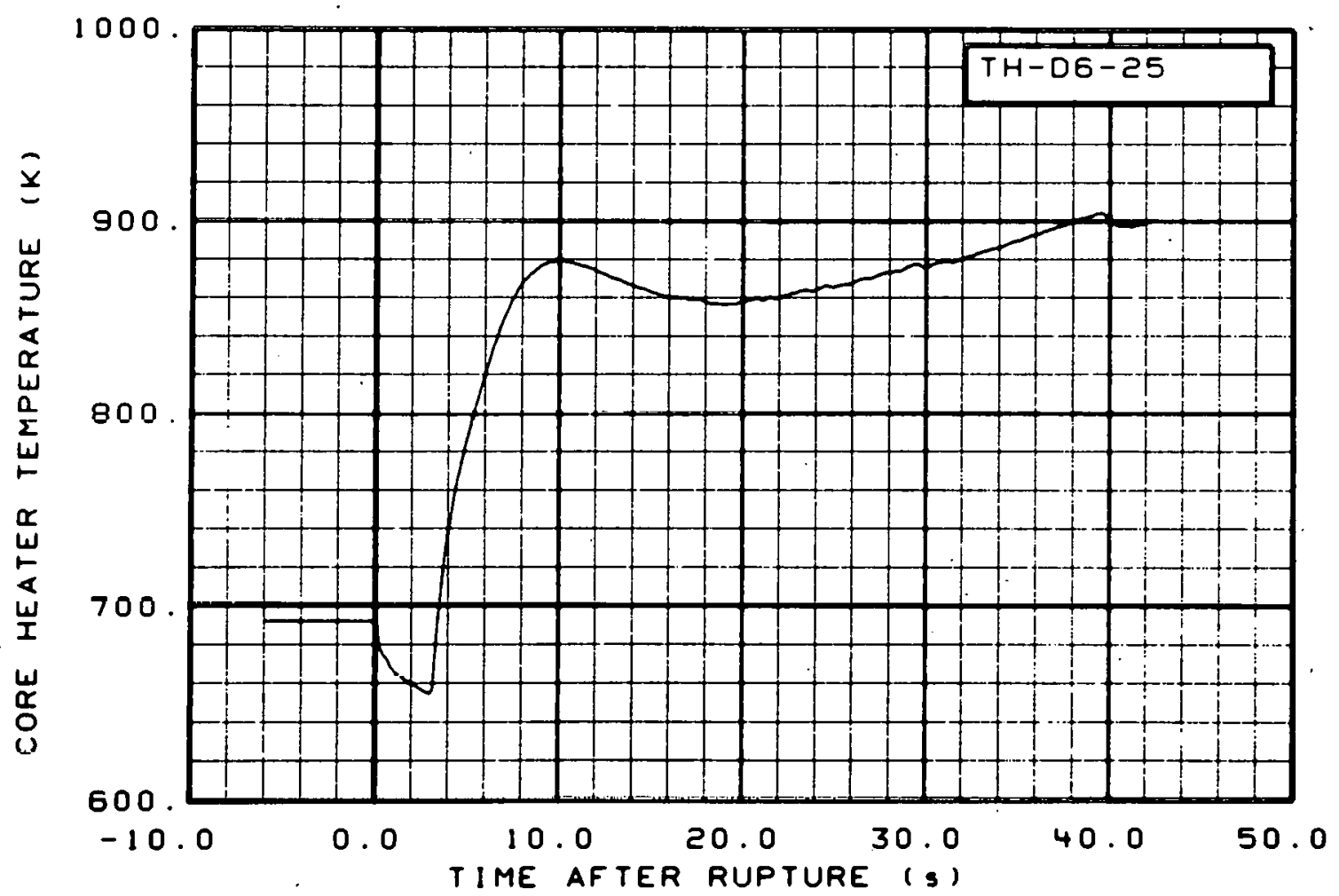

Fig. 112 Core heater temperature, Rod D-6 (TH-D6-25), from -6 to $42 \mathrm{~s}$. 


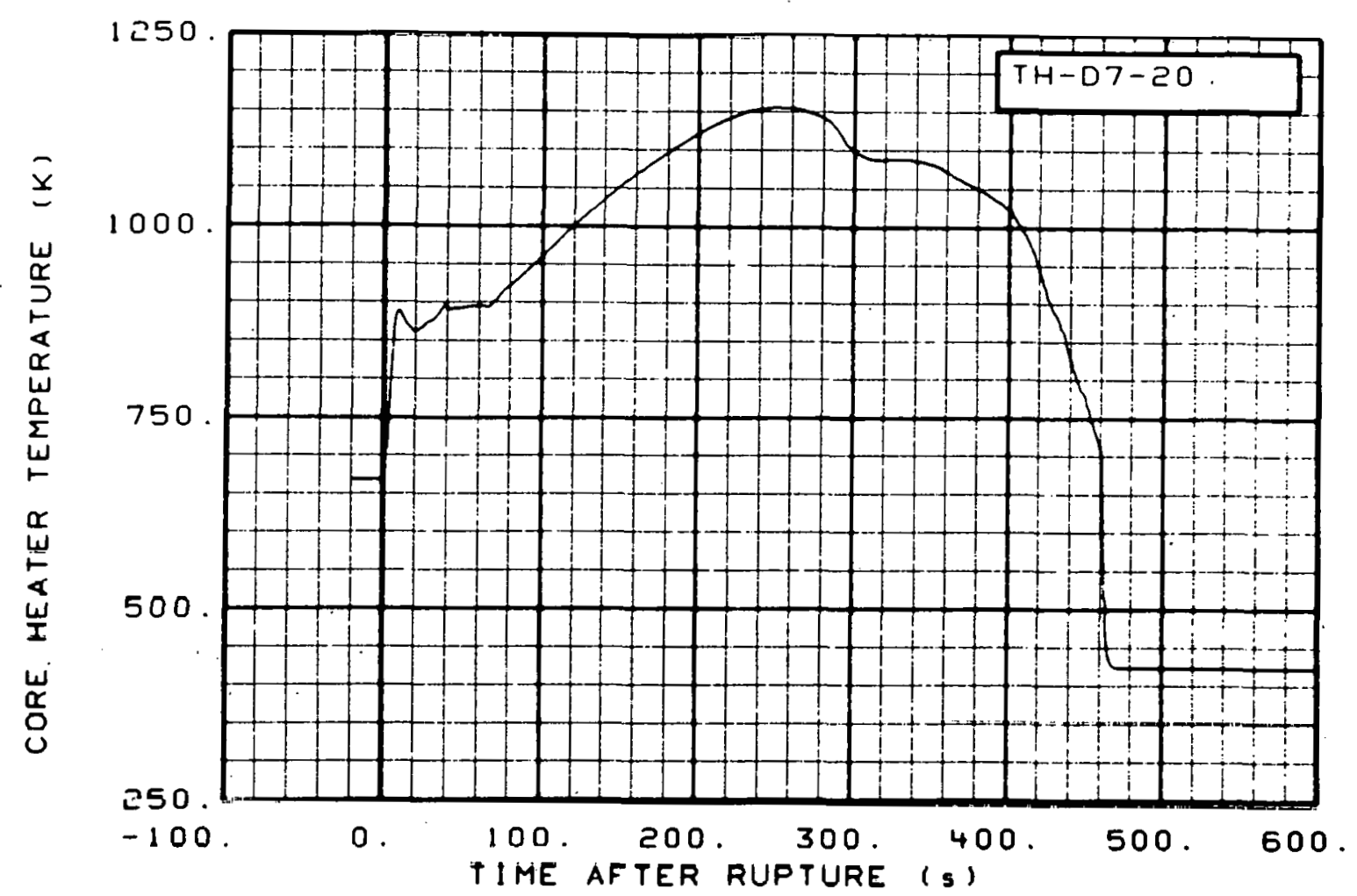

Fig. 113 Core heater temperature, Rod D-7 (TH-D7-20), from -20 to $600 \mathrm{~s}$.

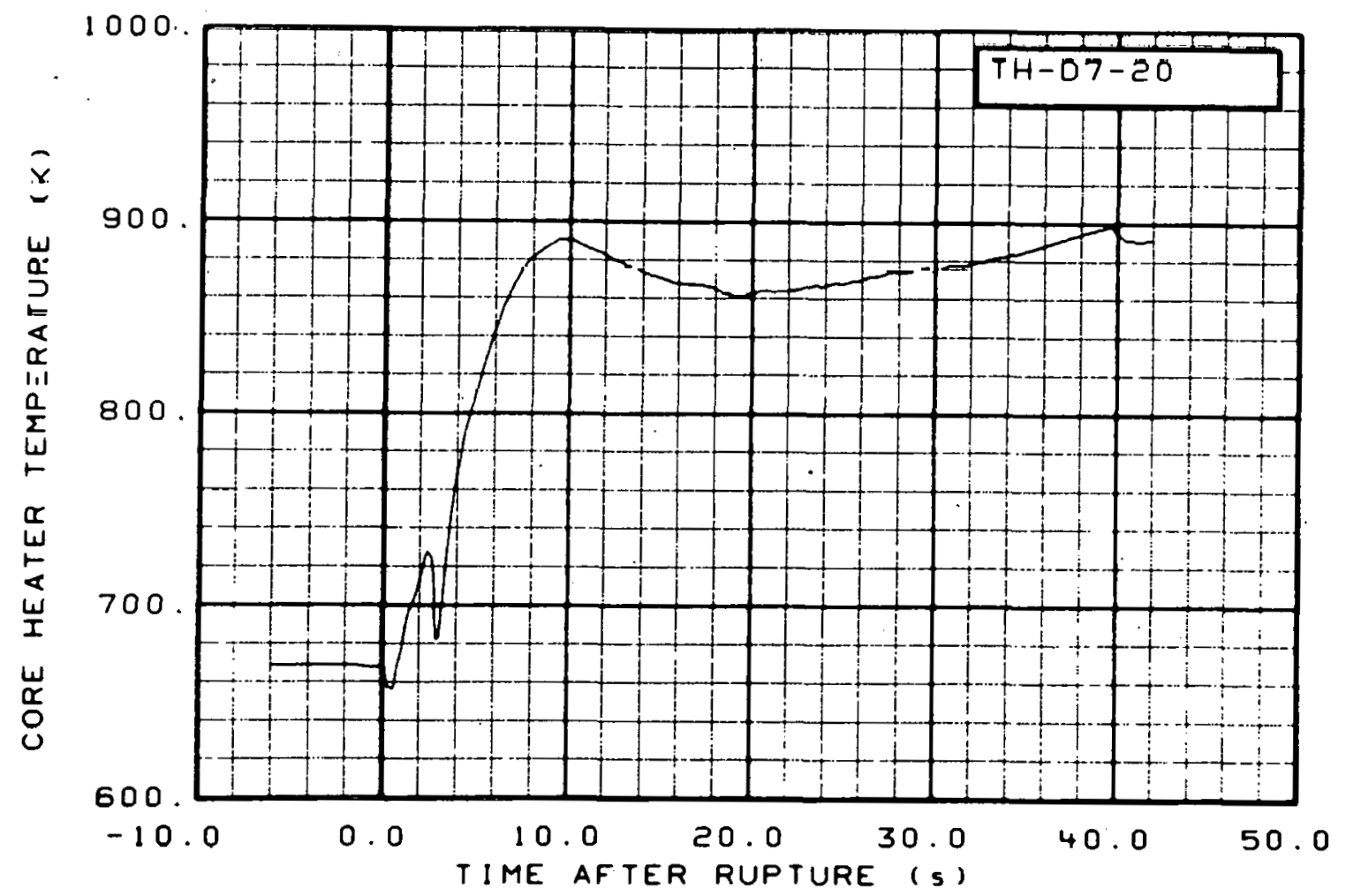

Fig. 114 Core heater temperature, Rod D-7 (TH-D7-20), from -6 to $42 \mathrm{~s}$. 


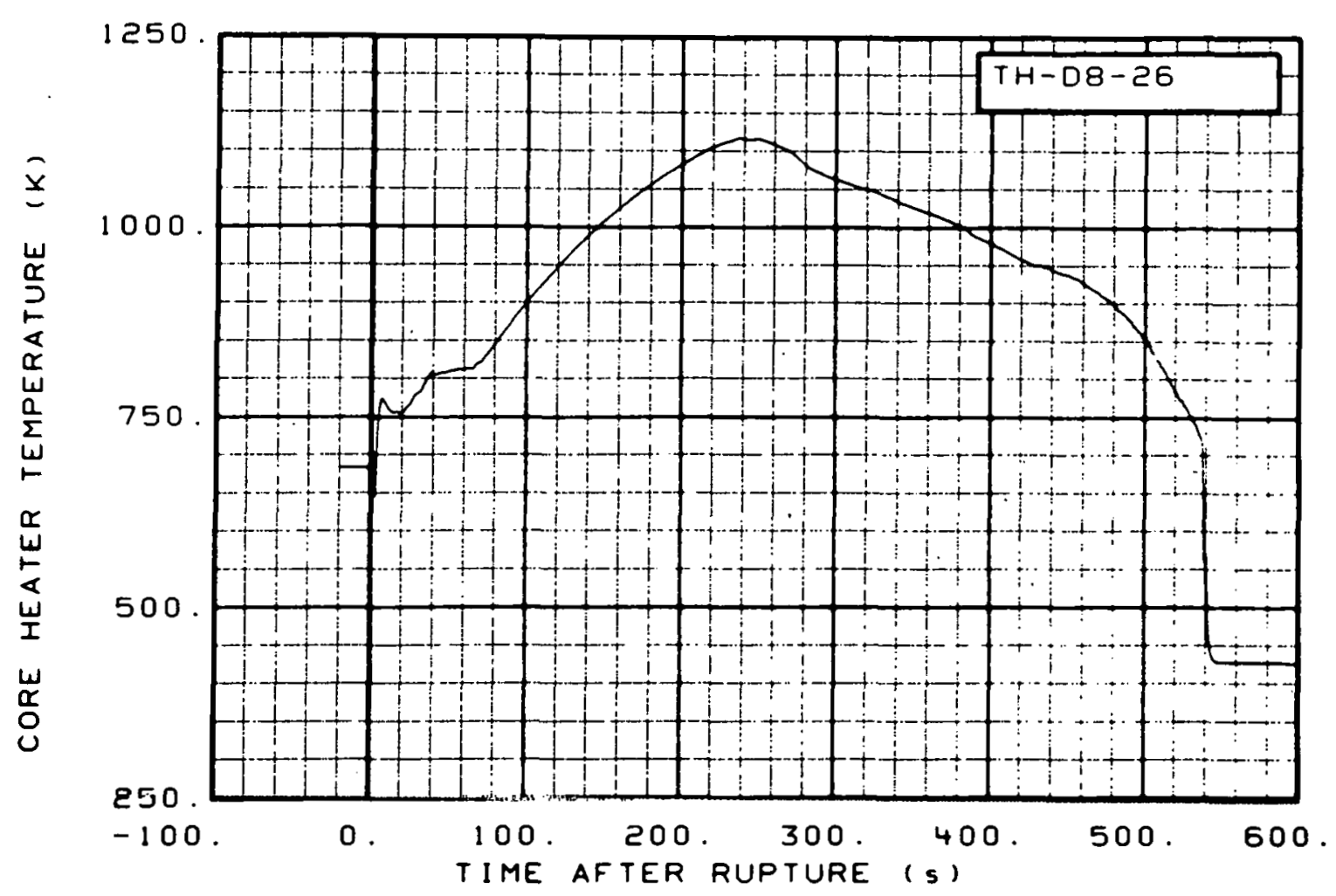

Fig. 115 Core heater temperature, Rod D-8 (TH-D8-26), from -20 to $600 \mathrm{~s}$.

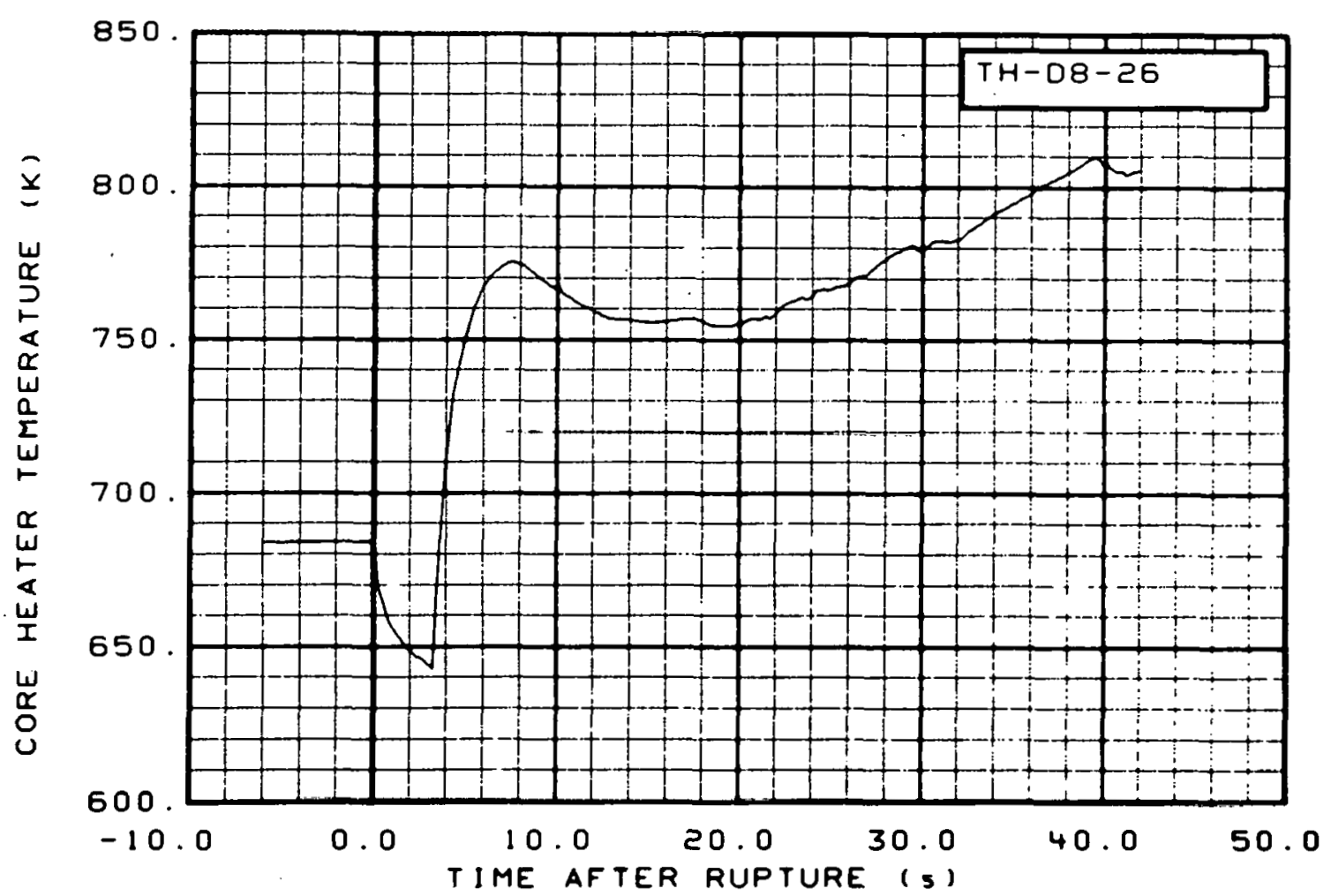

Fig. 116 Core heater temperature, Rod D-8 (TH-08-26), from -6 to $42 \mathrm{~s}$. 


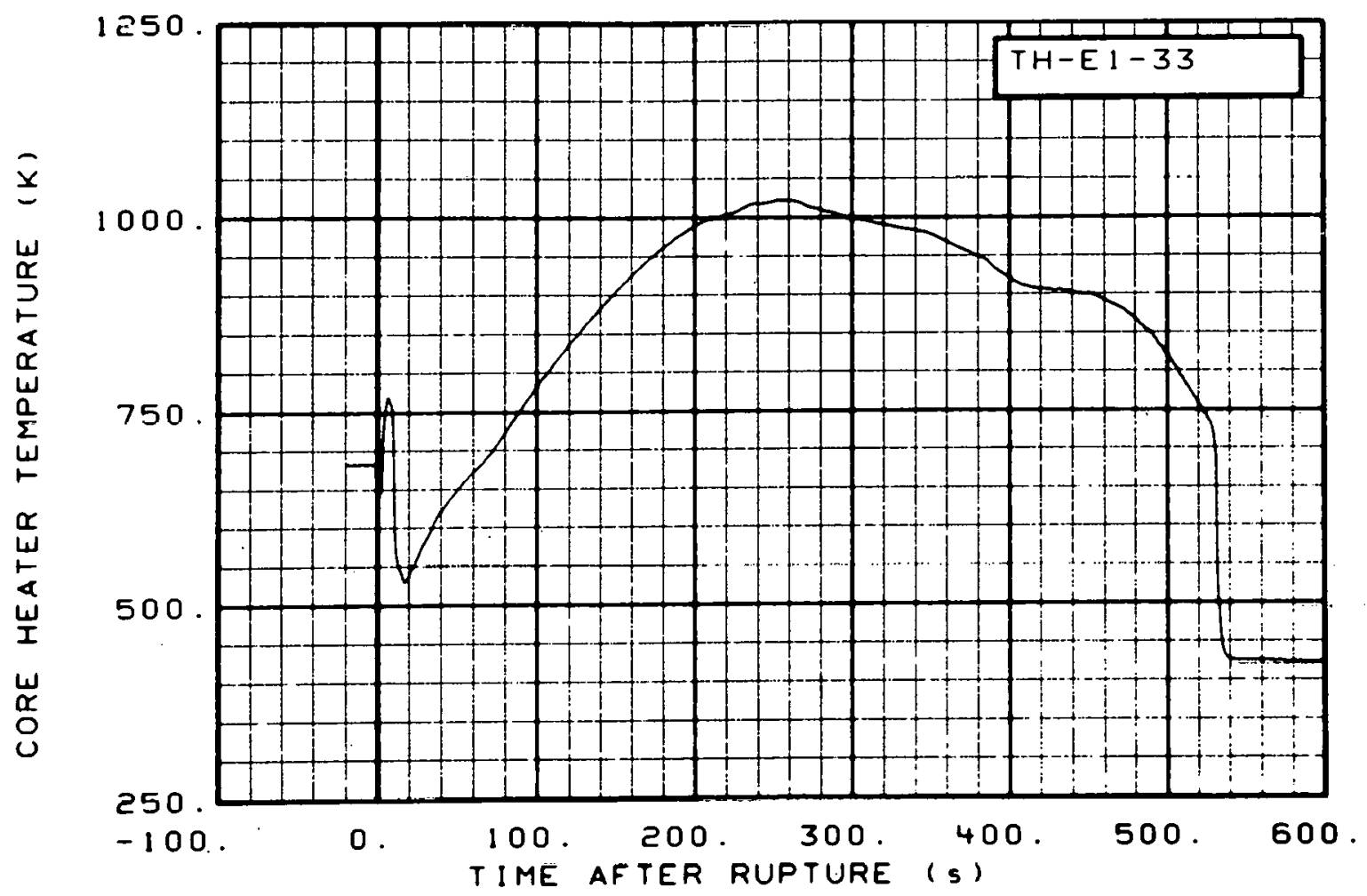

Fig. 117 Core heater temperature, Rod E-1 (TH-El-33), from -20 to $600 \mathrm{~s}$.

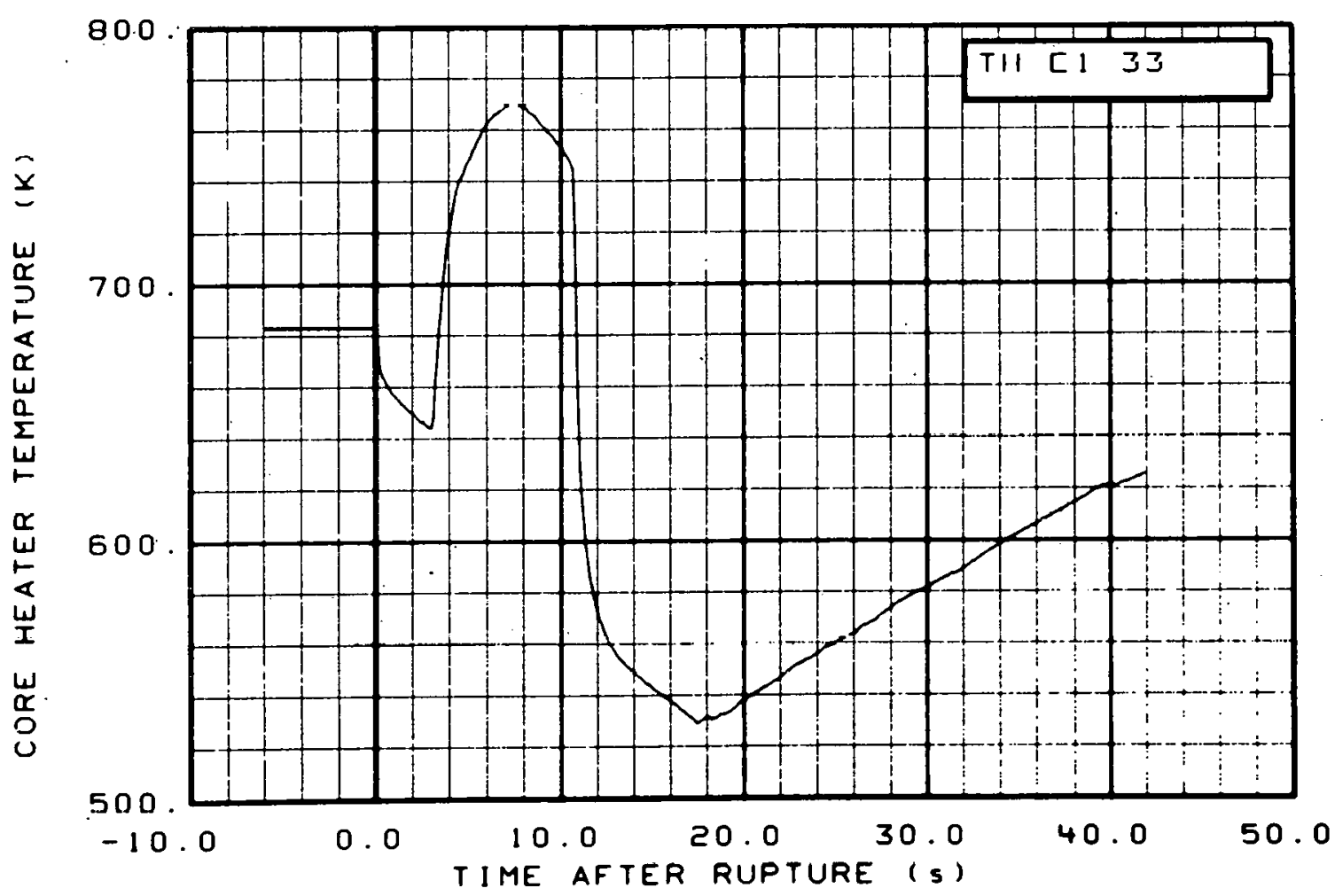

Fig. 118 Core heater temperature, Rod E-1 (TH-El-33), from -6 to $42 \mathrm{~s}$. 


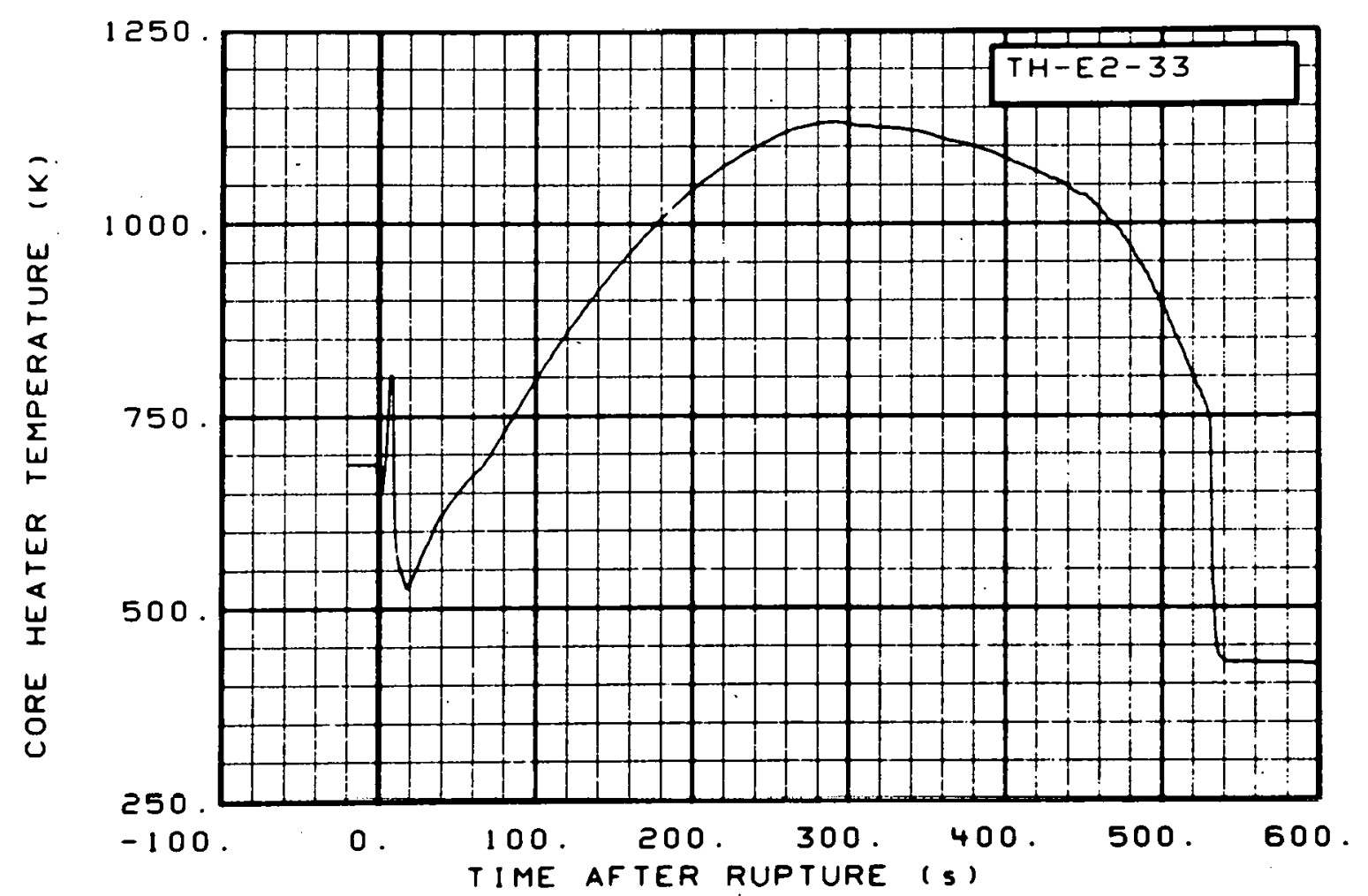

Fig. 119 Core heater temperature, Rod E-2 (TH-E2-33), from -20 to $600 \mathrm{~s}$.

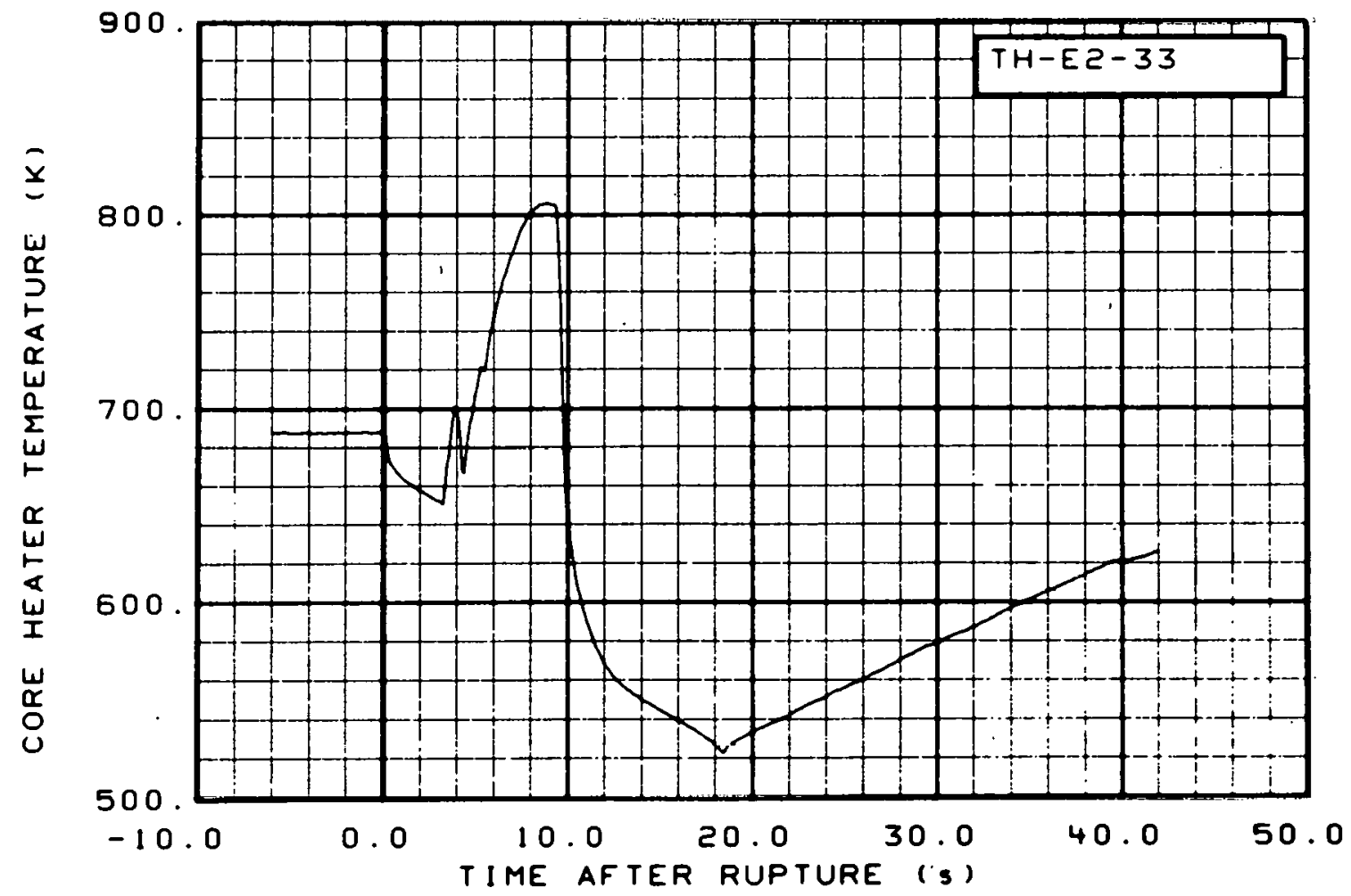

Fig. 120 Core heater temperature, Rod E-2 (TH-E2-33), from -6 to $42 \mathrm{~s}$. 


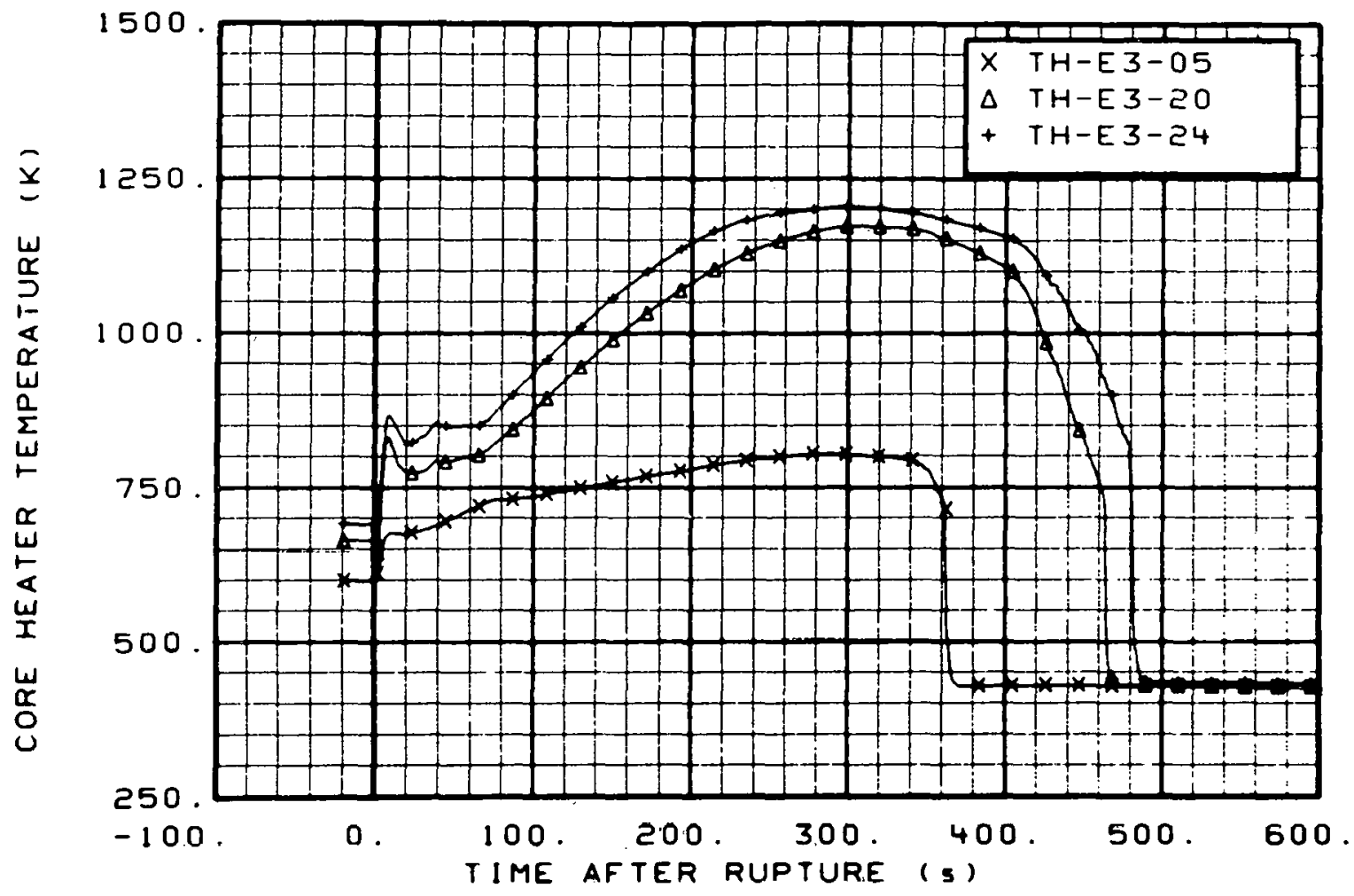

Fig. 121 Core heater temperature, Rod E-3 (TH-E3-05, TH-E3-20, and TH-E3-24), from -20 to $600 \mathrm{~s}$.

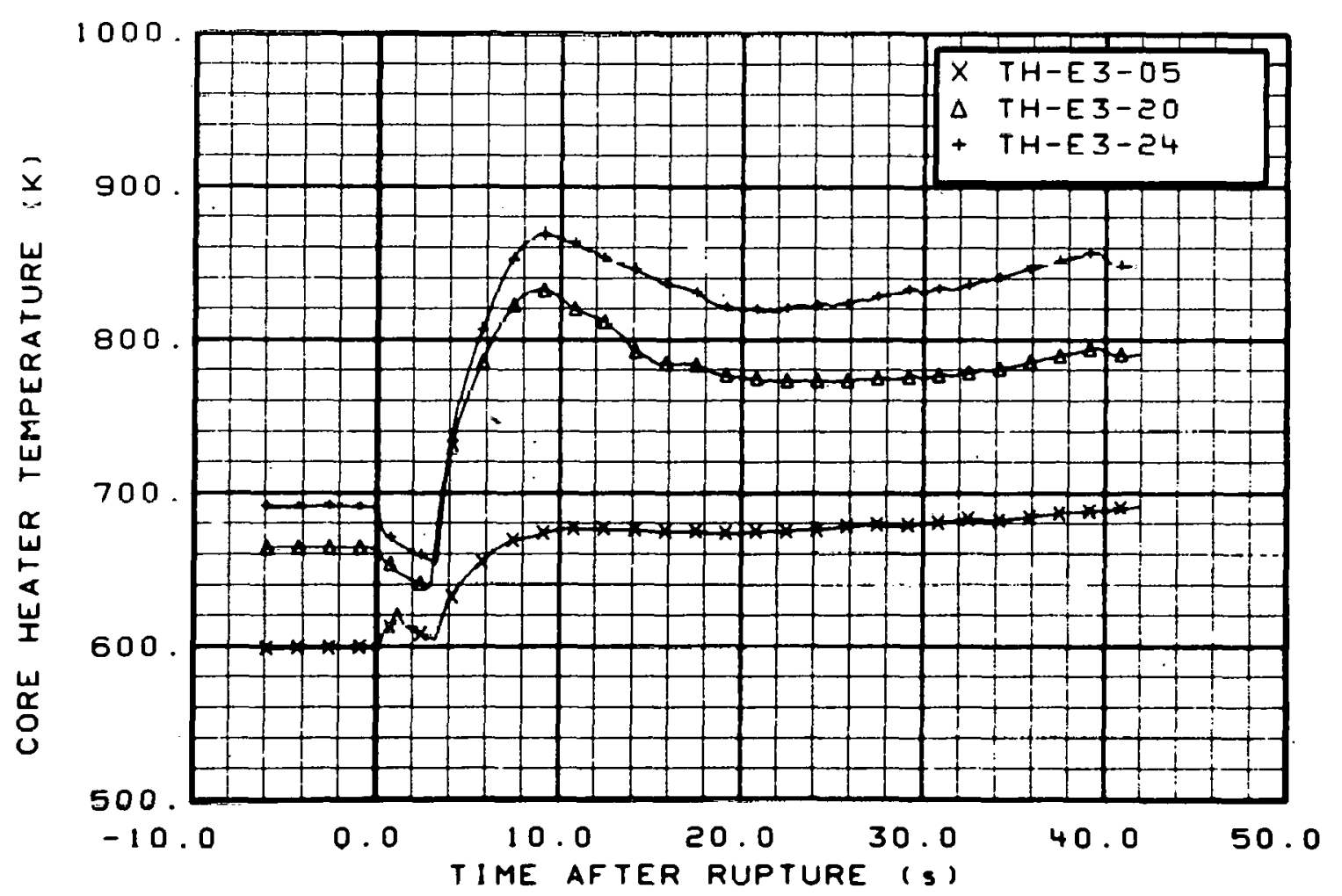

Fig. 122 Core heater temperature, Rod E-3 (TH-E3-05, TH-E3-20, and TH-E3-24), from -6 to $42 \mathrm{~s}$. 


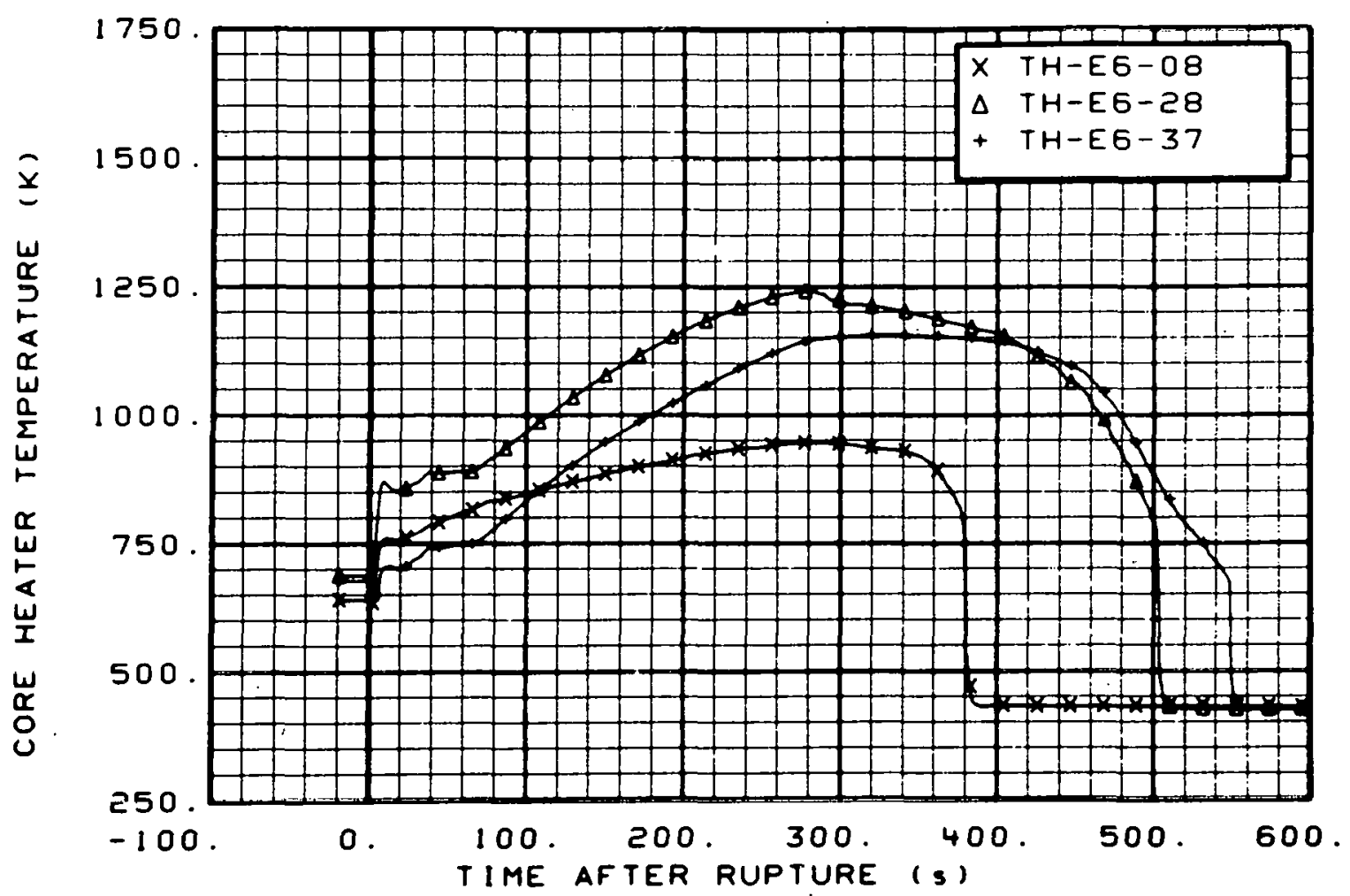

Fig. 123 Core heater temperature, Rod E-6 (TH-E6-08, TH-E6-28, and TH-E6-37), from -20 to $600 \mathrm{~s}$.

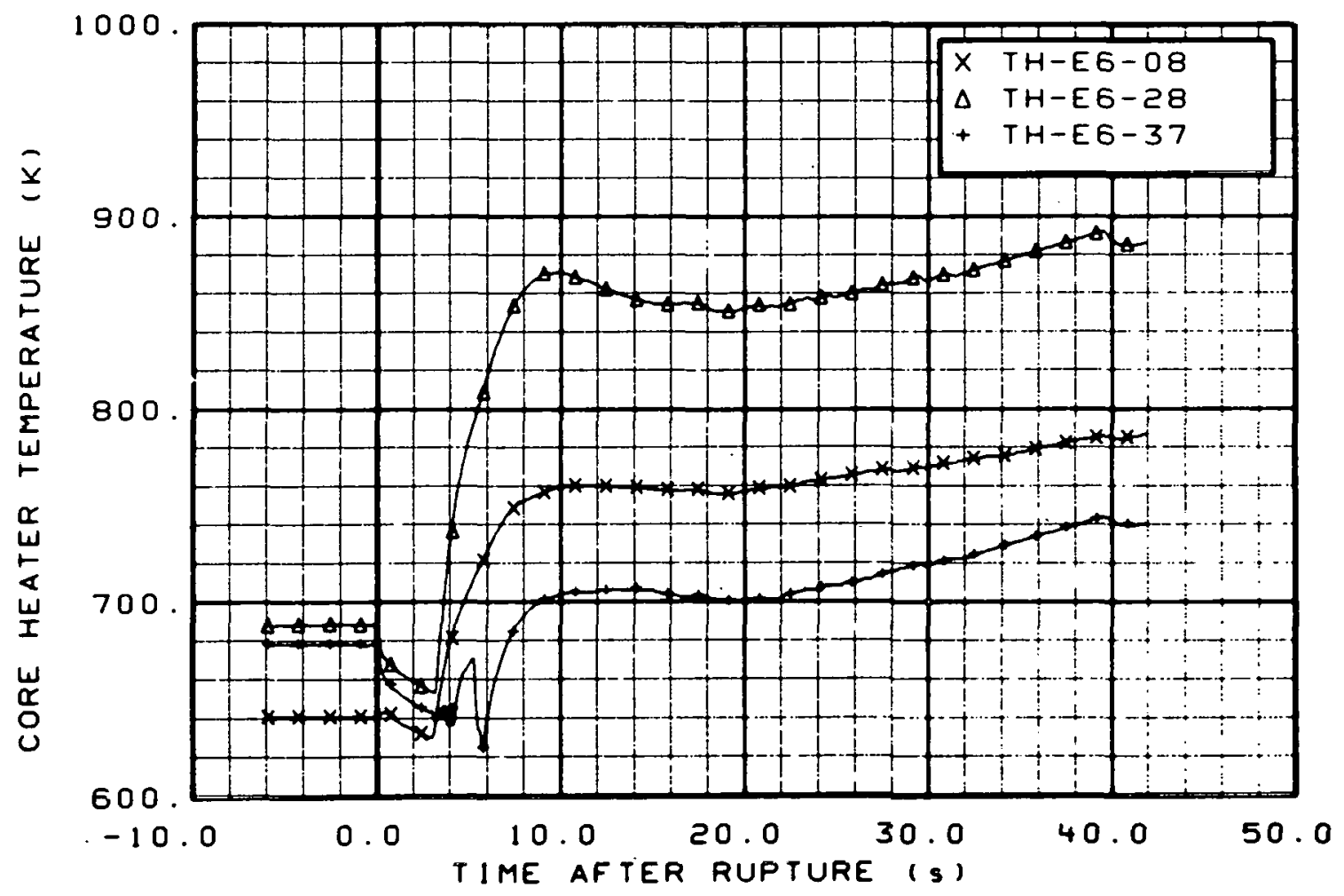

Fig. 124 Core heater temperature, Rod E-6 (TH-E6-08, TH-E6-28, and TH-E6-37), from -6 to $42 \mathrm{~s}$. 


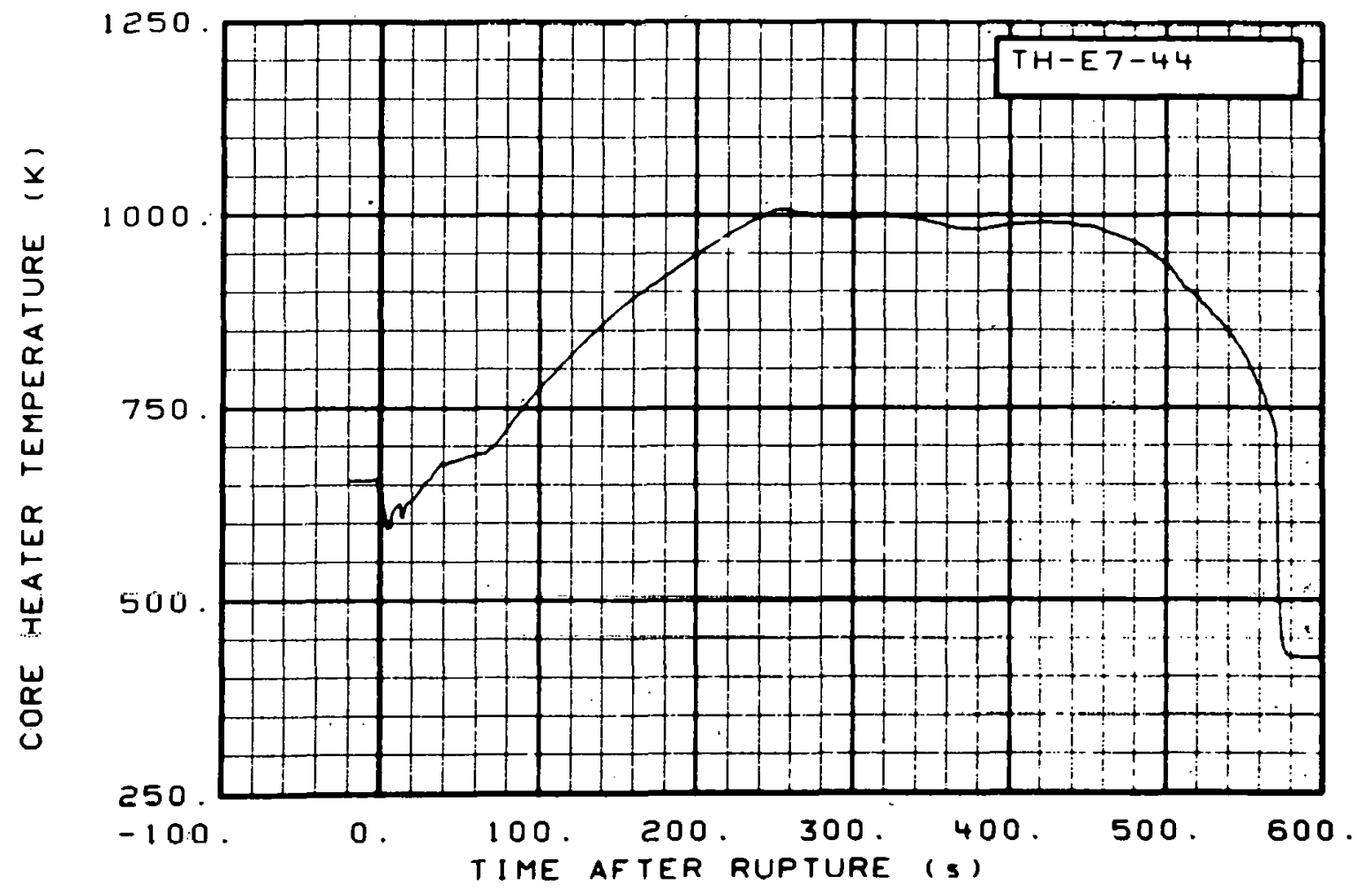

Fig. 125 Core heater temperature, Rod E-7 (TH-E7-44), from -20 to $600 \mathrm{~s}$.

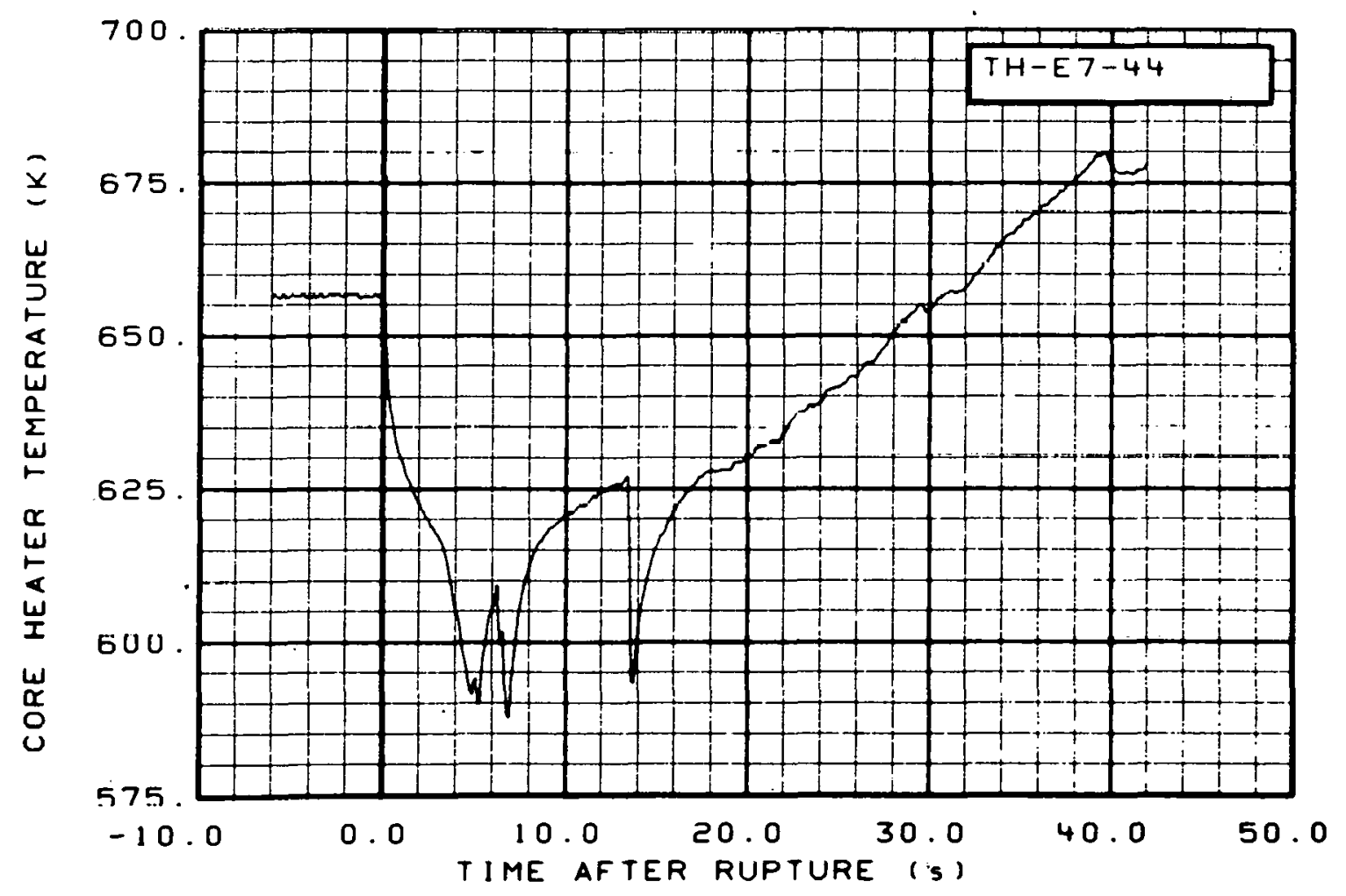

Fig. 126 Core heater temperature, Rod E-7 (TH-E7-44), from -6 to $42 \mathrm{~s}$. 


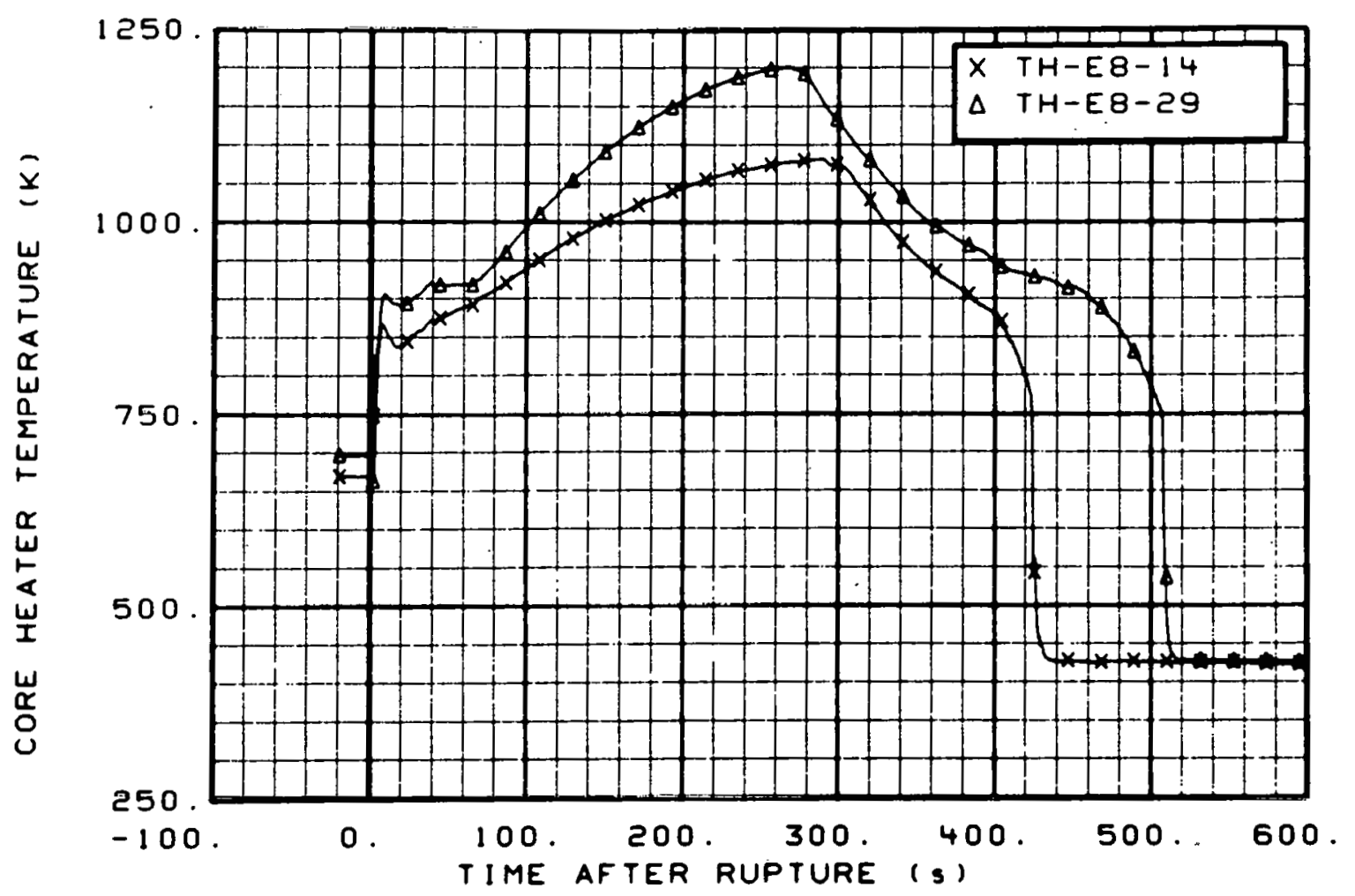

Fig. 127 Core heater temperature, Rod E-8 (TH-E8-14 and TH-E8-29), from -20 to $600 \mathrm{~s}$.

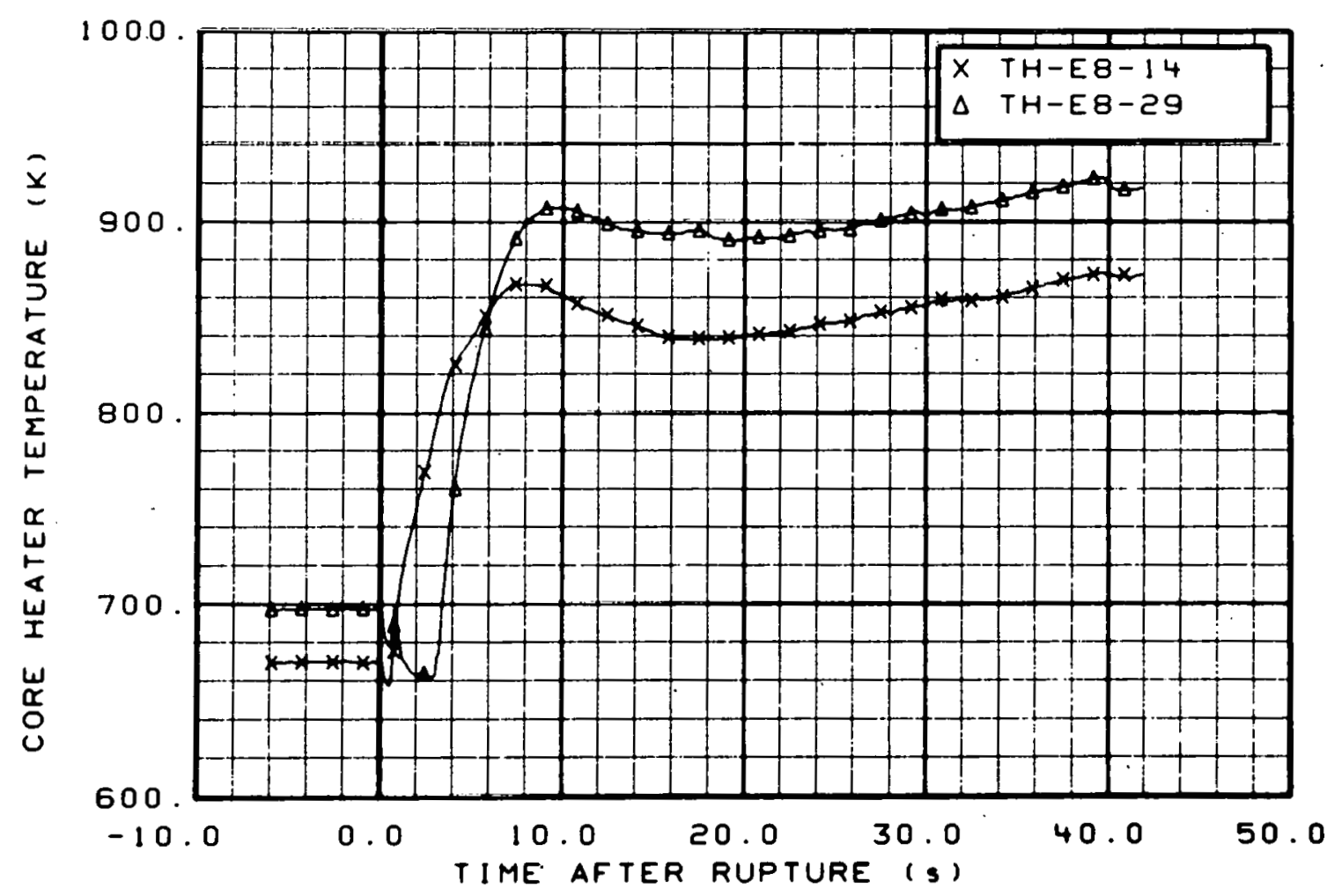

Fig. 128 Core heater temperature, Rod E-8 (TH-E8-14 and TH-E8-29), from -6 to $42 \mathrm{~s}$. 


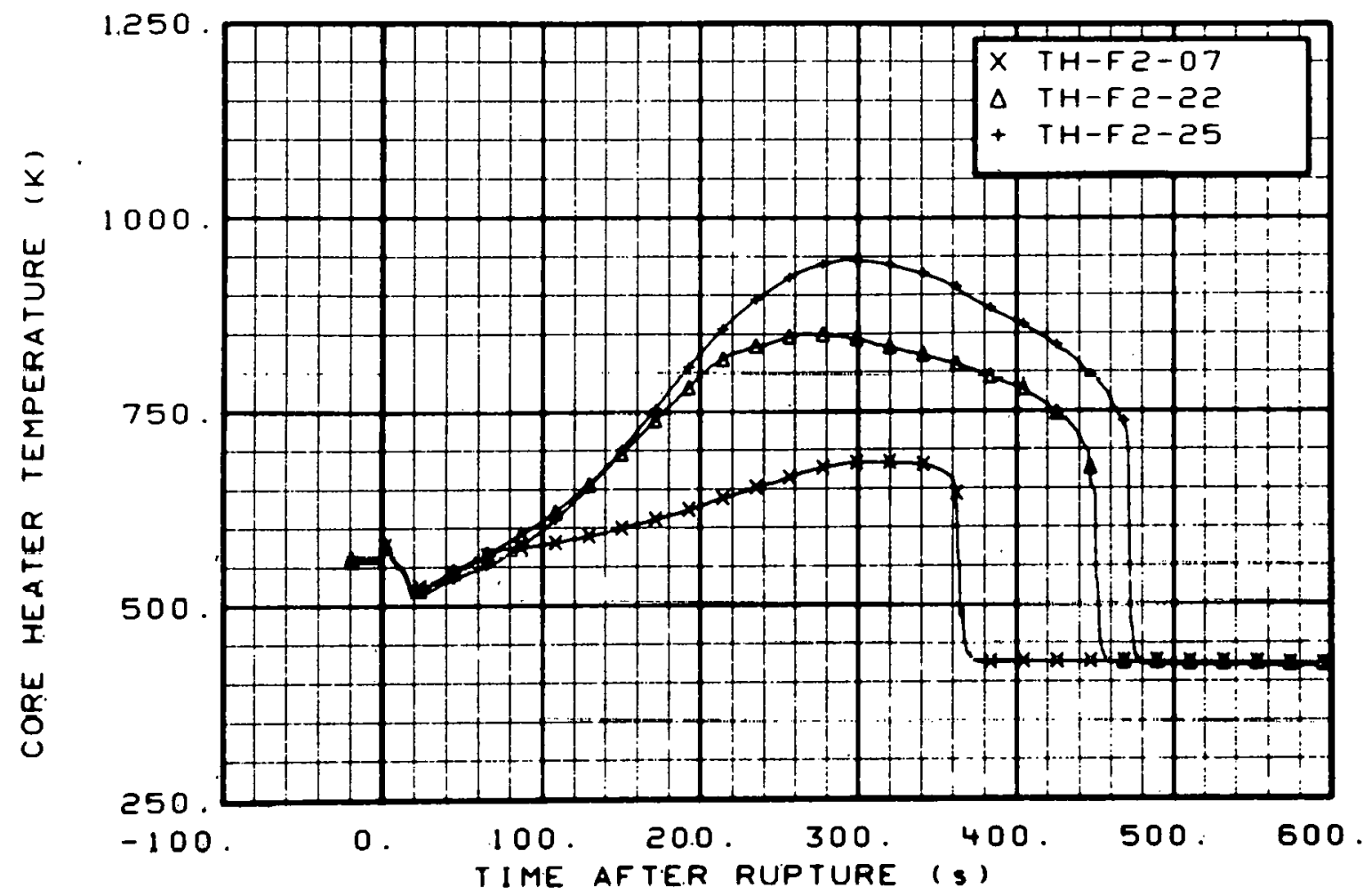

Fig. 129 Core heater temperature, Rod F-2 (TH-F2-07, TH-F2-22, and $\mathrm{TH}-\mathrm{F} 2-25)$, from -20 to $600 \mathrm{~s}$.

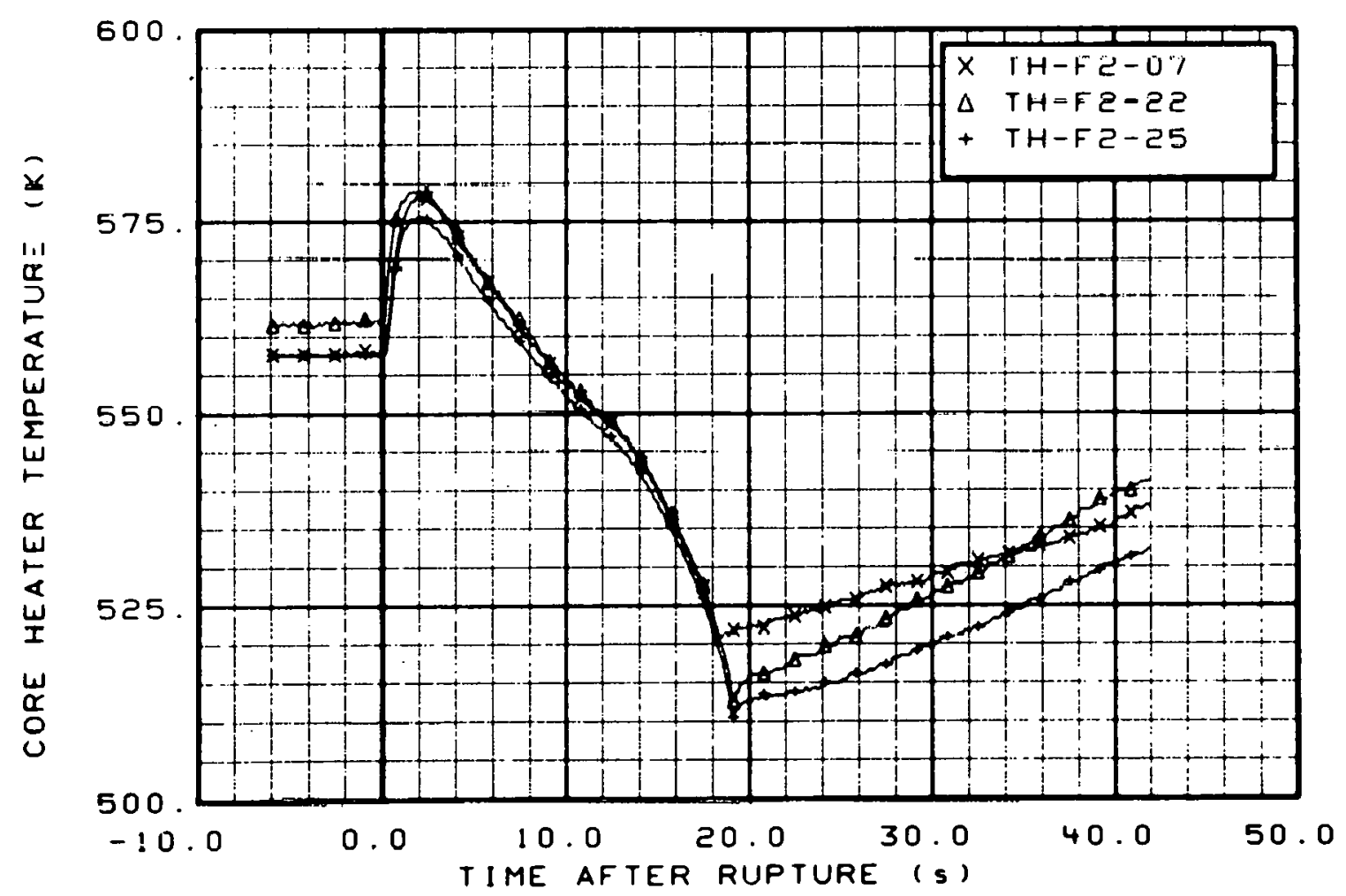

Fig. 130 Core heater temperature, Rod F-2 (TH-F2-07, TH-F2-22, and TH-F2-25), from -6 to $42 \mathrm{~s}$. 


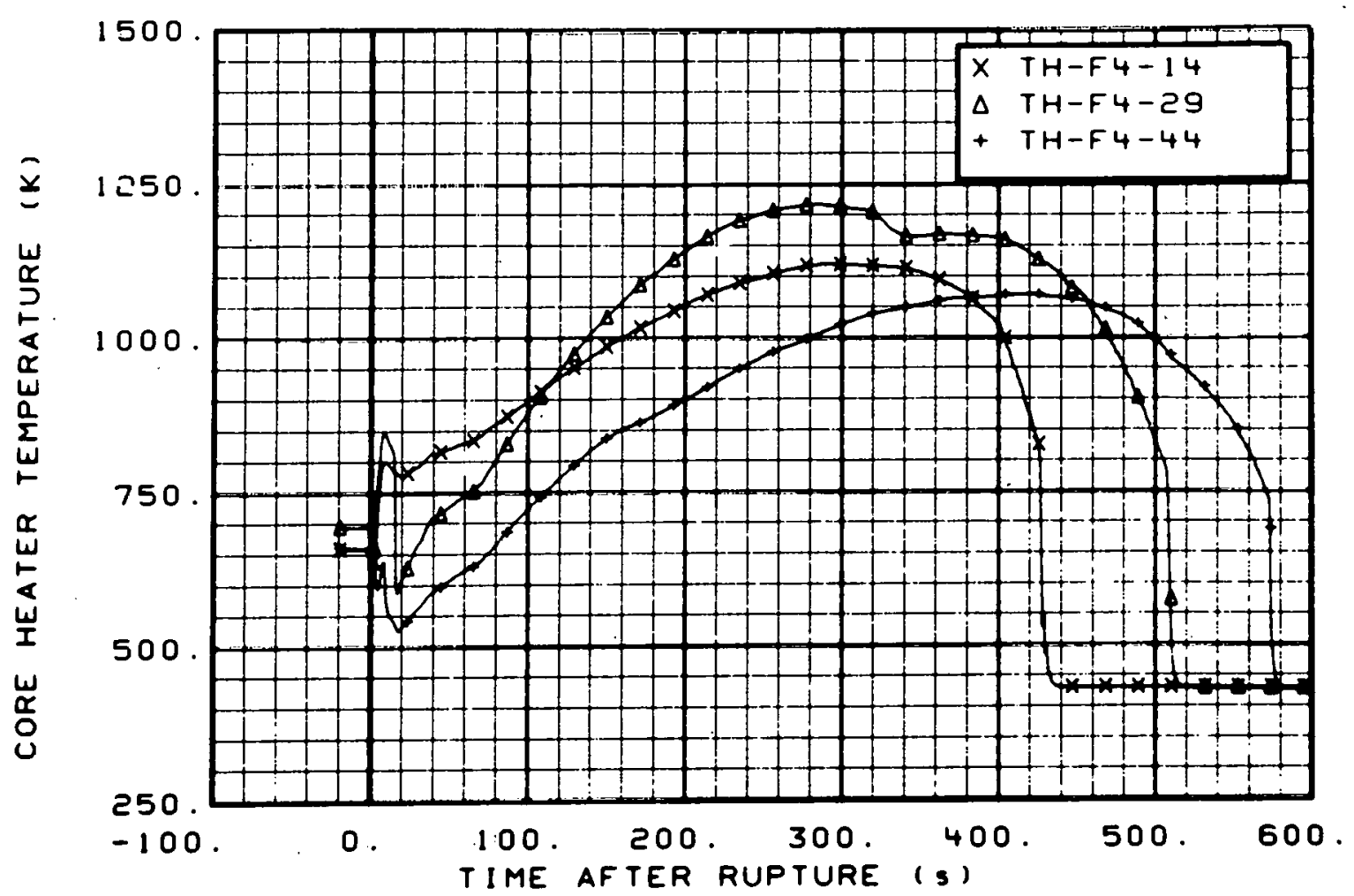

Fig. 131 Core heater temperature, Rod F-4 (TH-F4-14, TH-F4-29, and TH-F4-44), from -20 to $600 \mathrm{~s}$.

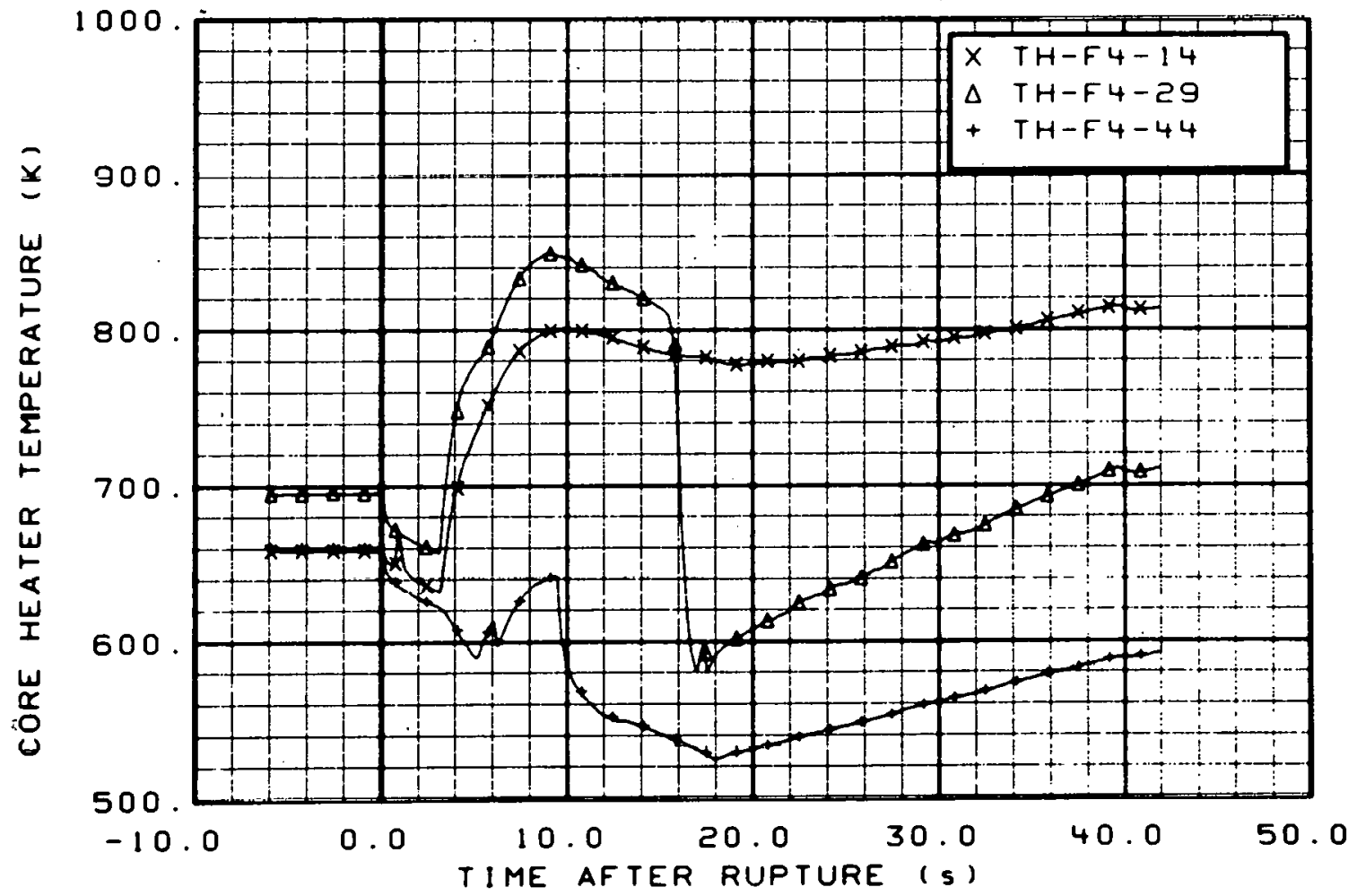

Fig. 132 Core heater temperature, Rod F-4 (TH-F4-14, TH-F4-29, and $\mathrm{TH}-\mathrm{F} 4-44)$, from -6 to $42 \mathrm{~s}$. 


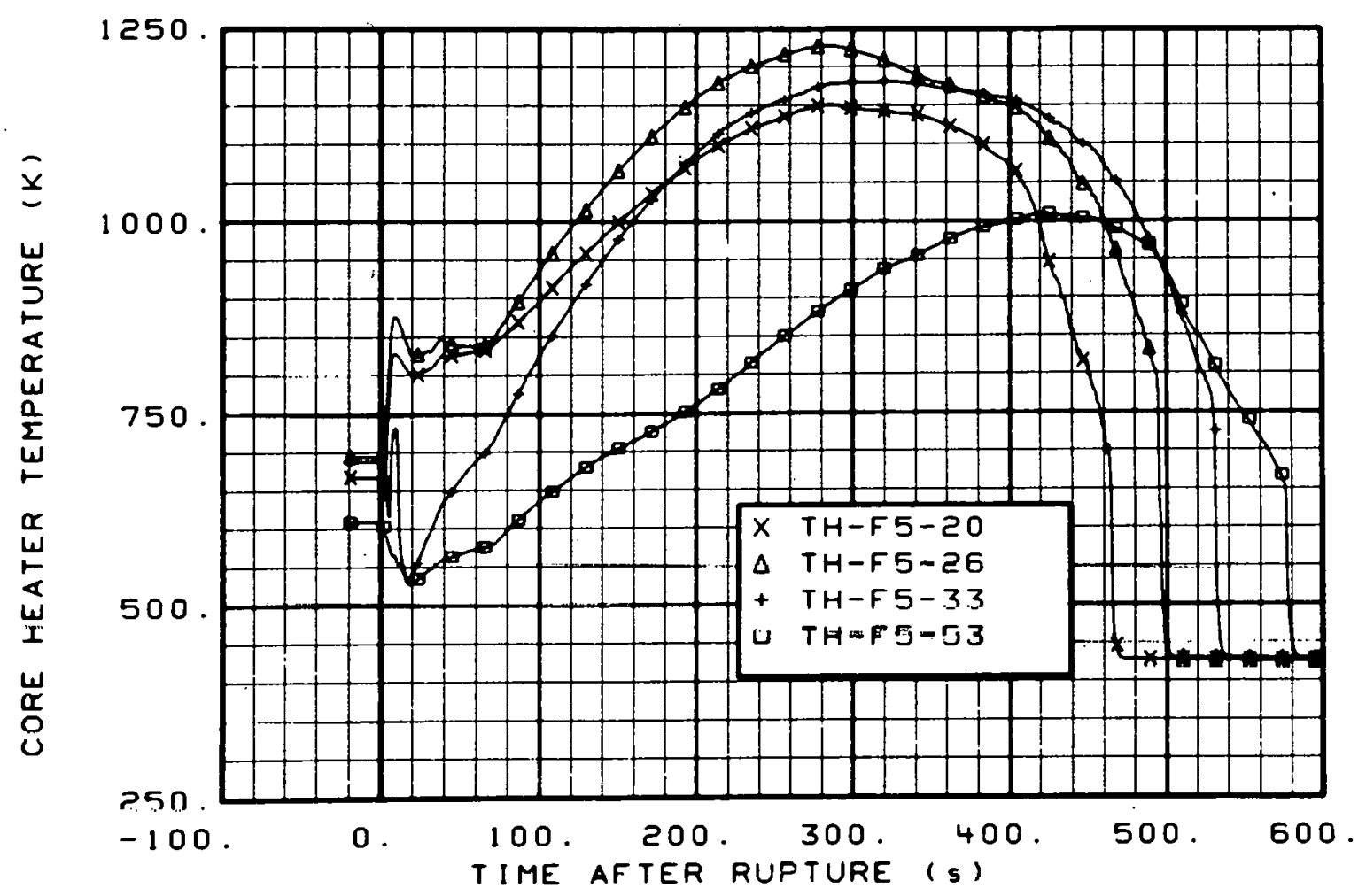

Fig. 133 Core heater temperature, Rod F-5 (TH-F5-20, TH-F5-26, TH-F5-33, and TH-F5-53), from -20 to $600 \mathrm{~s}$.

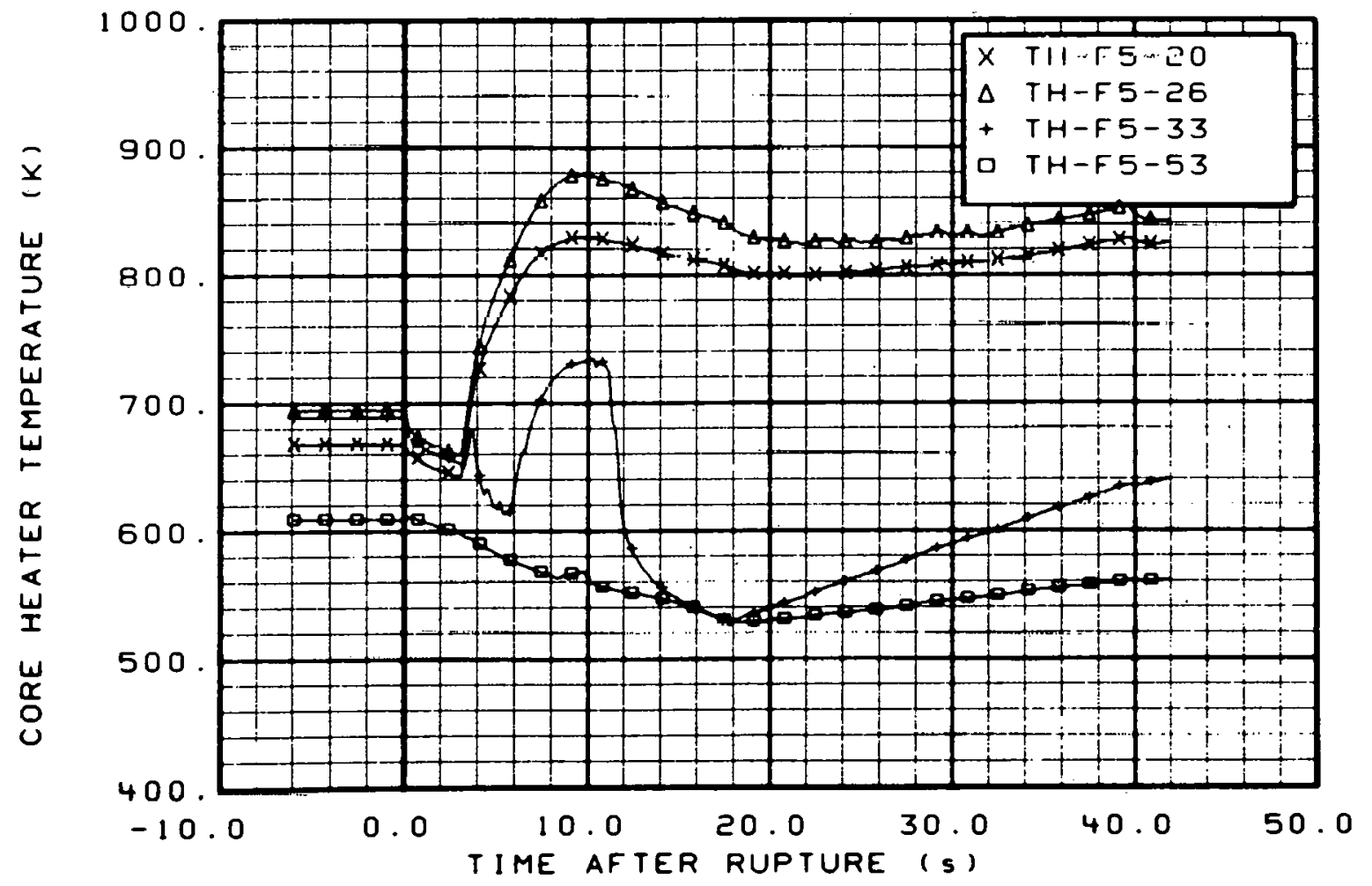

Fig. 134 Core heater temperature, Rod F-5 (TH-F5-20, TH-F5-26, TH-F5-33, and TH-F5-53), from -6 to $42 \mathrm{~s}$. 


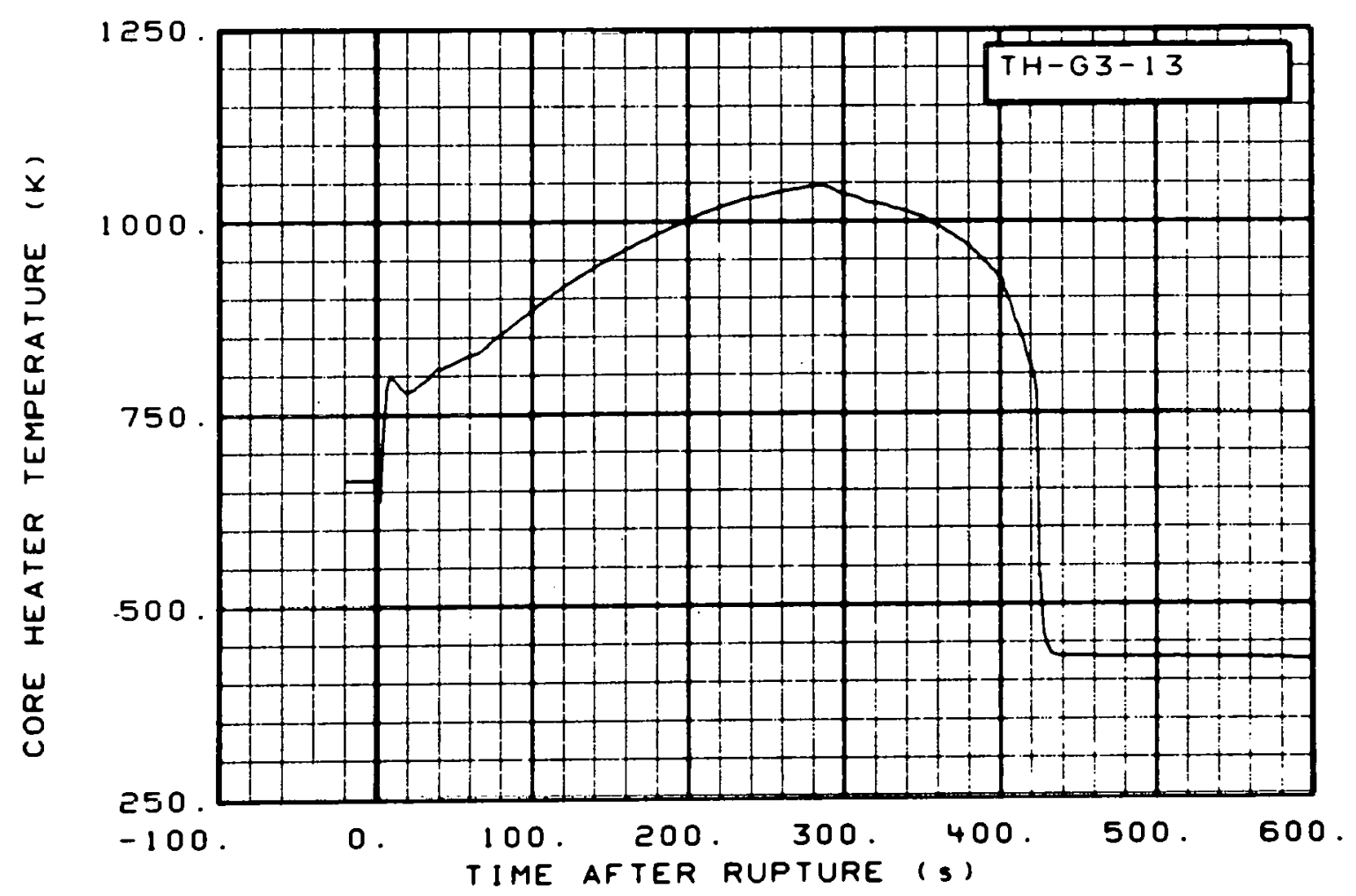

Fig. 135 Core heater temperature, Rod G-3 (TH-G3-13), from -20 to $600 \mathrm{~s}$.

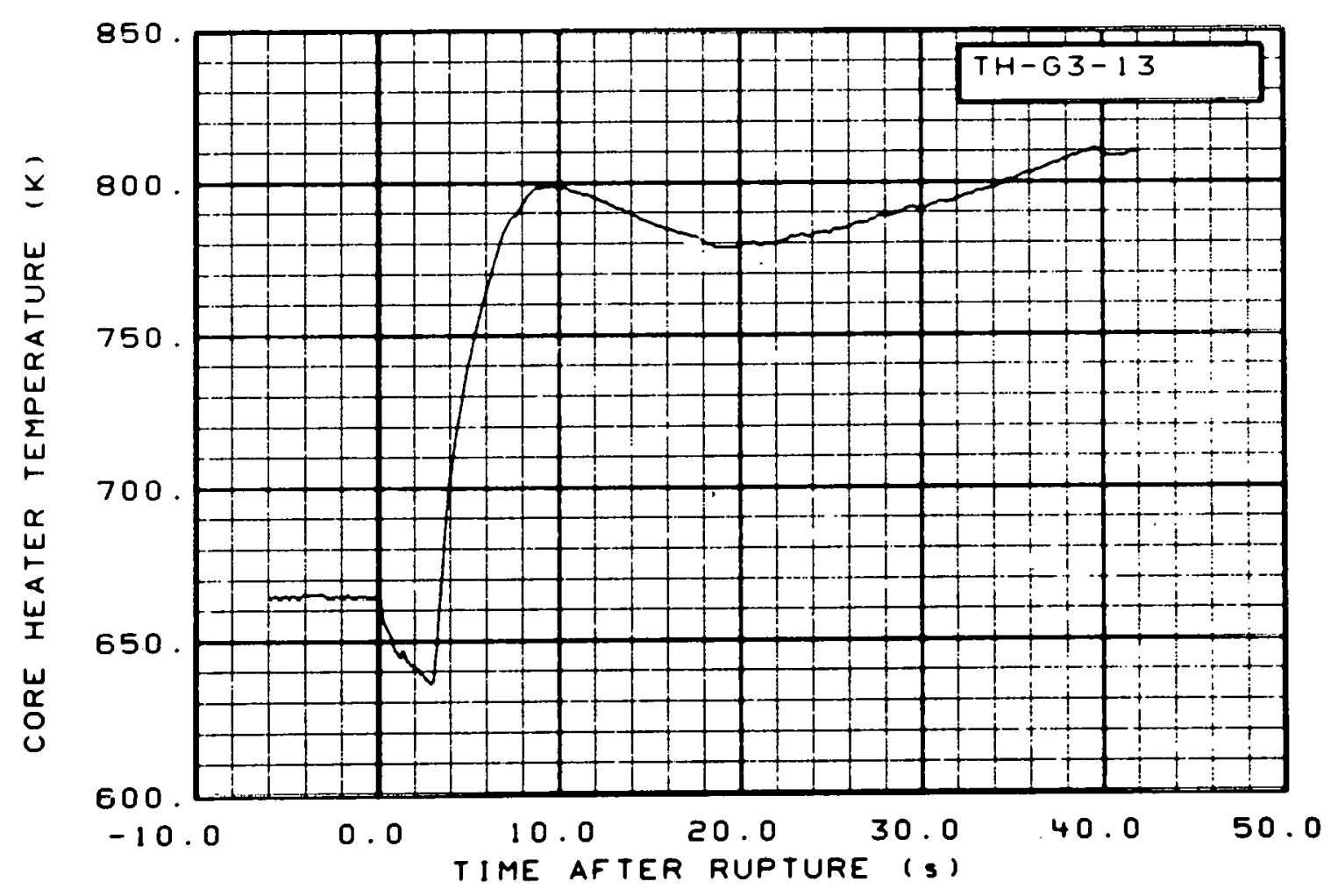

Fig. 136 Core heater temperature, Rod G-3 (TH-G3-13), from -6 to $42 \mathrm{~s}$. 


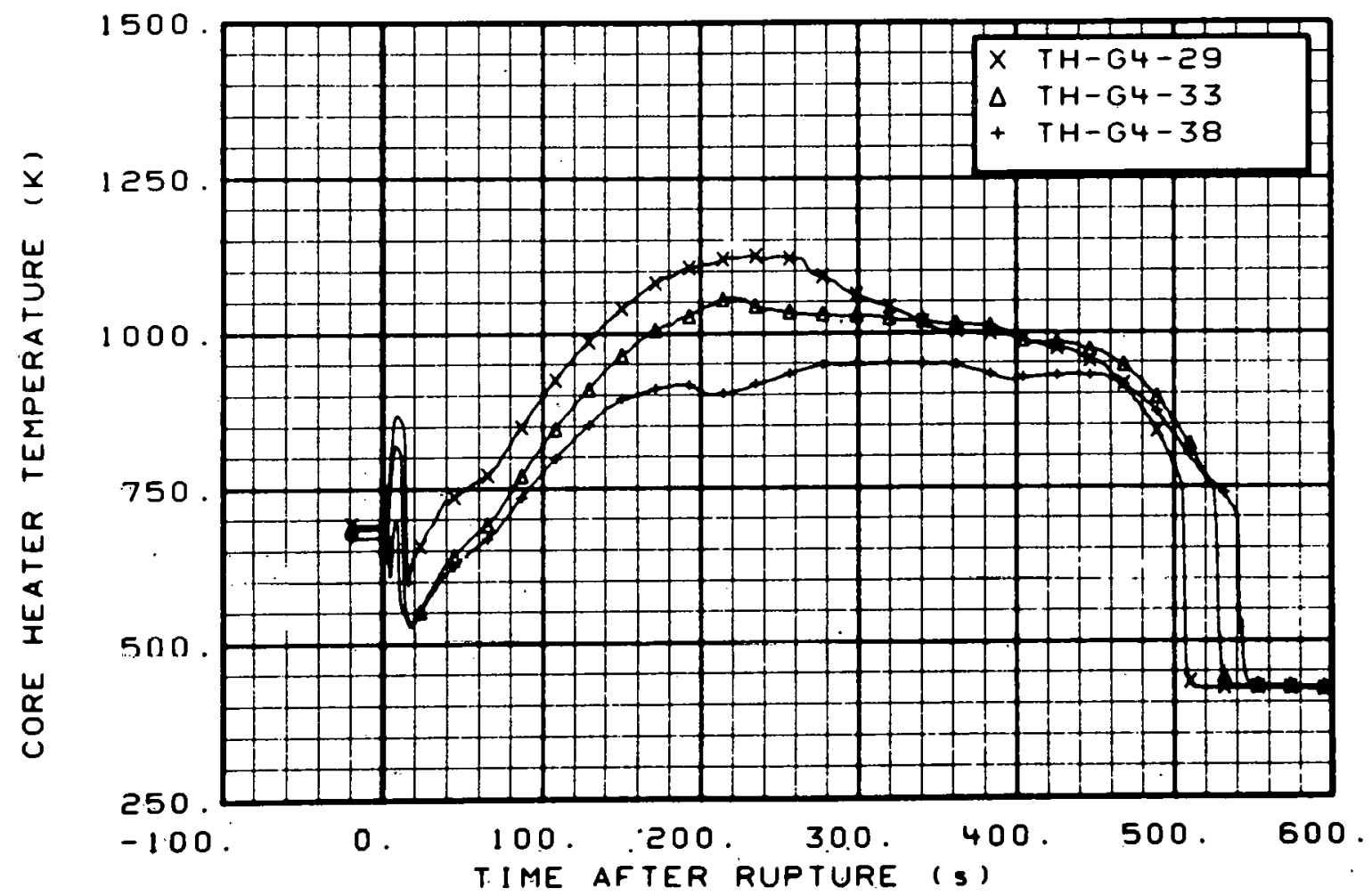

Fi.g. 137 Core heater temperature, Rod G-4 (TH-G4-29, TH-G4-33, and TH-G4-38), from -20 to $600 \mathrm{~s}$.

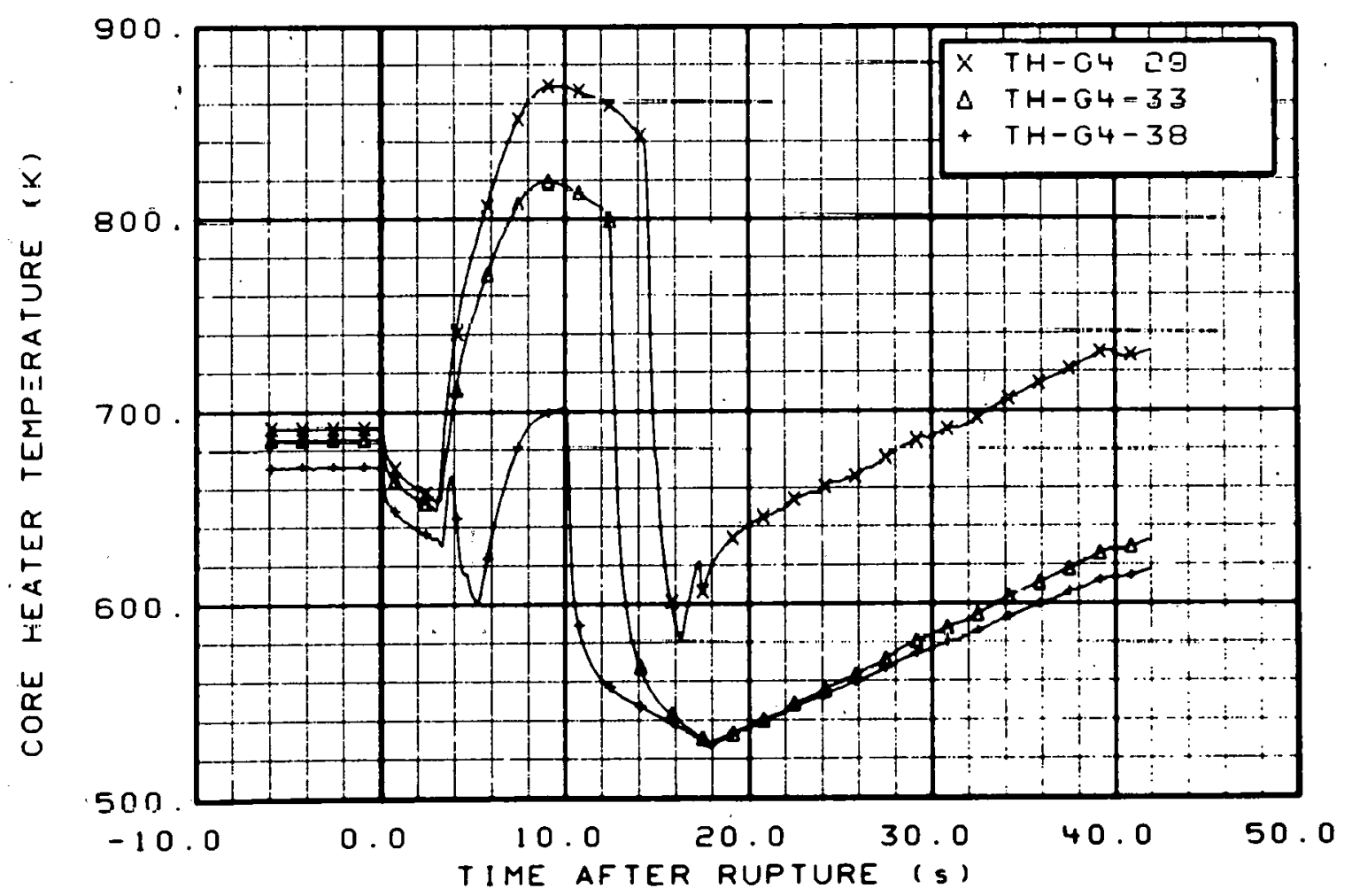

Fig. 138 Core heater temperature, Rod G-4 (TH-G4-29, TH-G4-33, and TH-G4-38), from -6 to $42 \mathrm{~s}$. 


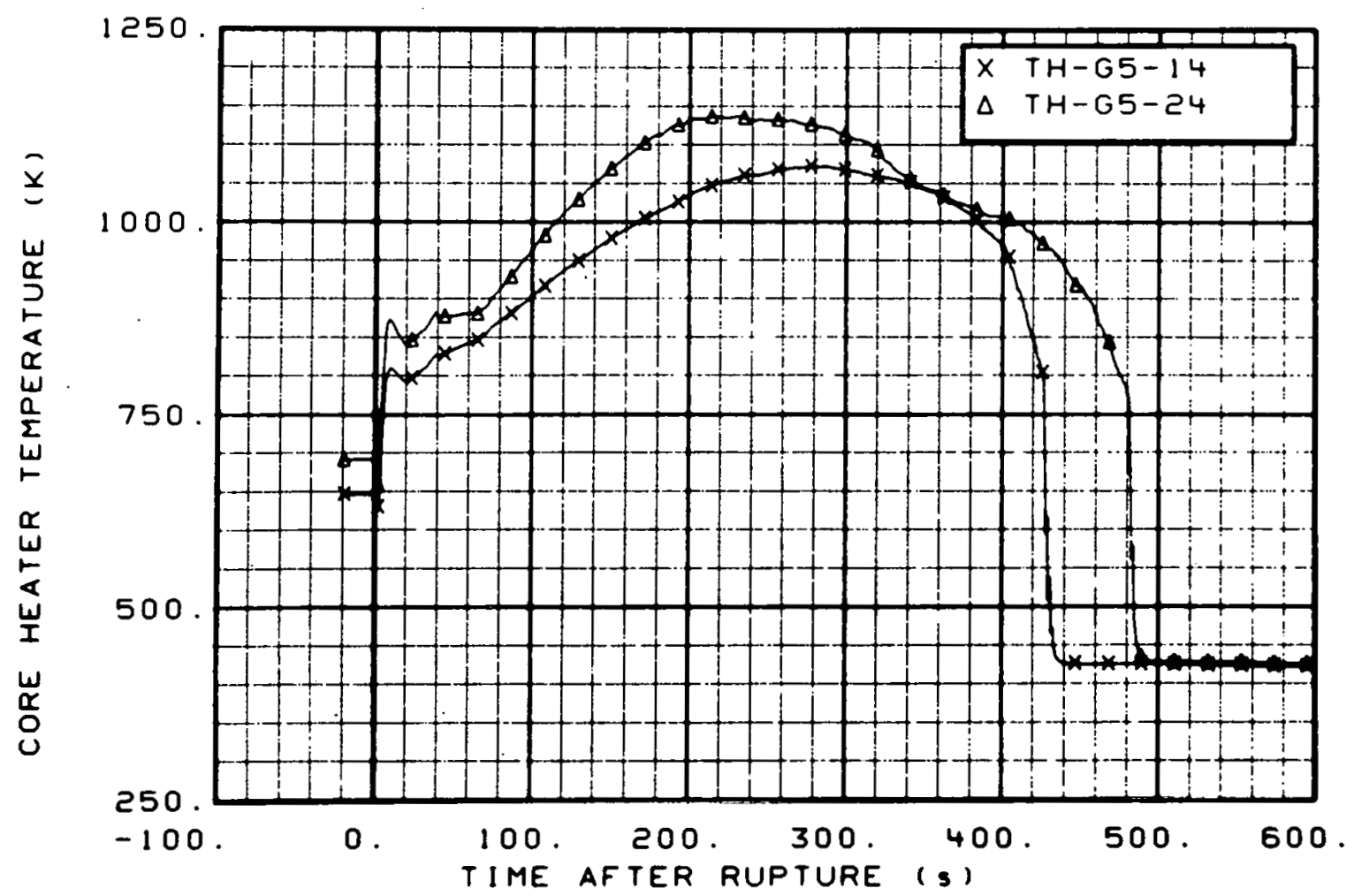

Fig. 139 Core heater temperature, Rod G-5 (TH-G5-14 and TH-G5-24), from -20 to $600 \mathrm{~s}$.

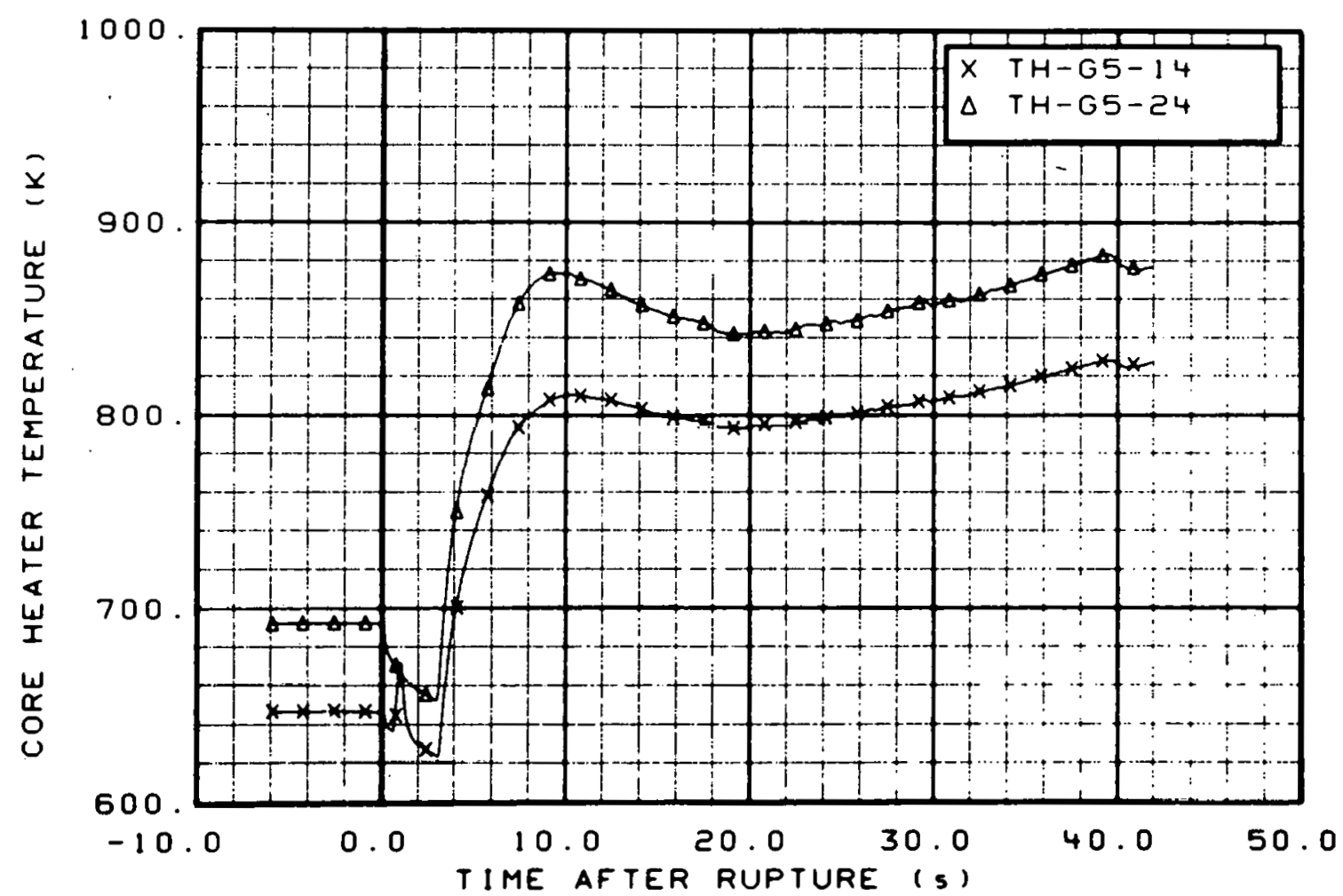

Fig. 140 Core heater temperature, Rod G-5 (TH-G5-14 and TH-G5-24), from -6 to $42 \mathrm{~s}$. 


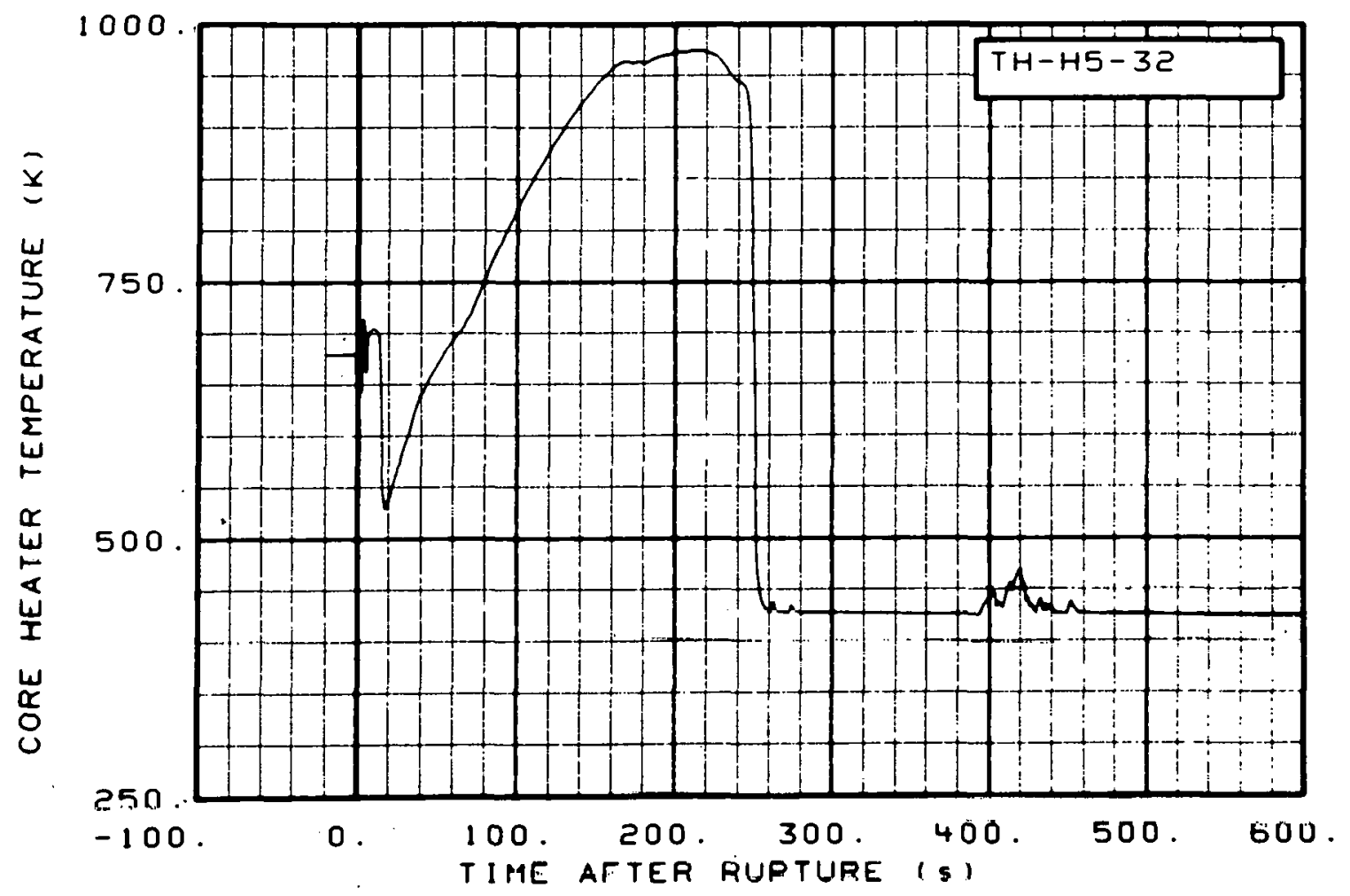

Fig. 1.41 Core heater temperature, Rod H-5 (TH-H5-32), from -20 to $600 \mathrm{~s}$.

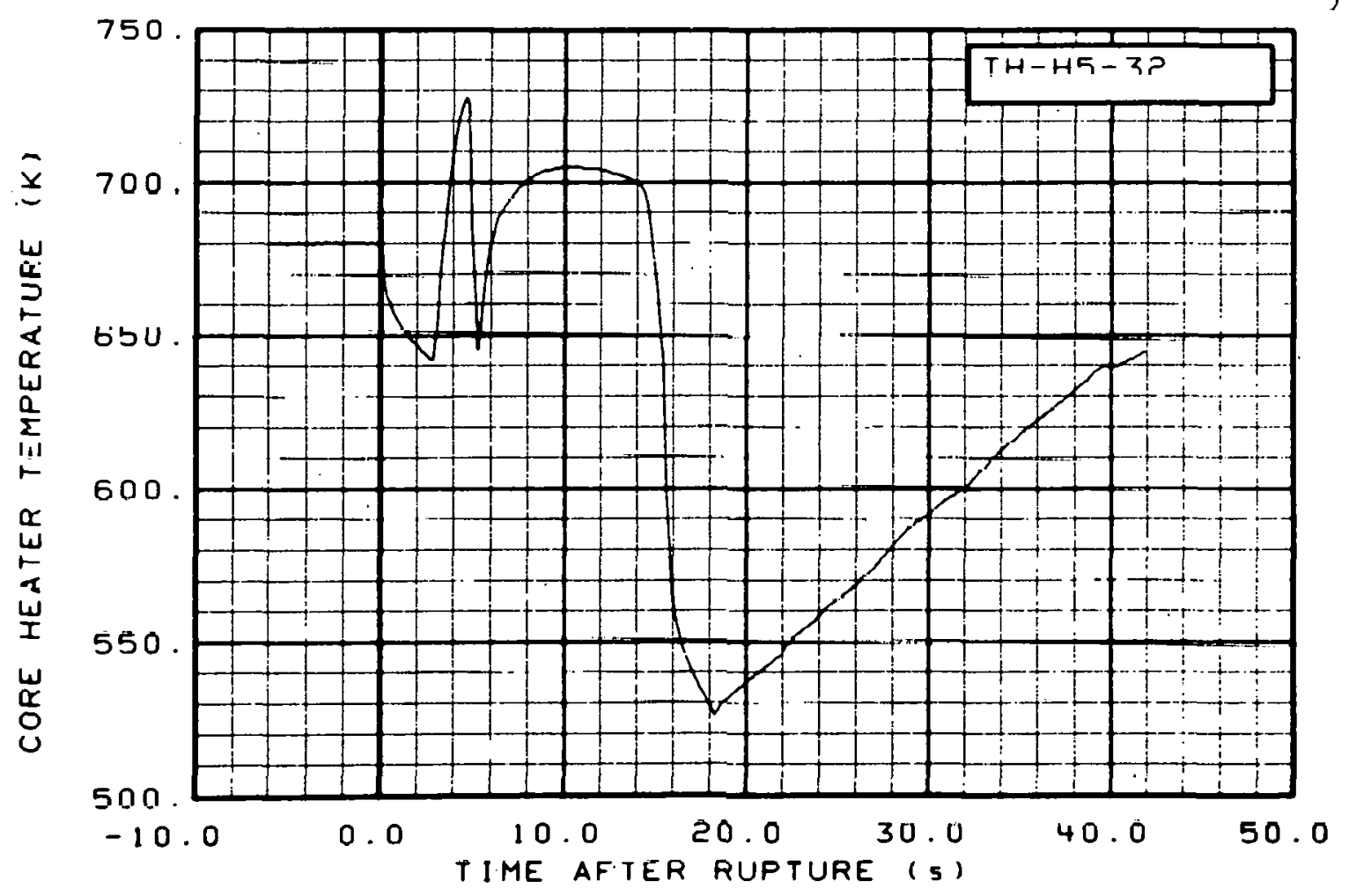

Fig. 142. Core heater temperature, Rod H-5 (TH-H5-32), from -6 to $42 \mathrm{~s}$. 


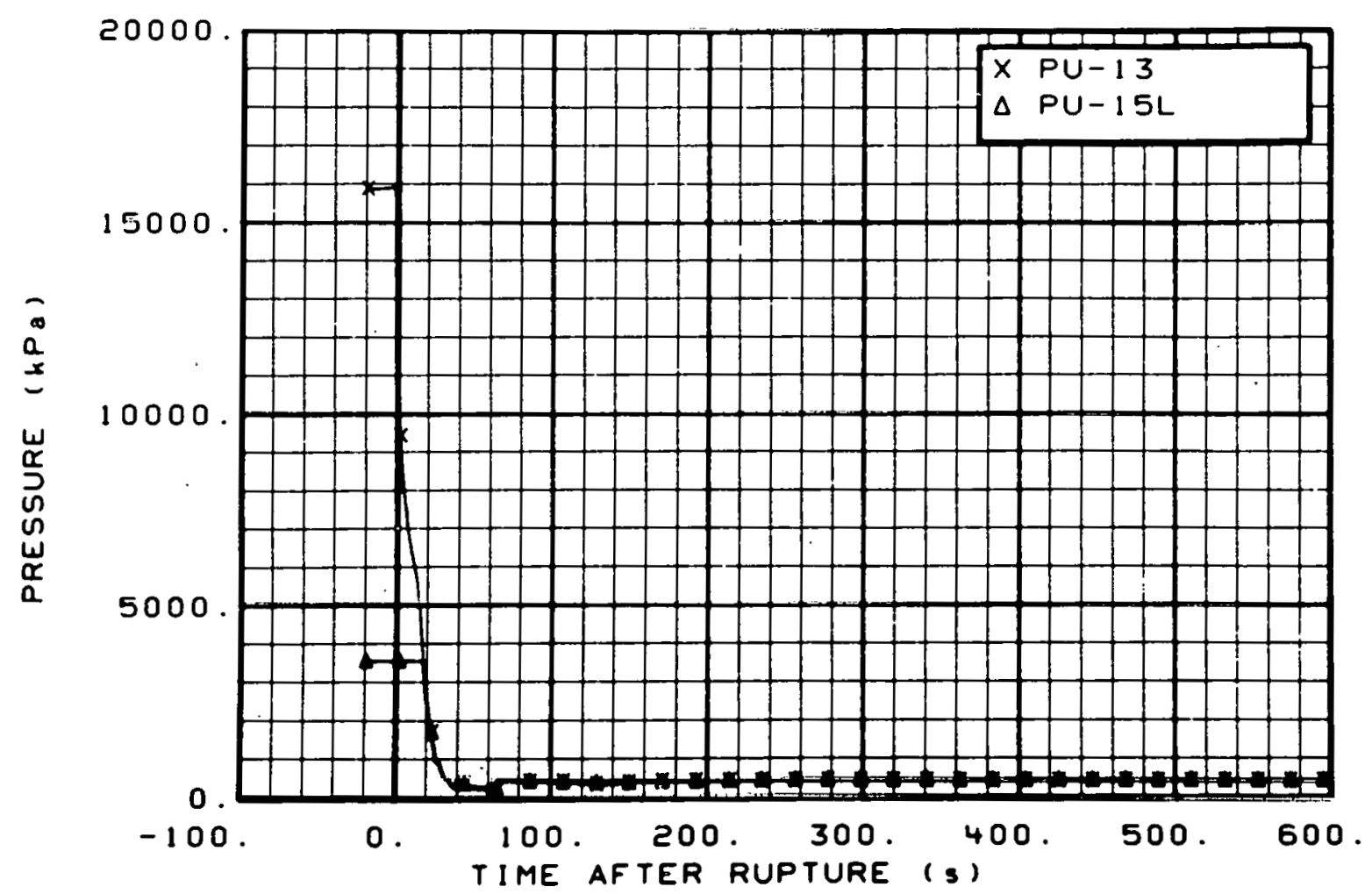

Fig. 143 Pressure in intact 10op (PU-13 and PU-15L), from -20 to $600 \mathrm{~s}$.

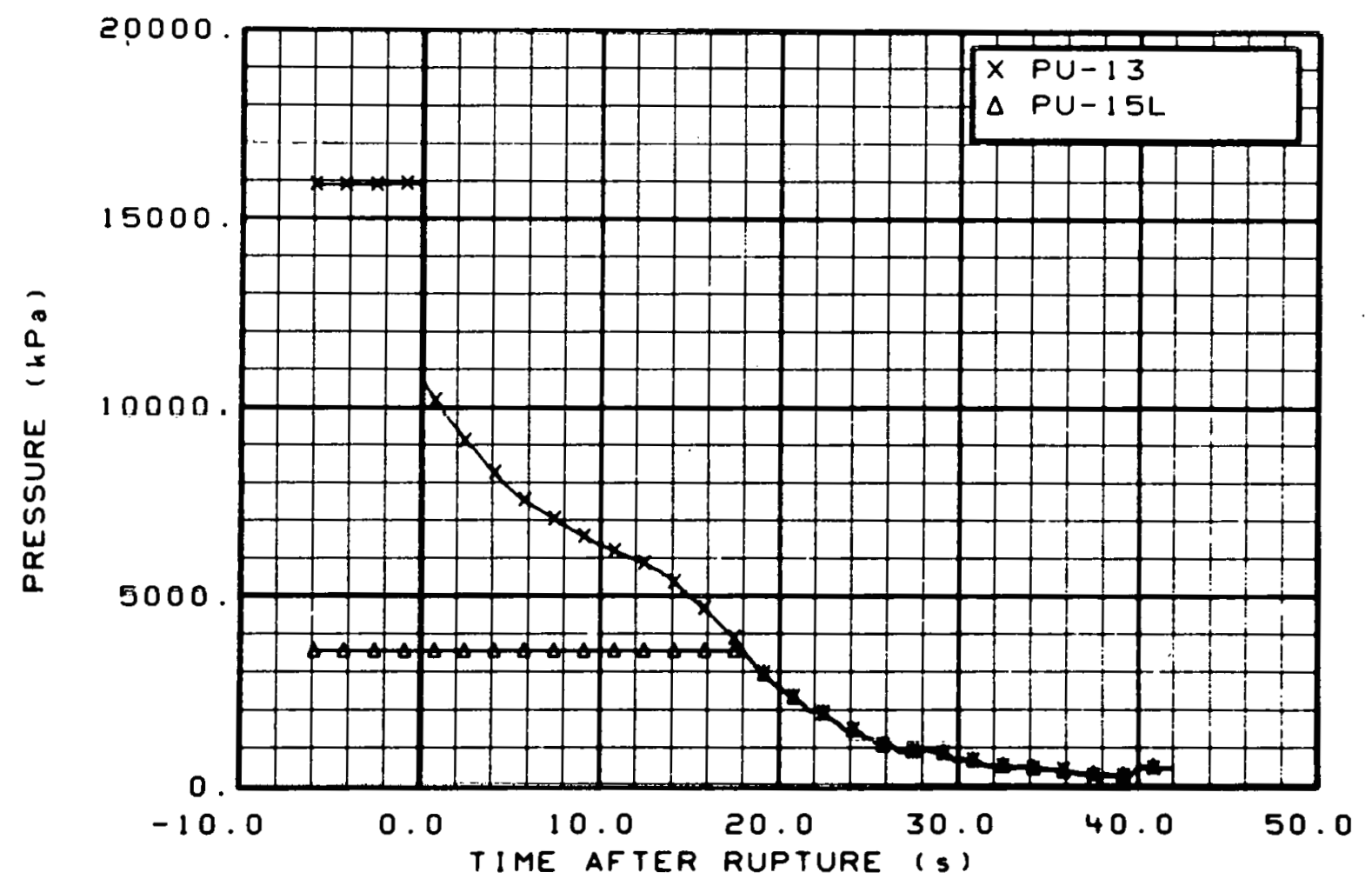

Fig. 144 Pressure in intact 10op (PU-13 and PU-15L), from -6 to $42 \mathrm{~s}$. 


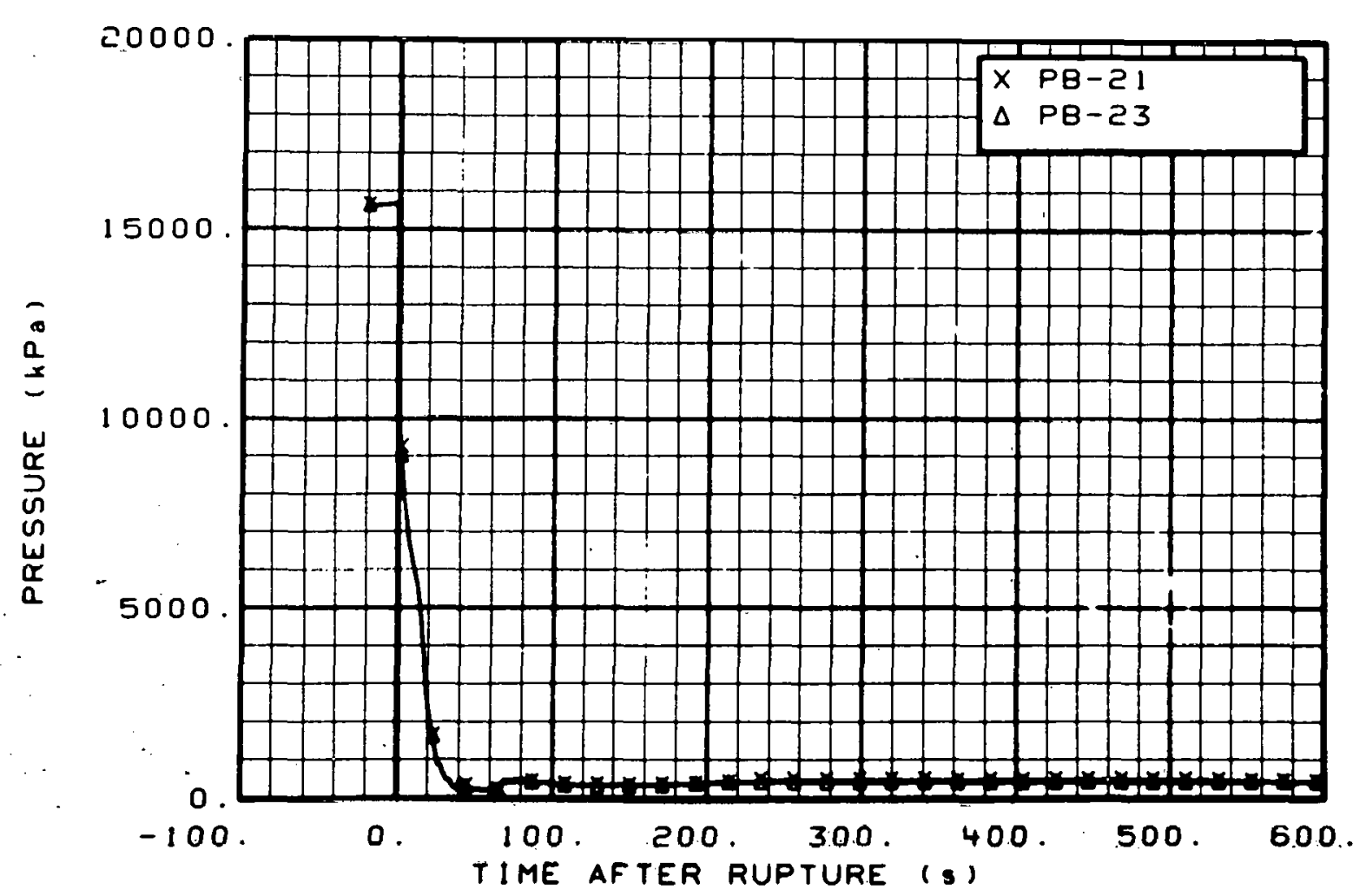

Fig. 145 Pressure in broken 1oop, vessel side (PB-21 and PB-23), from -20 to $600 \mathrm{~s}$.

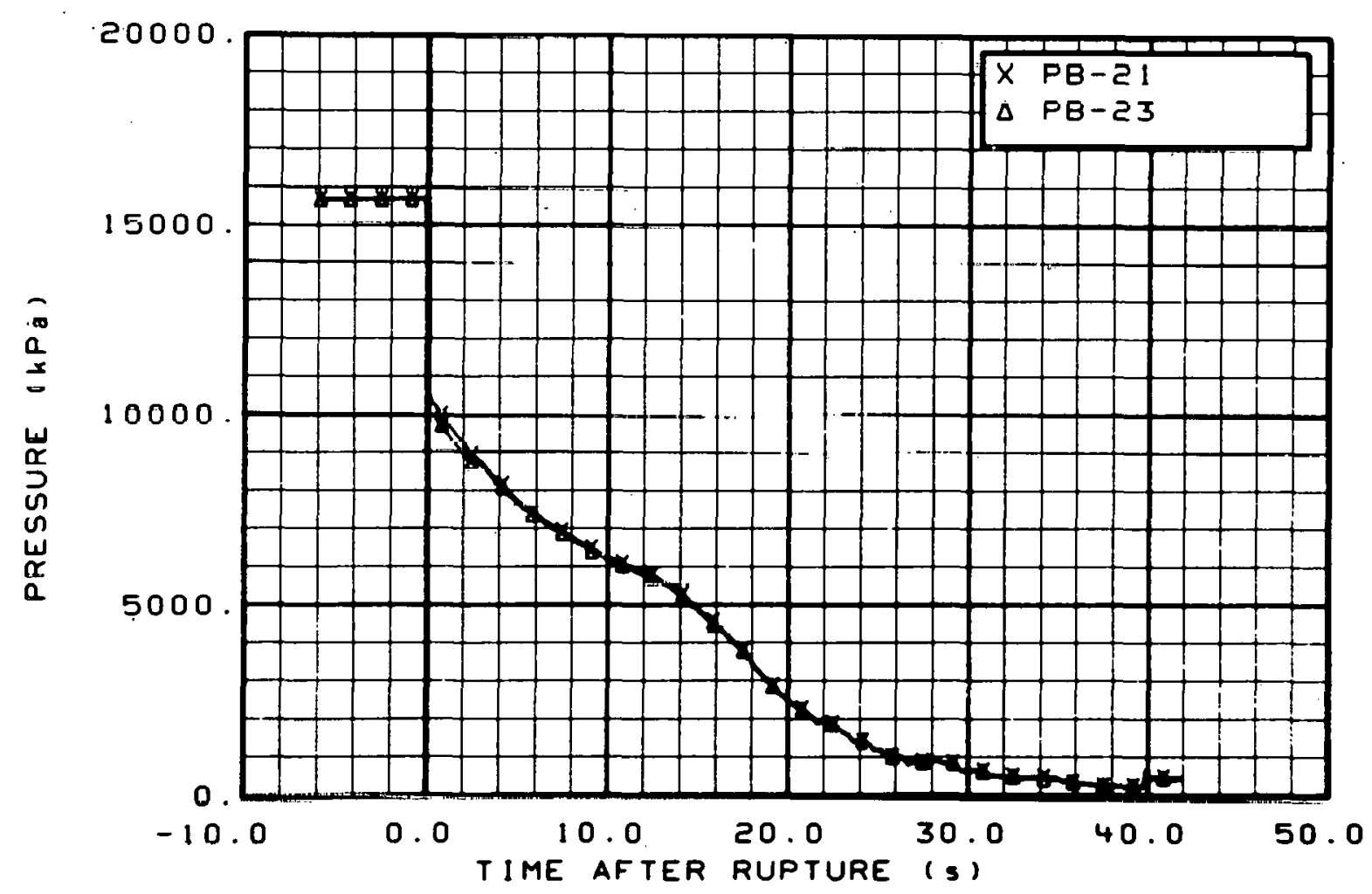

Fig. 146 Pressure in broken loop, vessel side (PB-21 and $P B-23)$, from -6 to $42 \mathrm{~s}$. 


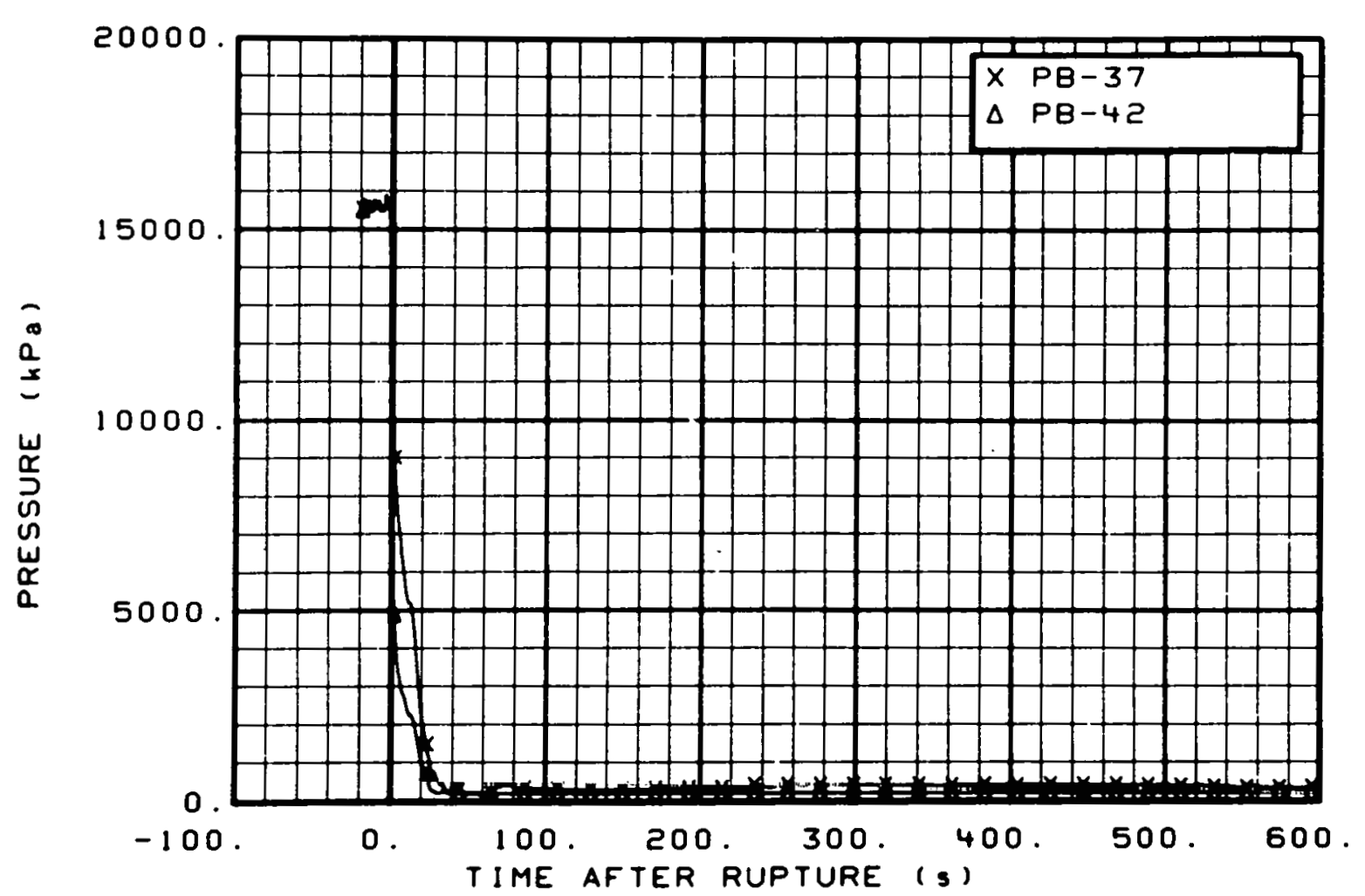

Fig. 147 Pressure in broken 10op, pump side (PB-37 and PB-42), from -20 to $600 \mathrm{~s}$.

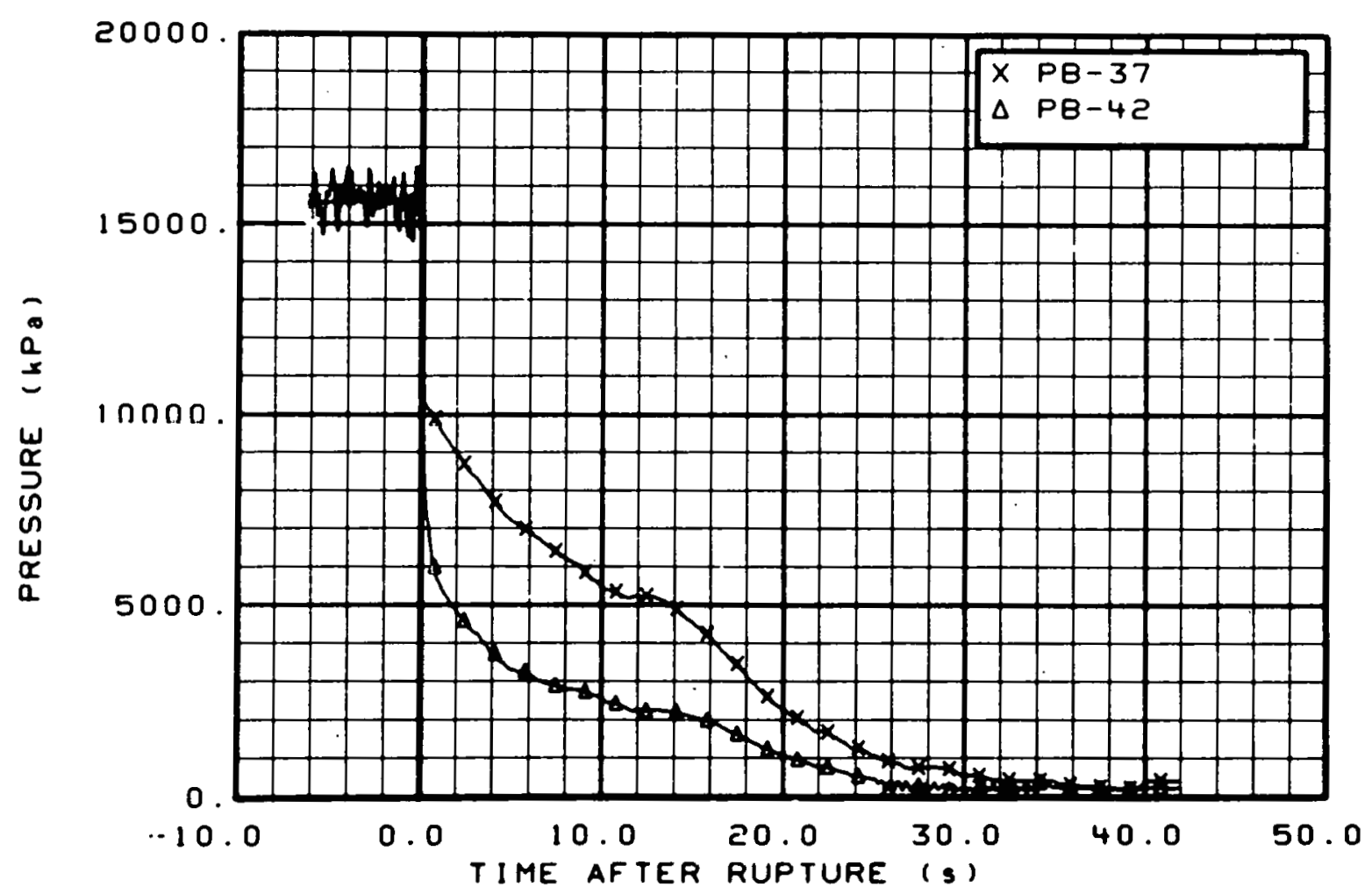

Fig. 148 Pressure in broken loop, pump side (PB-37 and PB-42), from -6 to $42 \mathrm{~s}$. 


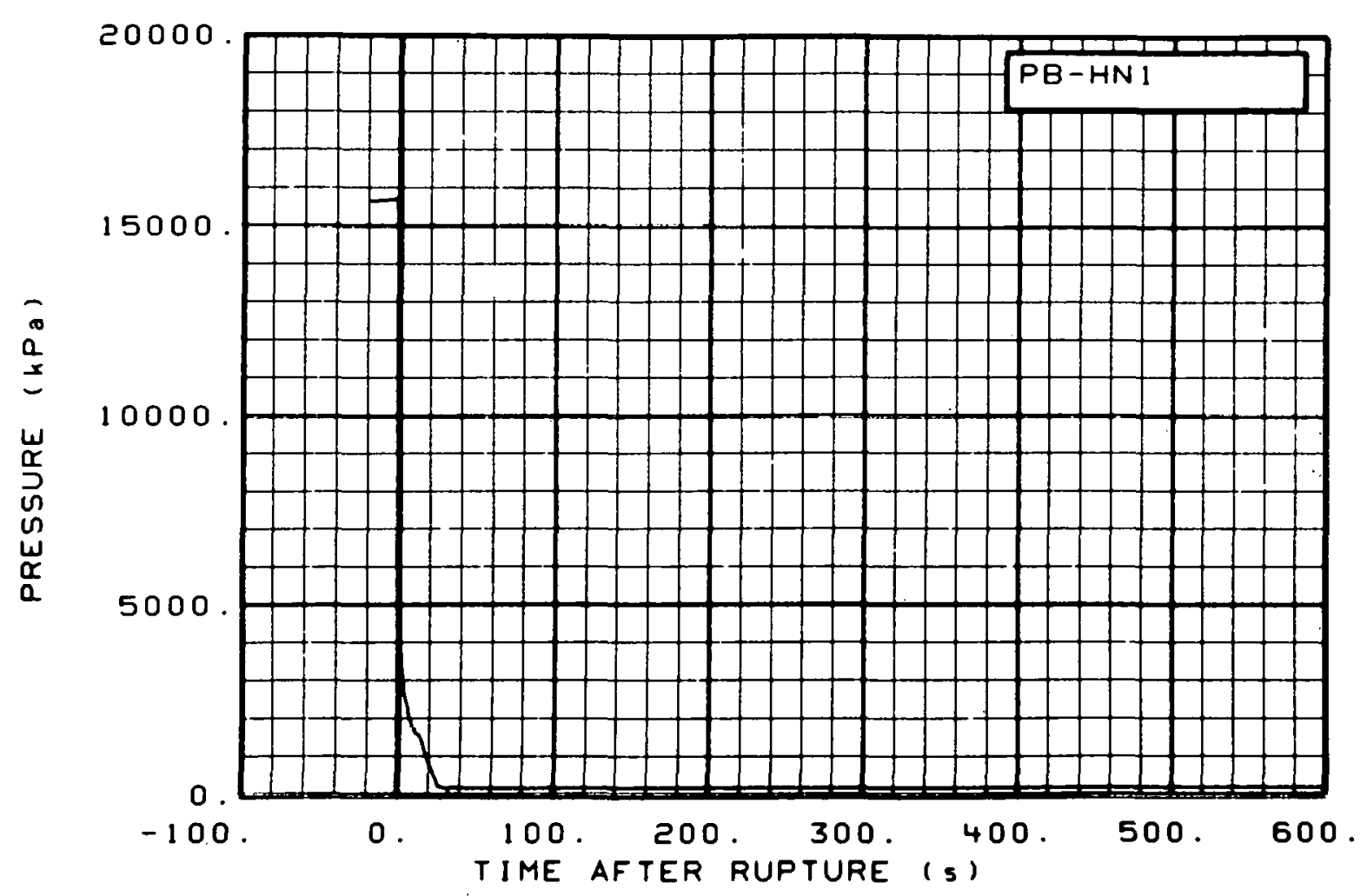

Fig. 149 Pressure in broken 10op, pump-side nozzle (PB-HN), from -20 to $600 \mathrm{~s}$.

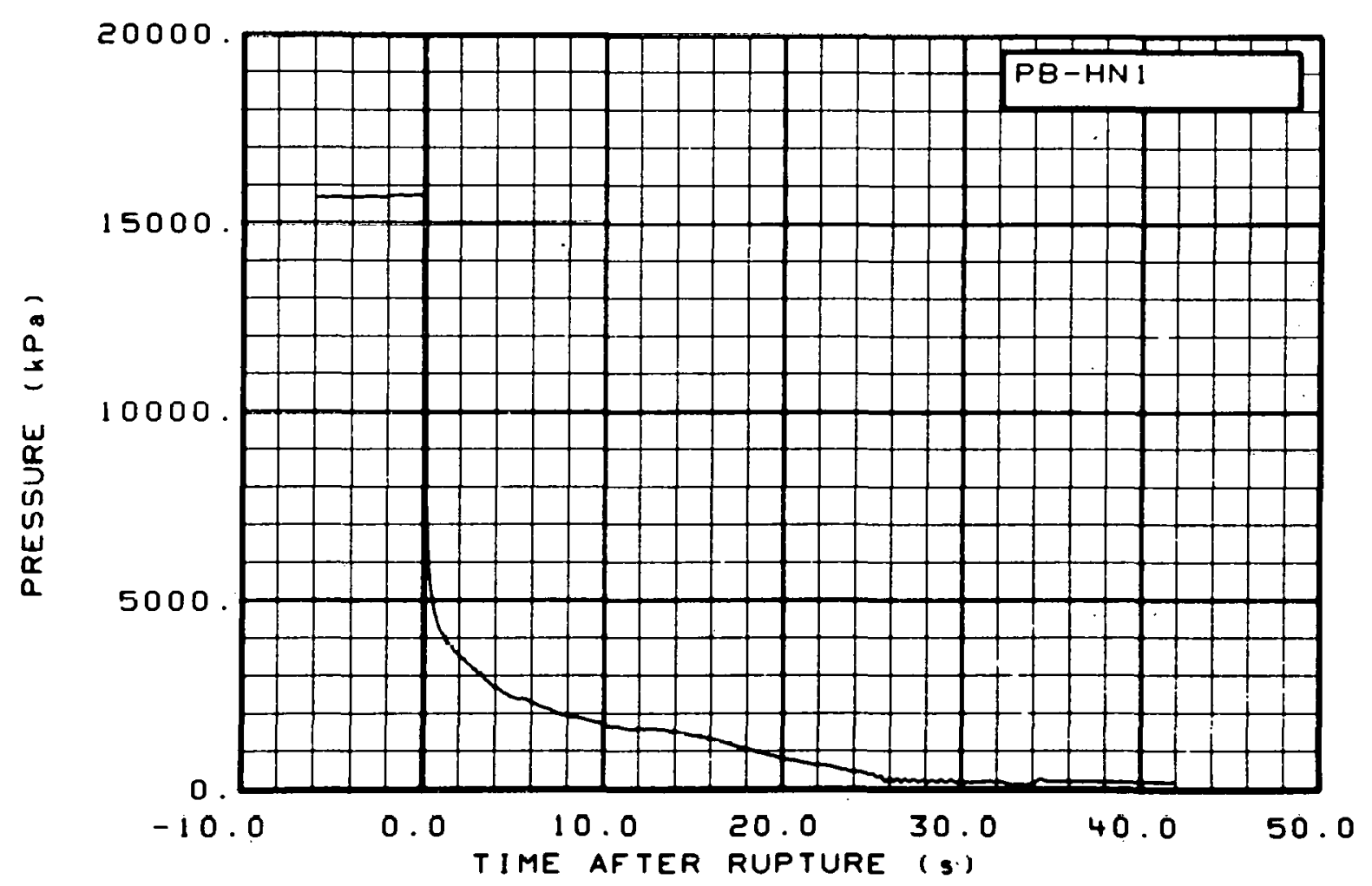

Fig. 150 Pressure in broken loop, pump-side nozzle (PB-HN1), from -6 to $42 \mathrm{~s}$. 


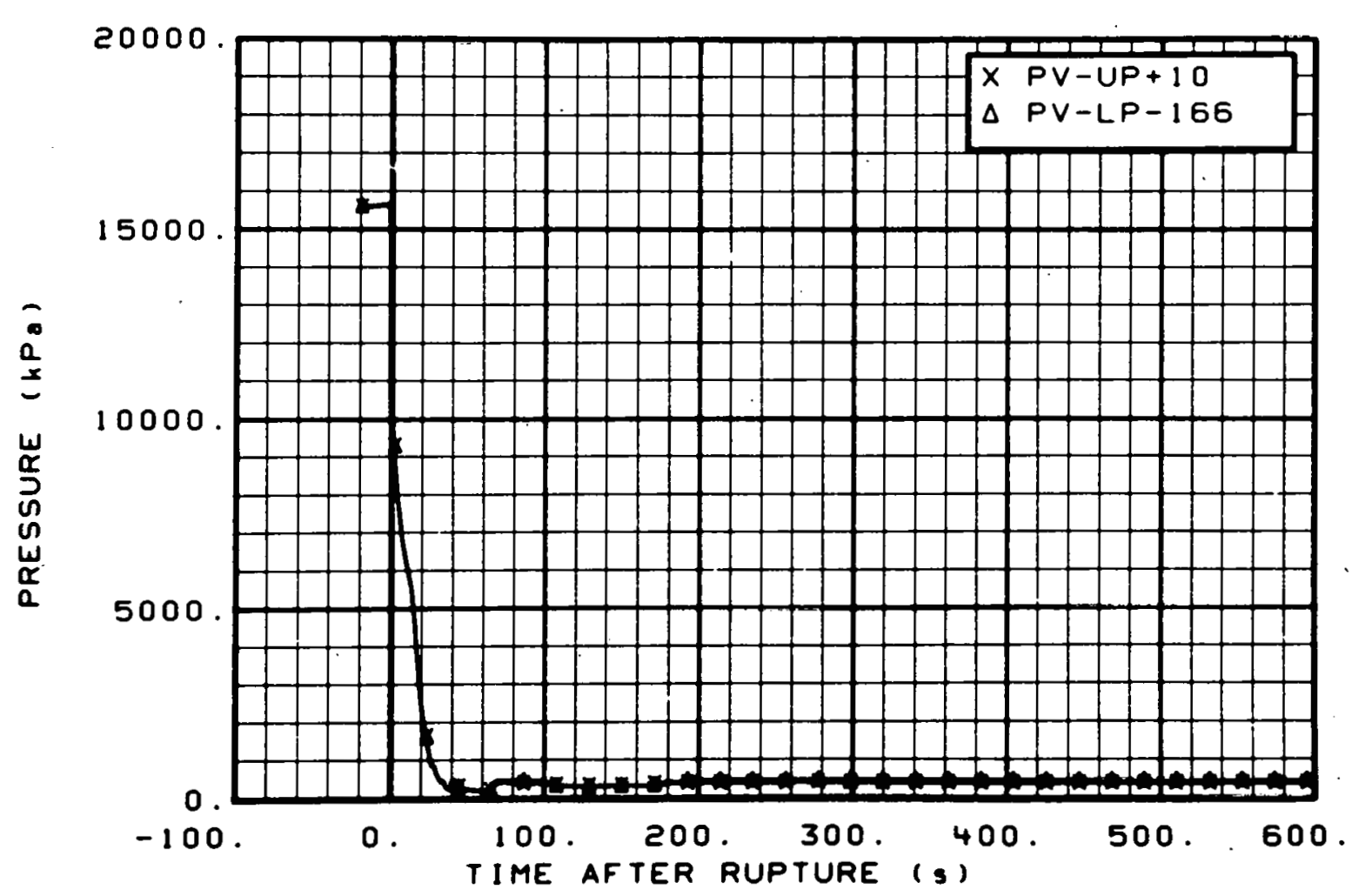

Fig. 151 Pressure in vesse1 (PV-UP+10 and PV-LP-166), from -20 to $600 \mathrm{~s}$.

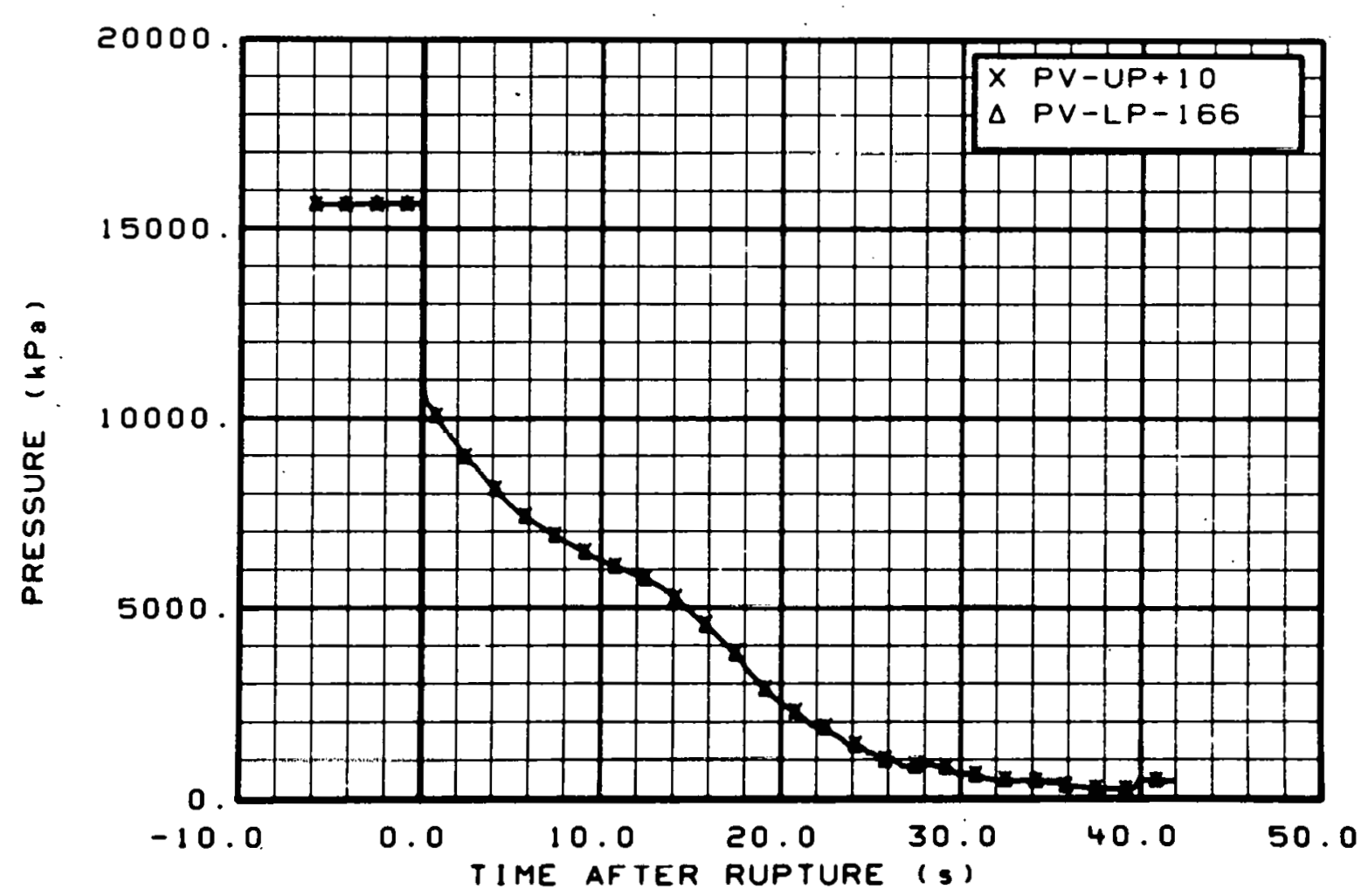

Fig. 152 Pressure in vessel (PV-UP+10 and PV-LP-166), from -6 to $42 \mathrm{~s}$. 


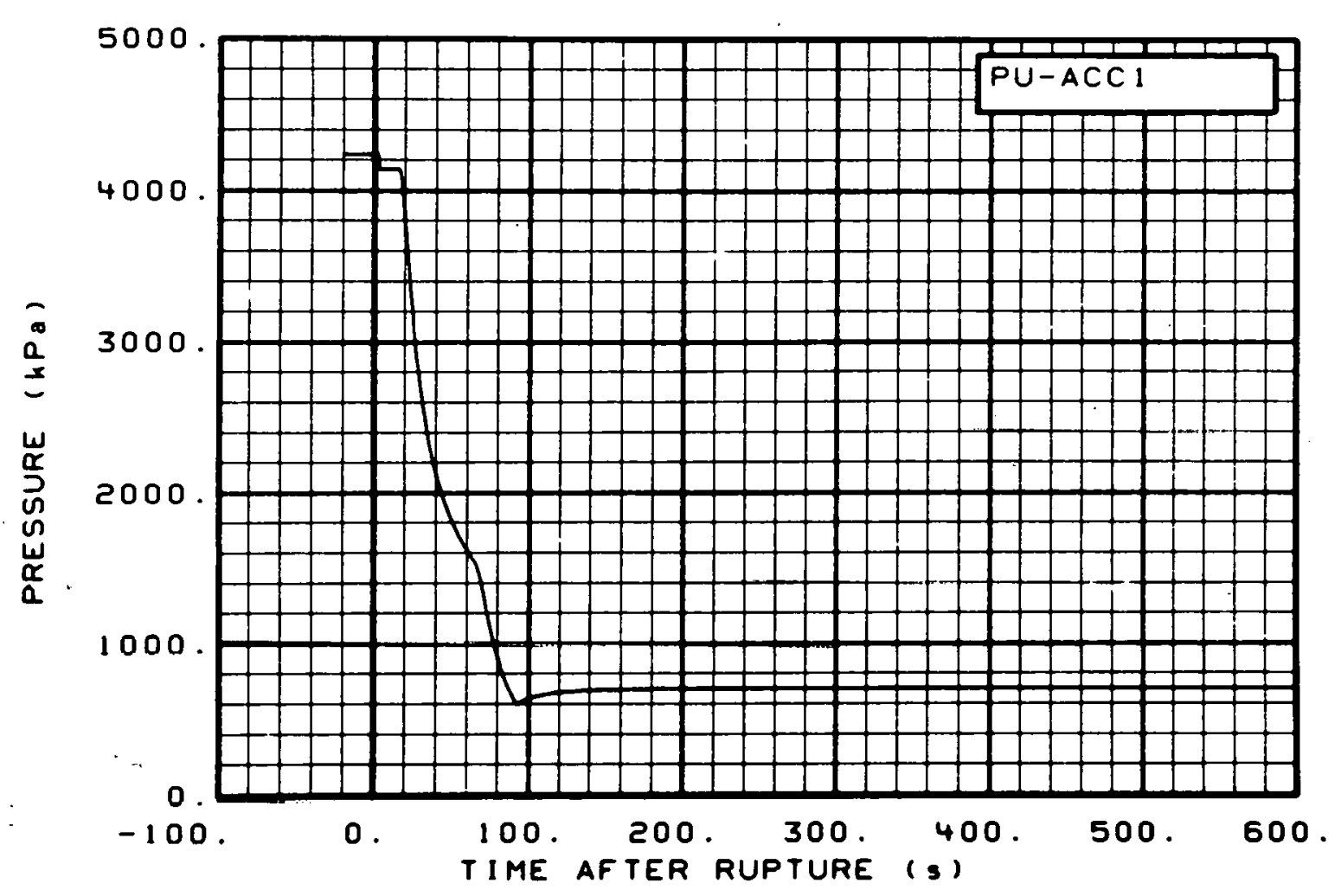

Fig. 153 Pressure in intact loop accumulator (PU-ACC1), from -20 to $600 \mathrm{~s}$.

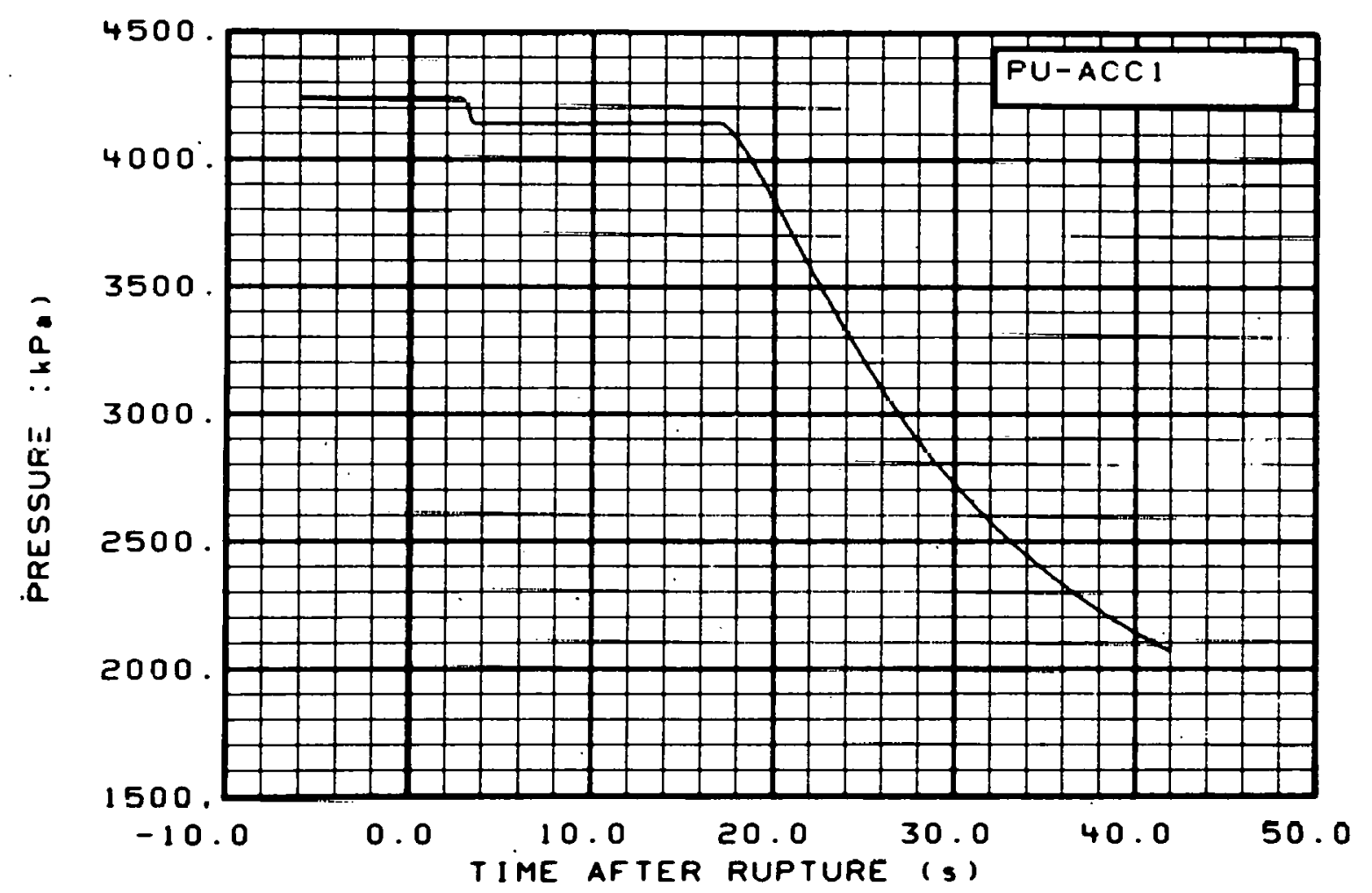

Fig. 154 Pressure in intact loop accumulator (PU-ACC1), from -6 to $42 \mathrm{~s}$. 


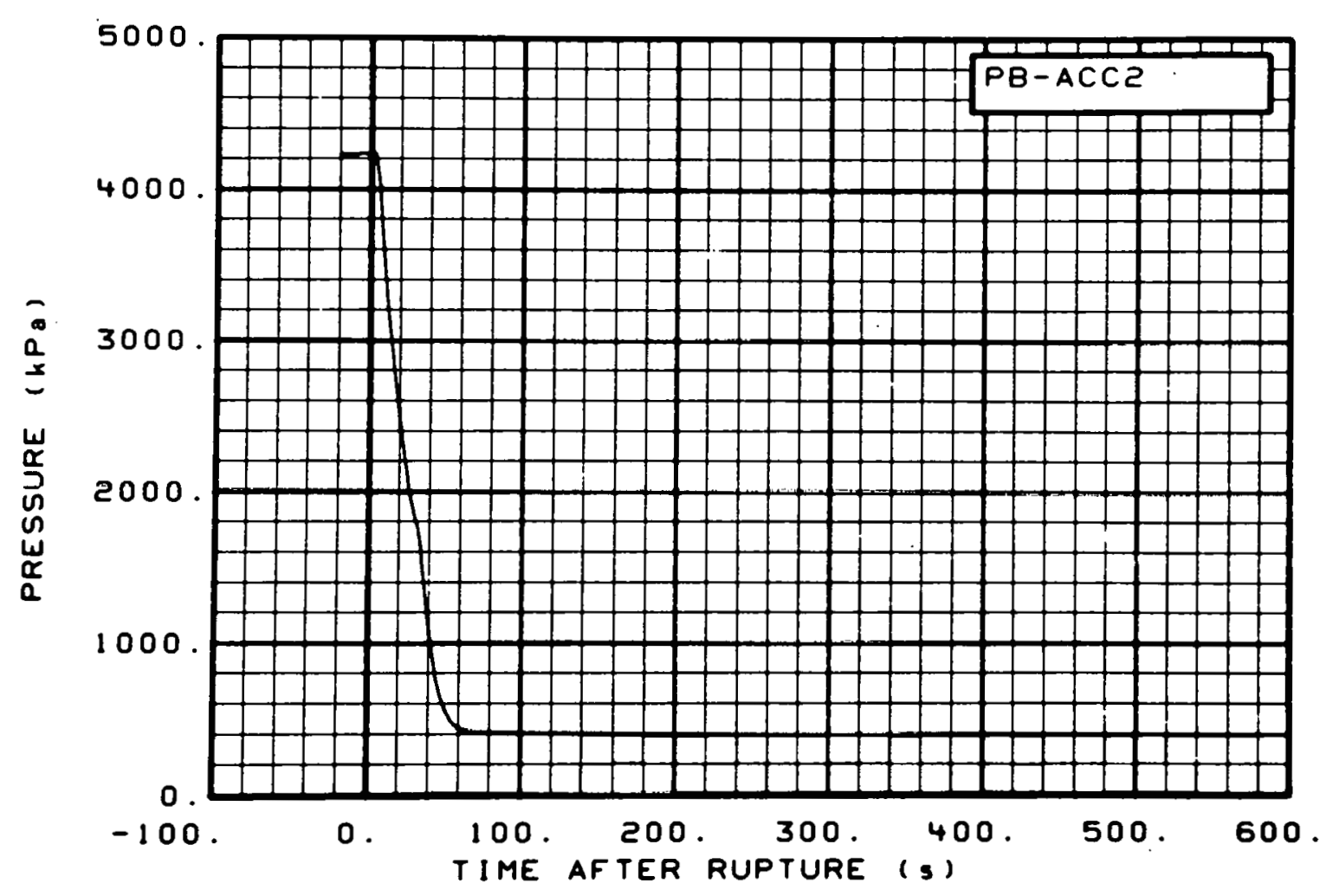

Fig. 155 Pressure in broken loop accumulator (PB-ACC2), from -20 to $600 \mathrm{~s}$.

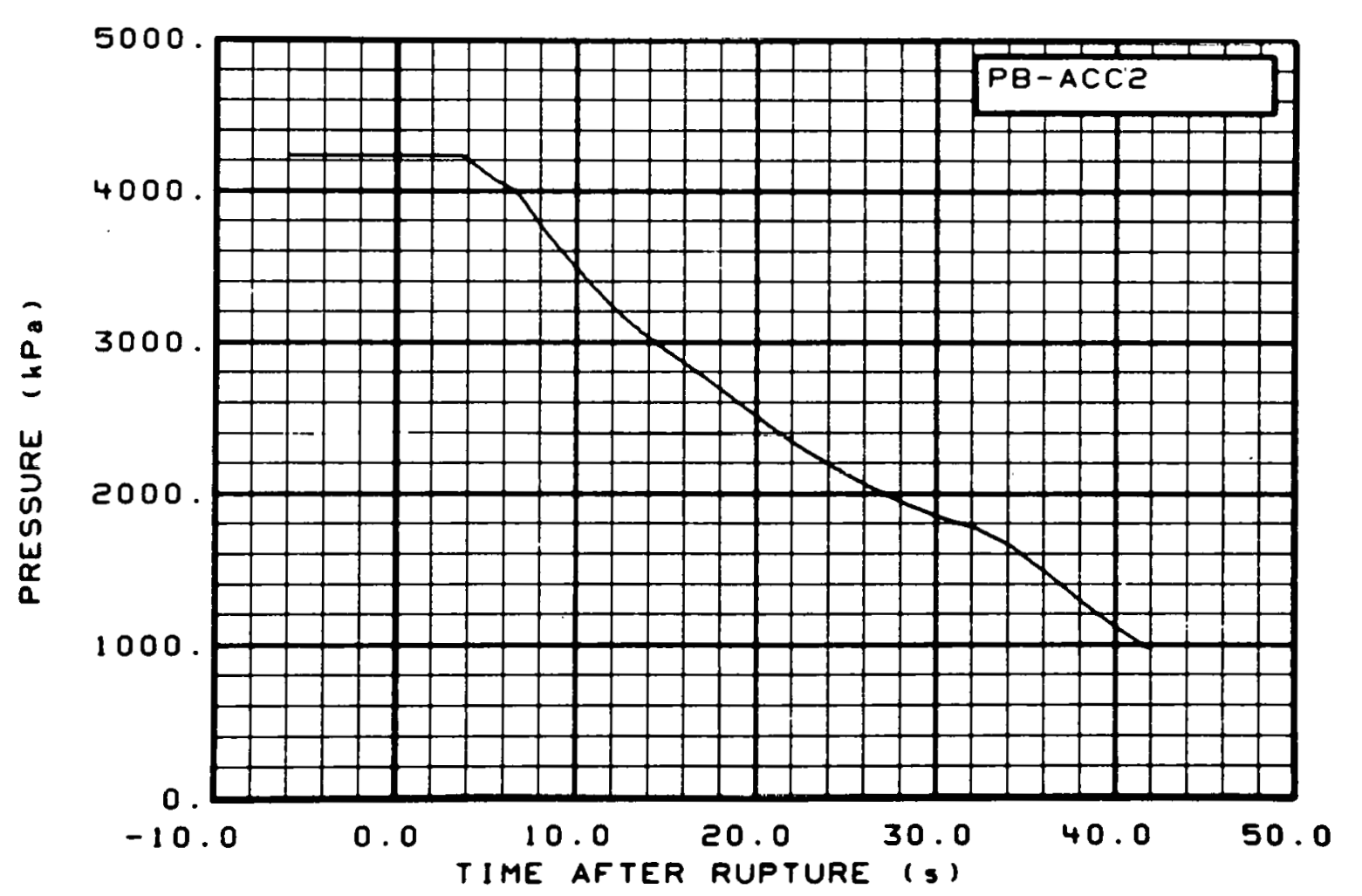

Fig. 156 Pressure in broken loop accumulator (PB-ACC2), from -6 to $42 \mathrm{~s}$. 


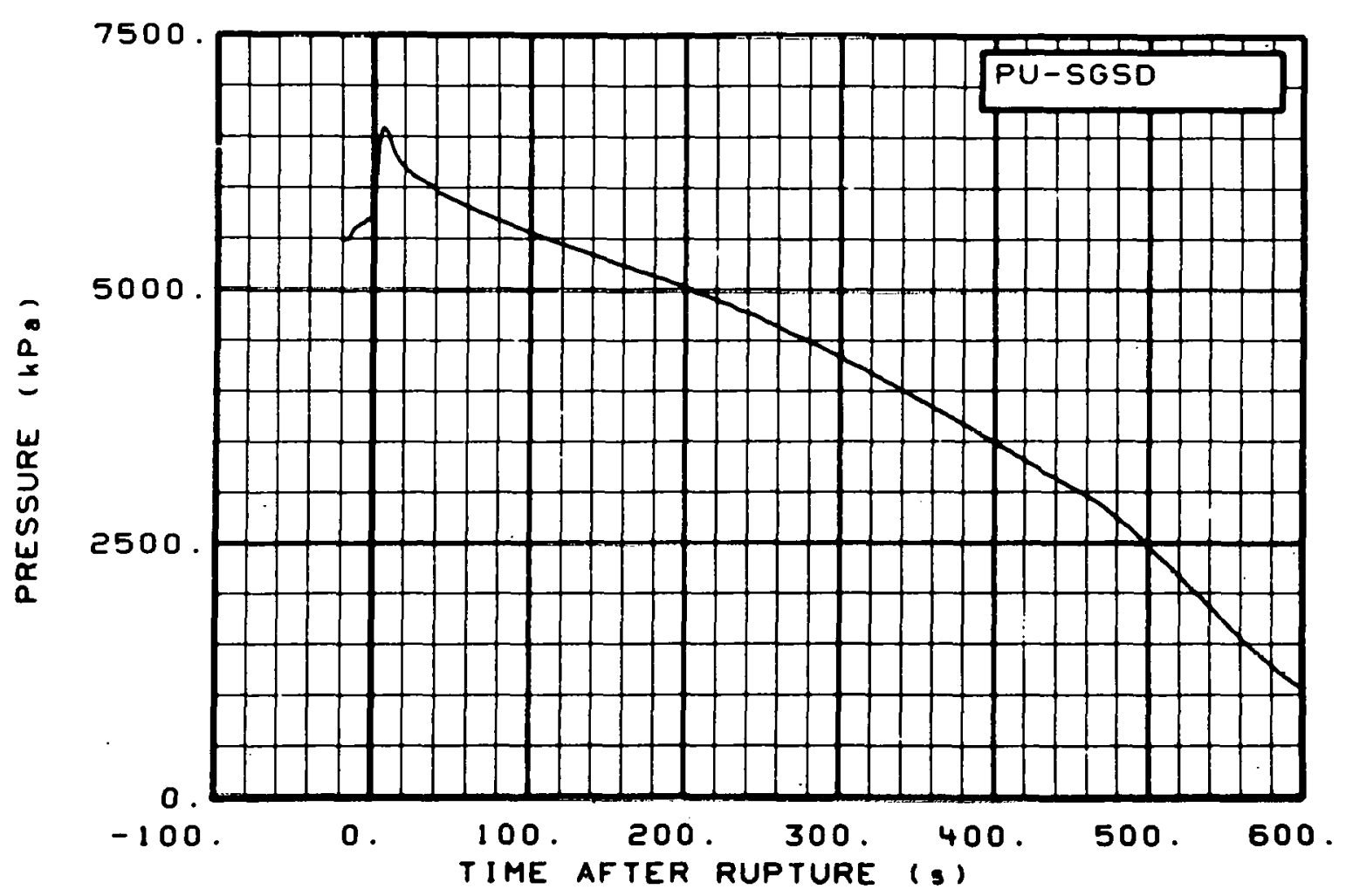

Fig. 157 Pressure in steam generator, secondary side (PU-SGSD), from -20 to $600 \mathrm{~s}$.

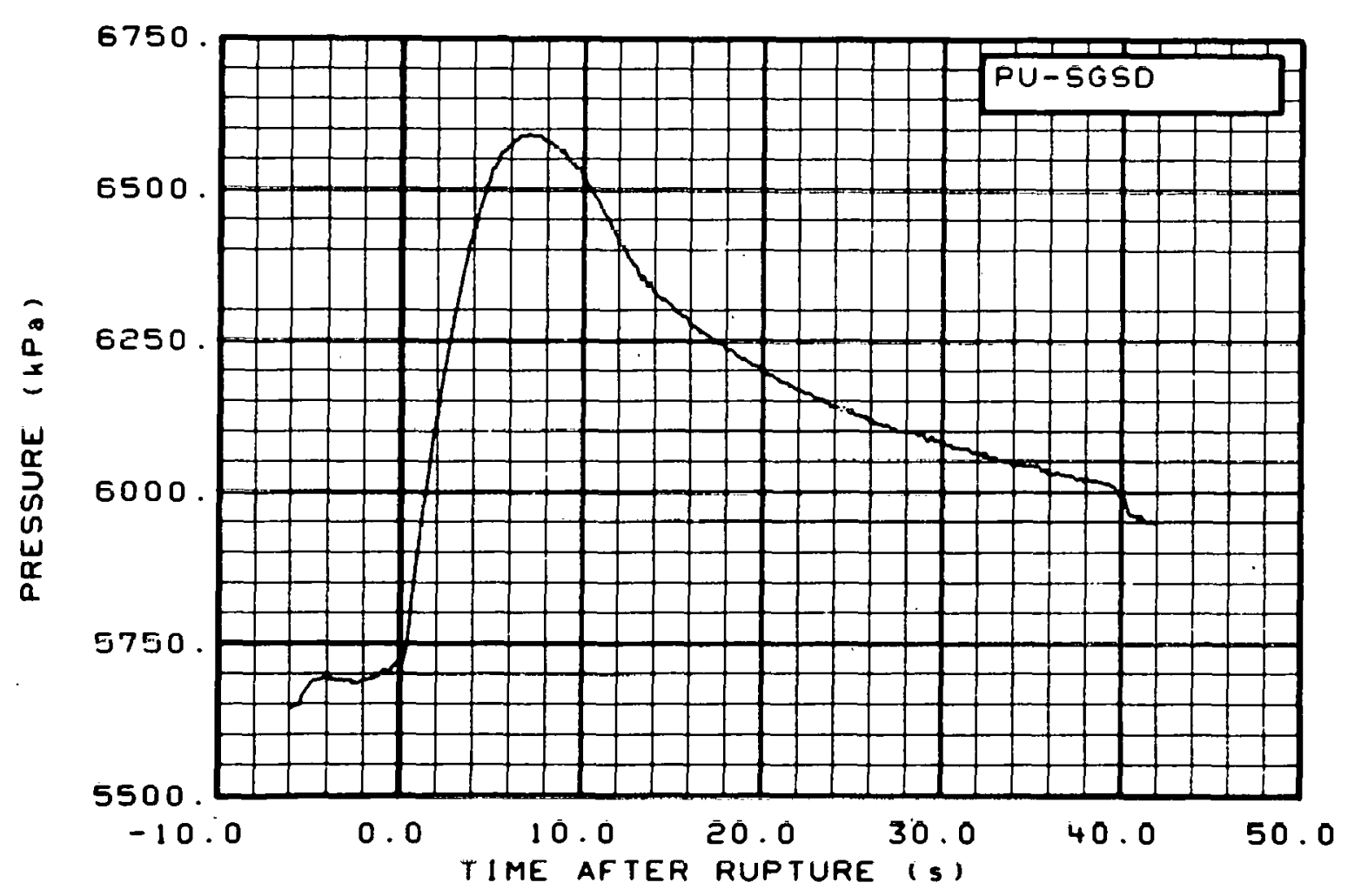

Fig. 158 Pressure in steam generator, secondary side (PU-SGSD), from -6 to $42 \mathrm{~s}$. 


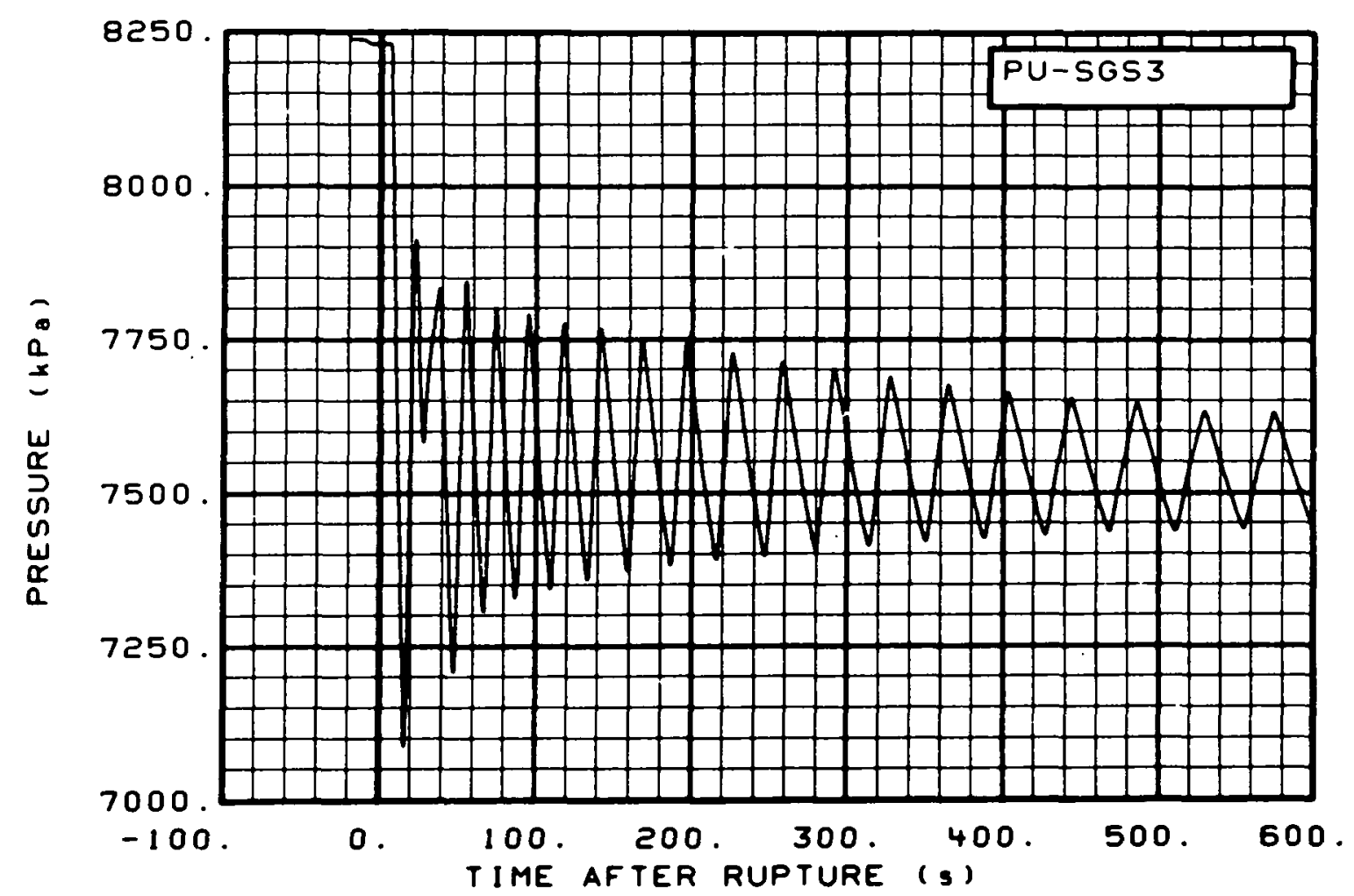

Fig. 159 Pressure in steam generator, tube rupture simulation accumulator (PU-SGS3), from -20 to $600 \mathrm{~s}$.

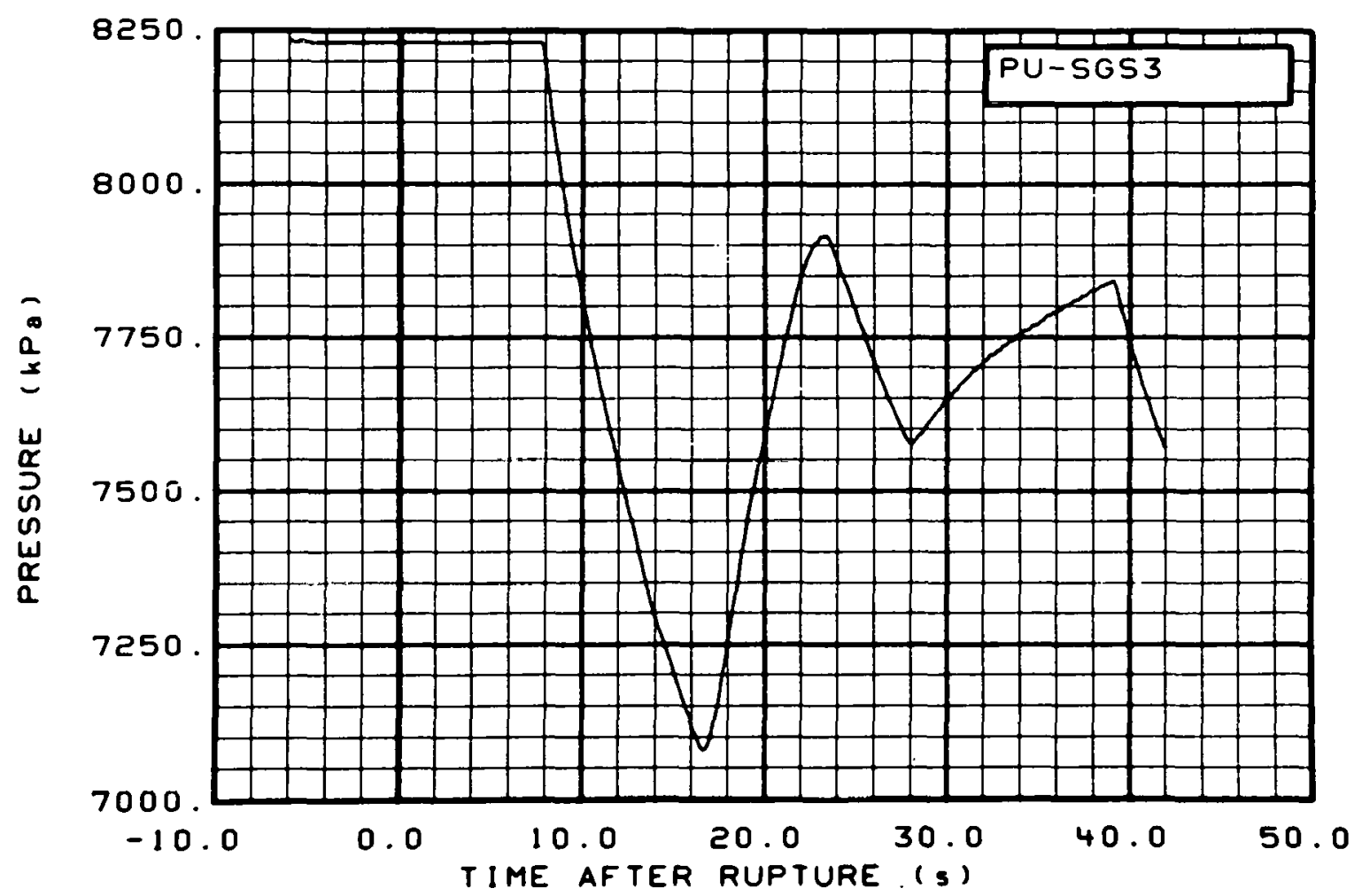

Fig. 160 Pressure in steam generator, tube rupture simulation accumulator (PU-SGS3), from -6 to $42 \mathrm{~s}$, 


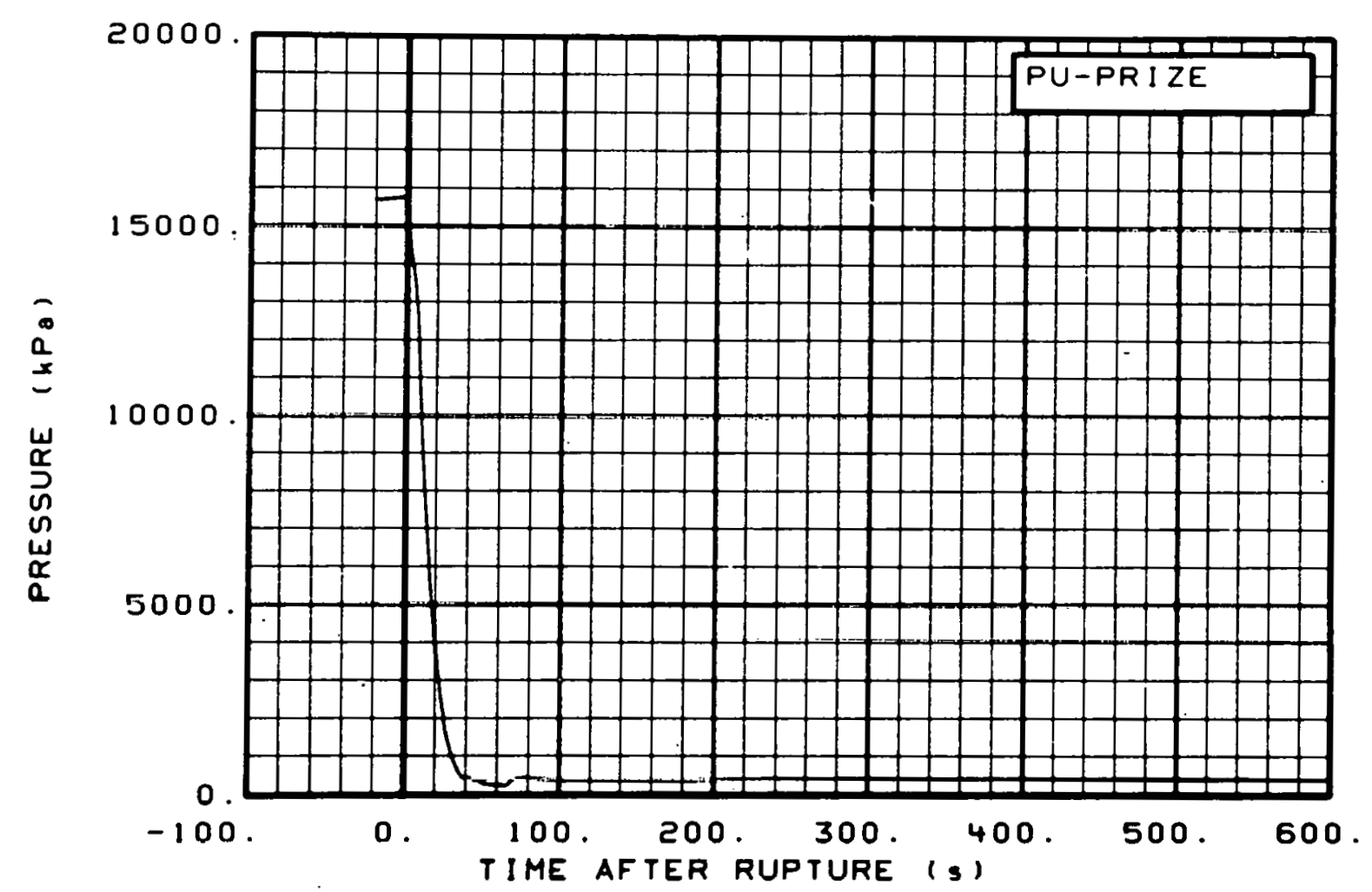

Fig. 161 Pressure in pressurizer (PU-PRIZE), from -20 to $600 \mathrm{~s}$.

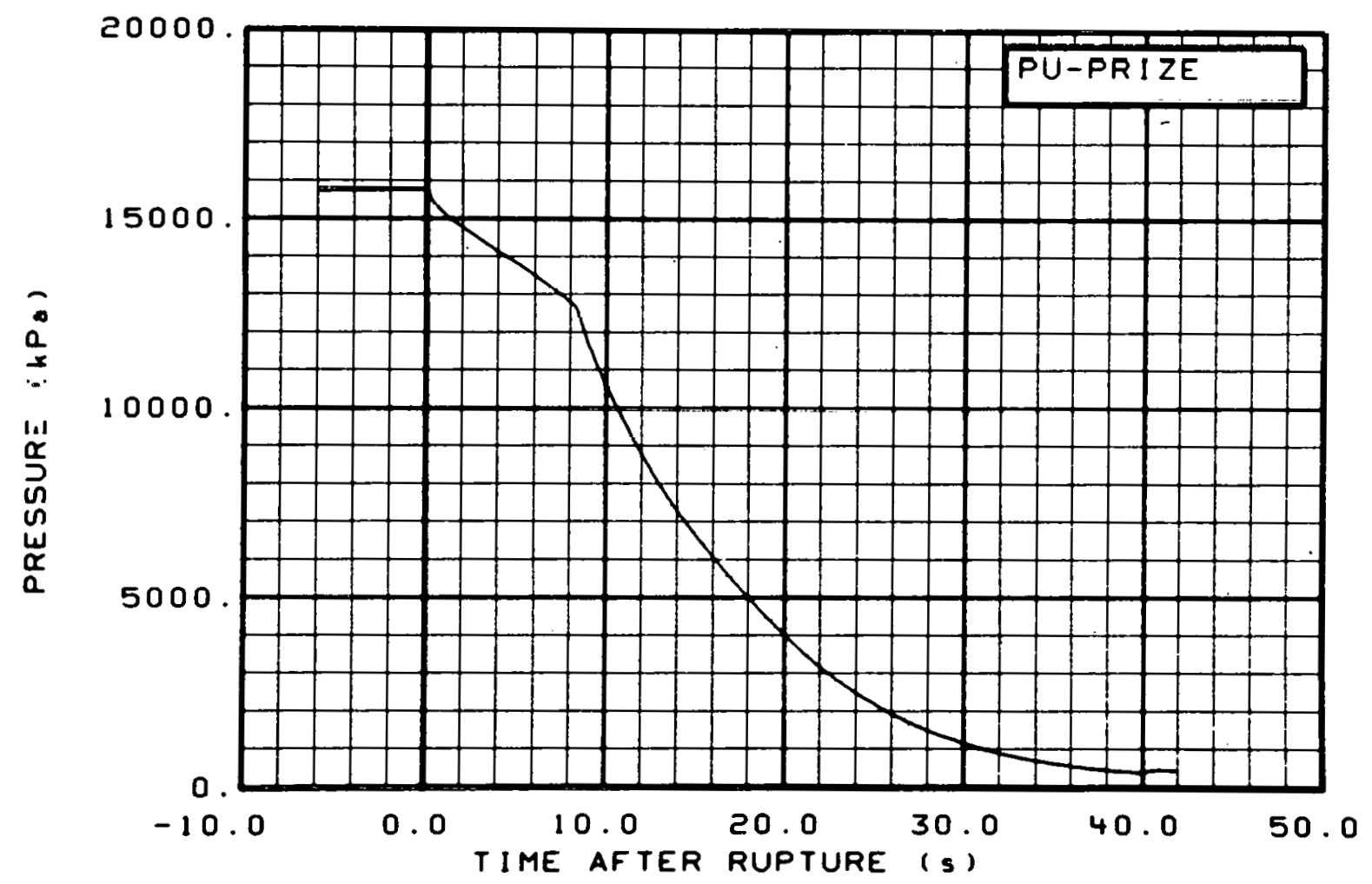

Fig. 162 Pressure in pressurizer (PU-PRIZE), from -6 to $42 \mathrm{~s}$. 


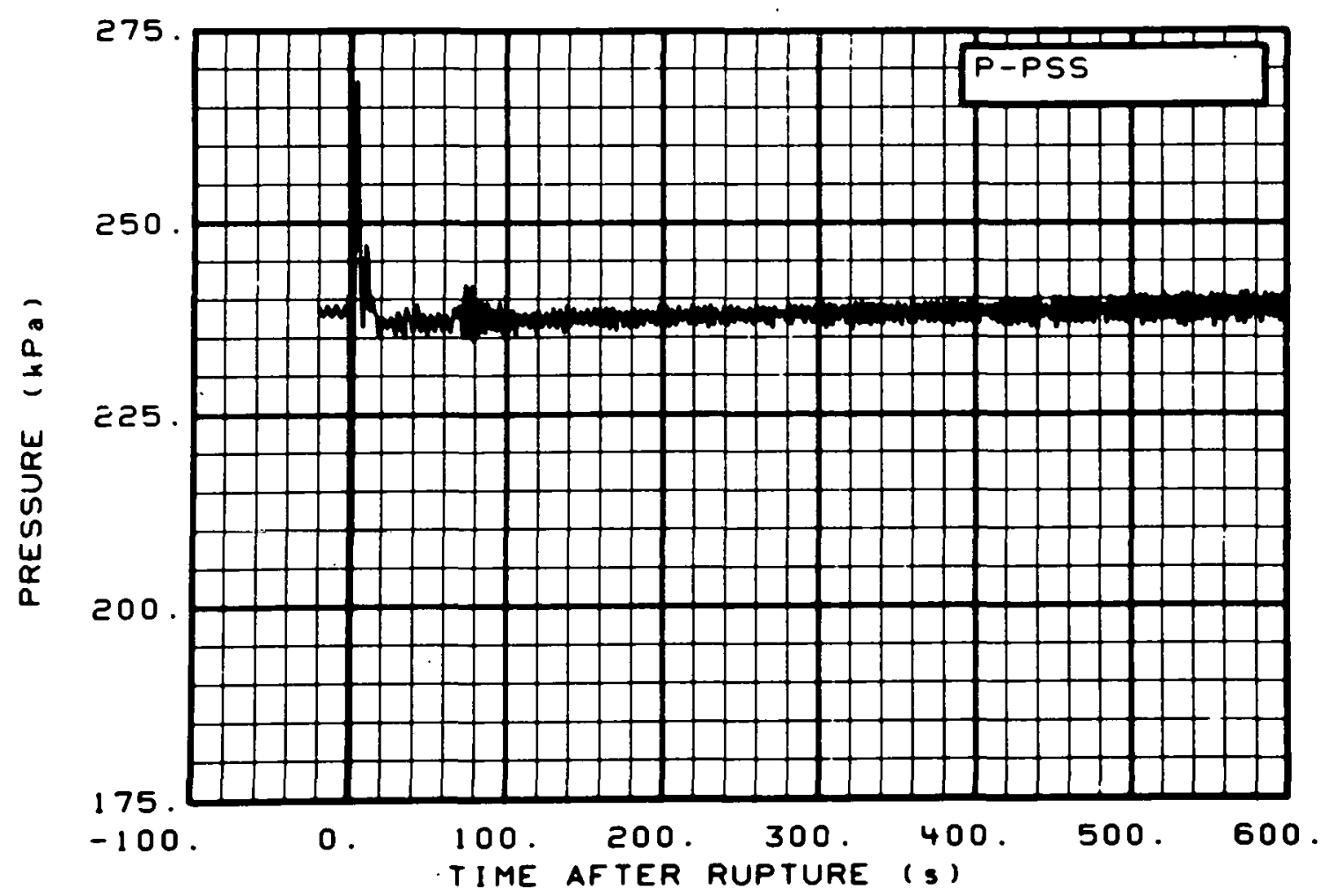

Fig. 163 Pressure in pressure suppression tank (P-PSS), from -20 to $600 \mathrm{~s}$.

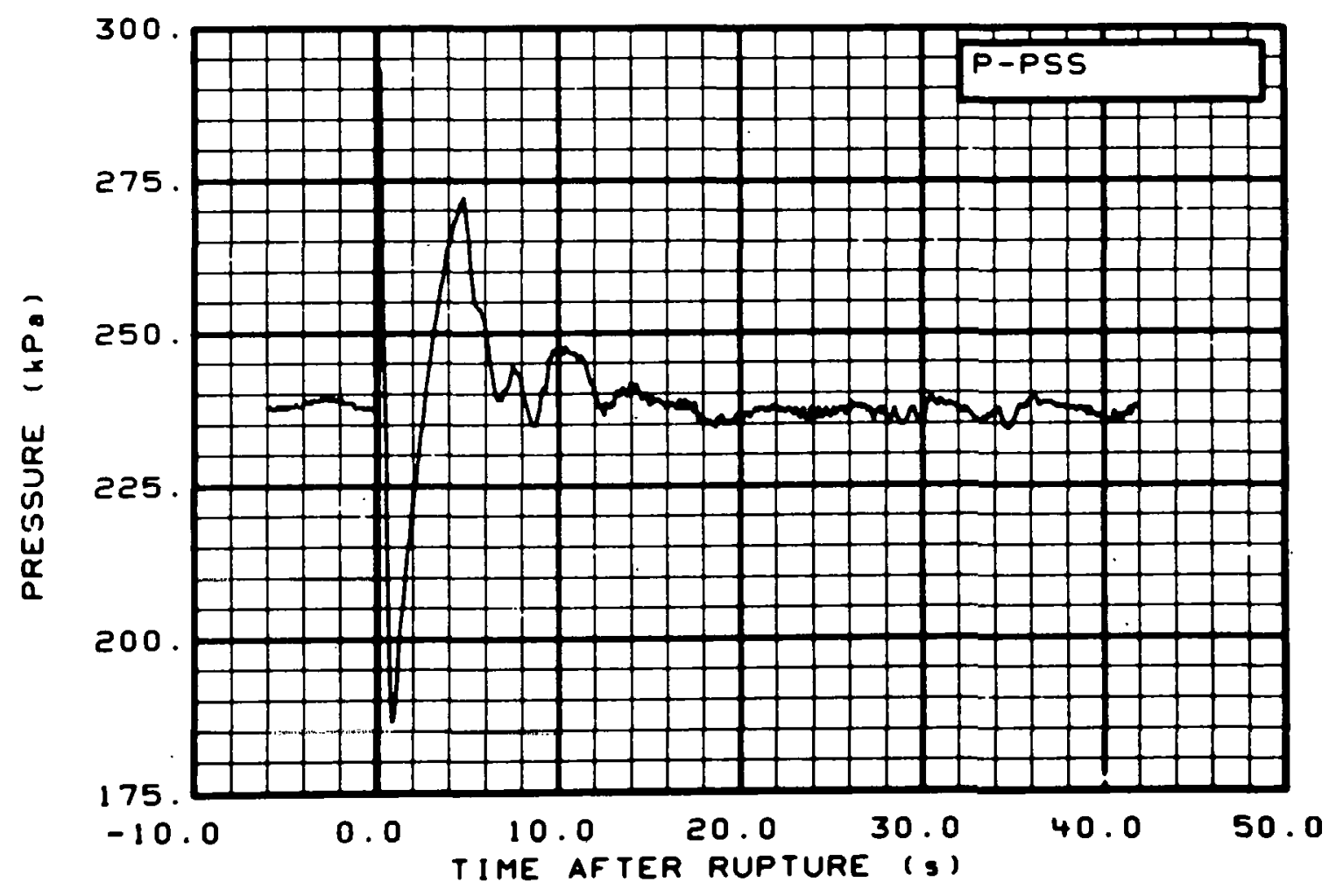

Fig. 164 Pressure in pressure suppression tank (P-PSS), from -6 to $42 \mathrm{~s}$. 


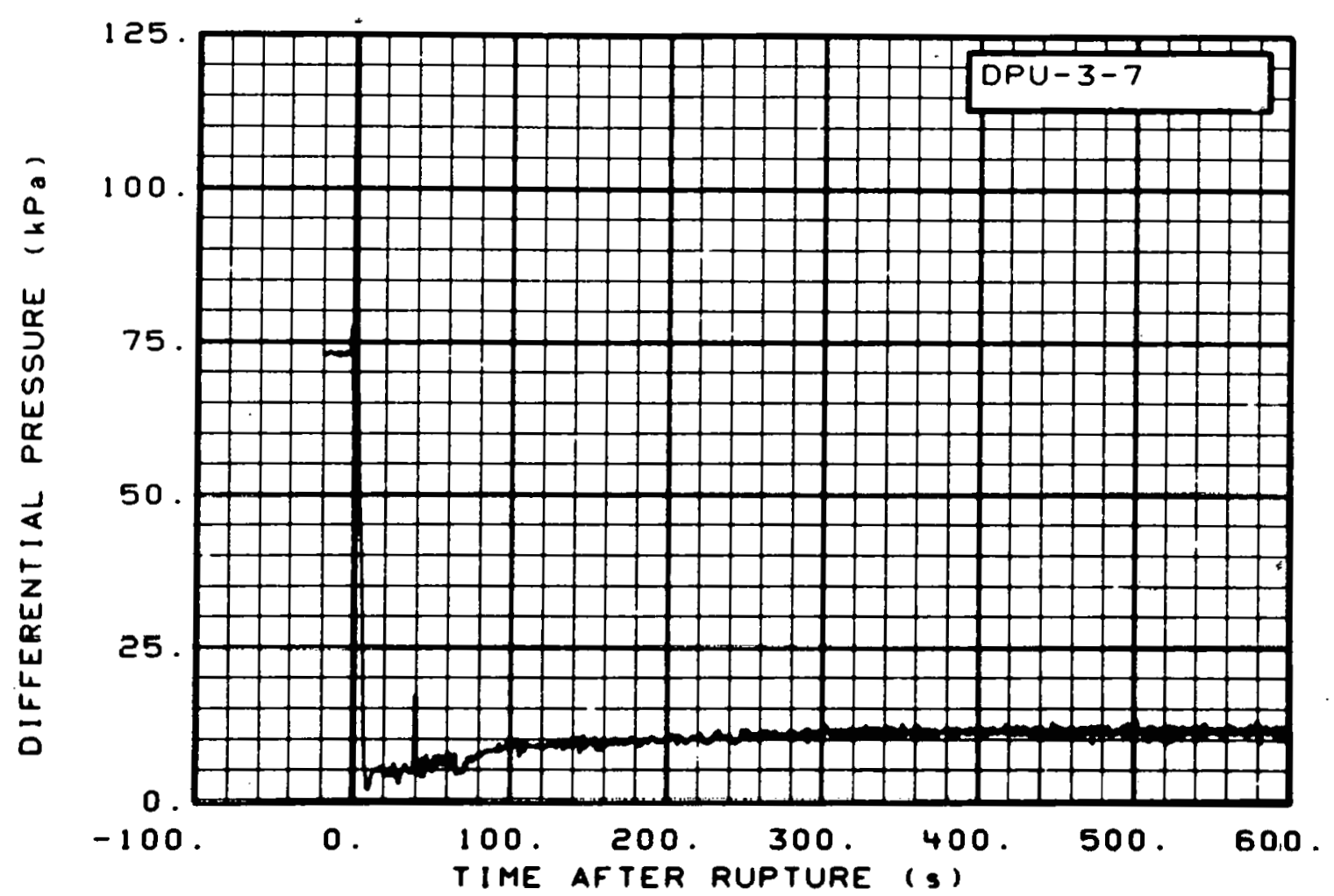

Fig. 165 Differential pressure in intact loop (DPU-3-7), from -20 to $600 \mathrm{~s}$.

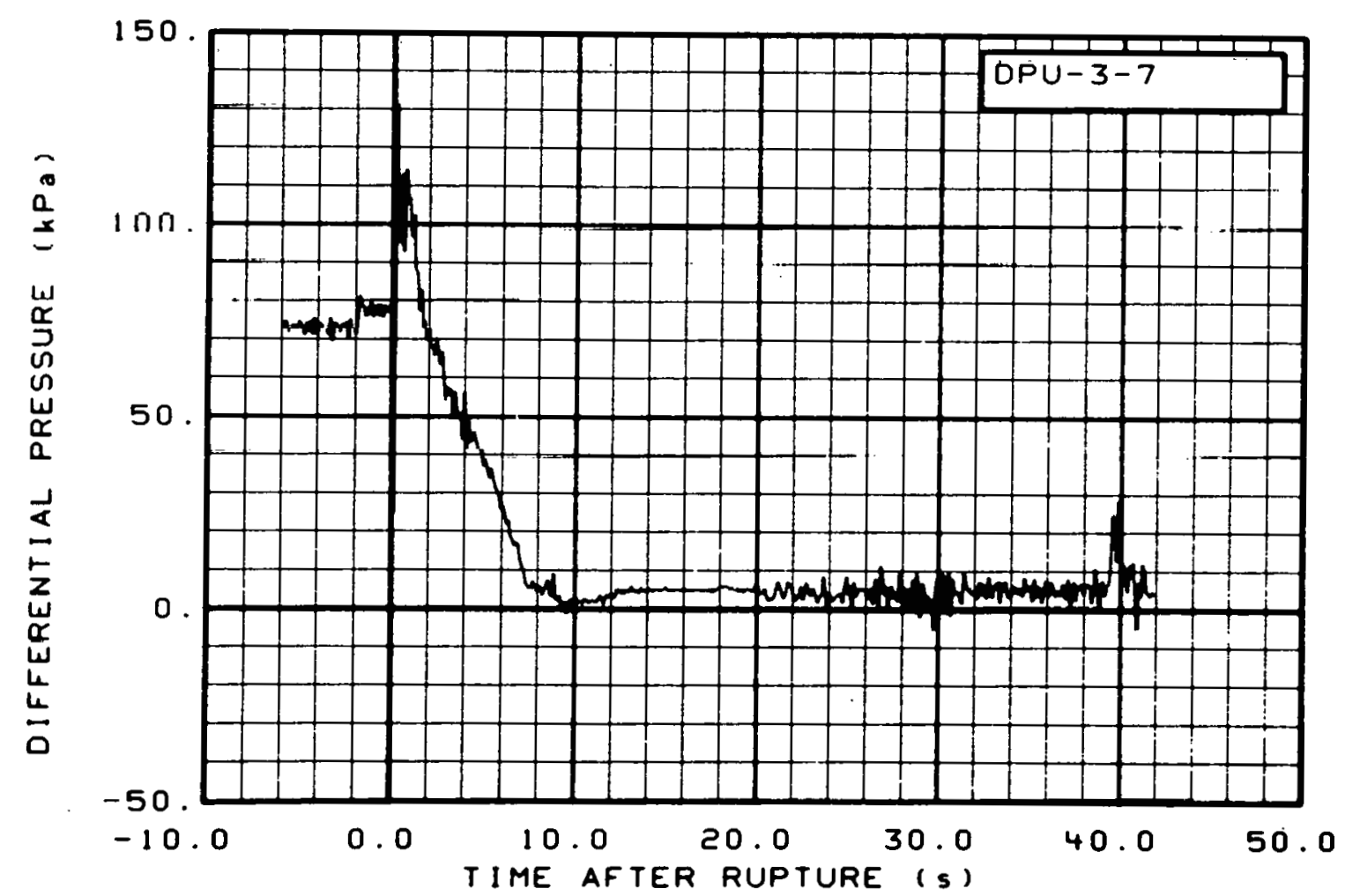

Fig. 166 Differential pressure in intact loop (DPU-3-7), from -6 to $42 \mathrm{~s}$. 


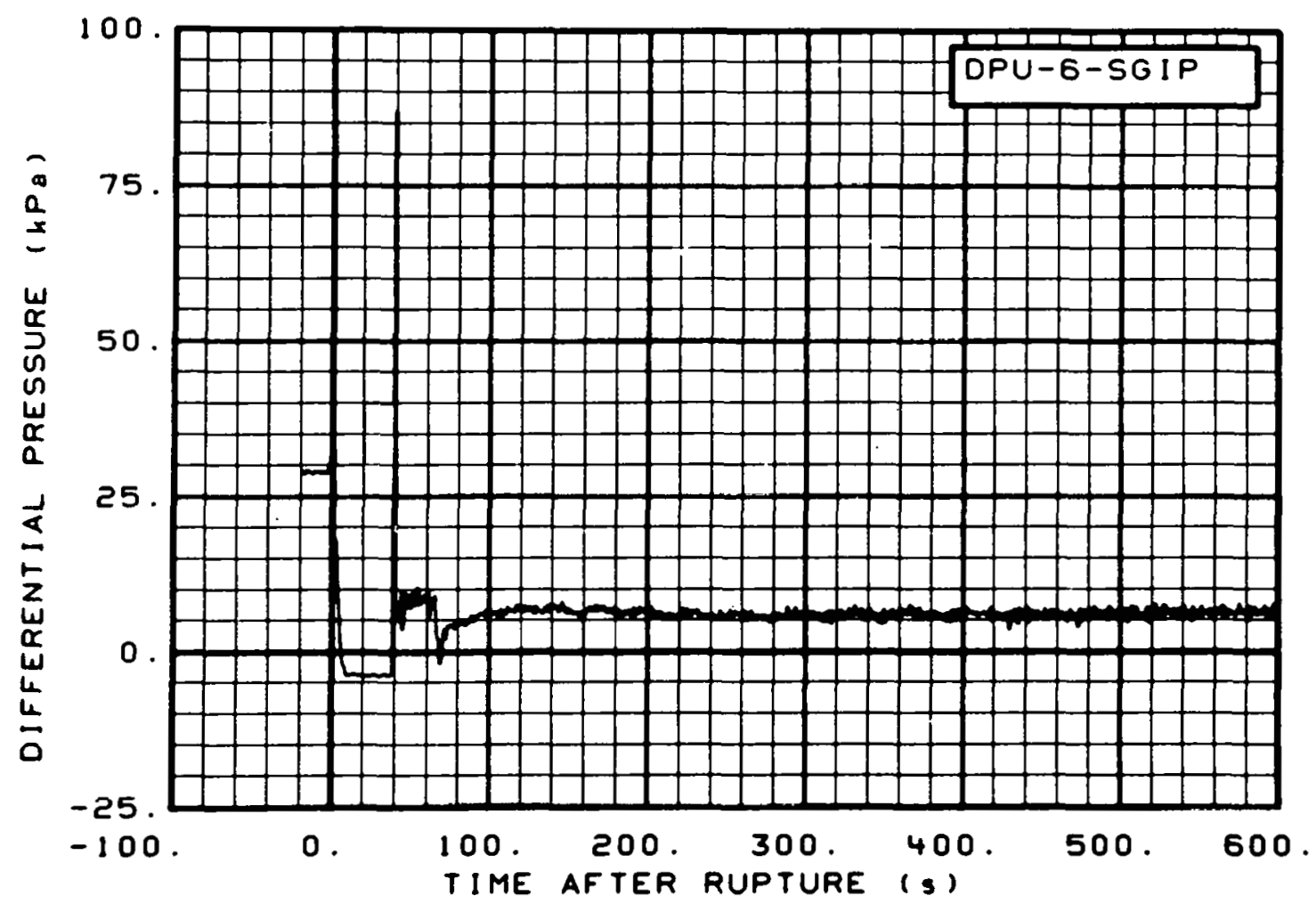

Fig. 167 Differential pressure in intact loop (DPU-6-SGIP), from -20 to $600 \mathrm{~s}$.

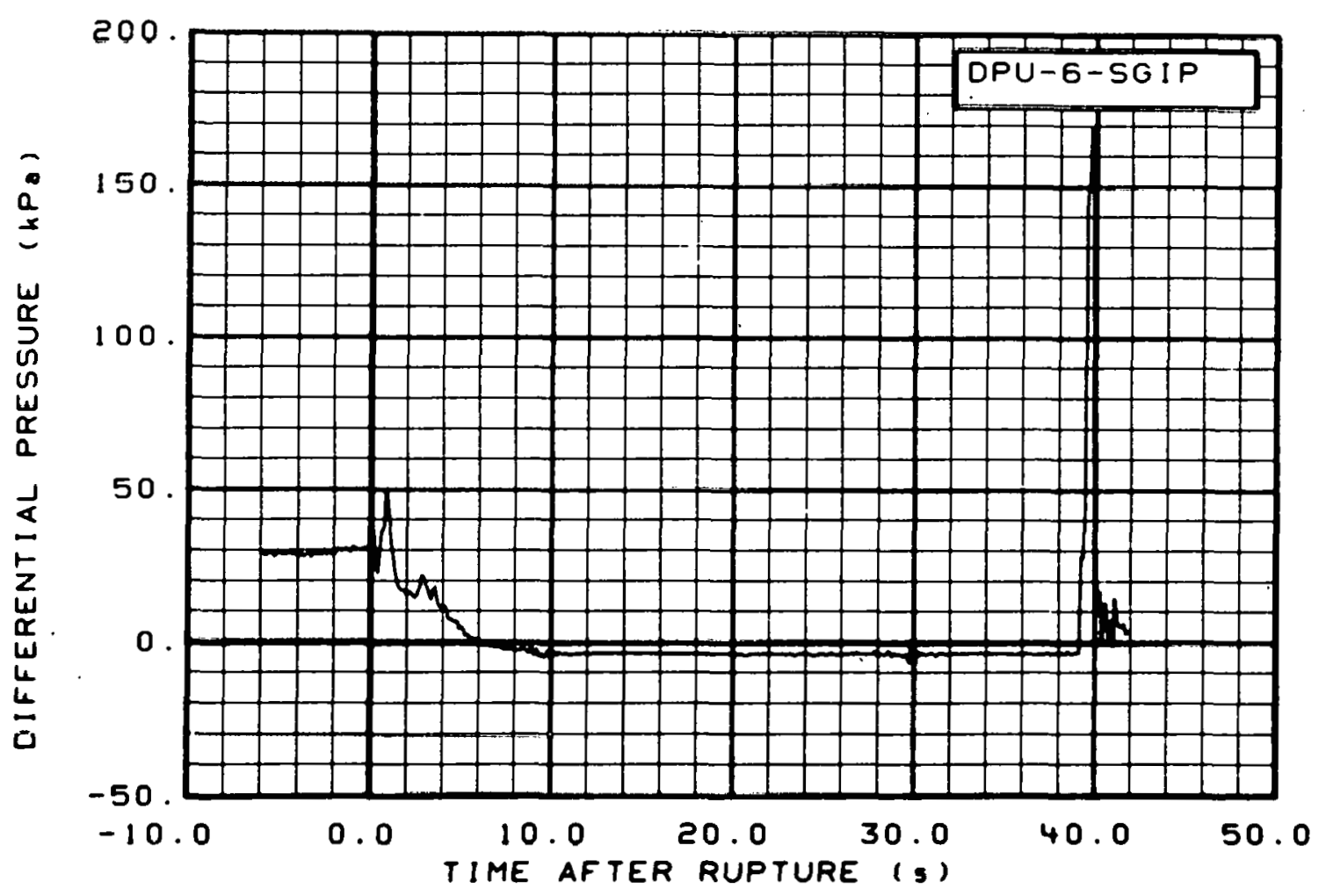

Fig. 168 Differential pressure in intact 10op (DPU-6-SGIP), from -6 to $42 \mathrm{~s}$. 


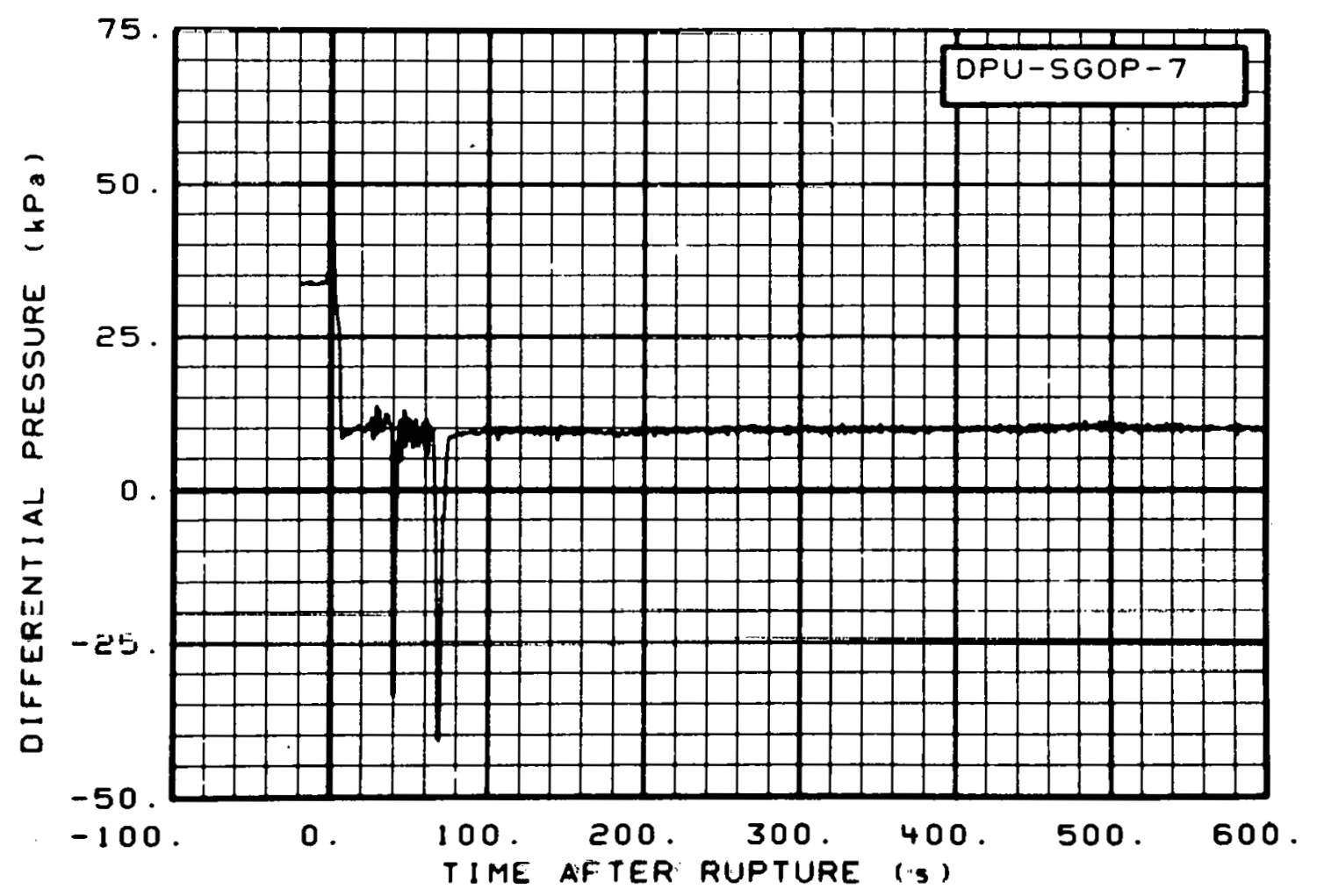

Fig. 169 Differential pressure in intact loop (DPU-SGOP-7), from -20 to $600 \mathrm{~s}$.

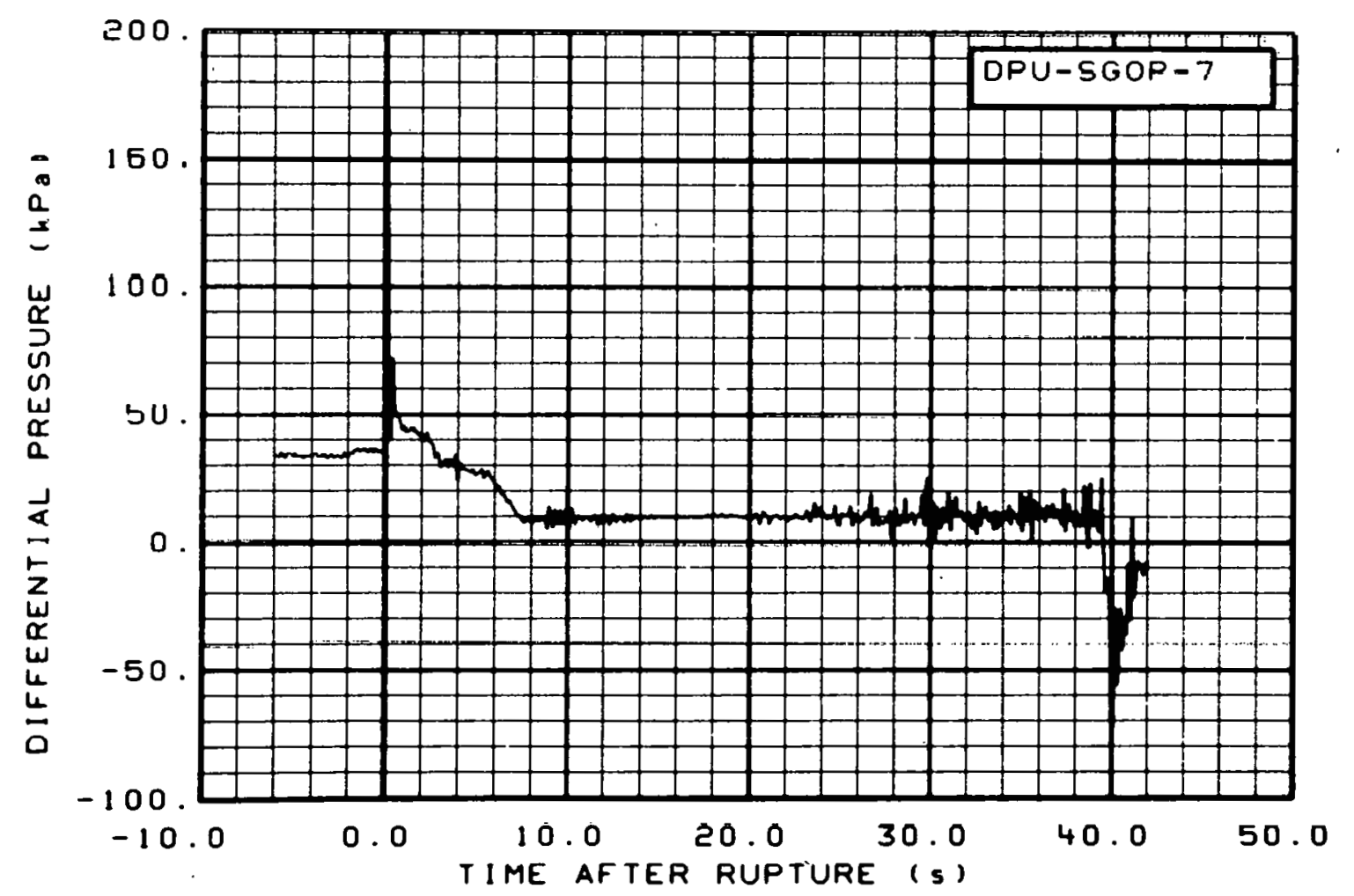

Fig. 170 Differential pressure in intact loop (DPU-SGOP-7), from -6 to $42 \mathrm{~s}$. 


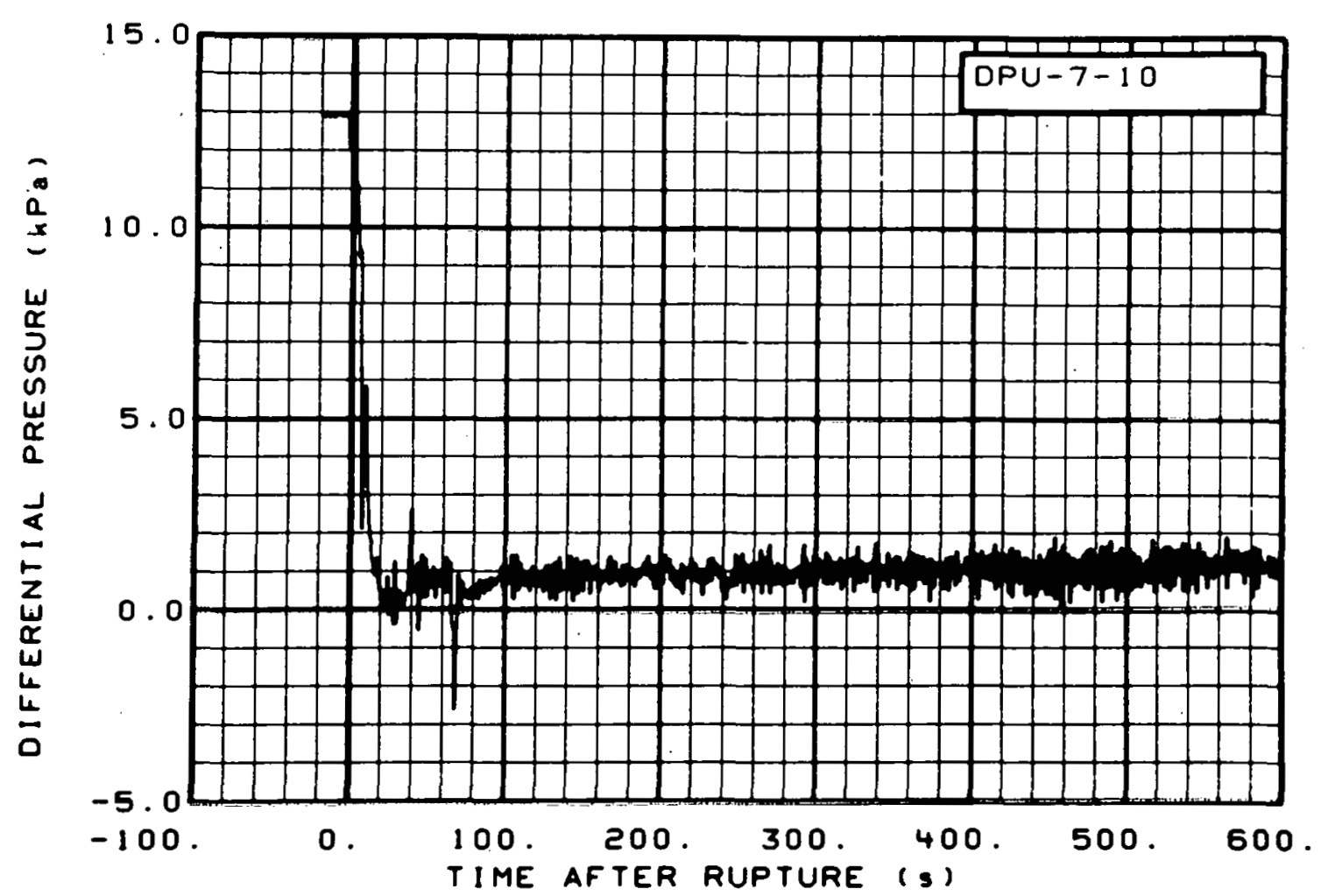

Fig. 171 Differential pressure in intact loop (DPU-7-10), from -20 to $600 \mathrm{~s}$.

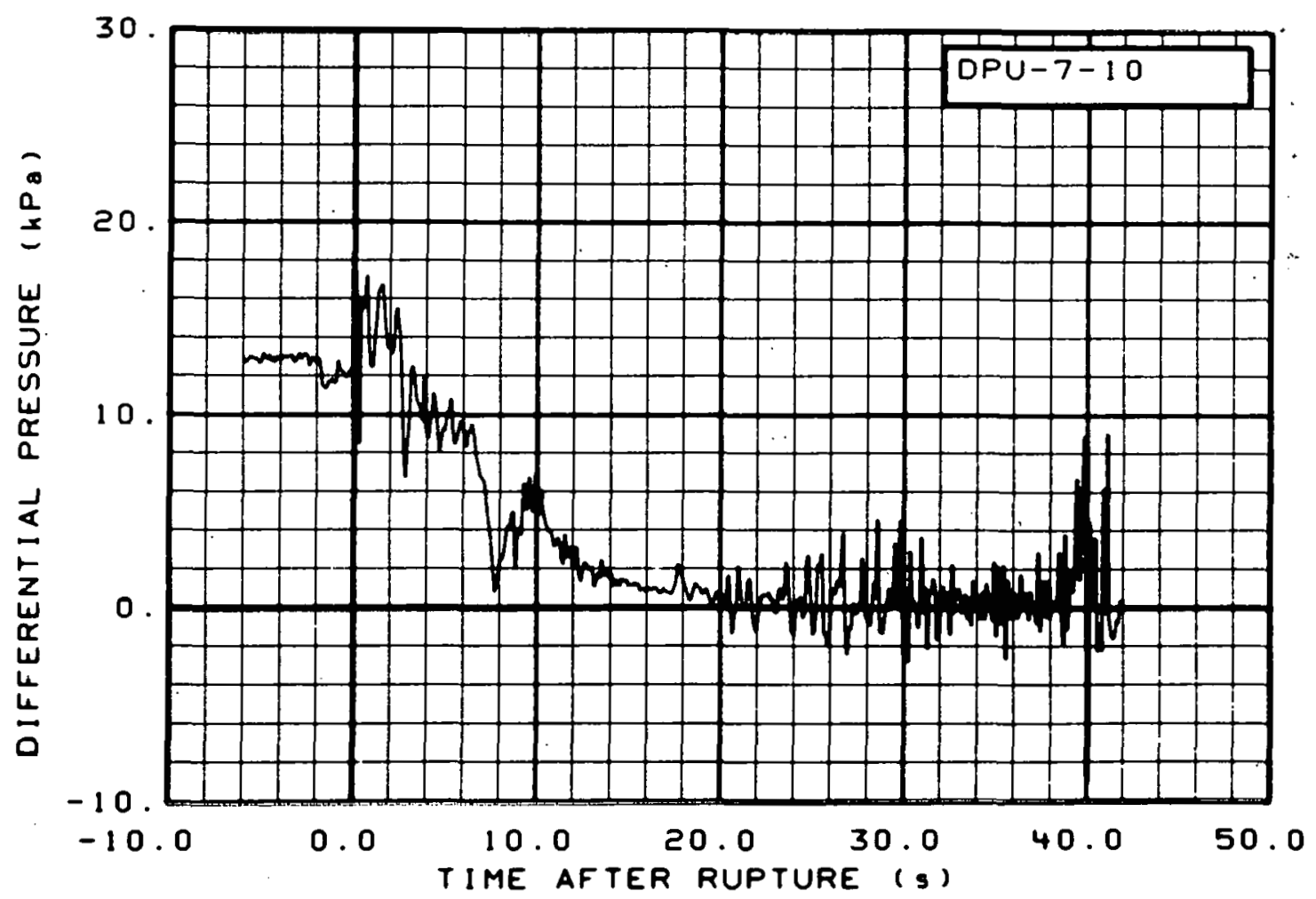

Fig. 172 Differential pressure in intact loop (DPU-7-10), from -6 to $42 \mathrm{~s}$. 


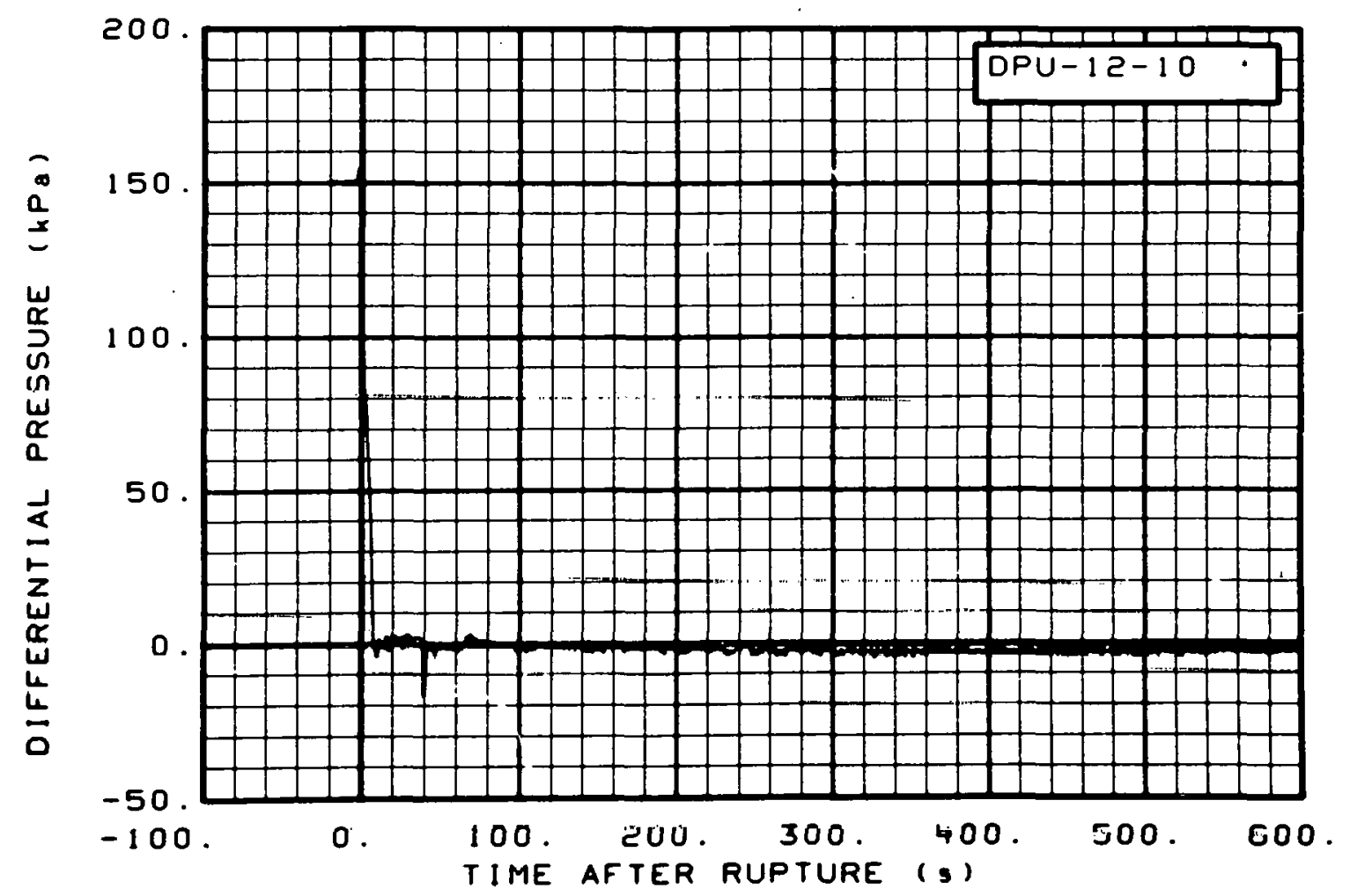

Fig. 173 Differential pressure in intact loop (DPU-12-10), from -20 to $600 \mathrm{~s}$.

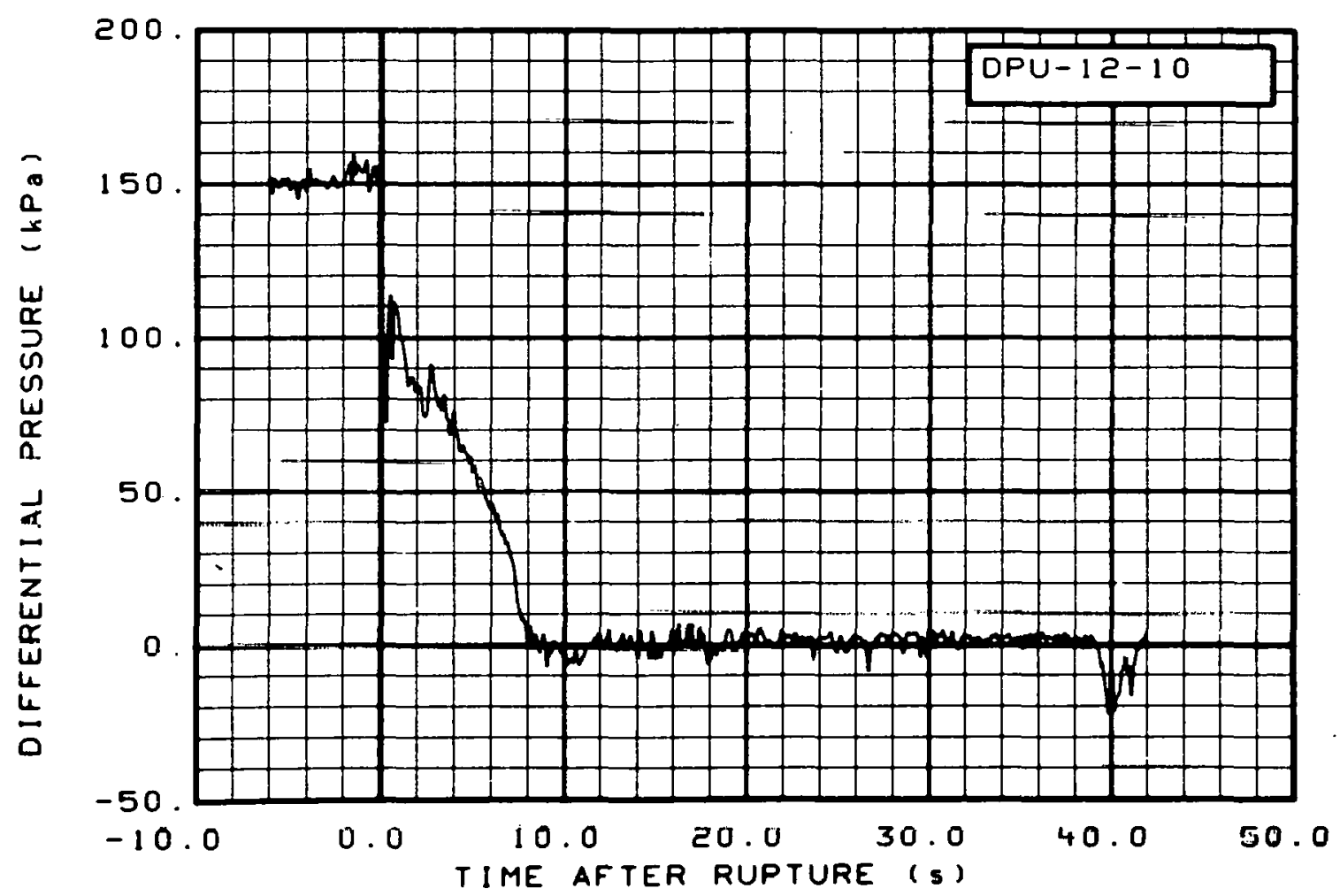

Fig. 174 Differential pressure in intact loop (DPU-12-10), from -6 to $42 \mathrm{~s}$. 


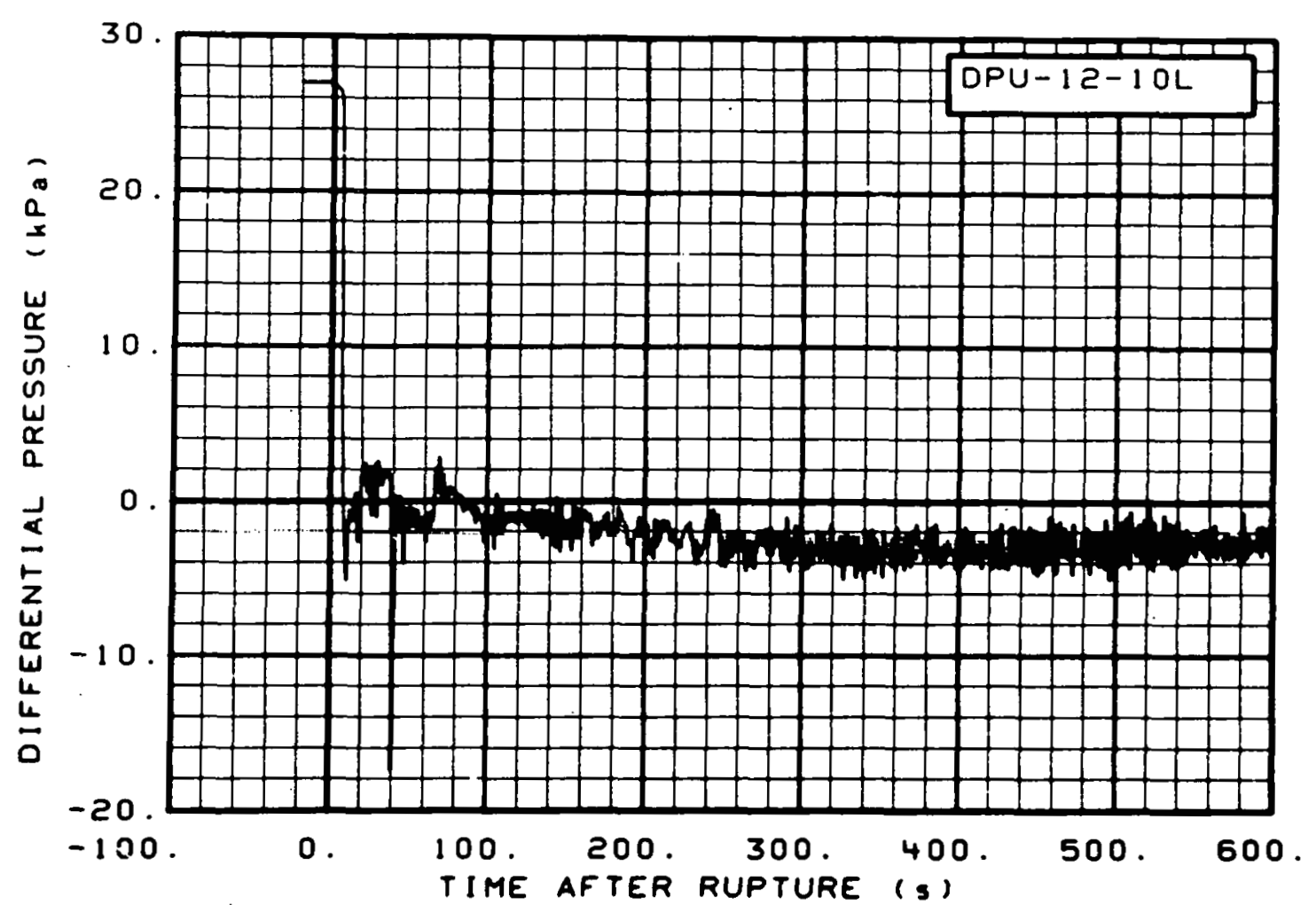

Fig. 175 Differential pressure in intact loop, low range (DPU-12-10L), from -20 to $600 \mathrm{~s}$.

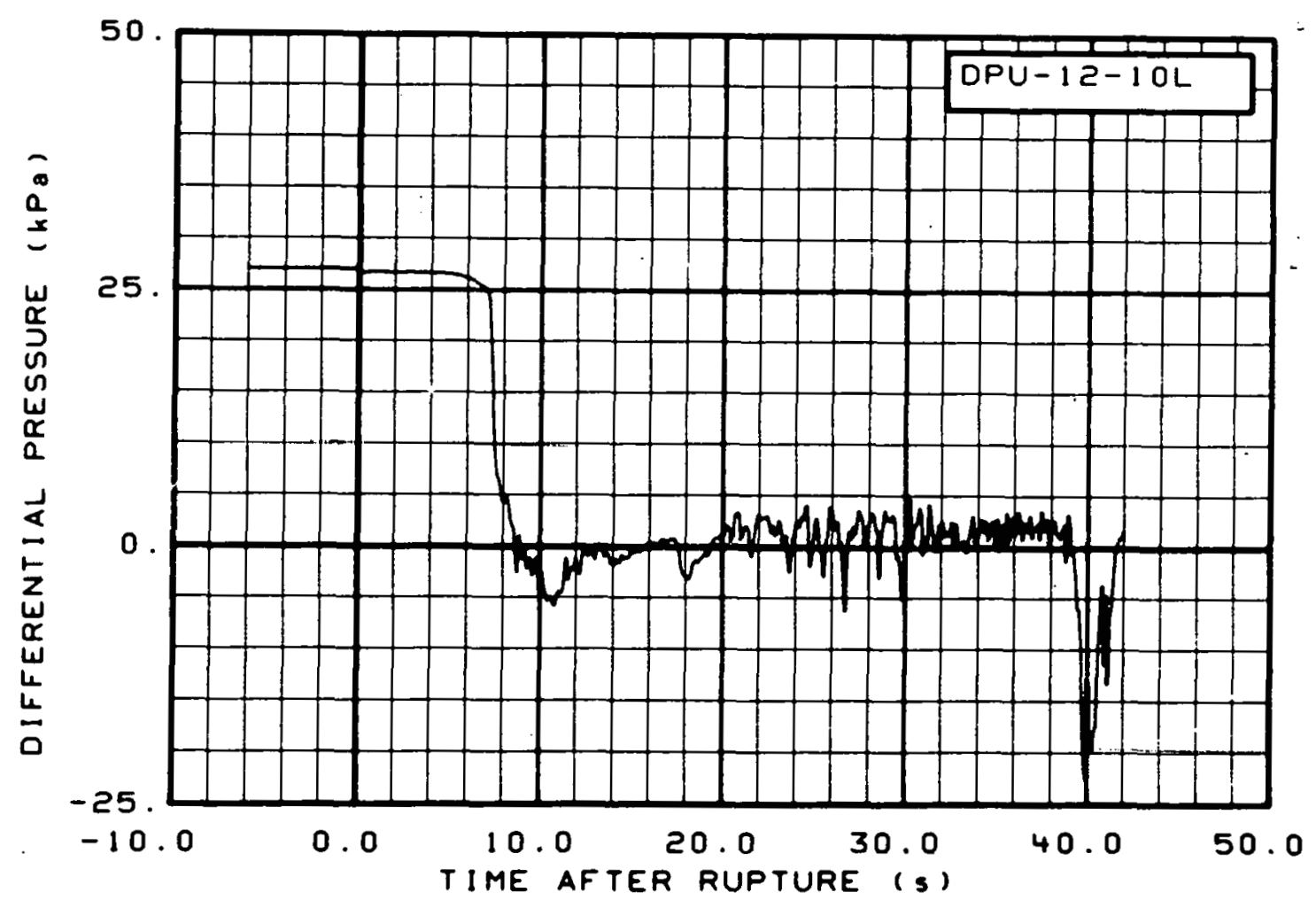

Fig. 176 Differential pressure in intact loop, low range (DPU-12-10L), from -6 to $42 \mathrm{~s}$. 


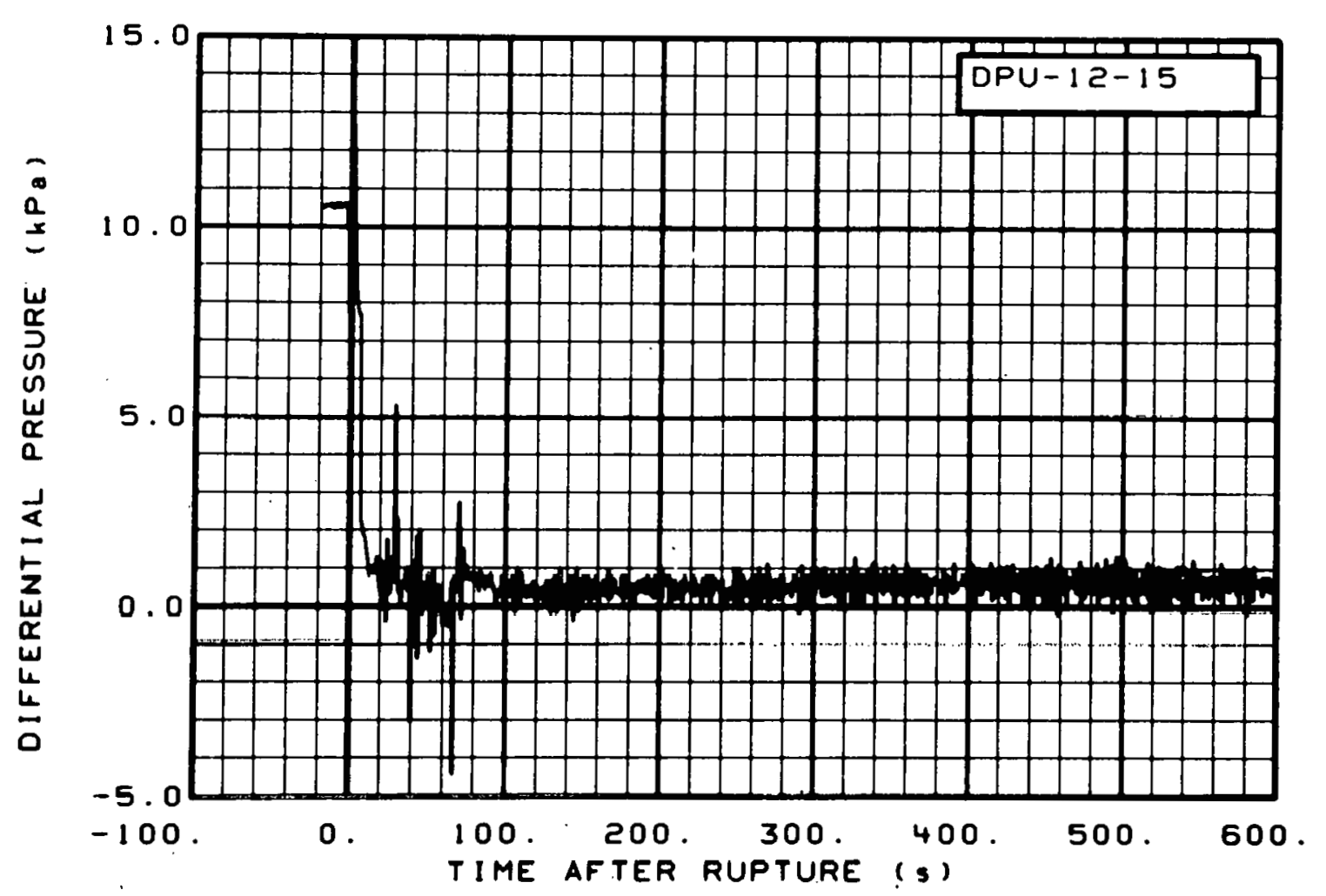

Fig. 177 Differential pressure in intact loop (DPU-12-15), from -20 to $600 \mathrm{~s}$.

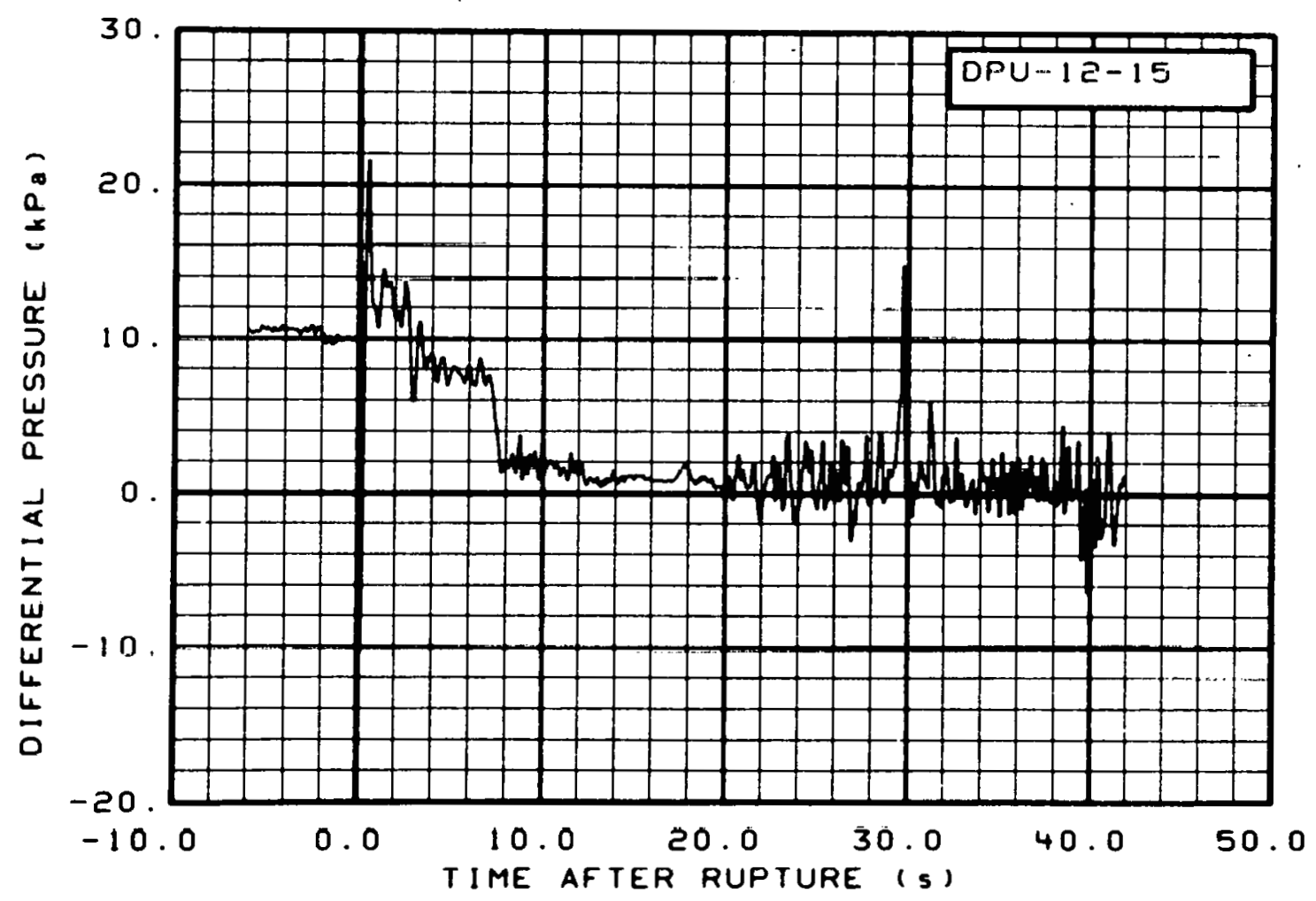

Fig. 178 Differential pressure in intact loop (DPU-12-15), from -6 to $42 \mathrm{~s}$. 


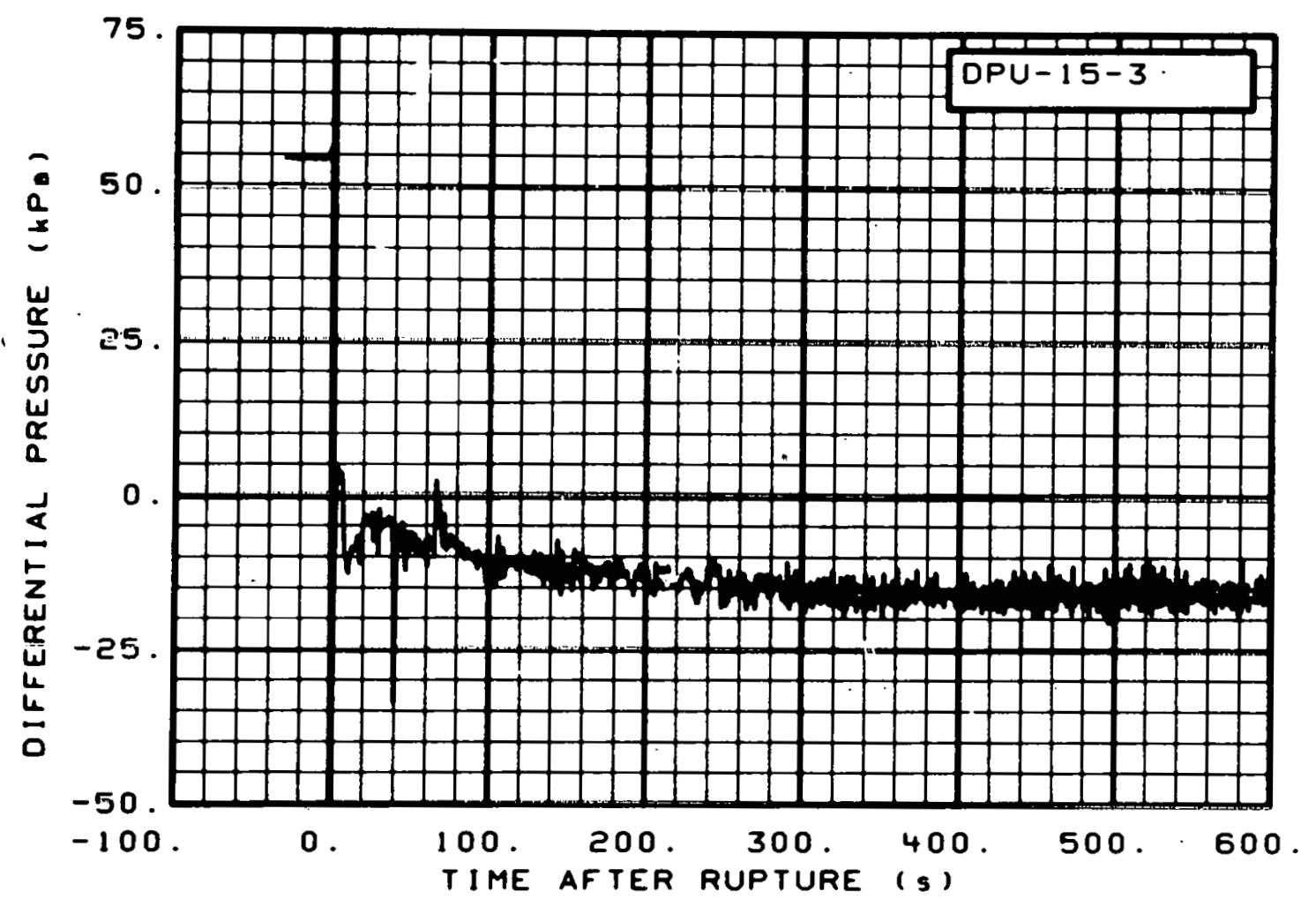

Fig. 179 Differential pressure in intact loop (DPU-15-3), from -20 to $600 \mathrm{~s}$.

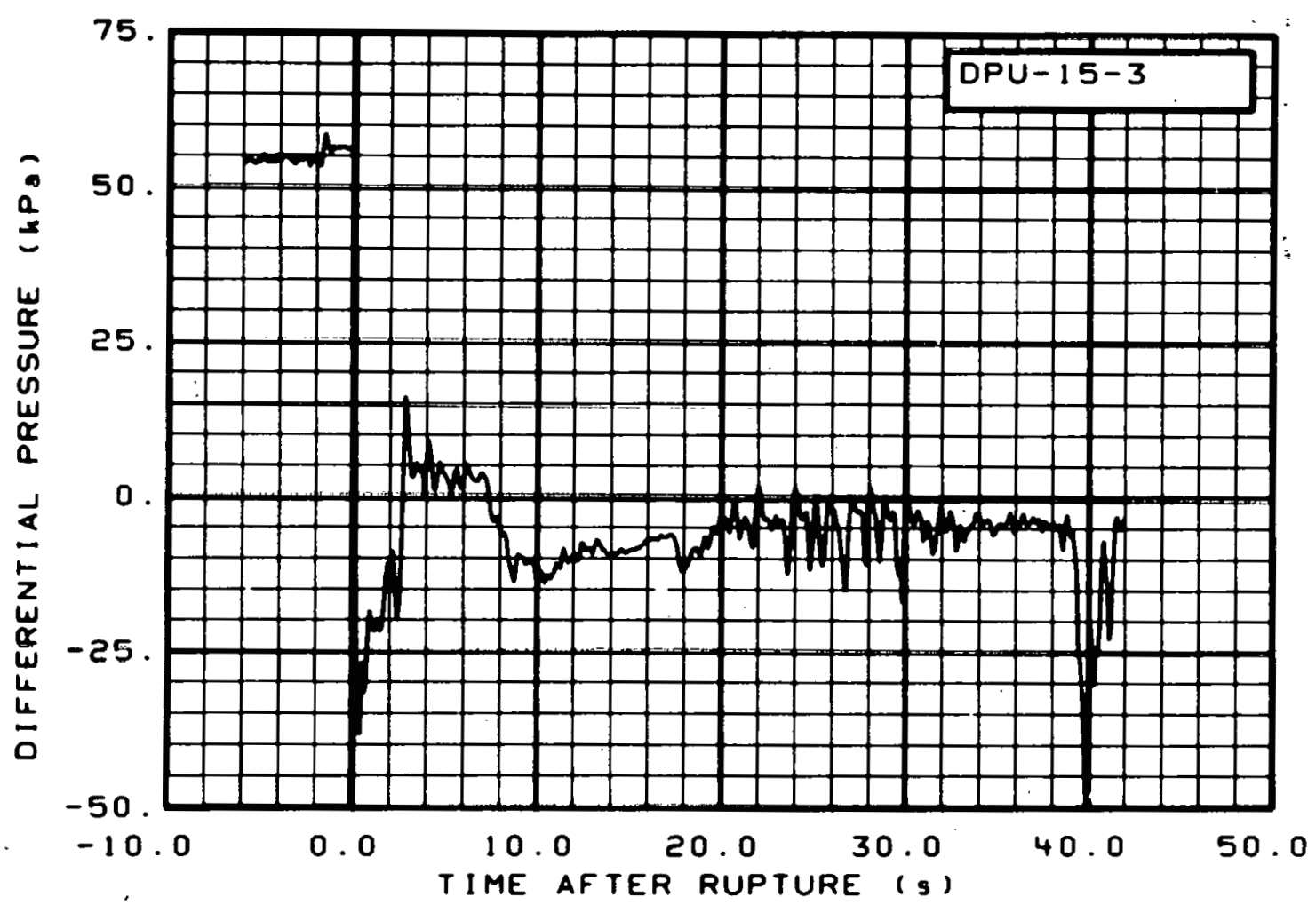

Fig. 180 Differential pressure in intact loop (DPU-15-3), from -6 to $42 \mathrm{~s}$. 


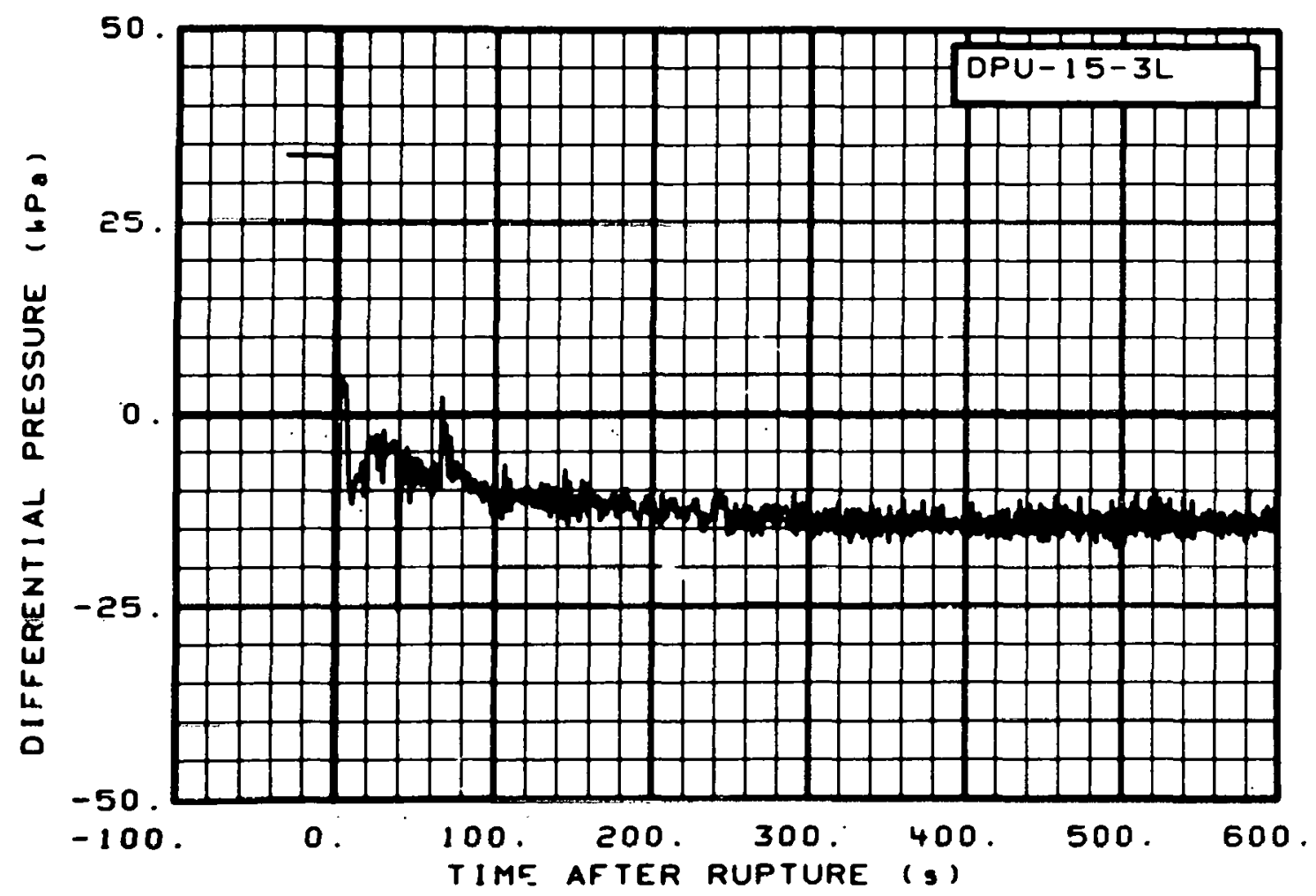

Fig. 181 Differential pressure in intact loop, low range (DPU-15-3L), from -20 to $600 \mathrm{~s}$.

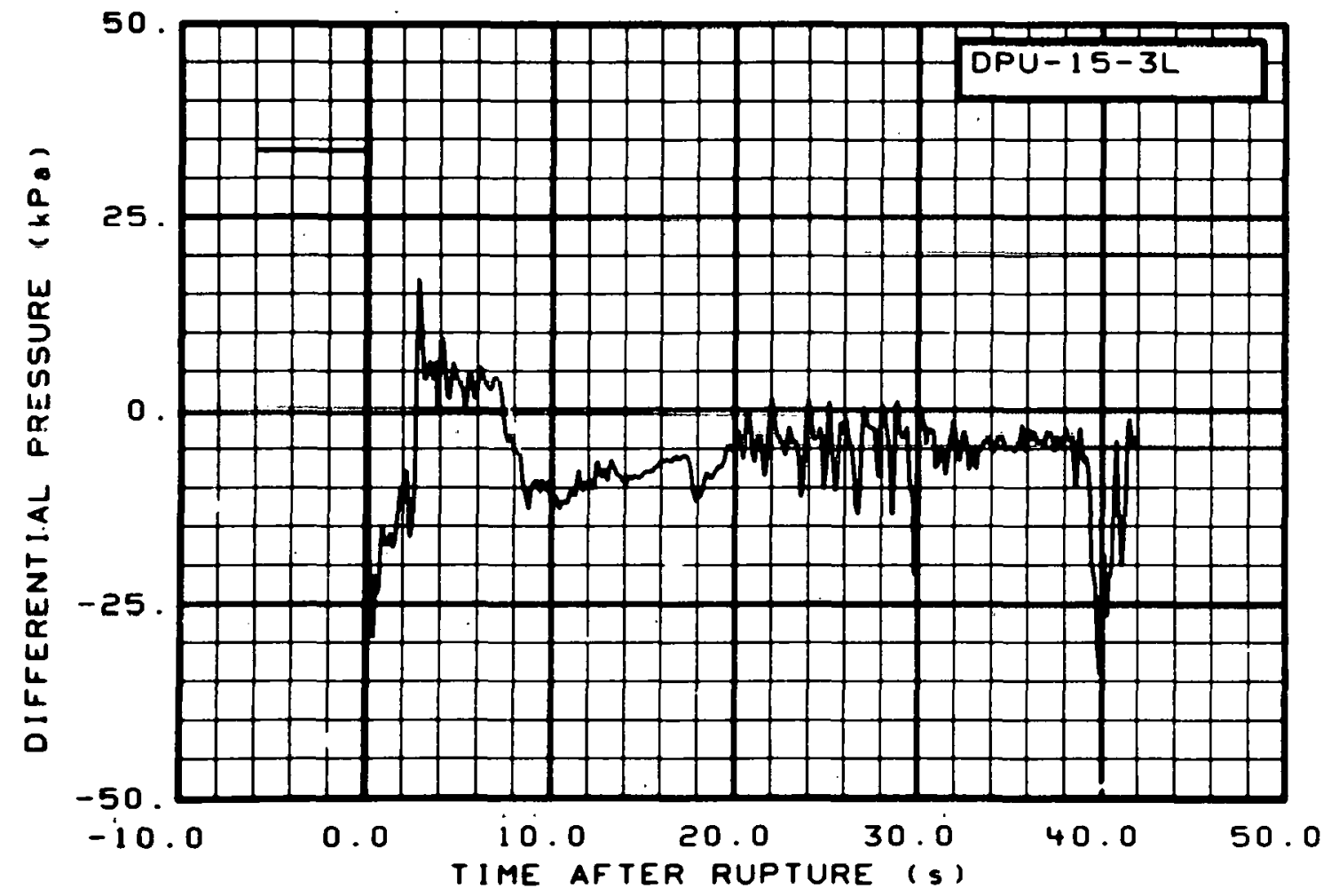

Fig. 182 Differential pressure in intact loop, low range (DPU-15-3L), from -6 to $42 \mathrm{~s}$. 


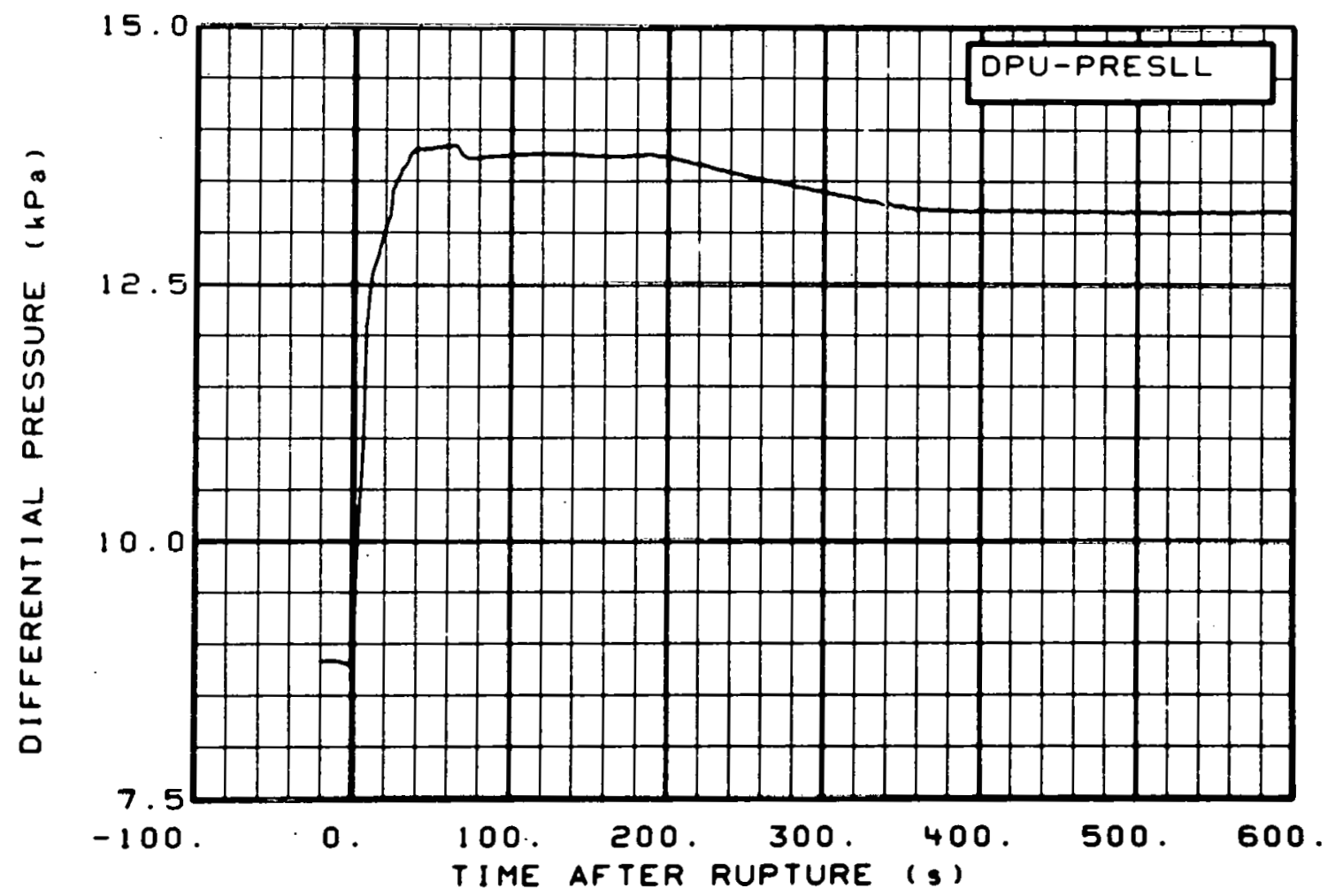

Fig. 183 Differential pressure in pressurizer (DPU-PRESLL), from -20 to $600 \mathrm{~s}$.

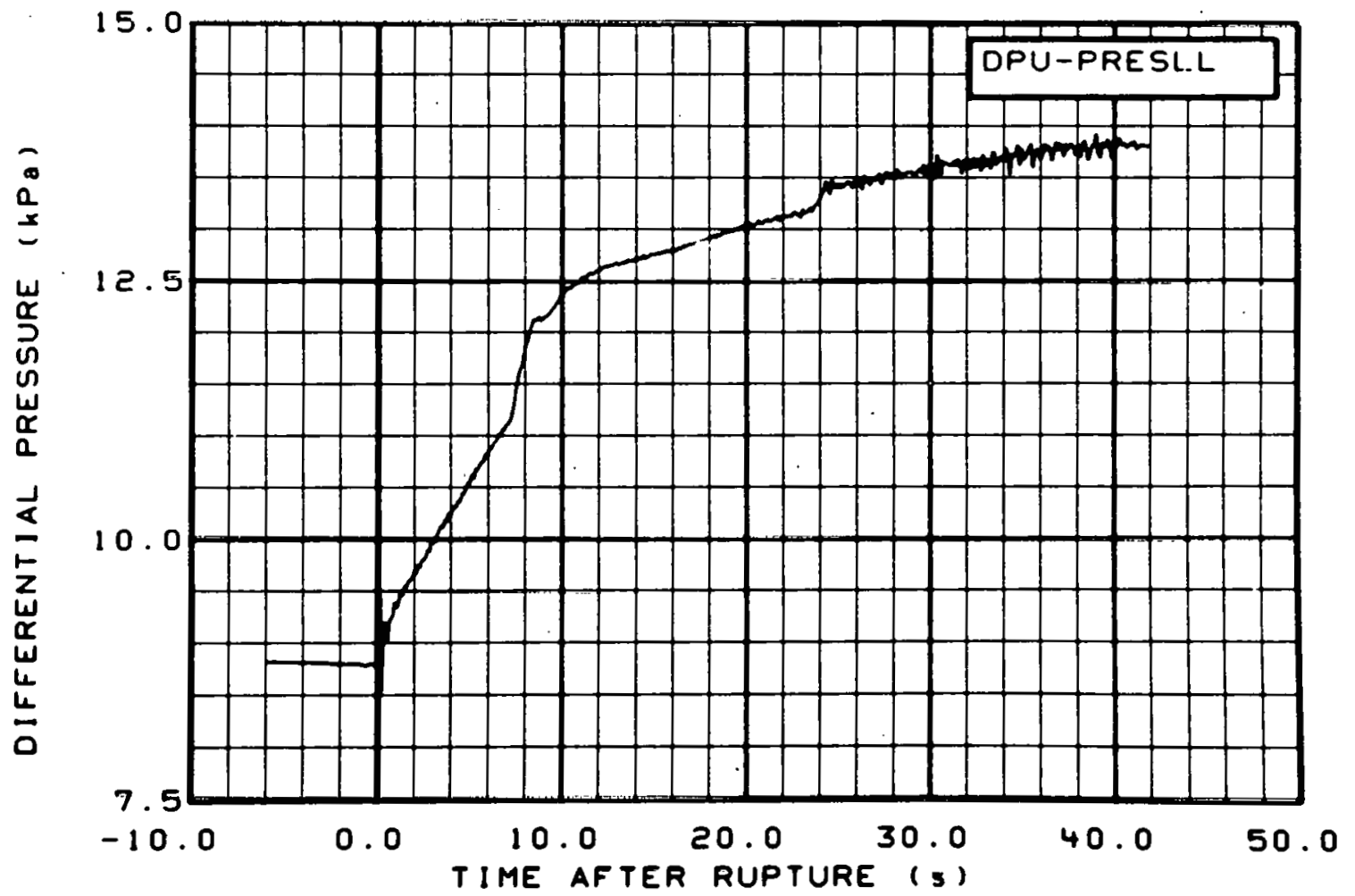

Fig. 184 Differential pressure in pressurizer (DPU-PRESLL), from -6 to $42 \mathrm{~s}$. 


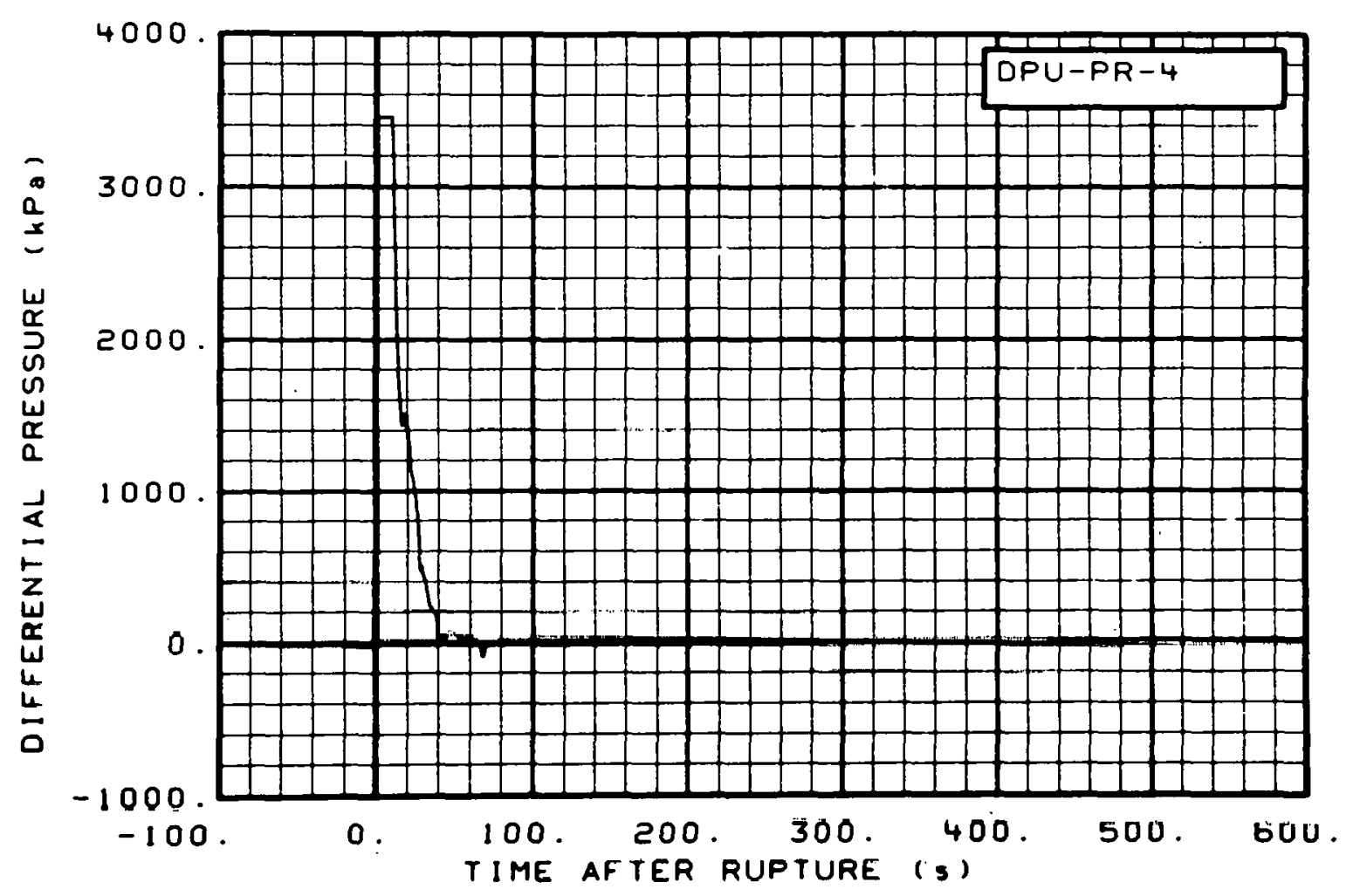

Fig. 185 Differential pressure in intact loop (DPU-PR-4), from -20 to $600 \mathrm{~s}$.

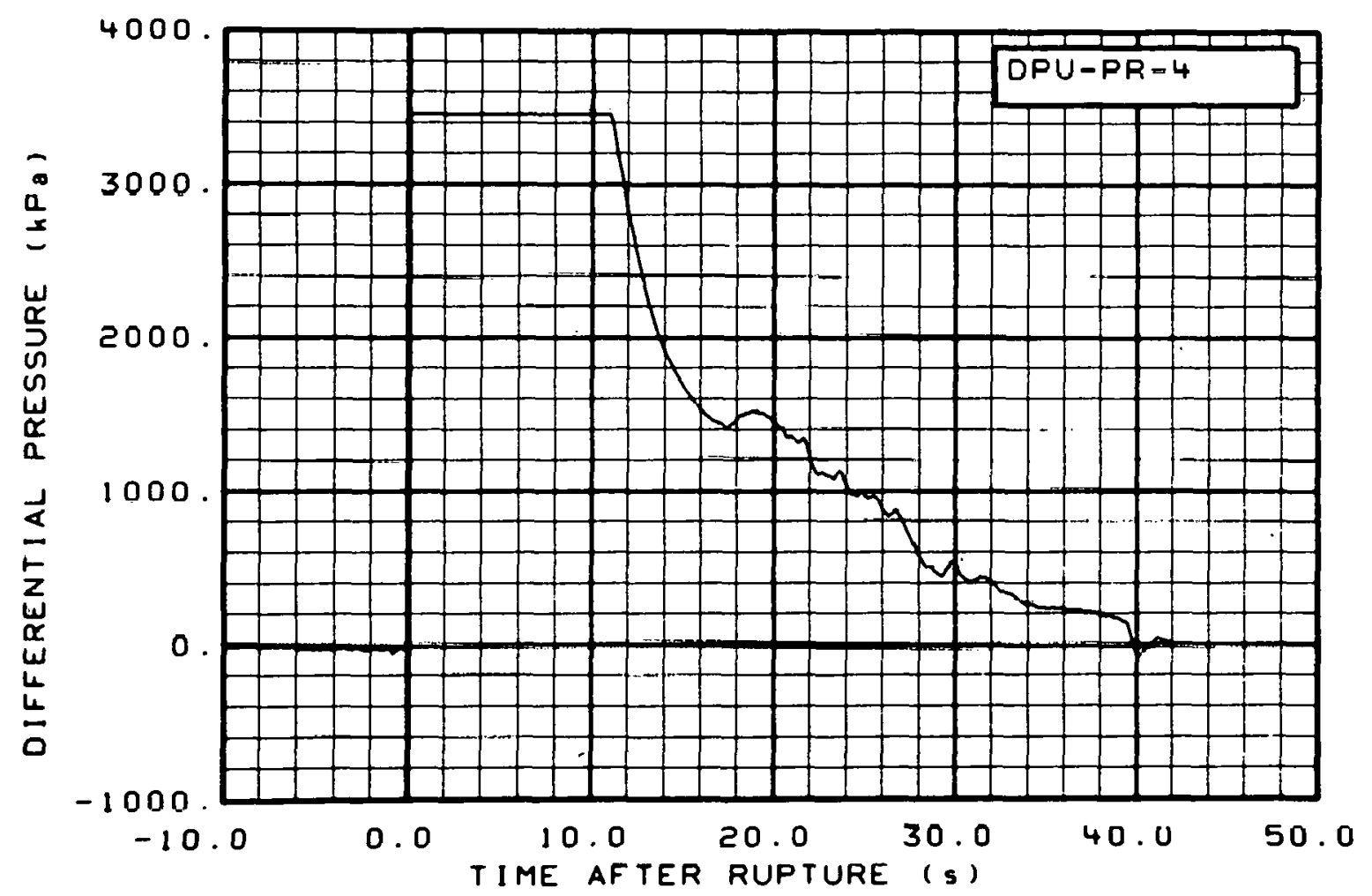

Fig. 186 Differential pressure in intact loop (DPU-PR-4), from -6 to $42 \mathrm{~s}$. 


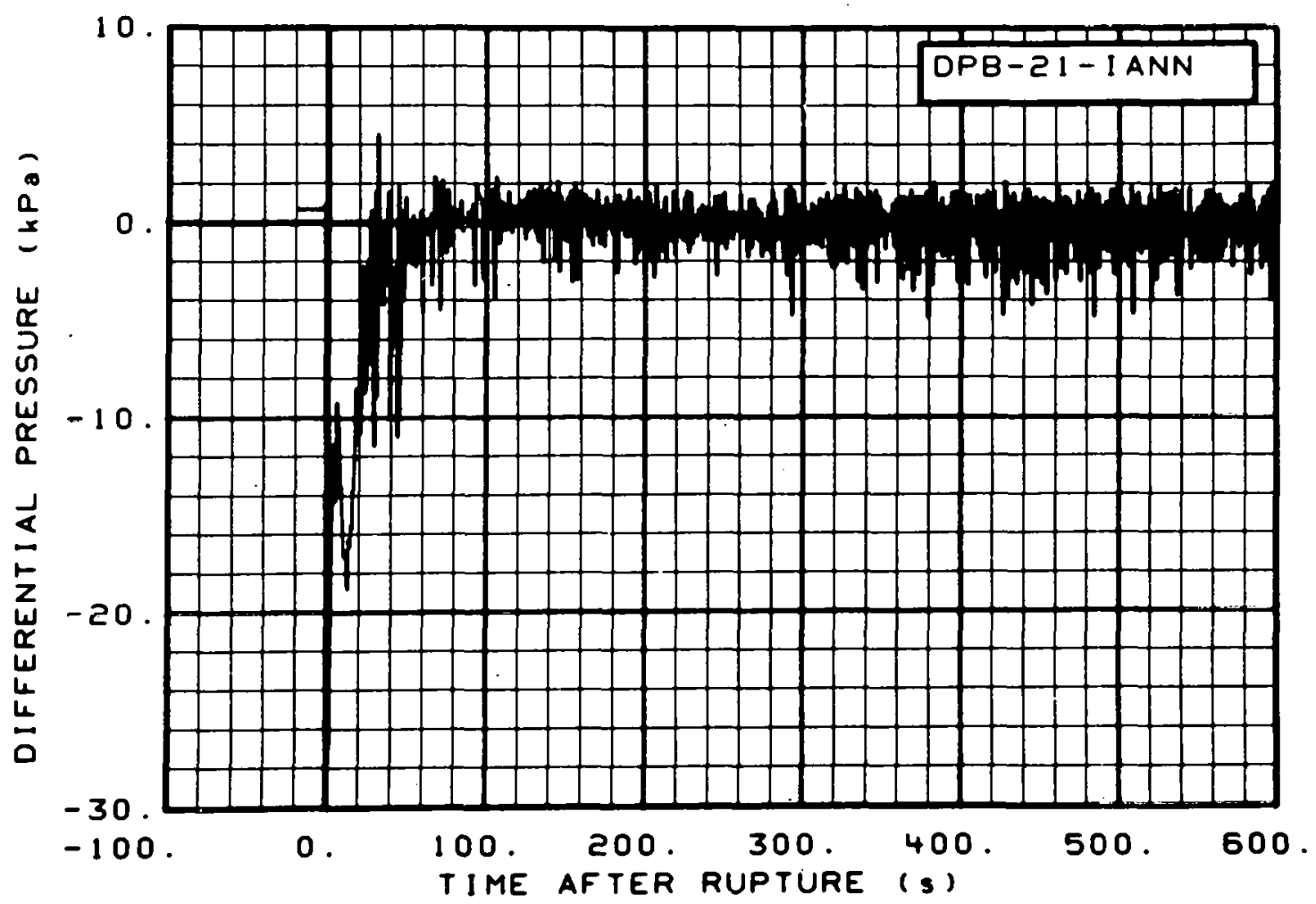

Fig. 187 Differential pressure in broken loop (DPB-21-IANN), from -20 to $600 \mathrm{~s}$.

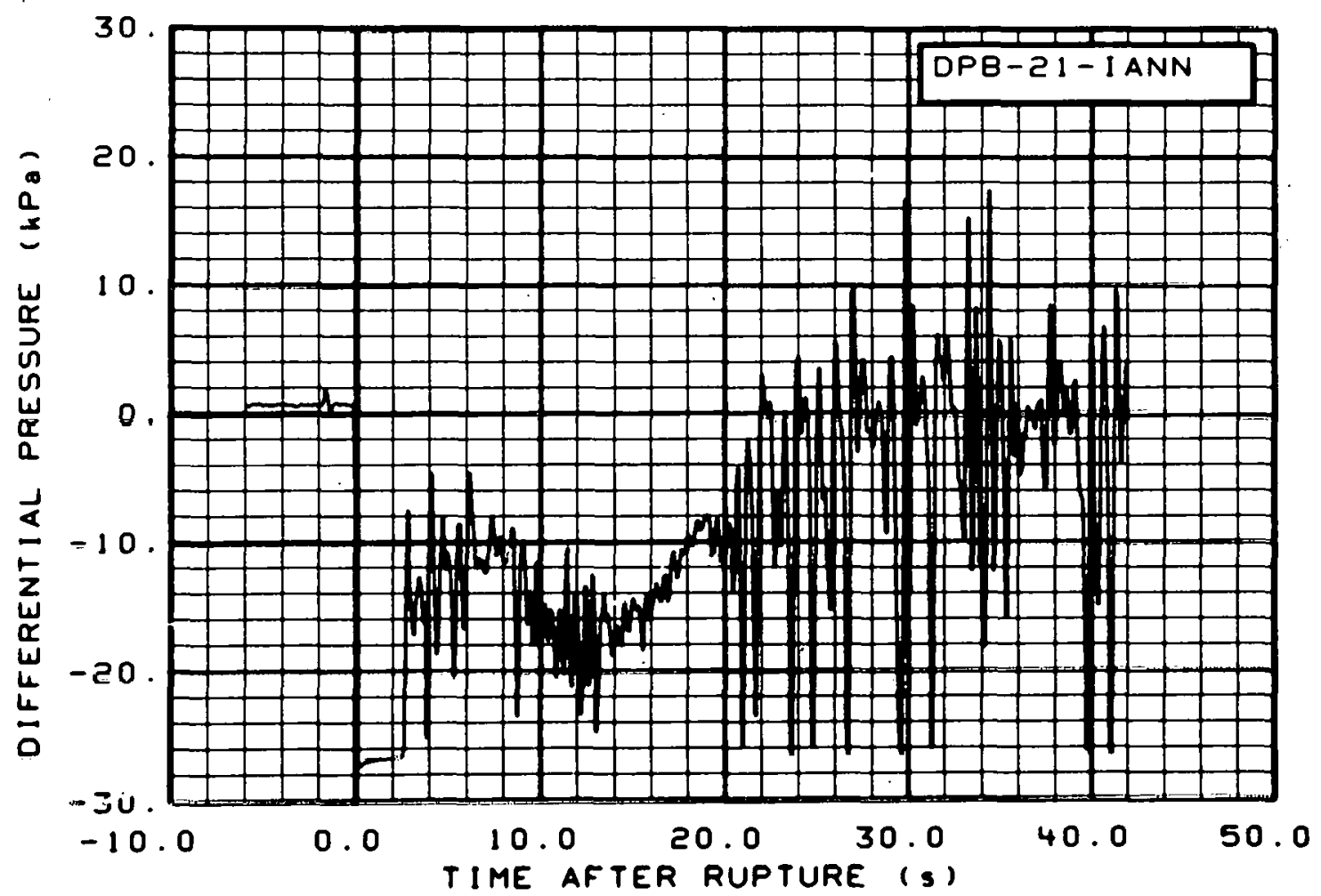

Fig. 188 Differential pressure in broken loop (DPB-21-IANN), from -6 to $42 \mathrm{~s}$. 


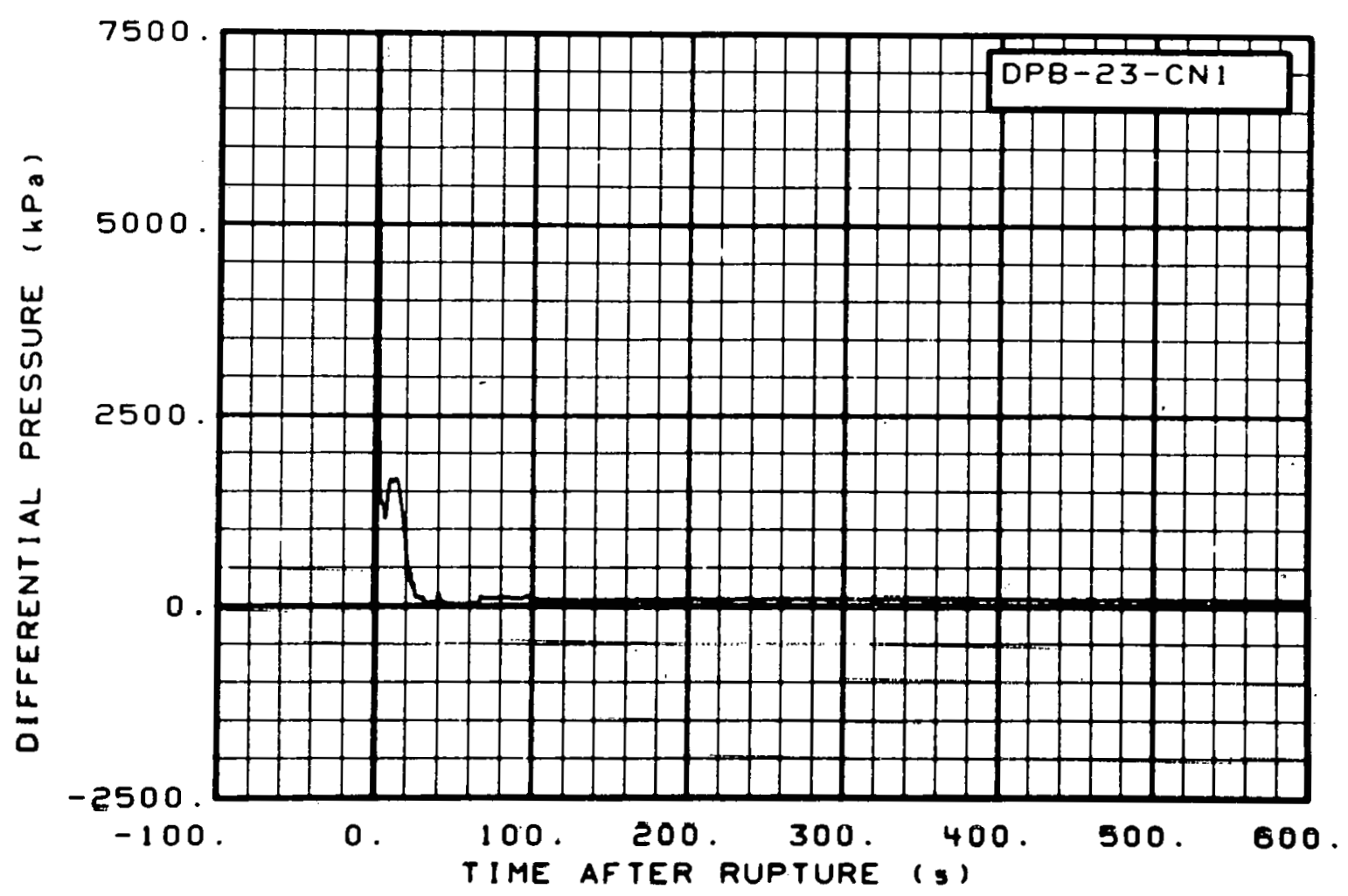

Fig. 189 Differential pressure in broken loop (DPB-23-CN1), from -20 to $600 \mathrm{~s}$.

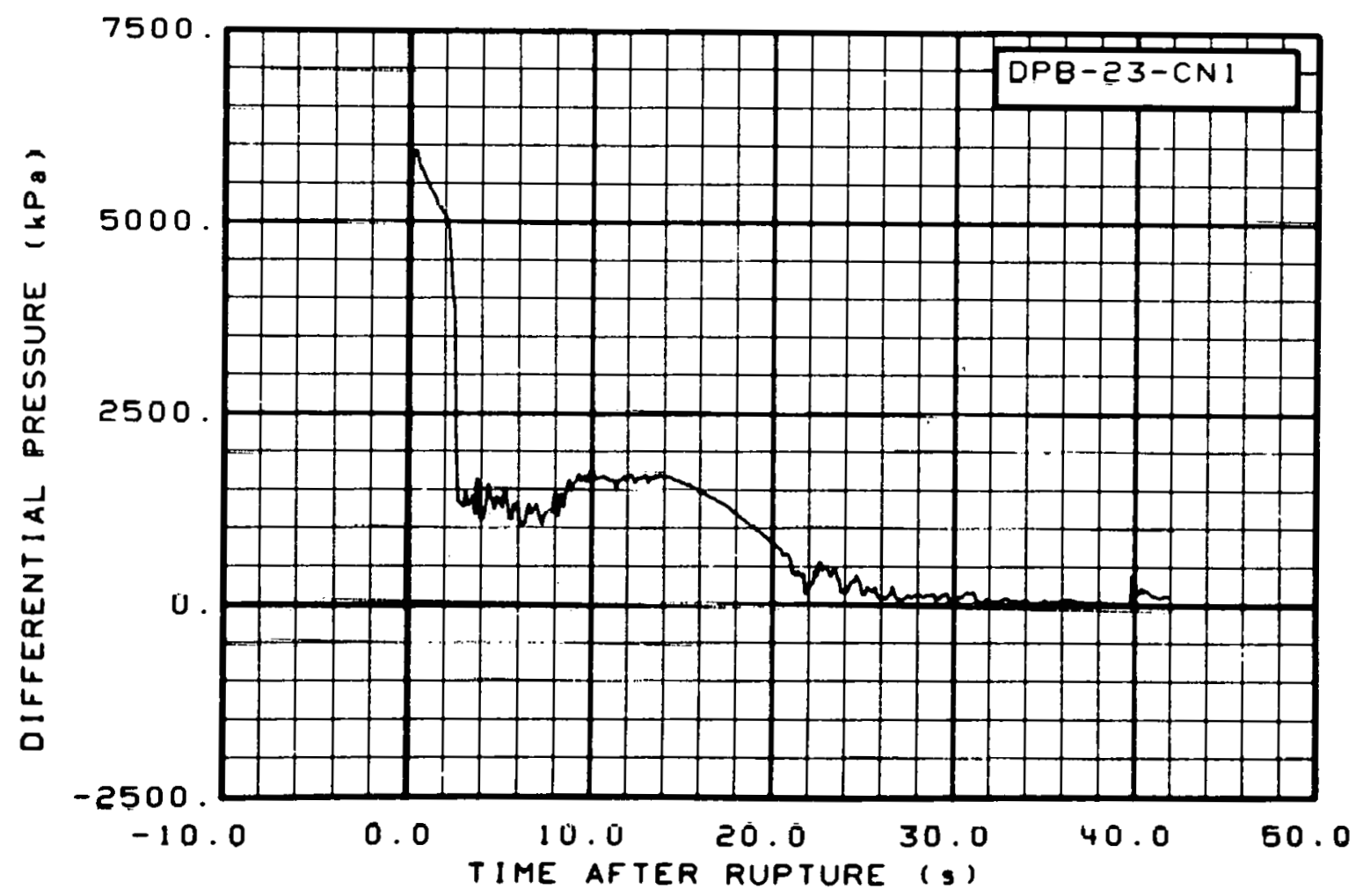

Fig. 190 Differential pressure in broken loop (DPB-23-CN1), from -6 to 42 s. 


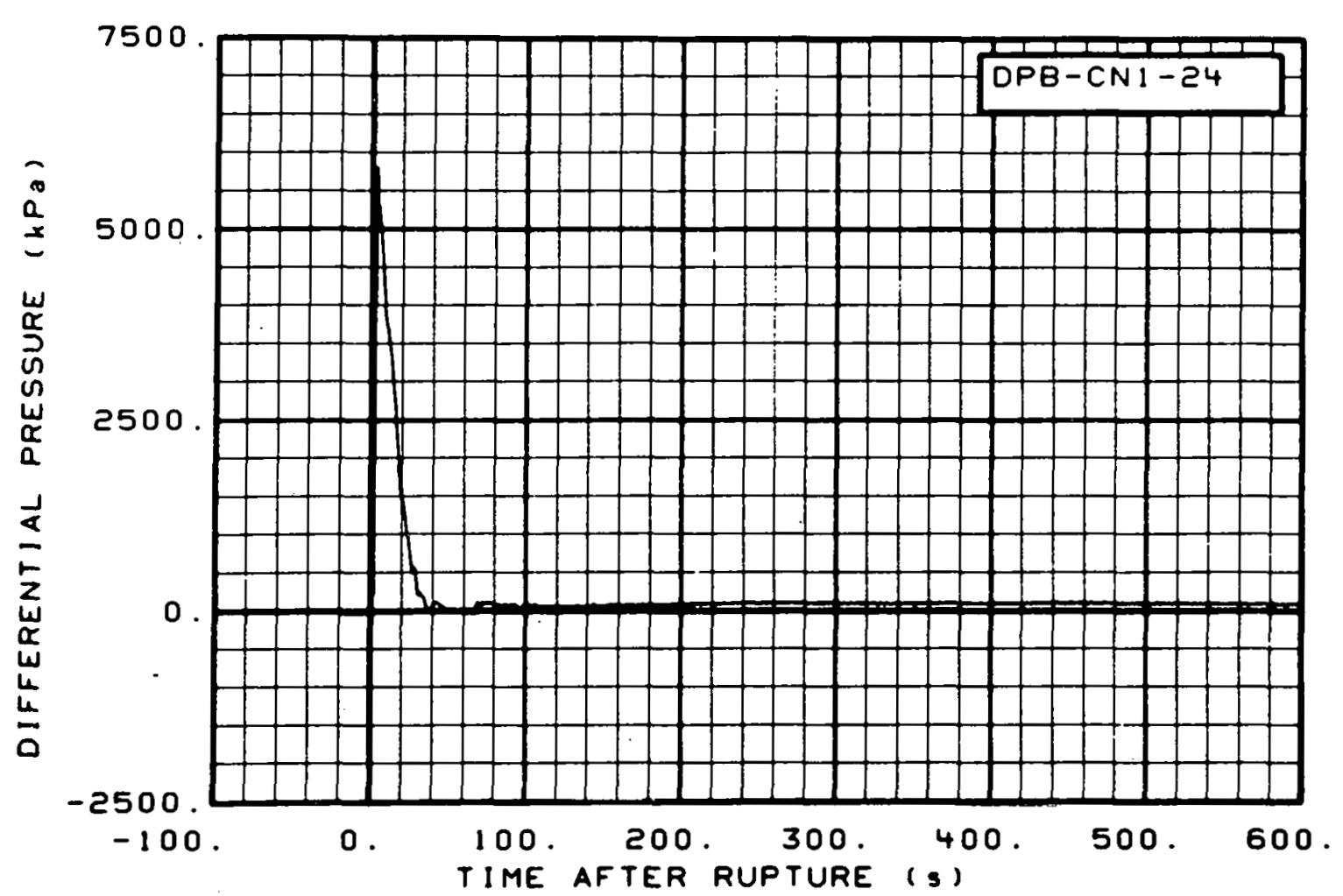

Fig. 191 Differential pressure in broken loop (DPB-CNT-24), from -20 to $600 \mathrm{~s}$.

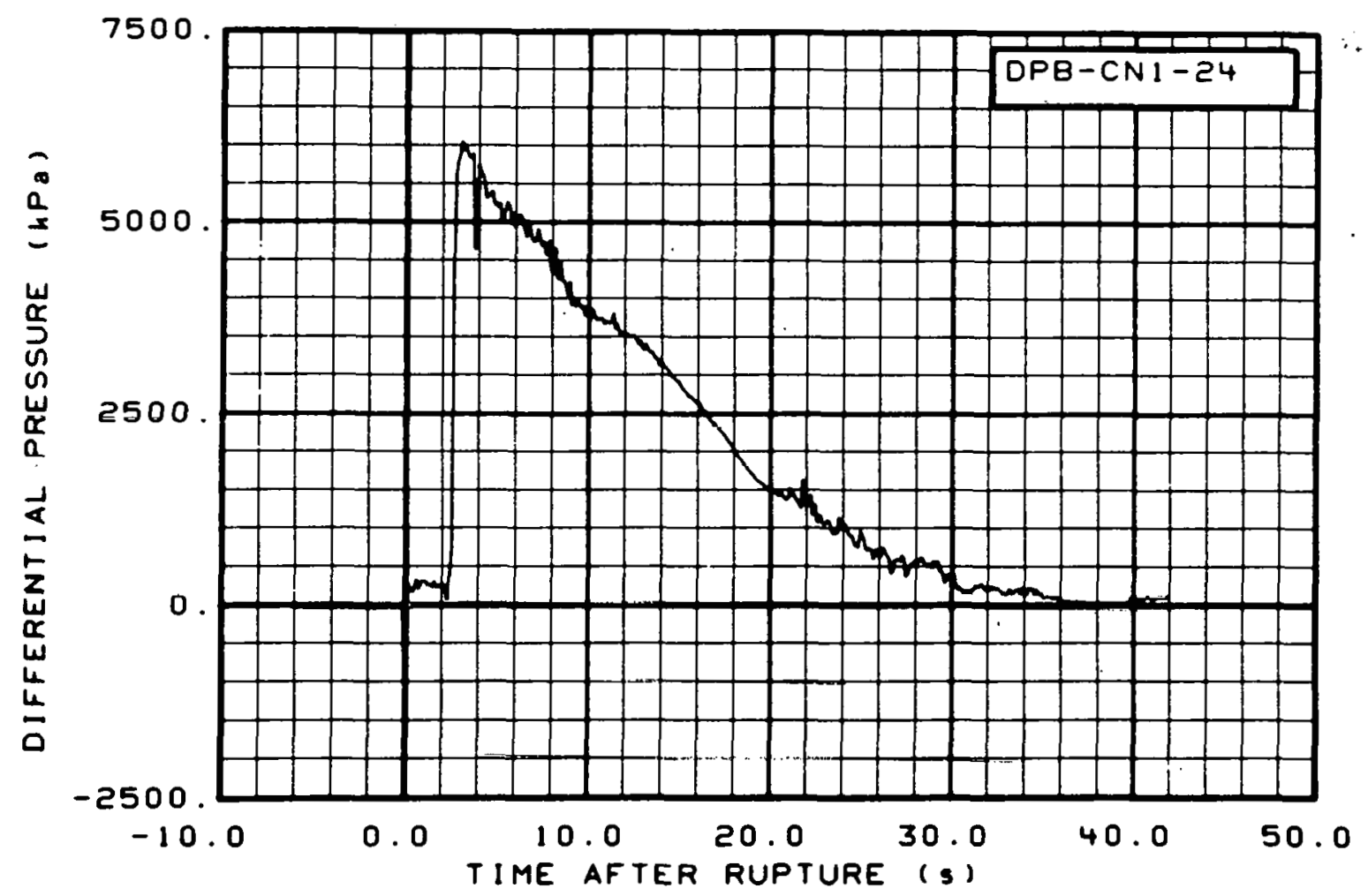

Fig. 192 Differential pressure in broken loop (DPB-CN1-24), from -6 to $42 \mathrm{~s}$. 


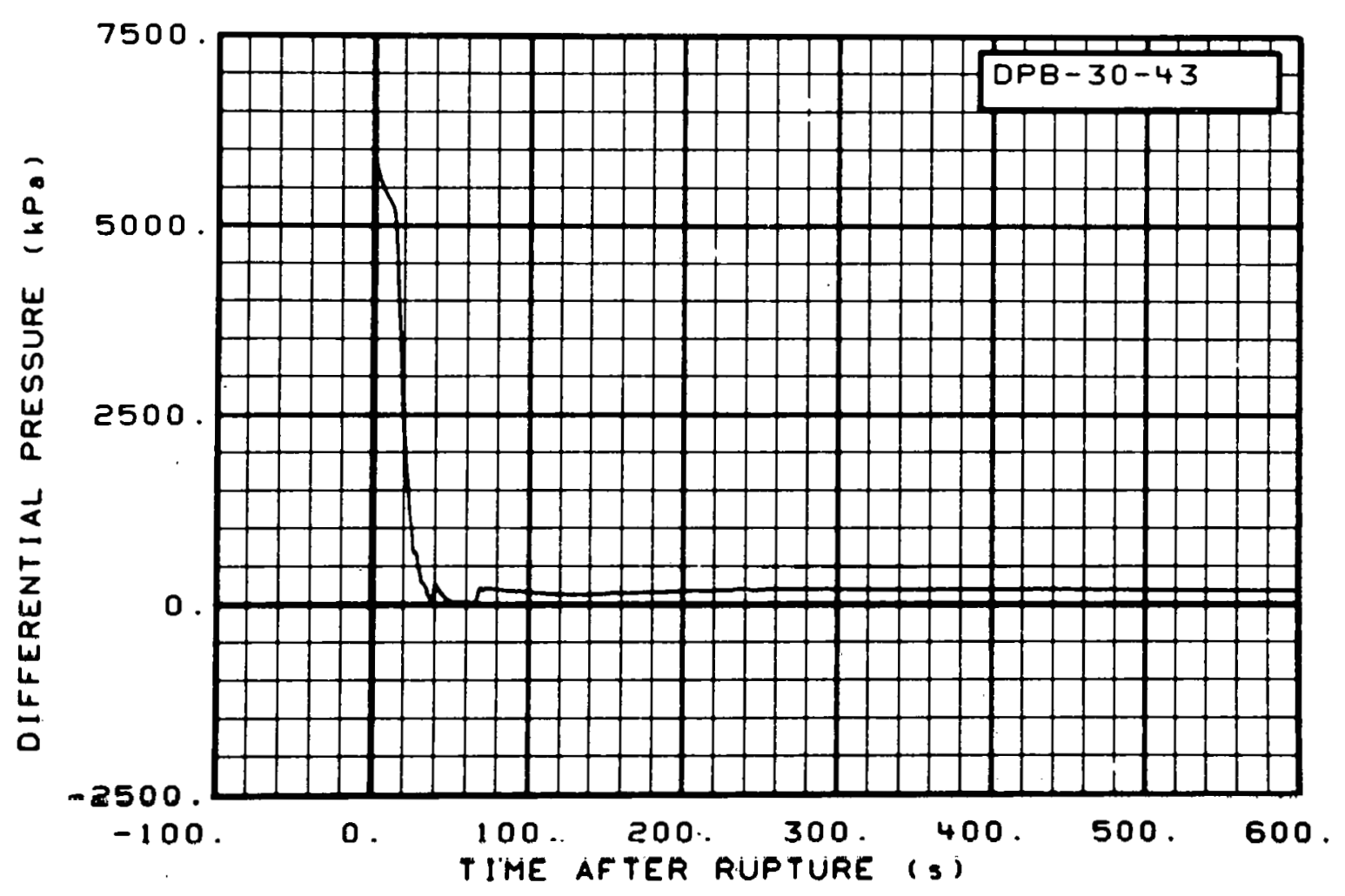

Fig. 193 Differential pressure in broken loop (DPB-30-43), from -20 to $600 \mathrm{~s}$.

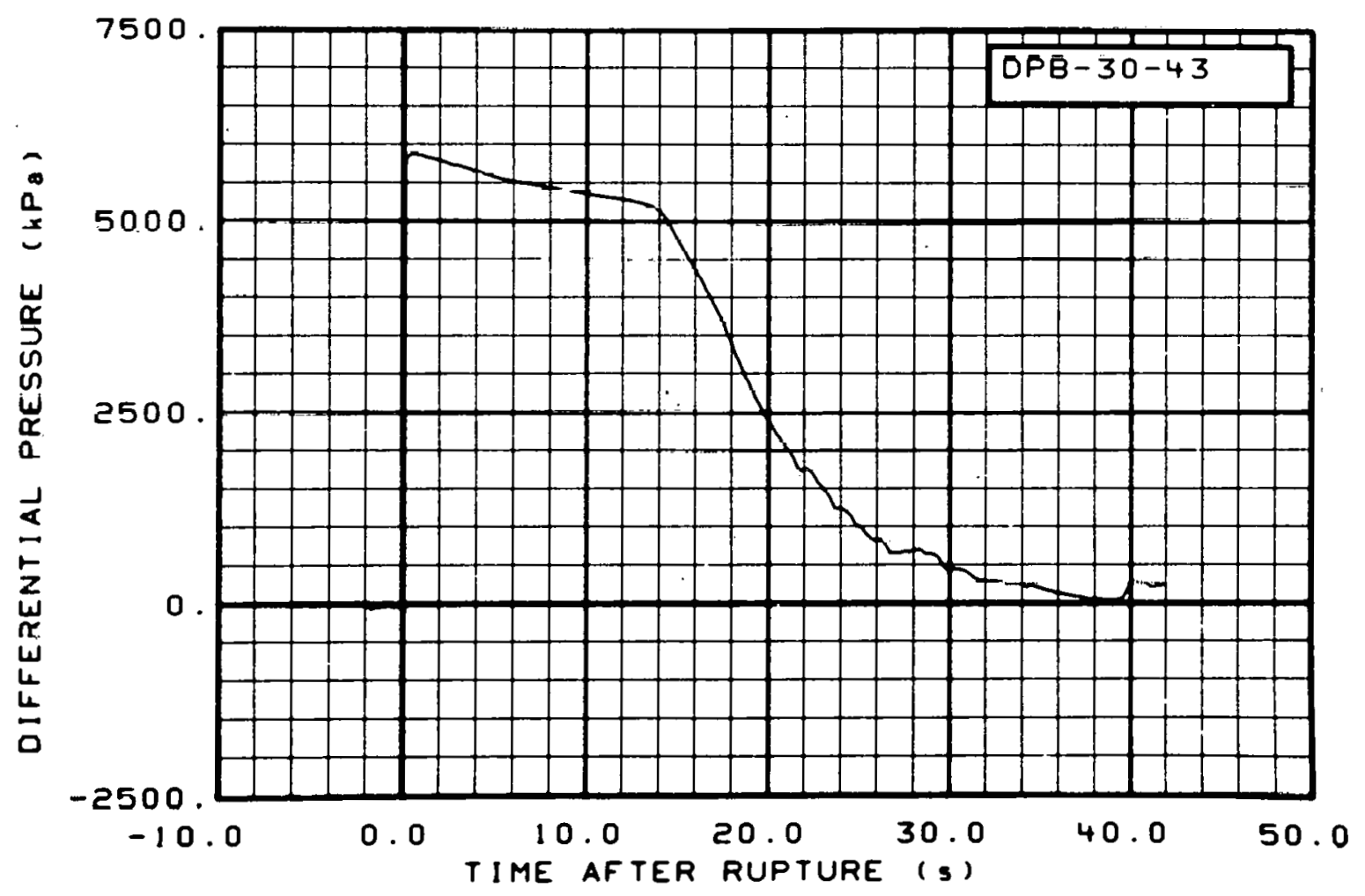

Fig. 194 Differential pressure in broken loop (DPB-30-43), from -6 to $42 \mathrm{~s}$. 


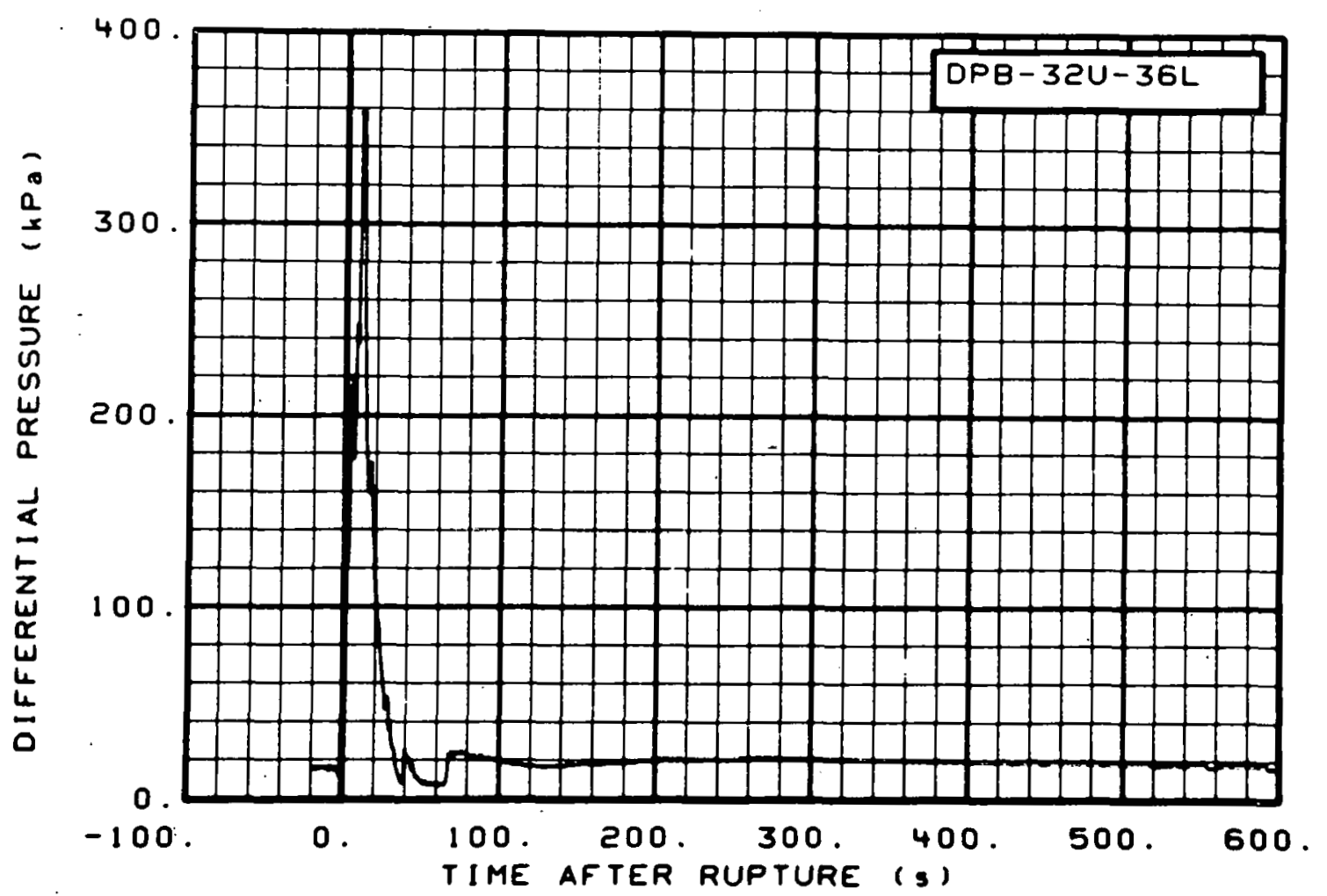

Fig. 195 Differential pressure in broken loop (DPB-32U-36L), from . -20 to $600 \mathrm{~s}$.

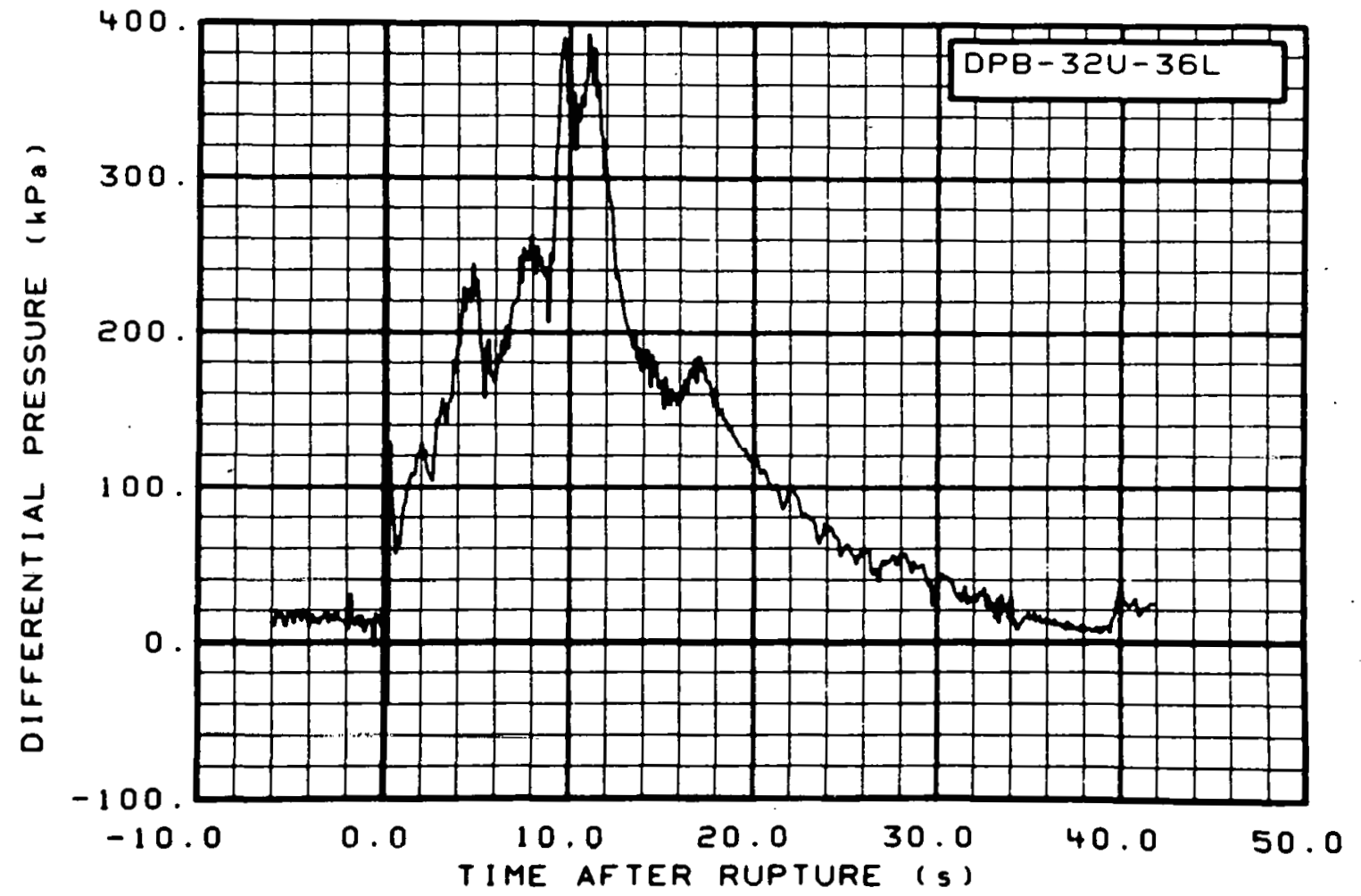

Fig. 196 Differential pressure in broken loop (DPB-32U-36L), from -6 to $42 \mathrm{~s}$. 


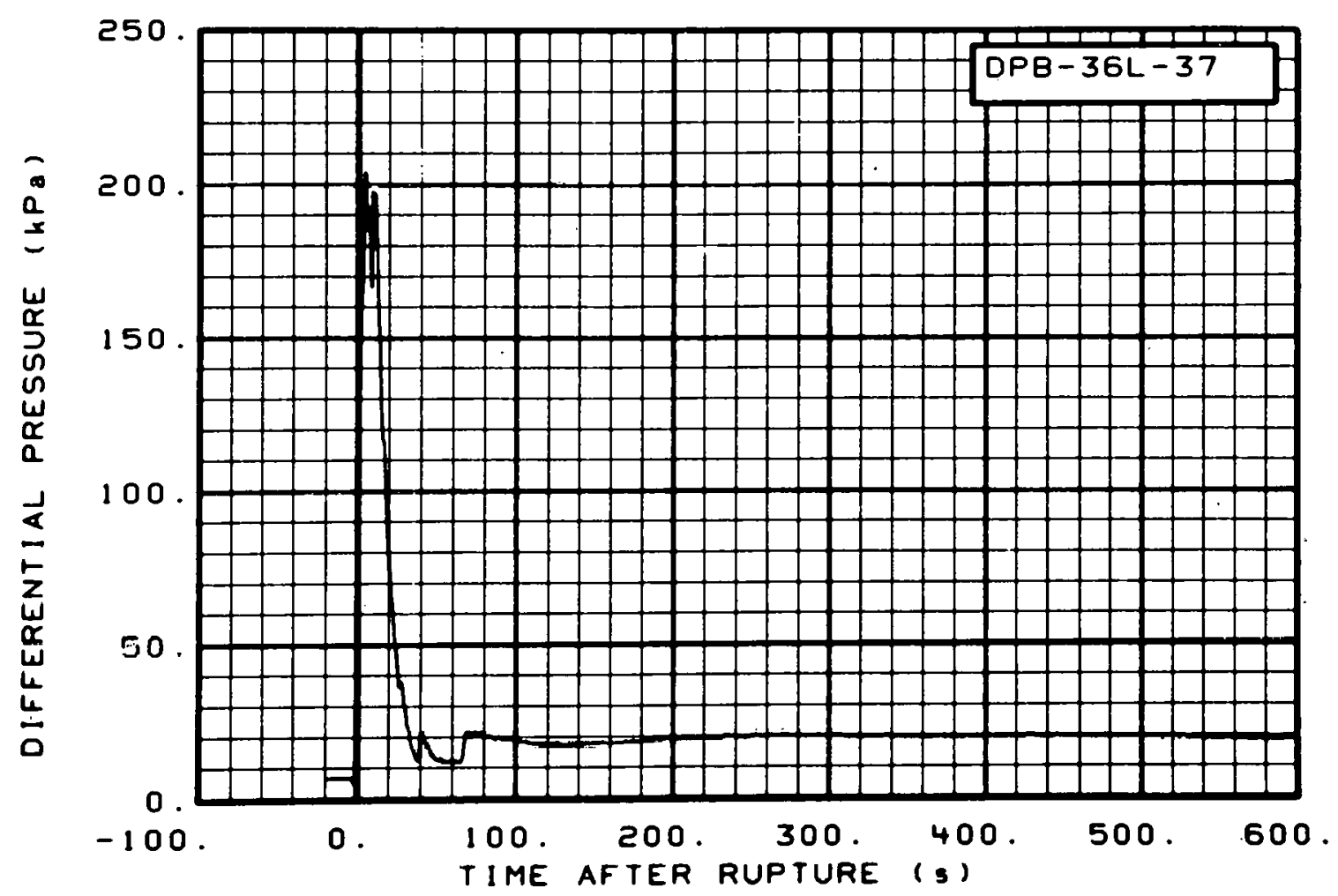

Fig. 197 Differential pressure in broken loop (DPB-36L-37), from -20 to $600 \mathrm{~s}$.

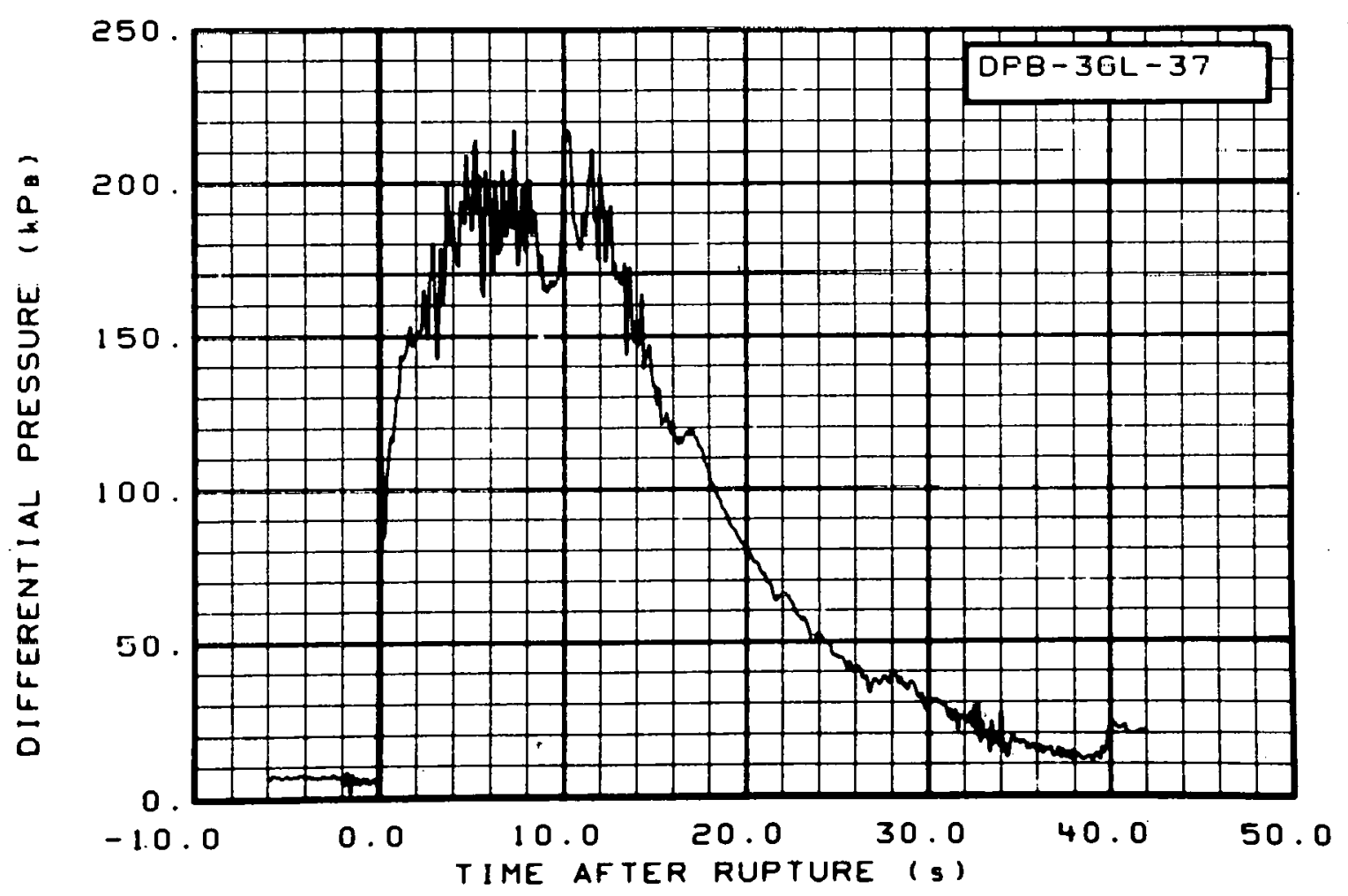

Fig. 198 Differential pressure in broken loop (DPB-36L-37), from -6 to $42 \mathrm{~s}$. 


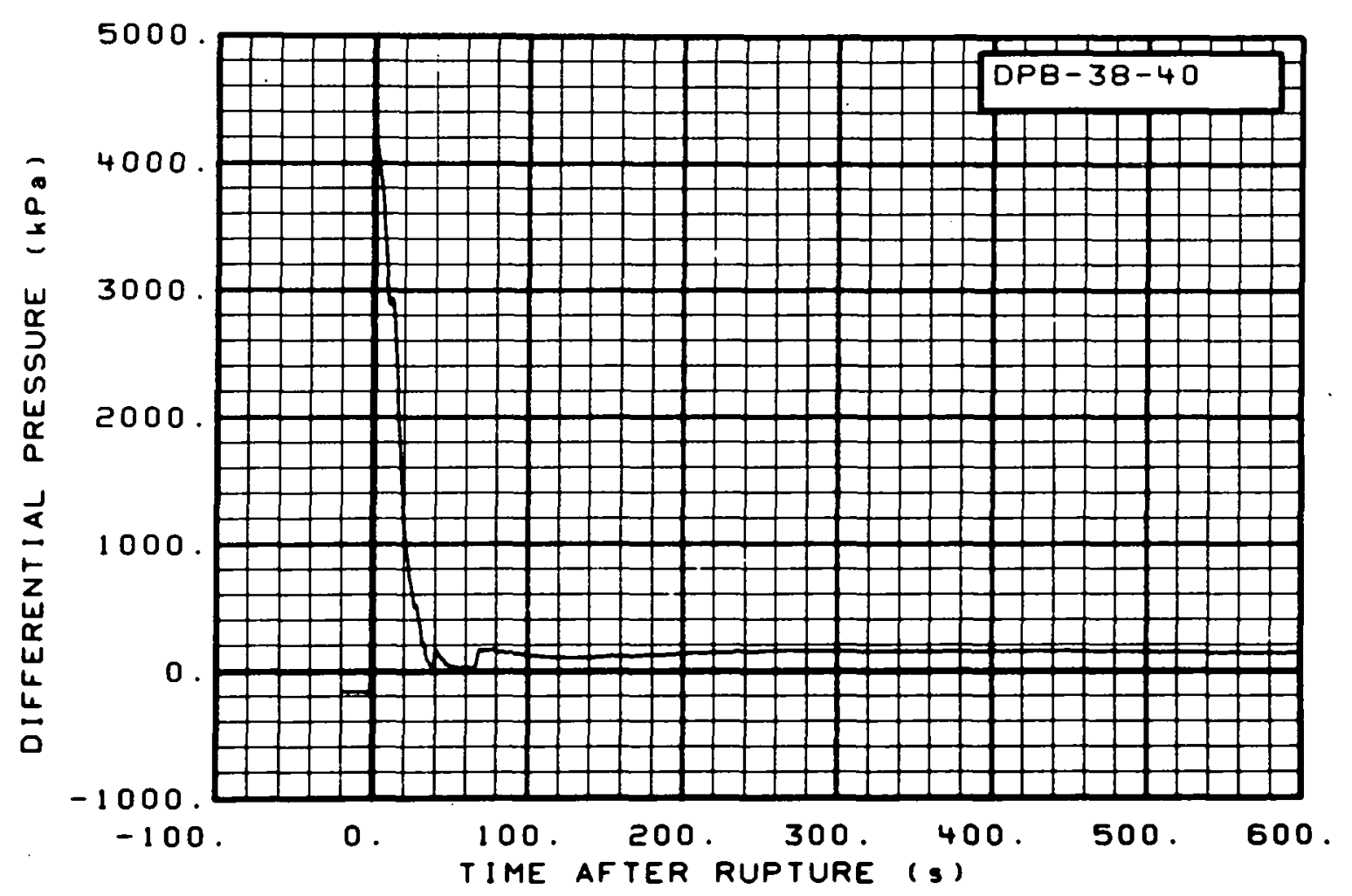

Fig. 199 Differential pressure in broken loop (DPB-38-40), from -20 to $600 \mathrm{~s}$.

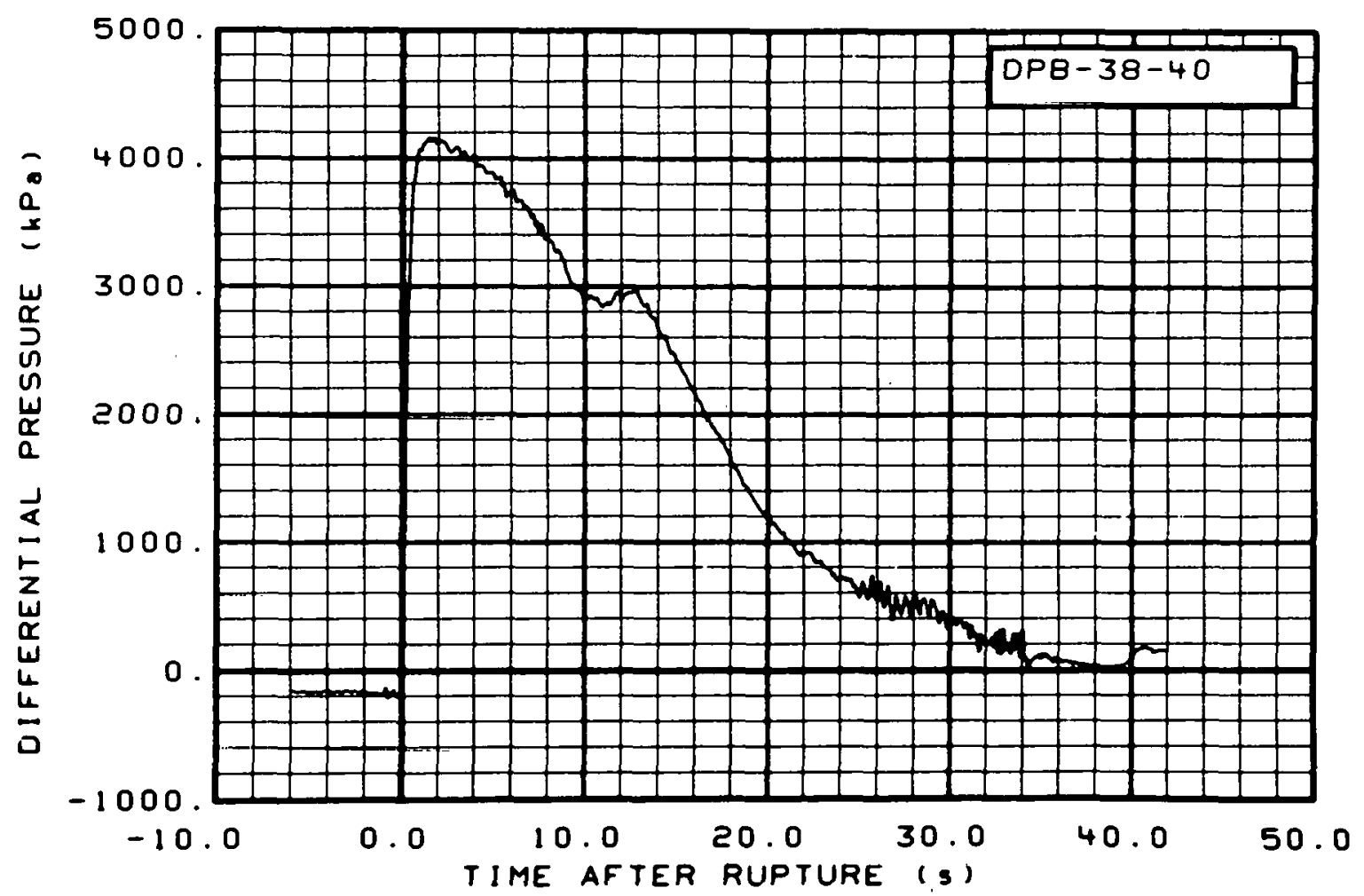

Fig. 200 Differential pressure in broken loop (DPB-38-40), from -6 t.n 42 s. 


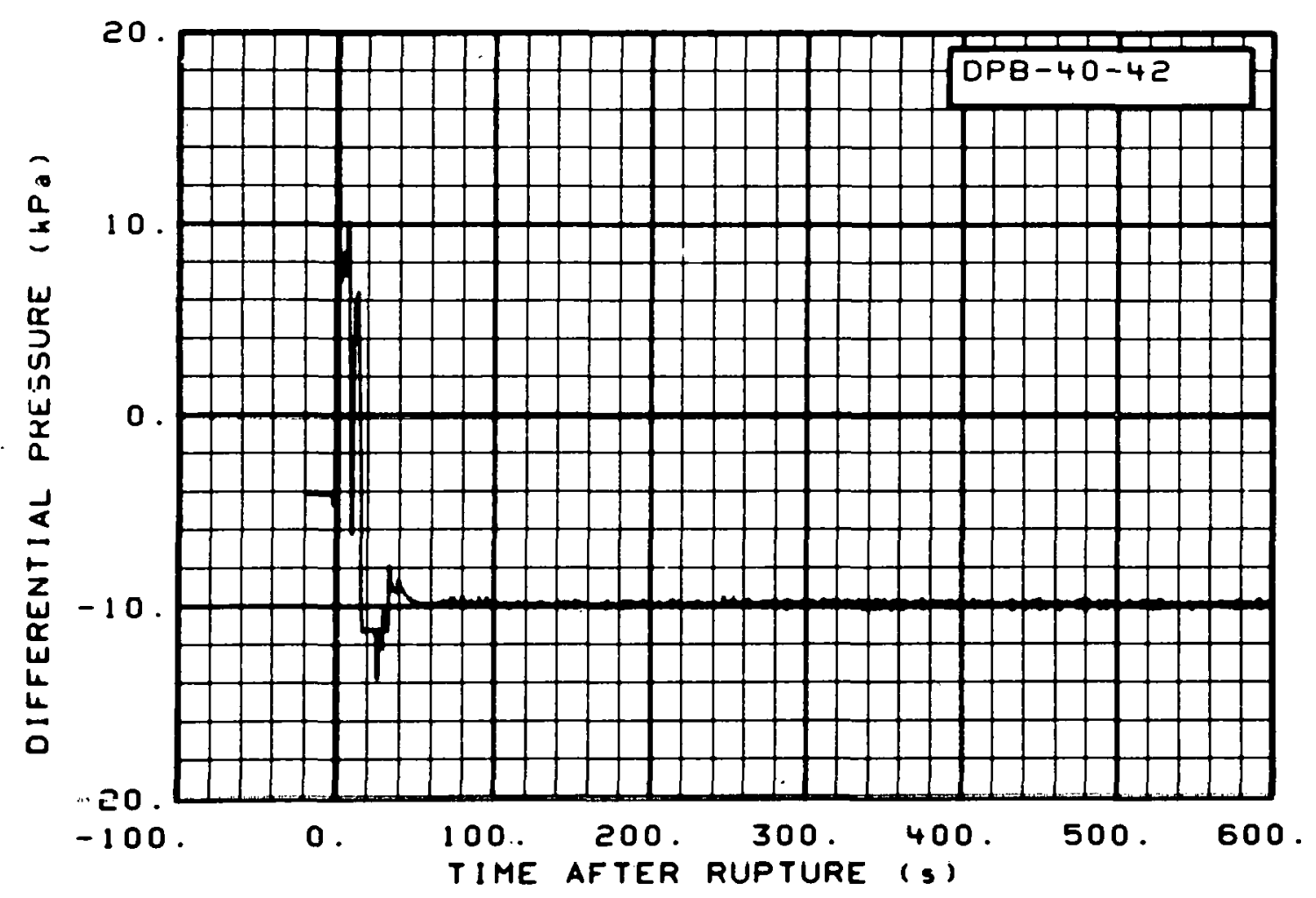

Fig. 201 Differential pressure in broken loop (DPB-40-42), from -20 to $600 \mathrm{~s}$.

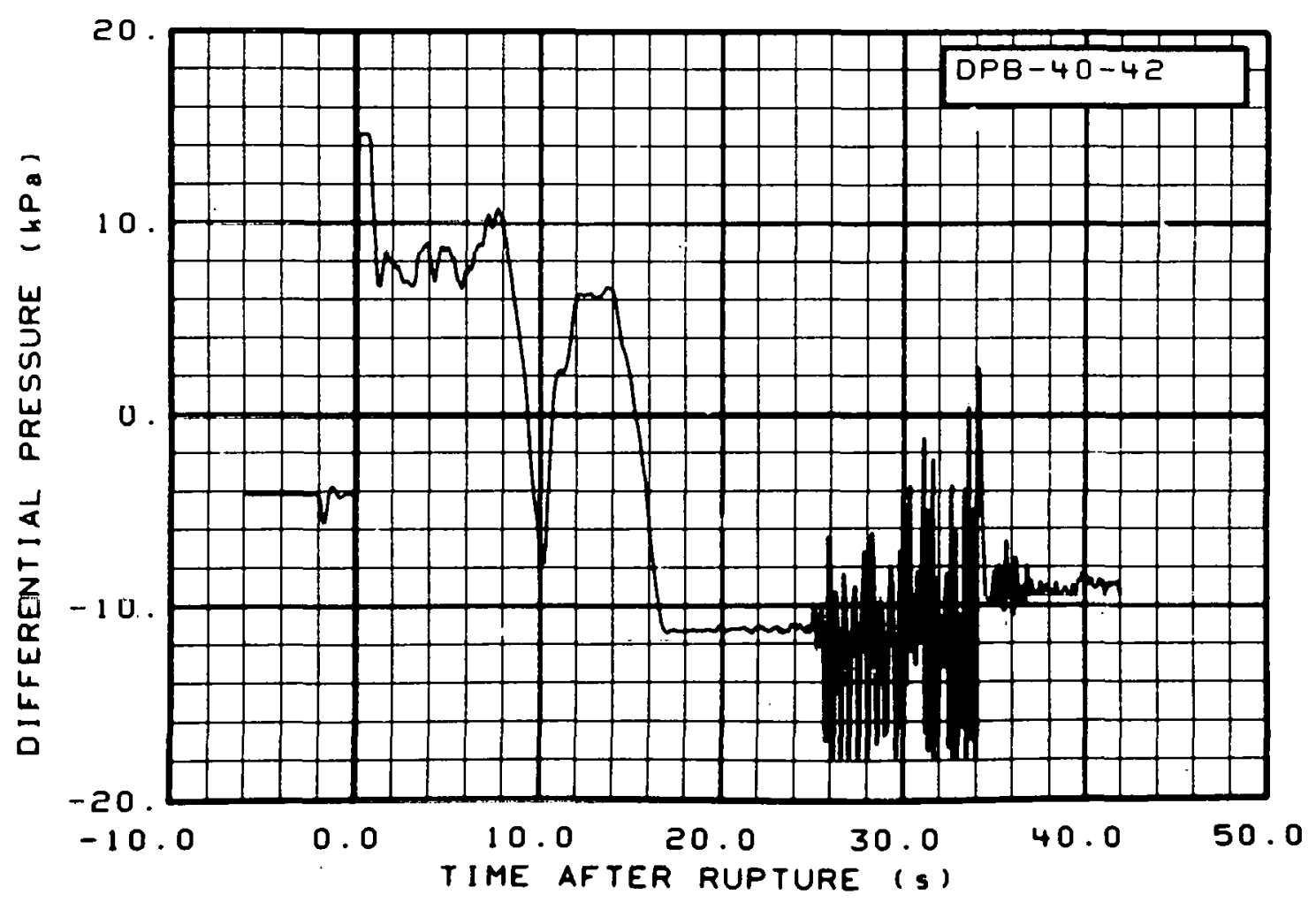

Fig. 202 Differential pressure in broken loop (DPB-40-42), from -6 to $42 \mathrm{~s}$. 


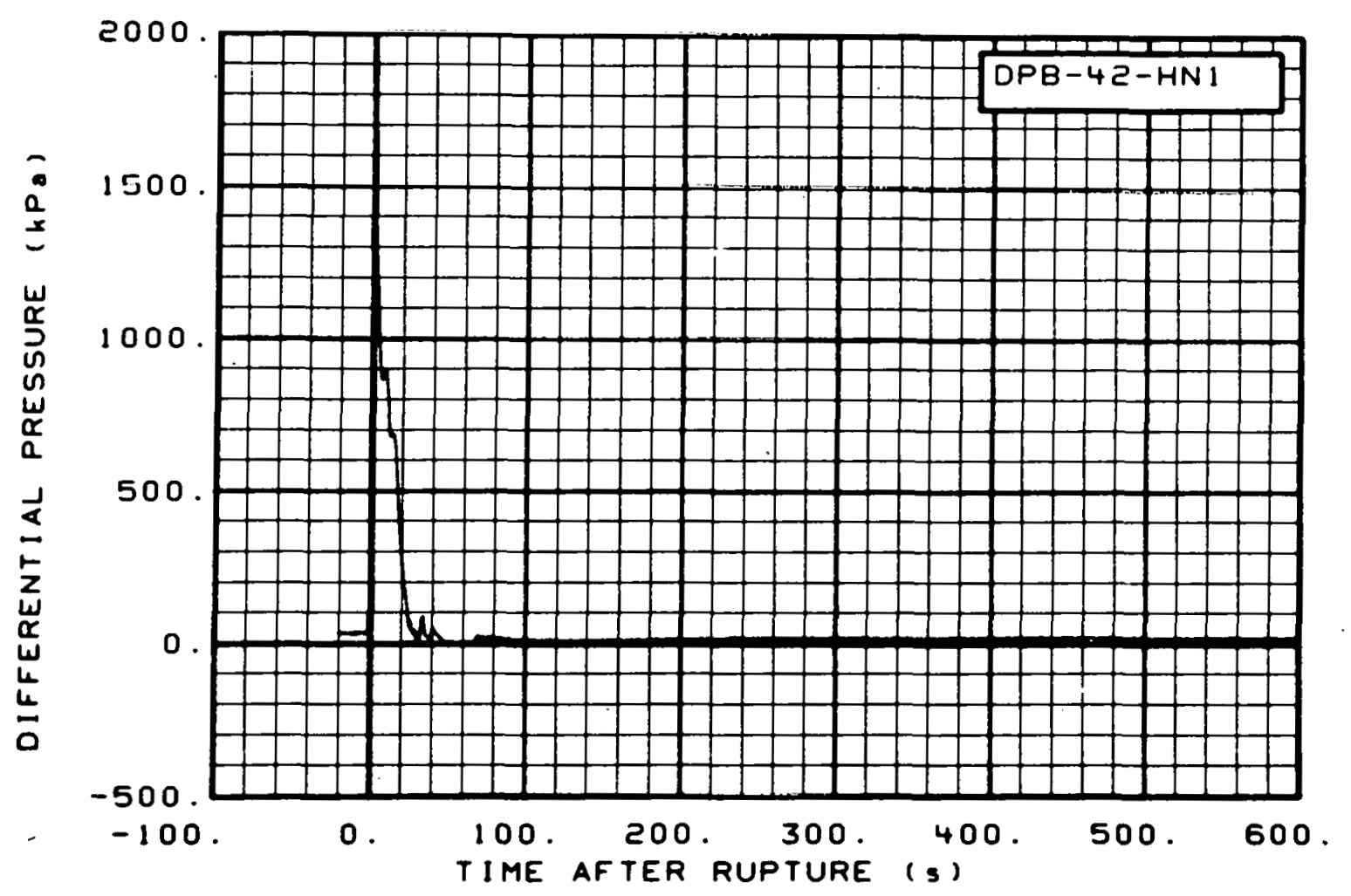

Fig. 203 Differential pressure in broken loop (DPB-42-HN1), from -20 to $600 \mathrm{~s}$.

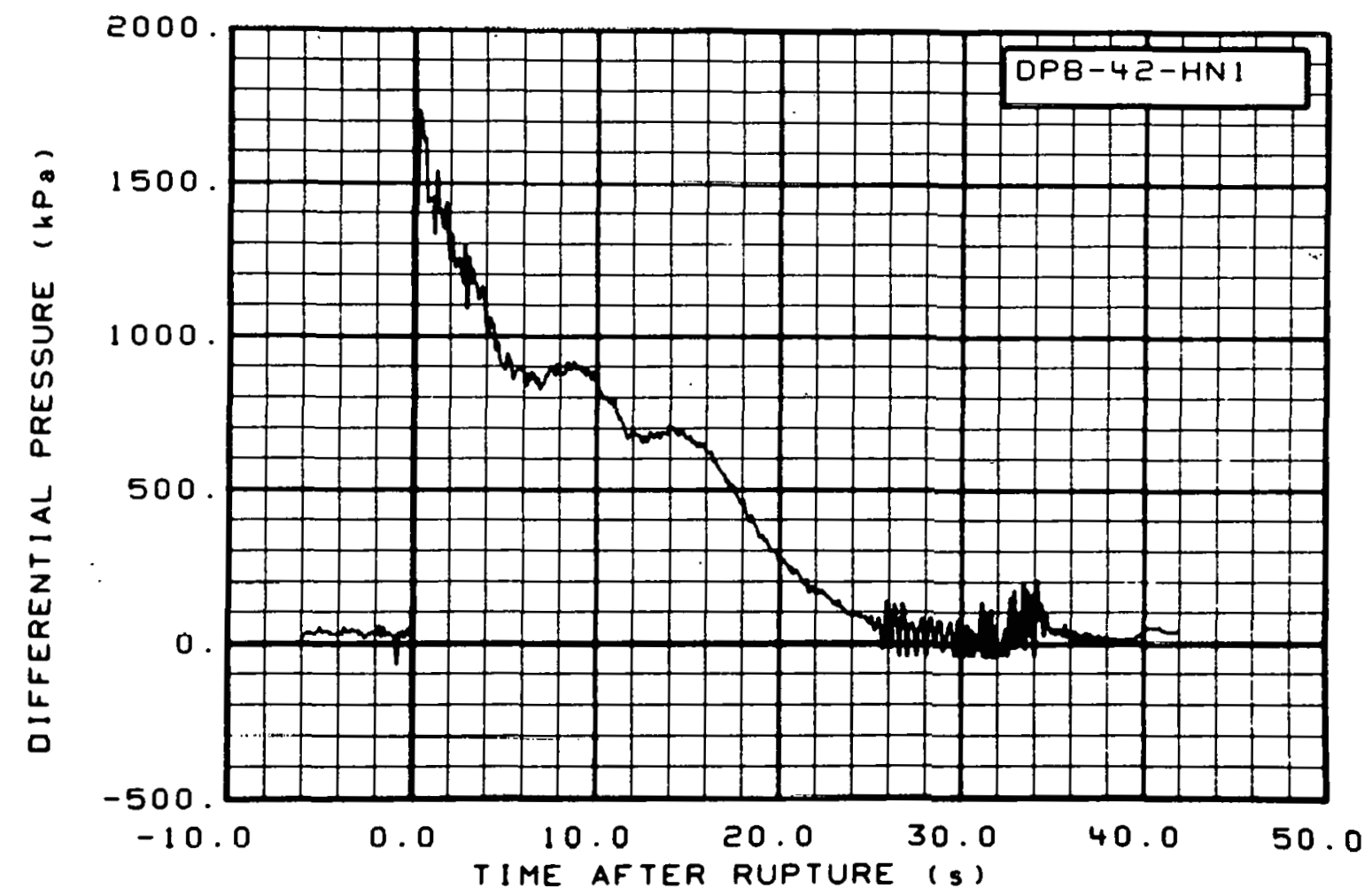

Fig. 204 Differential pressure in broken loop (DPB-42-HN1), from -6 to $42 \mathrm{~s}$. 


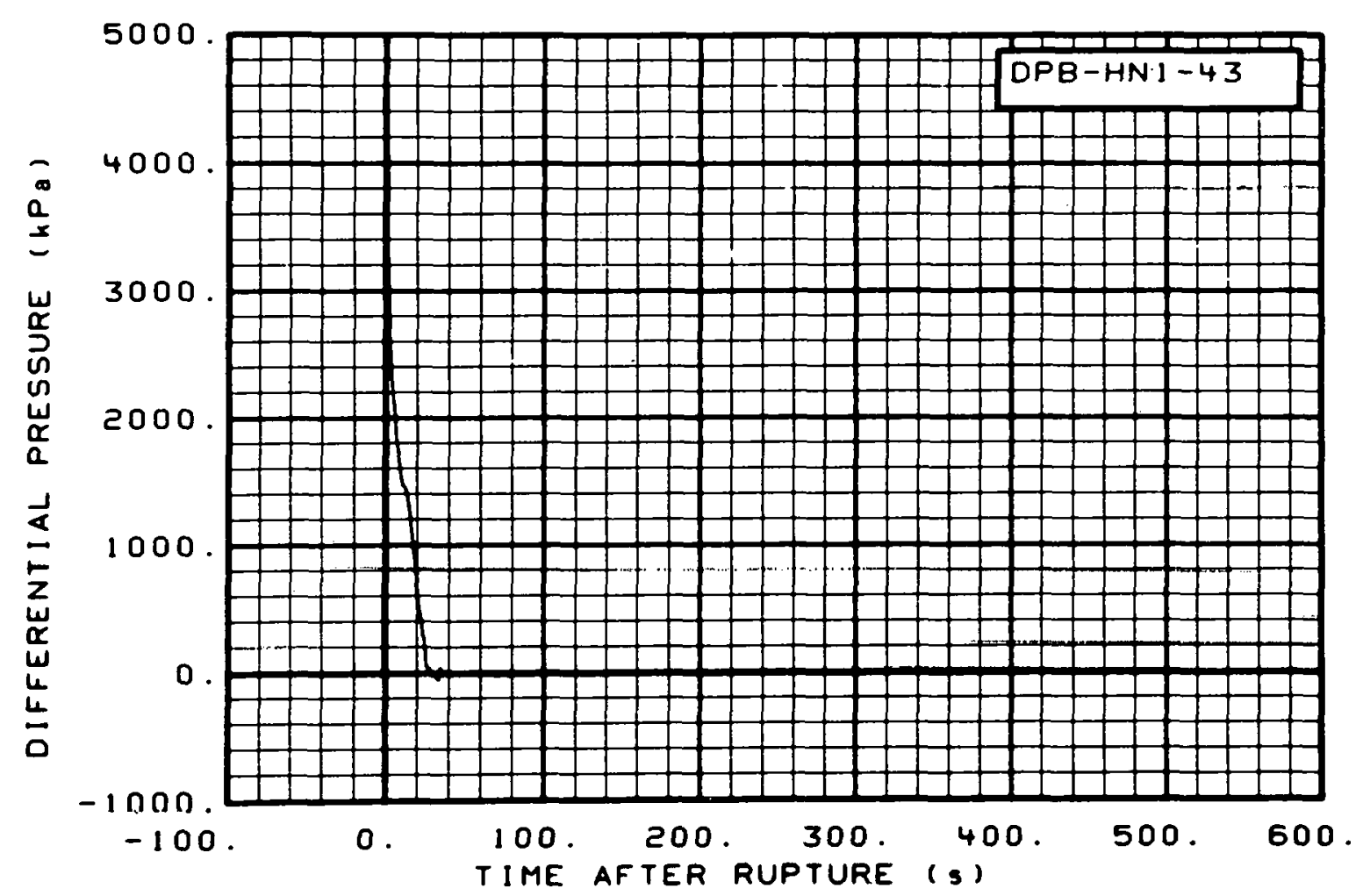

Fig. 205 Differential pressure in broken loop (DPB-HN1-43), from -20 to $600 \mathrm{~s}$.

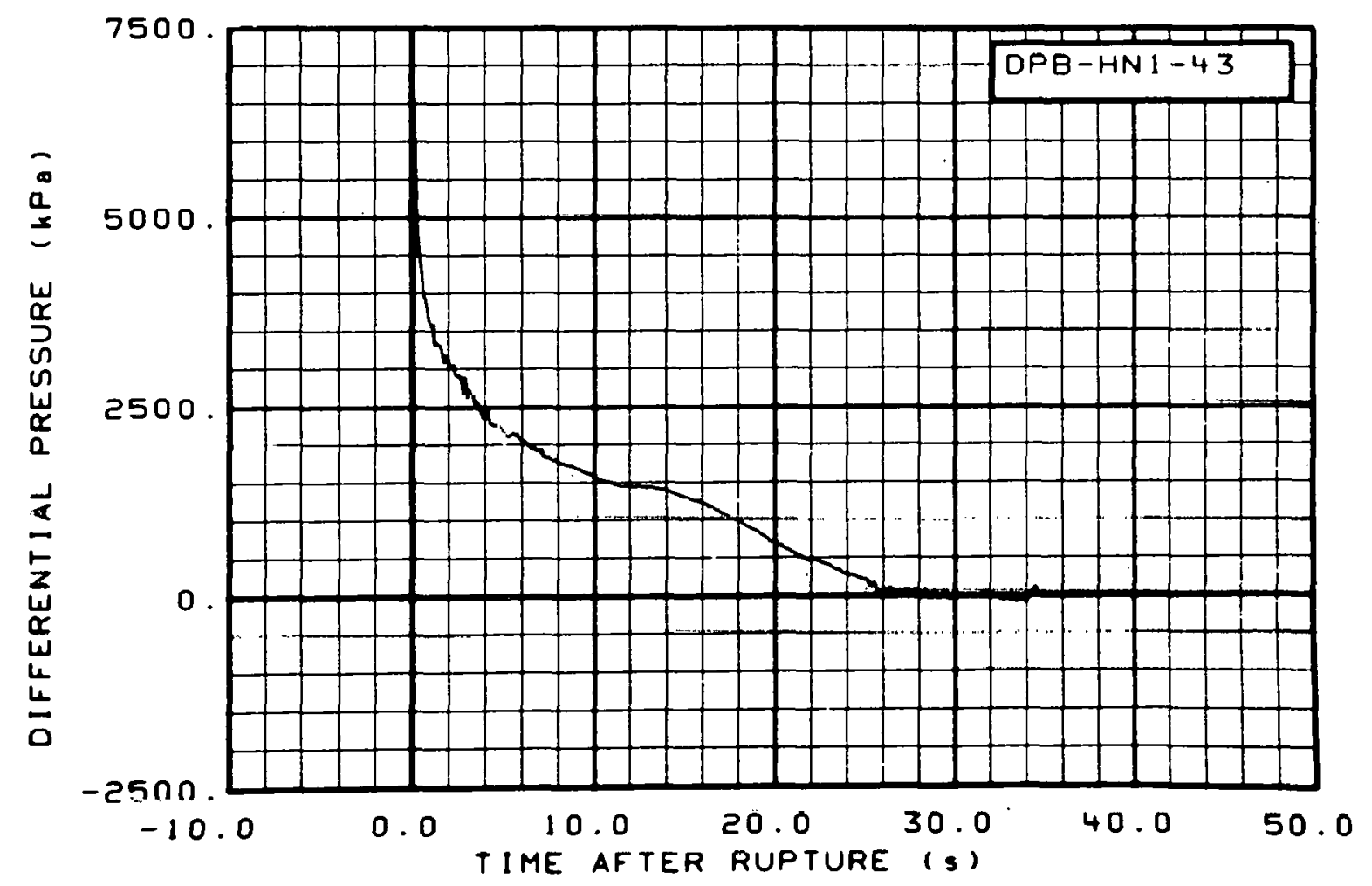

Fig. 206 Differential pressure in broken loop (DPB-HN1-43), from -6 to $42 \mathrm{~s}$. 


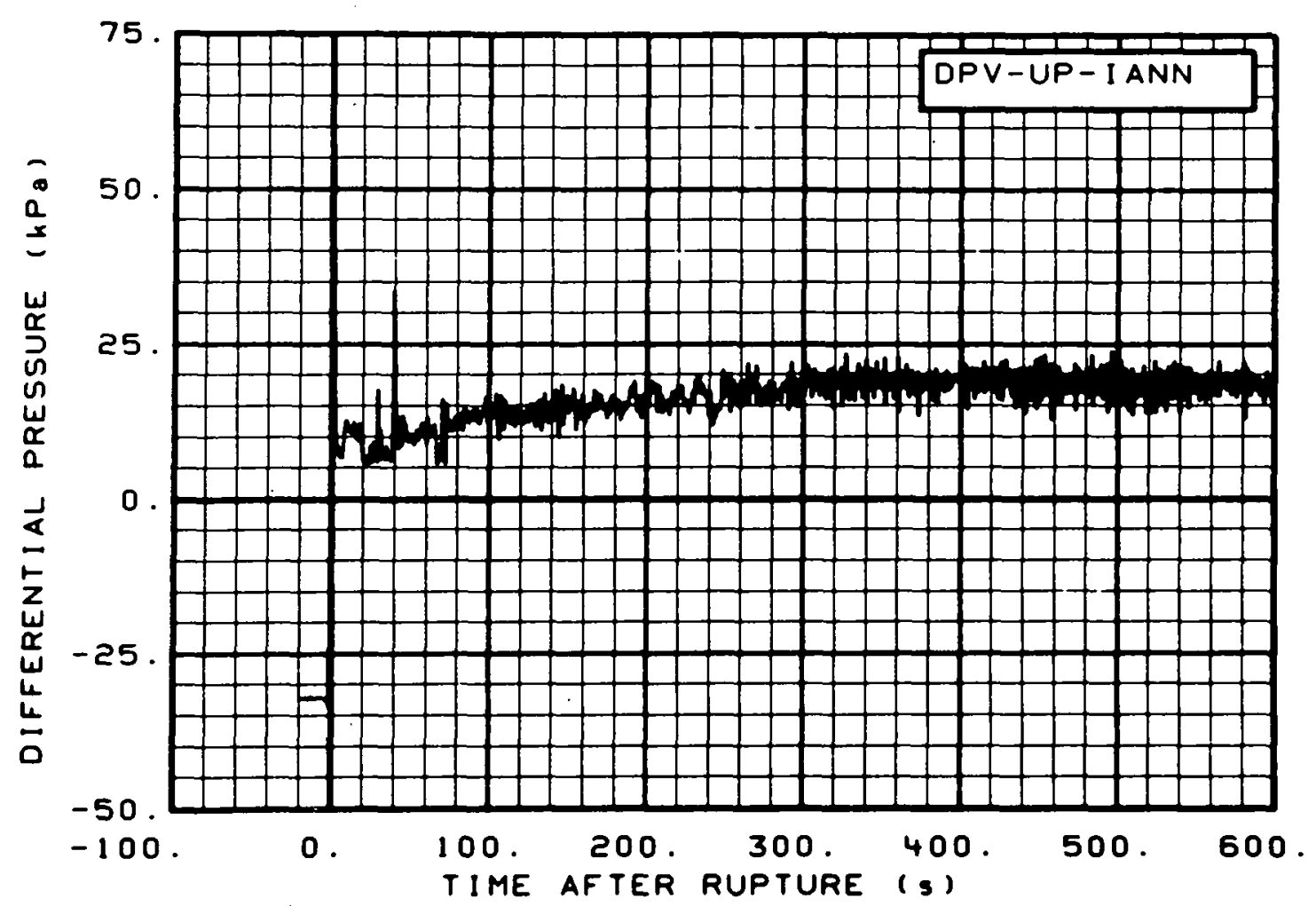

Fig. 207 Differential pressure in vessel (DPV-UP-IANN), from -20 to $600 \mathrm{~s}$.

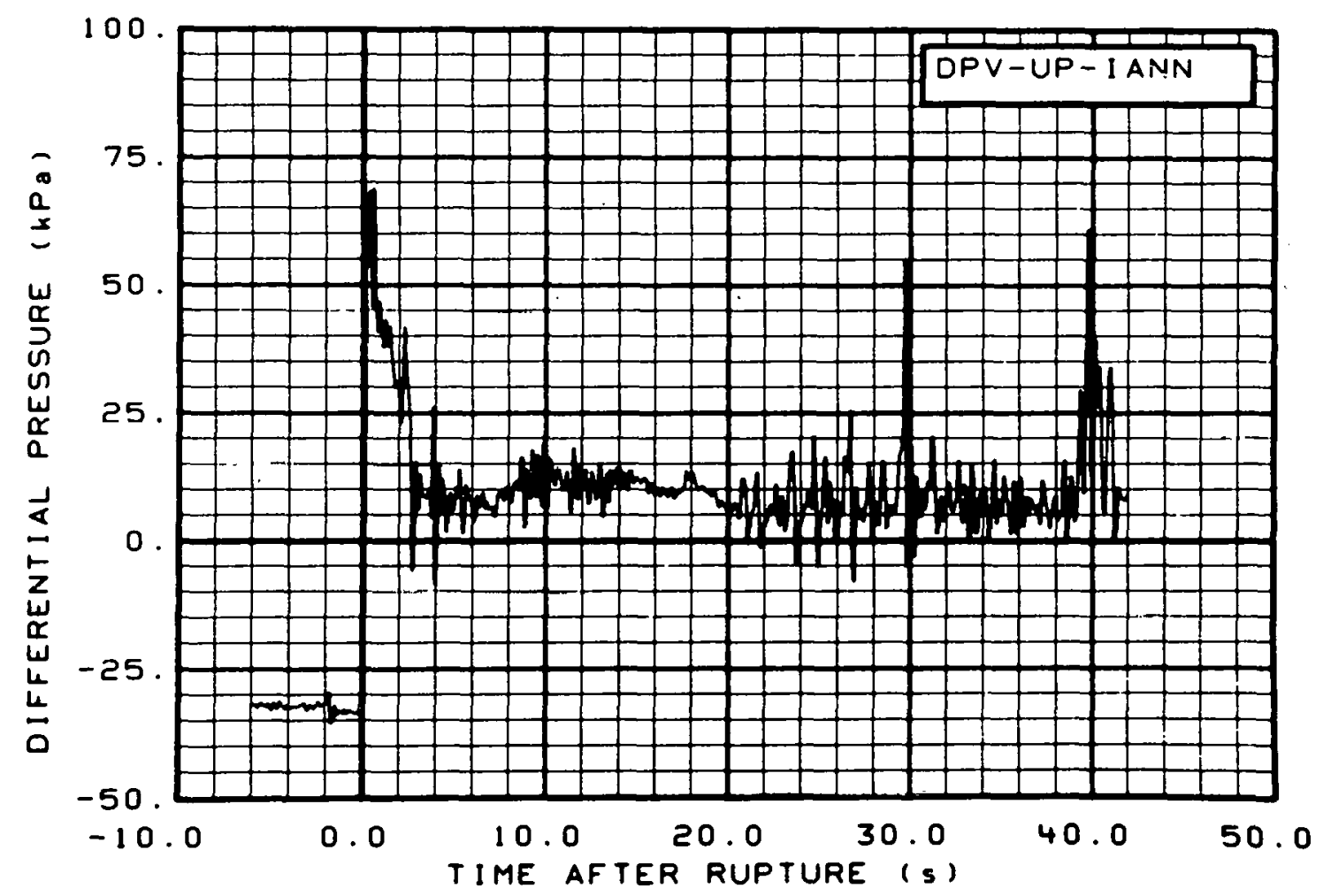

Fig. 208 Differential pressure in vessel (DPV-UP-IANN), from -6 to $42 \mathrm{~s}$. 


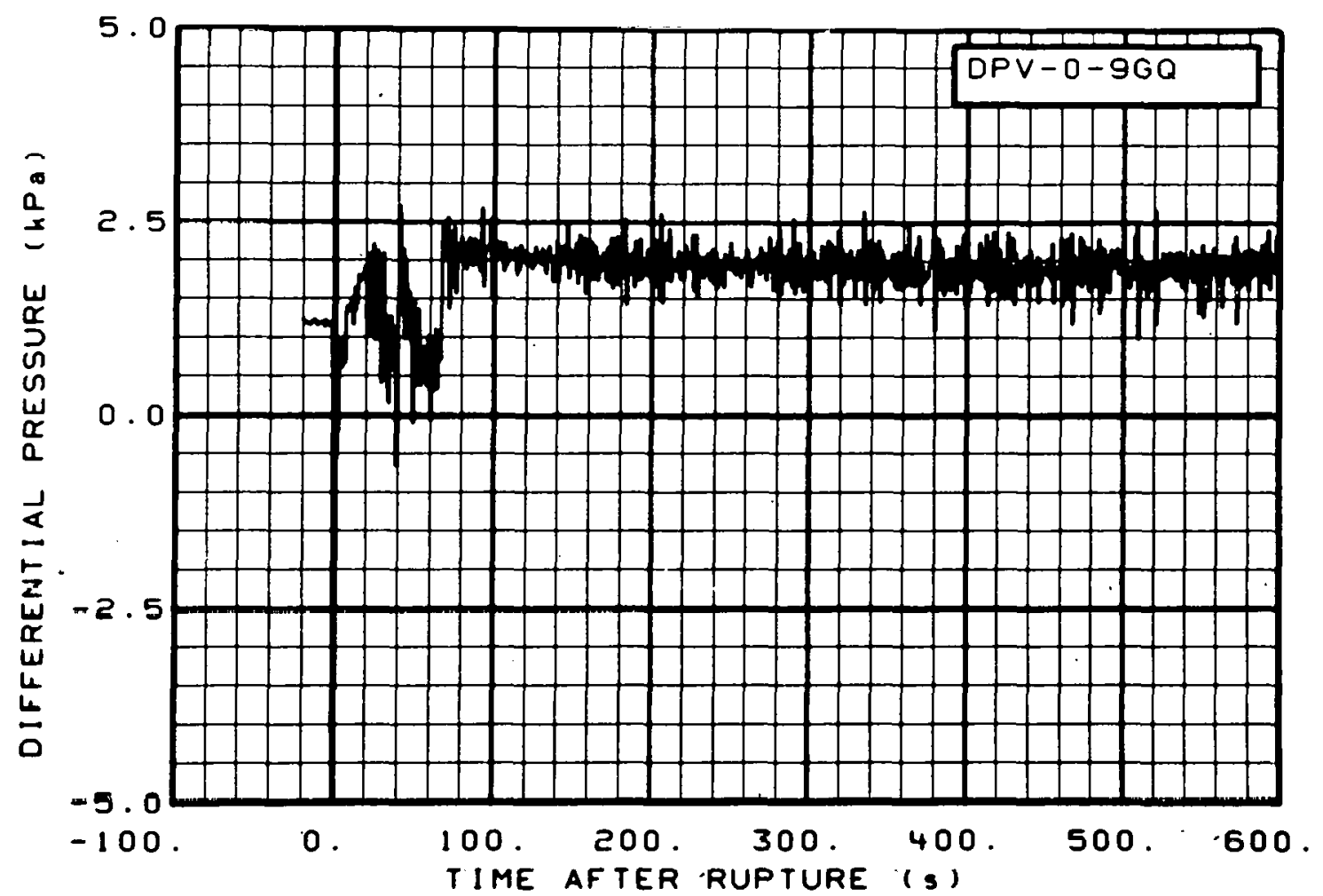

Fig. 209 Differential pressure in vessel (DPV-0-9GQ), from -20 to $600 \mathrm{~s}$.

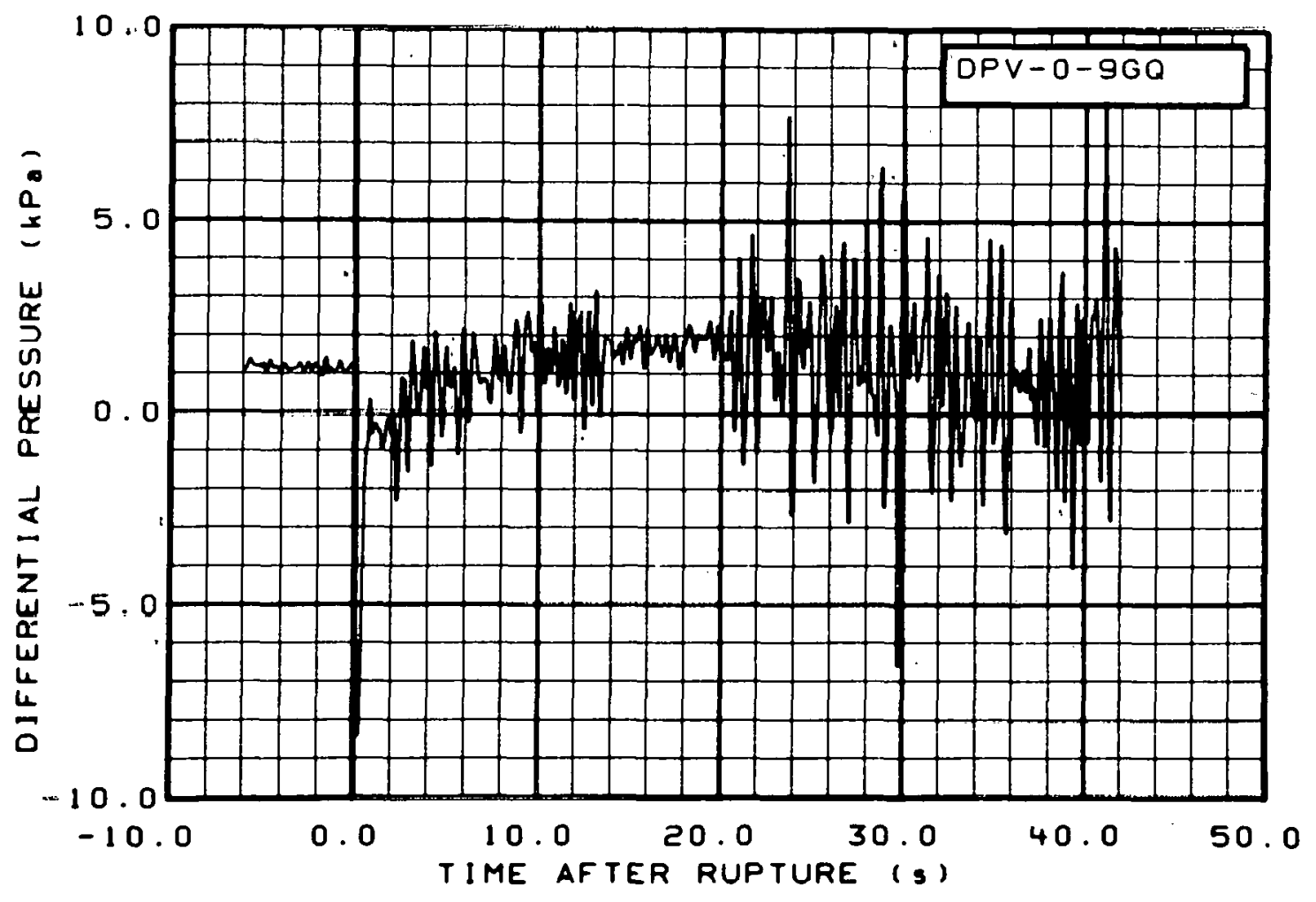

Fig. 210 Differential pressure in vessel (DPV-0-9GQ), from -6 to $42 \mathrm{~s}$. 


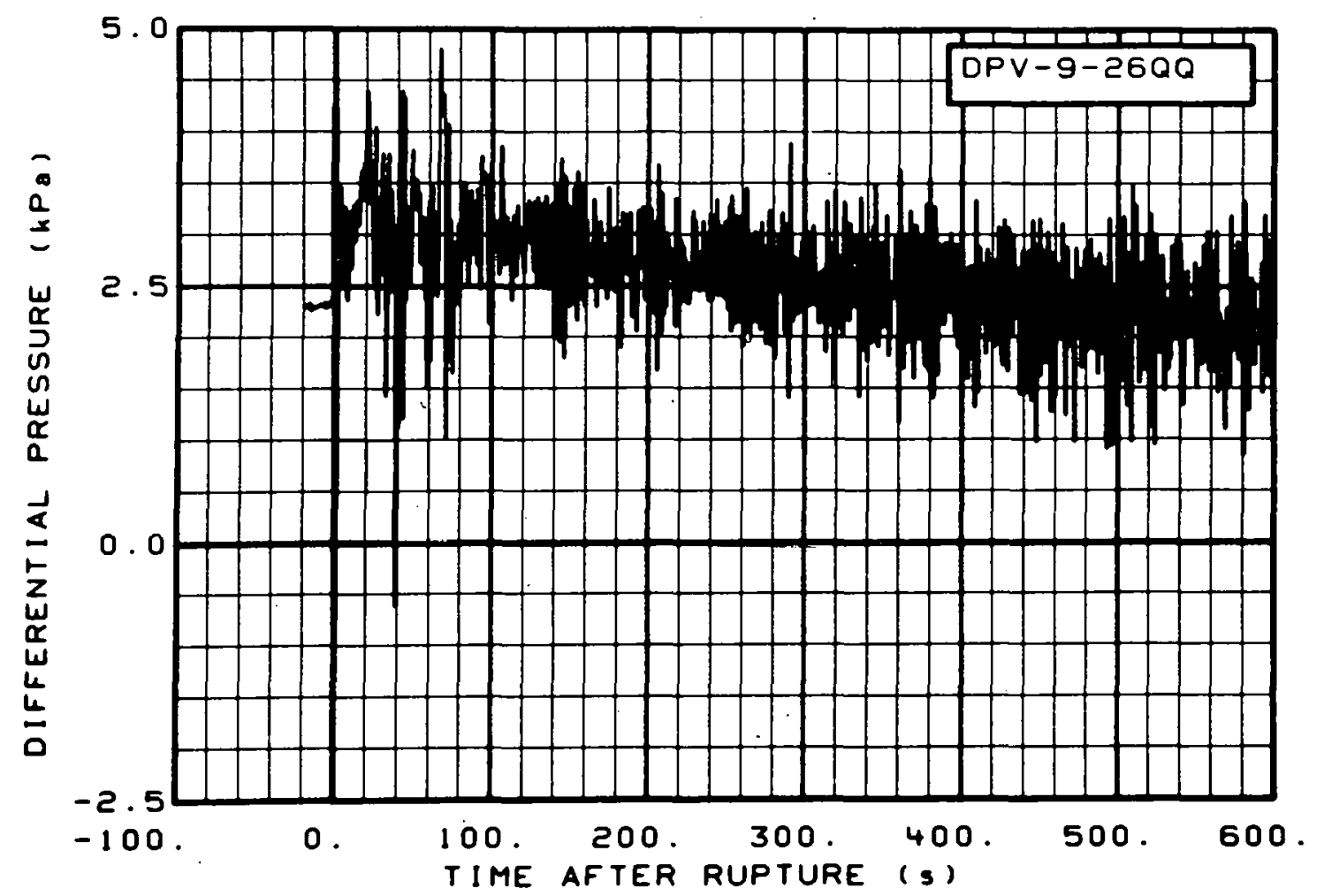

Fig. 211 Differential pressure in vessel (DPV-9-26QQ), from -20 to $600 \mathrm{~s}$.

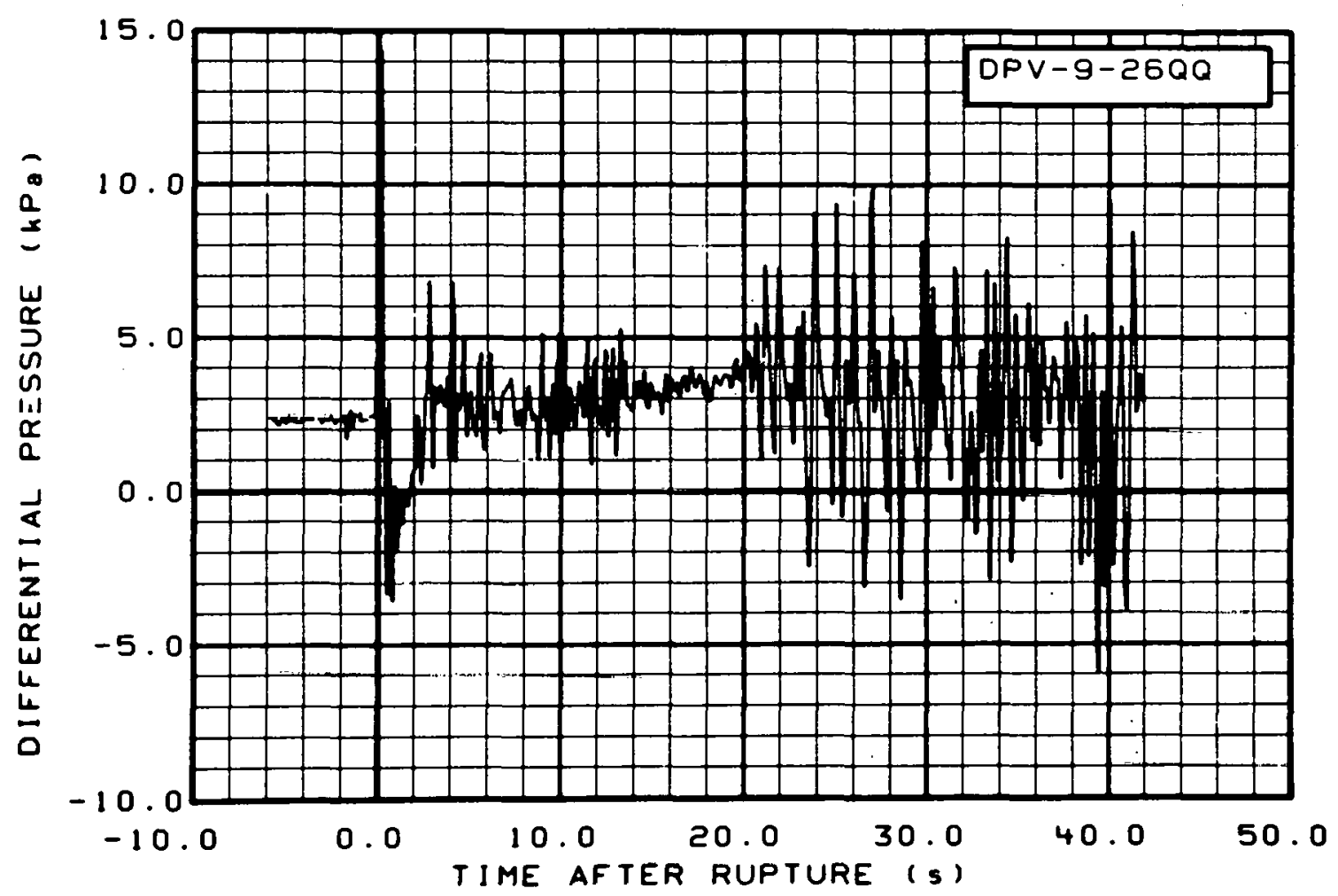

Fig. 212 Differential pressure in vessel (DPV-9-26QQ), from -6 to $42 \mathrm{~s}$. 


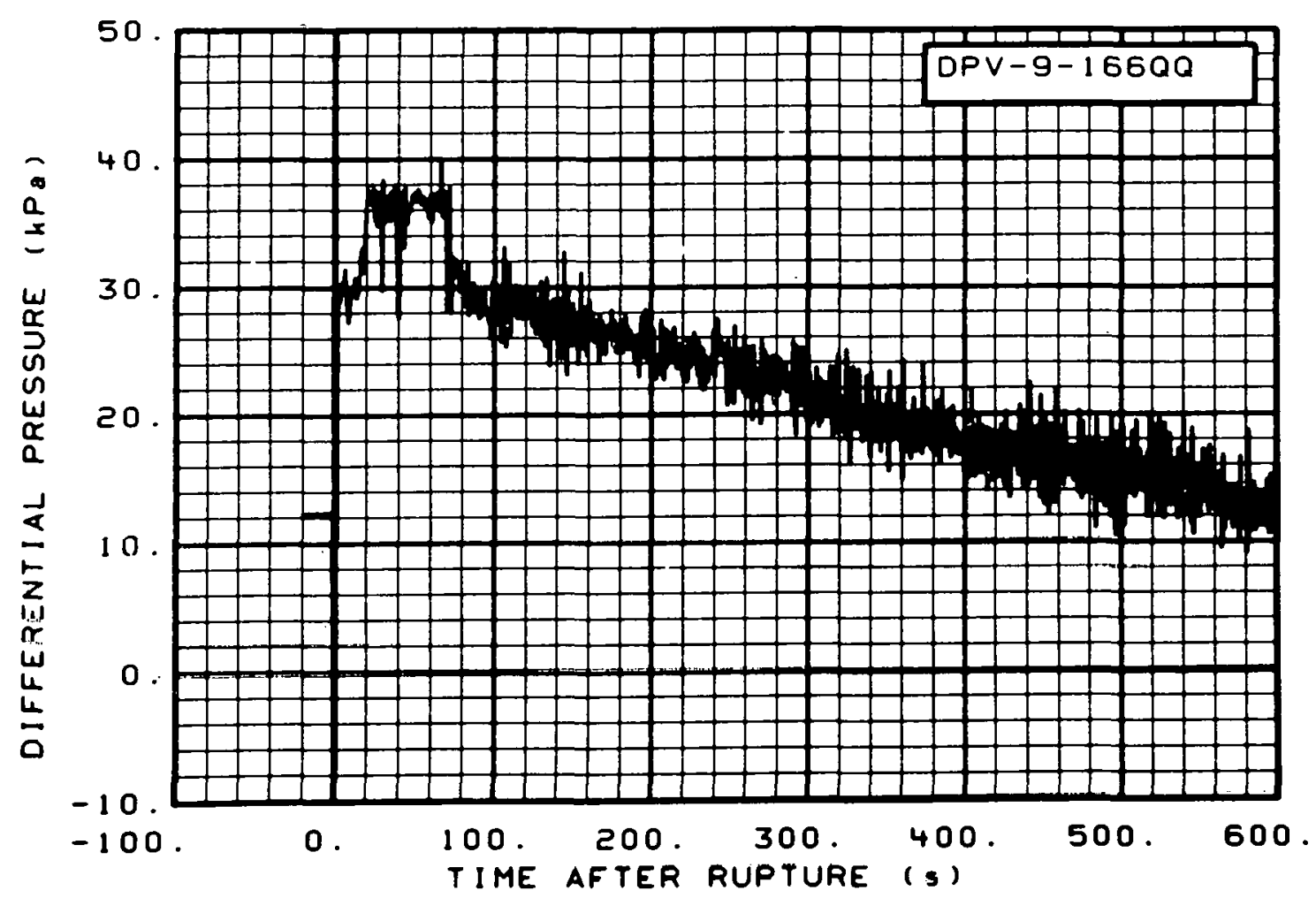

Fig. 213 Differential pressure in vessel (DPV-9-1660Q), from -20 to 600 s.

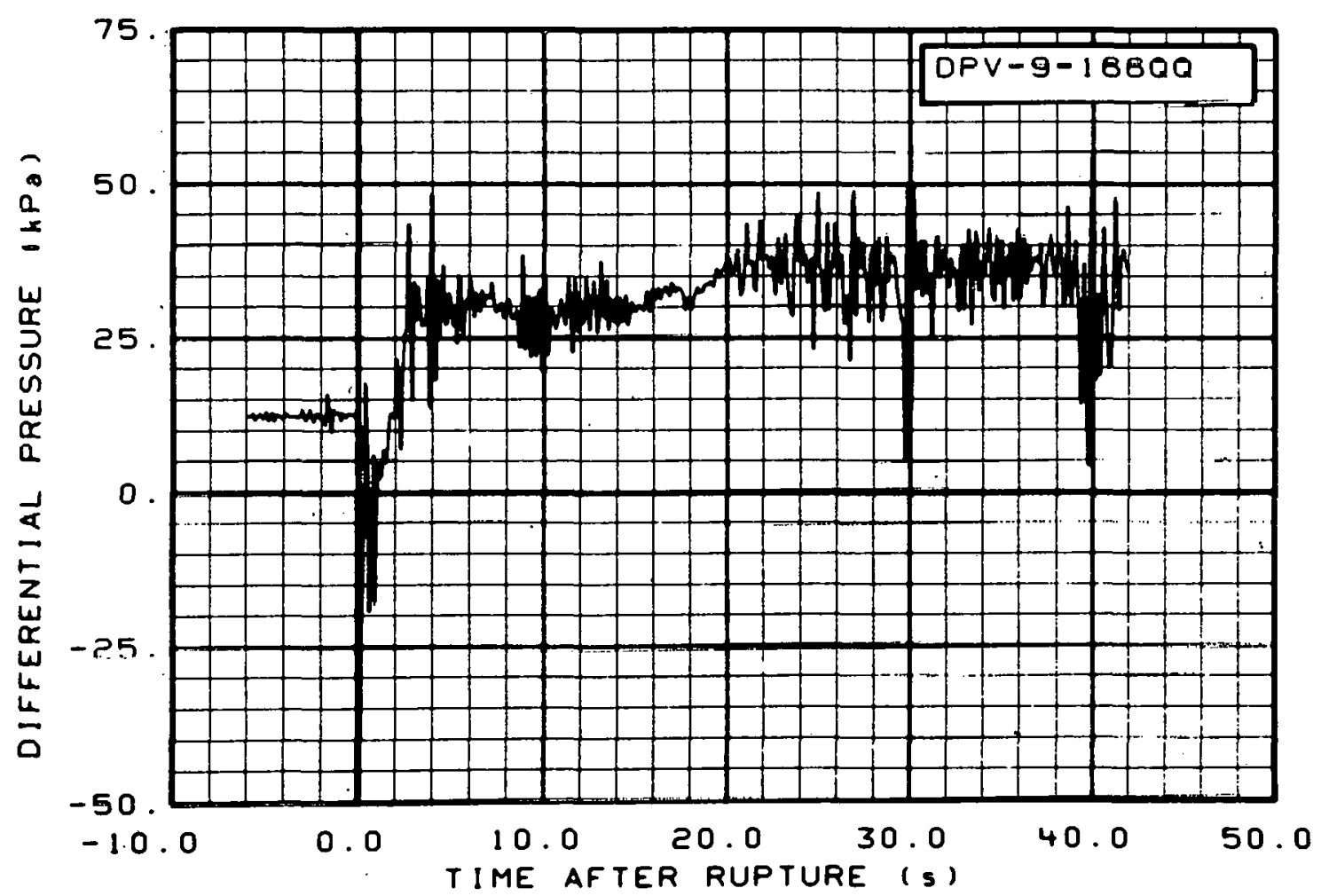

Fig. 214 Differential pressure in vesse1 (DP.V-9-166QQ), from -6 to $42 \mathrm{~s}$. 


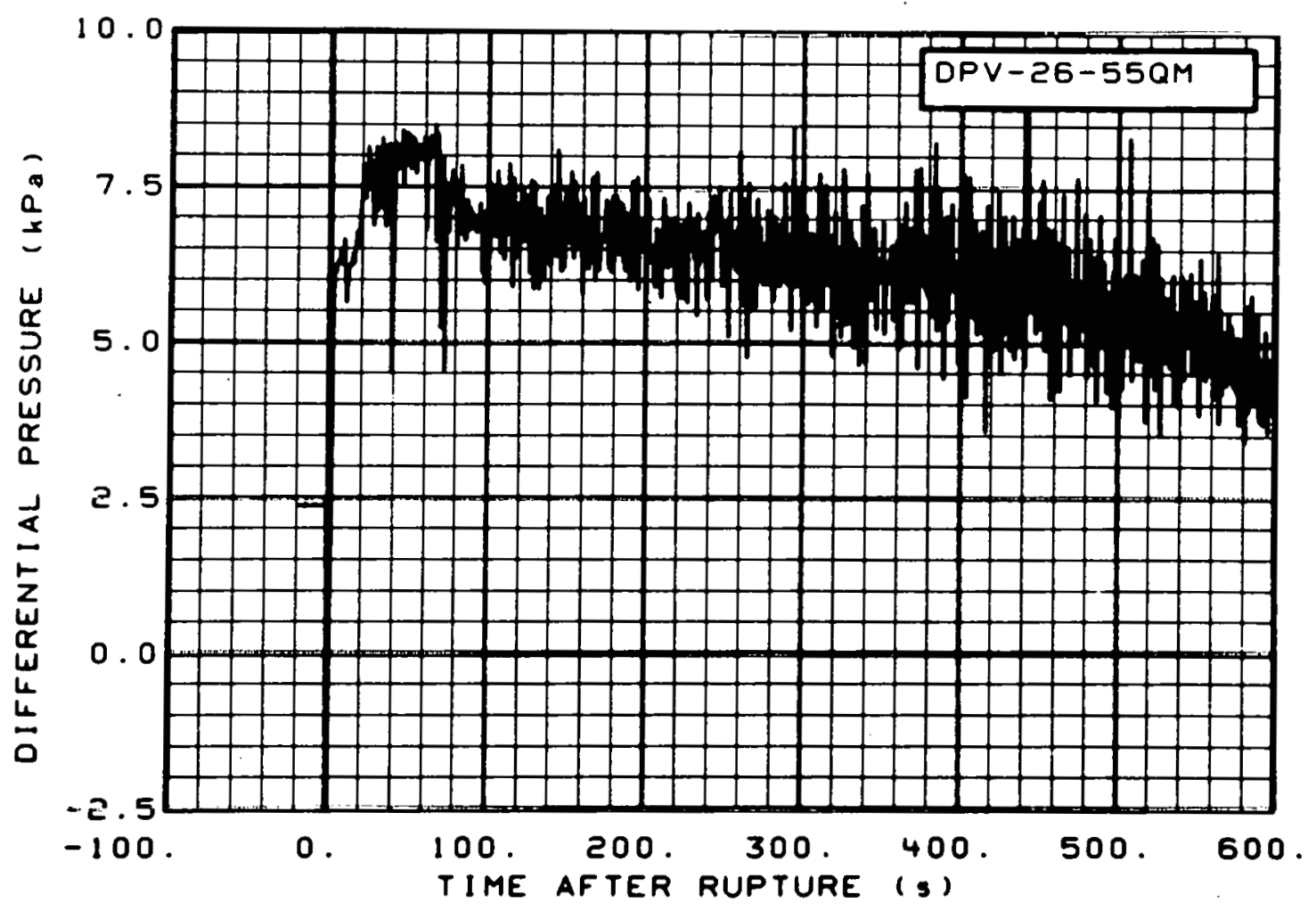

Fig. 215 Differential pressure in vessel (DPV-26-55QM), from -20 to $600 \mathrm{~s}$.

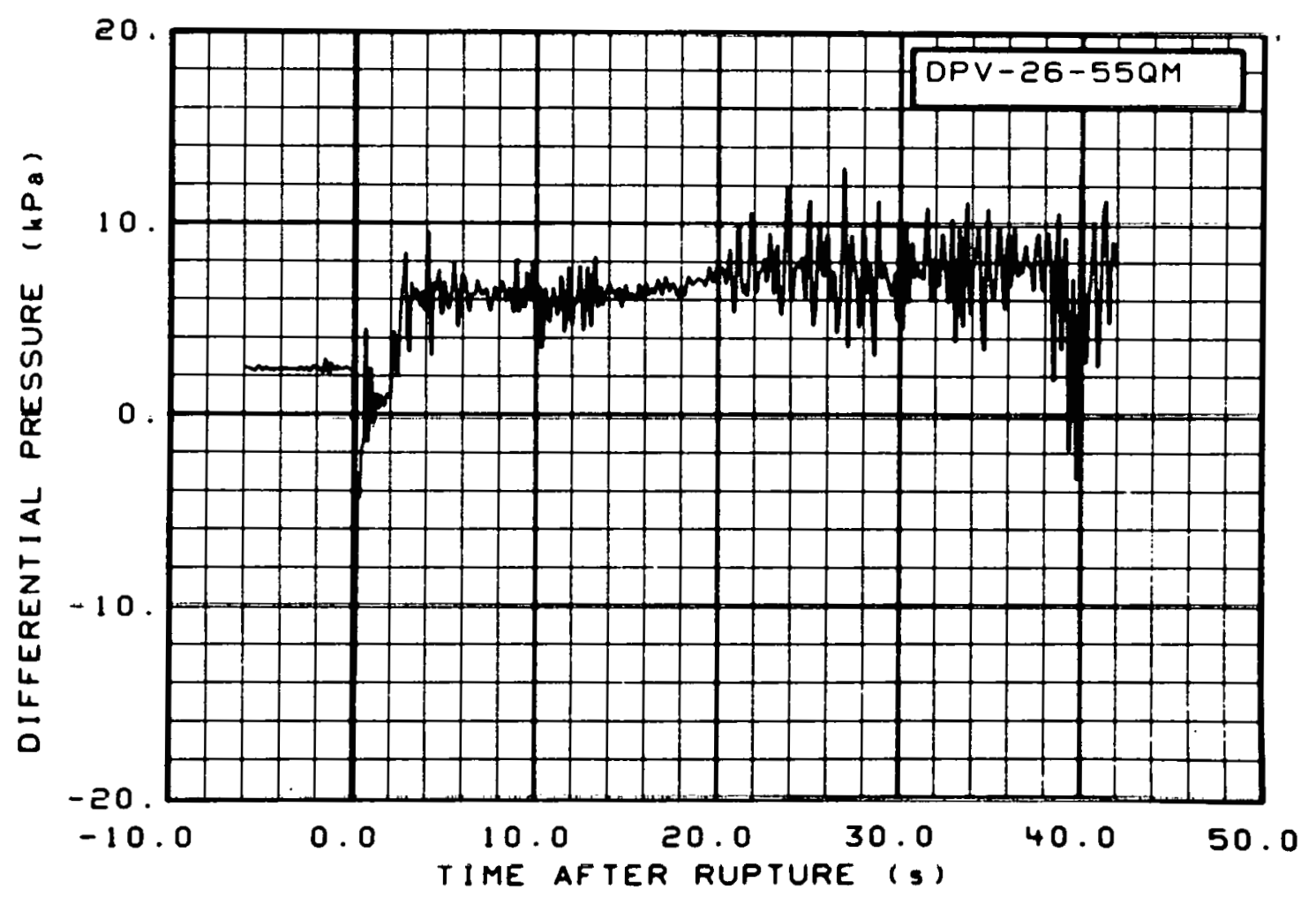

Fig. 216 Differential pressure in vessel (DPV-26-55QM), from -6 to $42 \mathrm{~s}$. 


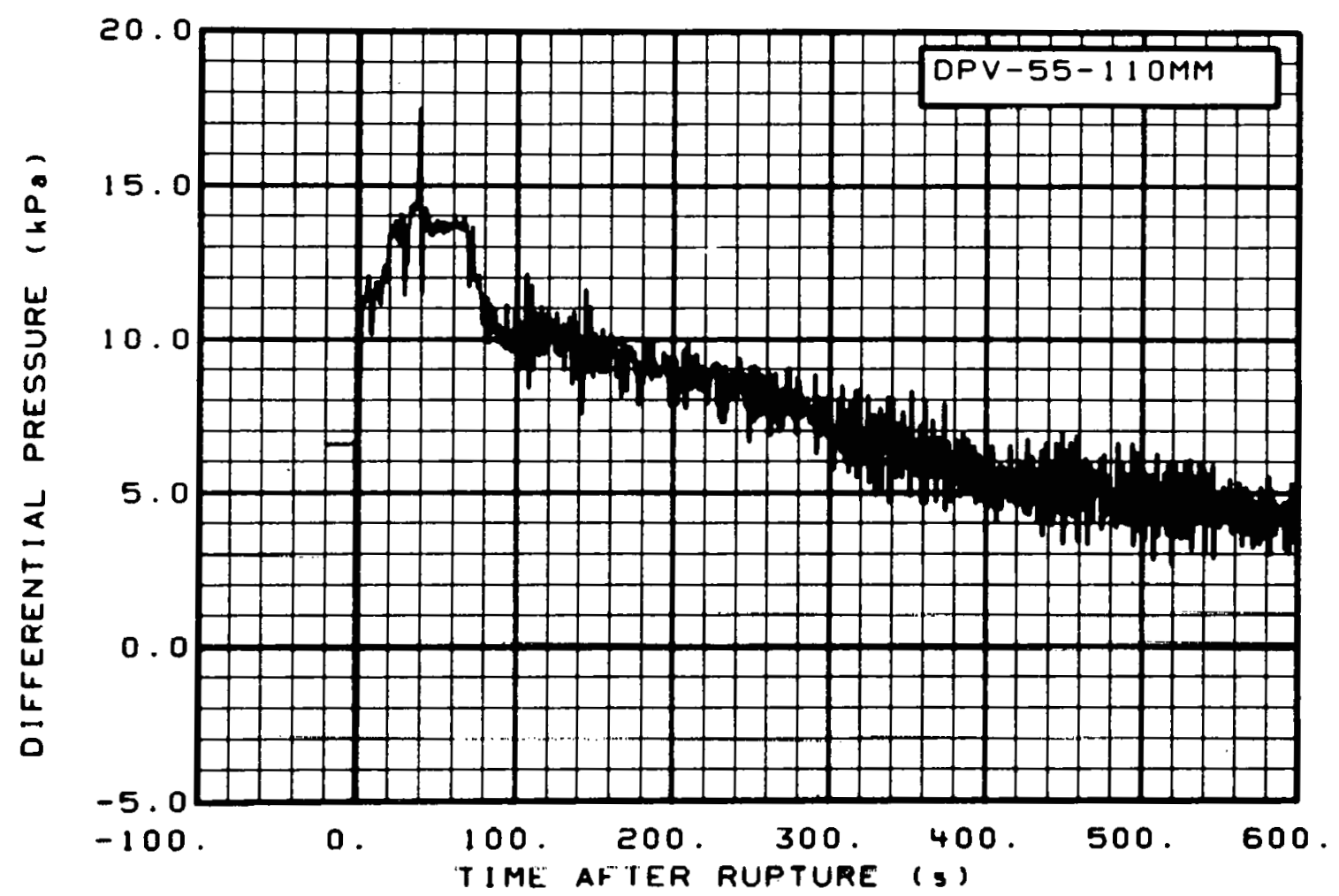

Fig. 217 Differential pressure in vessel (DPV-55-110MM), from -20 to $600 \mathrm{~s}$.

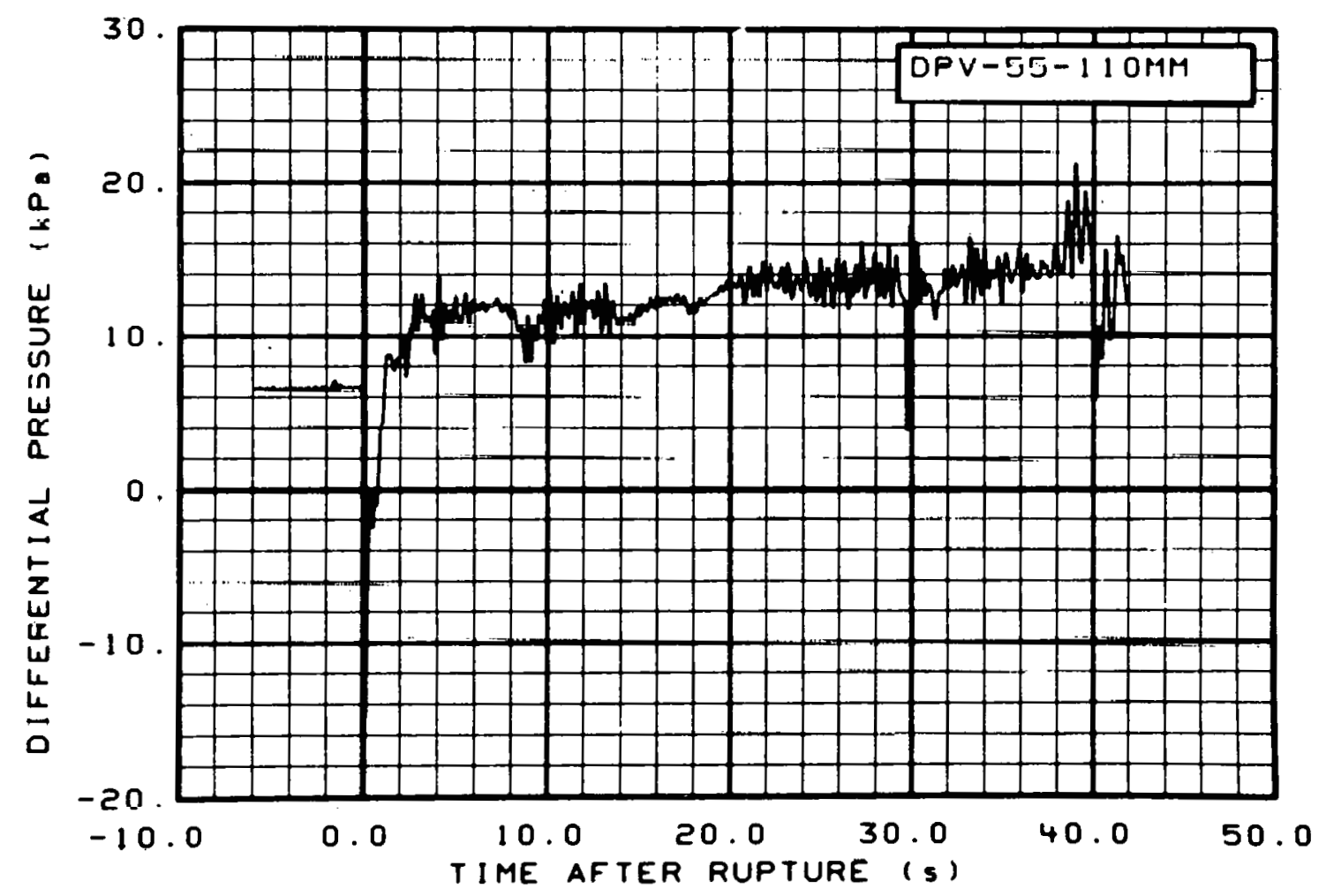

Fig. 218 Differential pressure in vessel (DPV-55-110MM), from -6 to $42 \mathrm{~s}$. 


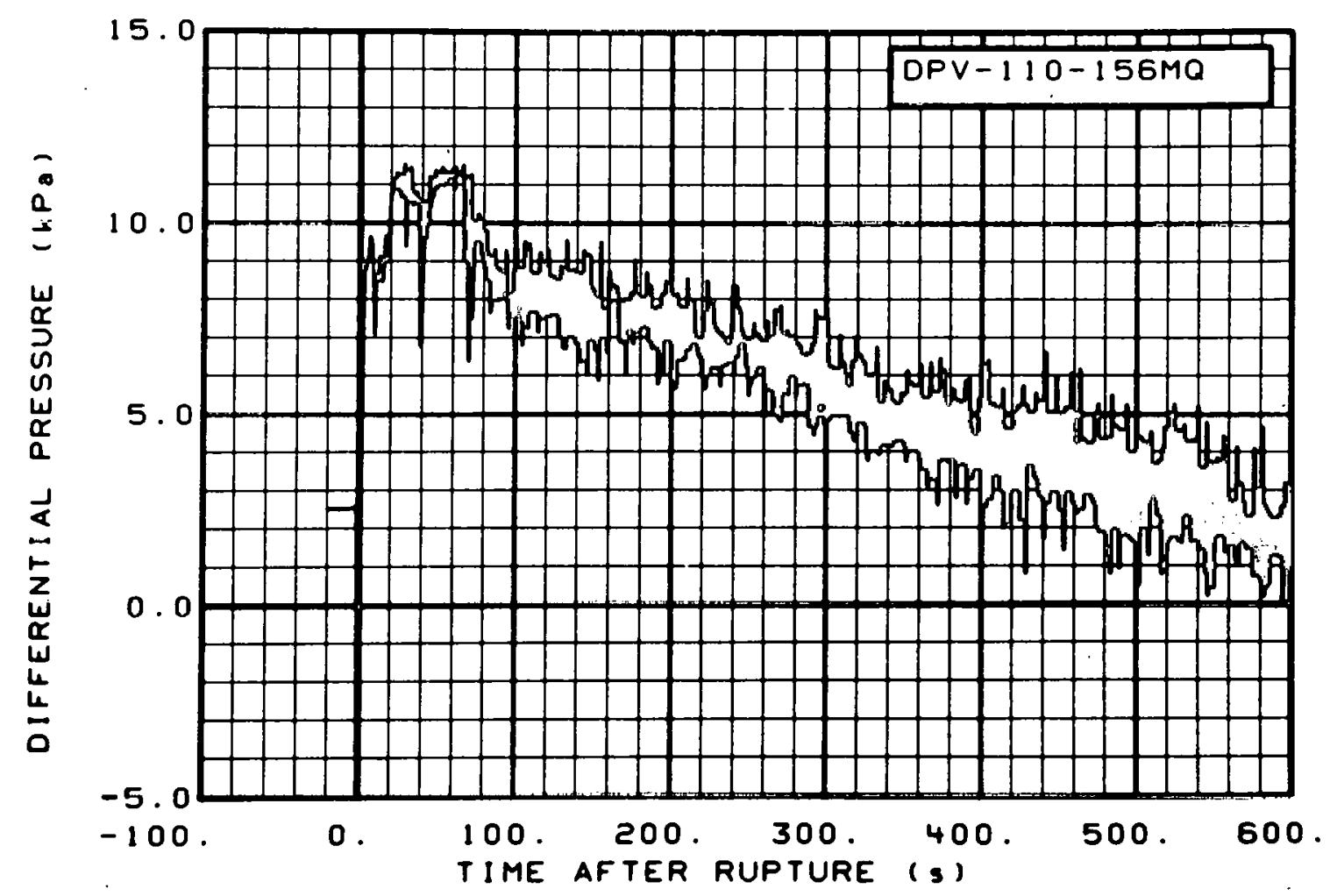

Fig. 219 Differential pressure in vessel (DPV-110-156MQ), from -20 to $600 \mathrm{~s}$.

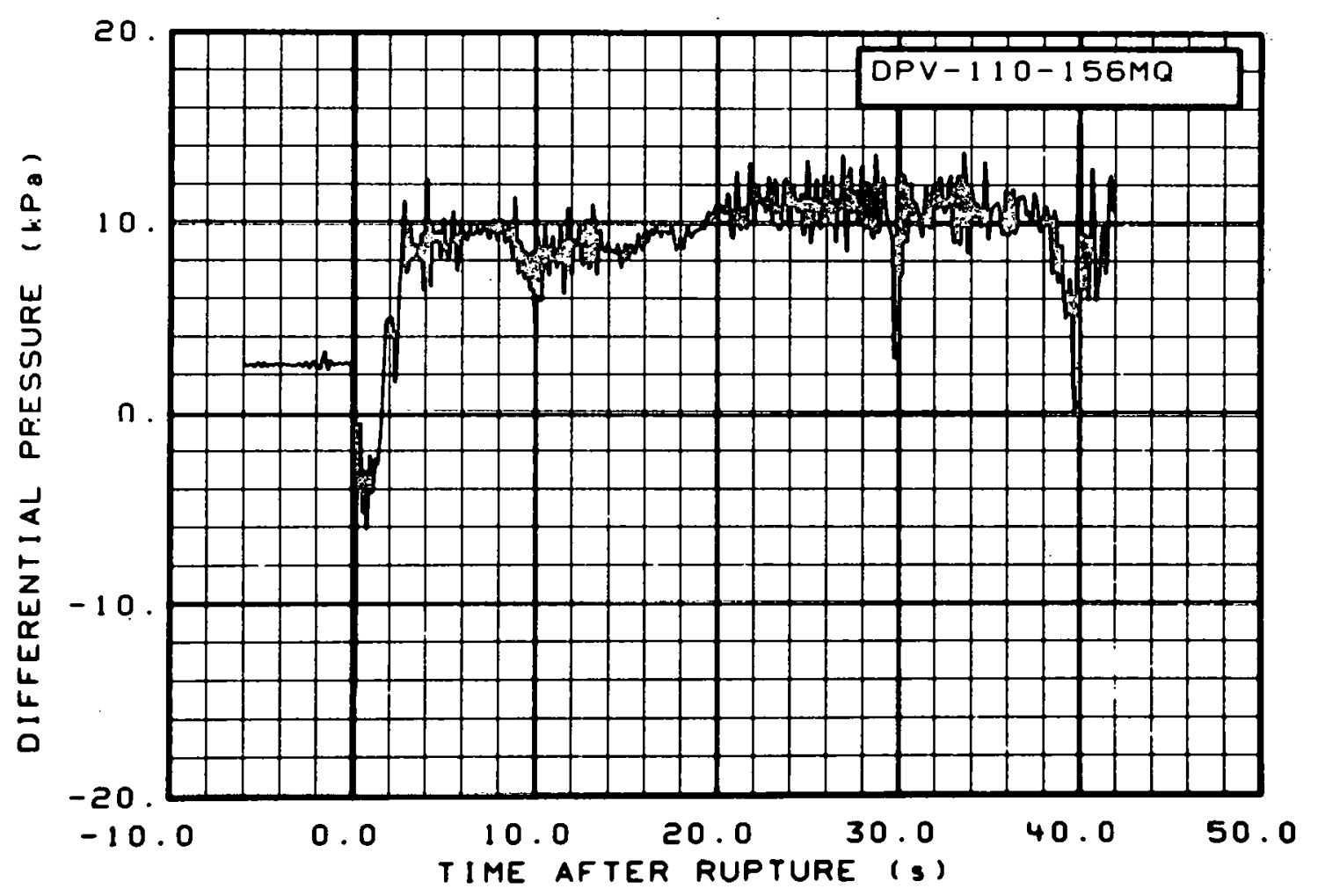

Fig. 220 Differential pressure in vessel (DPV-110-156MQ), from -6 to $42 \mathrm{~s}$. 


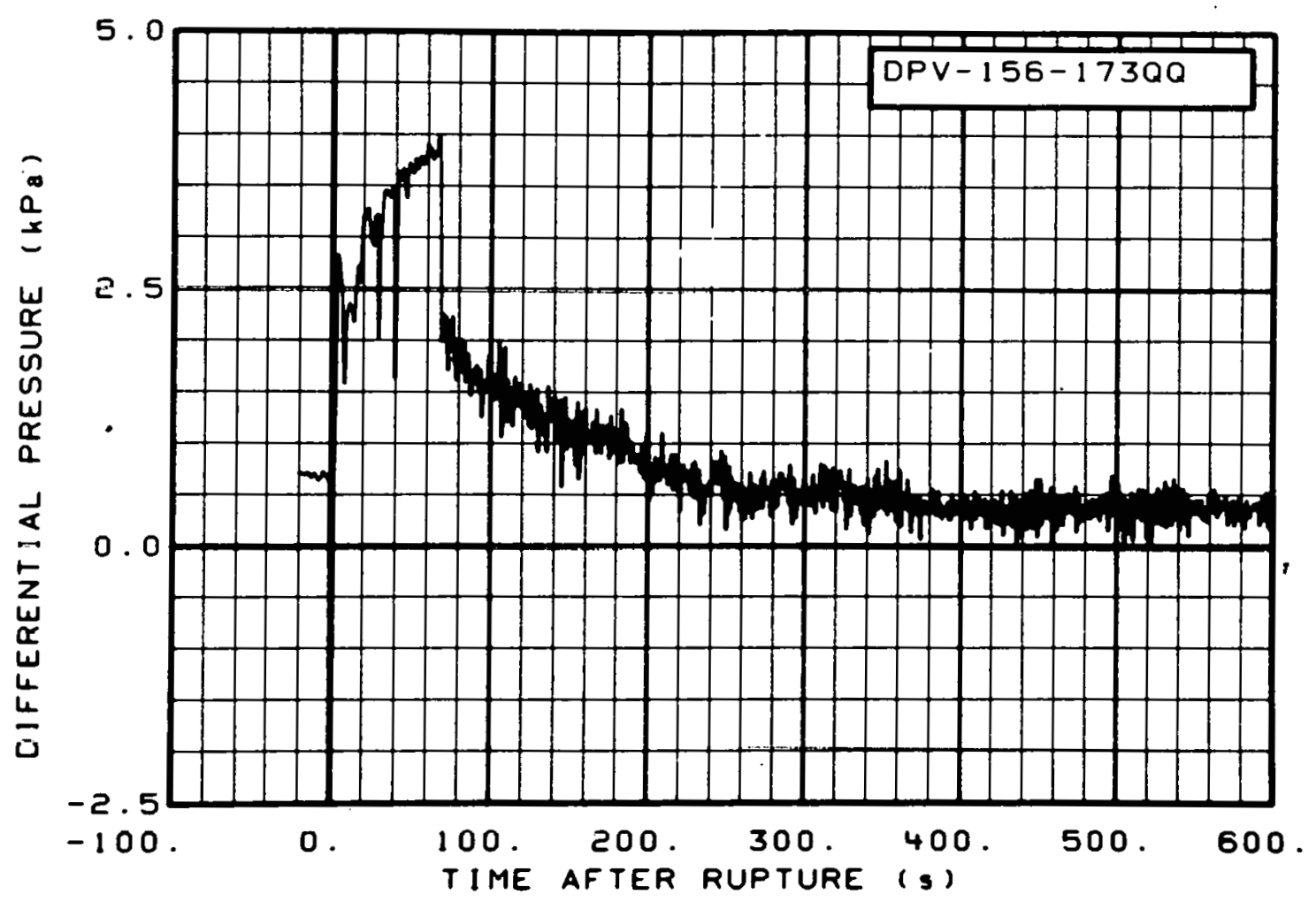

Fig. 221 Differential pressure in vessel (DPV-156-173QQ), from -20 to $600 \mathrm{~s}$.

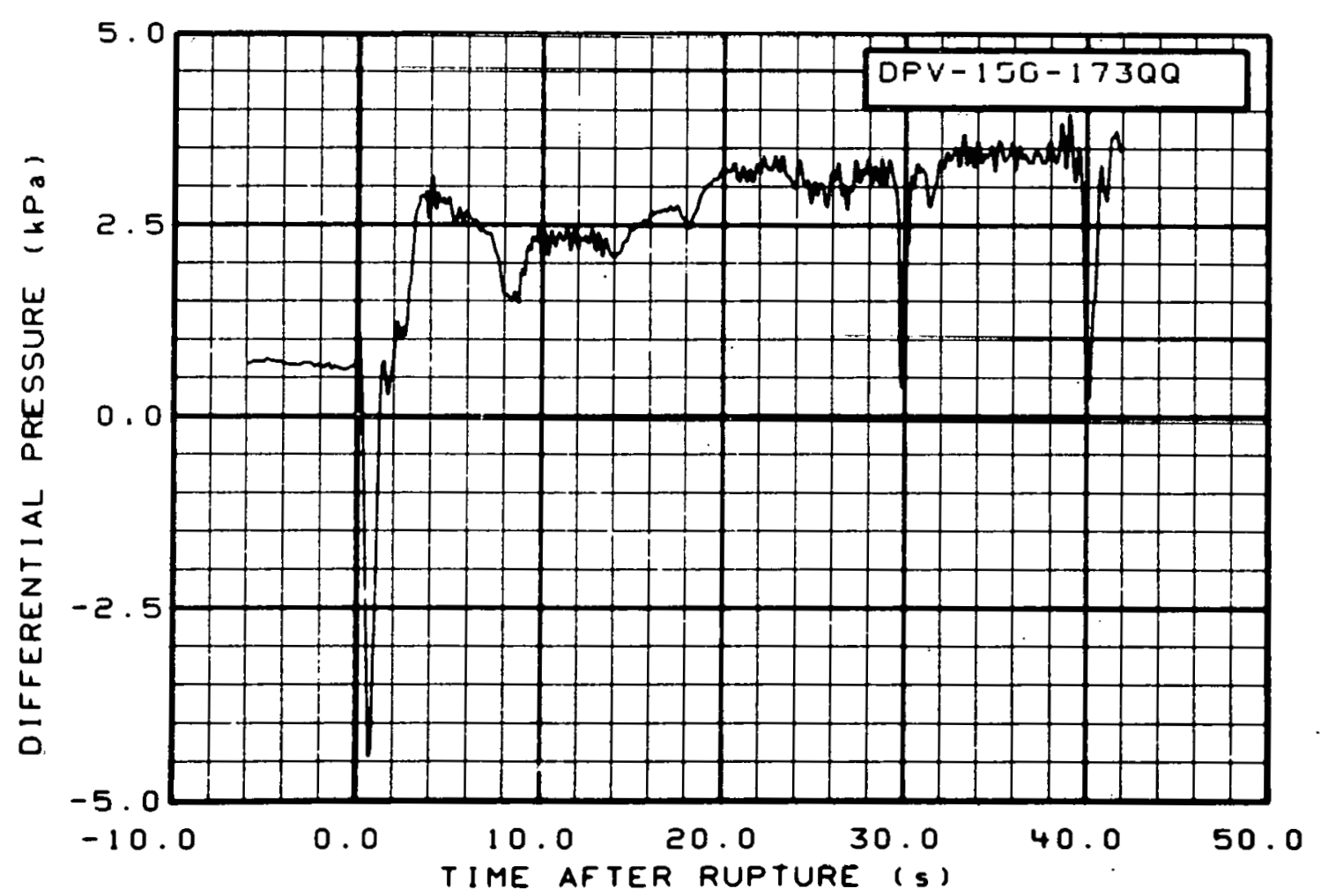

Fig. 222 Differential pressure in vessel (DPV-156-173QQ), from -6 to $42 \mathrm{~s}$. 


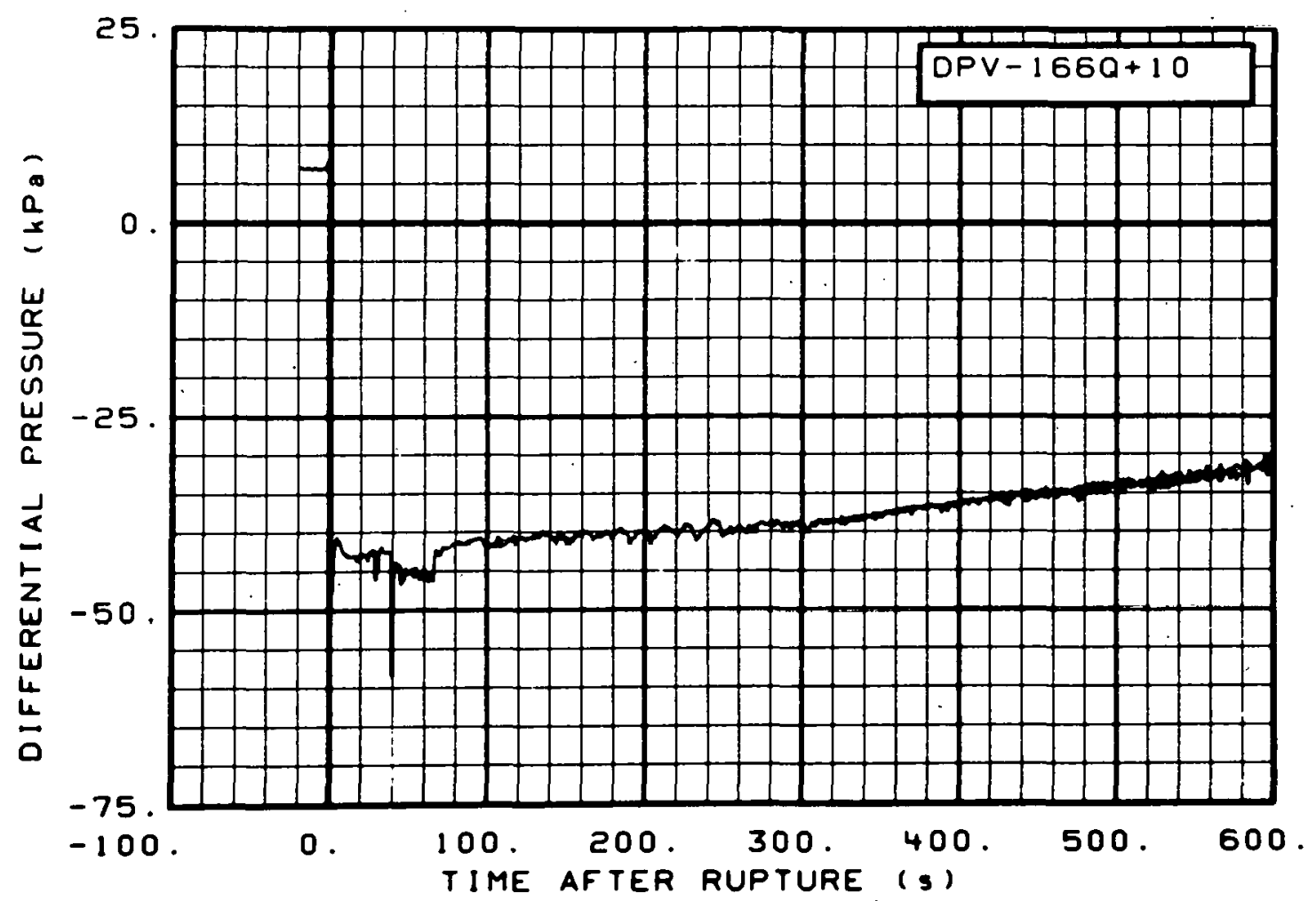

Fig. 223 Differential pressure in vessel (DPV-166Q+10), from -20 to . $600 \mathrm{~s}$.

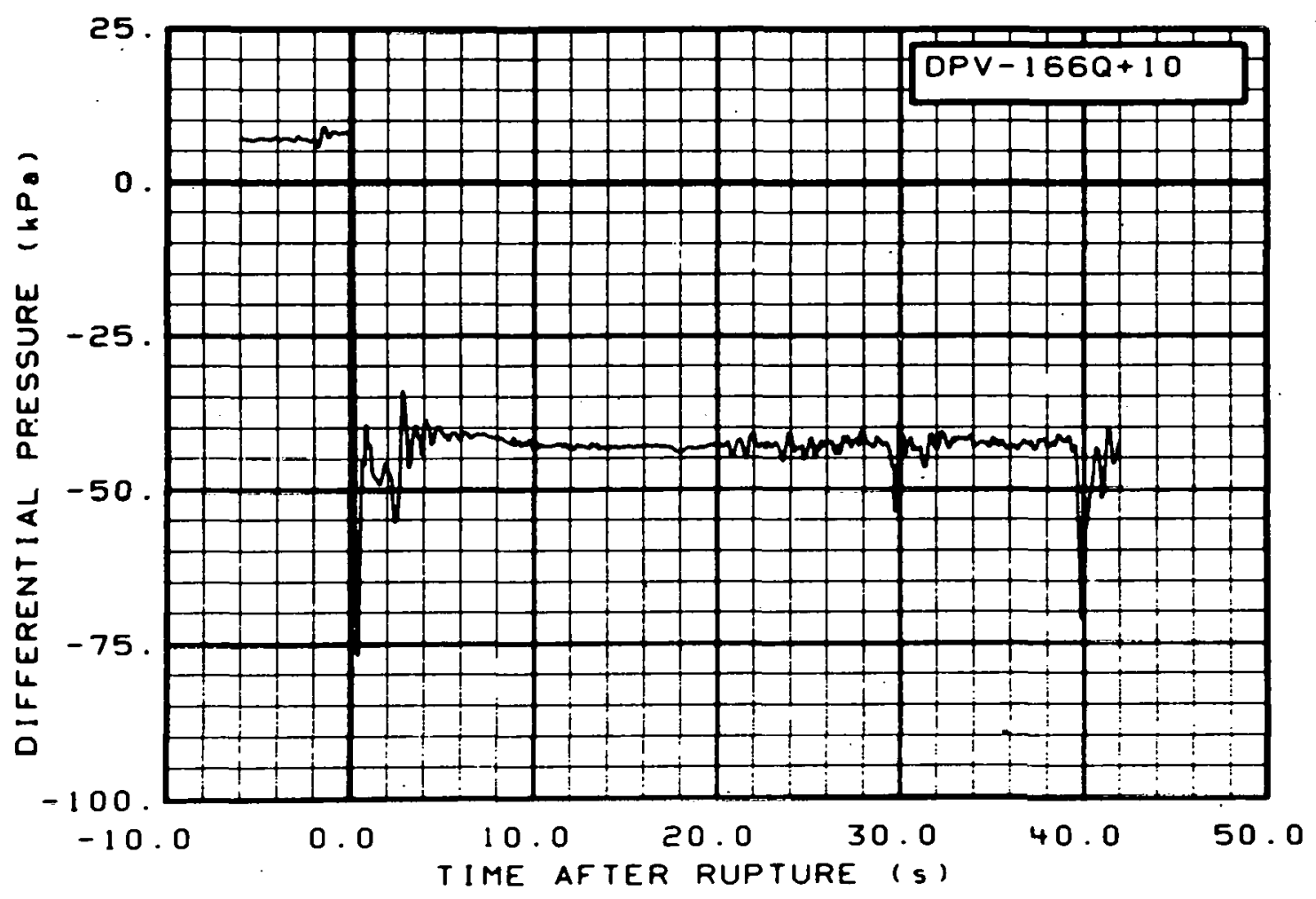

Fig. 224 Differential pressure in vessel (DPV-166Q+10), from -6 to $42 \mathrm{~s}$. 


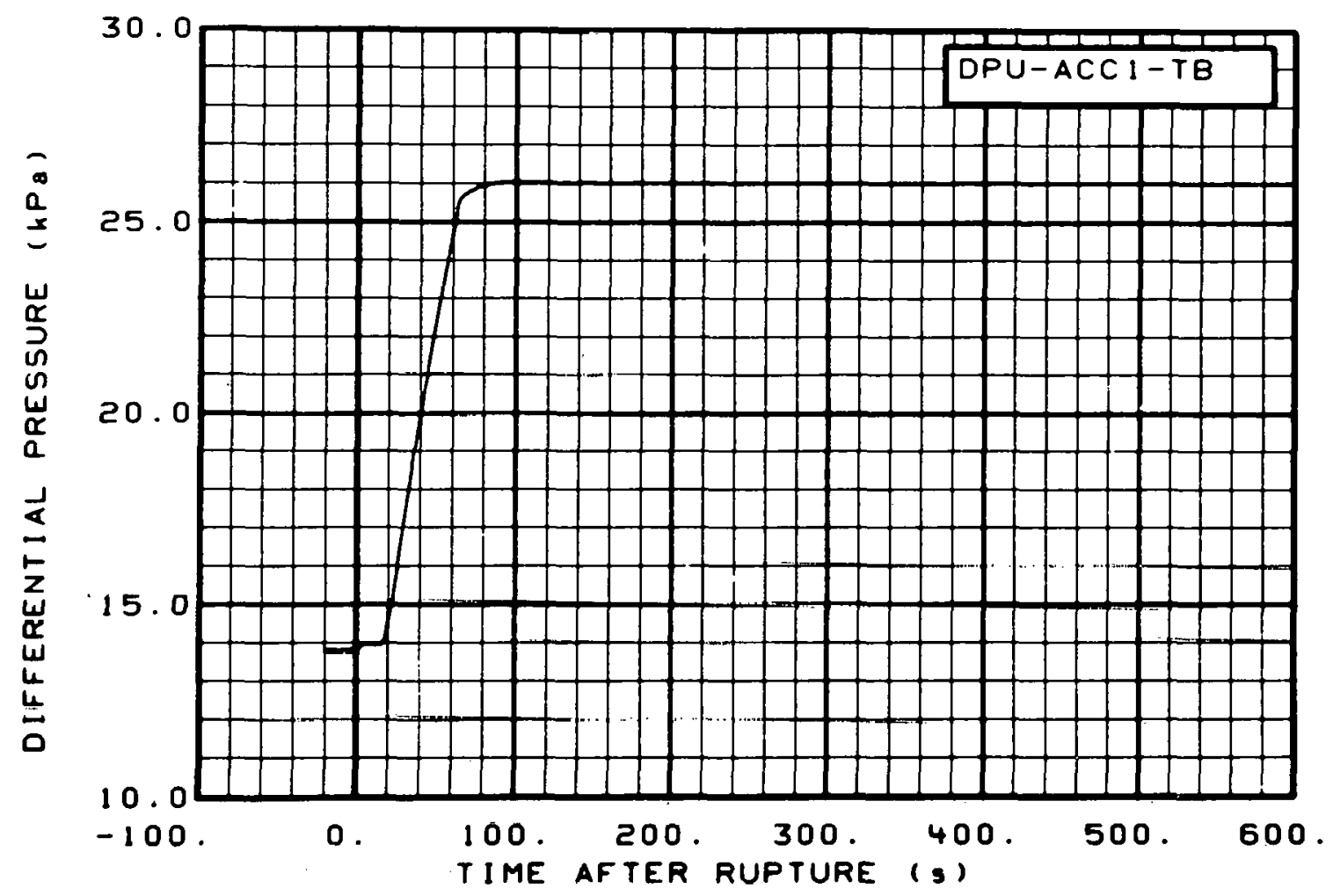

Fig. 225 Differential pressure in intact loop accumulator (DPU-ACCl-TB), from -20 to $600 \mathrm{~s}$.

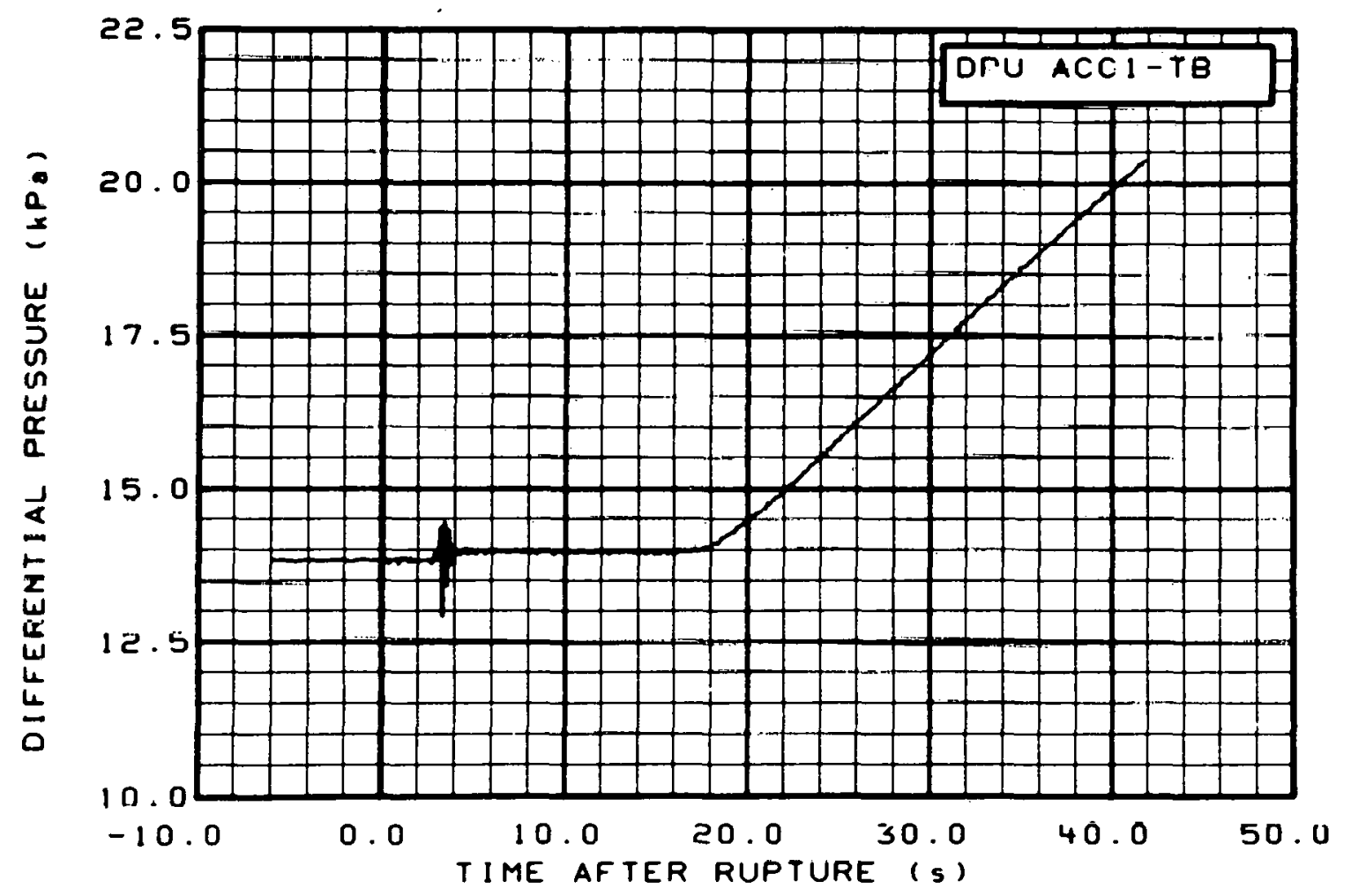

Fig. 226 Differential pressure in intact loop accumulator (DPU-ACC1-TB), from -6 to $42 \mathrm{~s}$. 


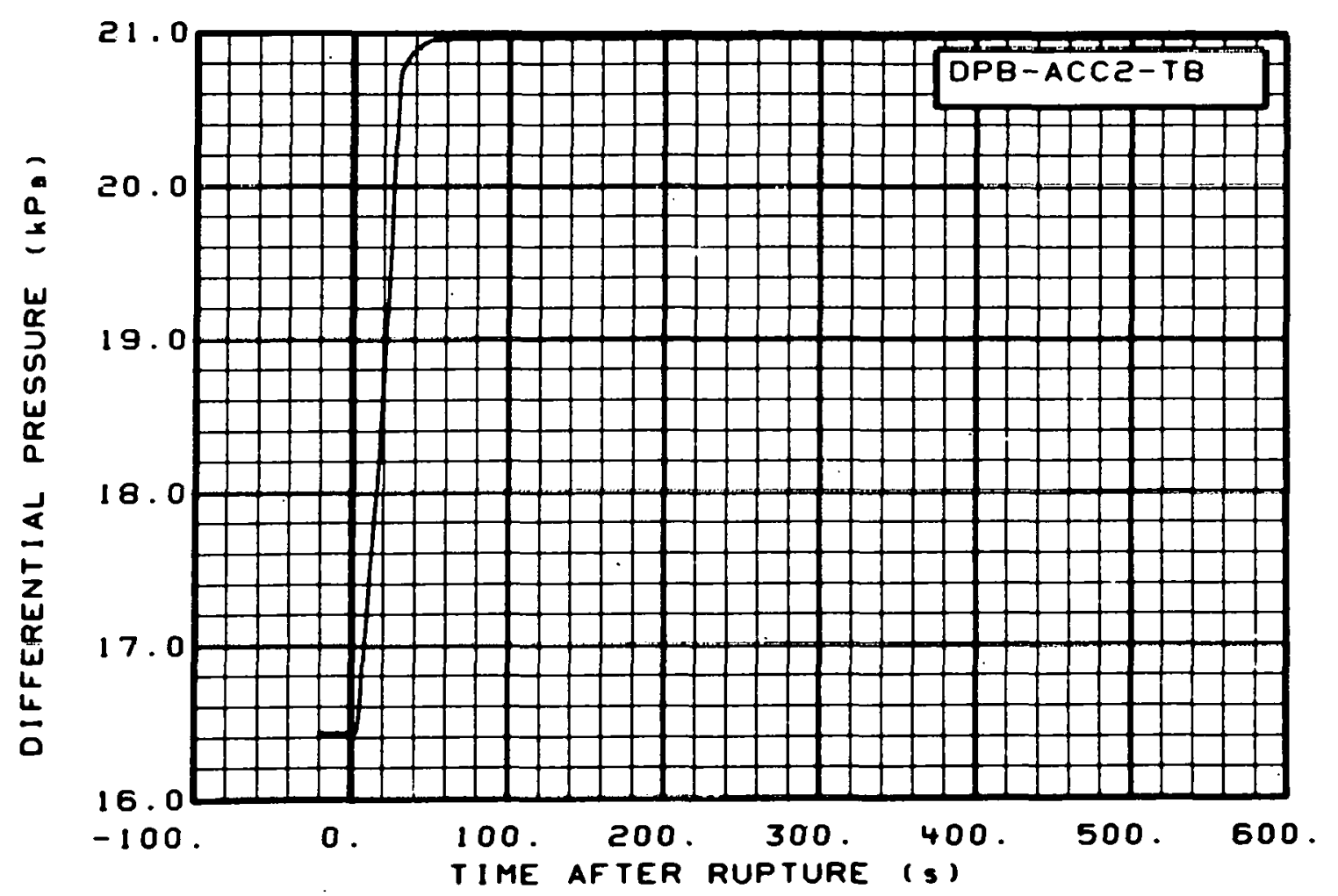

Fig. 227 Differential pressure in broken loop accumulator (DPB-ACC2-TB), from -20 to $600 \mathrm{~s}$.

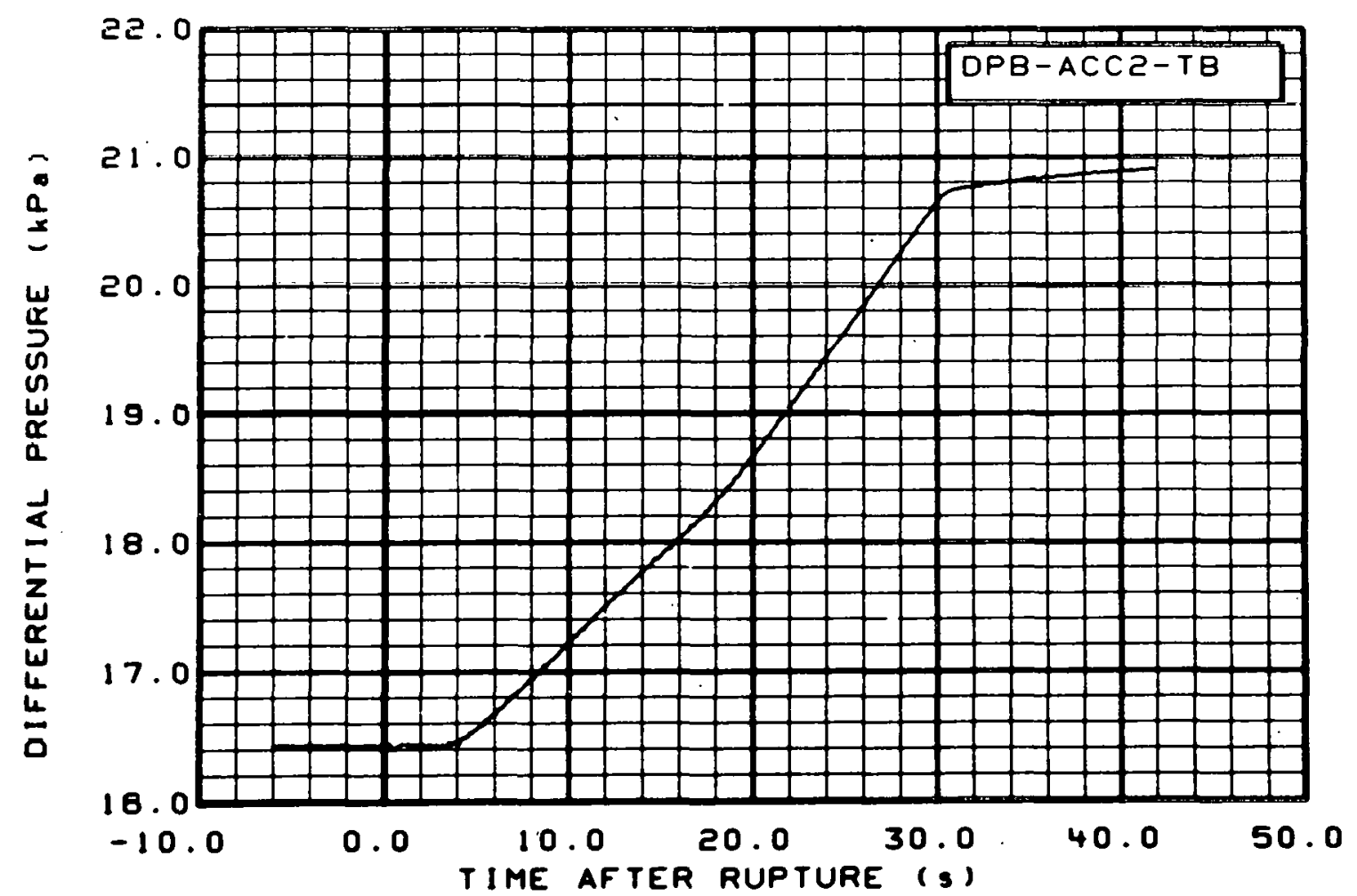

Fig. 228 Differential pressure in broken loop accumulator (DPB-ACC2-TB), from -6 to $42 \mathrm{~s}$. 


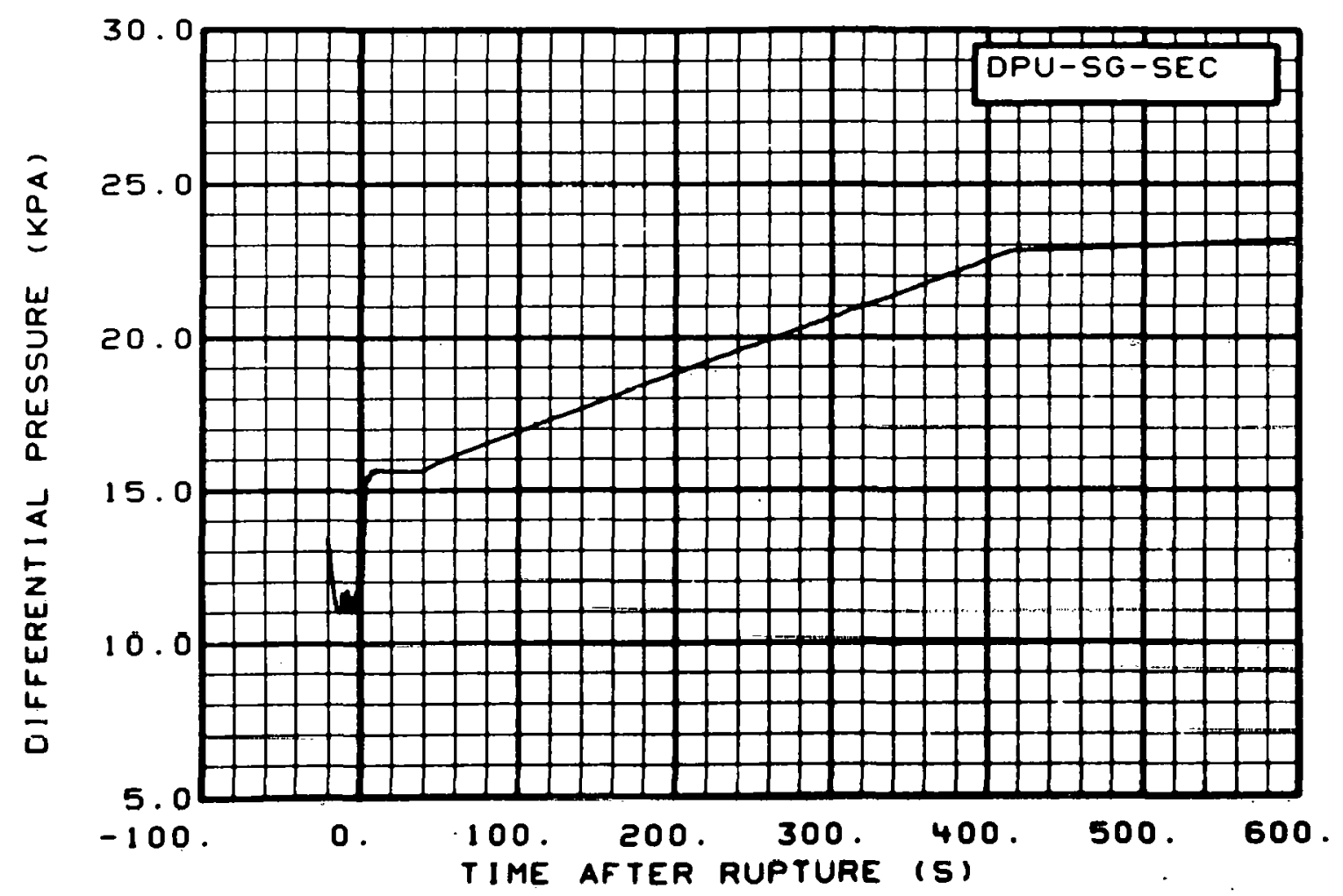

Fig. 229 Differential pressure in steam generator secondary (DPU-SG-SEC), from -20 to $600 \mathrm{~s}$.

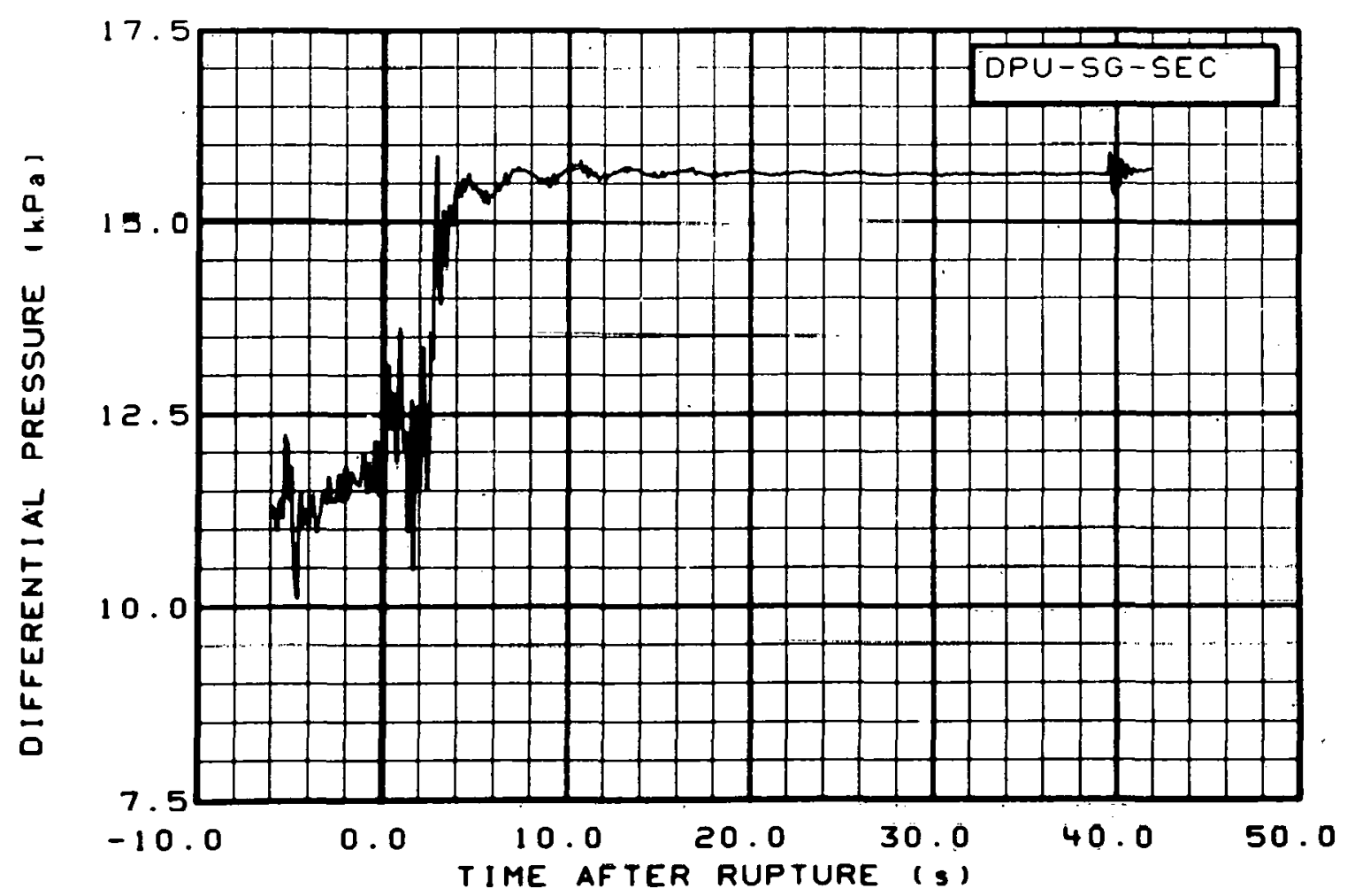

Fig. 230 Differential pressure in steam generator secondary (DPU-SG-SEC), from -6 to $42 \mathrm{~s}$. 


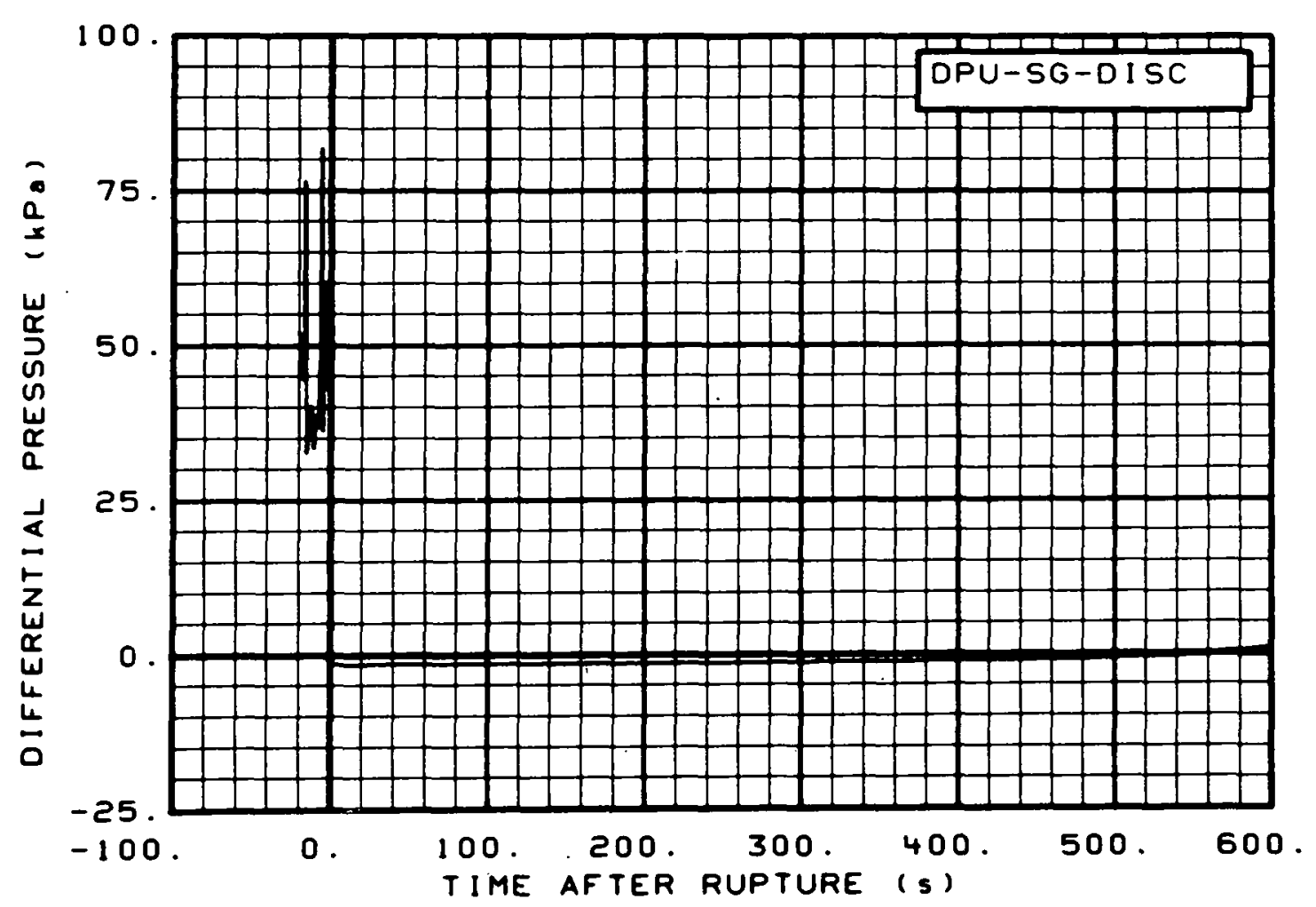

Fig. 231 Differential pressure across steam generator outlet orifice (DPU-SG-DISC), from -20 to $600 \mathrm{~s}$.

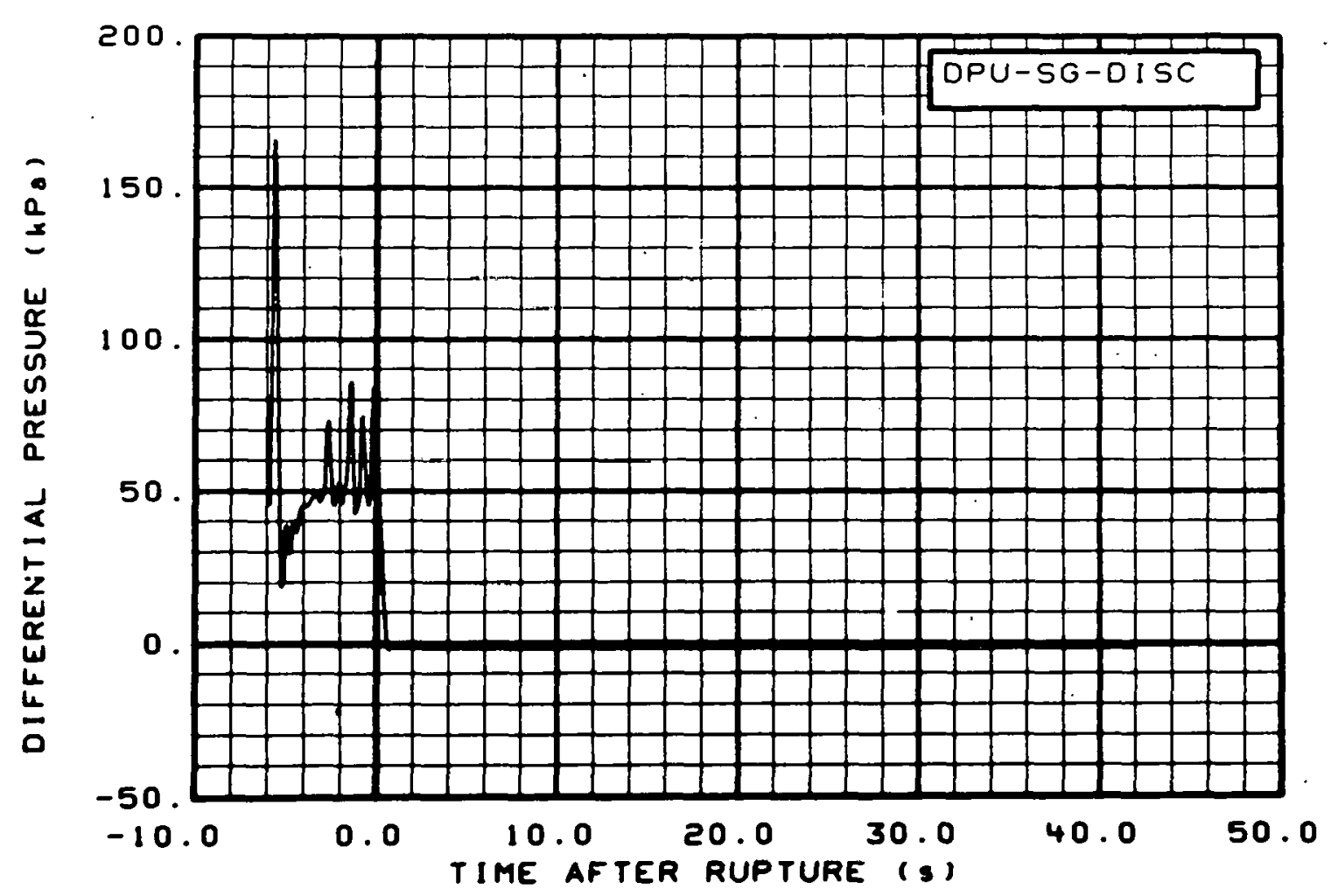

Fig. 232 Differential pressure across steam generator outlet orifice (DPII-SG-DISC), from -6 to $42 \mathrm{~s}$. 


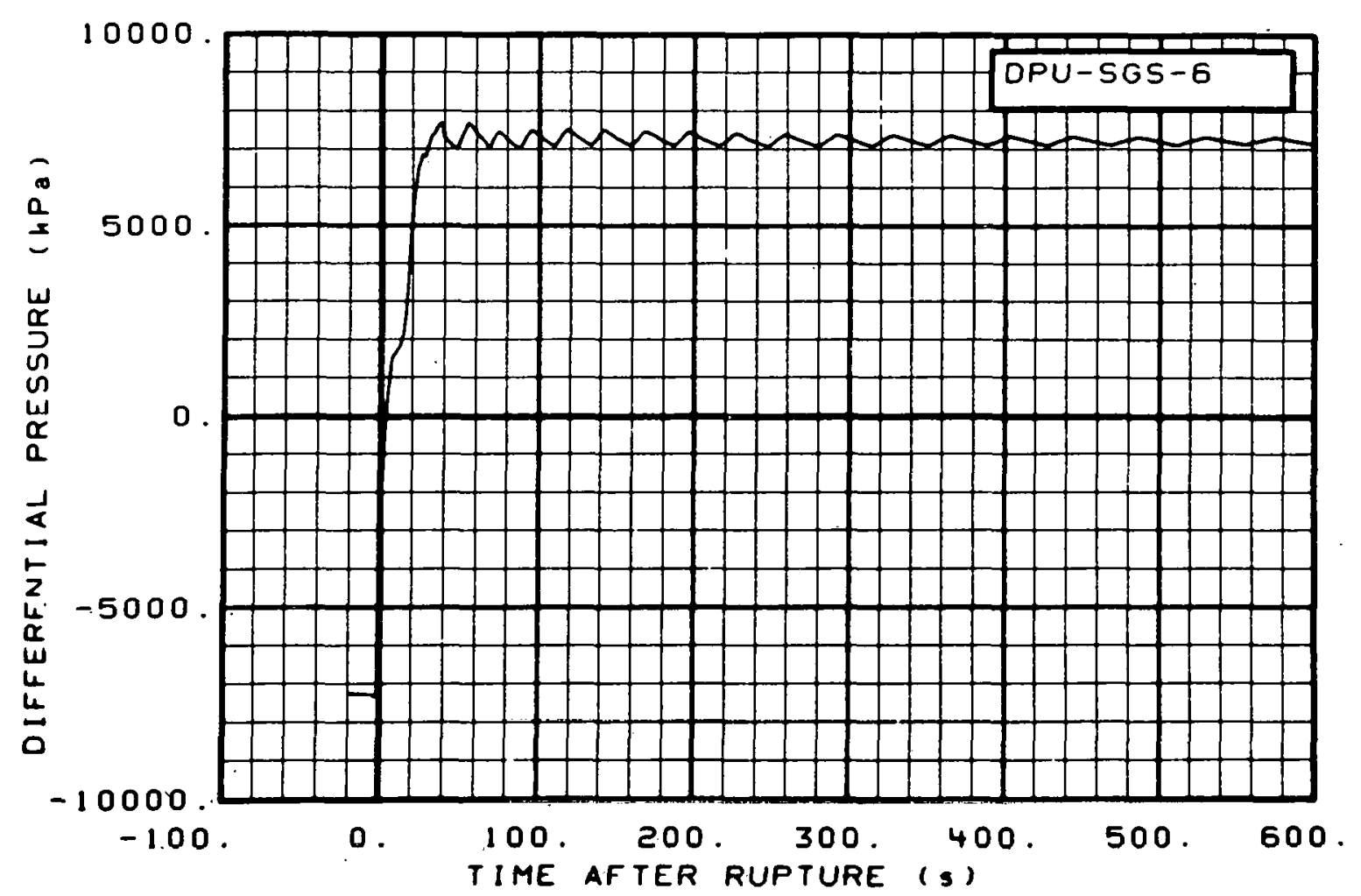

Fig. 233 Differential pressure between simulated rupture injection line and Spool 6 (DPU-SGS-6), from -20 to $600 \mathrm{~s}$.

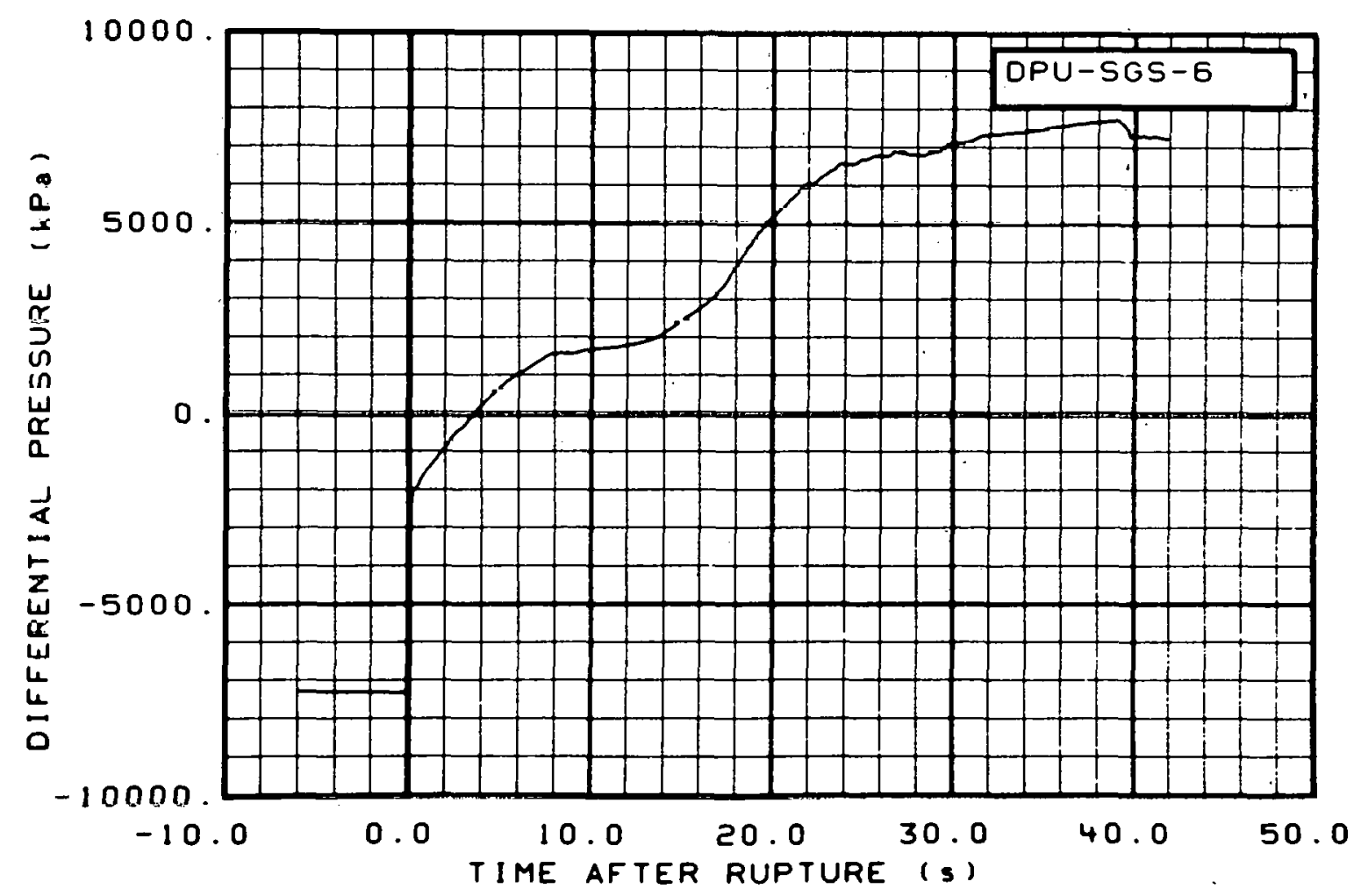

Fig. 234 Differential pressure between simulated rupture injection line and Spool 6 (DPU-SGS-6), from -6 to $42 \mathrm{~s}$. 


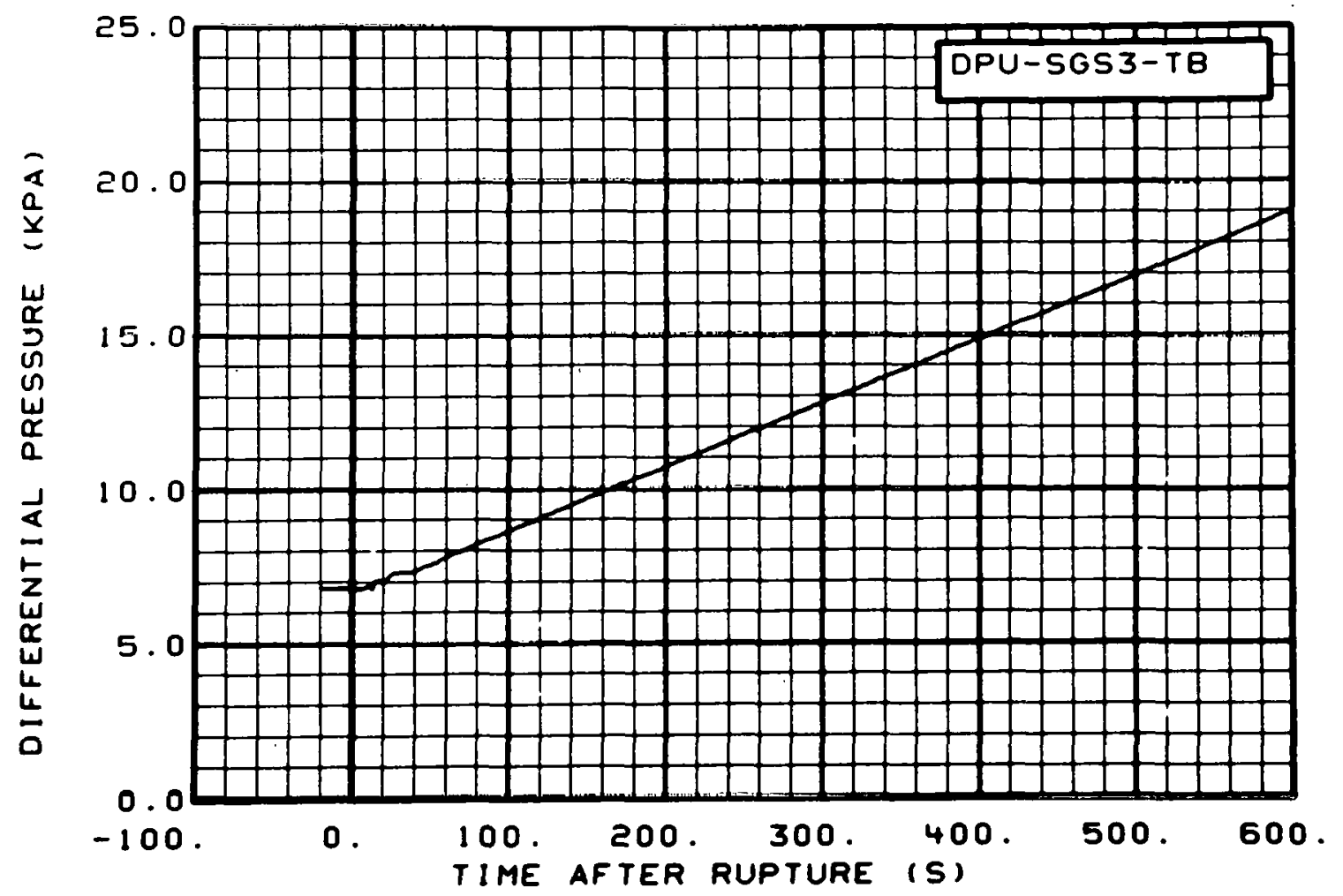

Fig. 235 Differential pressure in intact loop tube rupture simulation accumulator (DPU-SGS3-TB), from -20 to $600 \mathrm{~s}$.

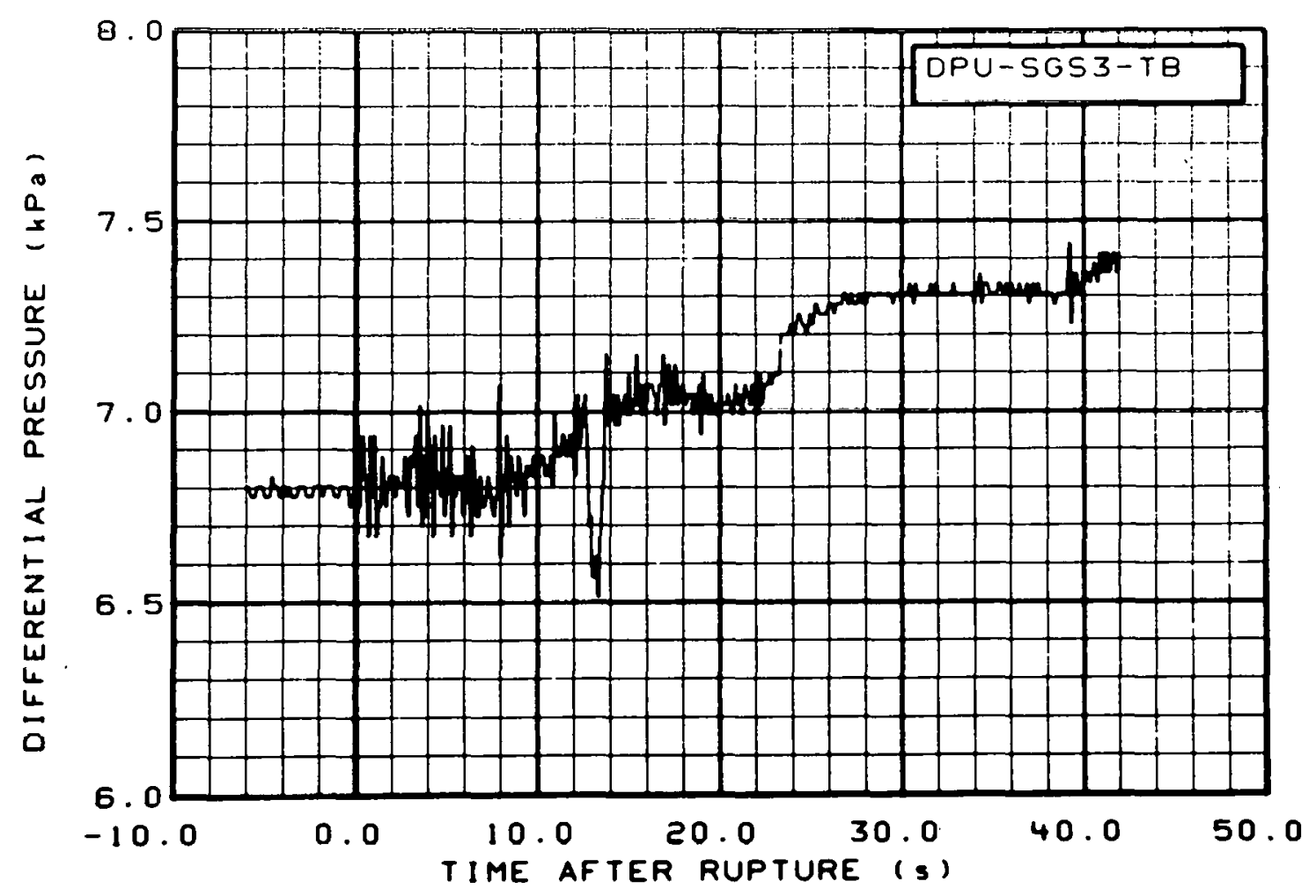

Fig. 236 Differential pressure in intact loop tube rupture simulation accumulator (DPU-SGS3-TB), from -6 to $42 \mathrm{~s}$. 


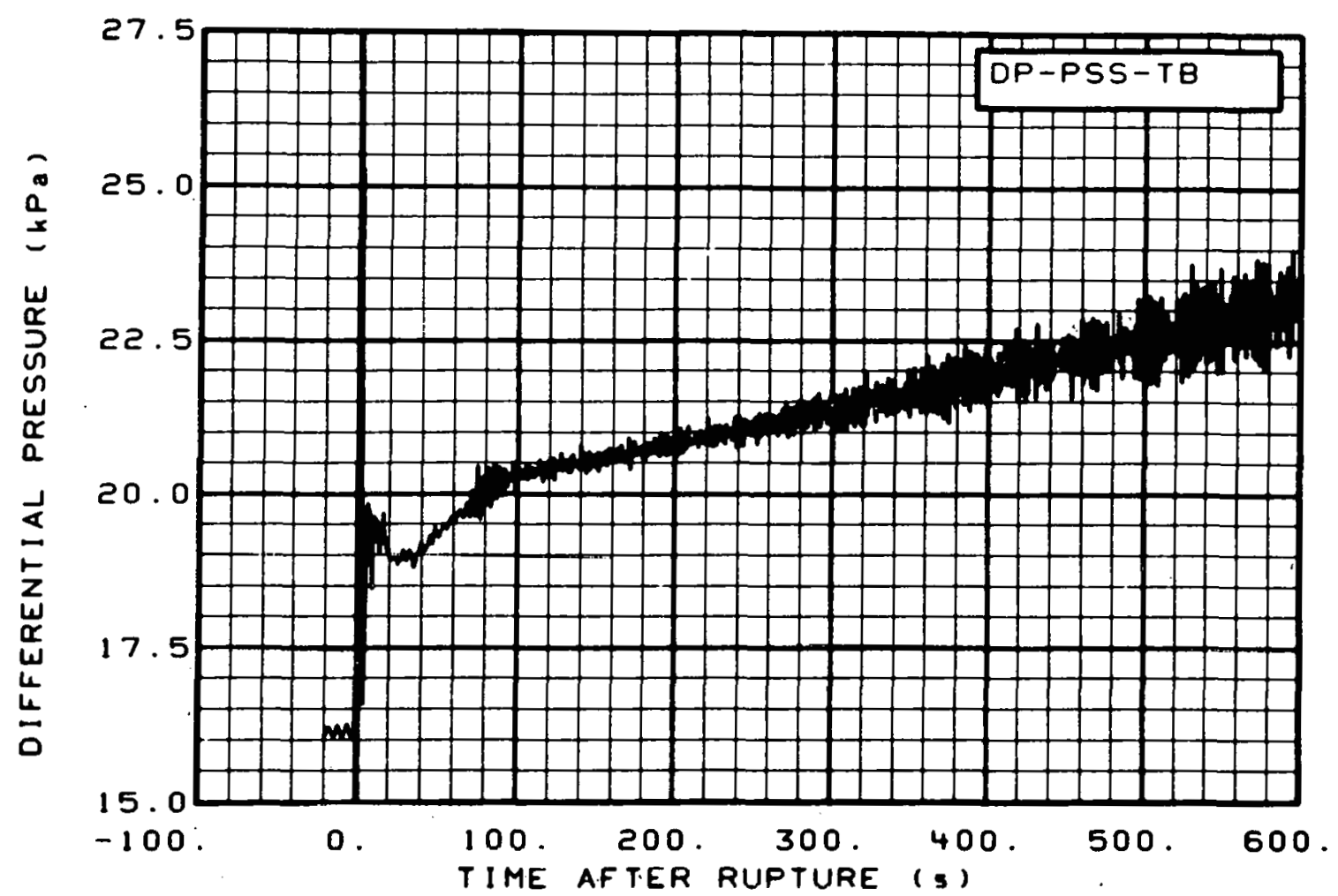

Fig. 237 Differential pressure in pressure suppression tank (DP-PSS-TB), from -20 to $600 \mathrm{~s}$.

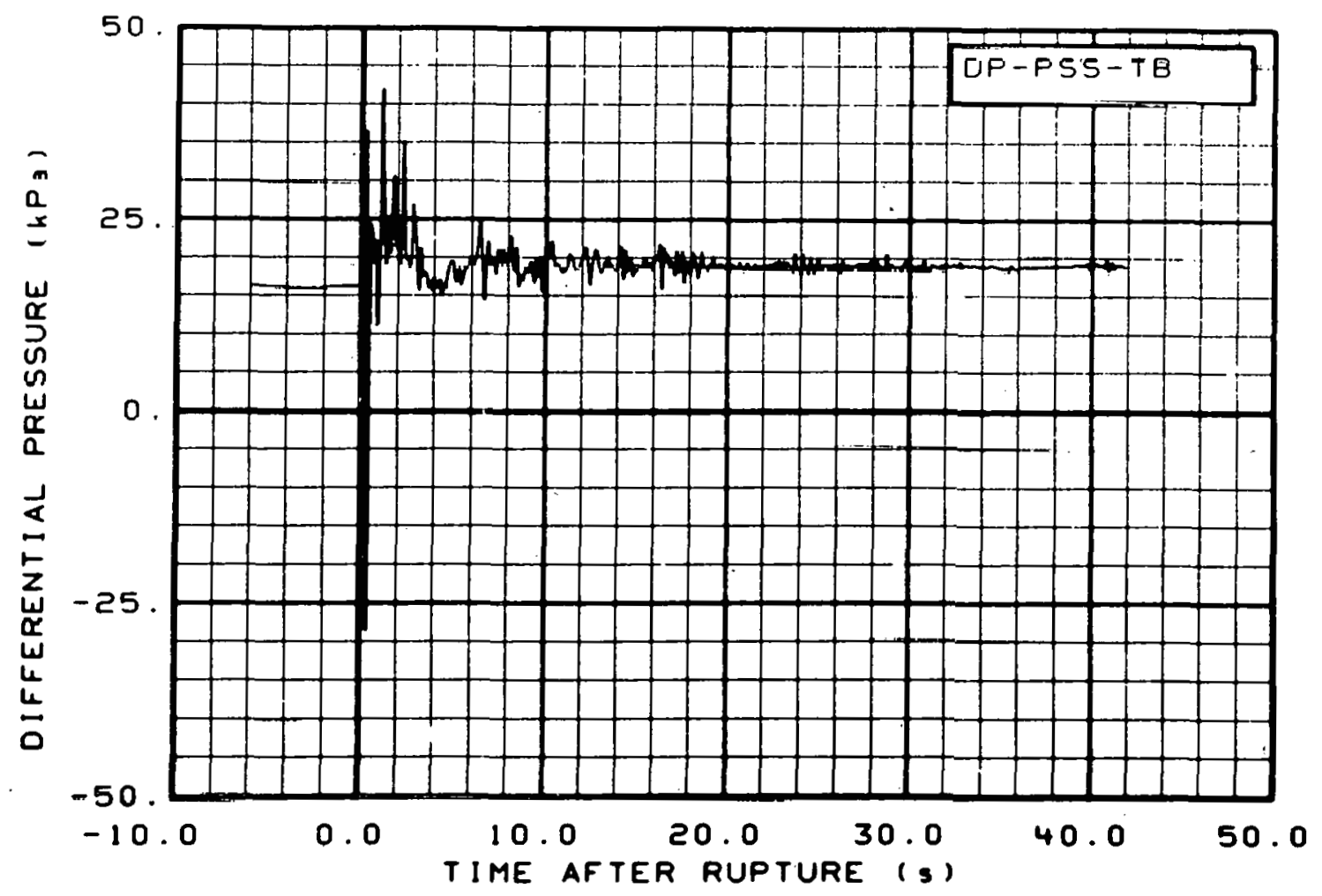

Fig. 238 Differential pressure in pressure suppression tank (DP-PSS-TB), from -6 to $42 \mathrm{~s}$. 


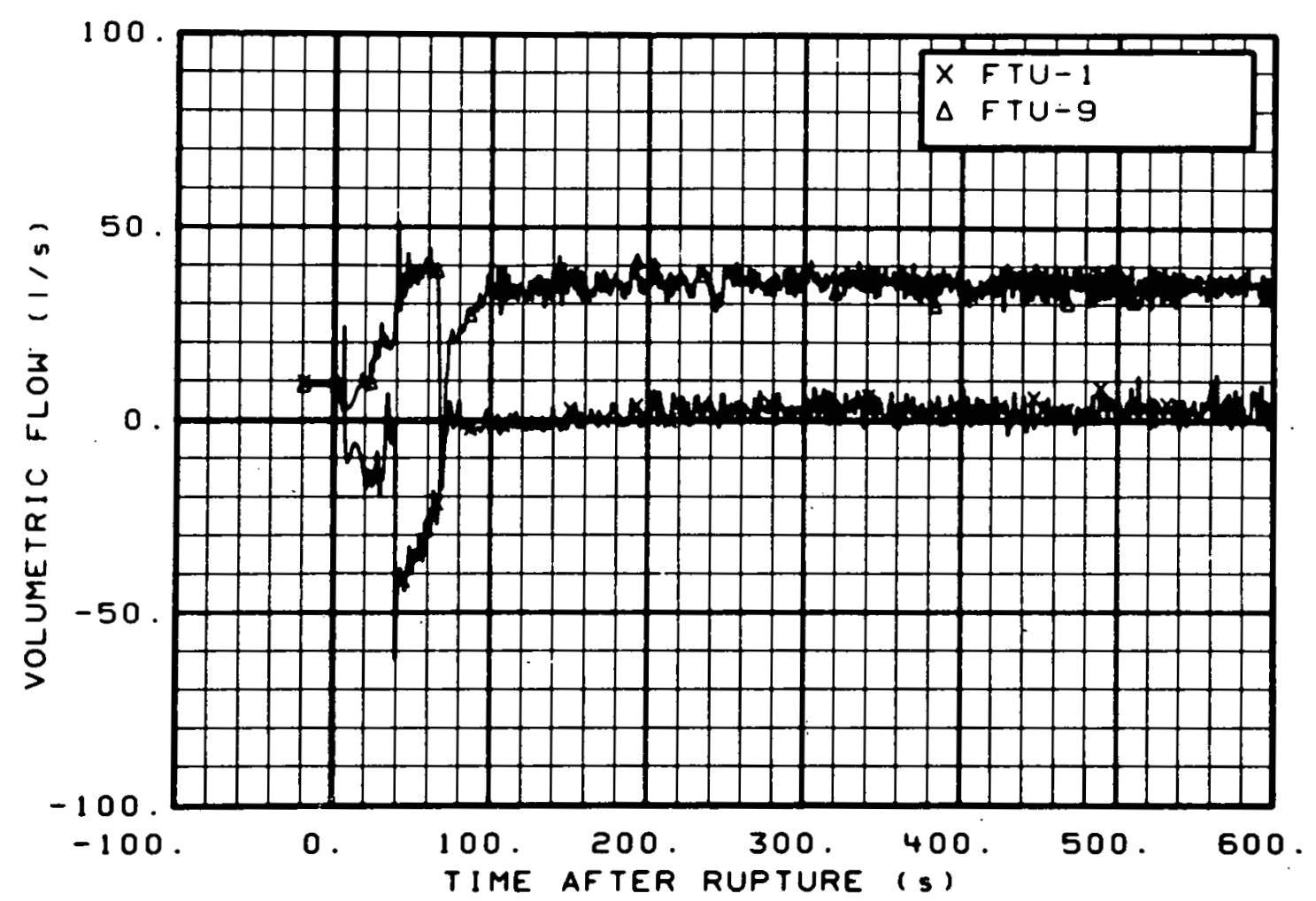

Fig. 239 Volumetric flow in intact loop (FTU-1 and FTU-9), from -20 to $600 \mathrm{~s}$.

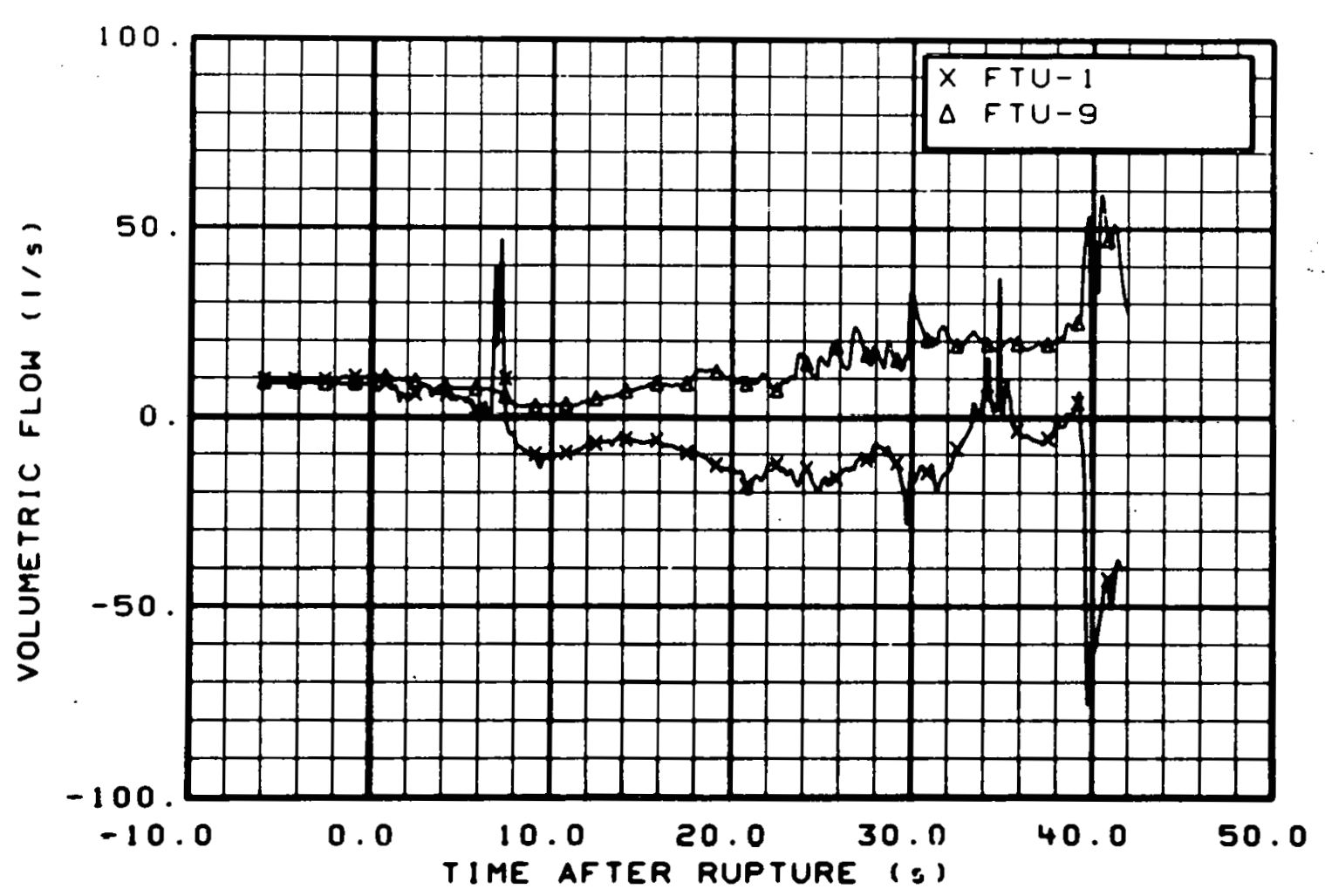

Fig. 240 Volumetric flow in intact loop (FTU-1 and FTU-9), from -6 to $42 \mathrm{~s}$. 


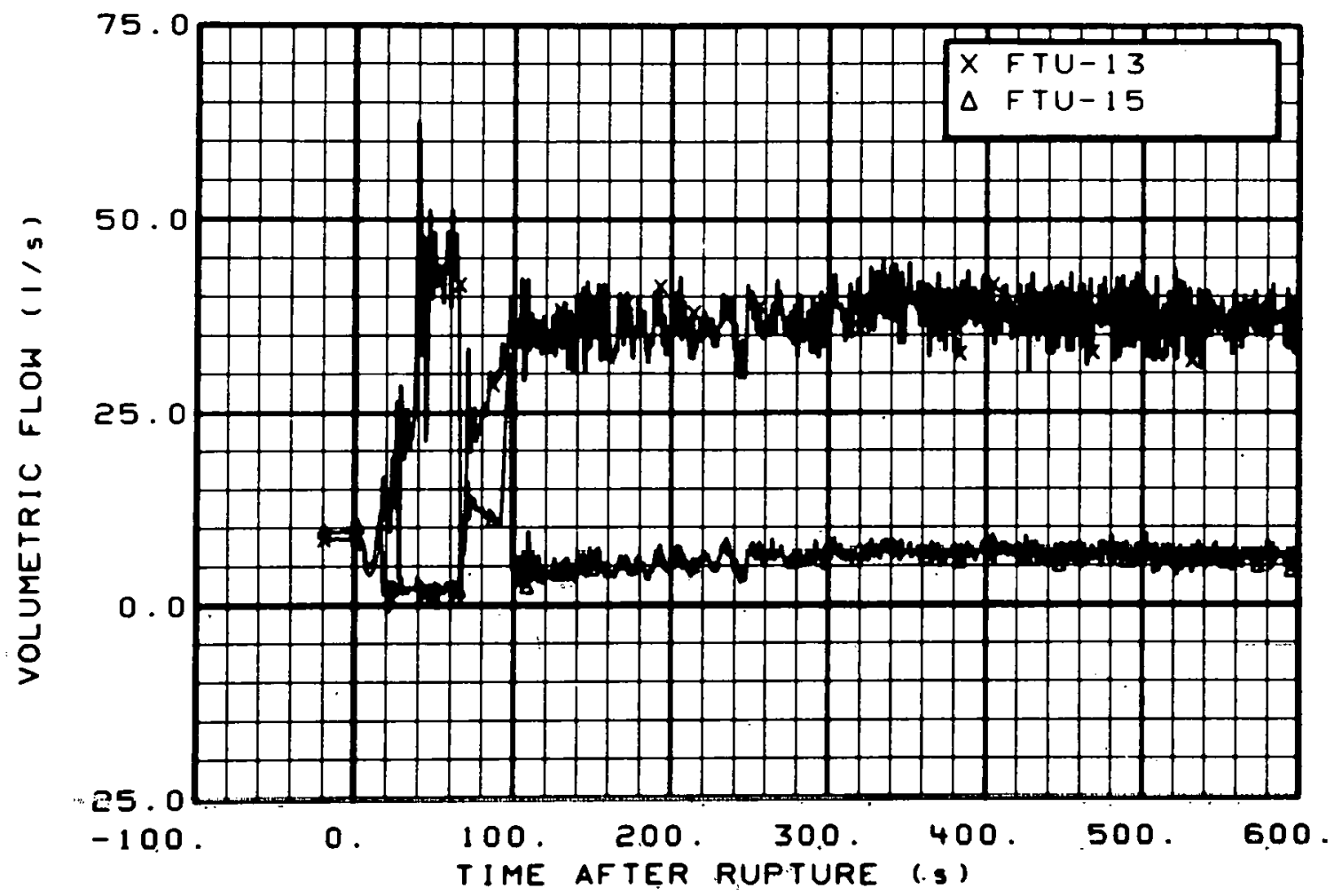

Fig. 241 Volumetric flow in intact loop (FTU-13 and FTU-15), from -20 to $600 \mathrm{~s}$.

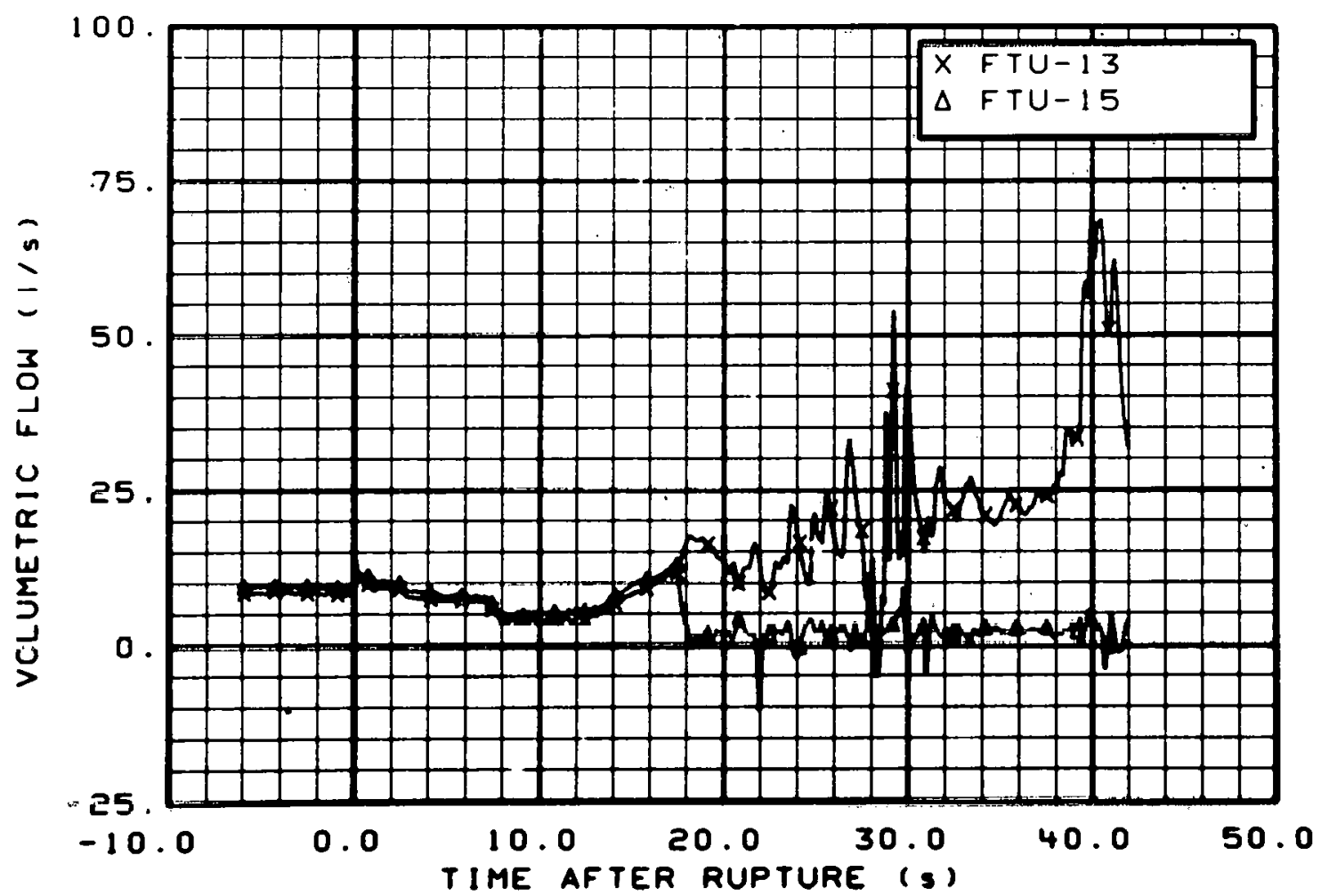

Fig. 242 Volumetric flow in intact loop (FTU-13 and FTU-15), from -6 to $42 \mathrm{~s}$. 


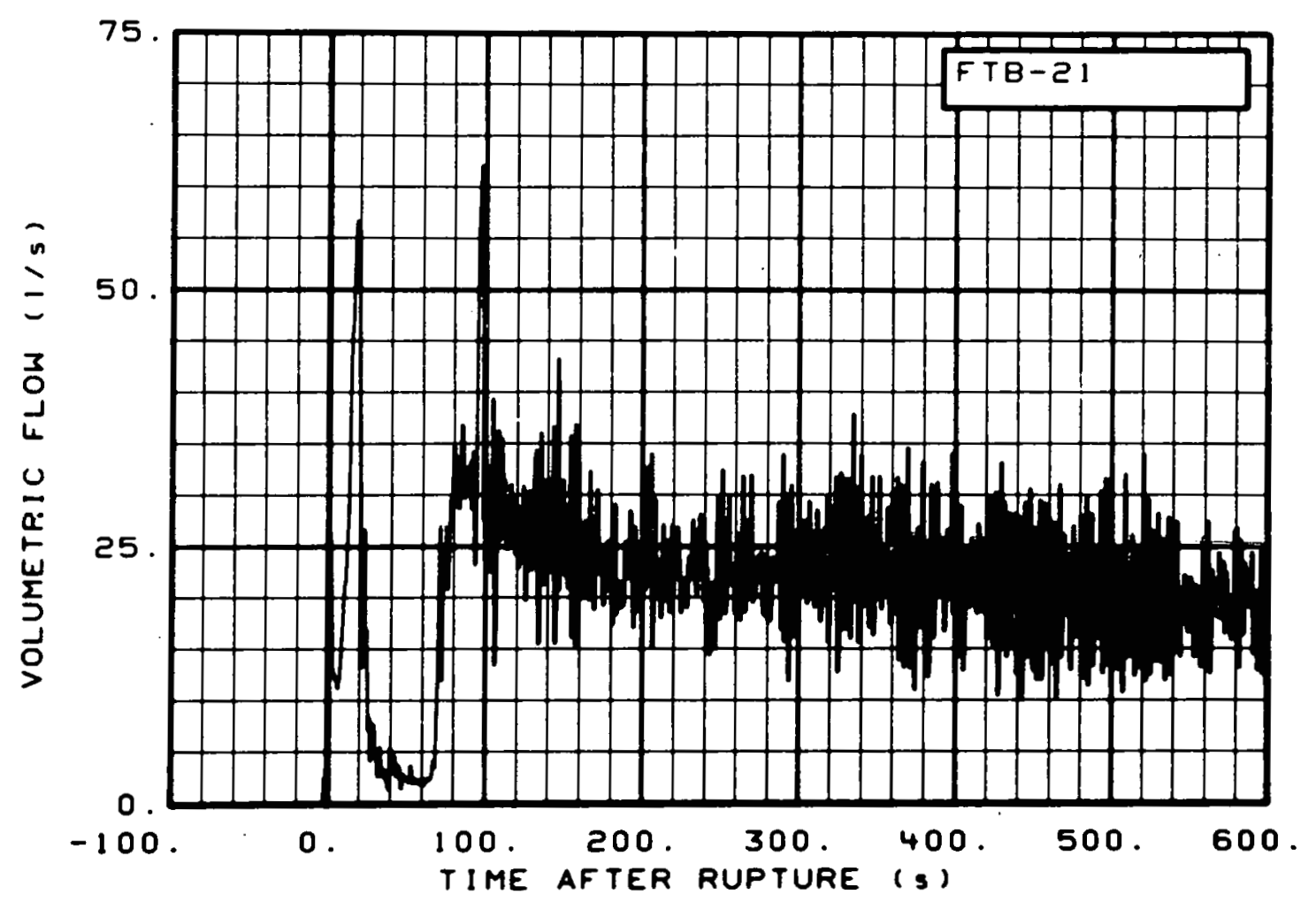

Fig. 243 Volumetric flow in broken loop (FTB-21), from -20 to $600 \mathrm{~s}$.

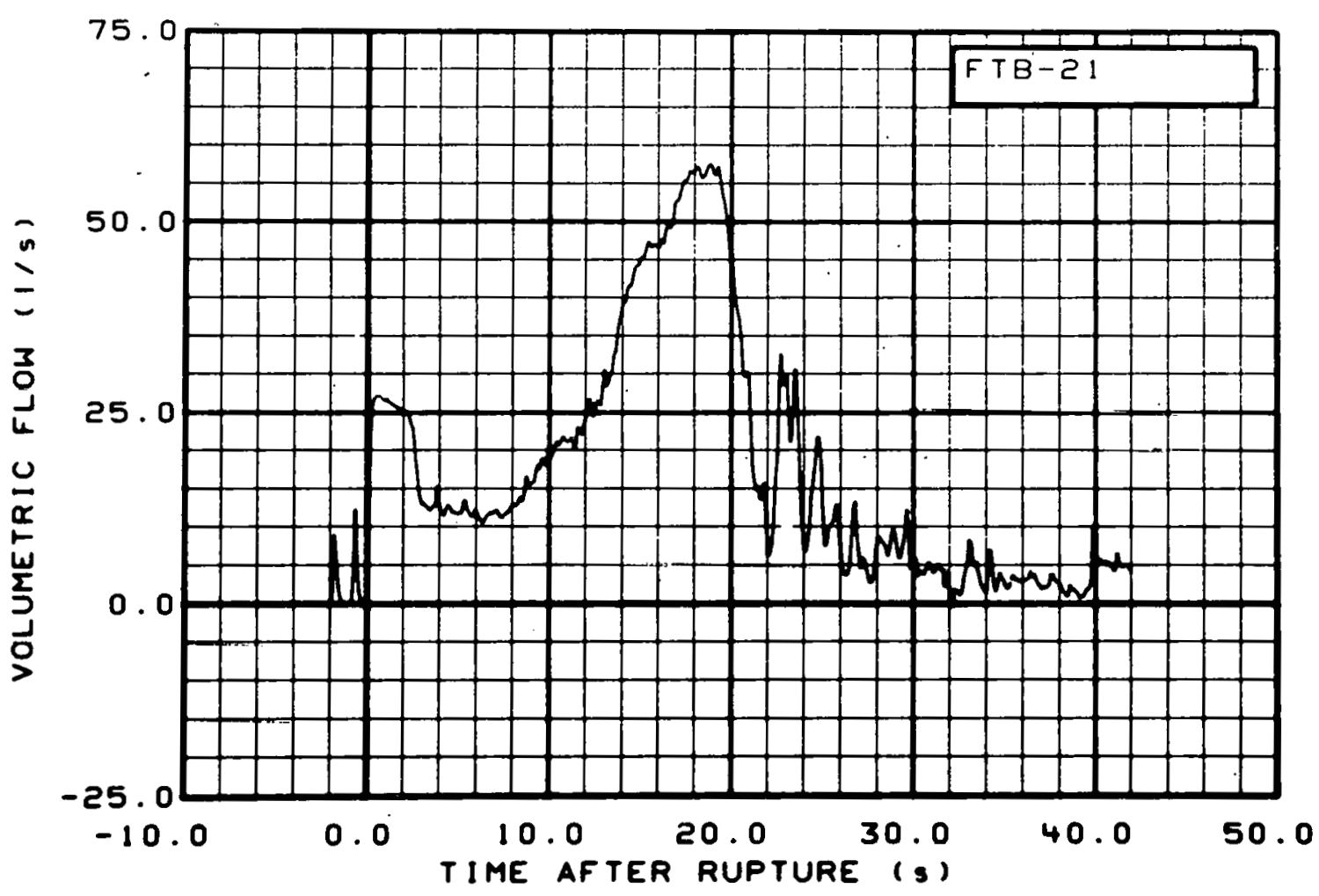

Fig. 244 Volumetric flow in broken loop (FTB-21), from -6 to $42 \mathrm{~s}$. 


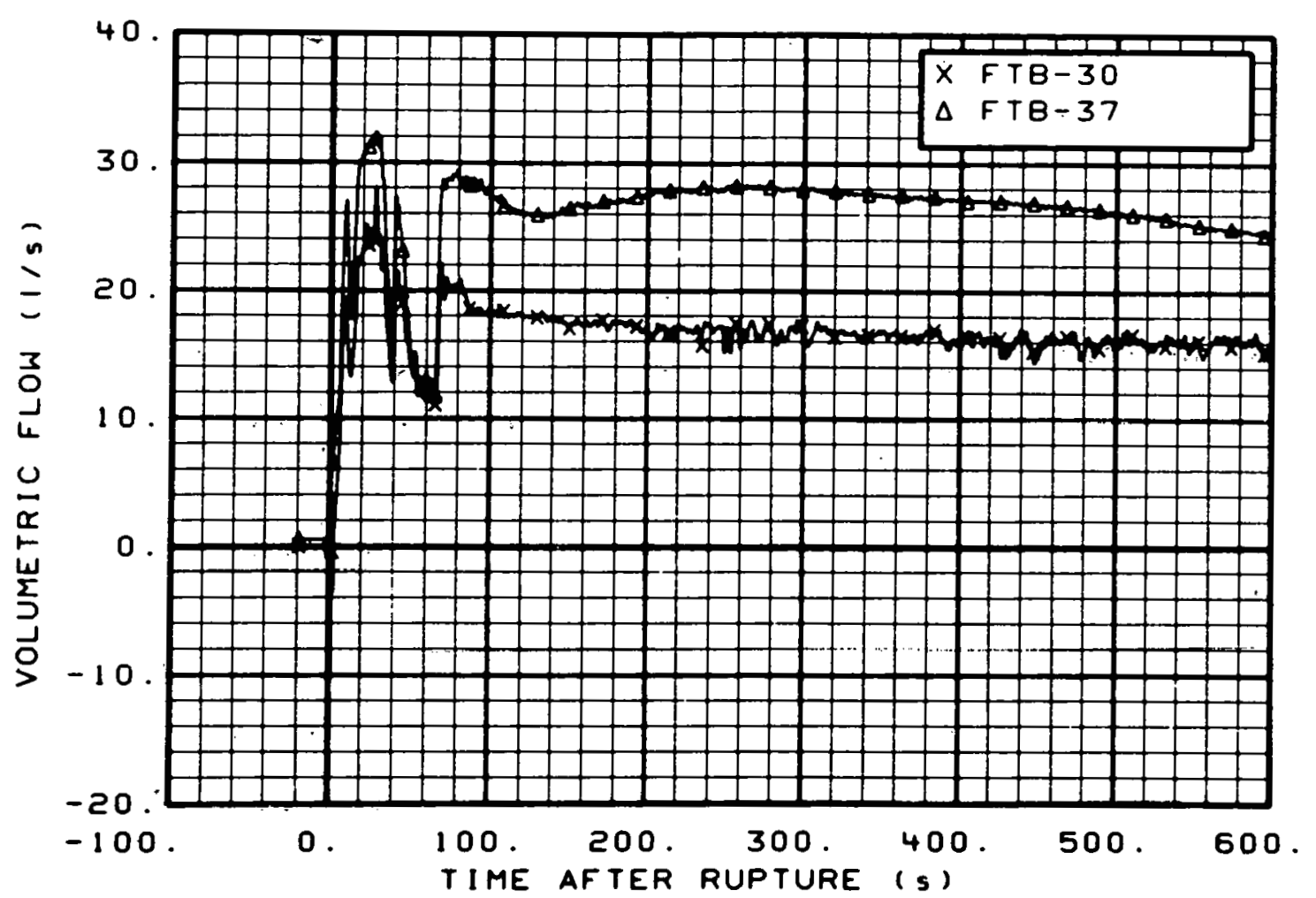

Fig. 245 Volumetric flow in broken loop (FTB-30 and FTB-37), from -20 to $600 \mathrm{~s}$.

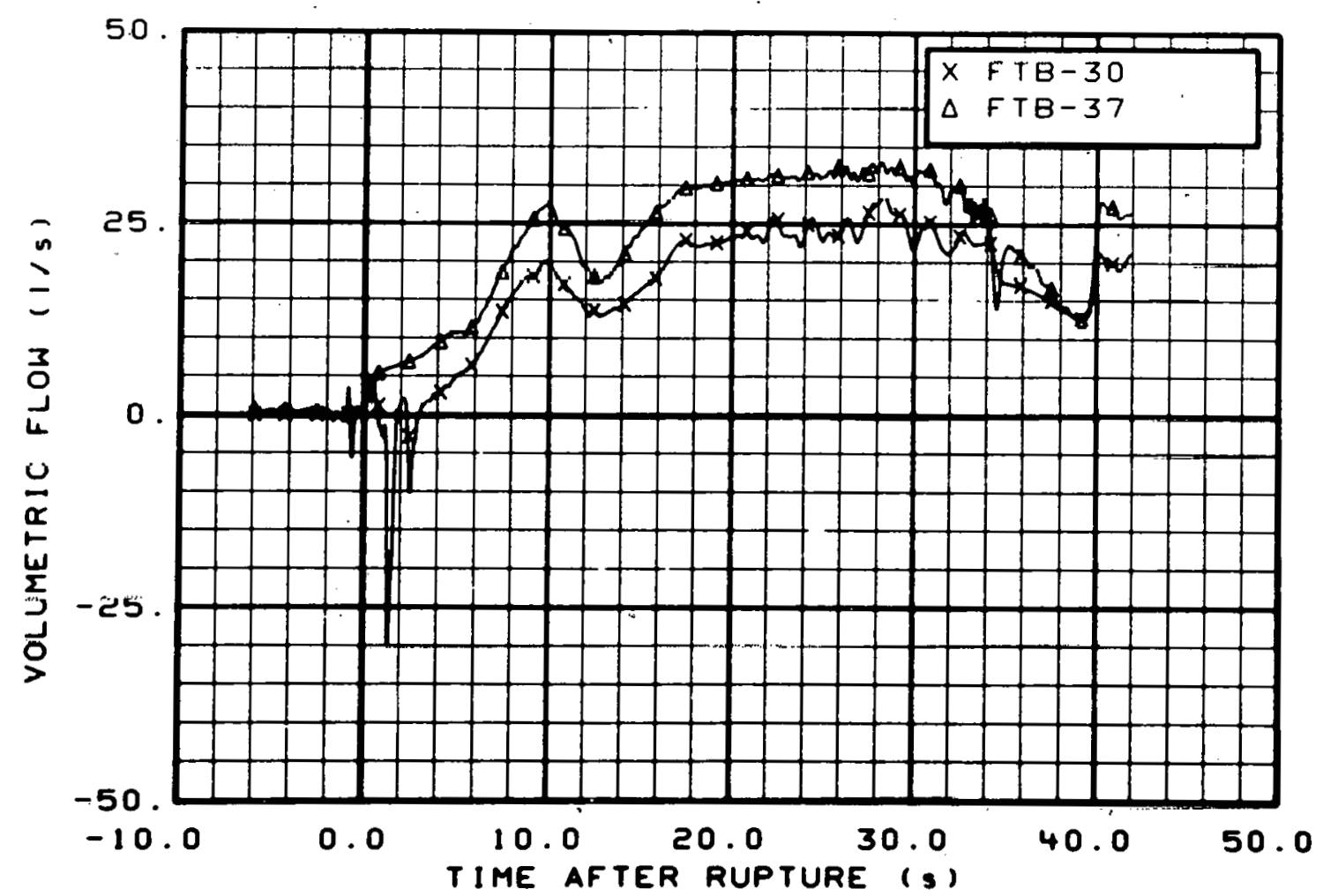

Fig. 246 Volumetric flow in broken loop (FTB-30 and FTB-37), from -6 to $42 \mathrm{~s}$. 


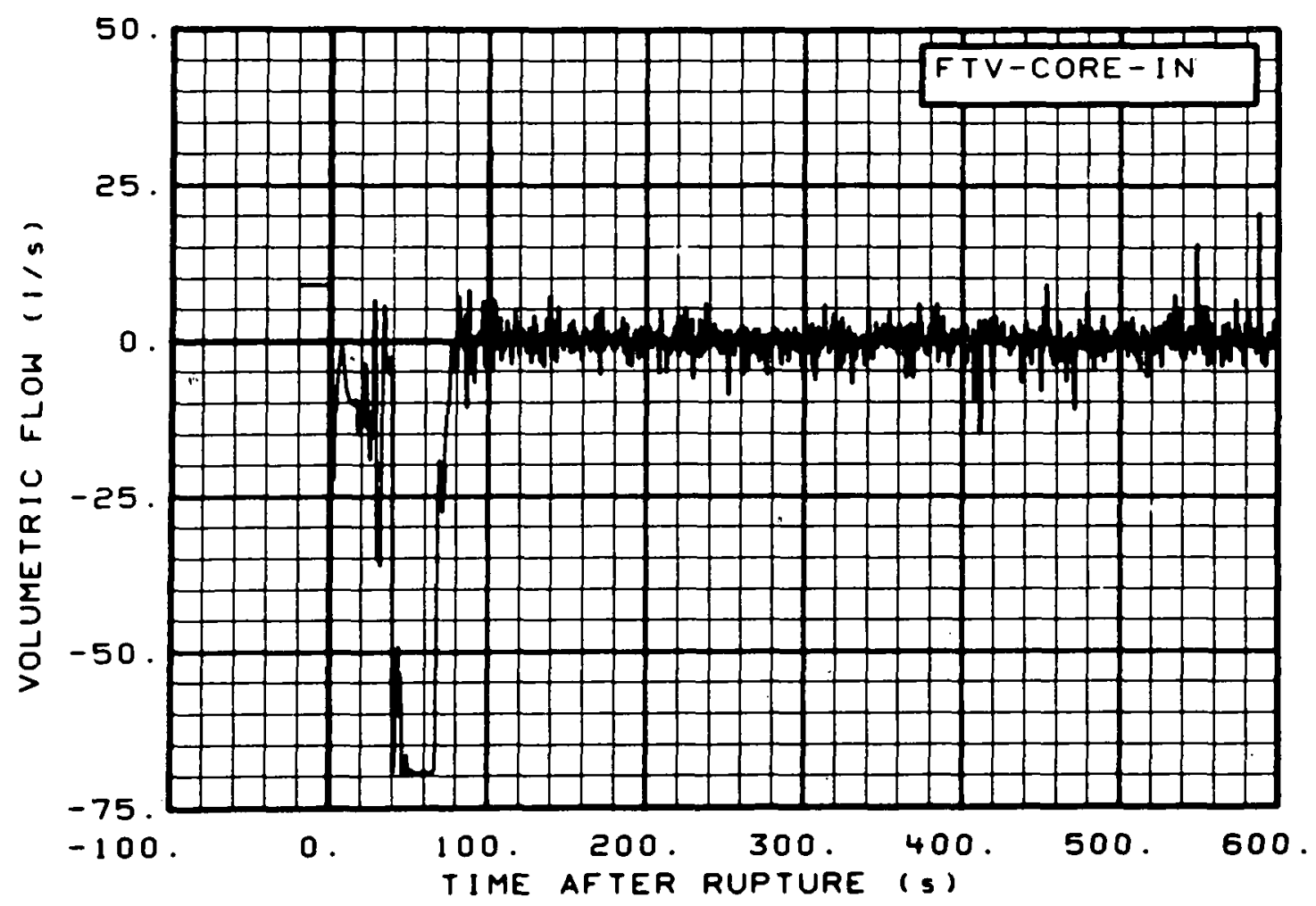

Fig. 247 Volumetric flow in core entrance (FTV-CORE-IN), from -20 to $600 \mathrm{~s}$.

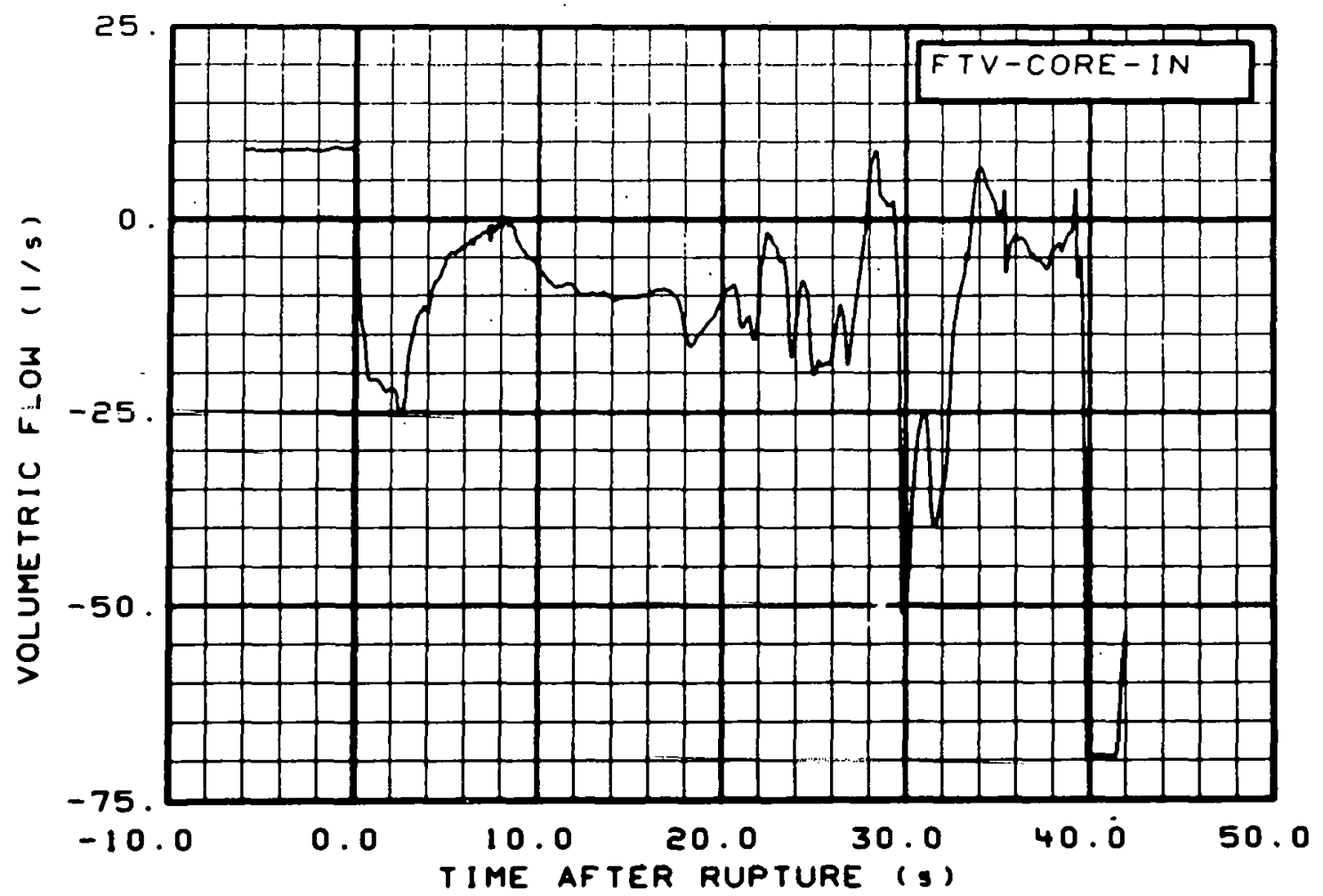

Fig. 248 Volumetric flow in core entrance (FTV-CORE-IN), from -6 to $42 \mathrm{~s}$. 


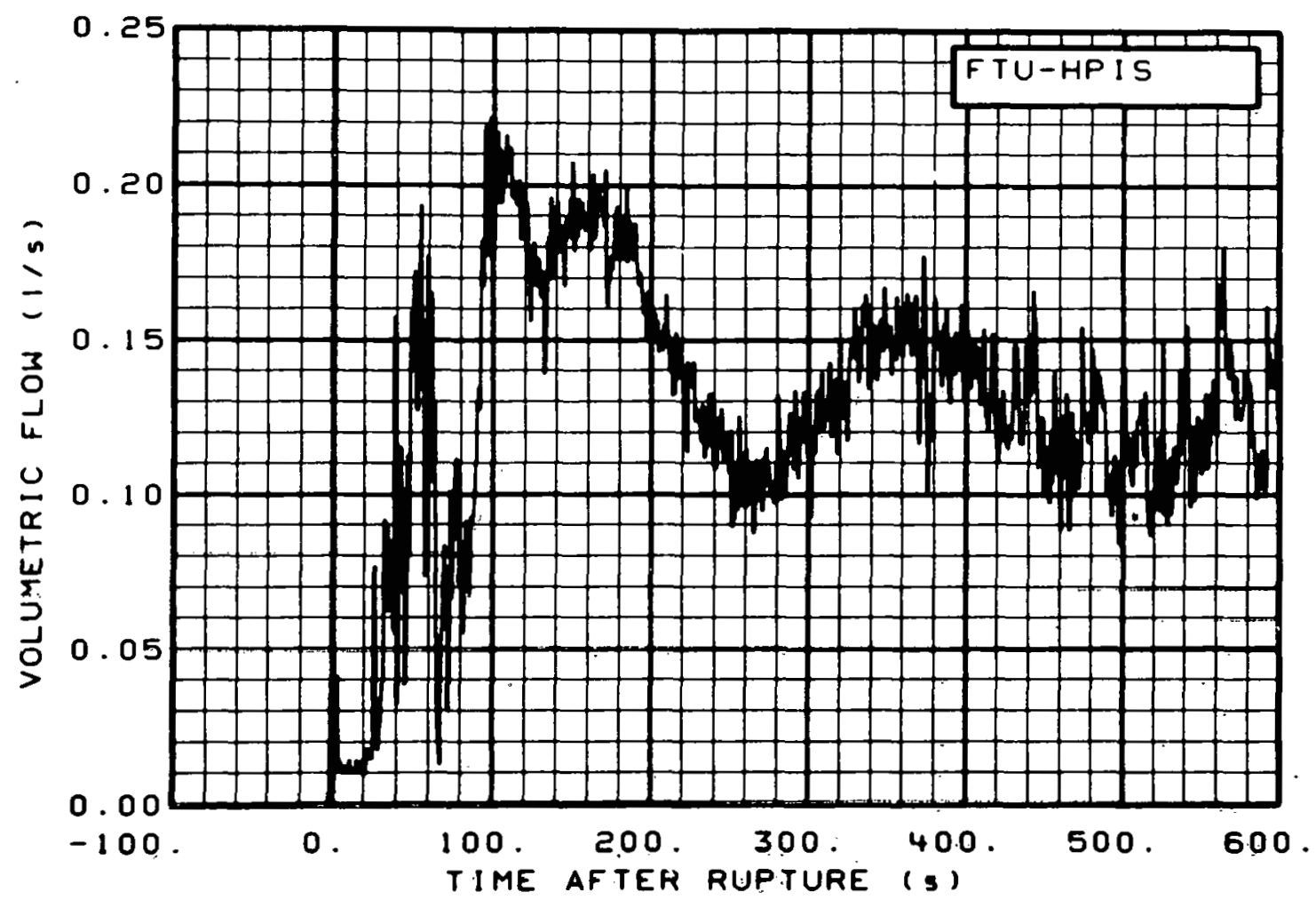

Fig. 249 Volumetric flow in intact loop high pressure injection line (FTU-HPIS), from -20 to $600 \mathrm{~s}$.

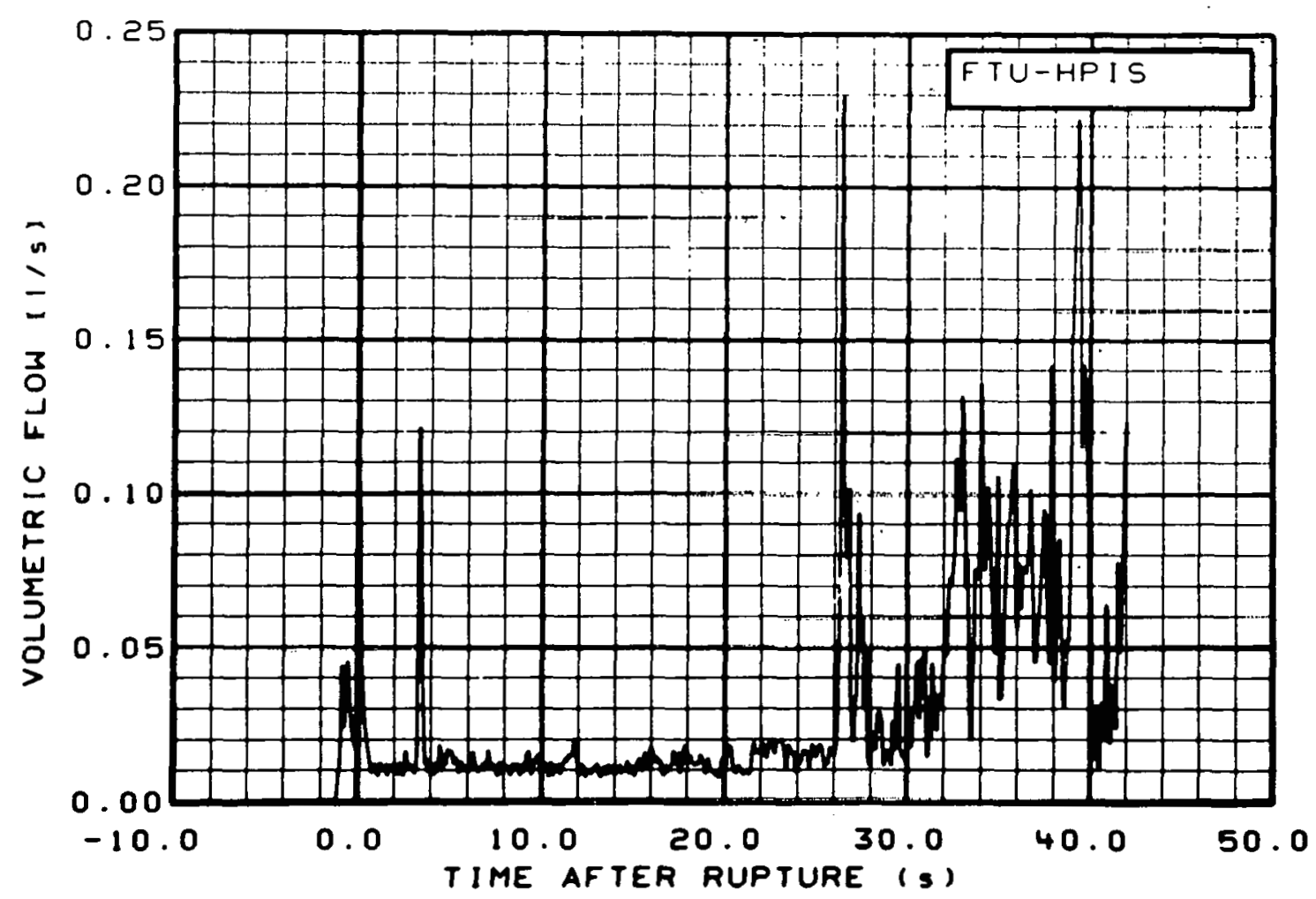

Fig. 250 Volumetric flow in intact loop high pressure injection line (FTU-HPIS), from -6 to $42 \mathrm{~s}$. 


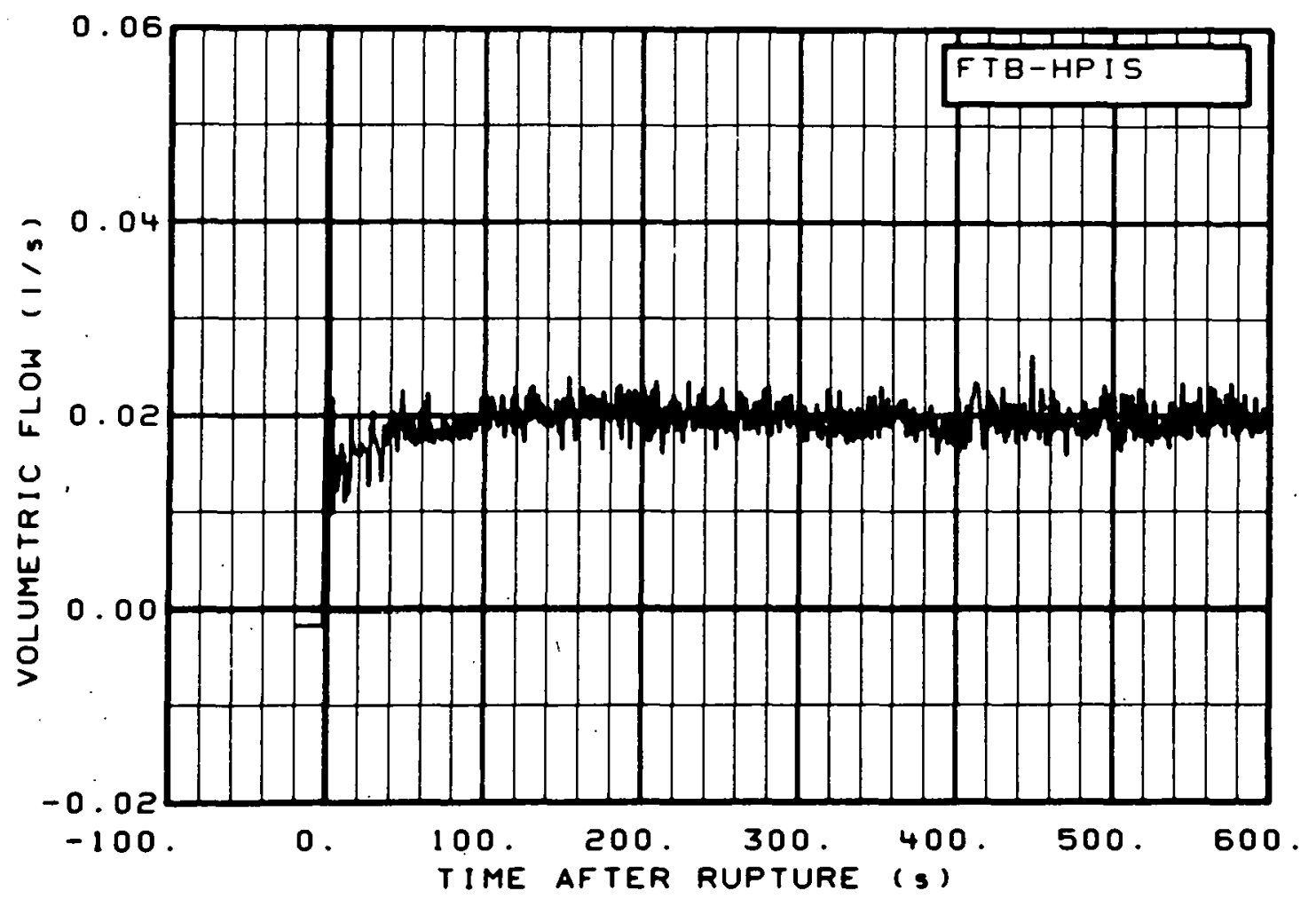

Fig. 251 Volumetric flow in broken loop high pressure injection line (FTB-HPIS), from -20 to $600 \mathrm{~s}$.

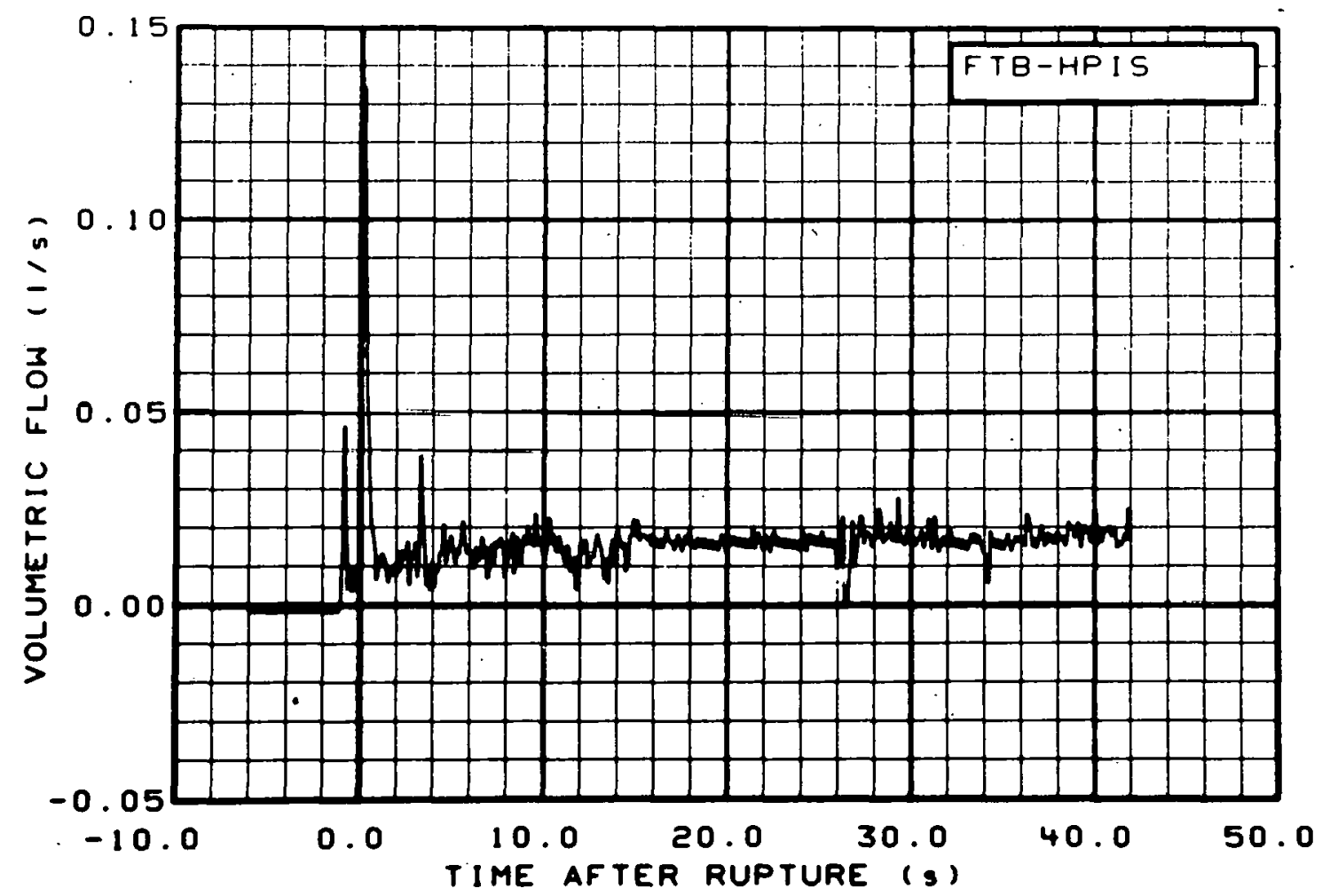

Fig. 252 Volumetric flow in broken loop high pressure injection line (FTB-HPIS), from -6 to $42 \mathrm{~s}$. 


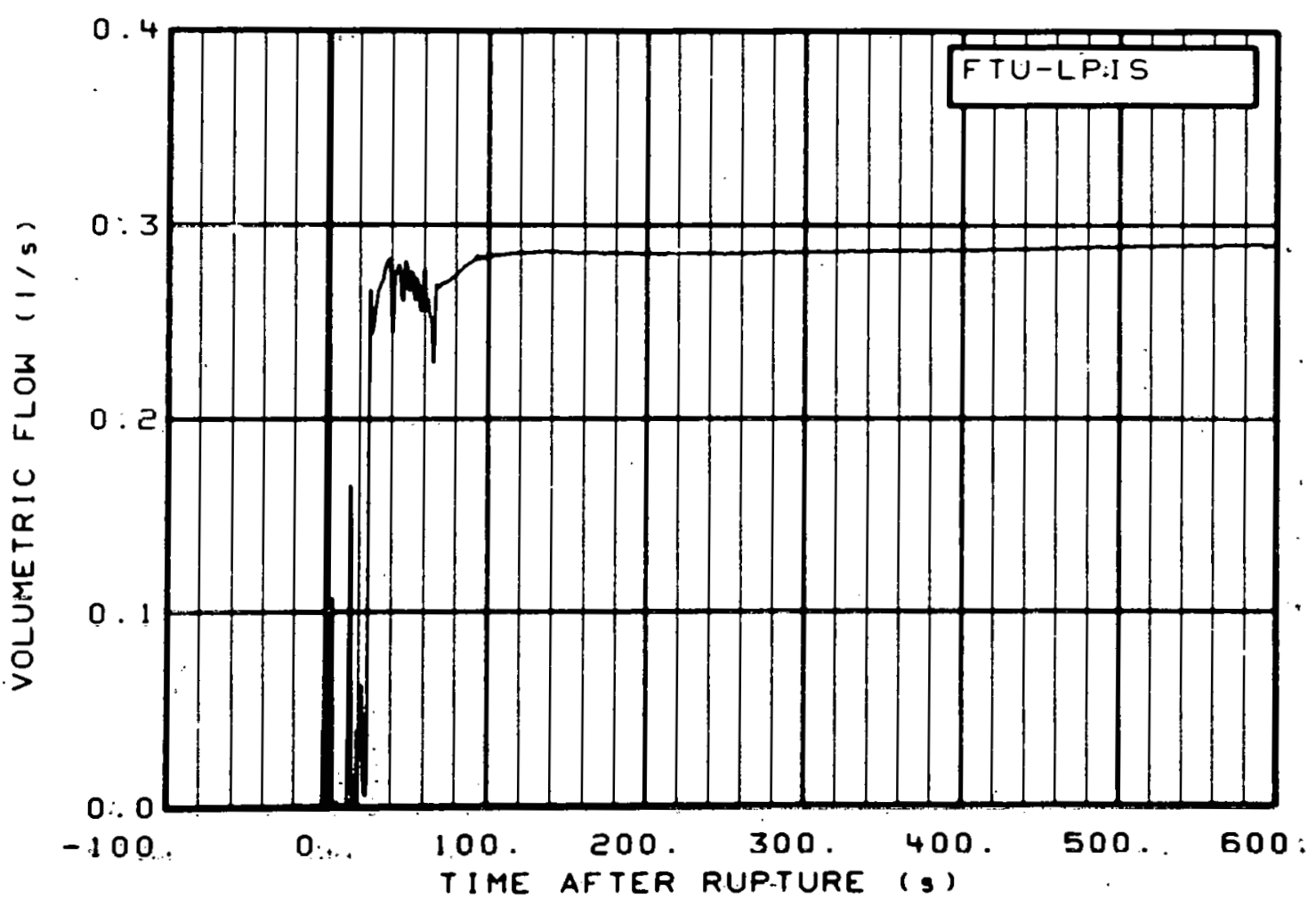

Fig. 253 Volumetric flow in intact loop low pressure injection line (FTU-LPIS), from -20. to $600 \mathrm{~s}$.

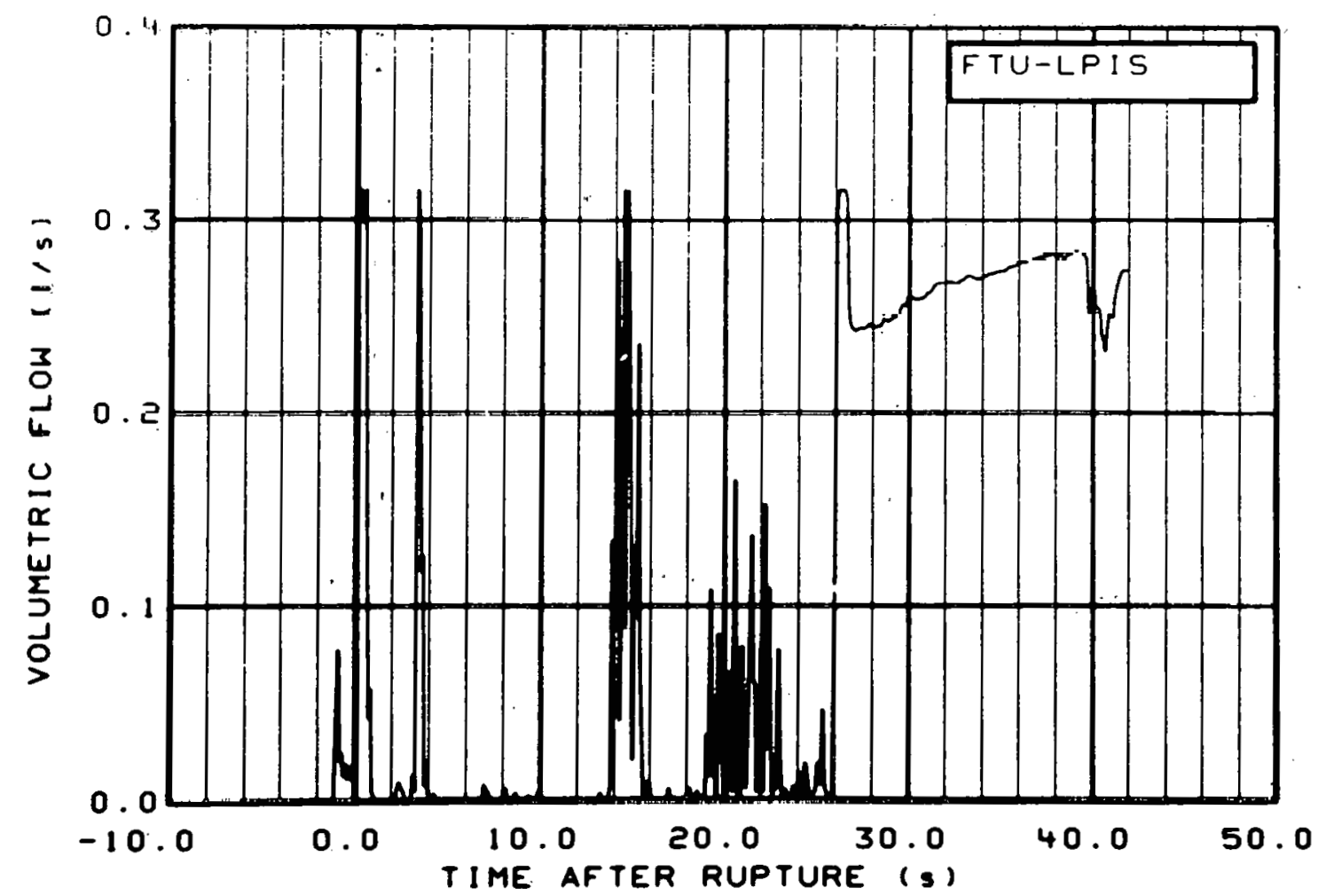

Fig. 254 Volumetric flow in intact loop low pressure injection line (FTU-LPIS), from -6 to $42 \mathrm{~s}$. 


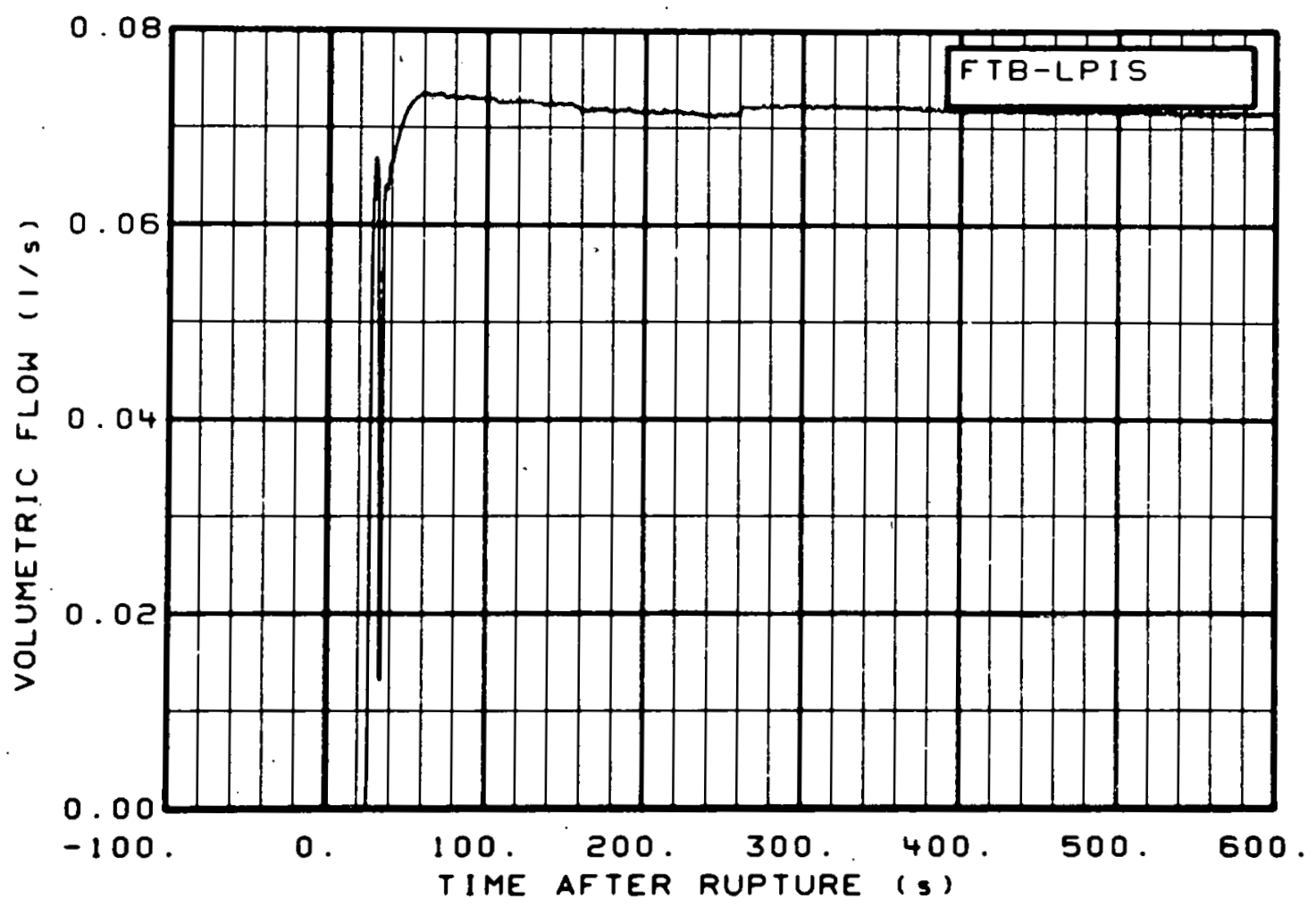

Fig. 255 Volumetric flow in broken loop low pressure injection line (FTB-LPIS), from -20 to $600 \mathrm{~s}$.

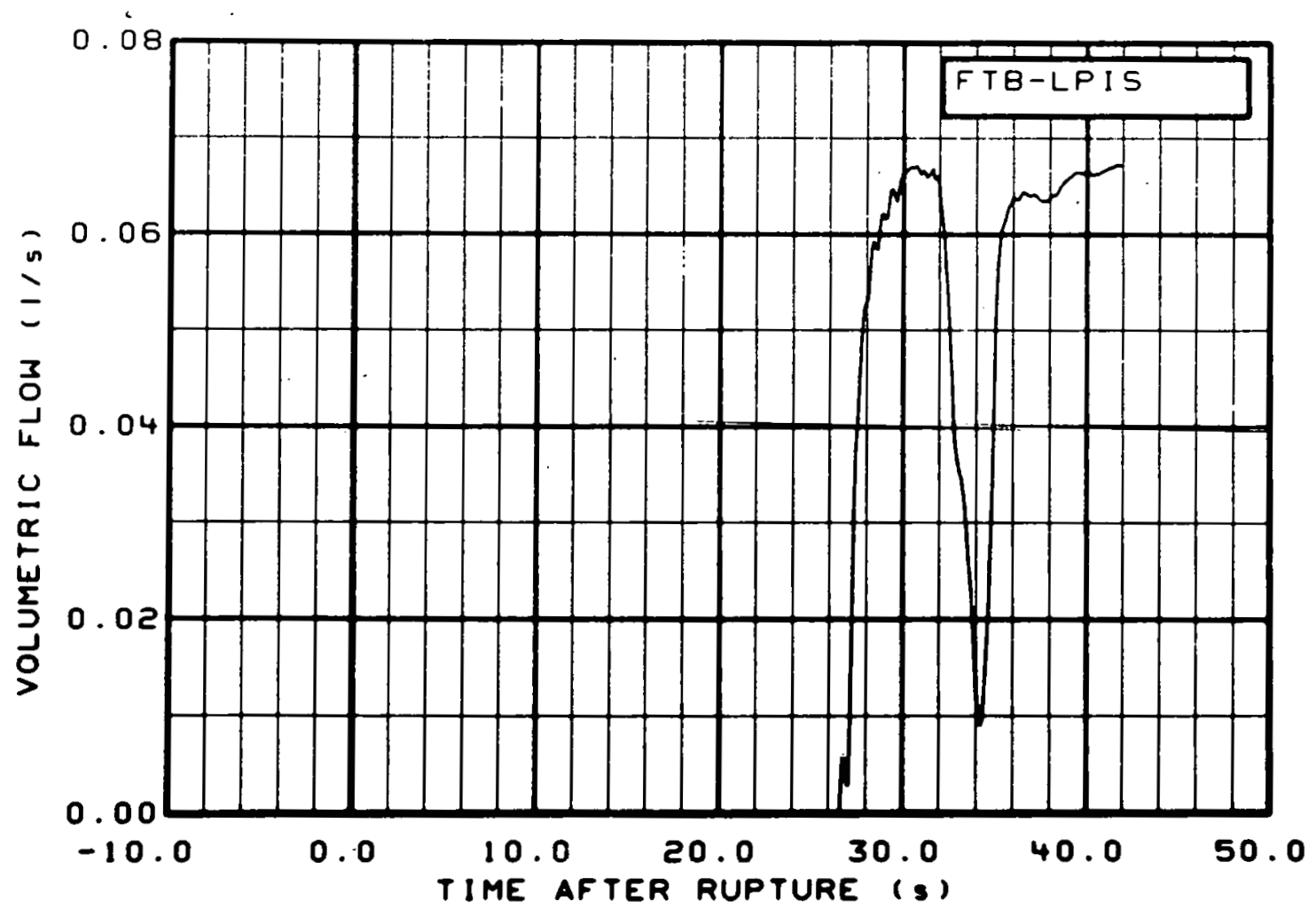

Fig. 256 Volumetric flow in broken loop low pressure injection line (FTB-LPIS), from -6 to $42 \mathrm{~s}$. 


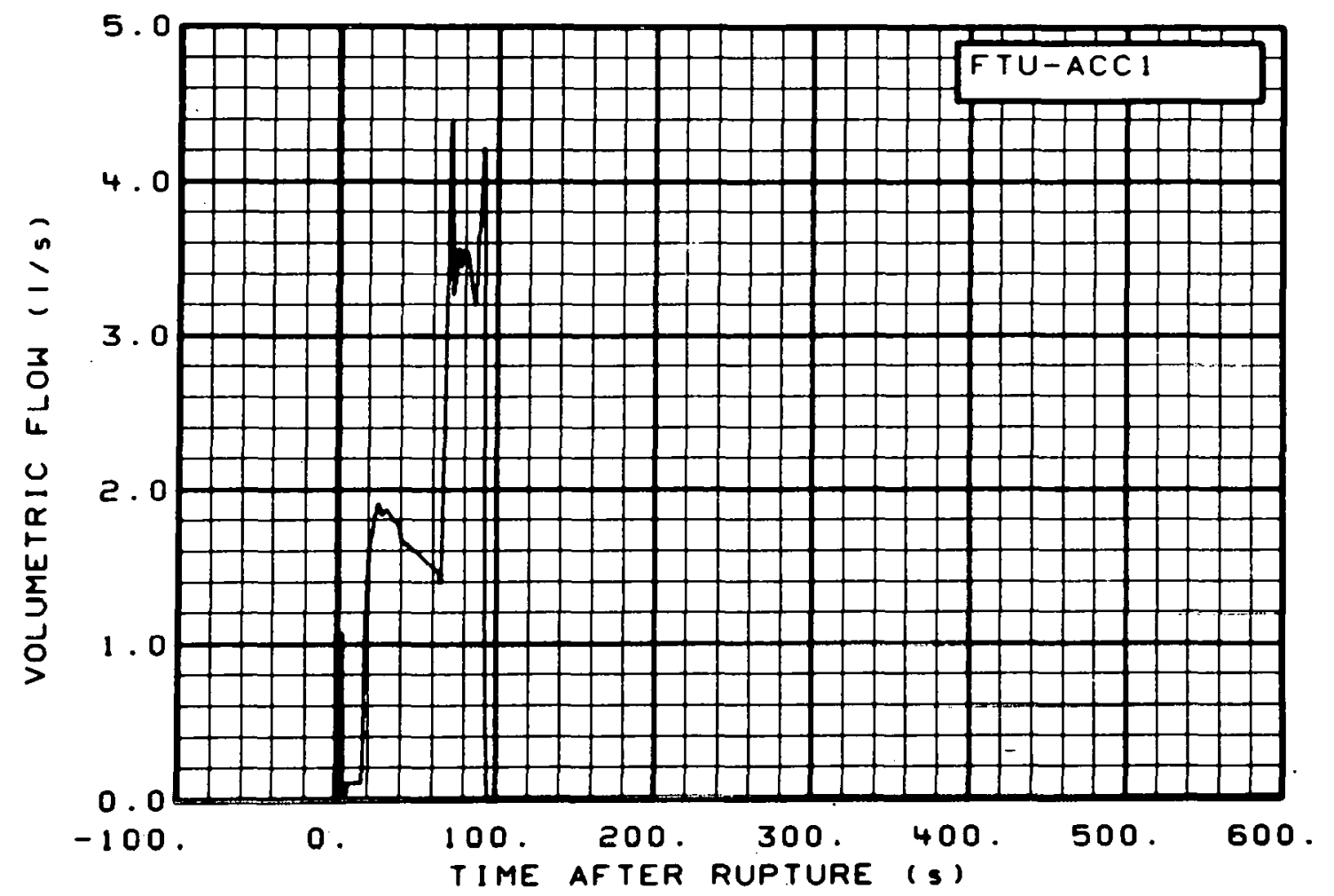

Fig. 257 Volumetric flow in intact loop accumulator discharge line (FTU-ACCl), from -20 to $600 \mathrm{~s}$.

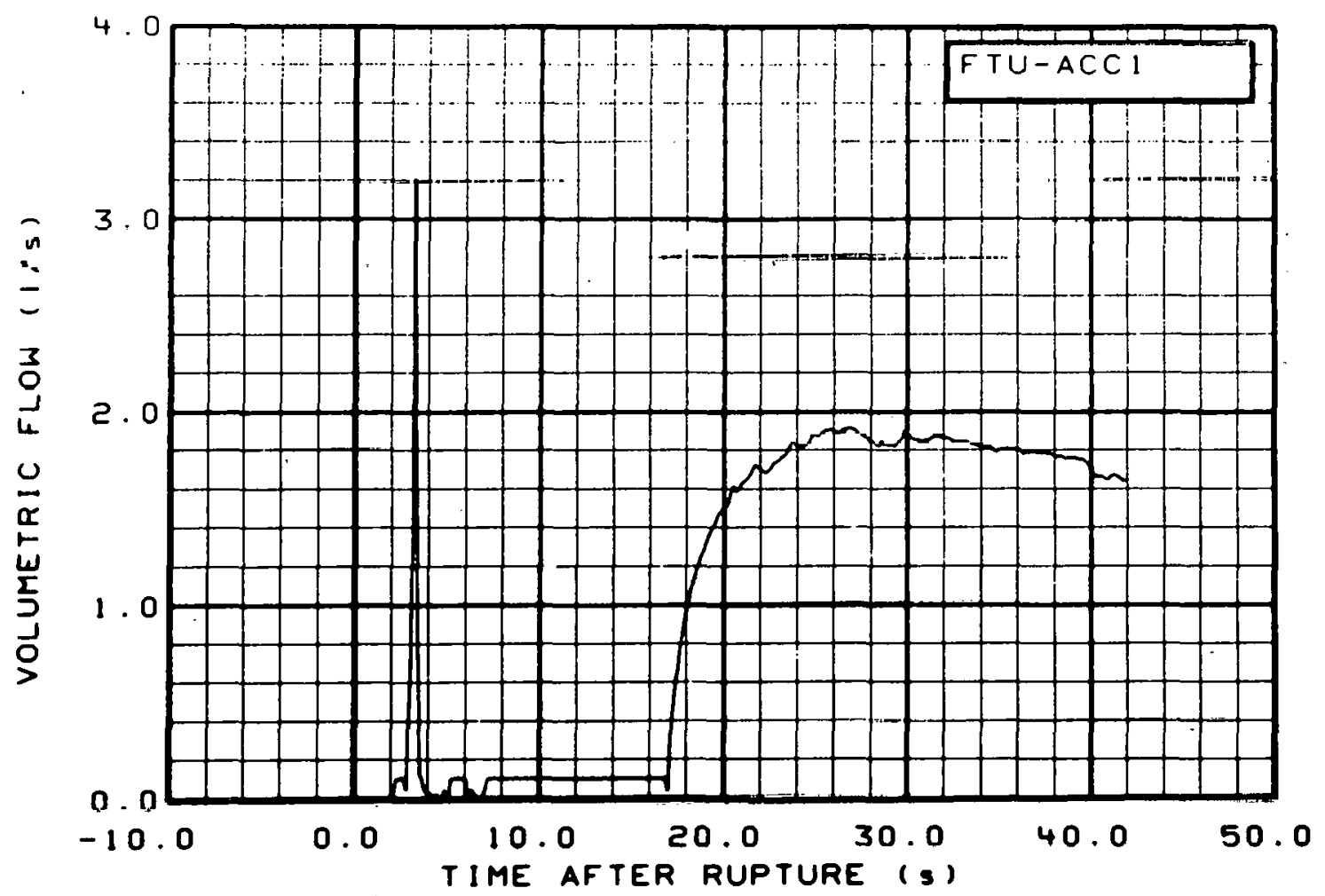

Fig. 258 Volumetric flow in intact loop accumulator discharge line (FTU-ACC 1), from -6 to $42 \mathrm{~s}$. 


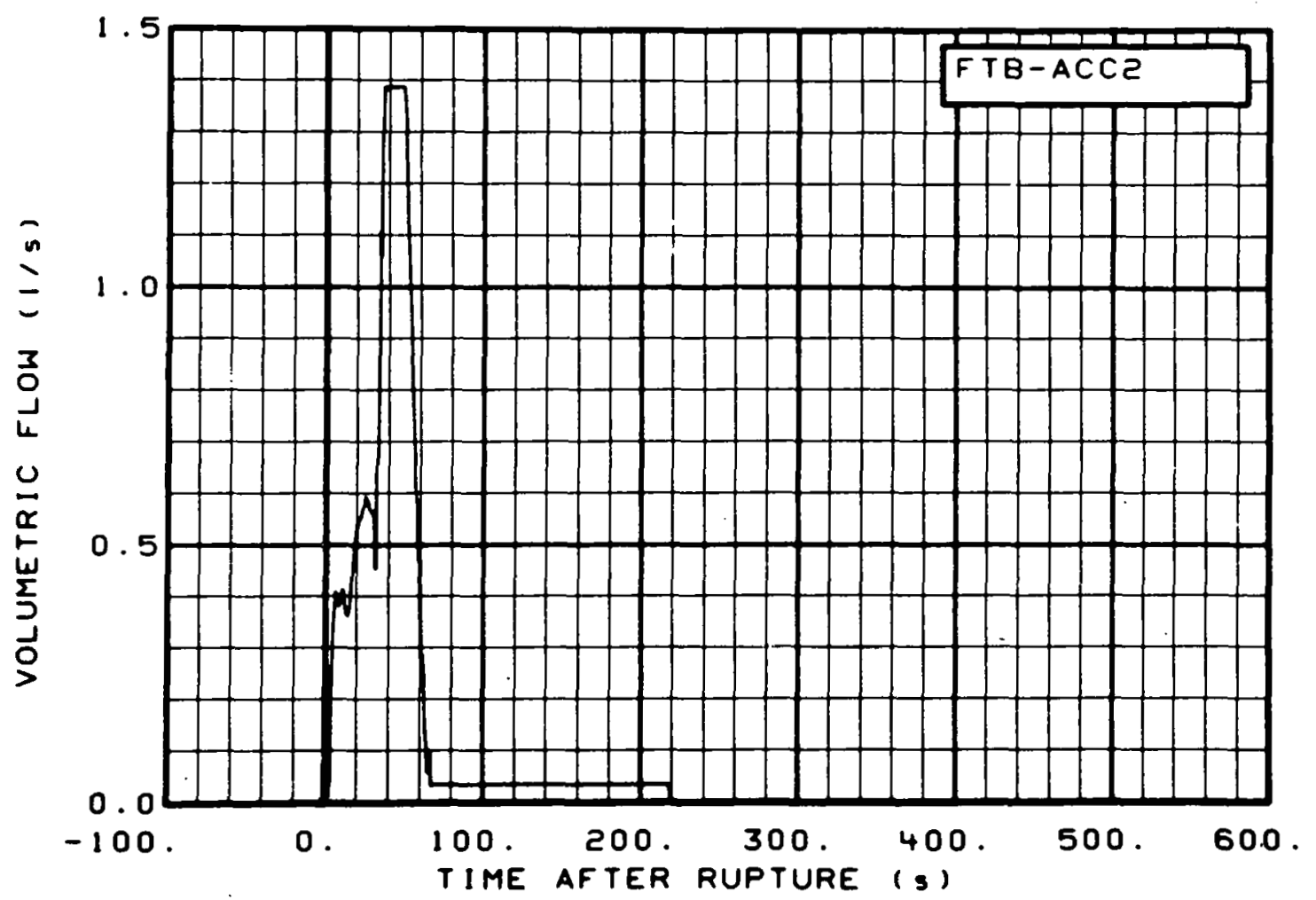

Fig. 259 Volumetric flow in broken loop accumulator discharge line (FTB-ACC2), from -20 to $600 \mathrm{~s}$.

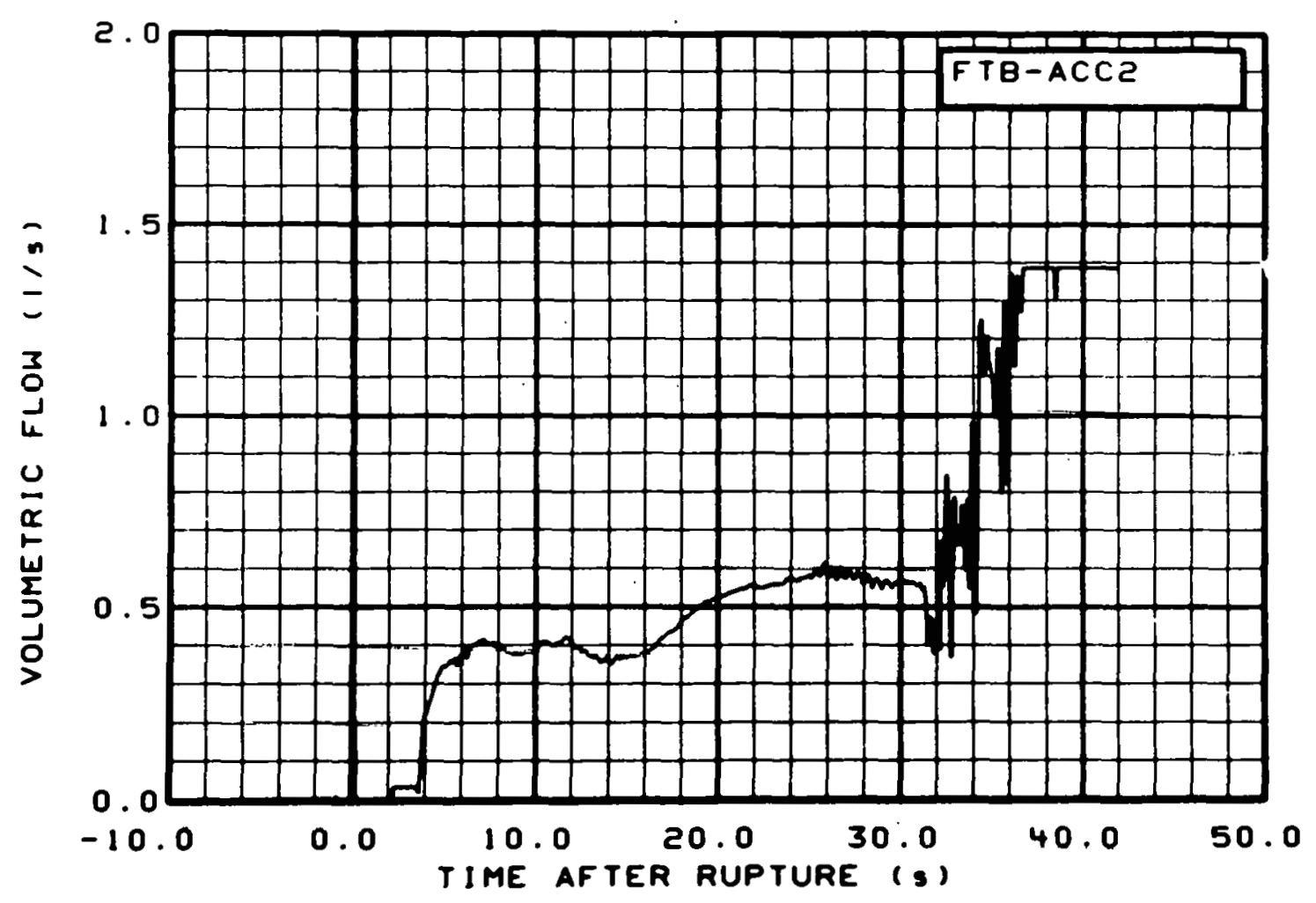

Fig. 260 Volumetric flow in broken loop accumulator discharge line (FTB-ACC2), from -6 to $42 \mathrm{~s}$. 


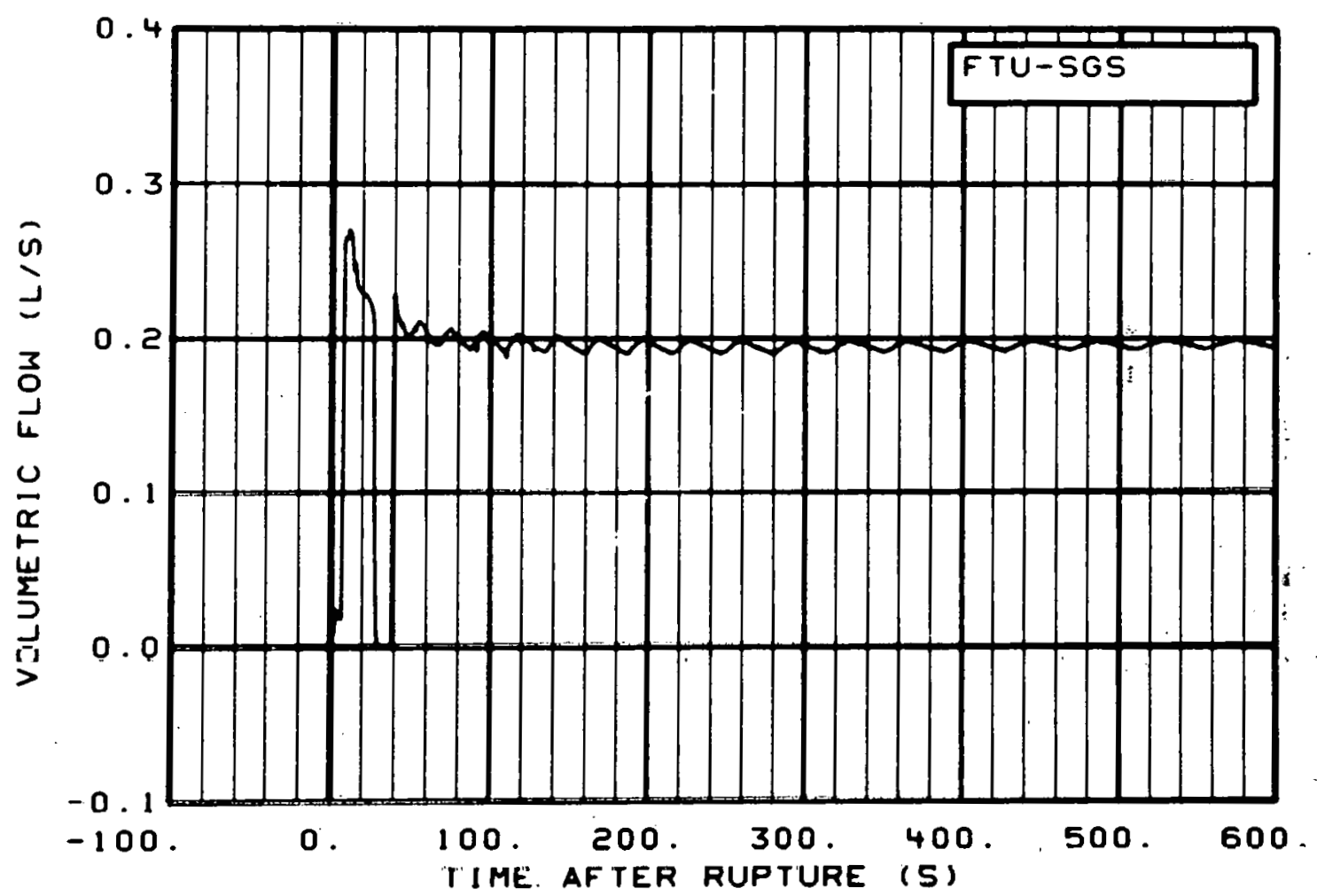

Fig. 261 Volumetric flow from intact loop steam generator tube rupture simulation accumulator (FTU-SGS), from -20 to $600 \mathrm{~s}$.

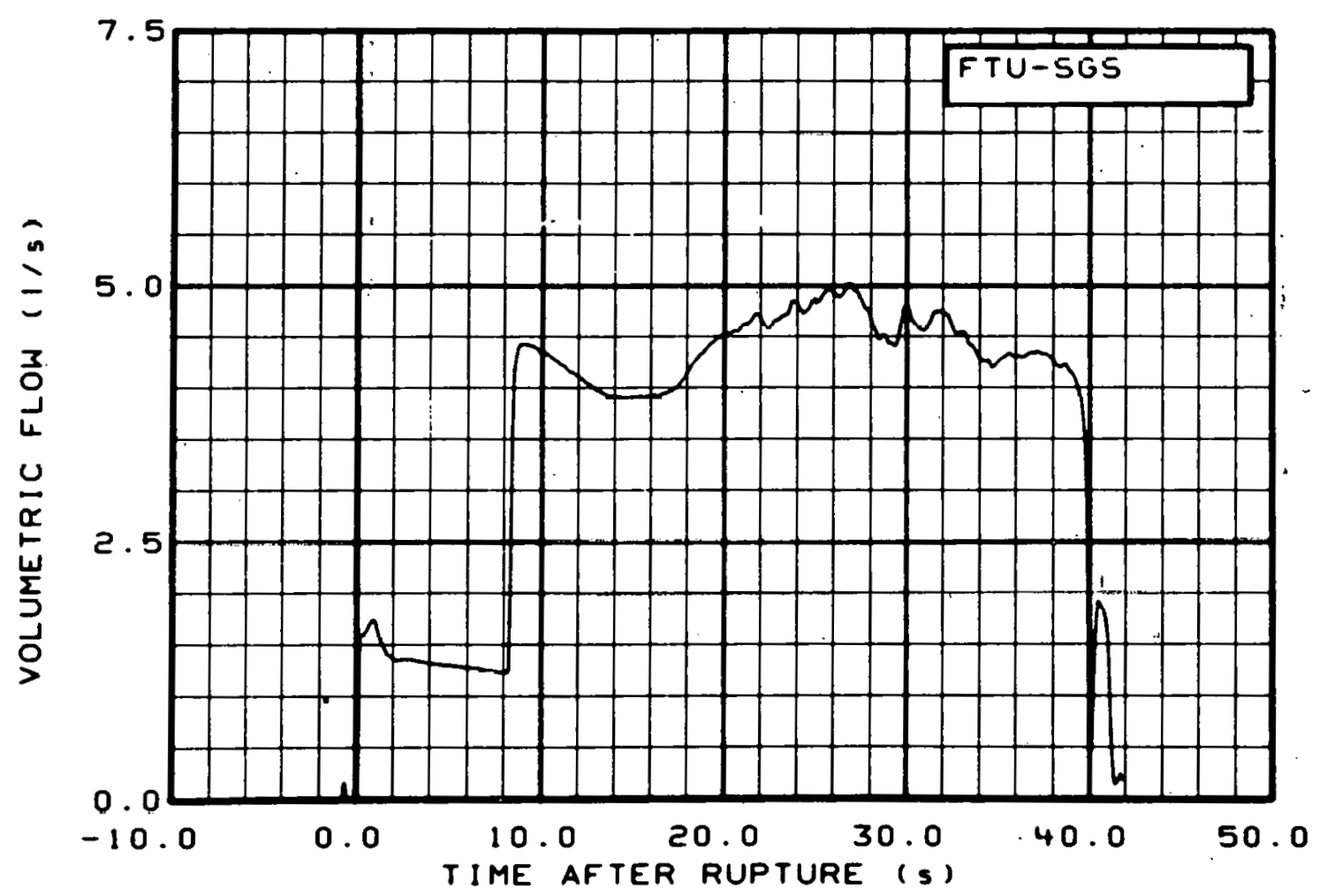

Fig. 262 Volumetric flow from intact loop steam generator tube rupture simulation accumulator (FTU-SGS), from -6 to $42 \mathrm{~s}$. 


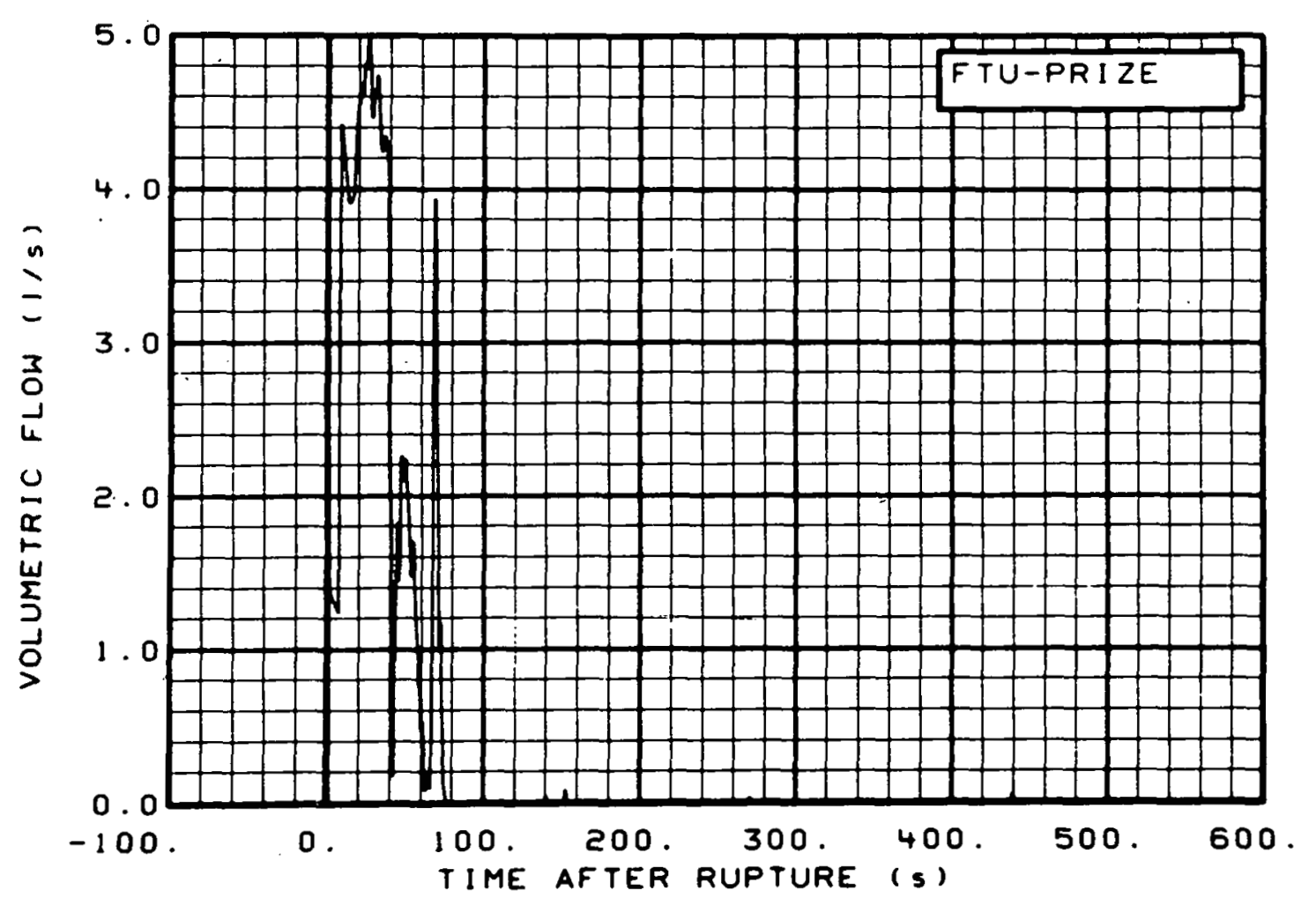

Fig. 263 Volumetric flow from pressurizer (FTU-PRIZE), from -20 to $600 \mathrm{~s}$.

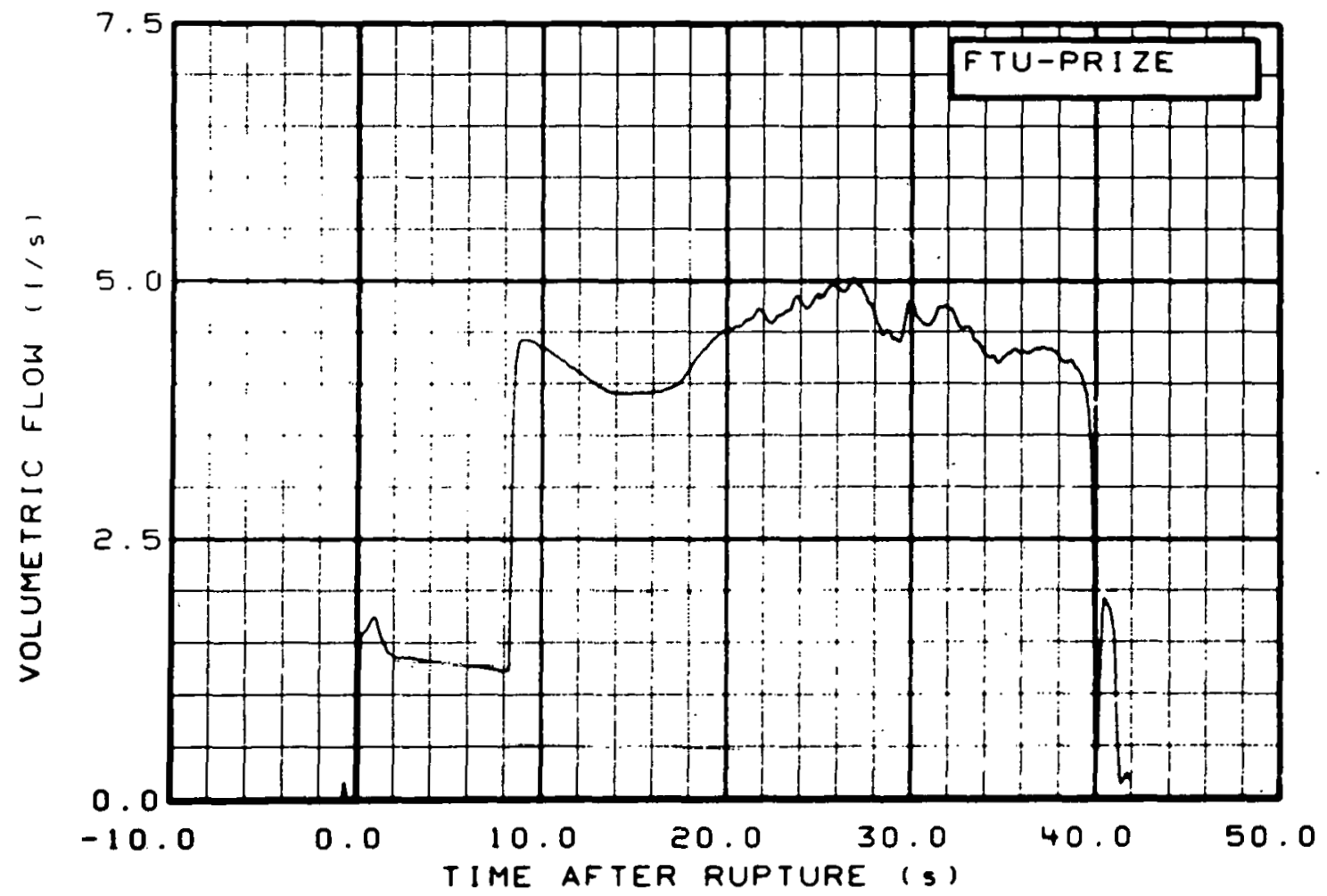

Fig. 264 Volumetric flow from pressurizer (FTU-PRIZE), from -6 to $42 \mathrm{~s}$. 


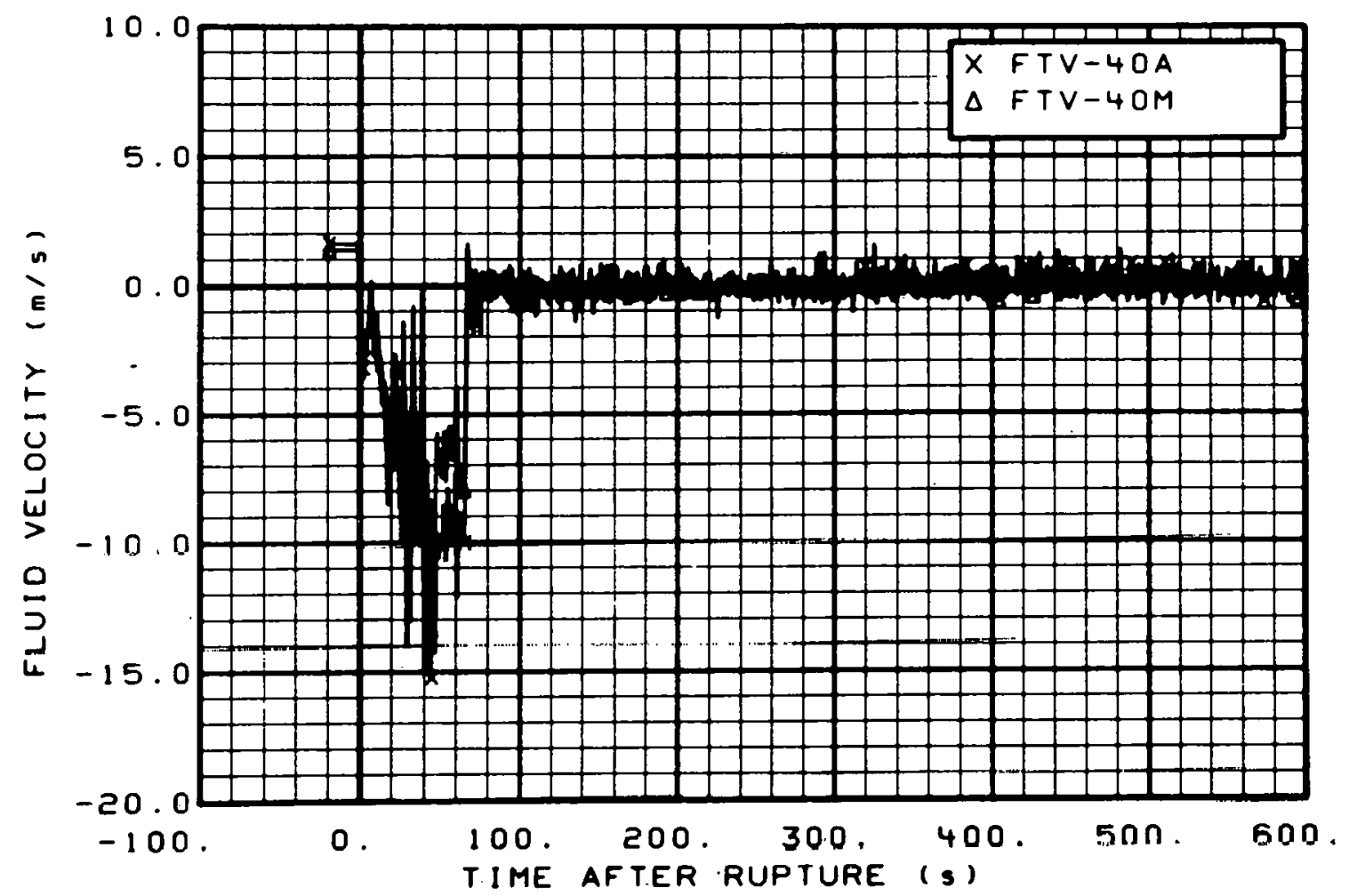

Fig. 265 Fluid velocity in vessel (FTV-40A and FTV-40M), from -20 to $600 \mathrm{~s}$.

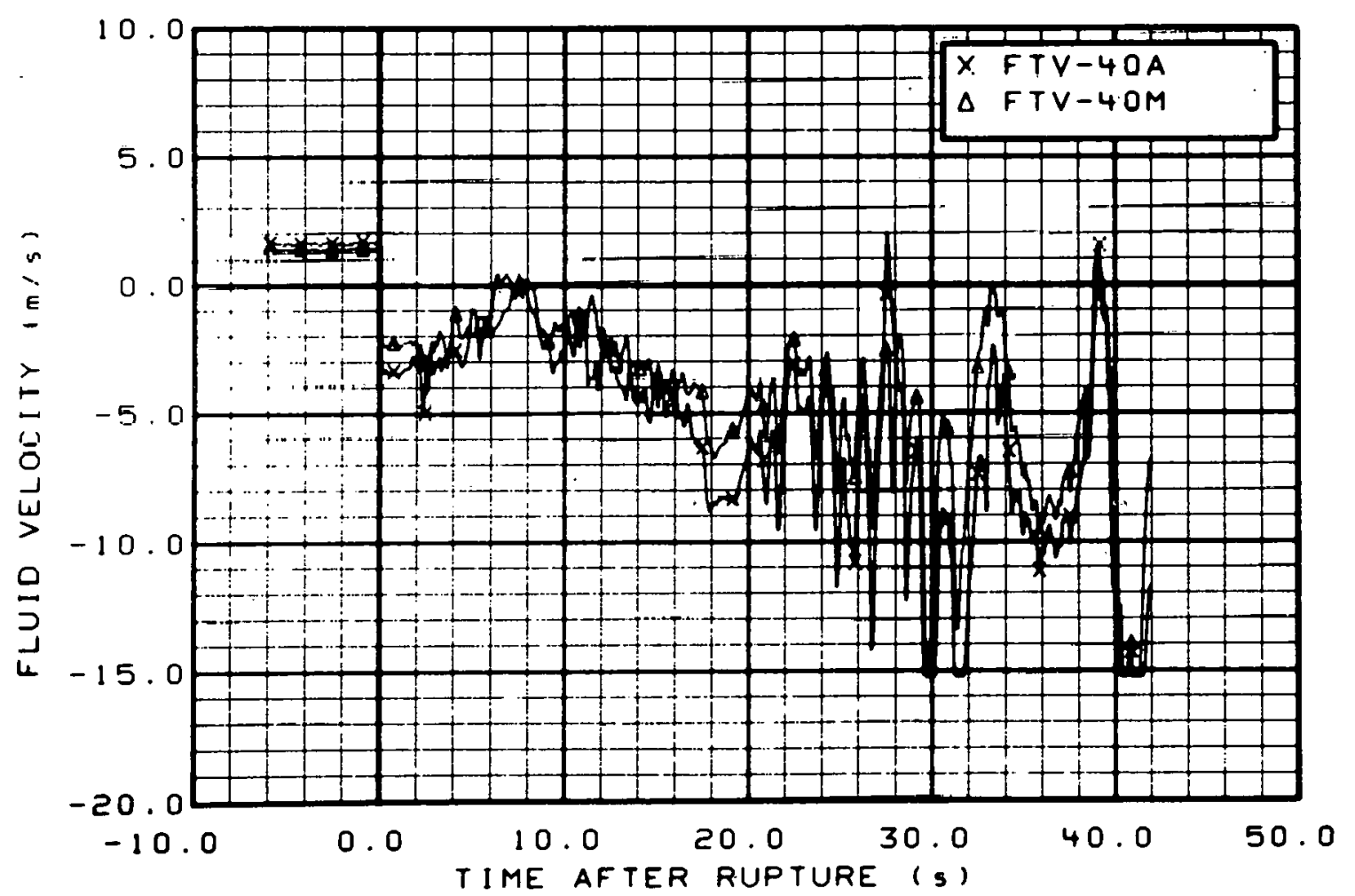

Fig. 266 Fluid velocity in vessel (FTV-40A and FTV-40M), from -6 to $42 \mathrm{~s}$. 


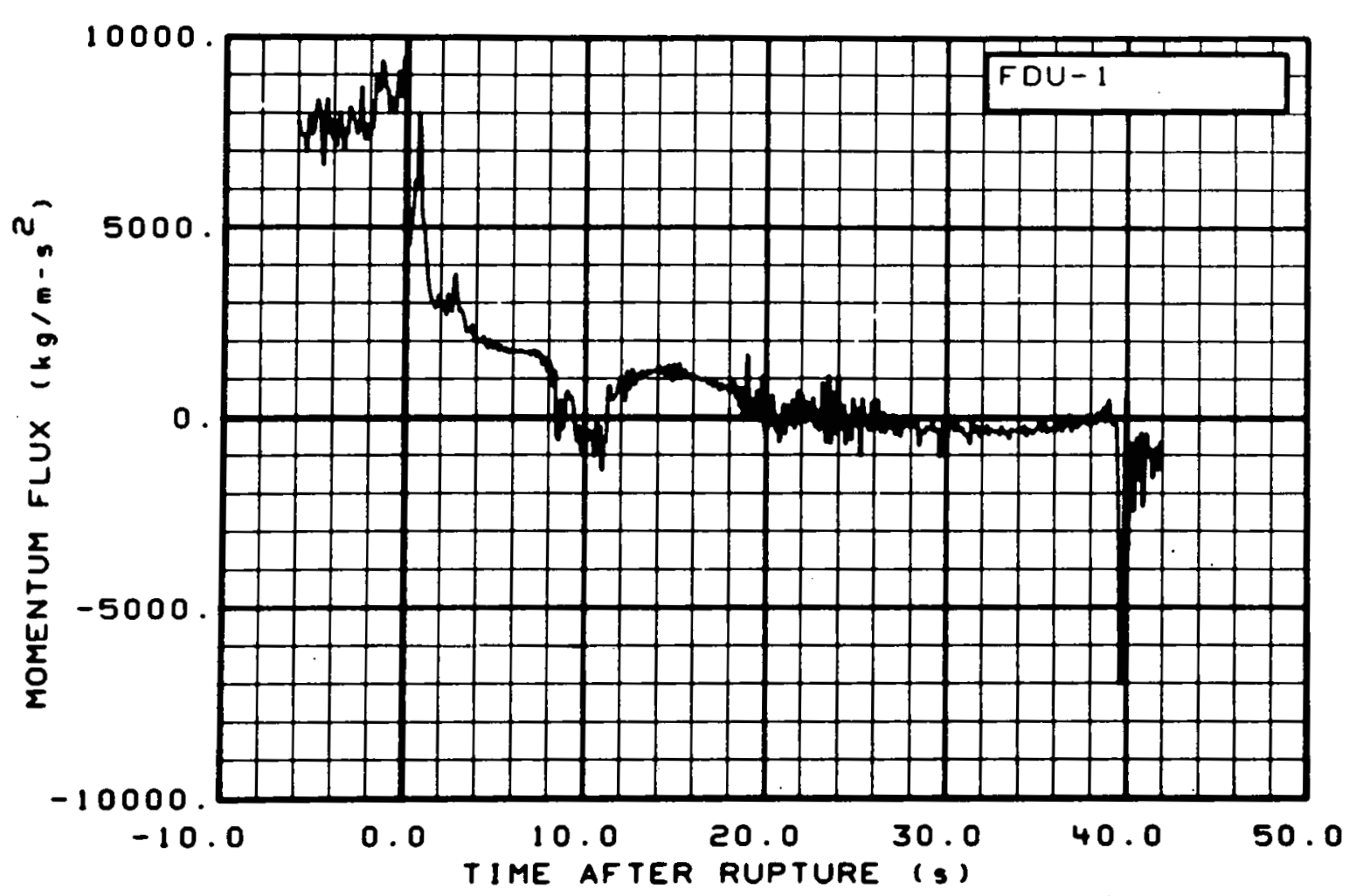

Fig. 267 Momentum flux in intact loop (FDU-1), from -6 to $42 \mathrm{~s}$.

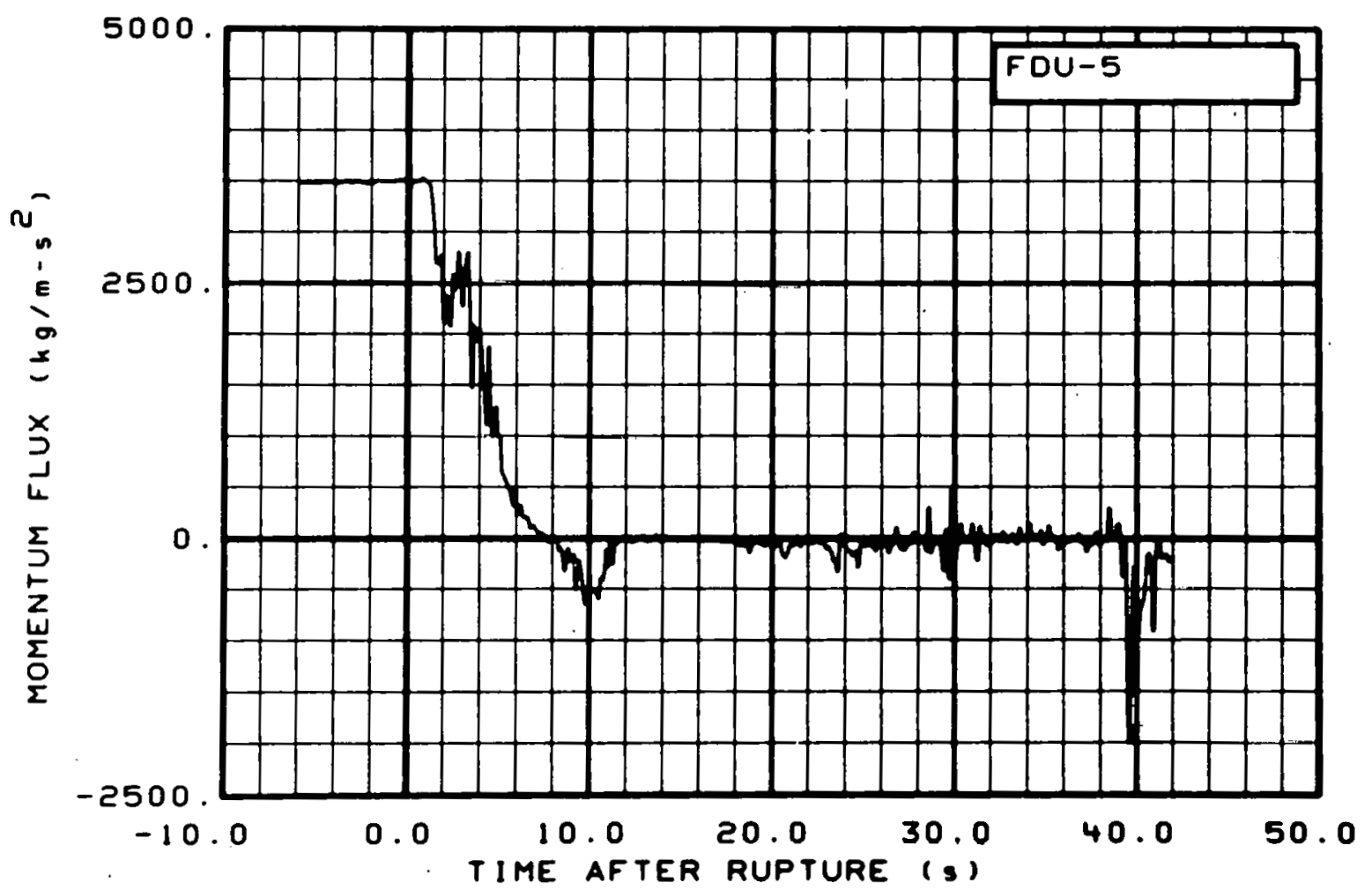

Fig. 268 Momentum flux in intact loop (FDU-5), from -6 to $42 \mathrm{~s}$. 


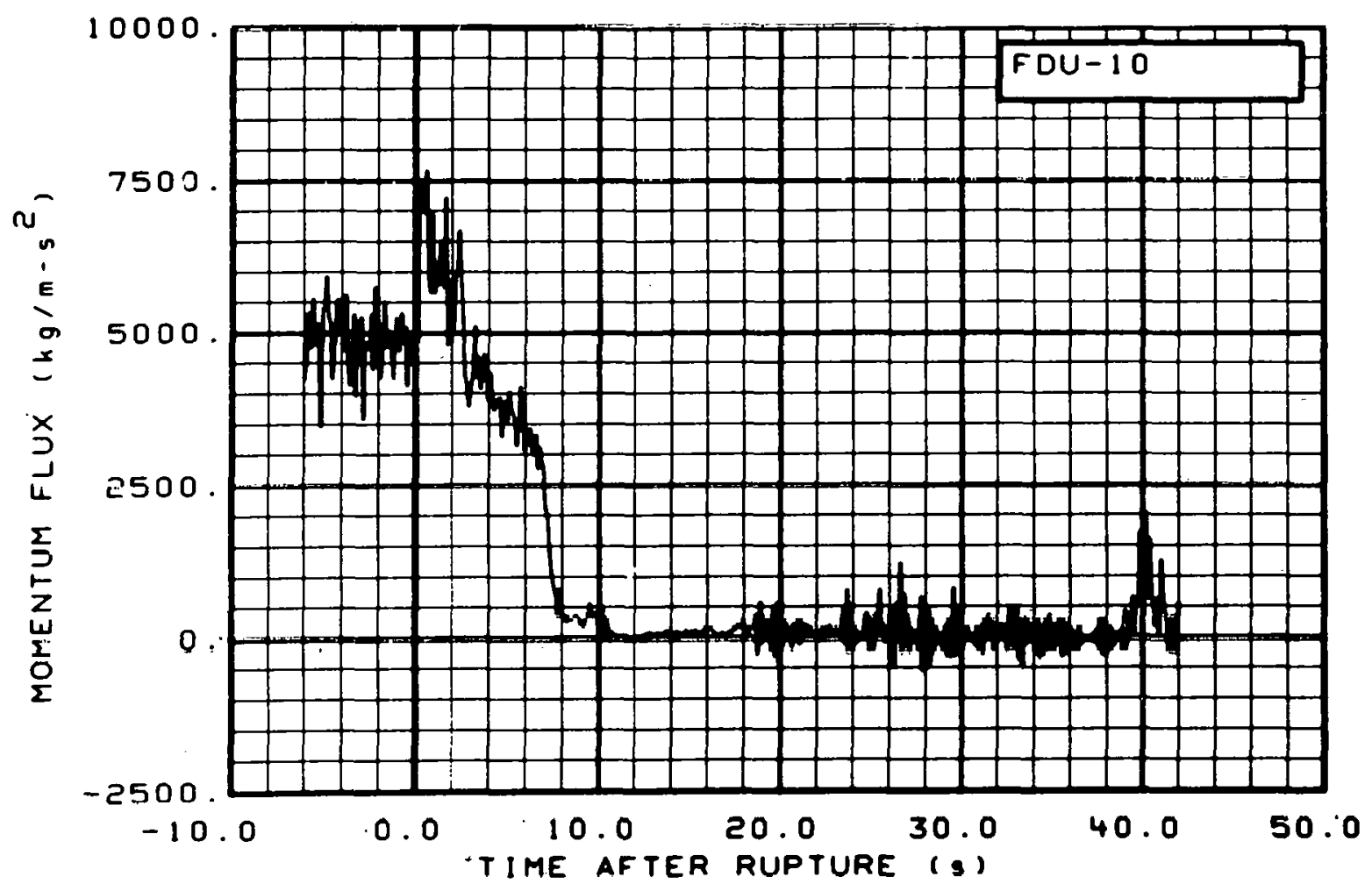

Fig. 269 Momentum flux in intact loop (FDU-10), from -6 to $42 \mathrm{~s}$.

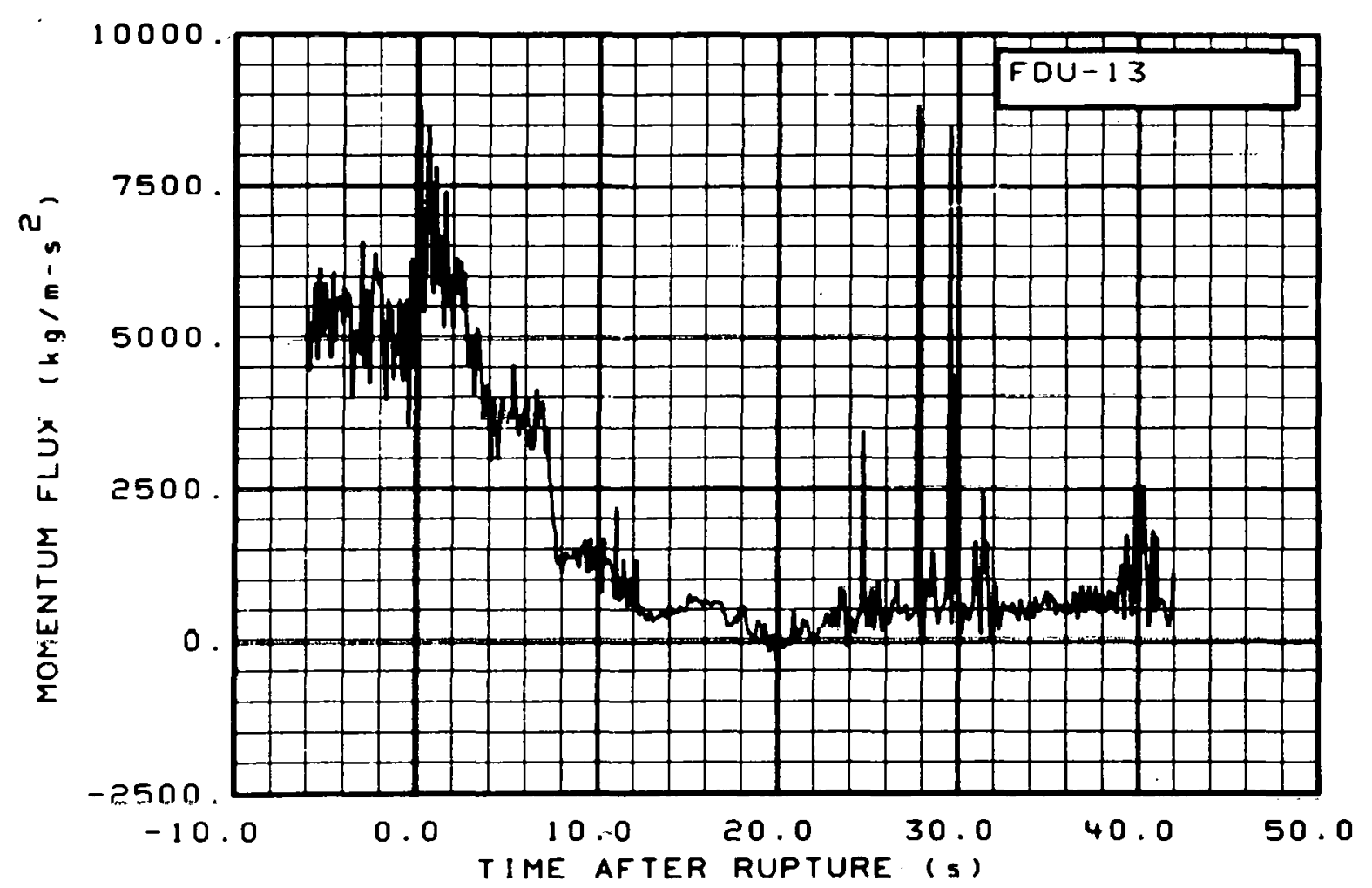

Fig. 270 Momentum flux in intact loop (FDU-13), from -6 to $42 \mathrm{~s}$. 


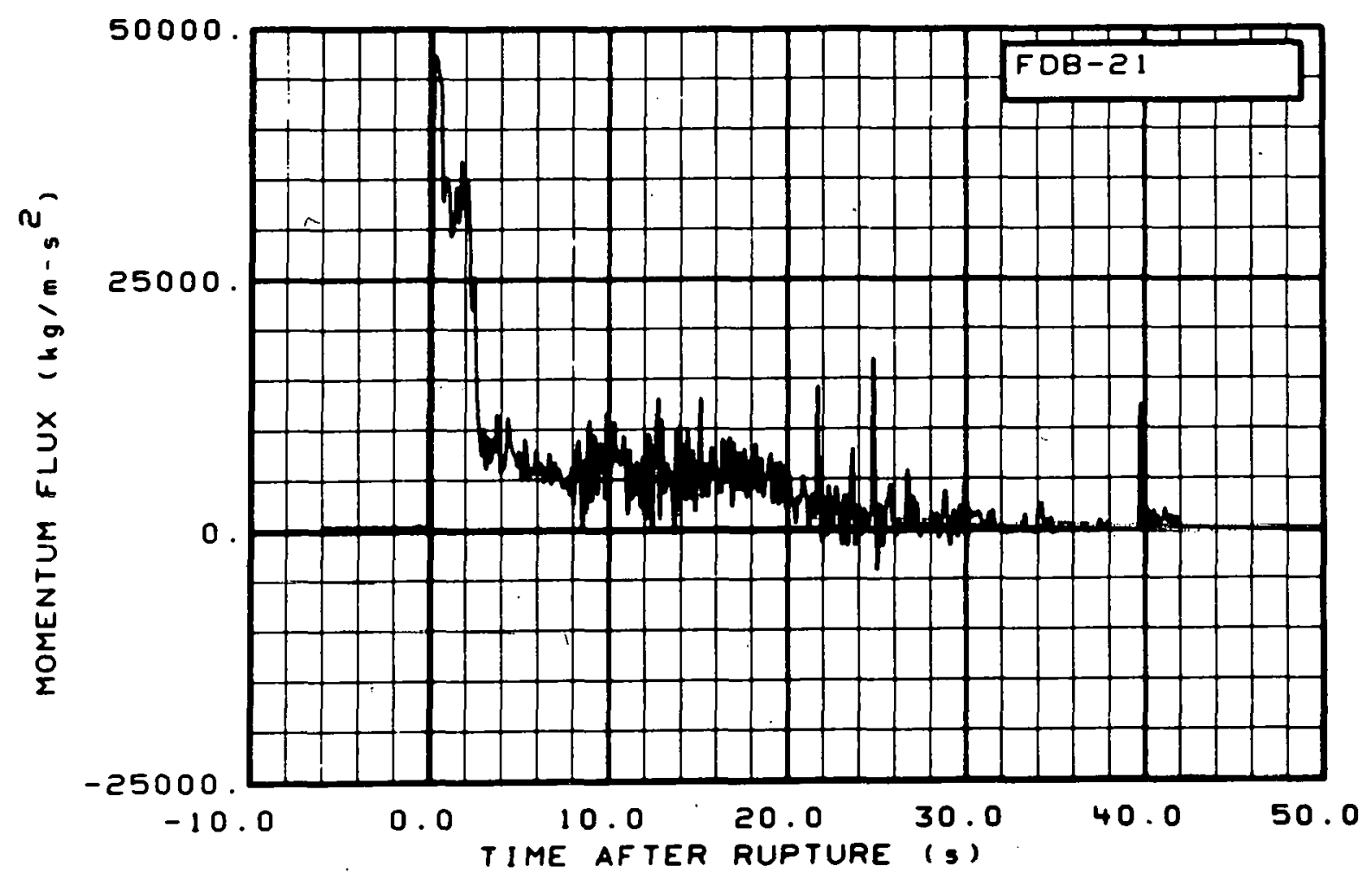

Fig. 271 Momentum flux in broken loop (FDB-21), from -6 to $42 \mathrm{~s}$.

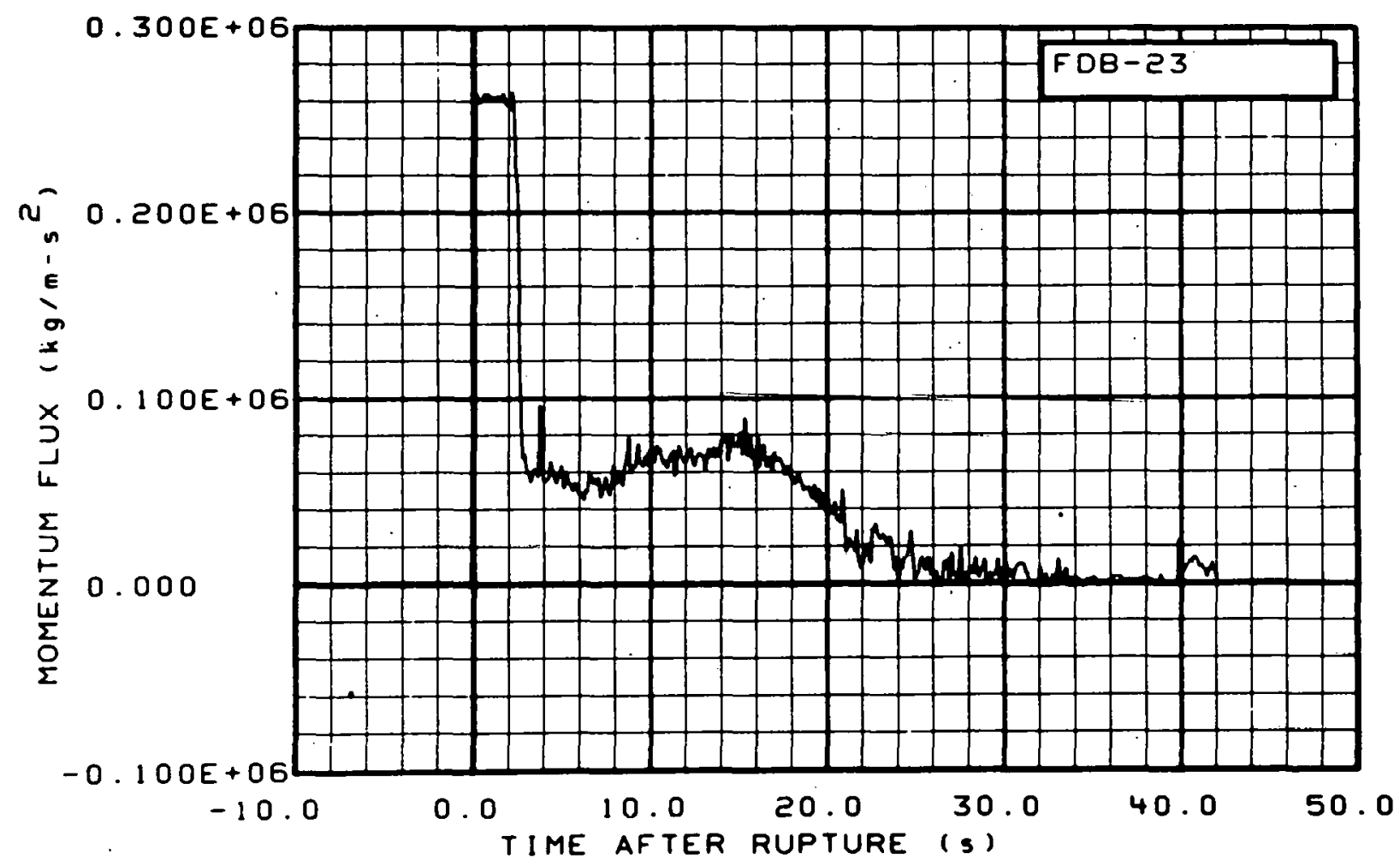

Fig. 272 Momentum flux in broken loop (FDB-23), from -6 to $42 \mathrm{~s}$. 


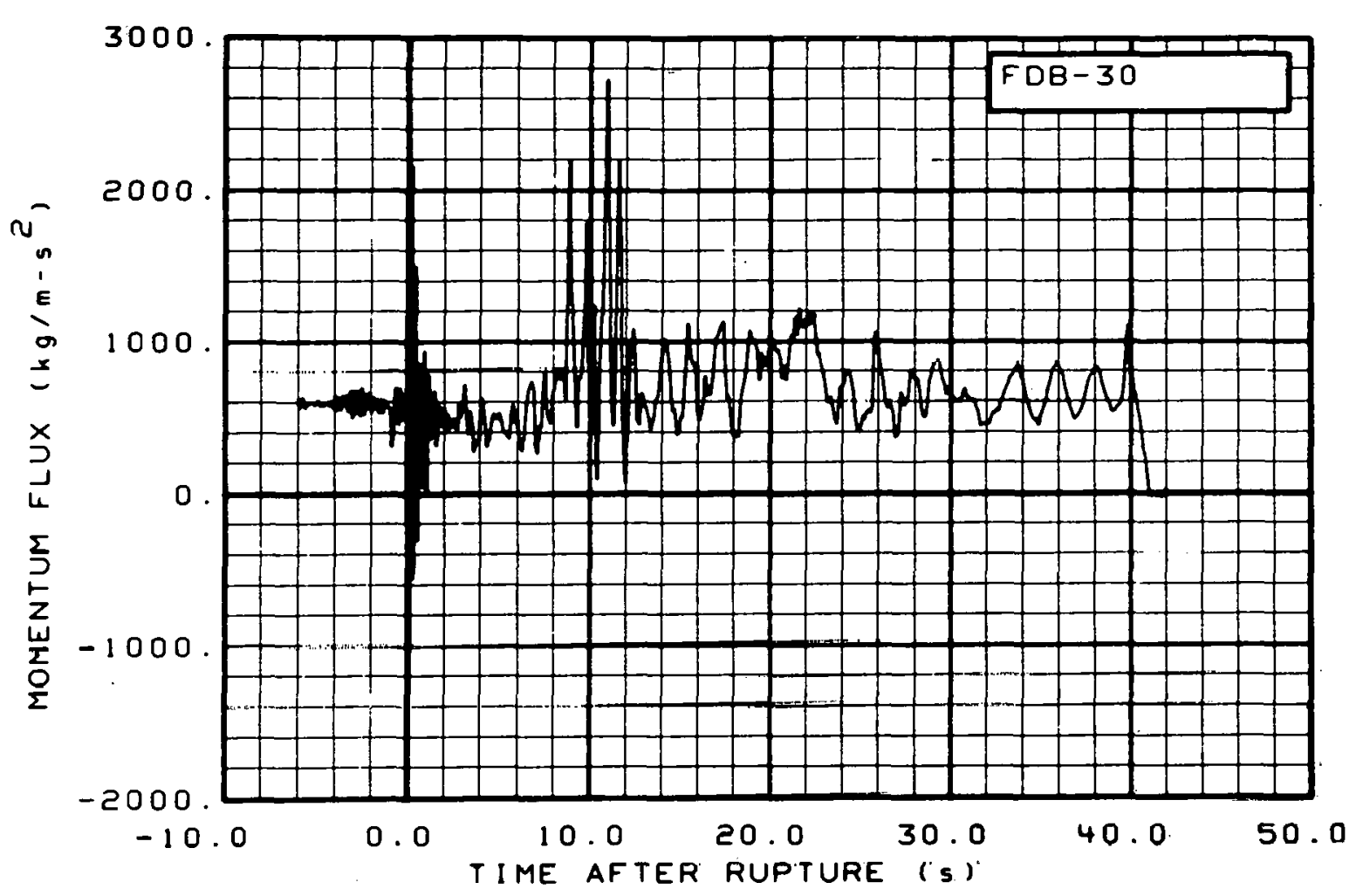

Fig. 273. Momentum flux in broken loop (FDB-30), from -6 to $42 \mathrm{~s}$.

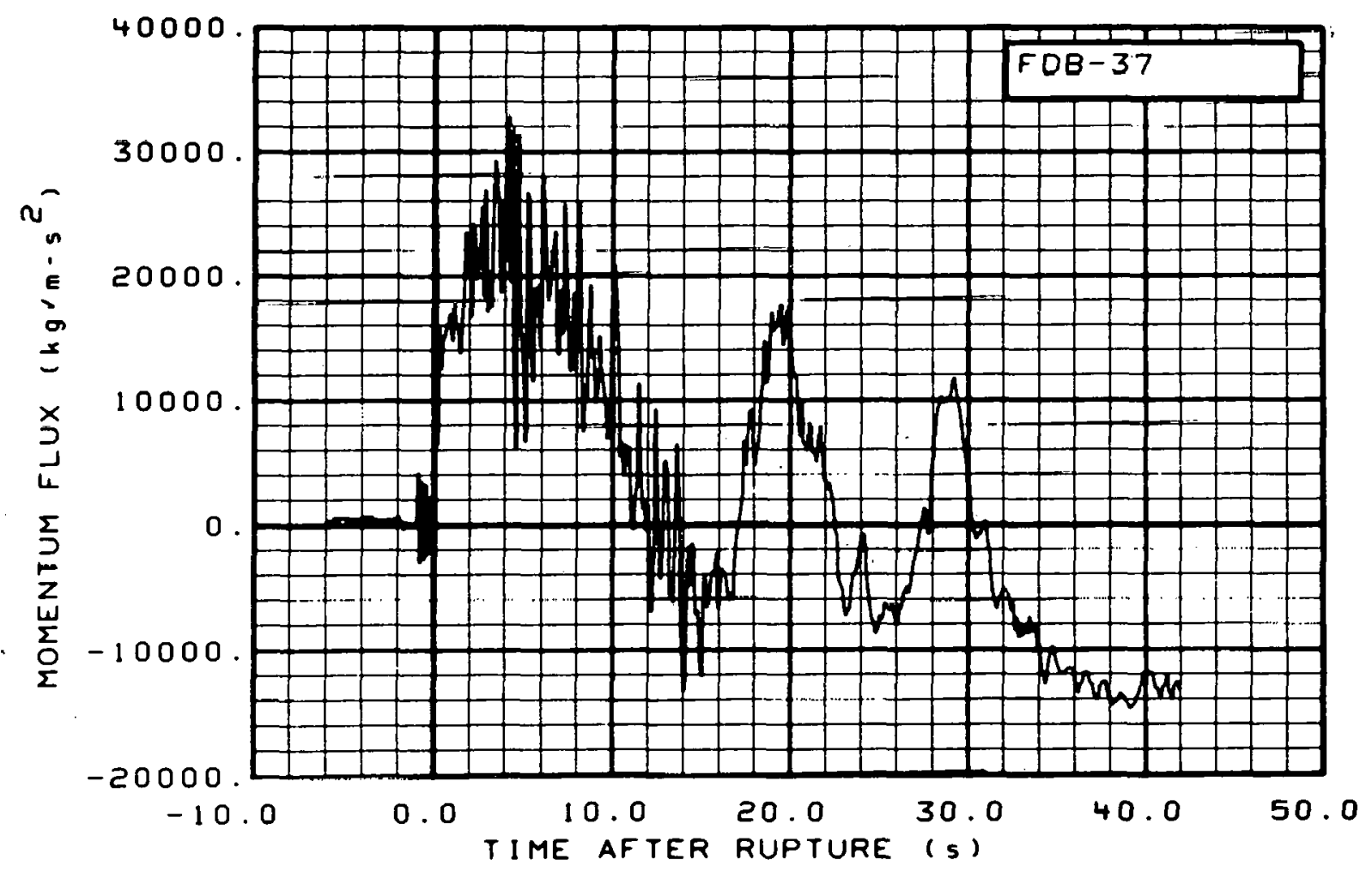

Fig. 274 Momentum flux in broken loop (FDB-37), from -6 to $42 \mathrm{~s}$. 


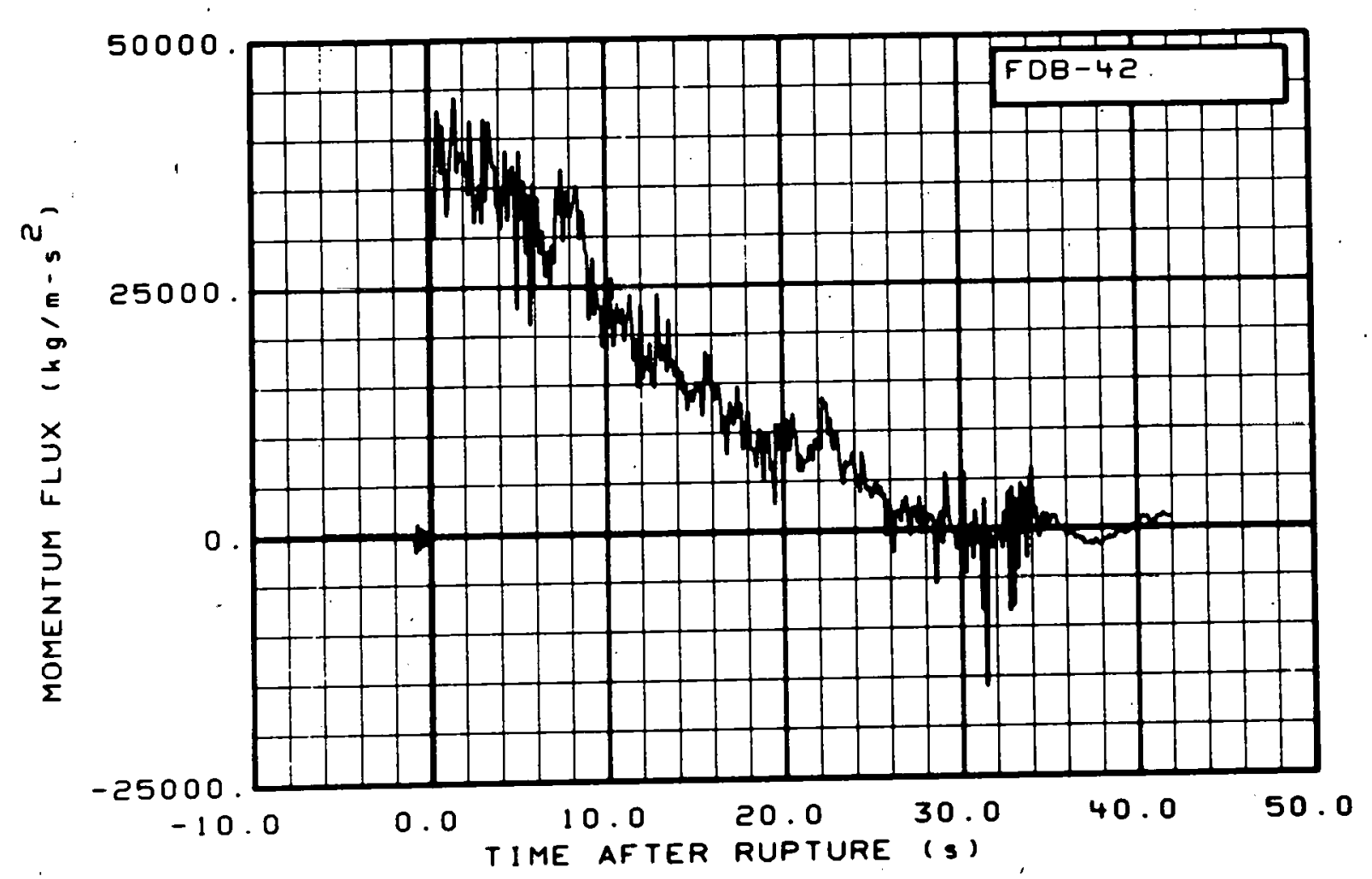

Fig. 275 Momentum flux in broken loop (FDB-42), from -6 to $42 \mathrm{~s}$. 


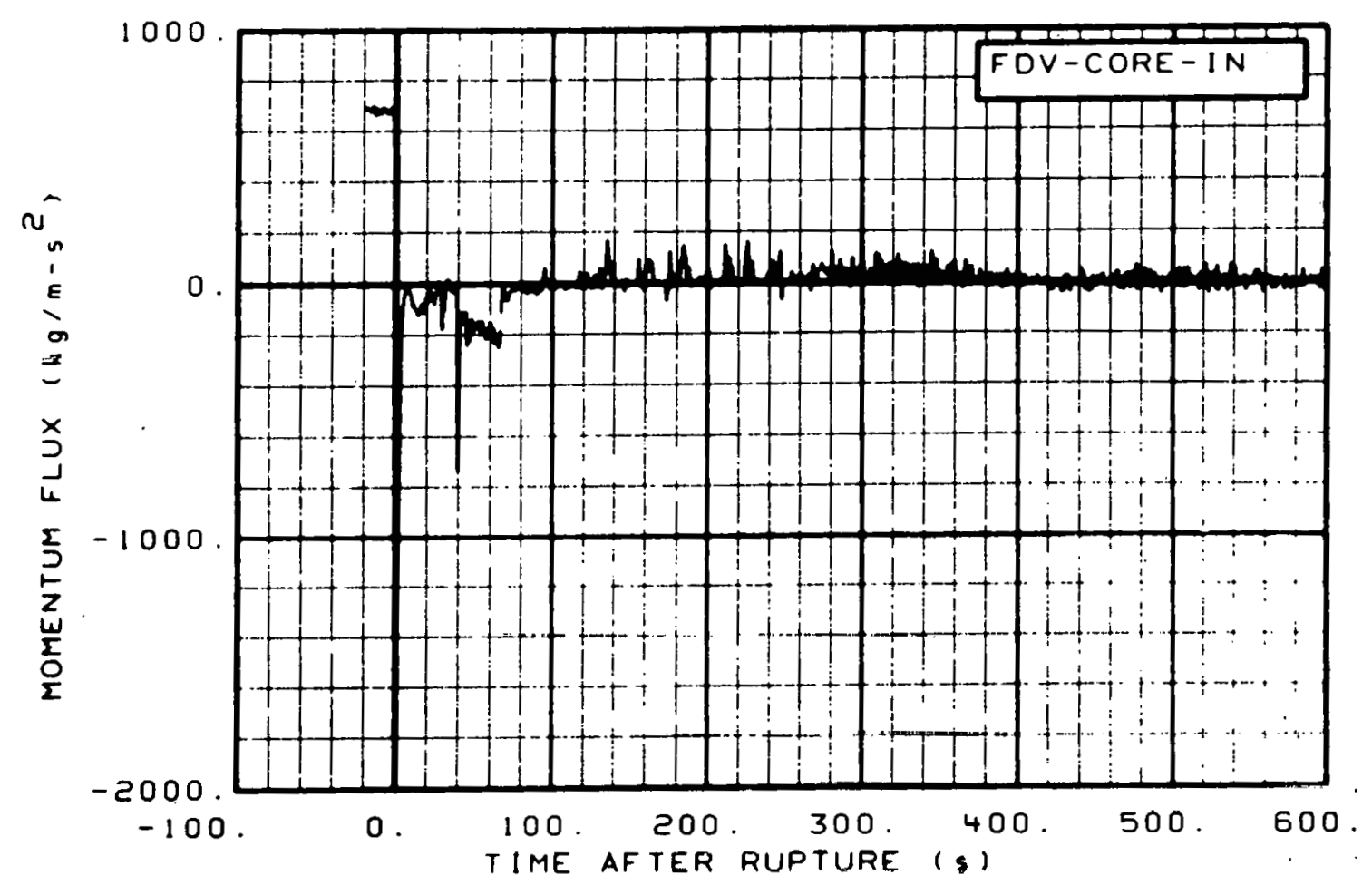

Fig. 276 Momentum flux in core entrance (FDV-CORE-IN), from -20 to $600 \mathrm{~s}$.

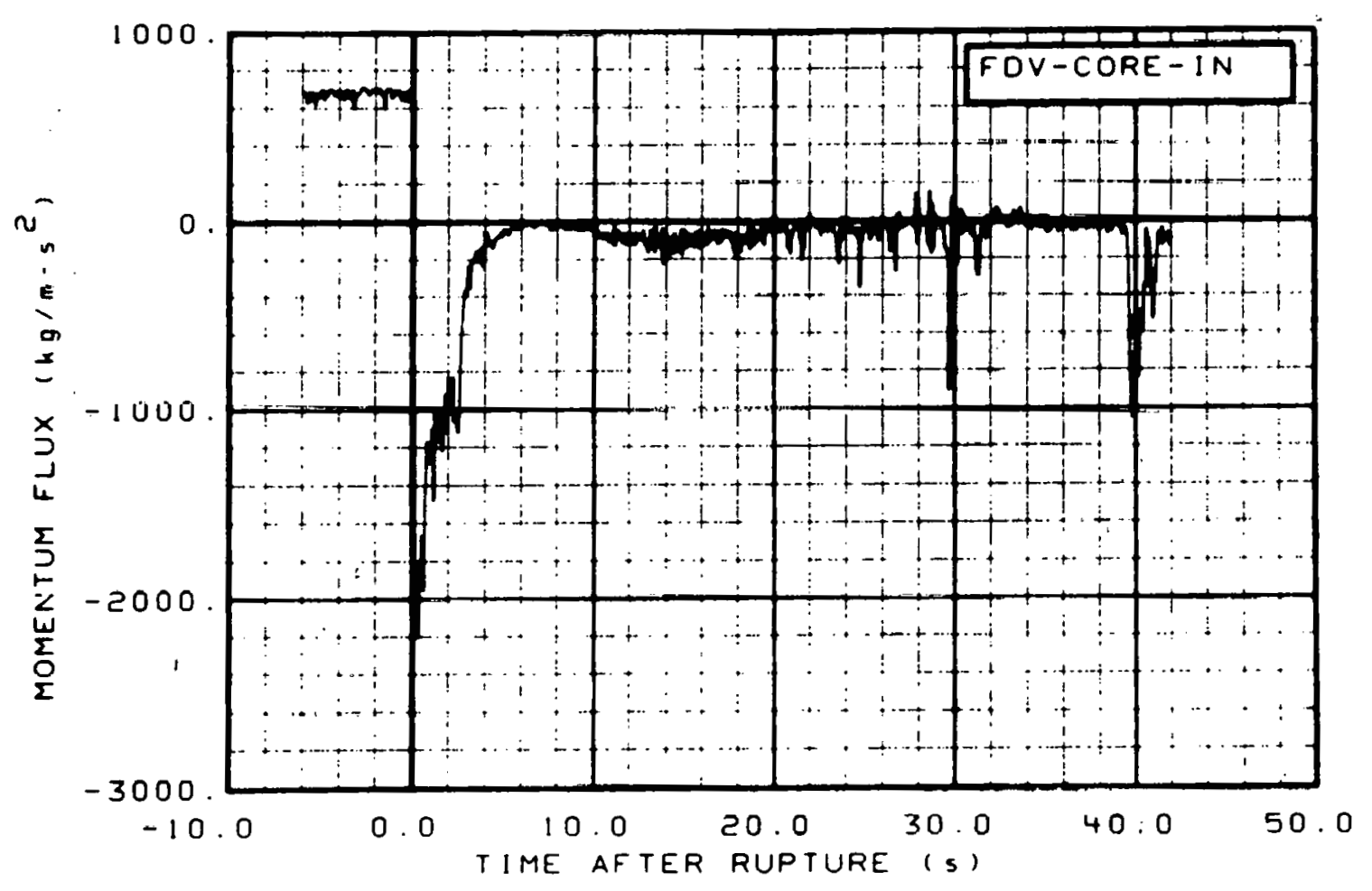

Fig. 277 iMomentum flux in core entrance (FOV-CORE-IN), from -6 to $42 \mathrm{~s}$. 


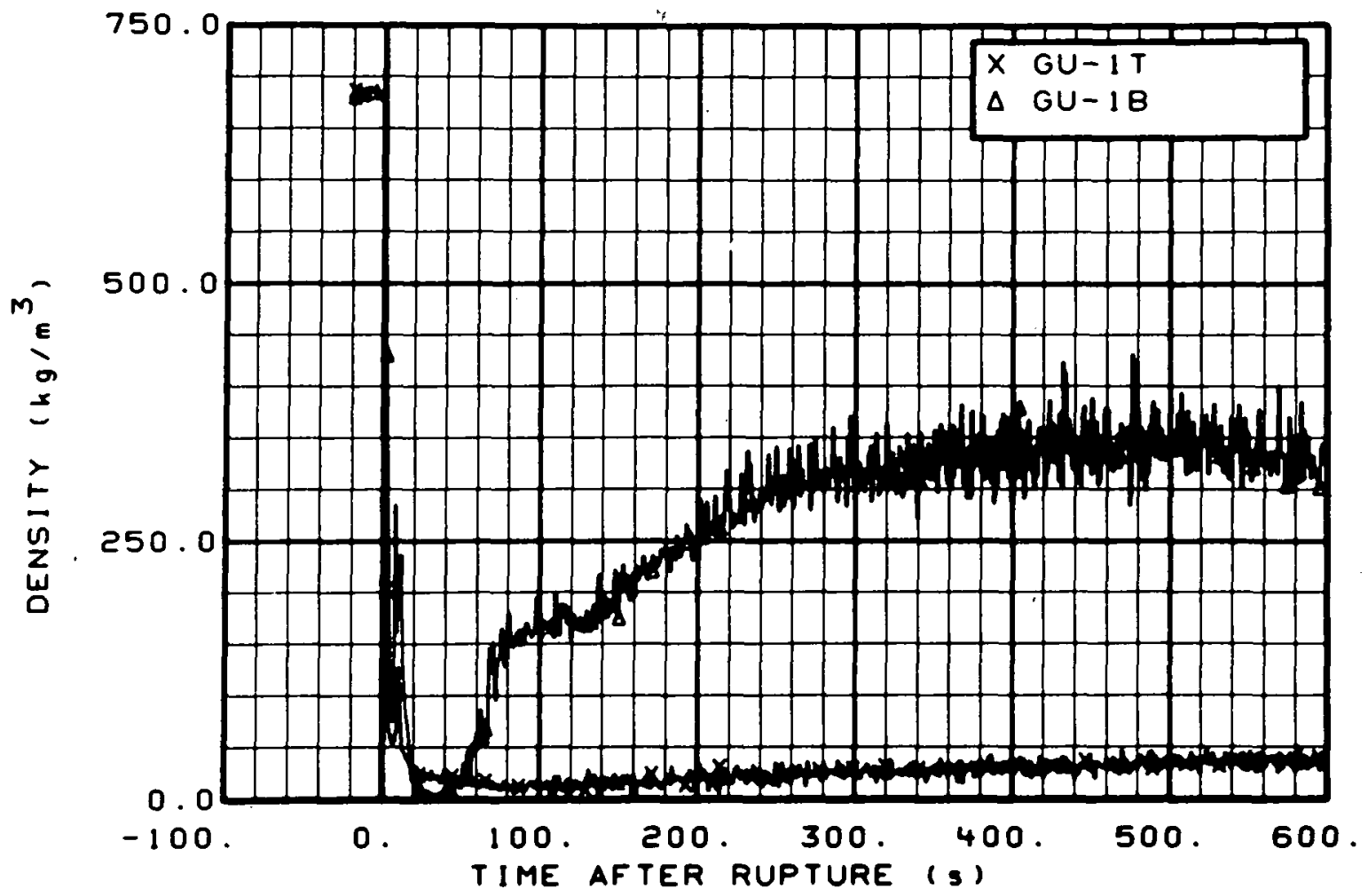

Fig. 278 Density in intact loop (GU-1T and GU-1B), from -20 to $600 \mathrm{~s}$.

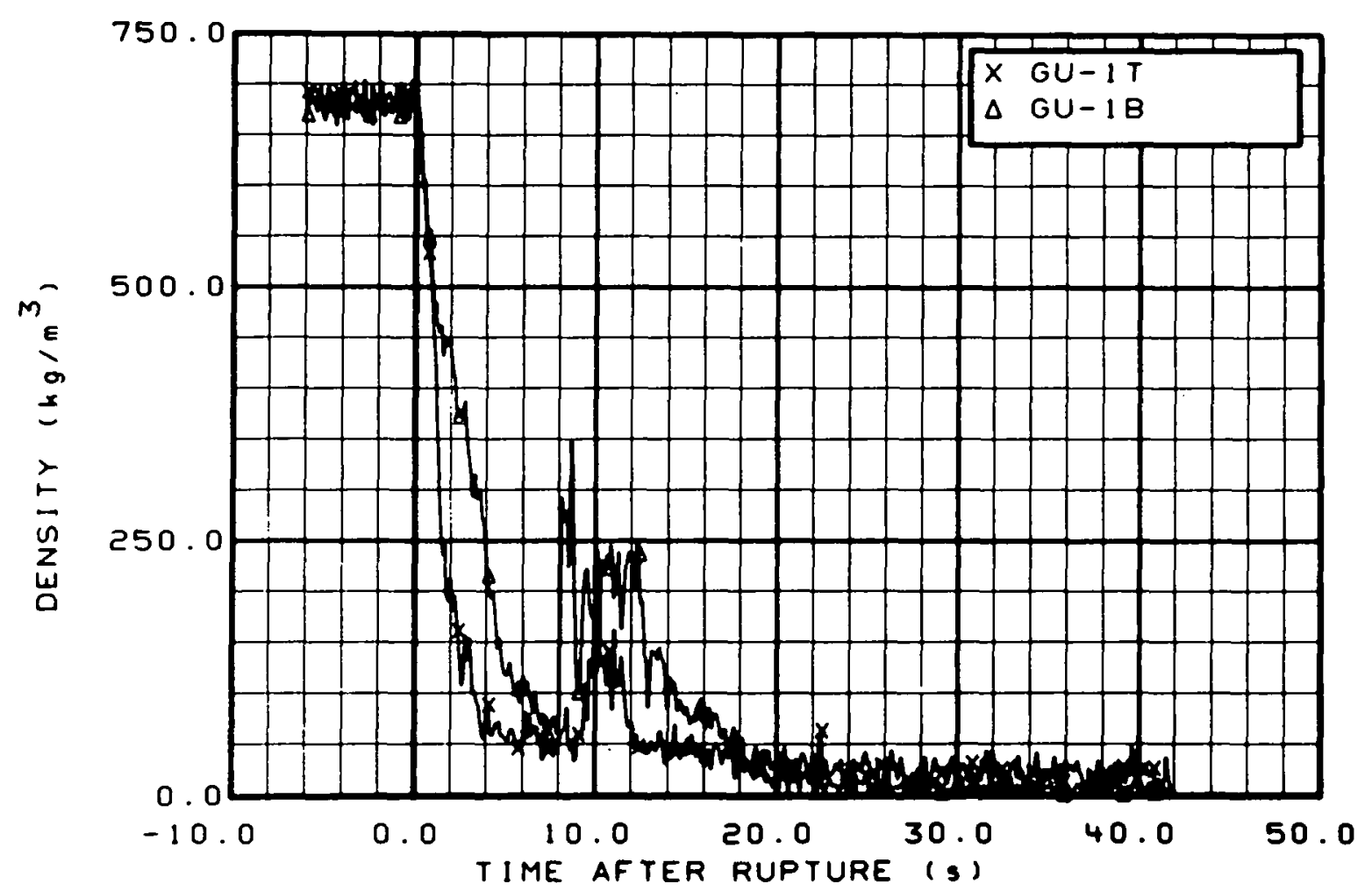

Fig. 279 Density in intact loop (GU-IT and GU-IB), from -6 to $42 \mathrm{~s}$. 


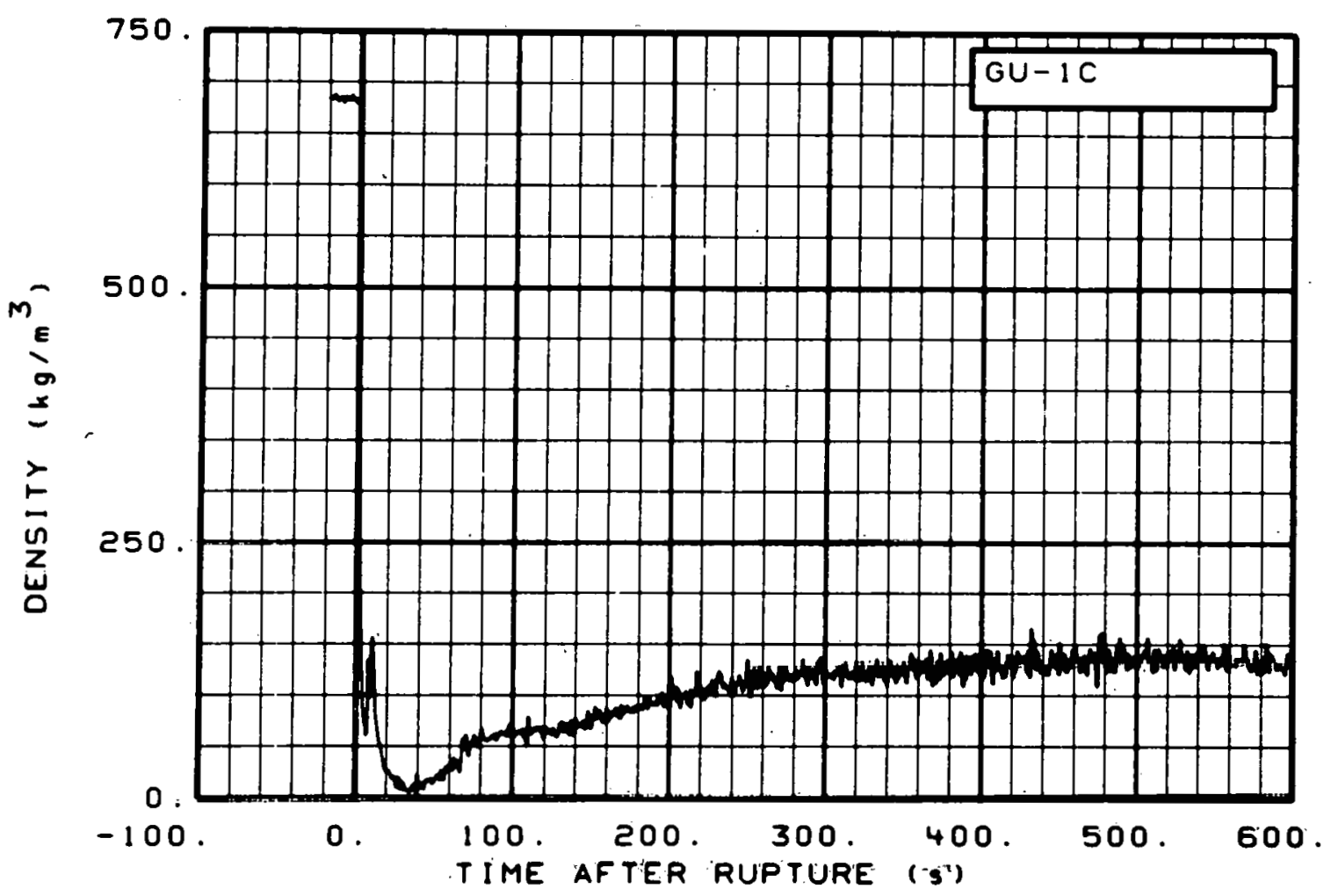

Fig. 280 Density in intact loop (GU-IC), from -20 to $600 \mathrm{~s}$.

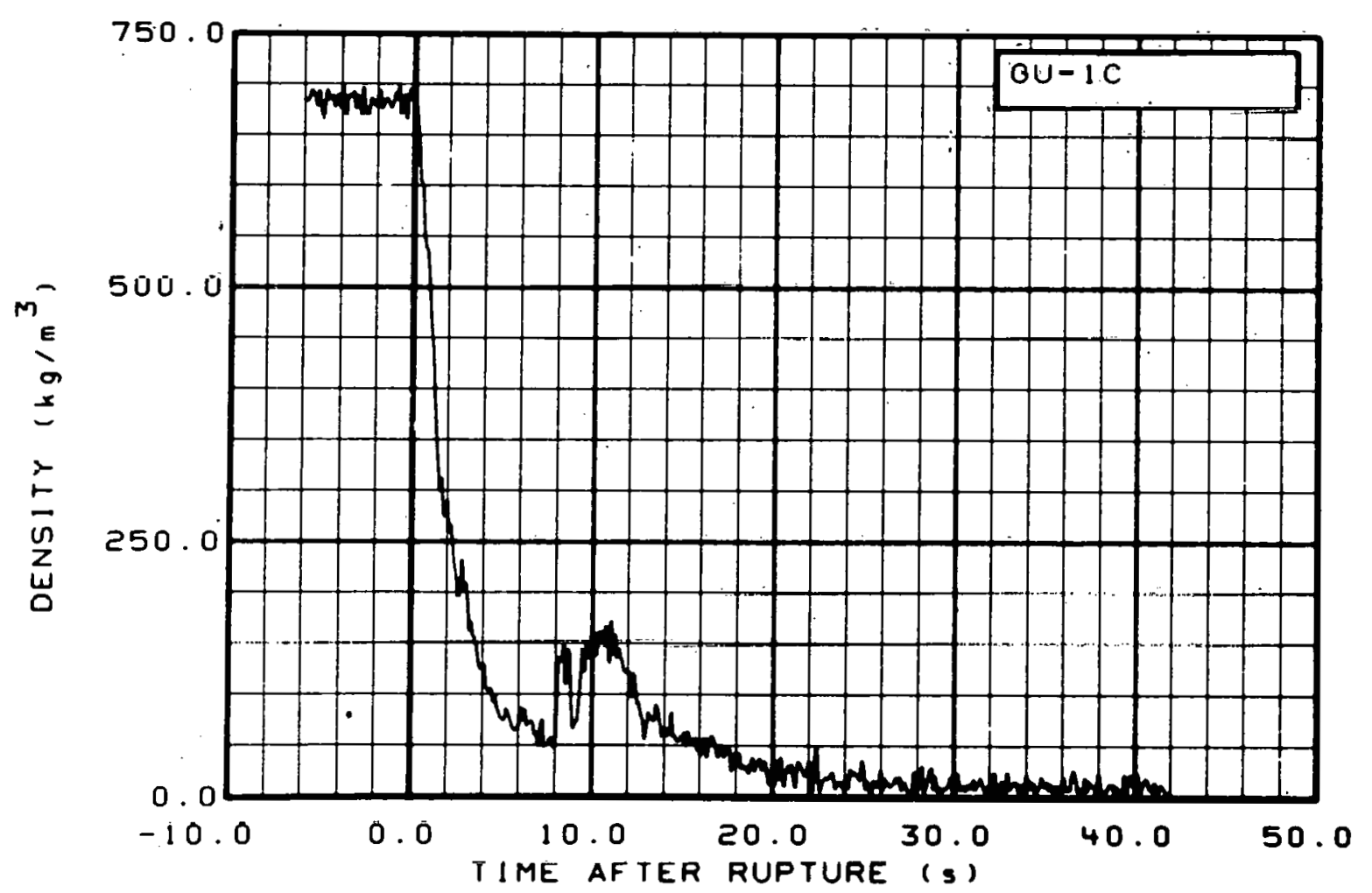

Fig. 281 Density in intact loop (GU-1C), from -6 to $42 \mathrm{~s}$. 


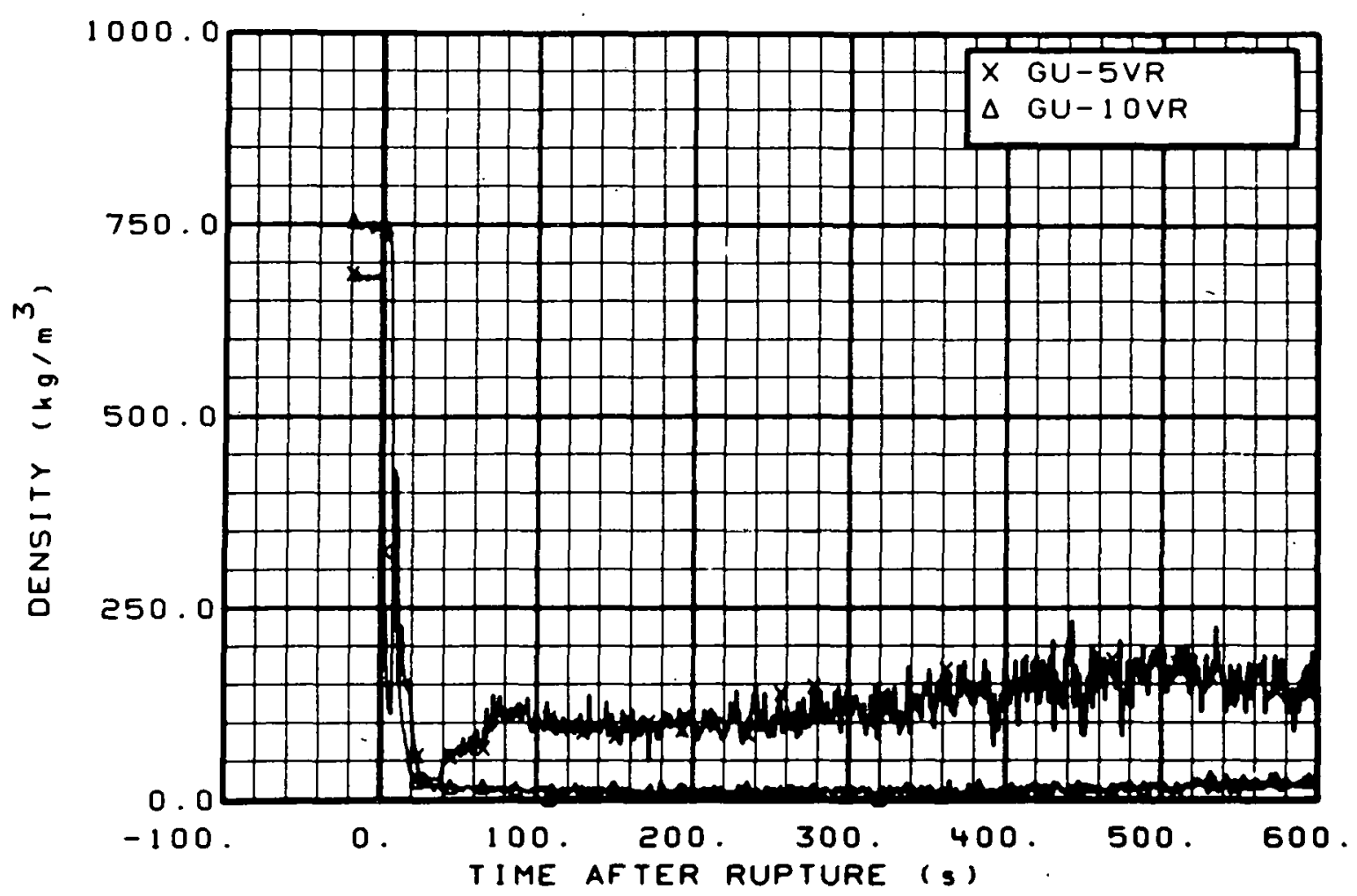

Fig. 282 Density in intact loop (GU-5VR and GU-10VR), from -20 to $600 \mathrm{~s}$.

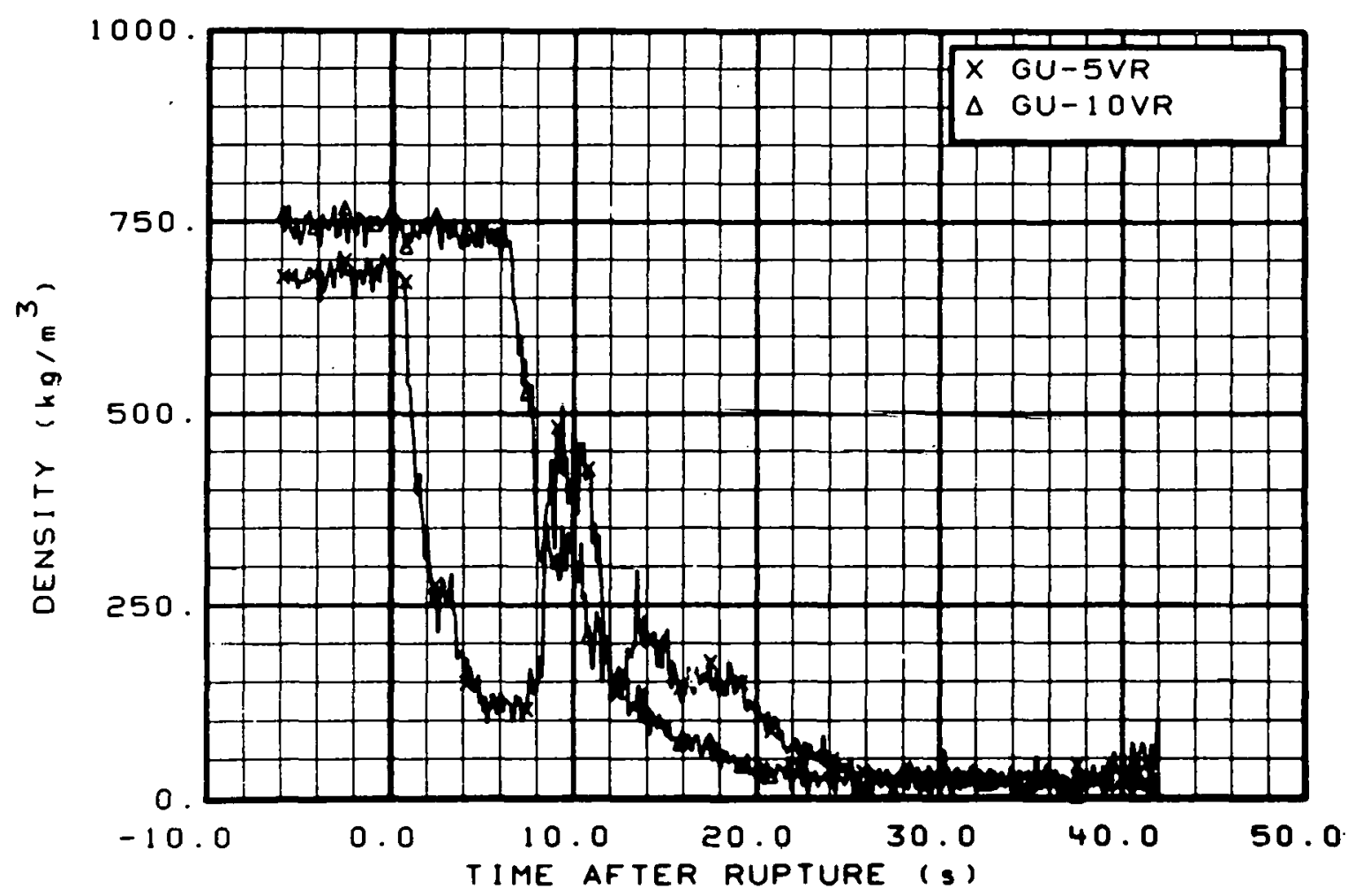

Fig. 283 Density in intact 10op (GU-5VR and GU-10VR), from -6 to $42 \mathrm{~s}$. 


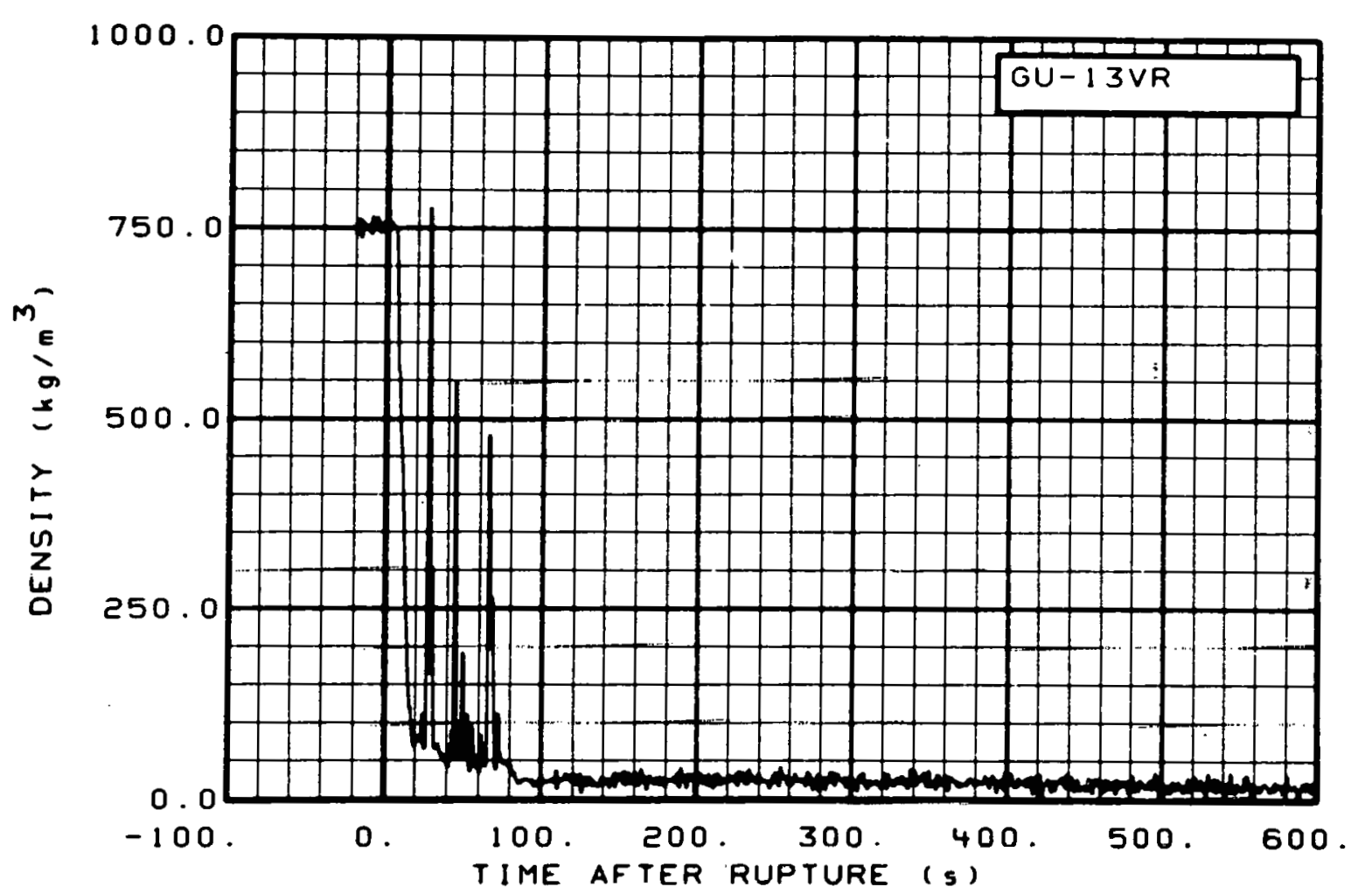

Fig. 284 Density in intact loop (GU-13VR), from -20 to $600 \mathrm{~s}$.

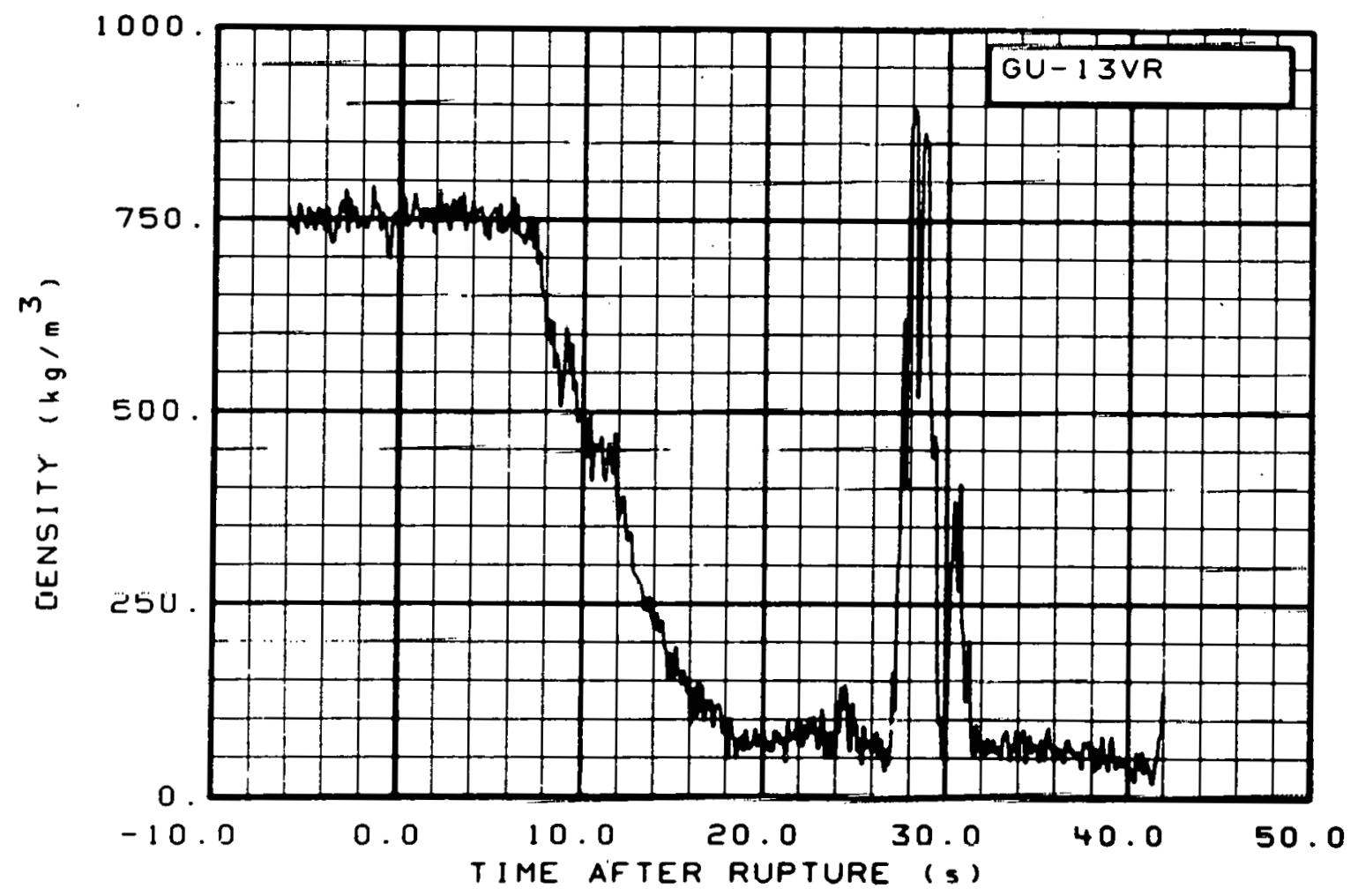

Fig. 285 Density in intact loop (GU-13VR), from -6 to $42 \mathrm{~s}$. 


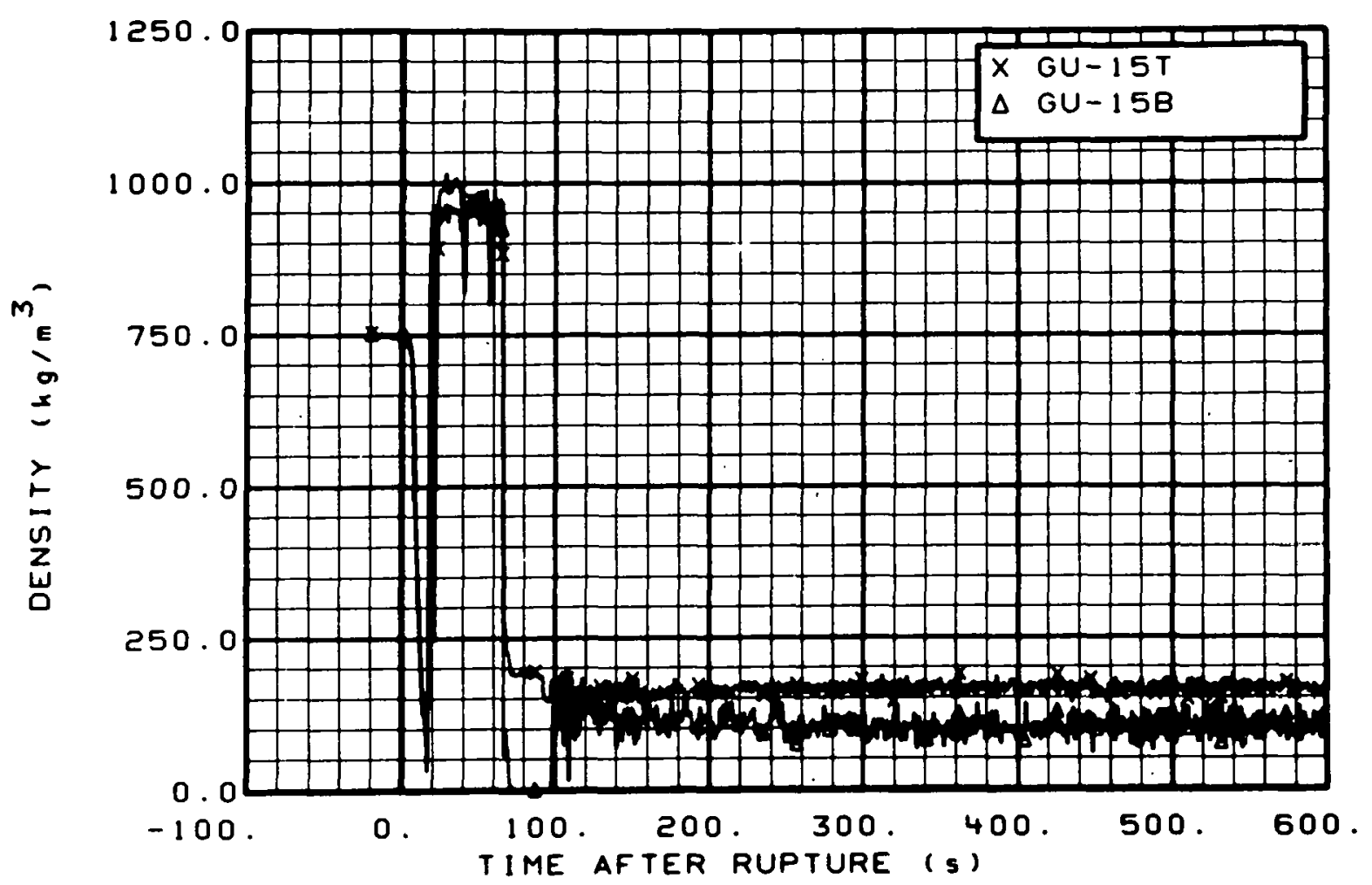

Fig. 286 Density in intact 10op (GU-15T and GU-15B), from -20 to $600 \mathrm{~s}$.

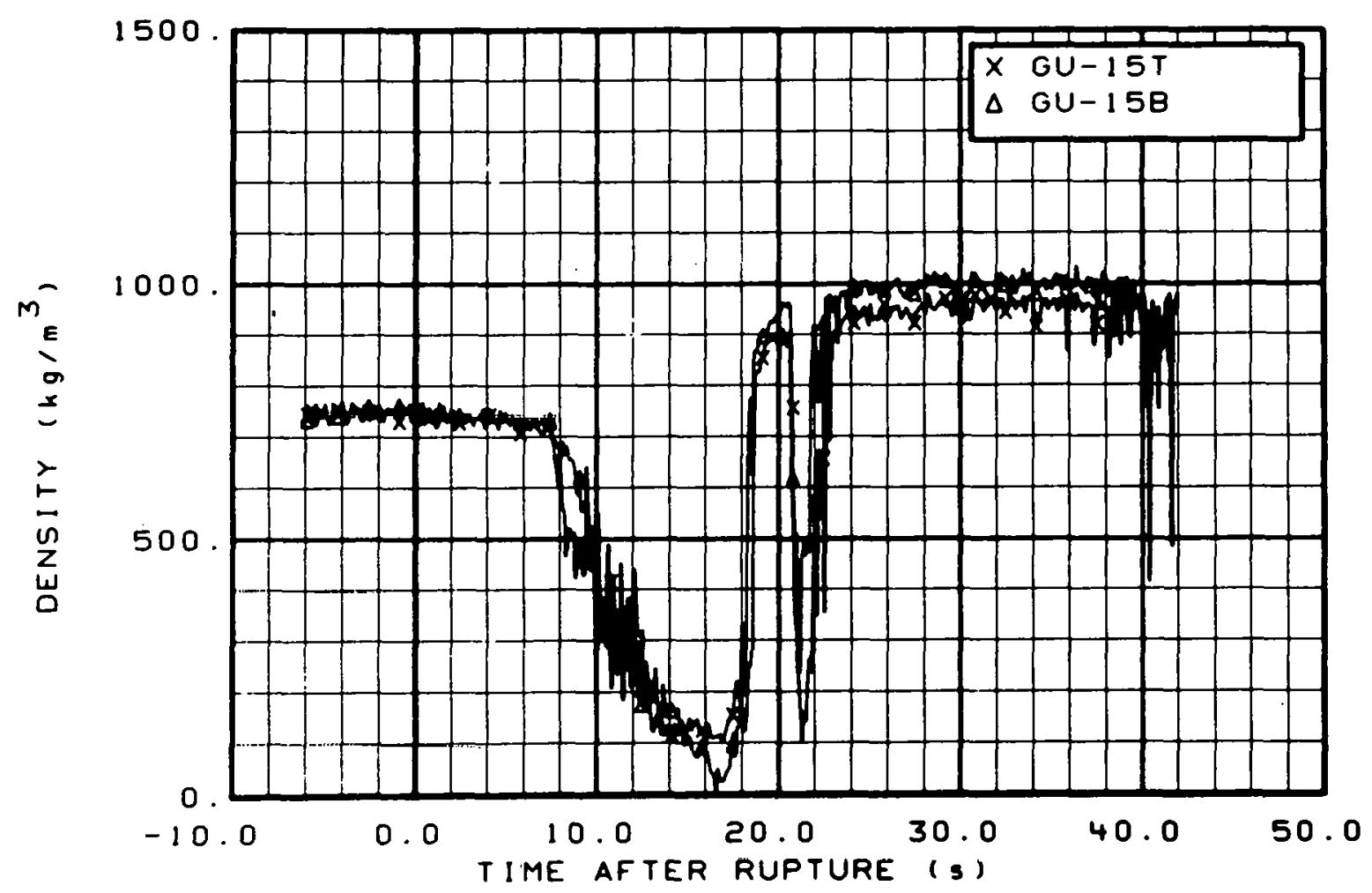

Fig. 287 Density in intact loop (GU-15T and GU-15B), from -6 to $42 \mathrm{~s}$. 


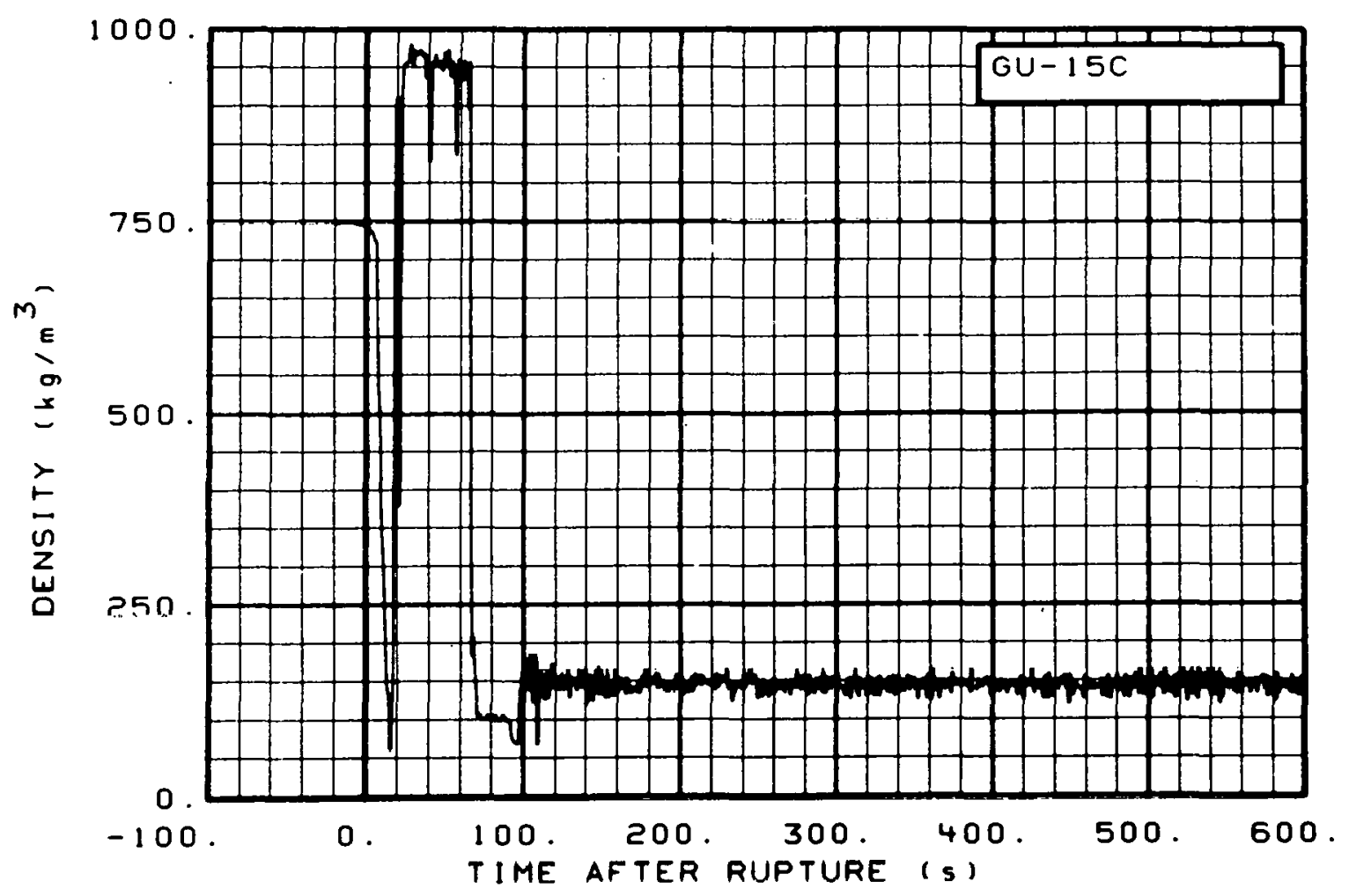

Fig. 288 Density in intact 100p (GU-15C), from -20 to $600 \mathrm{~s}$.

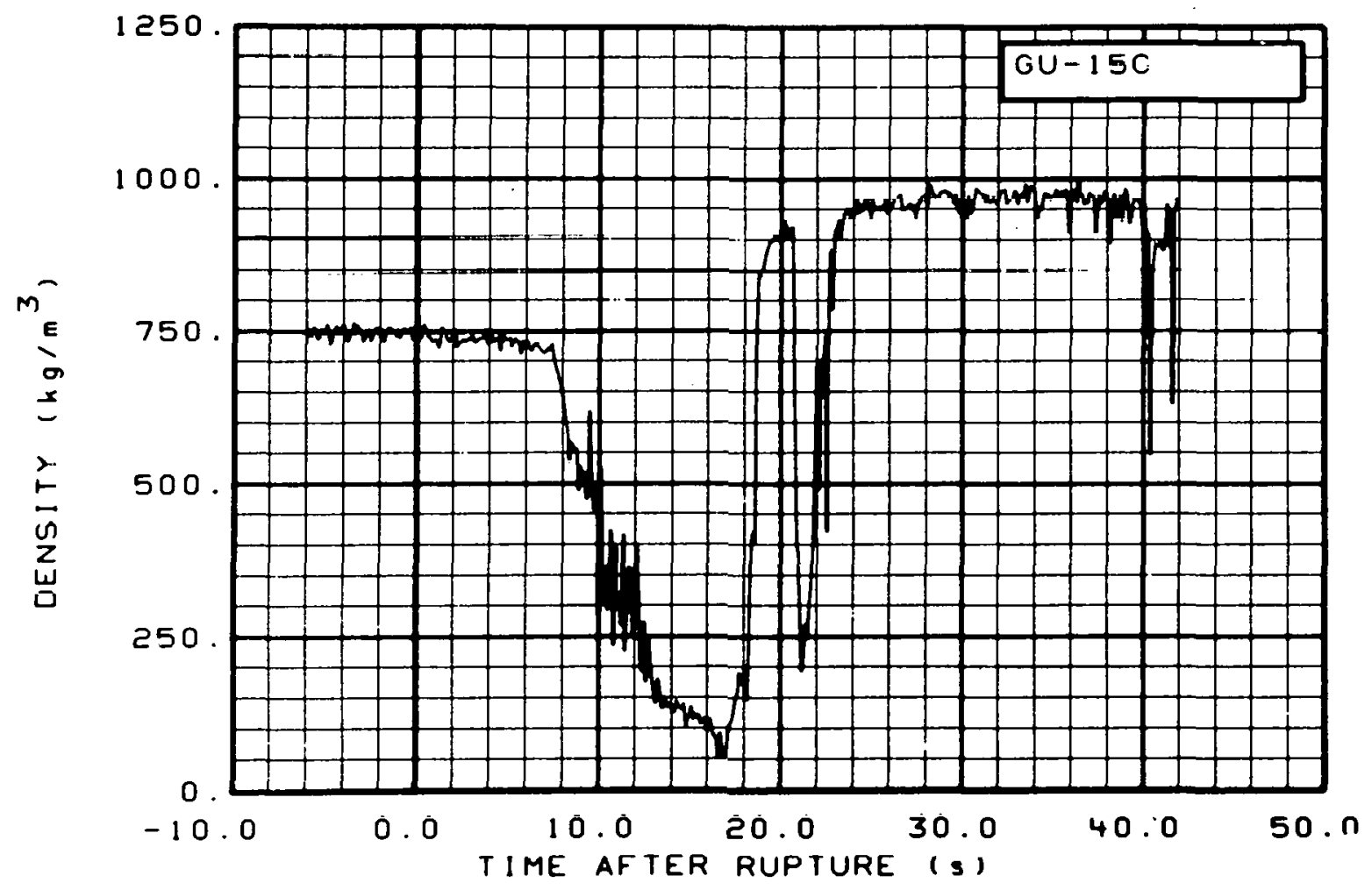

Fig. 289 Density in intact loop (GU-15C), from -6 to $42 \mathrm{~s}$. 


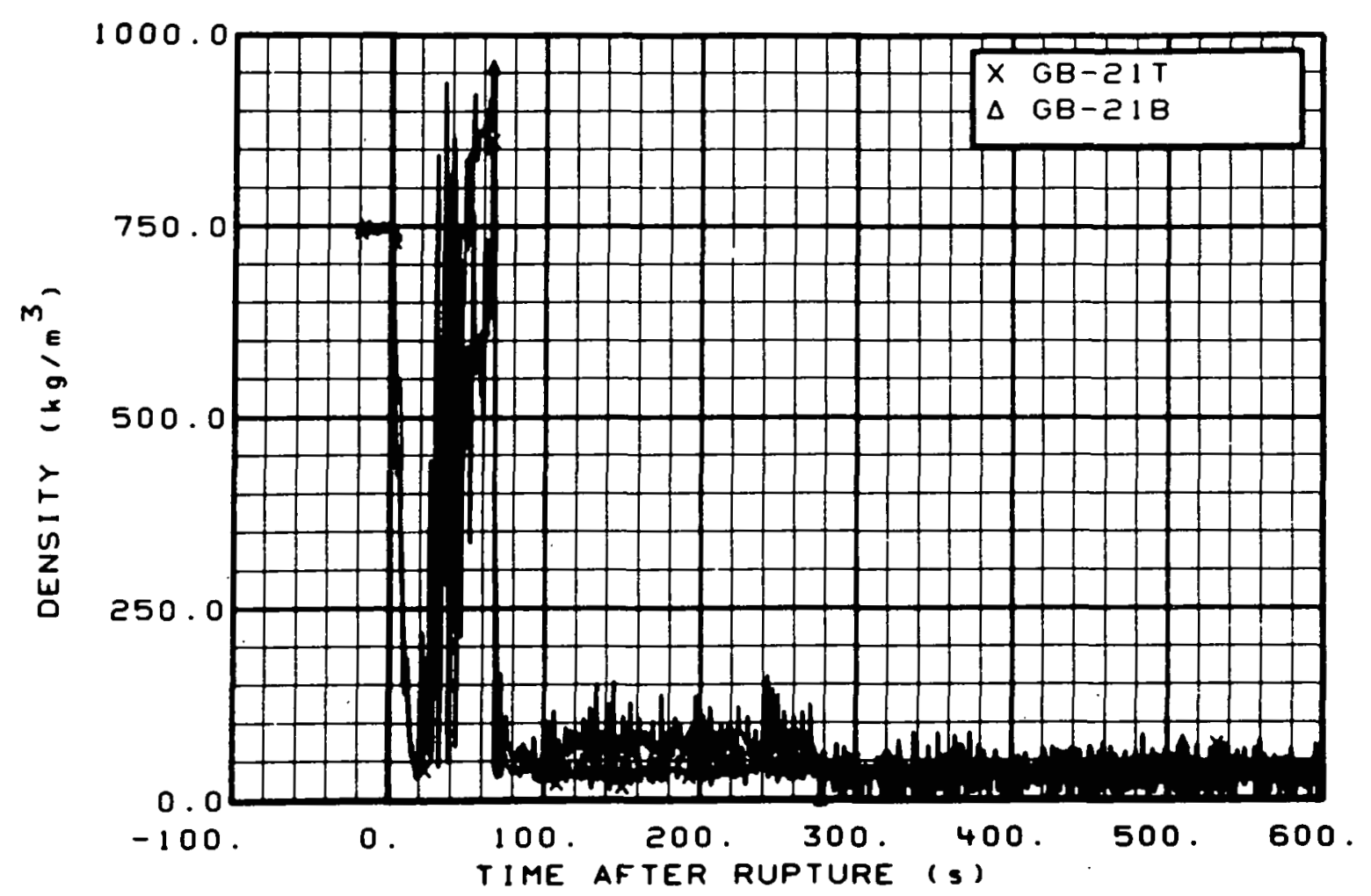

Fig. 290 Density in broken loop (GB-21T and GB-21B), from -20 to $600 \mathrm{~s}$.

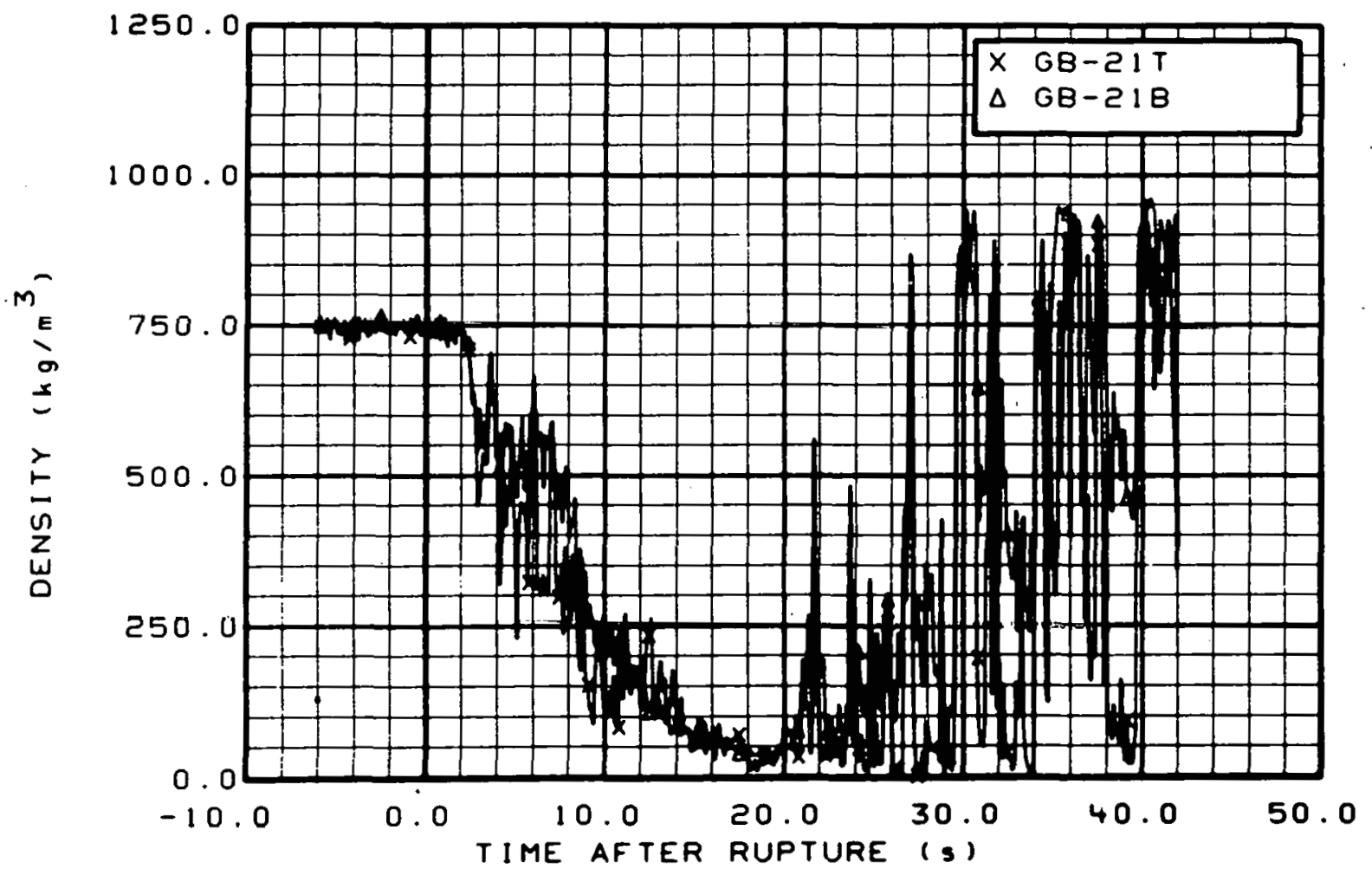

Fig. 291 Density in broken loop (GB-2IT and GB-21B), from -6 to $42 \mathrm{~s}$. 


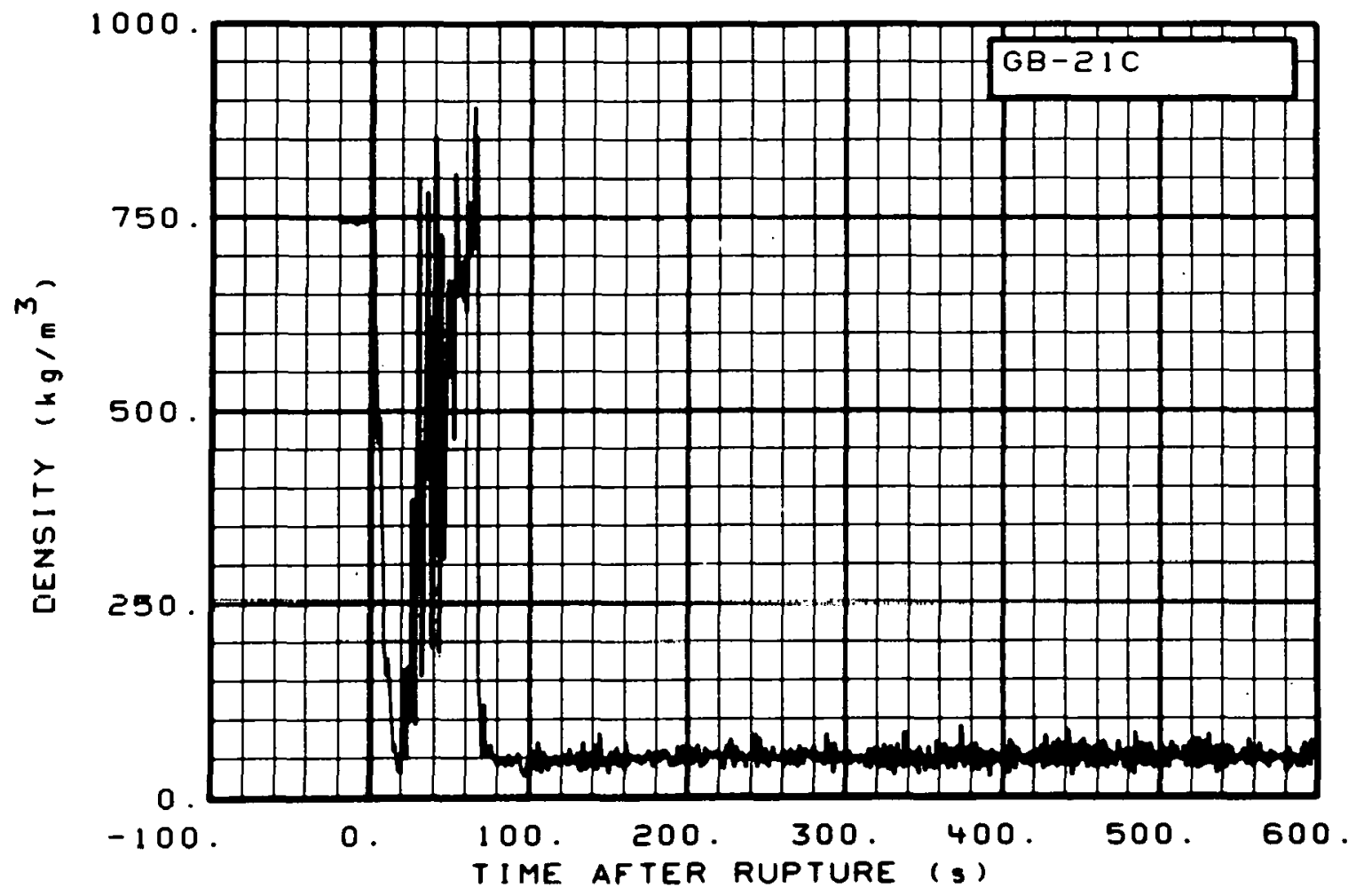

Fig. 292 Density in broken loop (GB-2lC), from -20 to $600 \mathrm{~s}$.

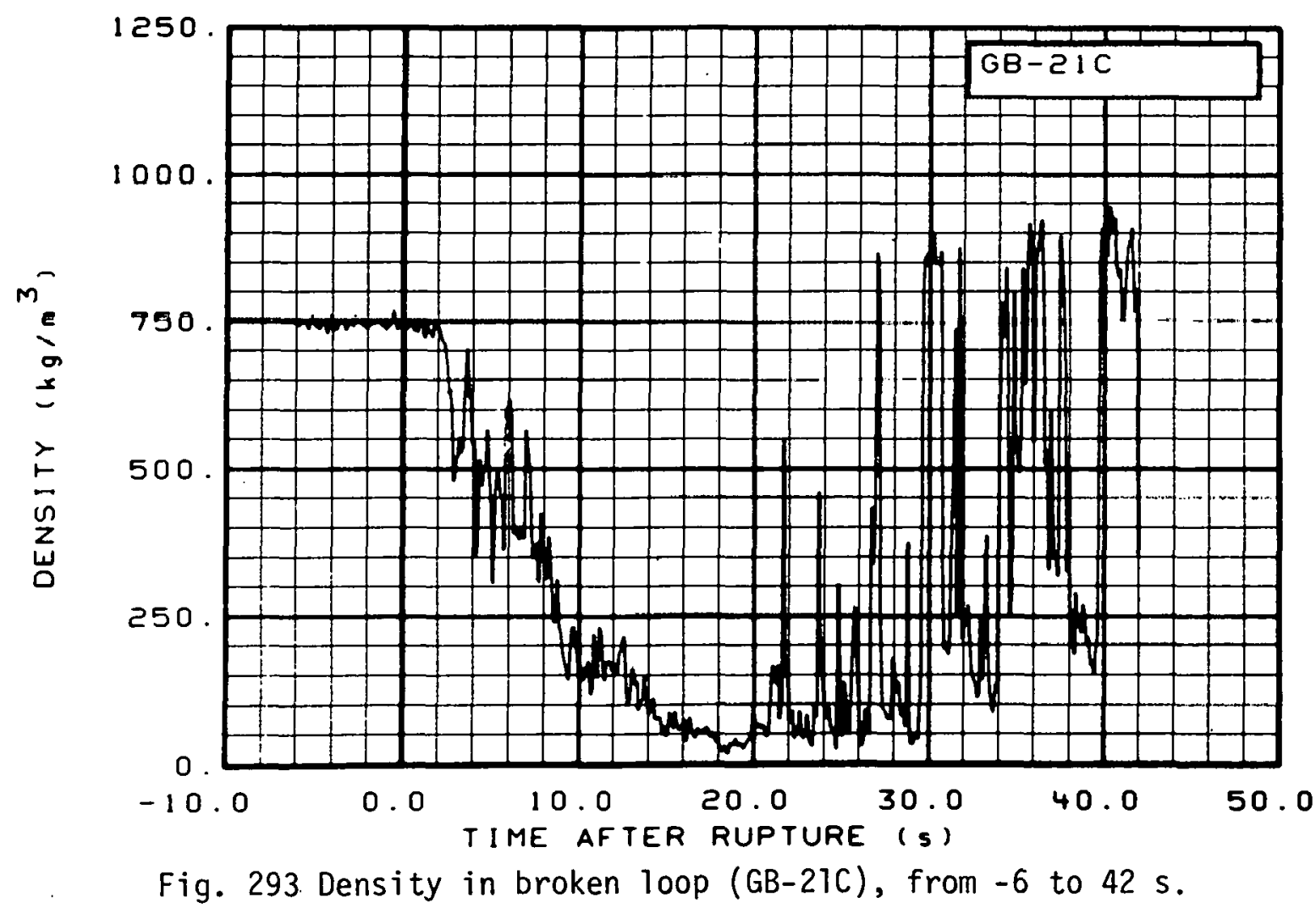




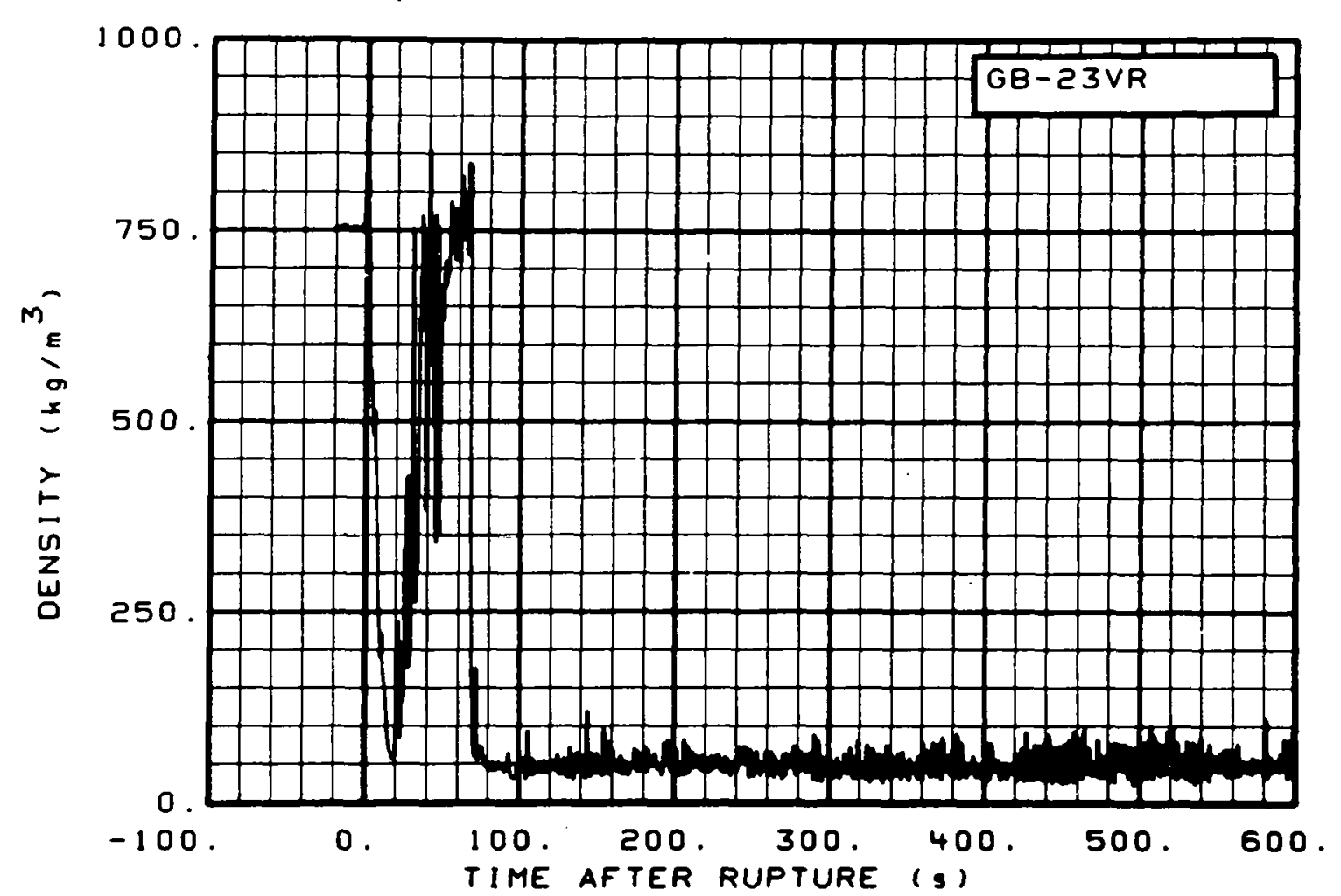

Fig. 294 Density in broken 100p (GB-23VR), from -20 to $600 \mathrm{~s}$.

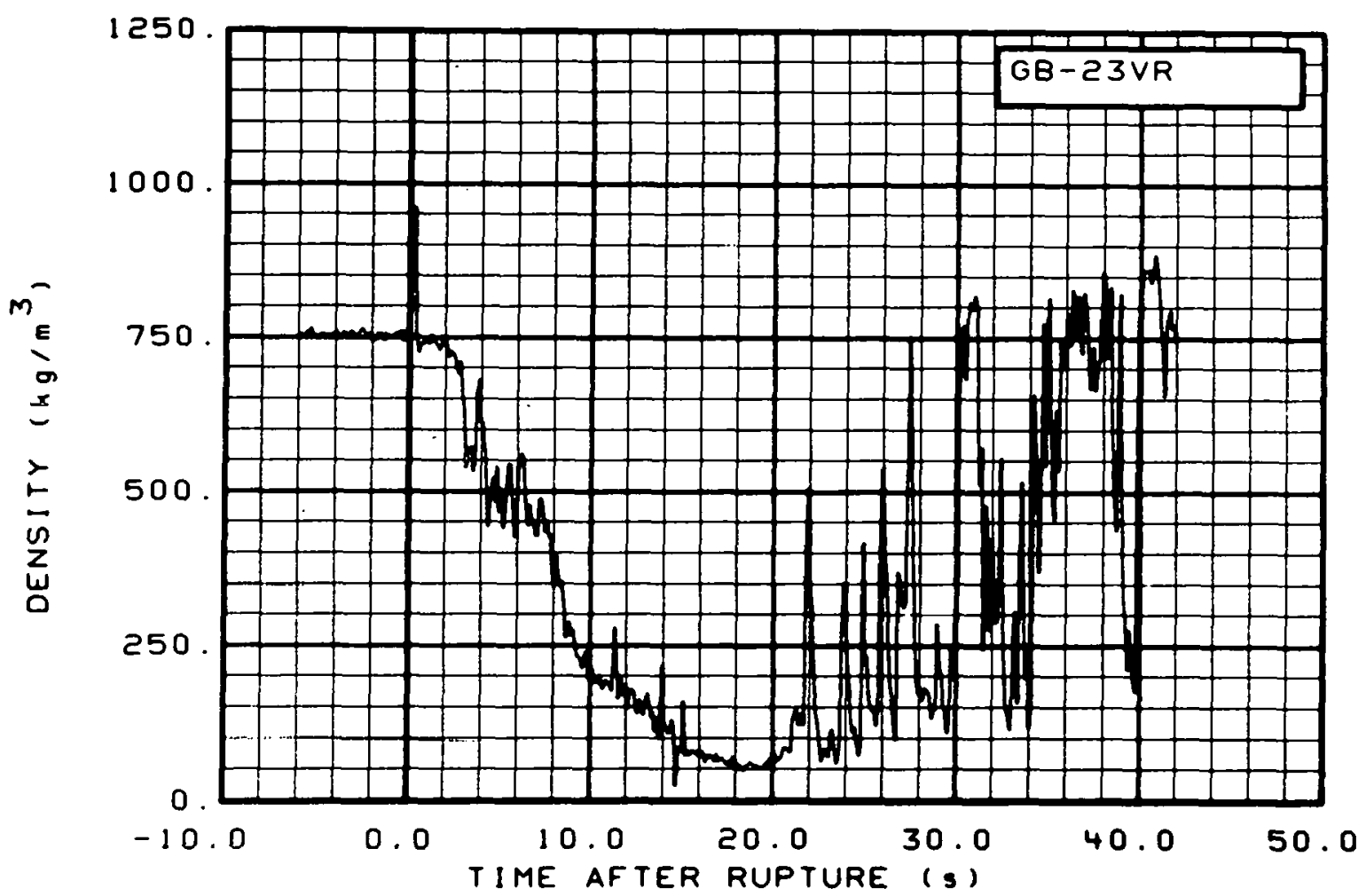

Fig. 295 Density in broken loop (GB-23VR), from -6 to $42 \mathrm{~s}$. 


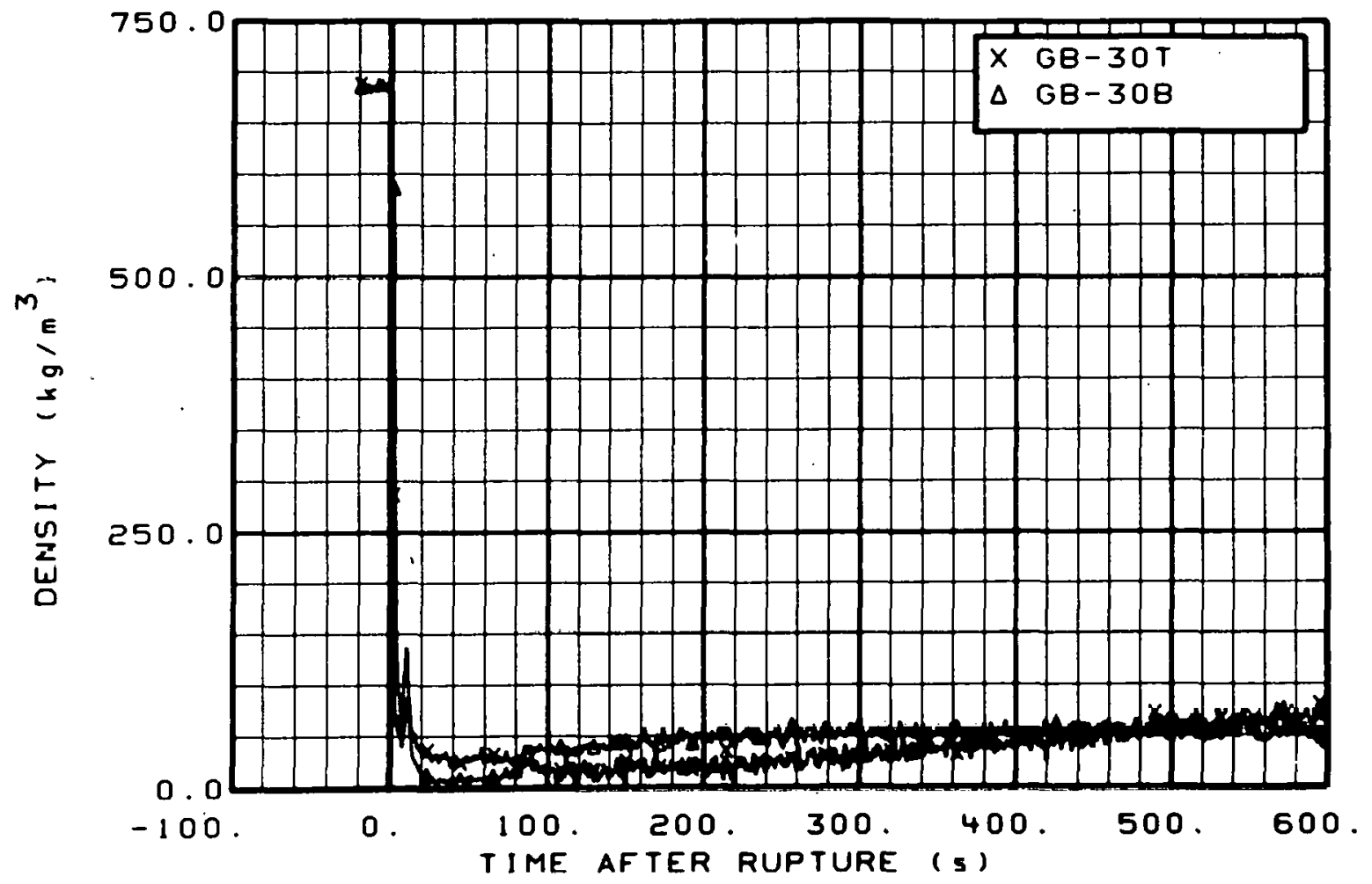

Fig. 296 Density in broken loop (GB-30T and GB-30B), from -20 to $600 \mathrm{~s}$.

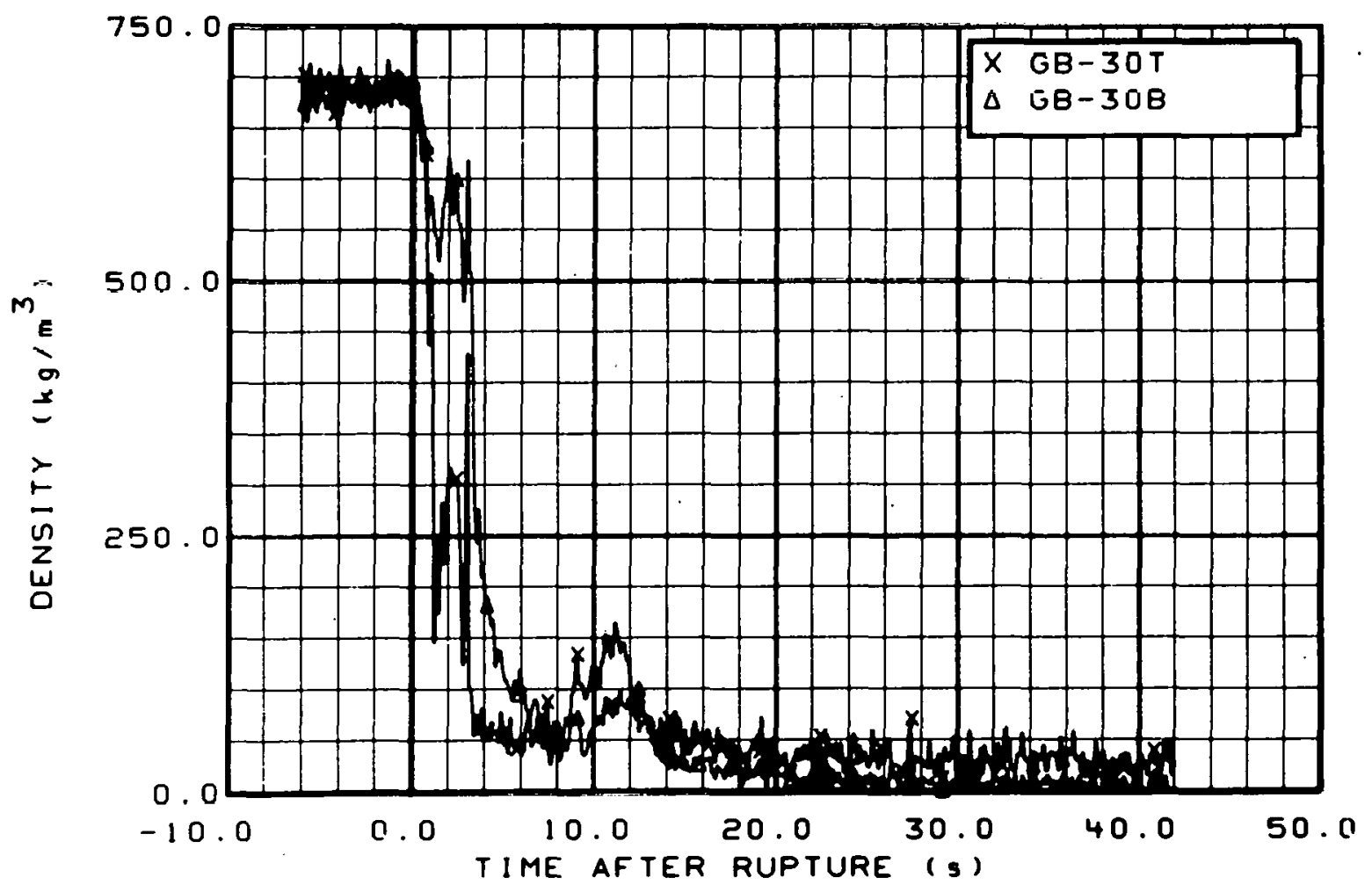

Fig. 297 Density in broken loop (GB-30T and GB-30B), from -6 to $42 \mathrm{~s}$. 


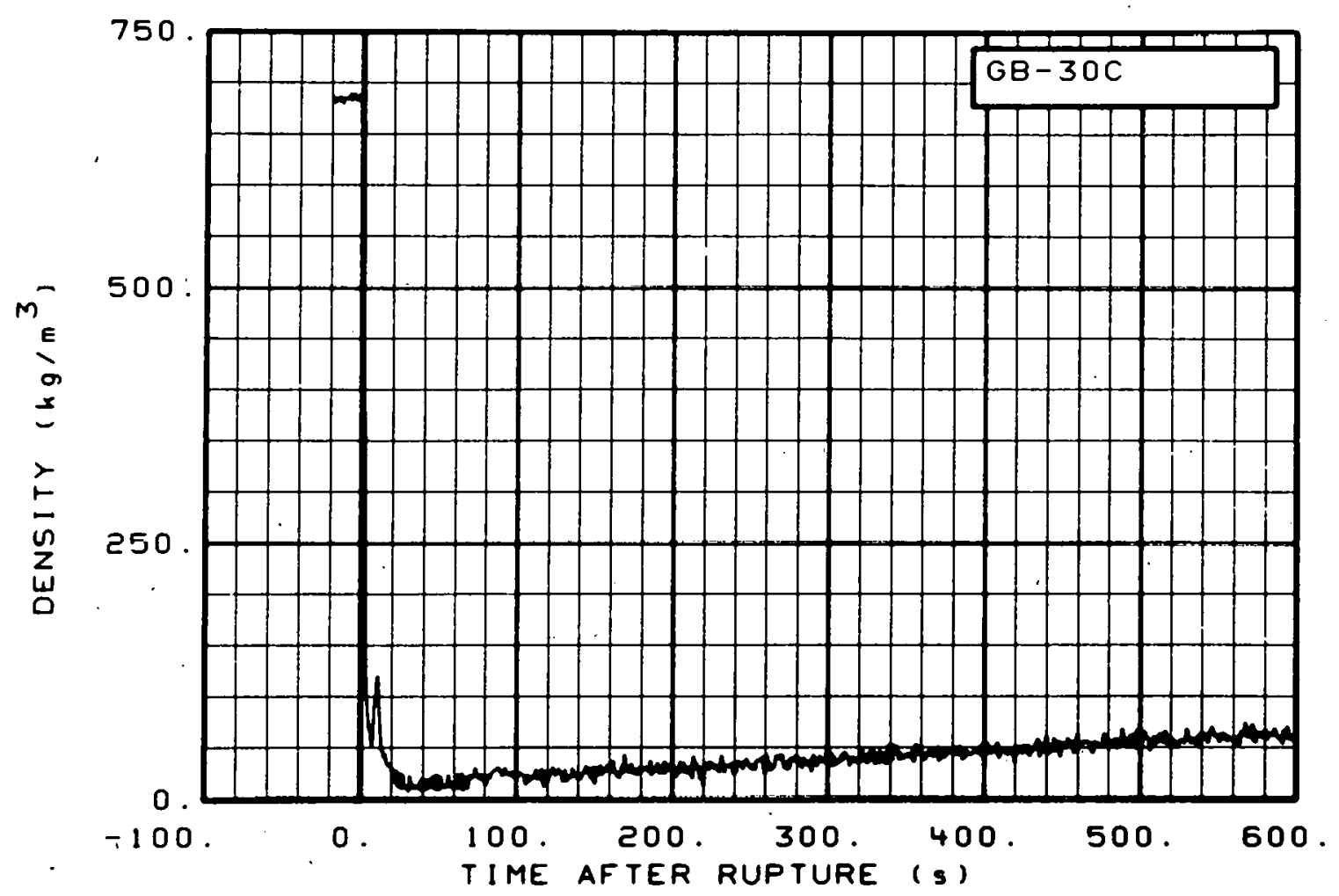

Fig. 298 Density in broken loop (GB-30C), from -20 to $600 \mathrm{~s}$.

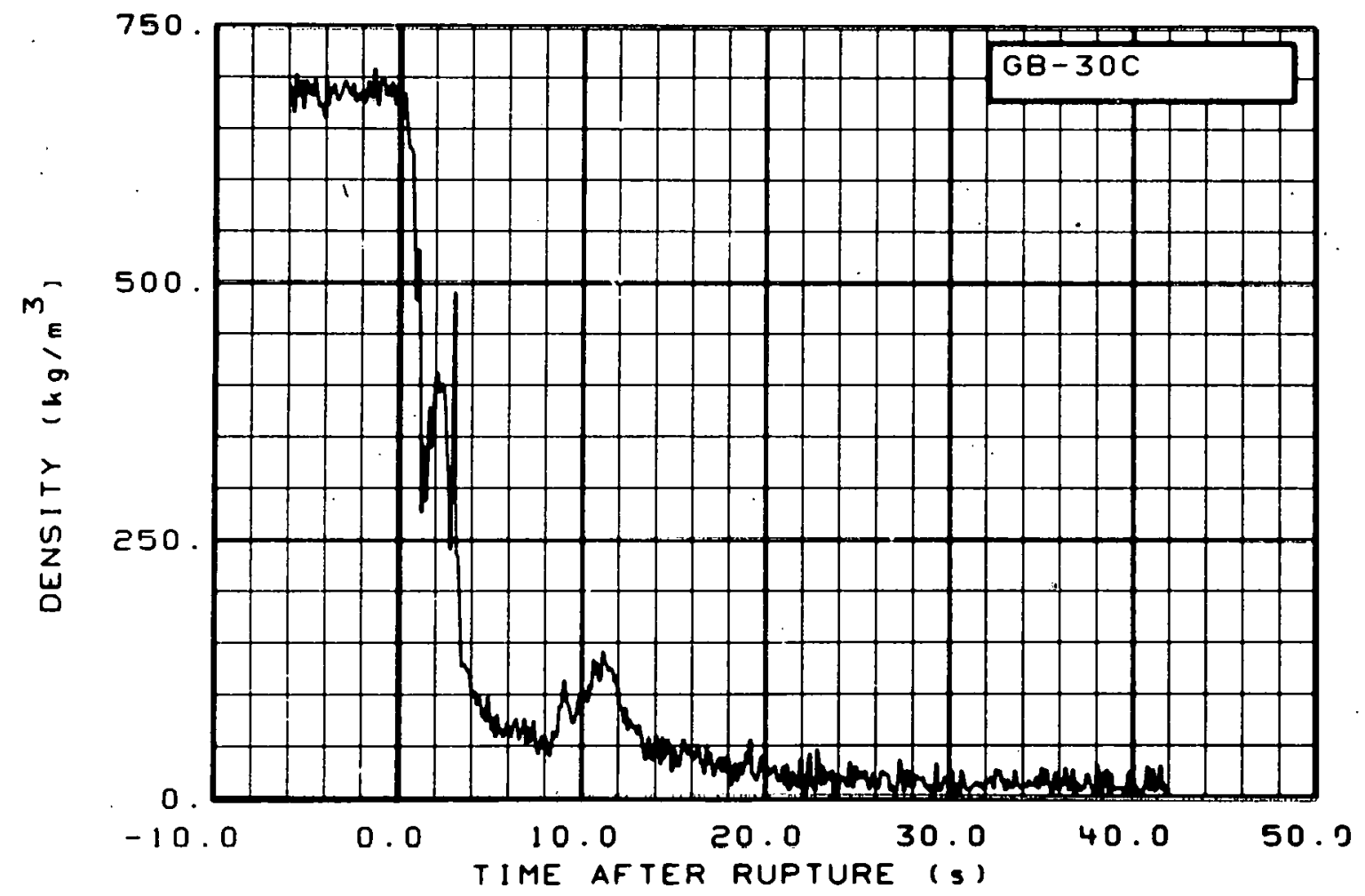

Fig. 299 Density in broken loop (GB-30C), from -6 to $42 \mathrm{~s}$. 


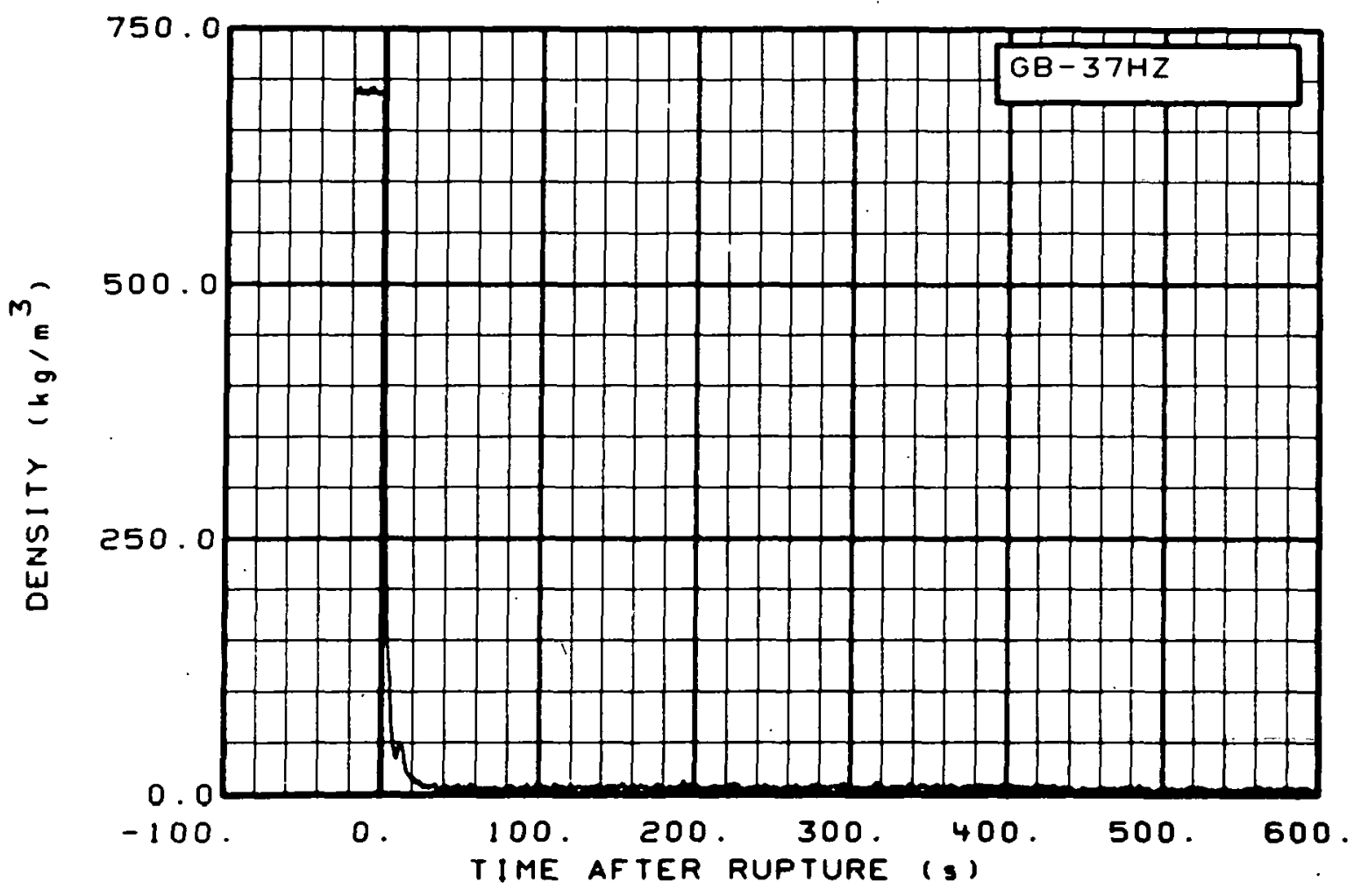

Fig. 300 Density in broken loop (GB-37HZ), from -20 to $600 \mathrm{~s}$.

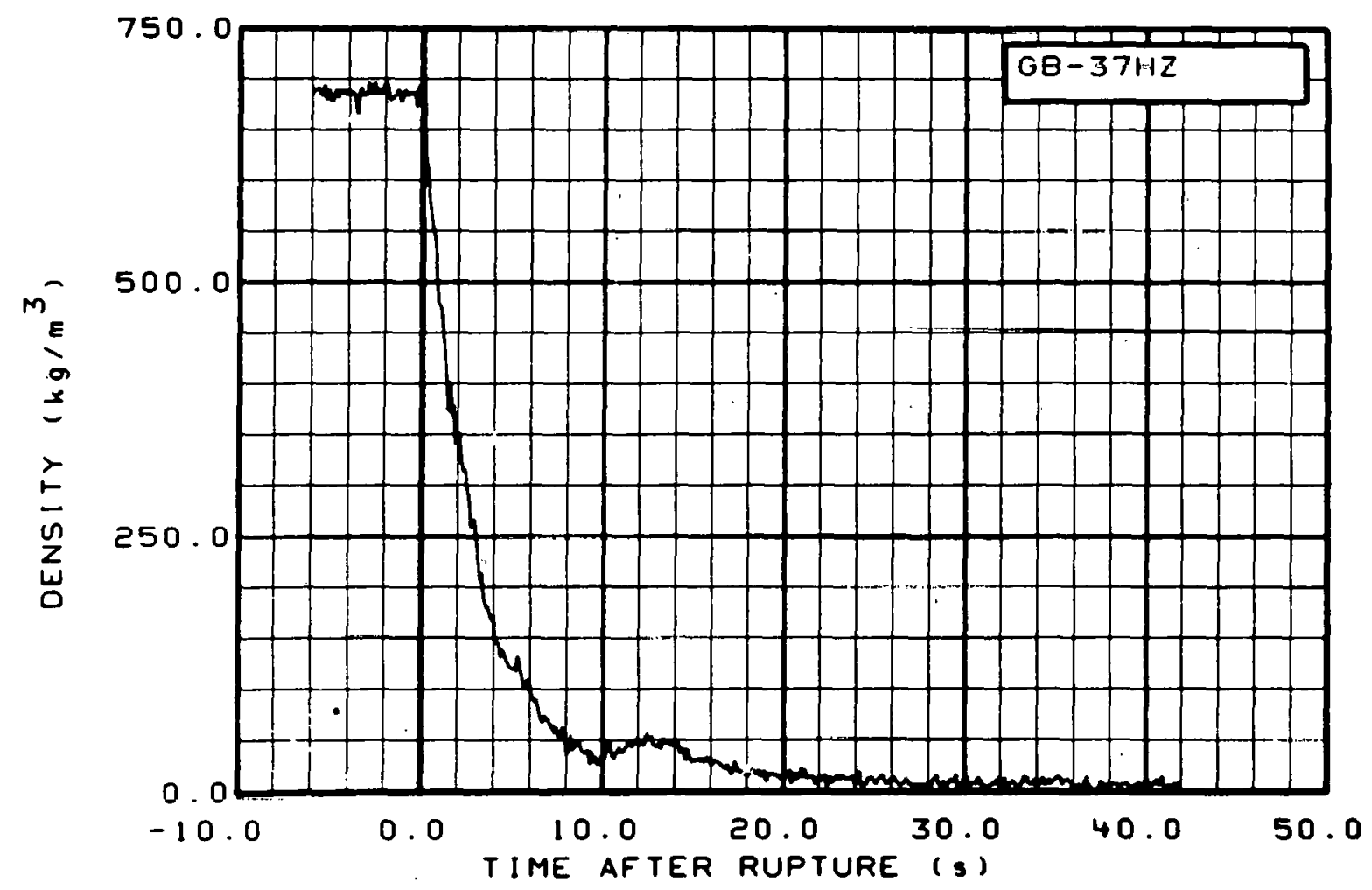

Fig. 301 Density in broken 100p (GB-37HZ), from -6 to $42 \mathrm{~s}$. 


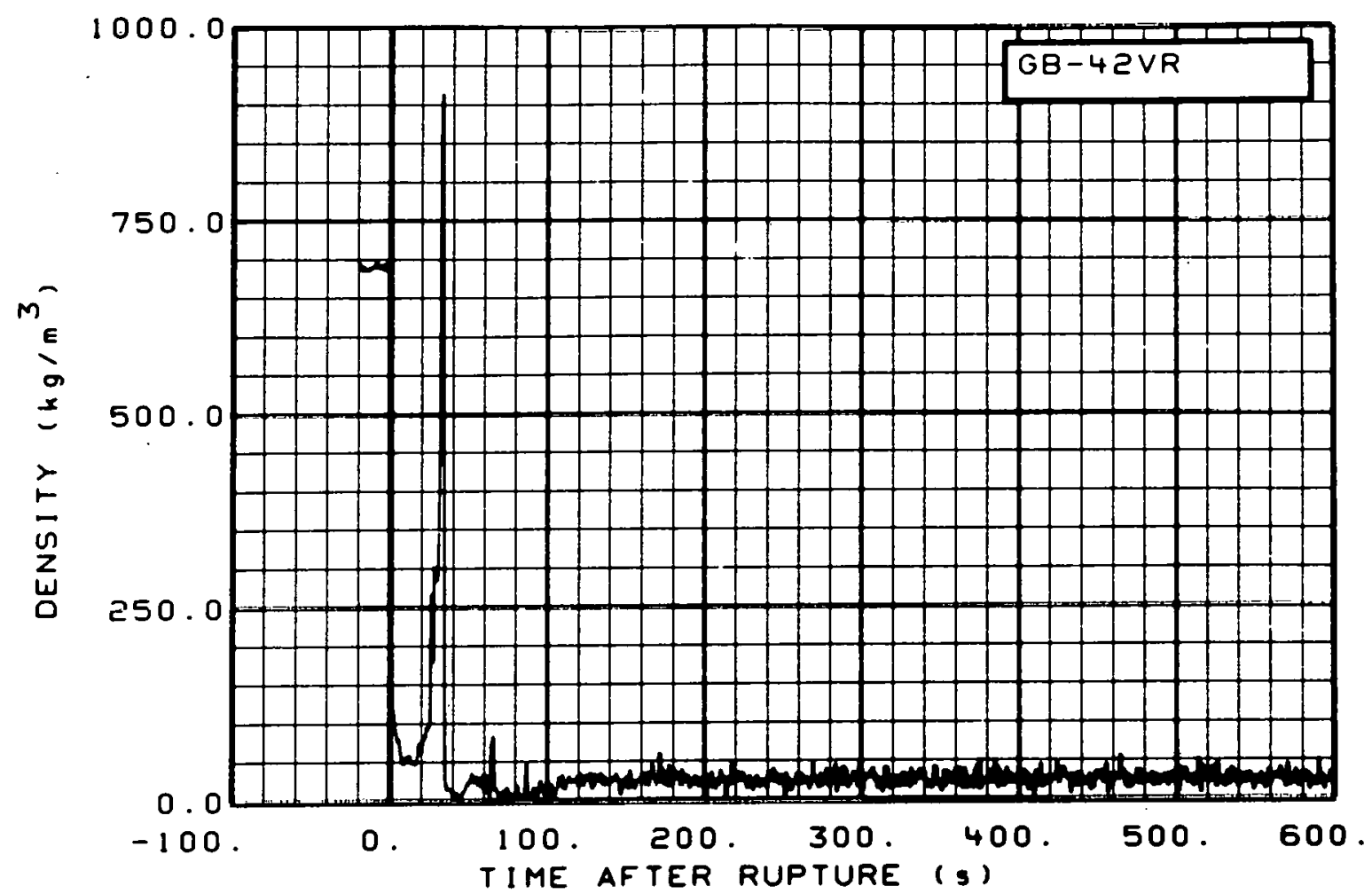

Fig. 302 Density in broken loop (GB-42VR), from -20 to $600 \mathrm{~s}$.

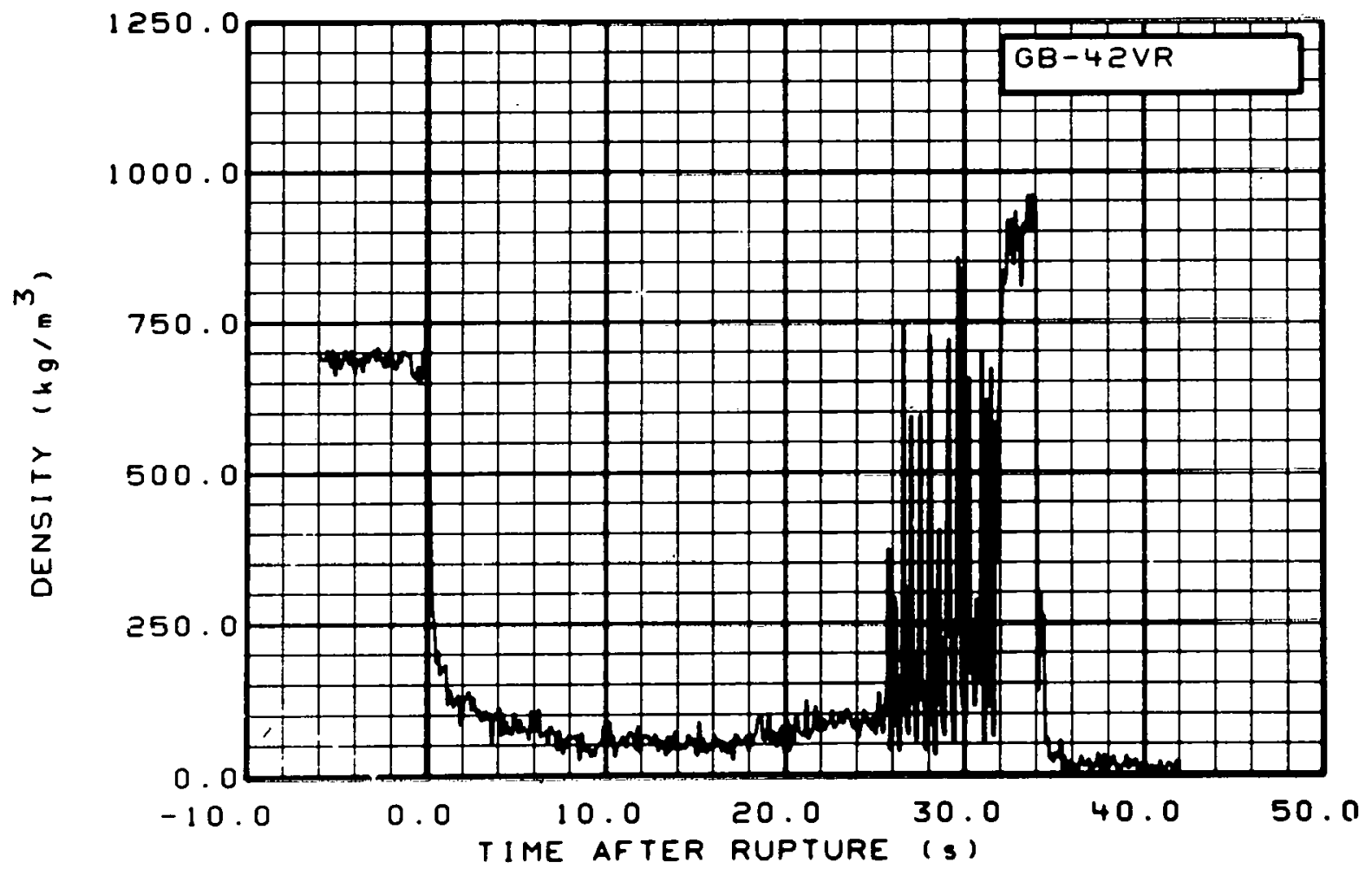

Fig. 303 Density in broken loop (GB-42VR), from -6 to $42 \mathrm{~s}$. 


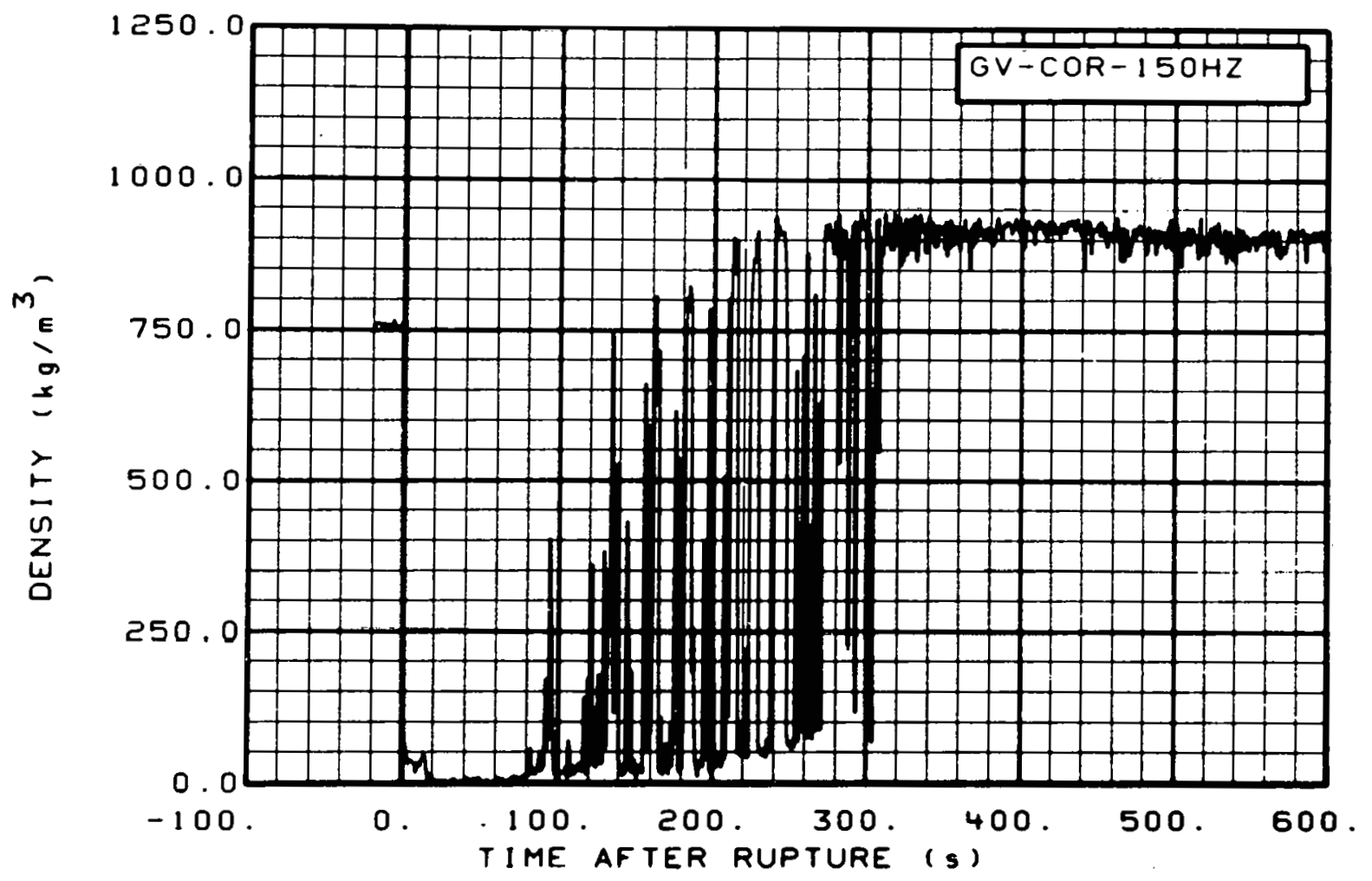

Fig. 304 Density in vessel (GV-COR-150HZ), from -20 to $600 \mathrm{~s}$.

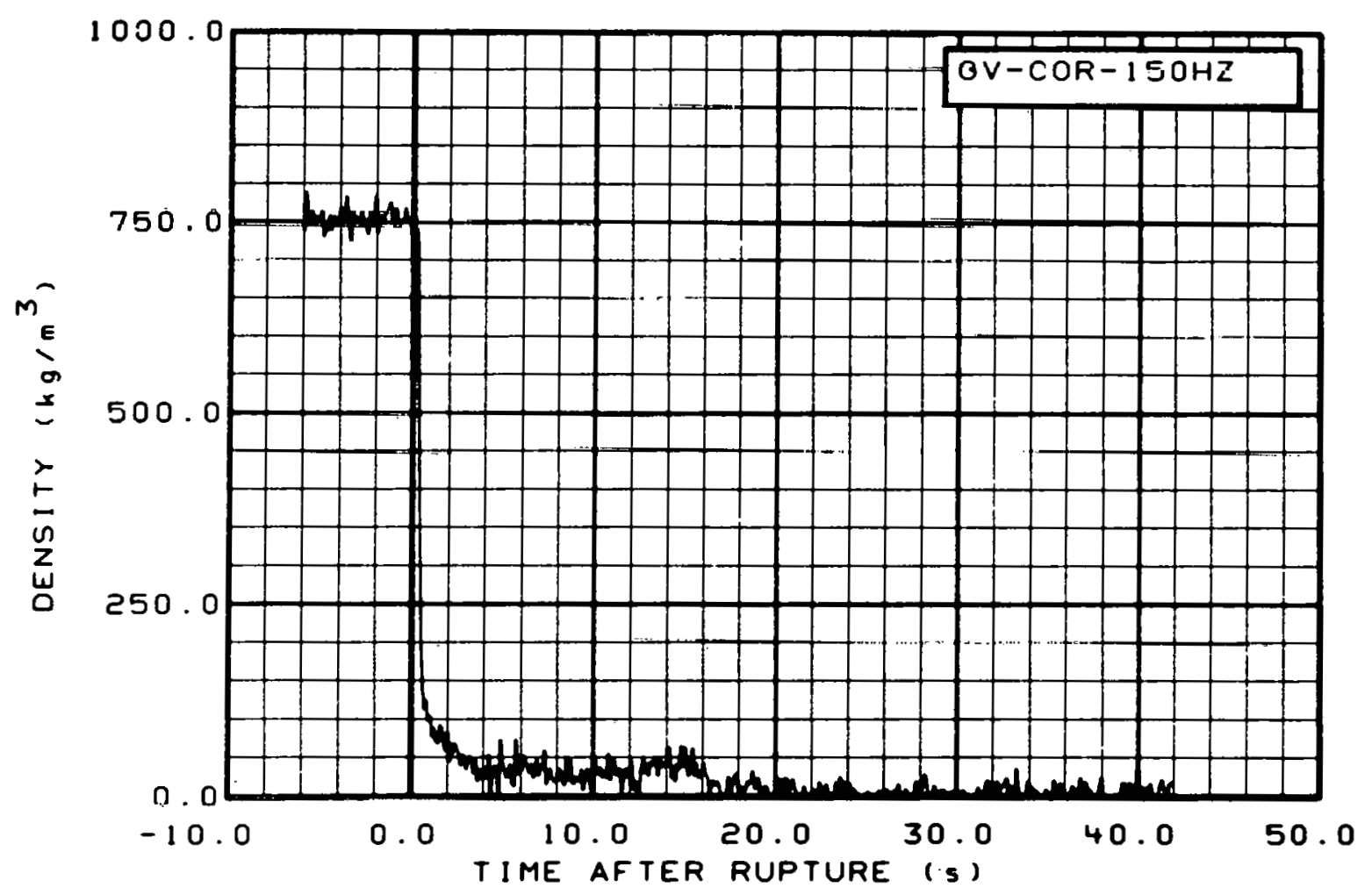

Fig. 305 Density in vessel (GV-COR-150HZ), from -6 to $42 \mathrm{~s}$. 


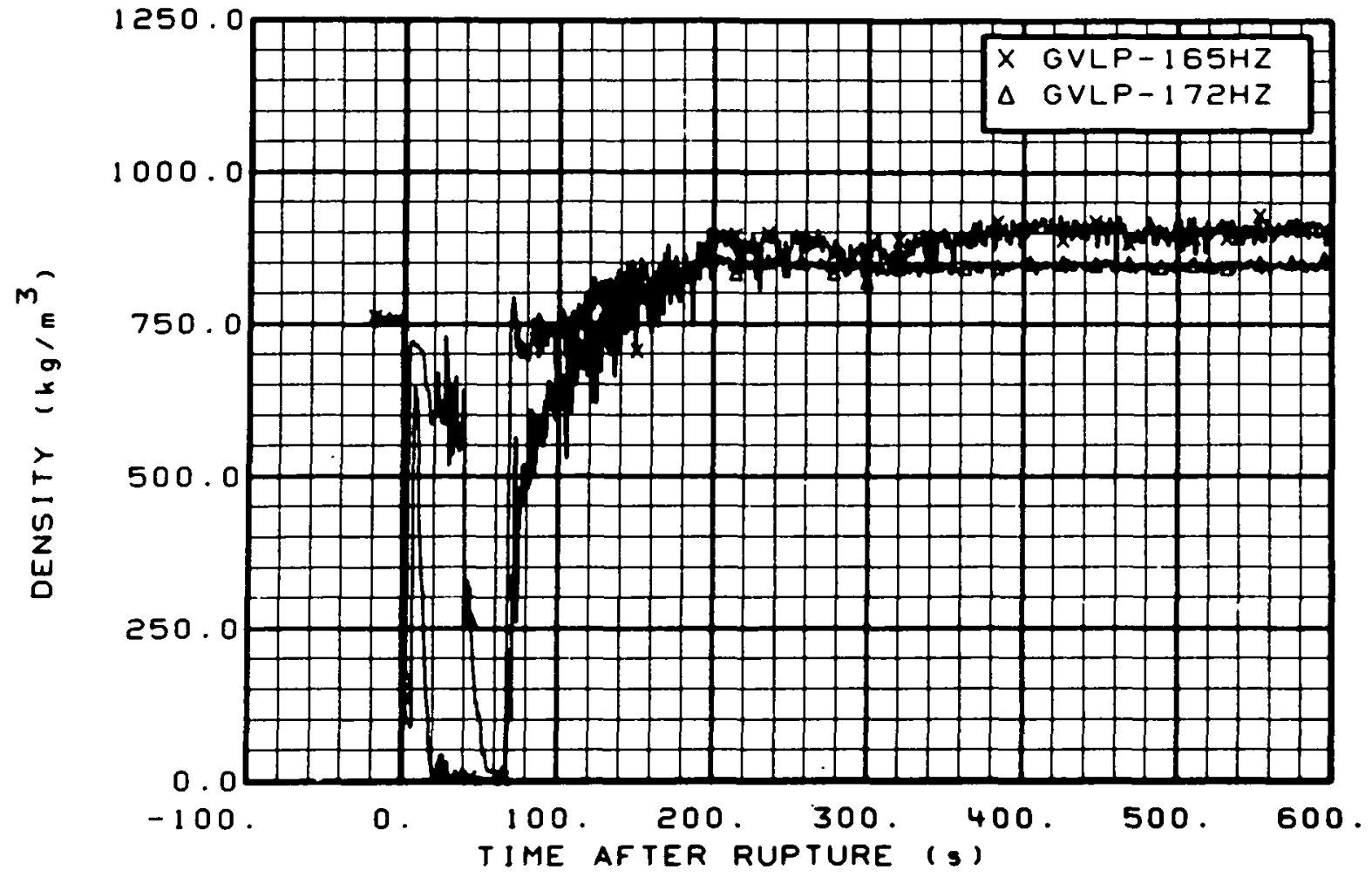

Fig. 306 Density in vessel (GVLP-165HZ and GVLP-172HZ), from -20 to $600 \mathrm{~s}$.

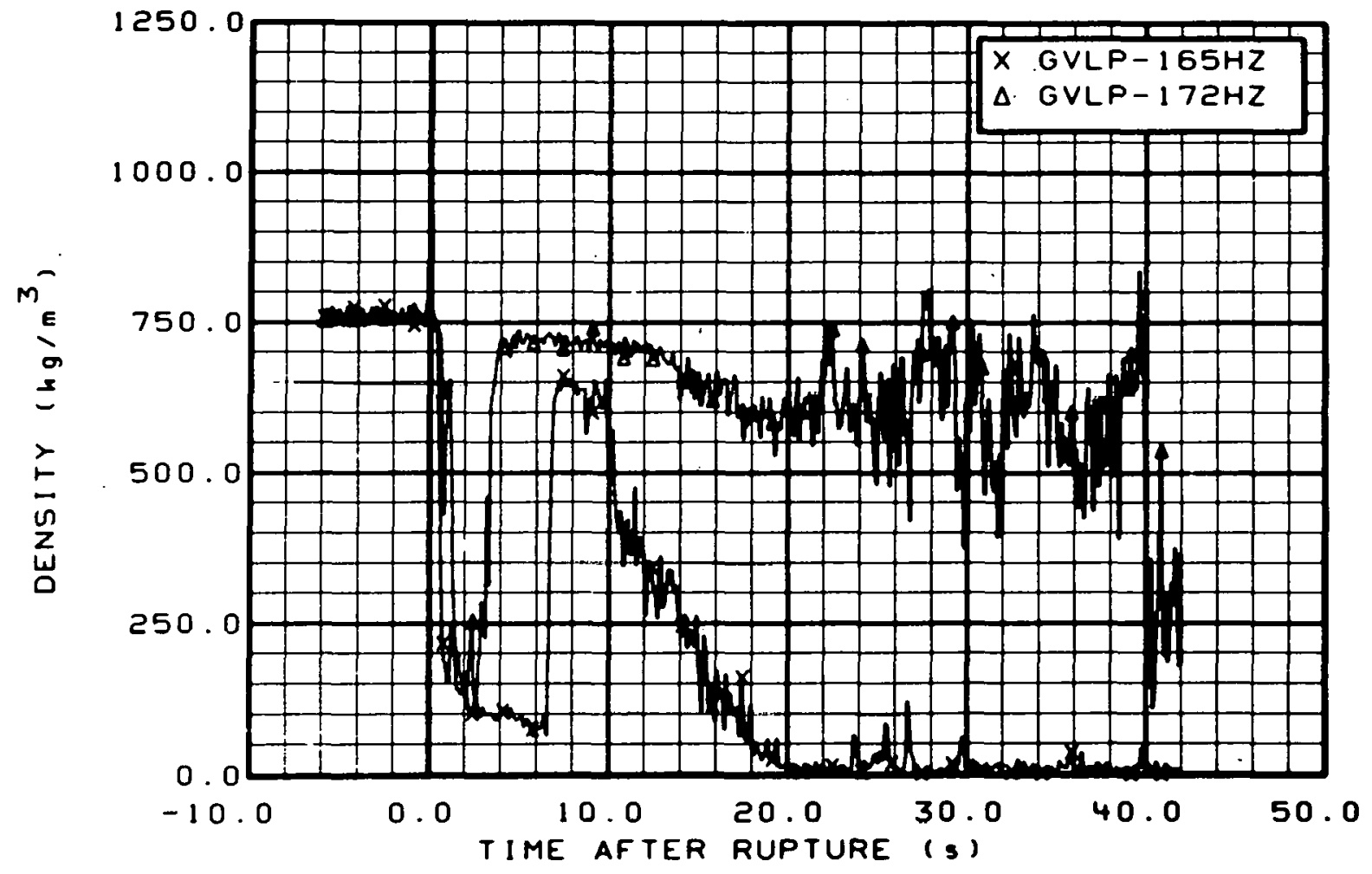

Fig. 307 Density in vessel (GVLP-165HZ and GVLP-172HZ), from -6 to $42 \mathrm{~s}$. 


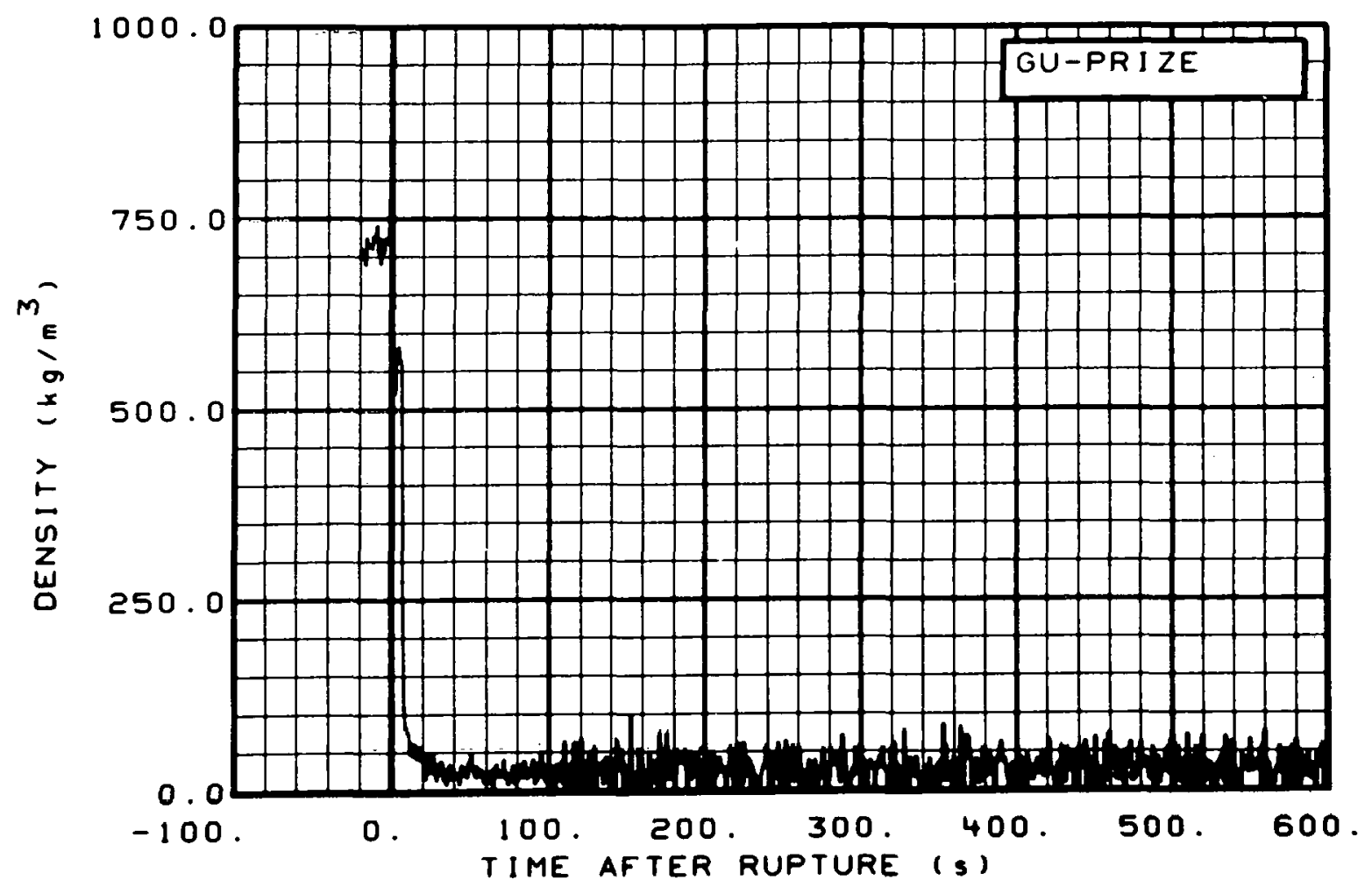

Fig. 308 Density in pressurizer (GU-PRIZE), from -20 to $600 \mathrm{~s}$.

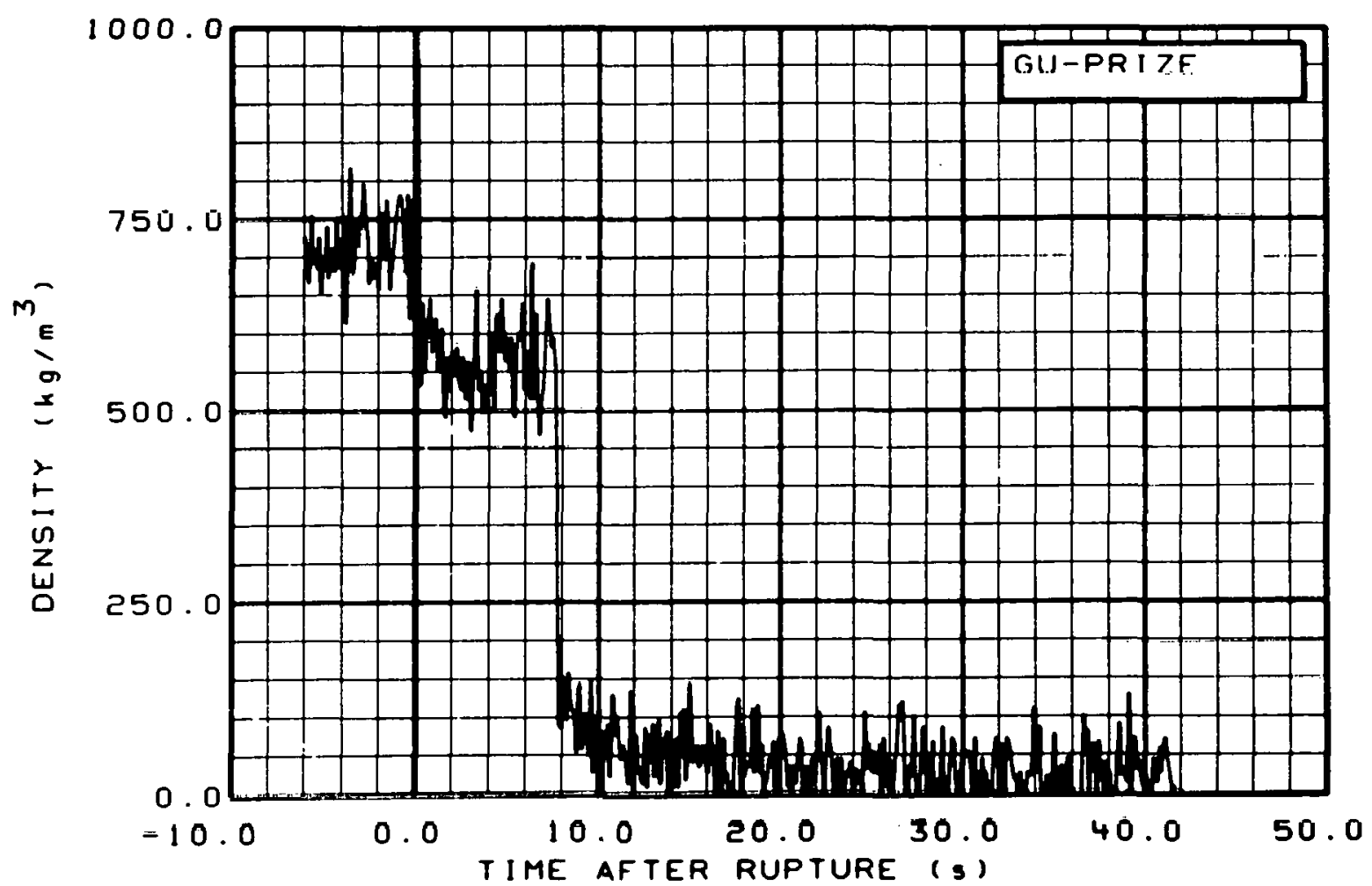

Fig. 309 Density in pressurizer (GU-PRIZE), from -6 to $42 \mathrm{~s}$. 


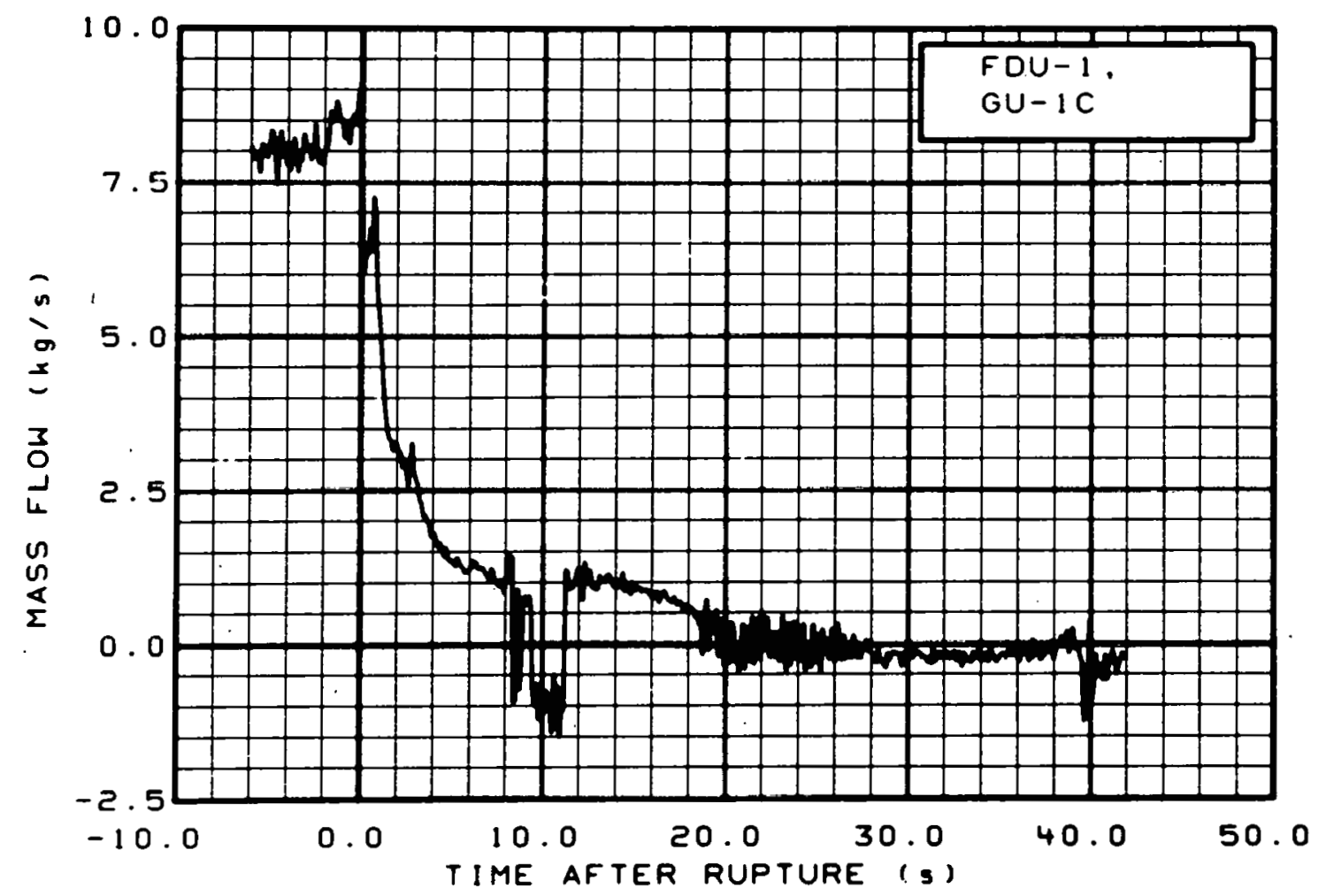

Fig. 310 Mass flow in intact loop (FDU-1, GU-1C), from -6 to $42 \mathrm{~s}$.

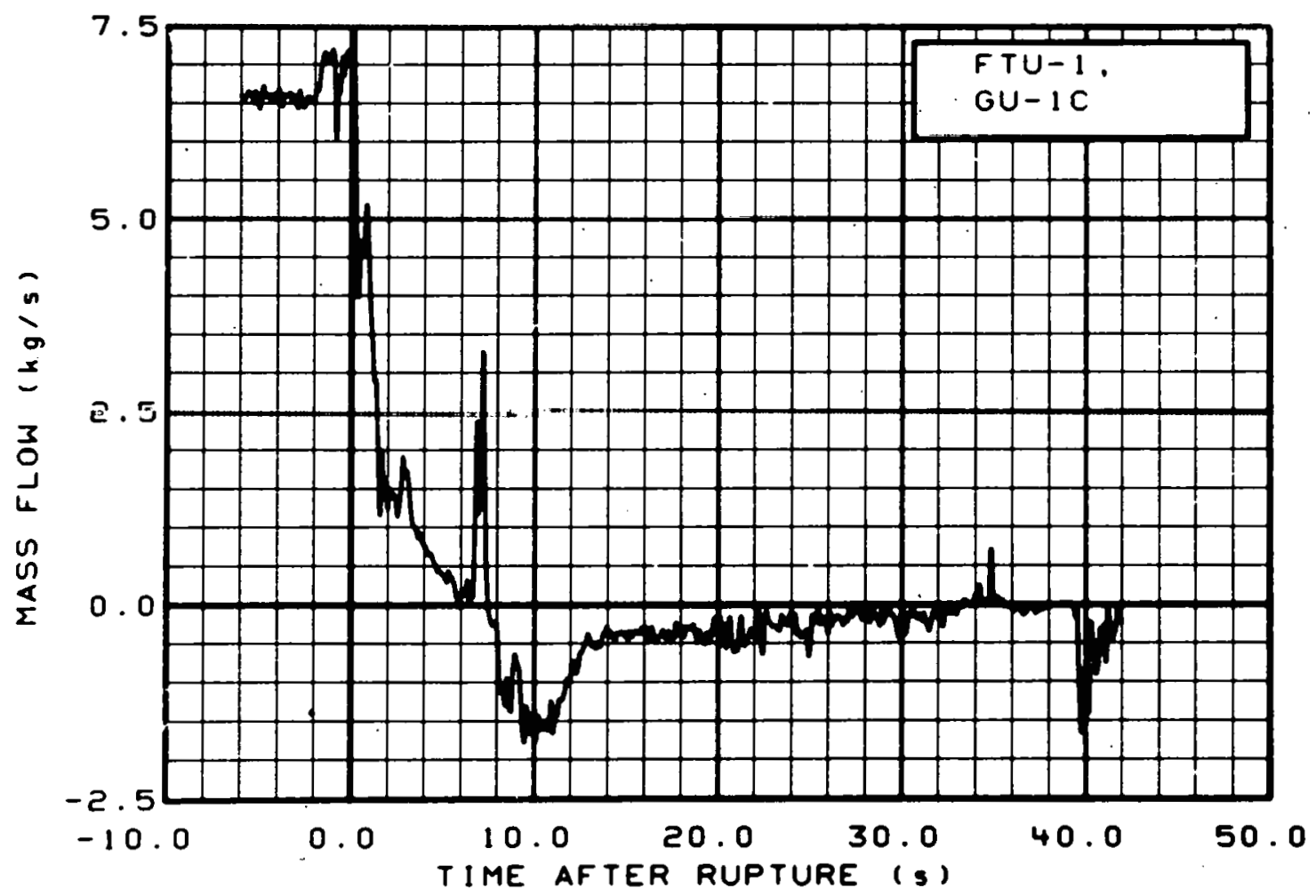

Fig. 311 Mass flow in intact loop (FTU-1, GU-1C), from -6 to $42 \mathrm{~s}$. 


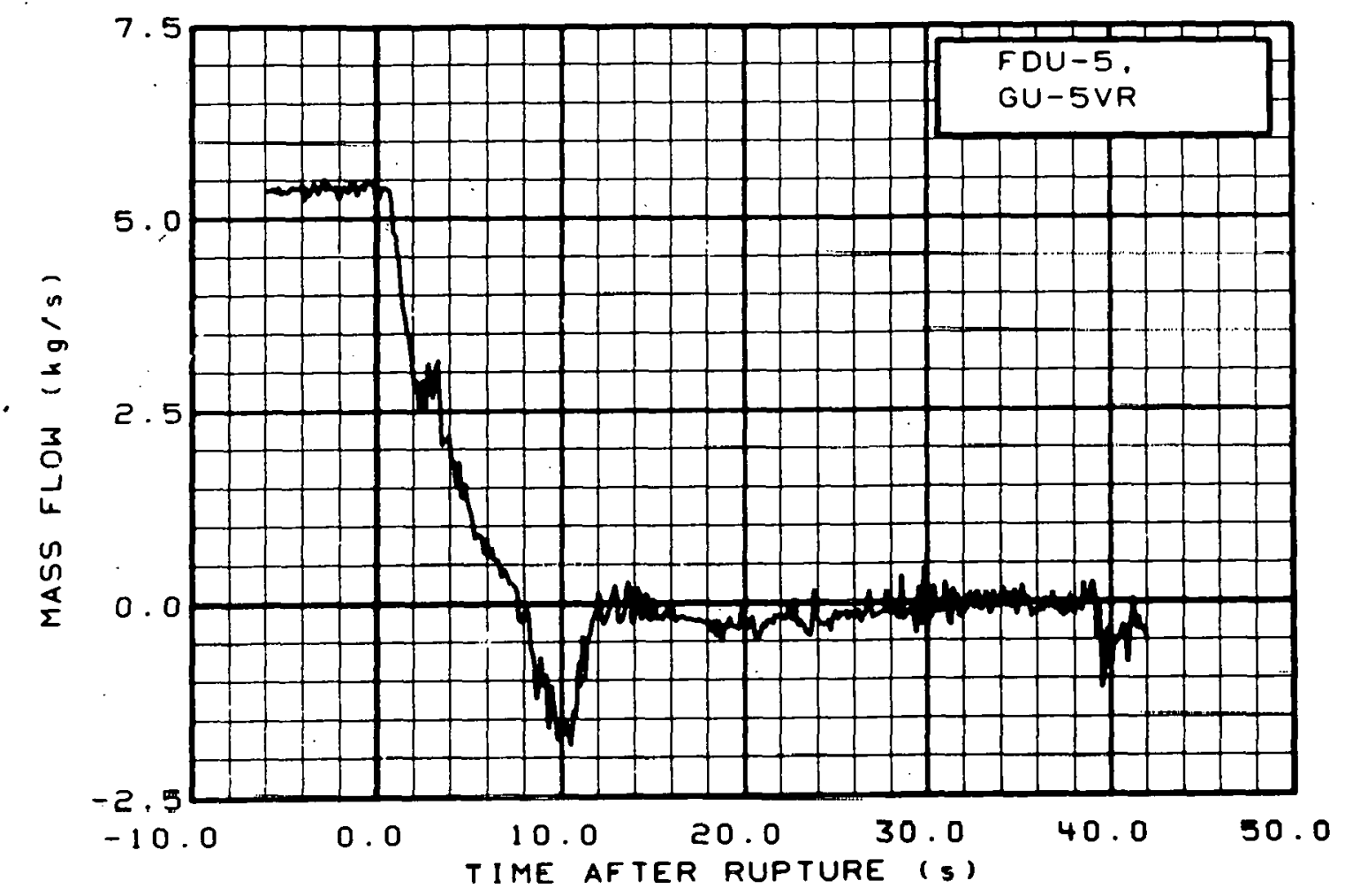

Fig. 312 Mass flow in intact loop (FDU-5, GU-5VR), from -6 to $42 \mathrm{~s}$. 


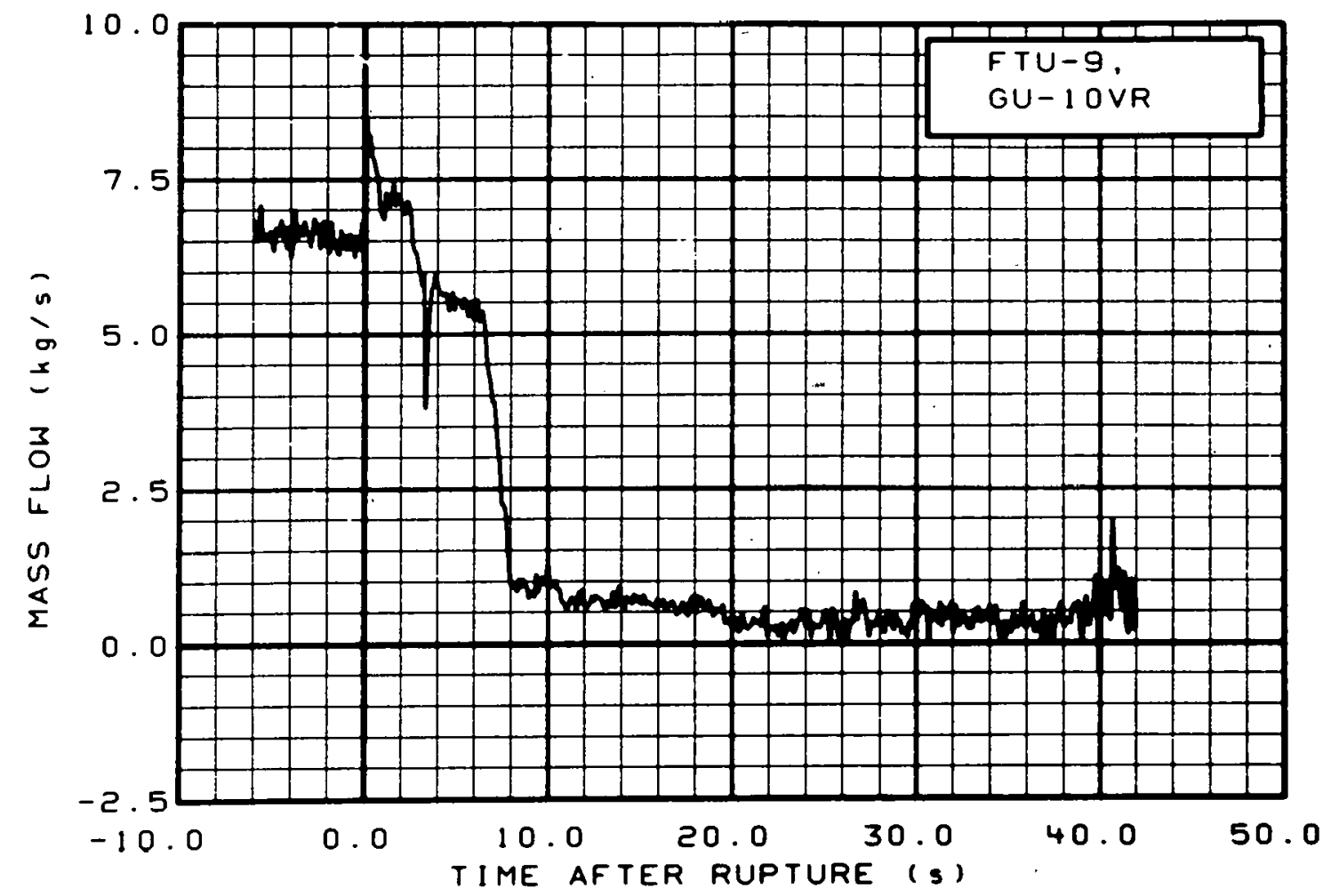

Fig. 313 Mass flow in intact loop (FTU-9, GU-10VR), from -6 to $42 \mathrm{~s}$.

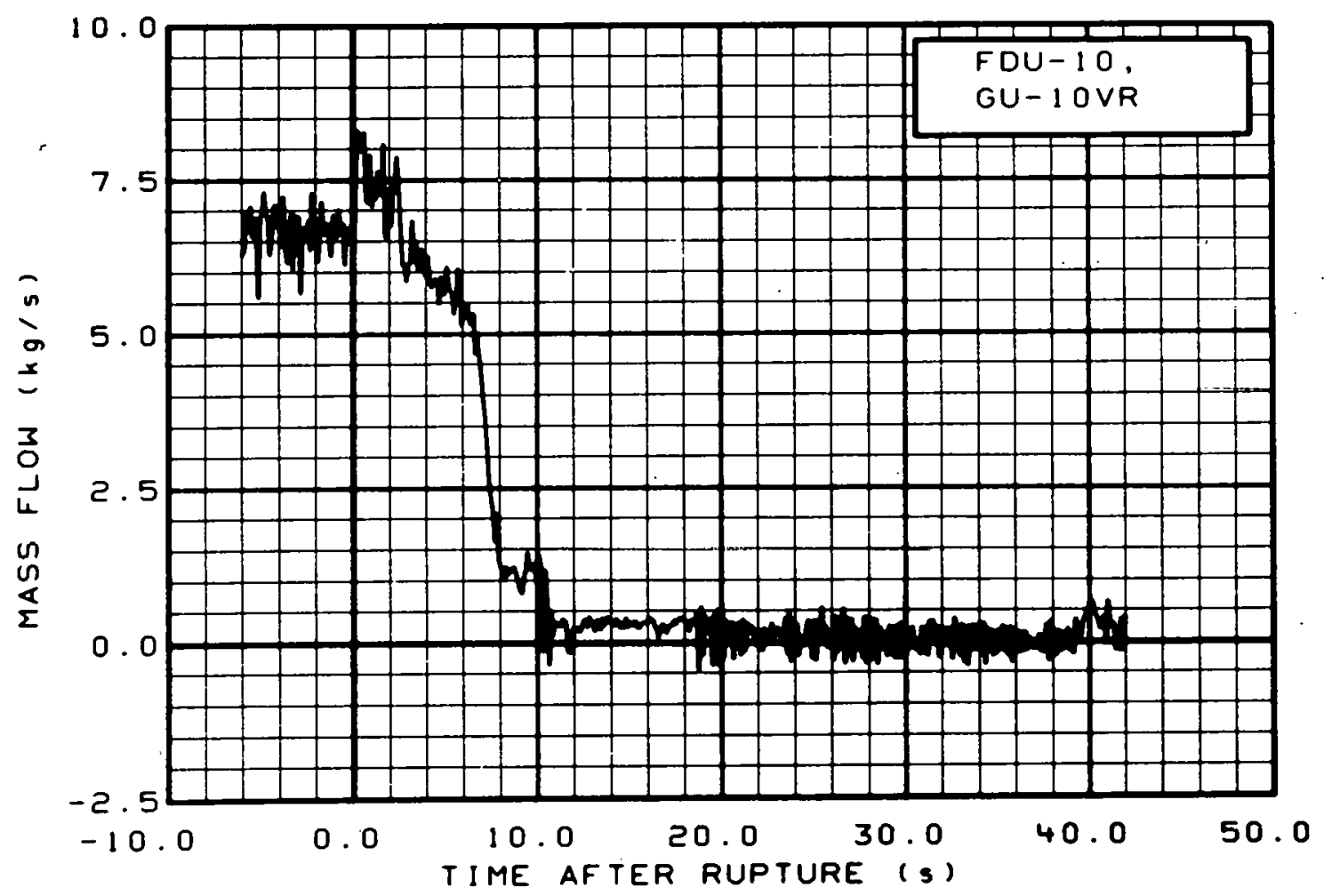

Fig. 314 Mass flow in intact loop (FDU-10, GU-10VR), from -6 to $42 \mathrm{~s}$. 


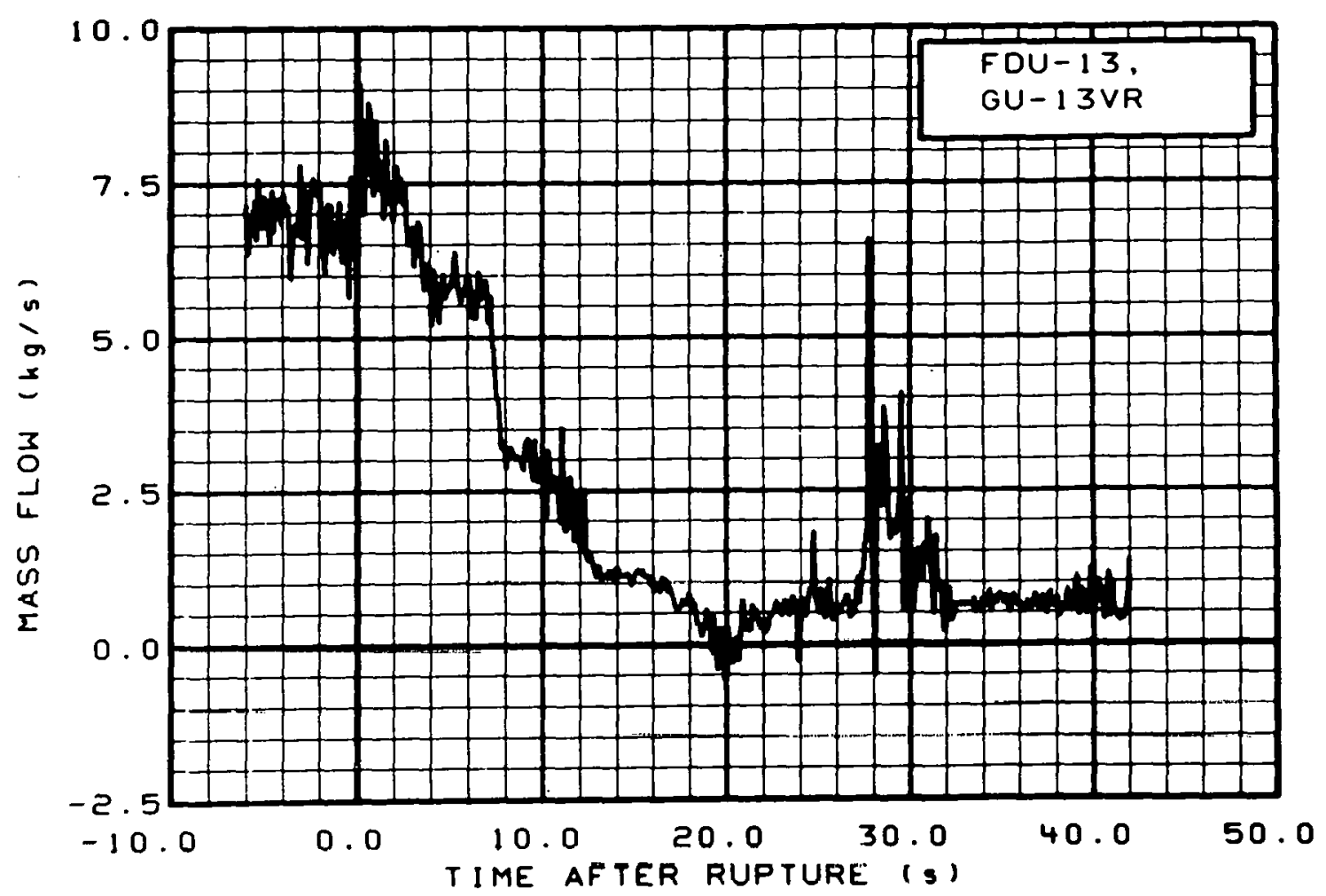

Fig. 315 Mass flow in intact loop (FDU-13, GU-13VR), from -6 to $42 \mathrm{~s}$.

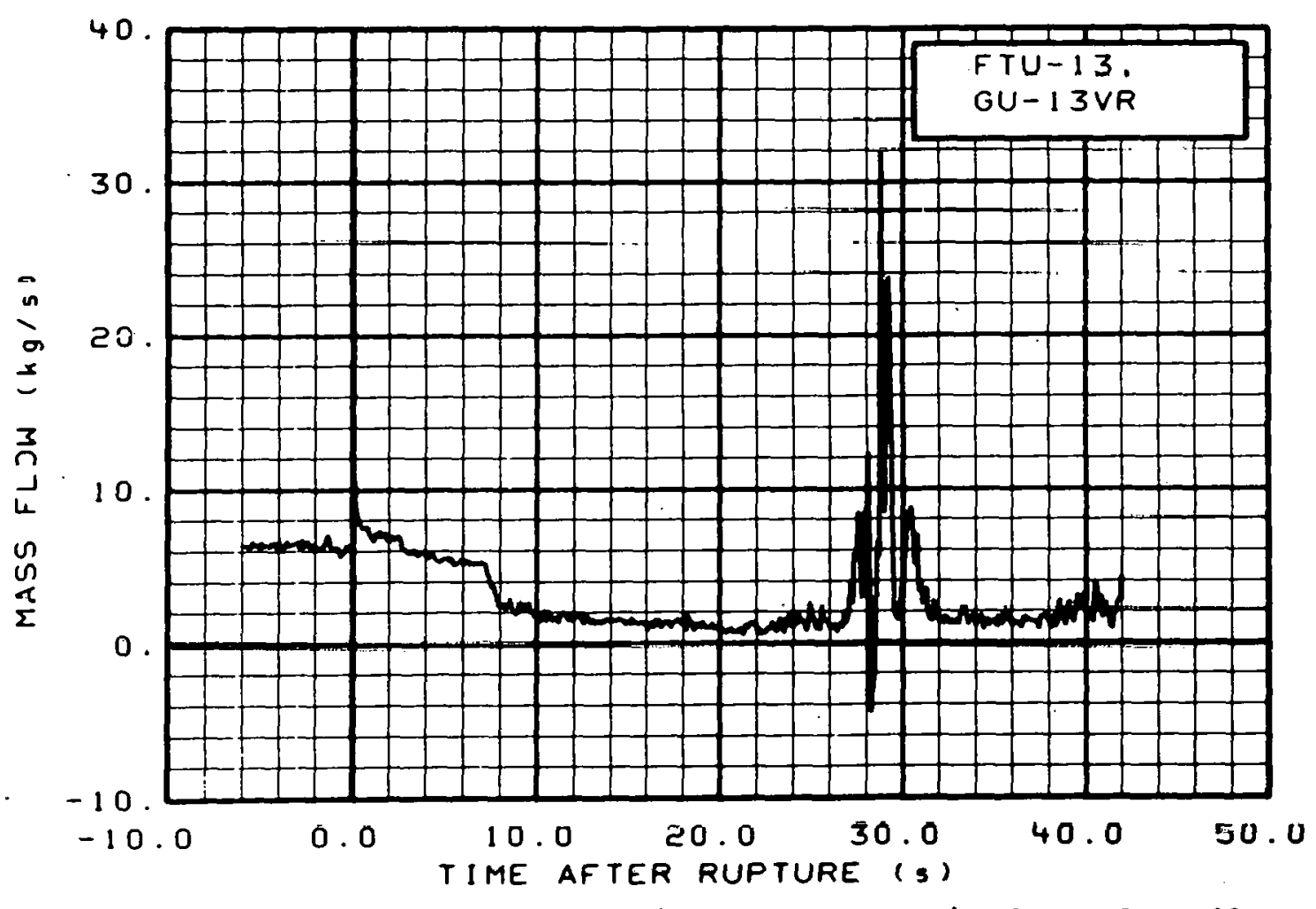

Fig. 316 Mass flow in intact loop (FTU-13, GU-13VR), from -6 to $42 \mathrm{~s}$. 


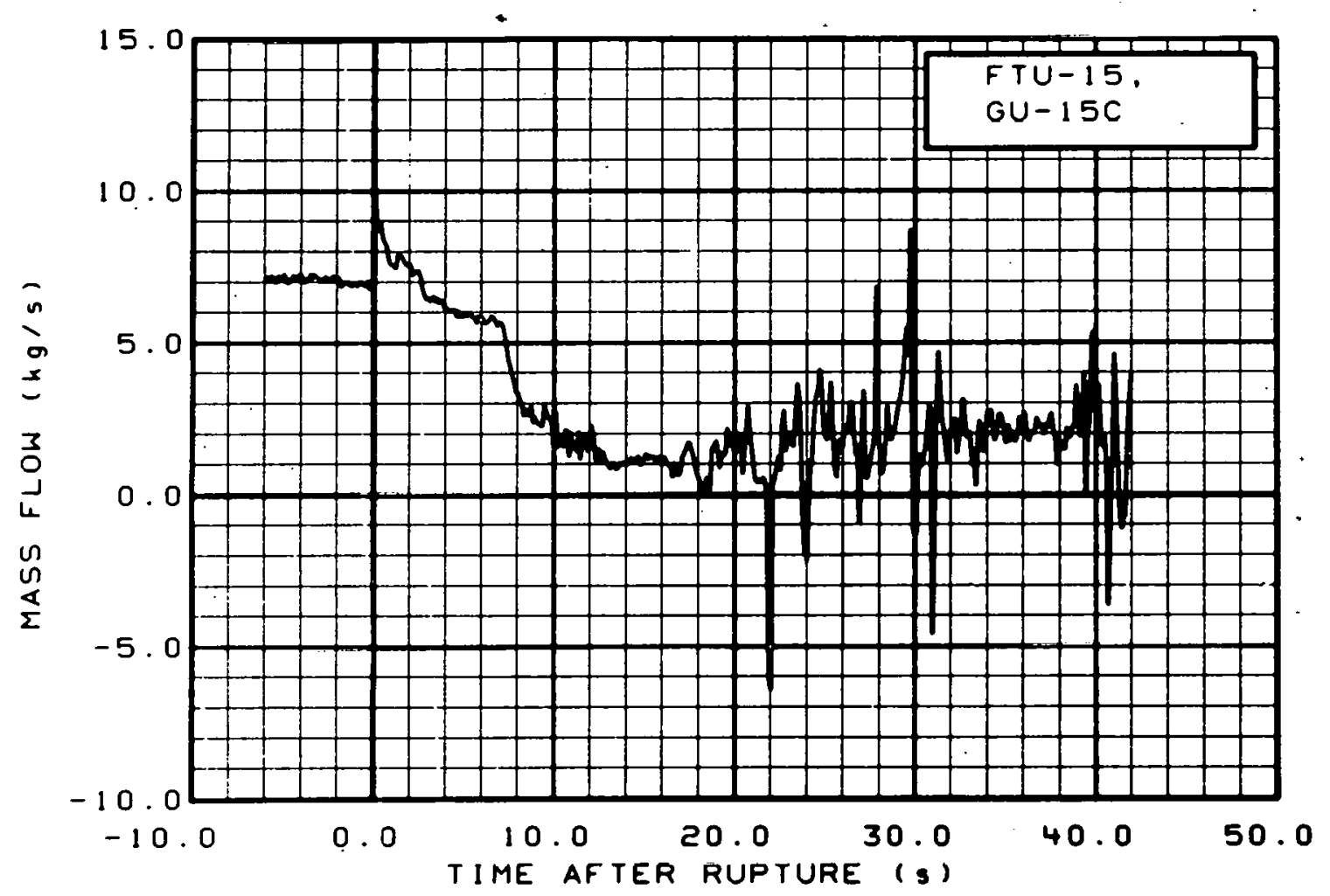

Fig. 317 Mass flow in intact loop (FTU-15, GU-15C), from -6 to $42 \mathrm{~s}$. 


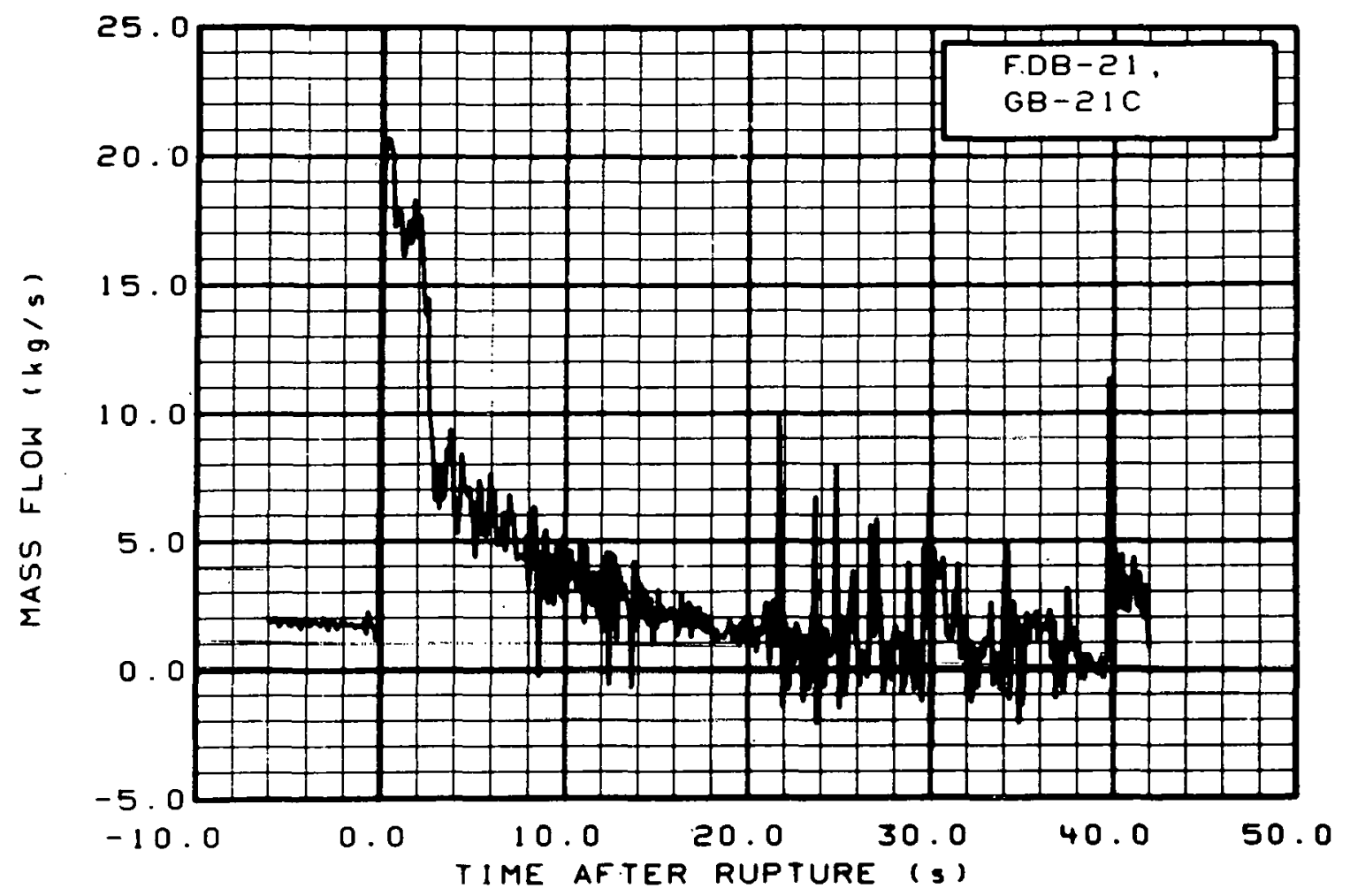

Fig. 318 Mass flow in broken loop (FDB-21, GB-21C), from -6 to $42 \mathrm{~s}$.

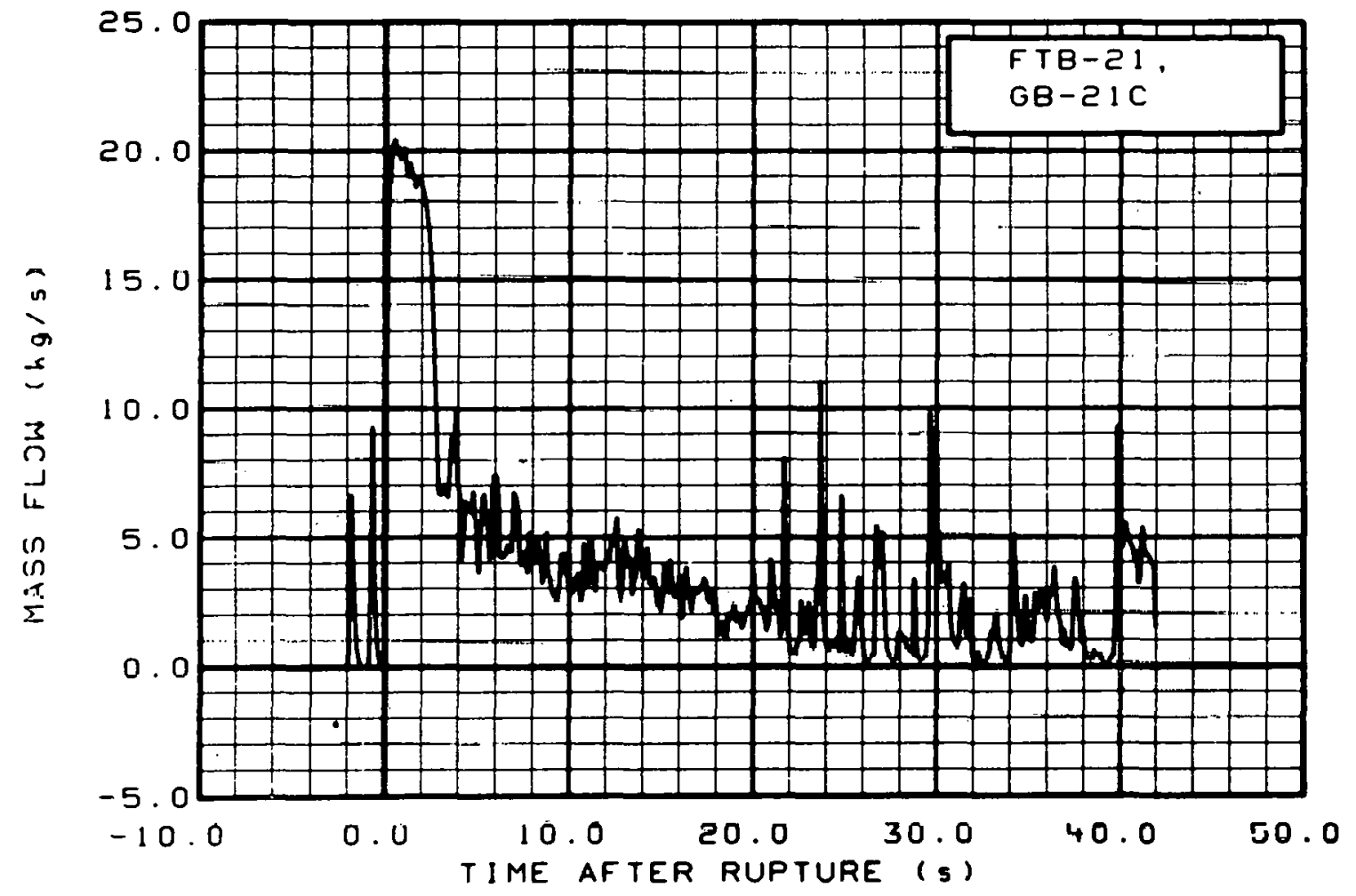

Fig. 319 Mass flow in broken loop (FTB-2l, GB-2TC), from -6 to $42 \mathrm{~s}$. 


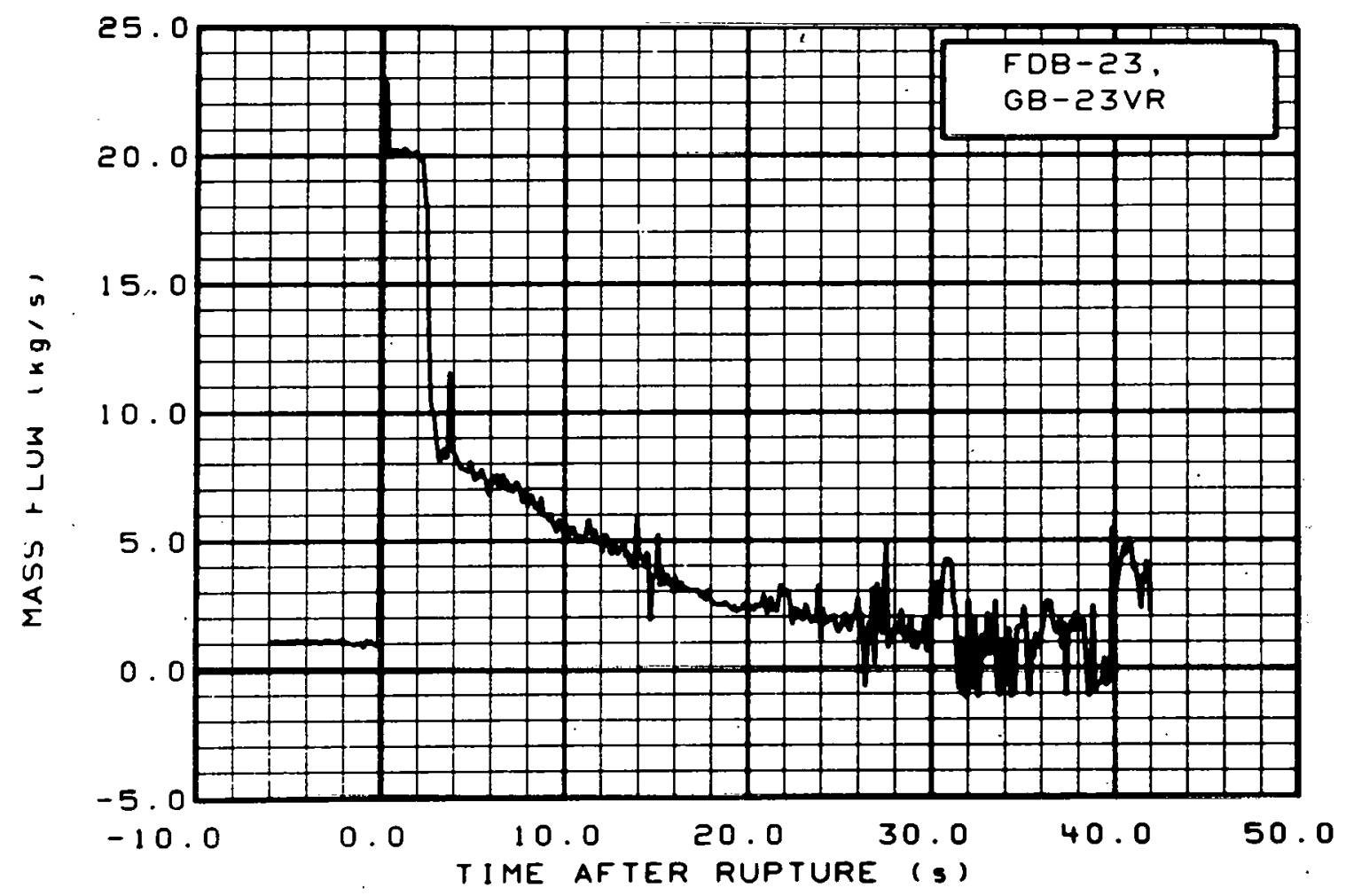

Fig. 320 Mass flow in broken loop (FDB-23, GB-23VR), from -6 to $42 \mathrm{~s}$. 


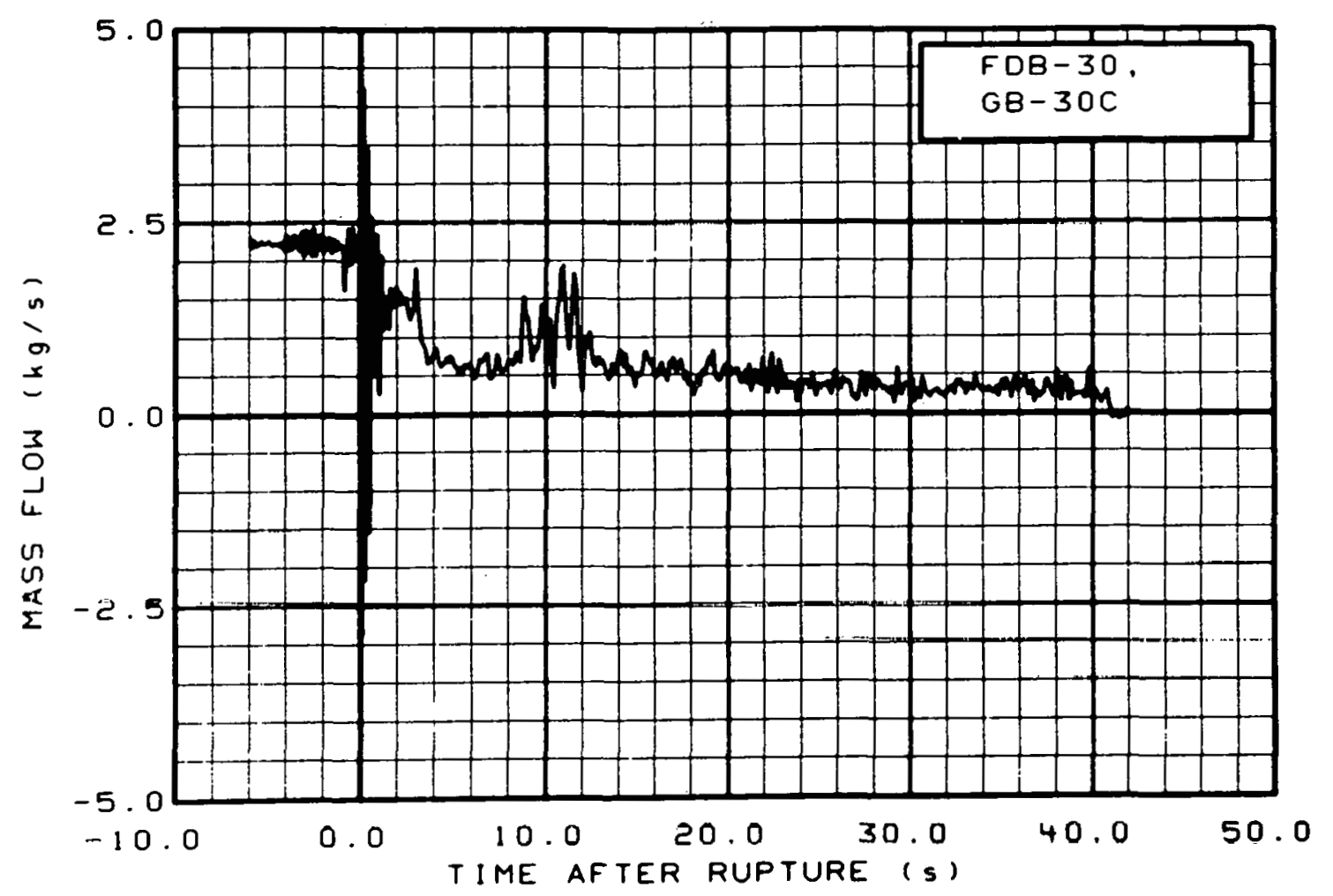

Fig. 321 Mass flow in broken loop (FDB-30, GB-30C), from -6 to $42 \mathrm{~s}$.

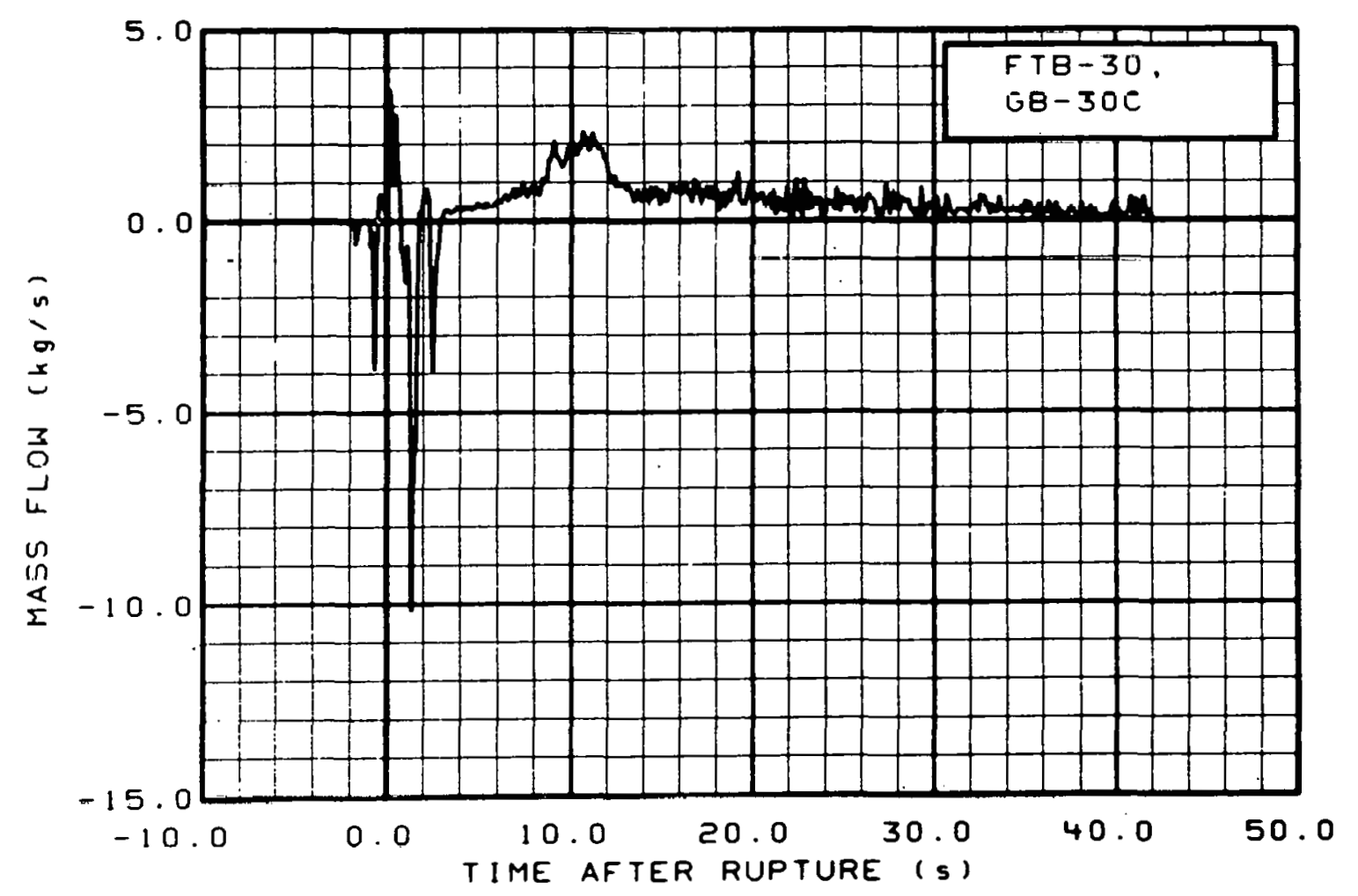

Fig. 322 Mass flow in broken loop (FTB-30, GB-30C), from -6 to $42 \mathrm{~s}$. 


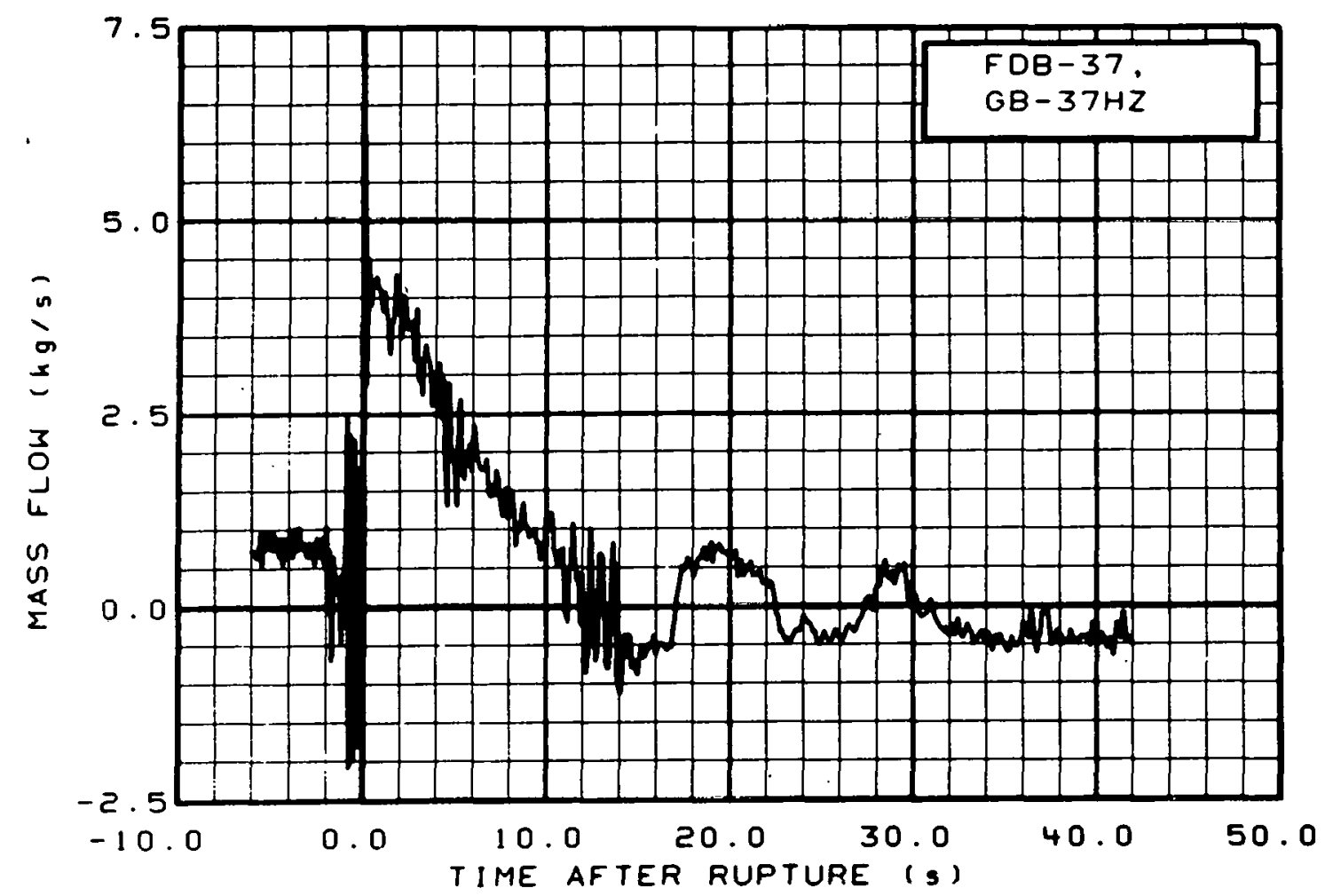

Fig. 323 Mass flow in broken loop (FDB-37, GB-37HZ), from -6 to $42 \mathrm{~s}$.

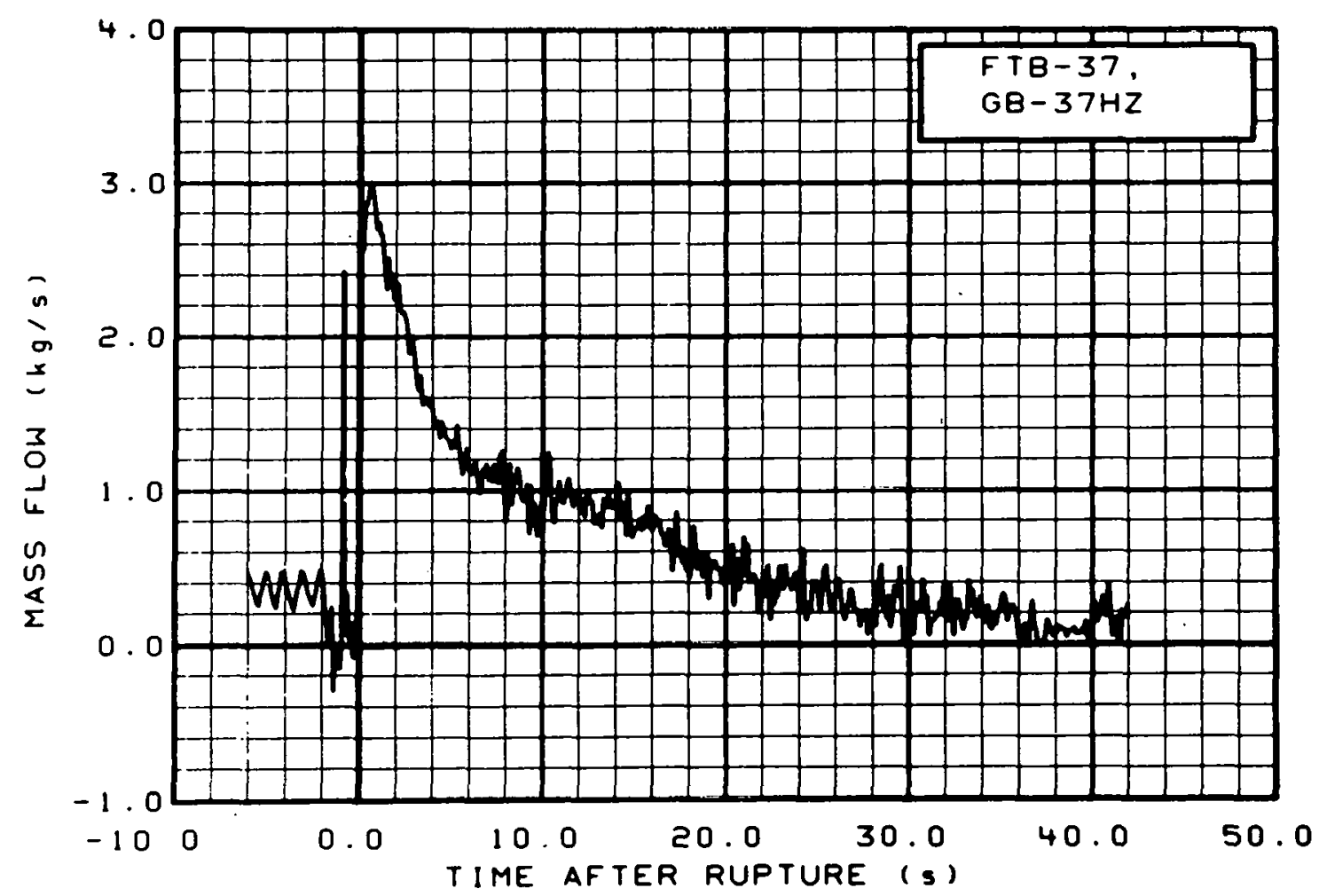

Fig. 324 Mass flow in broken loop (FTB-37, GB-37HZ), from -6 to $42 \mathrm{~s}$. 


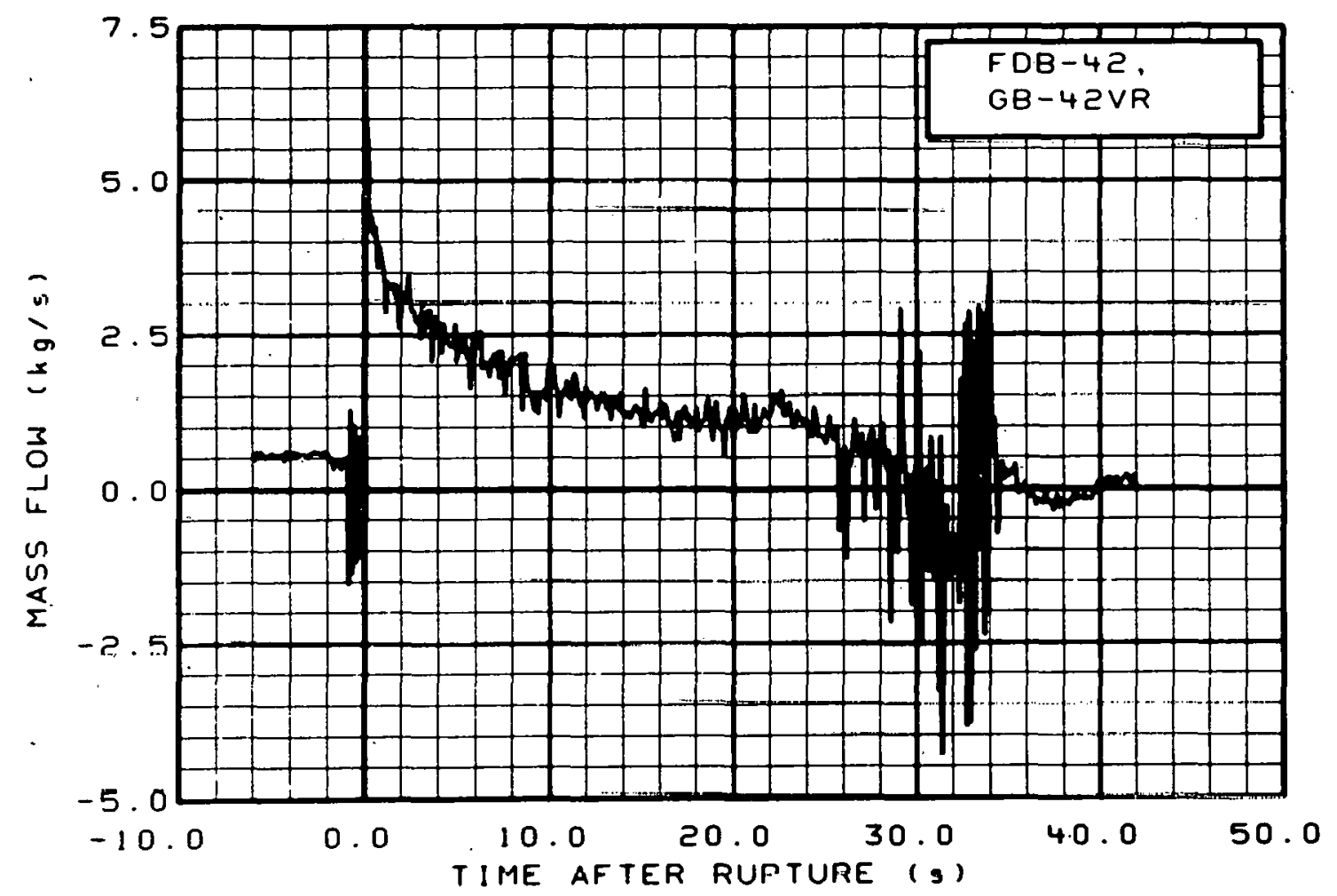

Fig. 325 Mass flow in broken loop (FDB-42, GB-42VR), from -6 to $42 \mathrm{~s}$. 


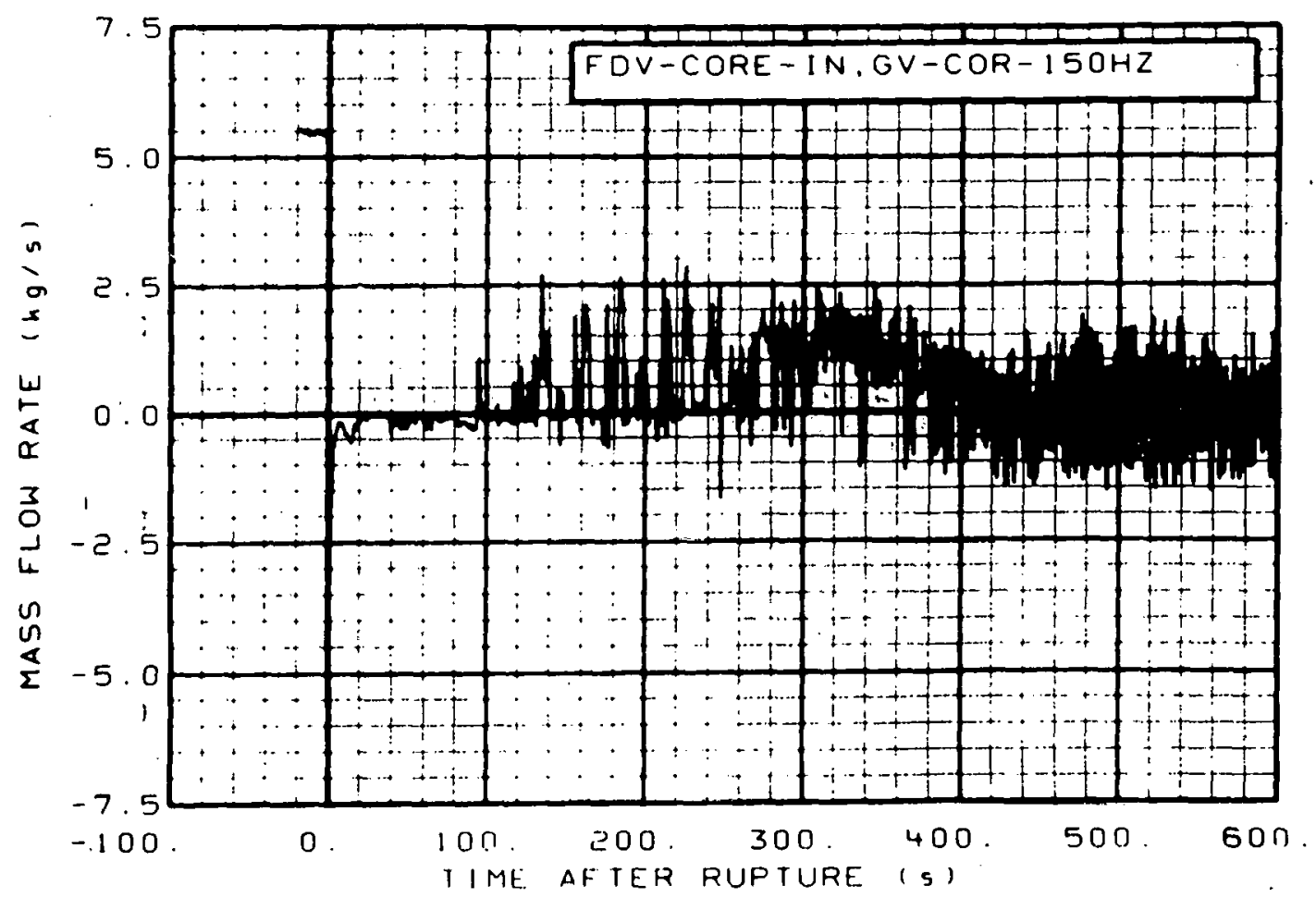

Fig. 326 Mass flow in vessel (FDV-CORF-IN, GV-COR-150HZ), from -20 to $600 \mathrm{~s}$.

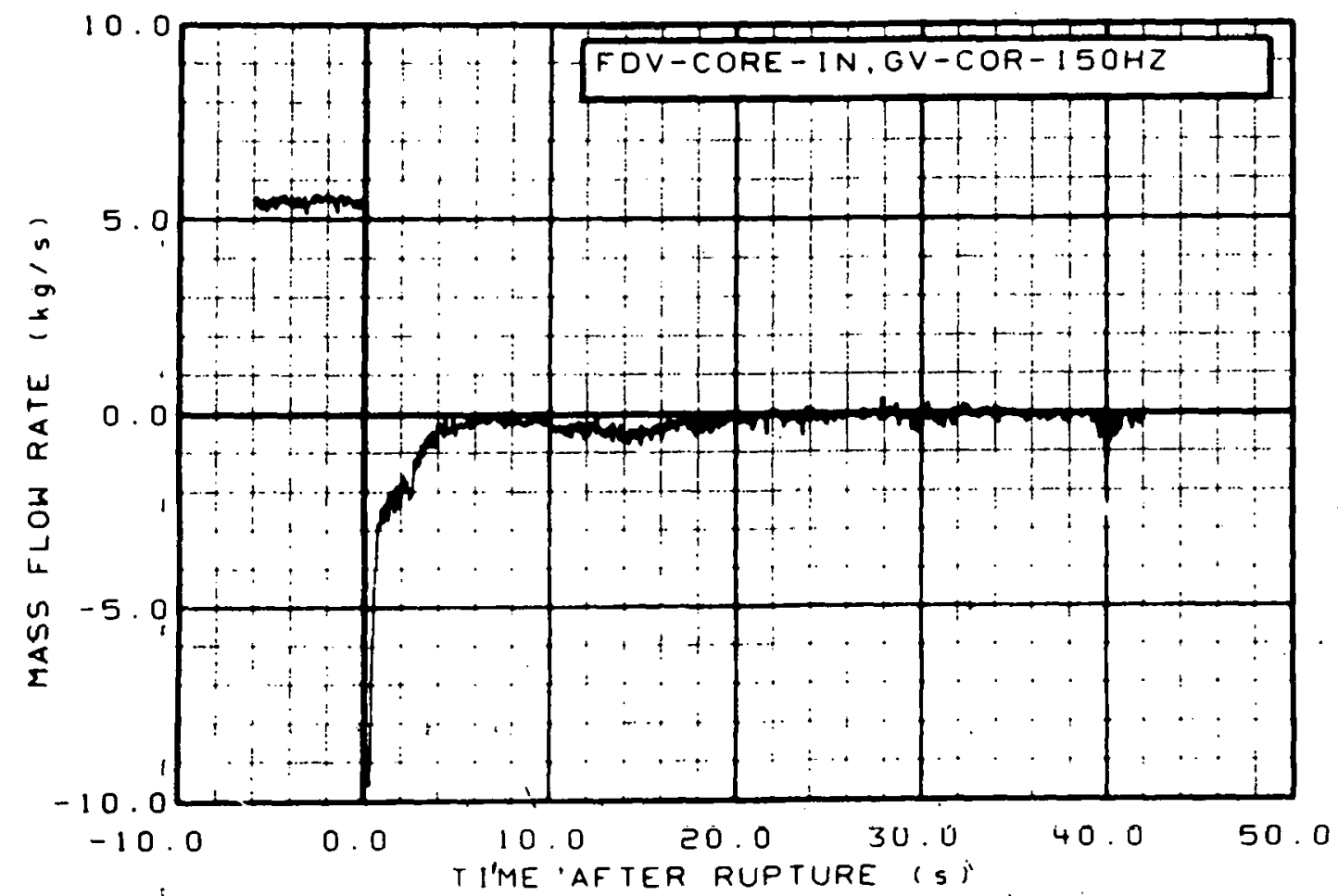

Fig. 327, Mass flow in vessel (FDV-CORE-IN, GV-COR $-150 H Z$ ), from -6 to $42 \mathrm{~s}$. 


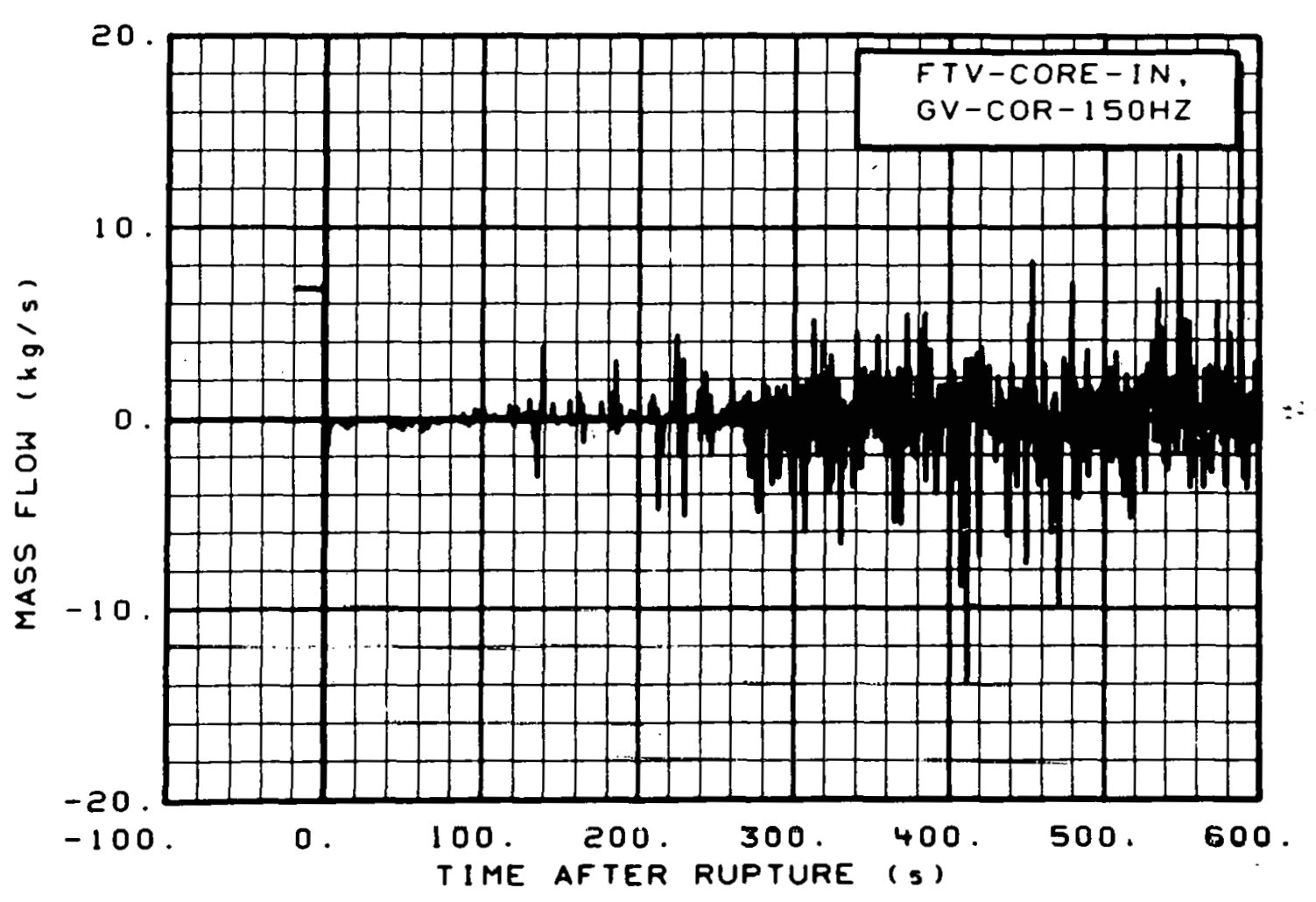

Fig. 328 Mass flow in vessel (FTV-CORE-IN, GV-COR-150HZ), from -20 to $600 \mathrm{~s}$.

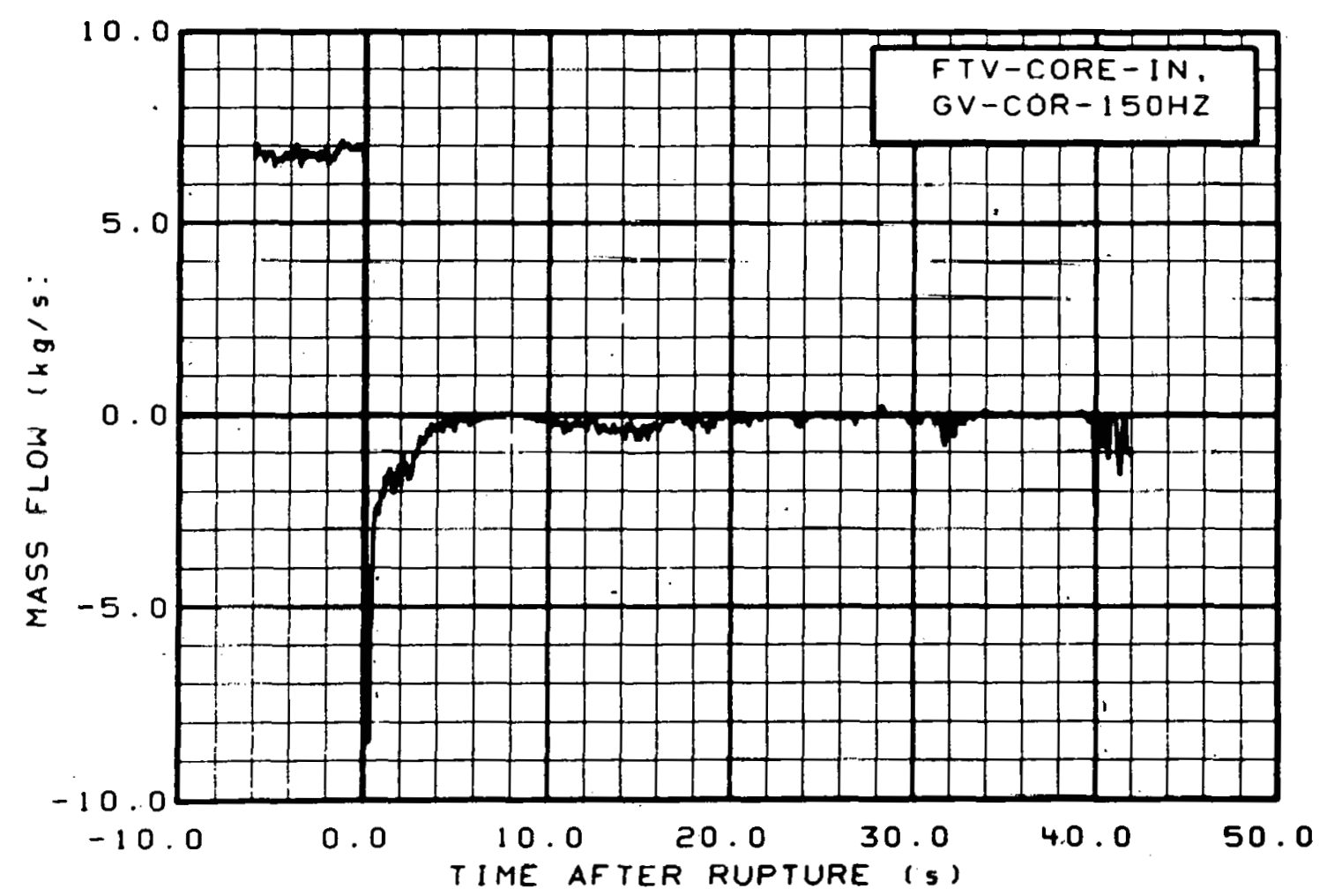

Fig. 329 Mass flow in vessel (FTV-CORE-IN, GV-COR-150HZ), from -6 to $42 \mathrm{~s}$. 


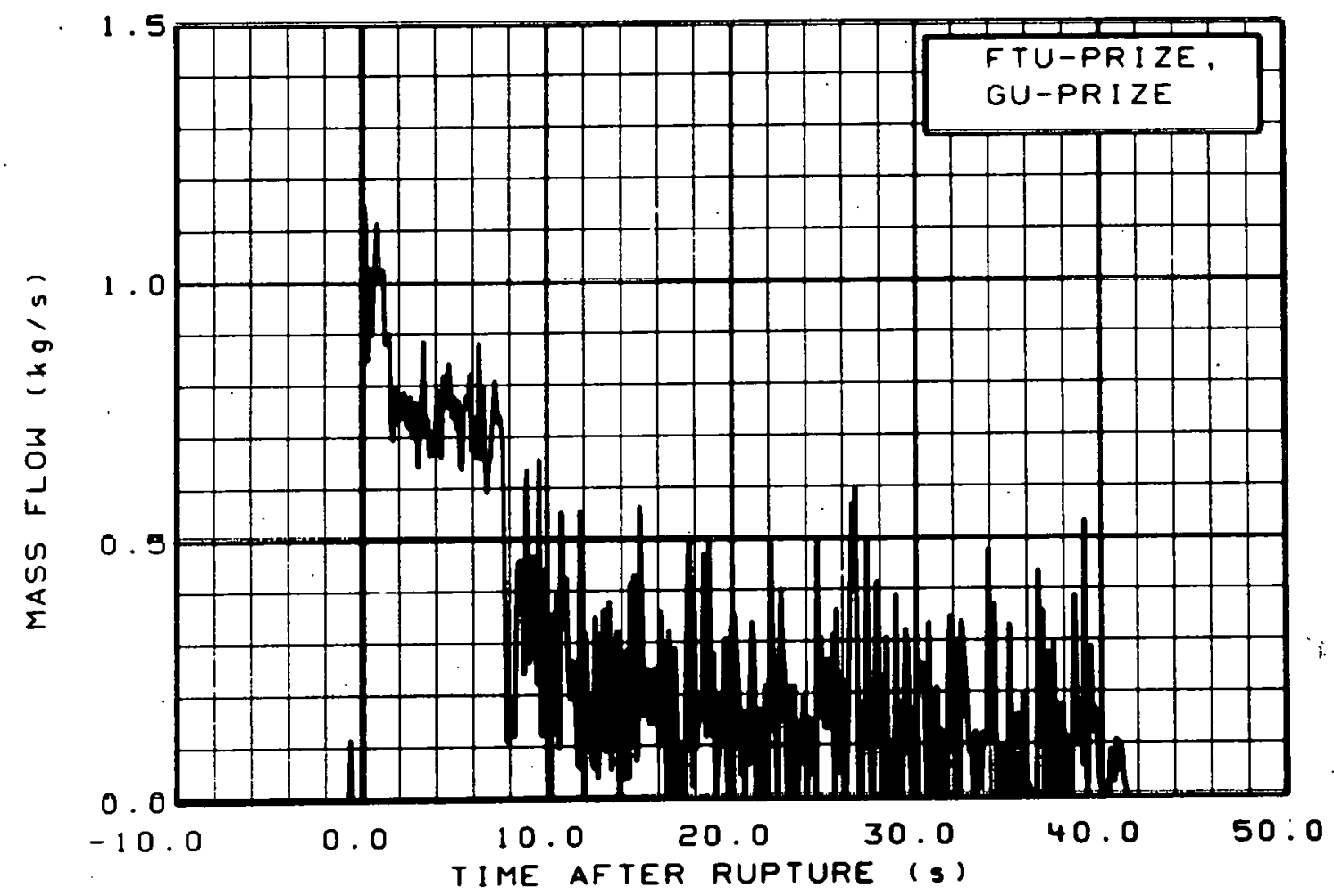

Fig. 330 Mass flow in pressurizer (FTU-PRIZE, GU-PRIZE), from -6 to $42 \mathrm{~s}$. 


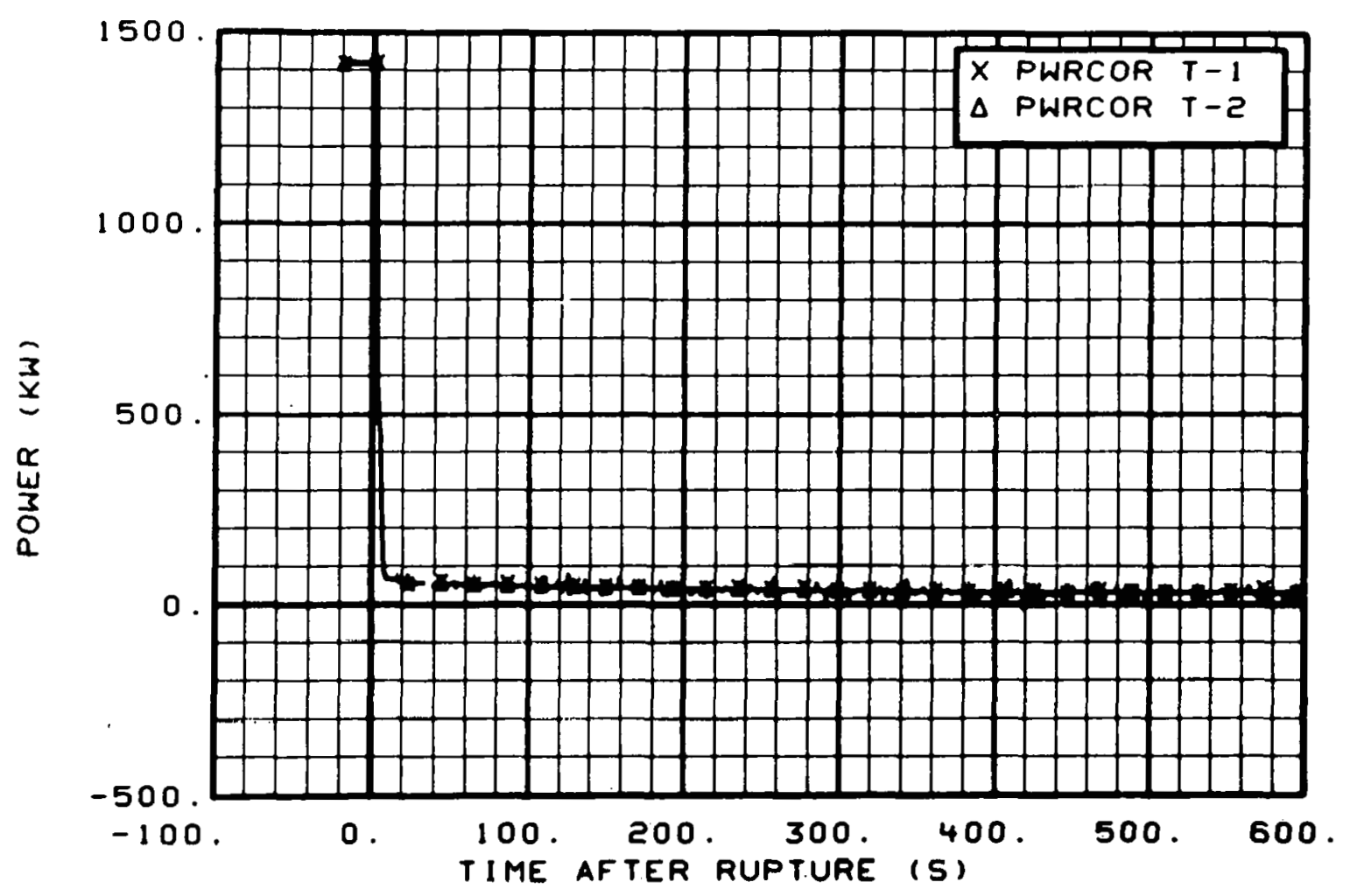

Fig. 331 Core heater rod total power (PWRCOR T-1 and PWRCOR T-2), from -20 to $600 \mathrm{~s}$.

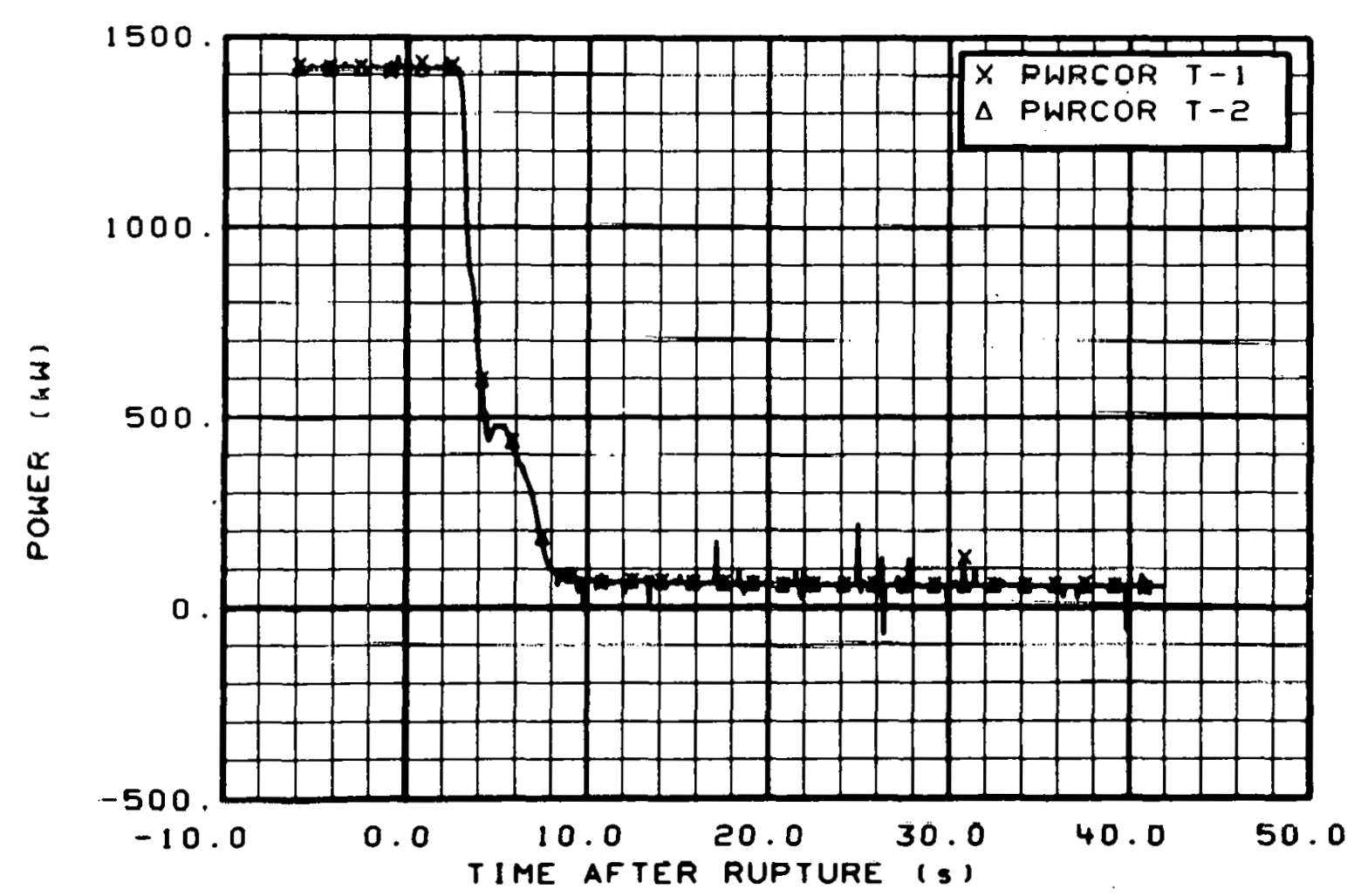

Fig. 332 Core heater rod total power (PWRCOR T-1 and PWRCOR T-2), from -6 to $42 \mathrm{~s}$. 


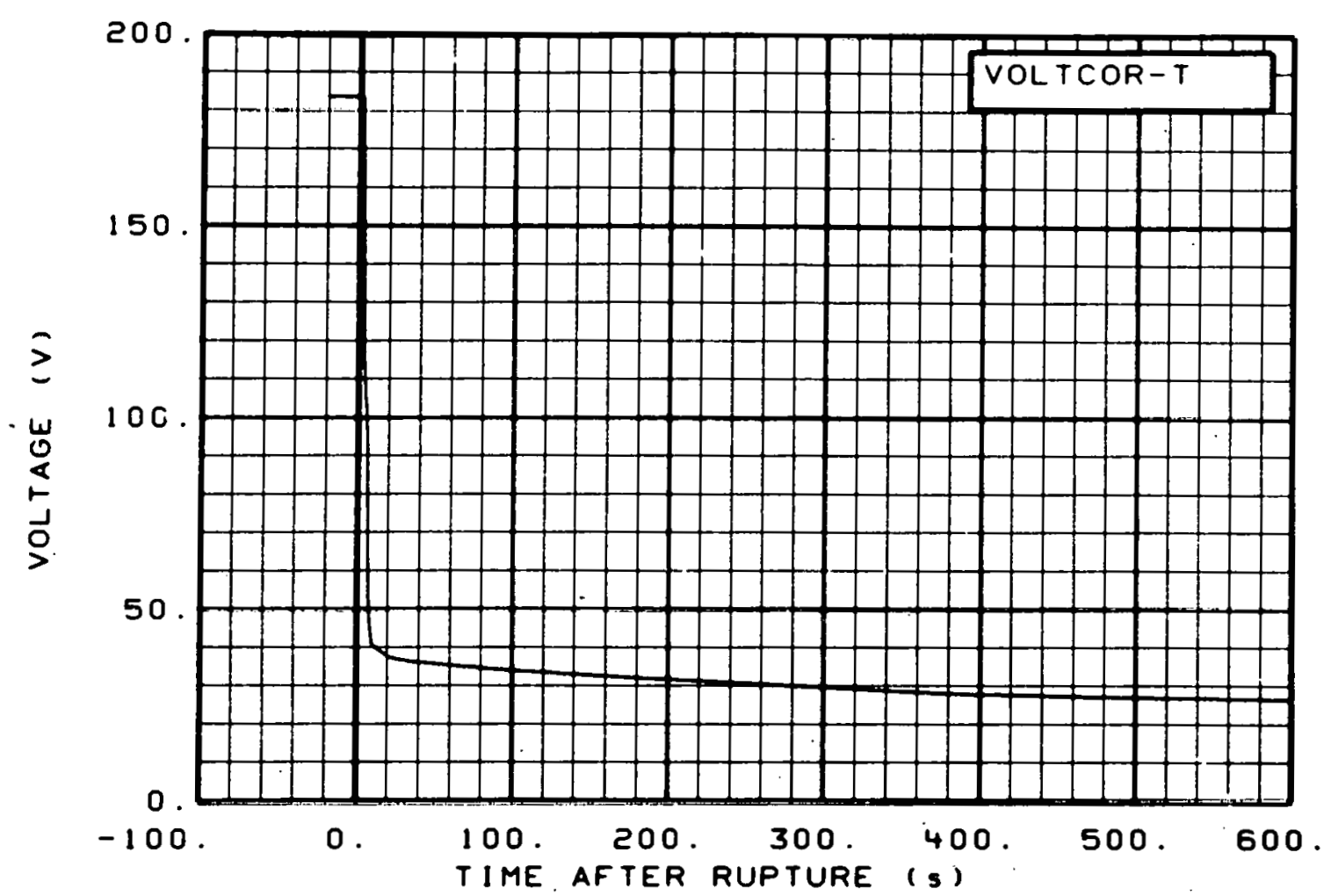

Fig. 333 Core heater voltage (VOLTCOR-T), from -20 to $600 \mathrm{~s}$.

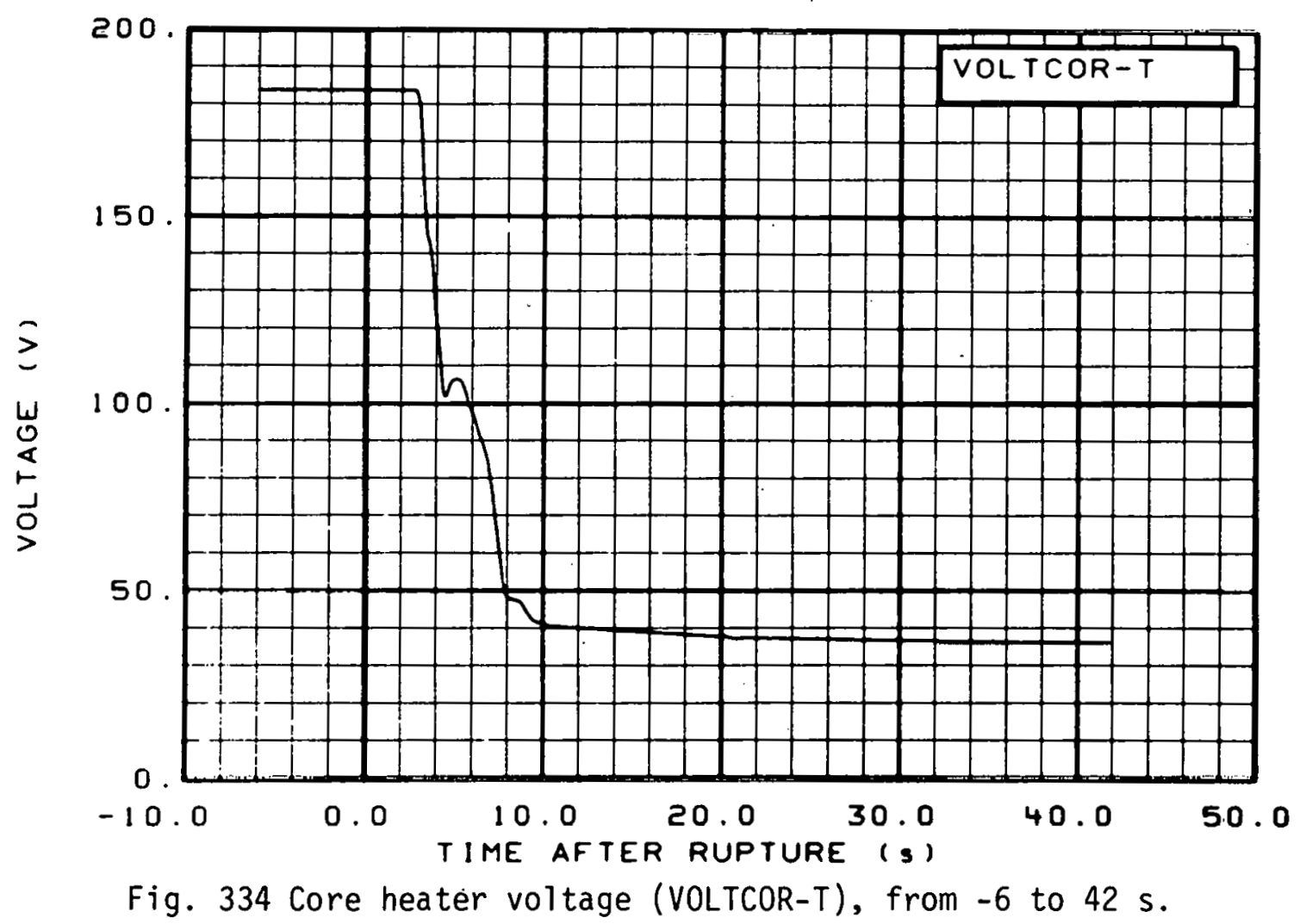




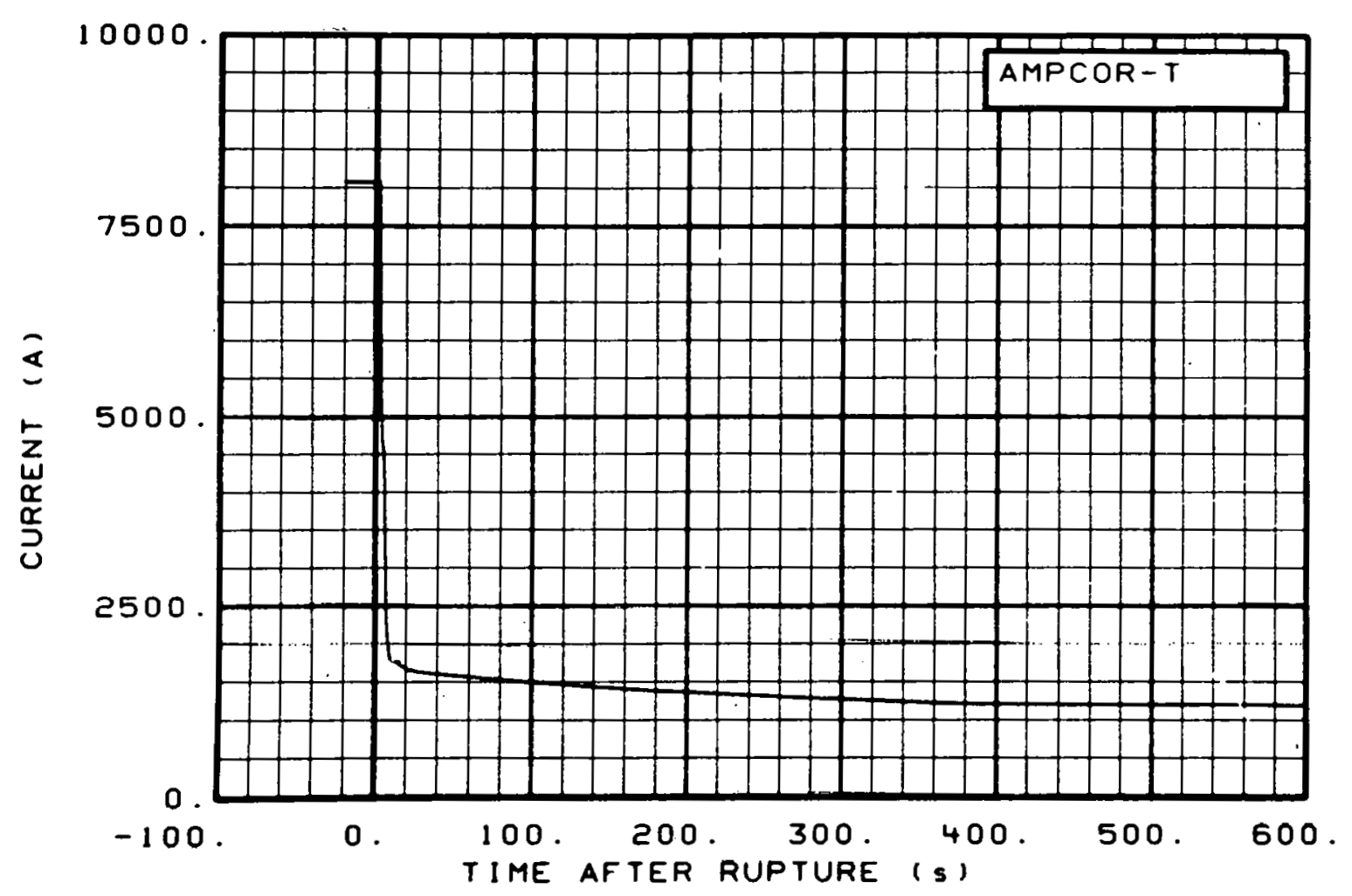

Fig. 335 Core heater total current (AMPCOR-T), from -20 to $600 \mathrm{~s}$.

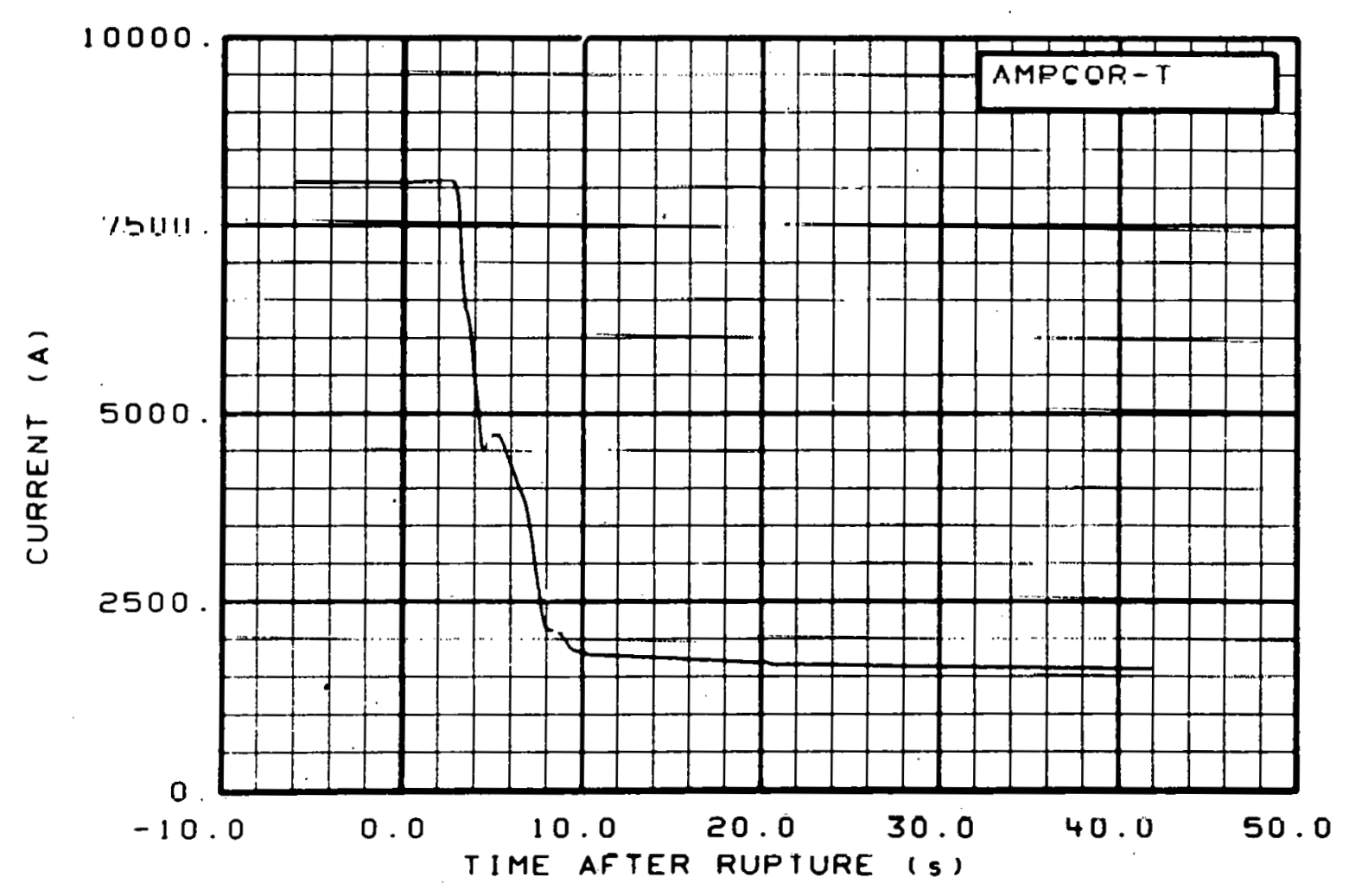

Fig. 336 Core heater total current (AMPCOR-T), from -6 to $42 \mathrm{~s}$. 


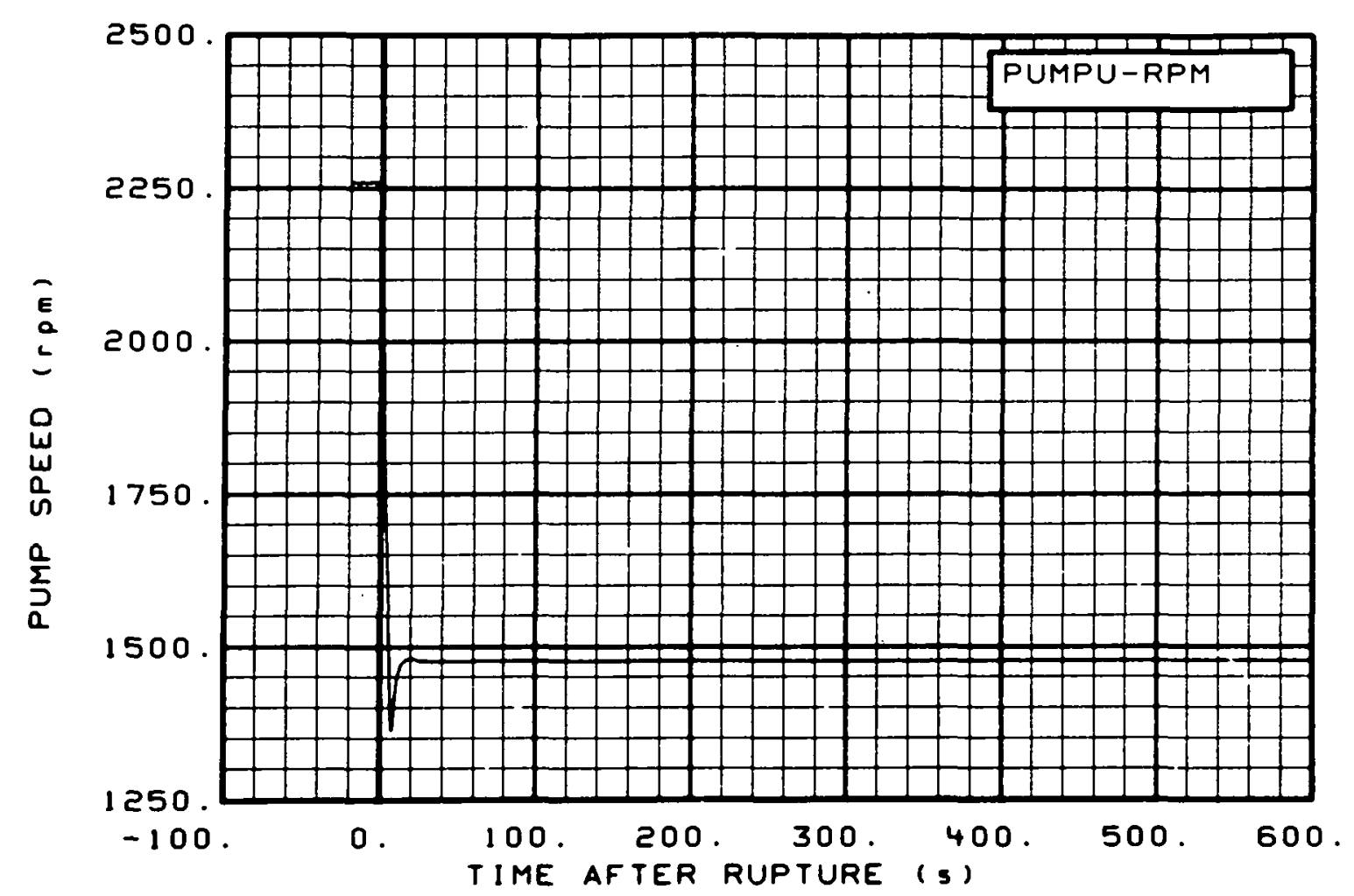

Fig. 337 Primary pump speed (PUMPU-RPM), from -20 to $600 \mathrm{~s}$.

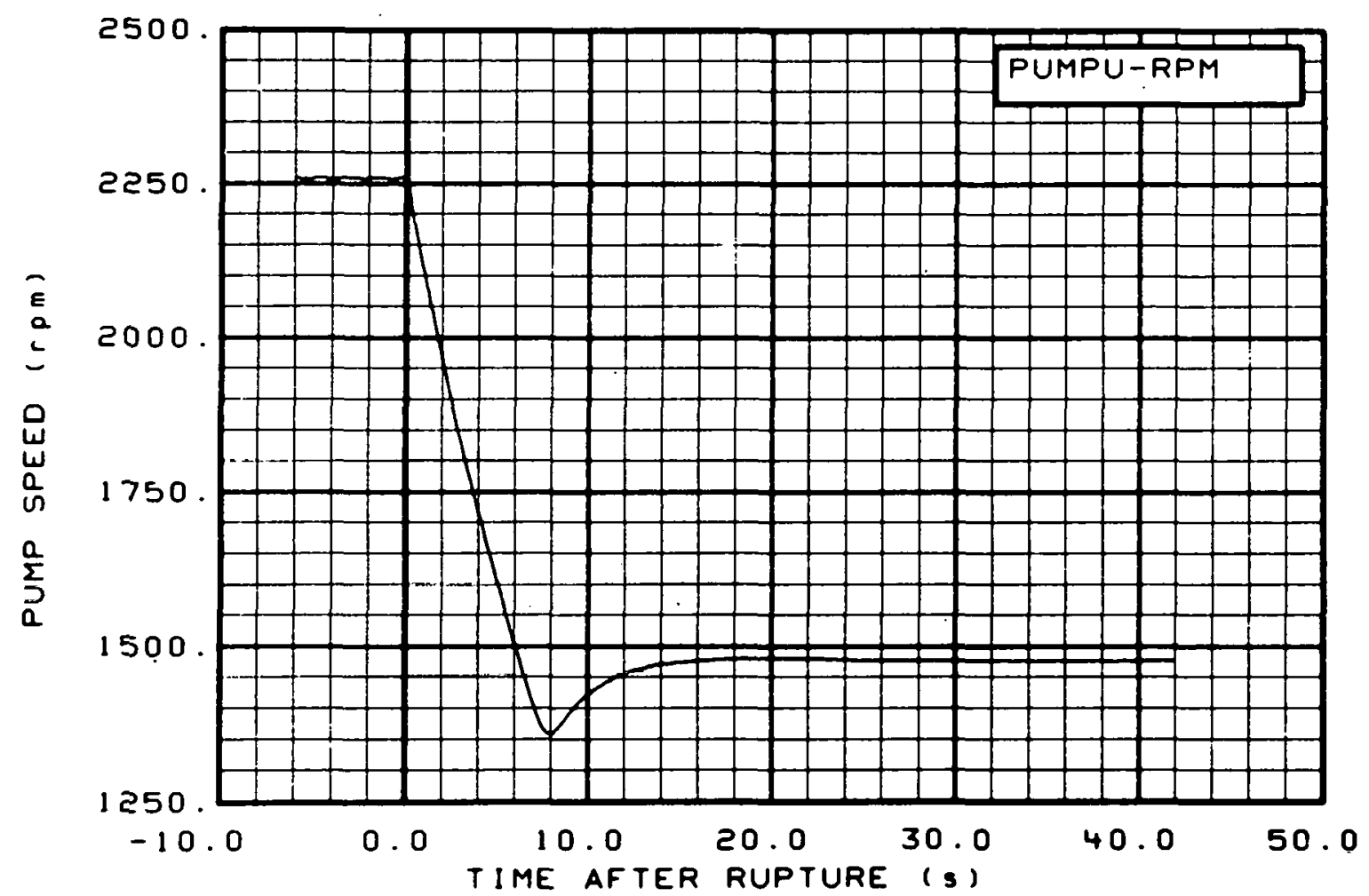

Fig. 338 Primary pump speed (PUMPU-RPM), from -6 to $42 \mathrm{~s}$. 


\section{REFERENCE}

1. E. M. Feldman and D. J. Olson, Semiscale Mod-1 Program and System Description for the Blowdown Heat Transfer Tests (Test Series 2), ANCR-1230 (August 1975). 
APPENDIX A

DATA ACQUISITION SYSTEM CAPABILITIES 
THIS PAGE

\section{WAS INTENTIONALLY LEFT BLANK}




\section{APPENDIX A \\ DATA ACQUISITION SYSTEM CAPABILITIES}

The Semiscale Mod-1 system provides for the acquisition, processing, and presentation of test data. Detectors, signal conditioners, signal processors, and recording and display equipment comprise the test data system. The data obtained are principally recorded on an on-line digital system. Selected data channels are also recorded on an analog system.

The on-line digital system is called the digital data acquisition and processing system (DDAPS). The DDAPS has dual and single speed capabilities with identical storage and data output limitations. The dual speed mode is used to extend the recording time when obtaining high frequency data.

From each of up to 240 data channels, the test data system stores 20 blocks of data. Each block of data contains 920 words (each word is the abscissa and ordinate of a data point) of digital information. These 920 words represent a fixed storage display.

The maximum measured throughput rate for the system is 24000 words per second. This throughput rate can be reduced in increments of 100 words per second. The throughput rate, the number of data channels recorded, and the fixed display of 920 words per block determine the time base for displaying the data.

After the data have been stored, data reduction can be made for presentation and analysis purposes. Because of hardware limitations and aesthetic considerations of data presentation, only certain time bases are used when the data are reduced. For data displayed from -20 to $300 \mathrm{~s}$, the recorded data are made to occupy a $320 \mathrm{~s}$ span. This yields a time base of $16 \mathrm{~s}$, which is the $320 \mathrm{~s}$ span divided by the 20 blocks of recorded data.

Generally, 920 words from a given data channel are displayed in the nominal time base of $16 \mathrm{~s}$. Integral ( 1 to 20 ) multiples of $16 \mathrm{~s}$ may be used as variations on the nominal

time base. Because the output is fixed at 920 words, data compression is made by averaging adjacent data points to give the desired compression. 


\section{THIS PAGE}

\section{WAS INTENTIONALLY LEFT BLANK}


APPENDIX B

POSTTEST ADJUSTMENTS TO DATA FROM SEMISCALE MOD-1 TEST S-28-6 
THIS PAGE

WAS INTENTIONALLY

LEFT BLANK 


\section{APPENDIX B}

\section{POSTTEST ADJUSTMENTS TO DATA FROM SEMISCALE MOD-1 TEST S-28-6}

Many of the transducers used in the Semiscale Mod-1 system exhibit significant sensitivity to one or more spurious inputs. Strain gage bridge circuits used in pressure transducers, differential pressure transducers, and drag discs are sensitive to changes in ambient temperature. Differential pressure cells are also sensitive to changes in system pressure. Photomultiplier tubes used as gamma ray detectors in the density transducers are sensitive to temperature changes, as well as to random variations in the locations of the radiation sources. Core power measurements depend on a calibrated resistor, whose resistance changes in value as a function of time and power level as it heats up.

Although the errors introduced into the data by spurious secondary inputs generally do not exceed the specified error ranges of the transducers, significant improvement in measurement accuracy can be achieved if the secondary sensitivity can be identified and removed. In the case of the drag discs, corrections are absolutely necessary because the signal due to temperature fluctuations can exceed that due to flow by several hundred percent. Since the exact values of the spurious inputs to which different transducers might be sensitive cannot often be easily predicted and are sometimes inconvenient to measure, secondary effects have been accounted for by correcting the data after the test rather than by using elaborate real time programs in the data acquisition system computer. The methods and results of the posttest data correction analysis for Test S-28-6 are presented in the following paragraphs and tables.

\section{PRESSURE MEASUREMENTS}

Corrections to pressure transducer measurements in the main system loop are based on data taken from the standard reference (Heise) gauge at Spool 4, taken $15 \mathrm{~s}$ before initiation of blowdown and at $300 \mathrm{~s}$ after initiation of blowdown. The pressure readings are adjusted to account for pressure variations around the main loop, using the readings of nearby differential pressure cells. A linear correction is then applied to the pressure data to match the data to the calculated reference data at the two specified time points.

Correction of the steam generator secondary pressure (PU-SGSD) and intact and broken loop emergency core coolant (ECC) accumulator pressure (PU-ACC1 and PB-ACC2) measurements are done in the same manner as for the main loop pressures using Heise gauges installed expressly for this purpose. The data from the pressure transducer for the pressure suppression system (P-PSS) are corrected to match the process instrumentation at preblowdown conditions. 
Pressure measurement corrections are performed using the data acquisition system (DAS) computer using the following equation:

$$
F^{\prime}(t)=C_{0}+C_{1}[F(t)]
$$

where

$$
\begin{aligned}
& F^{\prime}(t)=\text { corrected data, } \mathrm{kPa} \\
& \mathrm{F}(\mathrm{t})=\text { raw data, } \mathrm{kPa} \\
& \mathrm{C}_{\mathrm{O}}=\text { offset, } \mathrm{kPa} \\
& \mathrm{C}_{1}=\text { scaling factor: }
\end{aligned}
$$

The values of the offset and scaling factor are given.in Table B-I.

TABLE B-I

CONSTANTS FOR PRESSURE MEASUREMENT CORRECTIONS (TEST S-28-6)

\begin{tabular}{crc}
$\begin{array}{c}\text { Detectior } \\
\text { Identification }\end{array}$ & $\frac{C_{0}}{1.37}$ & $\frac{C_{1}}{0.1430}$ \\
PB-42 & 64.12 & 1 \\
\hline
\end{tabular}

\section{DIFFERENTIAL PRESSURE ME $\Lambda$ SUREMENTS}

Pressure sensitivity in the differential pressure cells in the main system loop is determined from the pretest system pressure check. Digital data are recorded for all measurements at ambient temperature, with no system flow, at pressures of ambient, 1380 , $3450,6900,10350,13800$, and $15500 \mathrm{kPa}$. The output of the differential pressure cells is plotted against system pressure, with the resulting plots used to describe the pressure response of the transducers.

The response of the differential pressure cells due to ambient temperature is determined from a digital data scan taken at $533 \mathrm{~K}$ and $12150 \mathrm{kPa}$ with. no system flow. The measured transducer outputs are corrected for pressure and compared with the values calculated due only to the density difference between the water inside the loop $(533 \mathrm{~K})$ and outside the loop in the sense lines ( 300 to $311 \mathrm{~K}$ ). 
The difference between the measured pressure corrected value and the calculated value is the thermal drift. After the data scan at $533 \mathrm{~K}$ is made, no more opportunities exist to obtain data with the pump stopped and the system full of fluid; therefore, for lack of later data, the thermal drift calculated from the $533 \mathrm{~K}$ data is assumed to be constant throughout the test.

For some differential pressure measurements, the data scan at $533 \mathrm{~K}$ cannot be used as a reference for thermal drift, so other references are used. The reading from the steam generator discharge venturi (DPU-SG-DISC) is shifted to read zero after flow is stopped. For these detectors, and those having nonlinear pressure sensitivities, the corrections are performed according to the following equations:

$$
F^{\prime}(t)=K F(t)+c_{1} \text { for } t<t_{1} \text { or when no } t_{j} \text { are listed }
$$

for time points $t$, where $t_{1} \leqslant t \leqslant t_{n}$

$$
F^{\prime}(t)=K F(t)+c_{i}+\frac{t-t_{i}}{t_{i+1}-t_{i}}\left(c_{i+1}-c_{i}\right) \text { for } t_{i} \leq t \leq t_{i+1}
$$

where $\mathrm{i}$ takes on values 1 to $n-1$

$$
F^{\prime}(t)=K F(t)+C_{n} \text { for } t>t_{n}
$$

where

$$
\begin{array}{lll}
\mathrm{t} & = & \text { time, } \mathrm{s} \\
\mathrm{F}^{\prime}(\mathrm{t}) & = & \text { corrected data, } \mathrm{kPa} \\
\mathrm{F}(\mathrm{t}) & = & \text { raw data, } \mathrm{kPa} \\
\mathrm{K} & = & \text { scaling factor } \\
\mathrm{C}_{\mathrm{i}} \text { and } \mathrm{t}_{\mathrm{i}} & = & \text { corrections }(\mathrm{kPa}) \text { and time points }(\mathrm{s}) .
\end{array}
$$

The values of the constants are given in Table B-II. 


\begin{tabular}{|c|c|c|c|c|c|c|c|}
\hline $\begin{array}{c}\text { Detector } \\
\text { Identification }\end{array}$ & $K$ & $c_{1}$ & $t_{1}$ & $\mathrm{C}_{2}$ & $t_{2}$ & $c_{3}$ & $t_{3}$ \\
\hline DPU-12-10 & 1 & -6.48 & 0 & -4.14 & 0.01 & 0 & 30 \\
\hline DPU-15-3 & 1 & -4.27 & & & & & \\
\hline DPU- $15-3 L^{\circ}$ & 1 & -4.27 & & & & & \\
\hline $\mathrm{DPB}-23-\mathrm{CN} 1$ & 1 & 114.45 & 0 & 102.73 & 0.01 & 0 & 30 \\
\hline DPB-HN1-43. & 1 & 78.6 & 0 & $55.85^{\prime}$ & 0.01 & 0 & 30 \\
\hline DPV-26-55 & 1 & -1.59 & 0 & -1.03 & 0.01 & 0 & 30 \\
\hline
\end{tabular}

\section{MOMENTUM FLUX MEASUREMENTS}

The temperature sensitivity of drag discs is determined from pretest warmup data taken at 366 and $533 \mathrm{~K}$ with no system flow: The temperature sensitivity is removed hefore the data are converted to momentum flux. The temperature of each transducer is taken from the signal of a nearby fluid or metal temperature thermocouple. Slight corrections for errors in setting the transducer output to zero at ambient conditions are also made at this time. Corrections are made using the following equation:

$$
F^{\prime}(t)=F(t)+C_{n}-T_{1} T(t)=P_{1} P(t)
$$

where

$$
\begin{aligned}
& F^{\prime}(t)=\text { corrected data, DAS volts } \\
& F(t)=\text { raw data, DAS volts } \\
& \mathrm{l}_{1}=\text { temperature sensitivity, LAS volts } / \mathrm{K} \\
& \mathrm{T}(\mathrm{t})=\quad \begin{array}{l}
\text { temperature data from the transduccr uscd for tempera- } \\
\mathrm{C}_{\mathrm{O}}=
\end{array}
\end{aligned}
$$




$$
\begin{aligned}
& \mathrm{P}_{1}=\text { pressure sensitivity, DAS volts } / \mathrm{kPa} \\
& \mathrm{P}(\mathrm{t})=\begin{array}{l}
\text { pressure data from the indicated transducer used for } \\
\text { pressure correction sensitivity, } \mathrm{kPa} .
\end{array}
\end{aligned}
$$

Values of the constants are given in Table B-III.

TABLE B-I II

\begin{tabular}{|c|c|c|c|c|c|}
\hline $\begin{array}{c}\text { Detector } \\
\text { Identification }\end{array}$ & $c_{0}$ & $T_{1}$ & $T(t)^{[a]}$ & $\mathrm{P}_{1}$ & $P(t)^{[c]}$ \\
\hline FDU-1 & 0.095 & 0.002363 & RBU-2 & 0.0001089 & $P V-U P+10$ \\
\hline FDU -5 & 0 & -0.000248 & TFU-6 & $0.000073 \quad 1$ & $P V-U P+10$ \\
\hline FDU- 10 & 0 & 0.000301 & TFU- 10 & & \\
\hline FDU $=13$ & 0.100 & 0.001616 & RBU-14A & 0.0000965 & PU-13 \\
\hline FDB-21 & 0 & -0.001337 & TFB-20 & 0.0000745 & $P B-21$ \\
\hline FDB-23 & 0 & -0.001341 & TFB-23 & 0.0000496 & PB-23 \\
\hline $\mathrm{FDB}-30^{[\mathrm{b}]}$ & 0 & -0.061643 & TFB-30 & & \\
\hline $\mathrm{FDB}-3^{[\mathrm{b}]}$ & -0.085 & 0.000806 & TFB- 37 & & \\
\hline FDB -42 & 0.110 & 0.000905 & TFB- 42 & 0.0000338 & PB-42 \\
\hline FDV-CORE-IN & 0 & 0 & TFV-CORE-IN & & \\
\hline
\end{tabular}

CONTANTS FOR MOMENTUM FLUX

MEASUREMENT CORRECTIONS (TEST S-28-6)

[a] $T(t)$ is the temperature data used for temperature sensitivity correction. The symbols listed identify the thermocouples from which the data are obtained.

[b] Temperature sensitivity corrections were applied as usual; however, because FDB- 30 and FDB-37 are mounted horizontally, during blowdown they were partially filled with subcooled water which affected the temperature sensitivity. Therefore, the temperature sensitivity correction is more uncertain than that applied to other temperature sensitivity corrections.

[c] $P(t)$ is the pressure data from the indicated transducer used for pressure sensitivity correction. The symbols listed identify the pressure transducers from which the data are obtained. 


\section{DENSITY MEASUREMENTS}

Density calculations are based on the voltage output of the photomultiplier tubes in the gamma-attenuation densitometer assemblies. The equation used for converting voltage to density is as follows:

$$
\rho=(1 / C) \ln \{D /[A F(t)+B]\}
$$

where

o. $=$ the density in $\mathrm{kg} / \mathrm{m}^{3}$

$\mathrm{C}=\mathrm{a}$ constant based on the length of the gamma beam path

$\mathrm{D}=\mathrm{a}$ theoretical voltage for zero attenuation inside the vessel

A $=$ an amplification factor

$\mathrm{B}^{\prime \prime} \quad=\quad$ a biasing factor

$F(t)^{-}=\quad$ the transducer voltage output.

Constants A and B are adjusted to match the final data to density values calculated from measured pressure and temperature values at the preblowdown and postdrain conditions, effectively giving the data an in-place calibration. The values of the constants for various transducers are given in Table B-IV.

The density measurements GVLP-172HZ and GV-COR-150HZ use amplifiers which precalculate the logarithm function, and hence have a simpler conversion formula:

$$
\begin{array}{ll}
\text { GVLP-172HZ } & \rho=-22.99 F(t)-587.86 \\
\text { GV-COR-15OHZ } & \rho=-60.98 F(t)-2908.87
\end{array}
$$




\begin{tabular}{|c|c|c|c|c|}
\hline $\begin{array}{c}\text { Detector } \\
\text { Identification } \\
\end{array}$ & A & B & C & $D$ \\
\hline GU-1T & 1.057 & -0.122 & 0.0004 & 6.68 \\
\hline GU-1B & 0.9 .98 & 0.258 . & 0.0005 & 7.27 \\
\hline GU-5VR & 1.041 & -0.040 & 0.0006 & 4.67 \\
\hline GU-10VR & 1.078 & -0.318 & 0.0006 & 6.95 \\
\hline GU-13VR & 0.894 & 0.263 & 0.0006 & 2 \\
\hline GU-15T & 1.029 & 0.057 & 0.0004 & 6.65 \\
\hline GU-15B & 1.068 & -0.220 & 0.0005 & 6.75 \\
\hline$G B-21 T$ & 1.047 & -0.056 & 0.0004 & 6.63 \\
\hline$G B-21 B$ & 1.078 & -0.329 & 0.0005 & 7.68 \\
\hline$G B-23 V R$ & 0.993 & +0.281 & 0.0004 & 6.65 \\
\hline GB-30T & 1.130 & -0.531 & 0.0004 & 6.04 \\
\hline$G B-30 B$ & 1.033 & +0.005 & 0.0005 & 7.89 \\
\hline $\mathrm{GB}-37 \mathrm{HZ}$ & 1.616 & -2.340 & 0.0006 & 4.21 \\
\hline$G B-42 V R$ & 1.072 & -0.297 & 0.0004 & 9.22 \\
\hline CVLP $-165 \mathrm{HZ}$ & 1.020 & -0.067 & 0.0015 & 9.89 \\
\hline GU-PRIZE & 1.131 & -0.099 & 0.0006 & 1.05 \\
\hline
\end{tabular}

Some density measurements are obtained using a two-beam gamma densitometer which operates on the same basic principle of gamma attenuation as does the single-beam gamma densitometer. Each beam originates from the same gamma source and is allowed to pass through separate portions of the piping cross-sectional flow area to obtain an average density measurement in that particular region. The geometrical relationship of the gamma beam path through the piping and geometrically related variables used for processing of data from a two-beam gamma densitometer are shown in Figure B-1. 


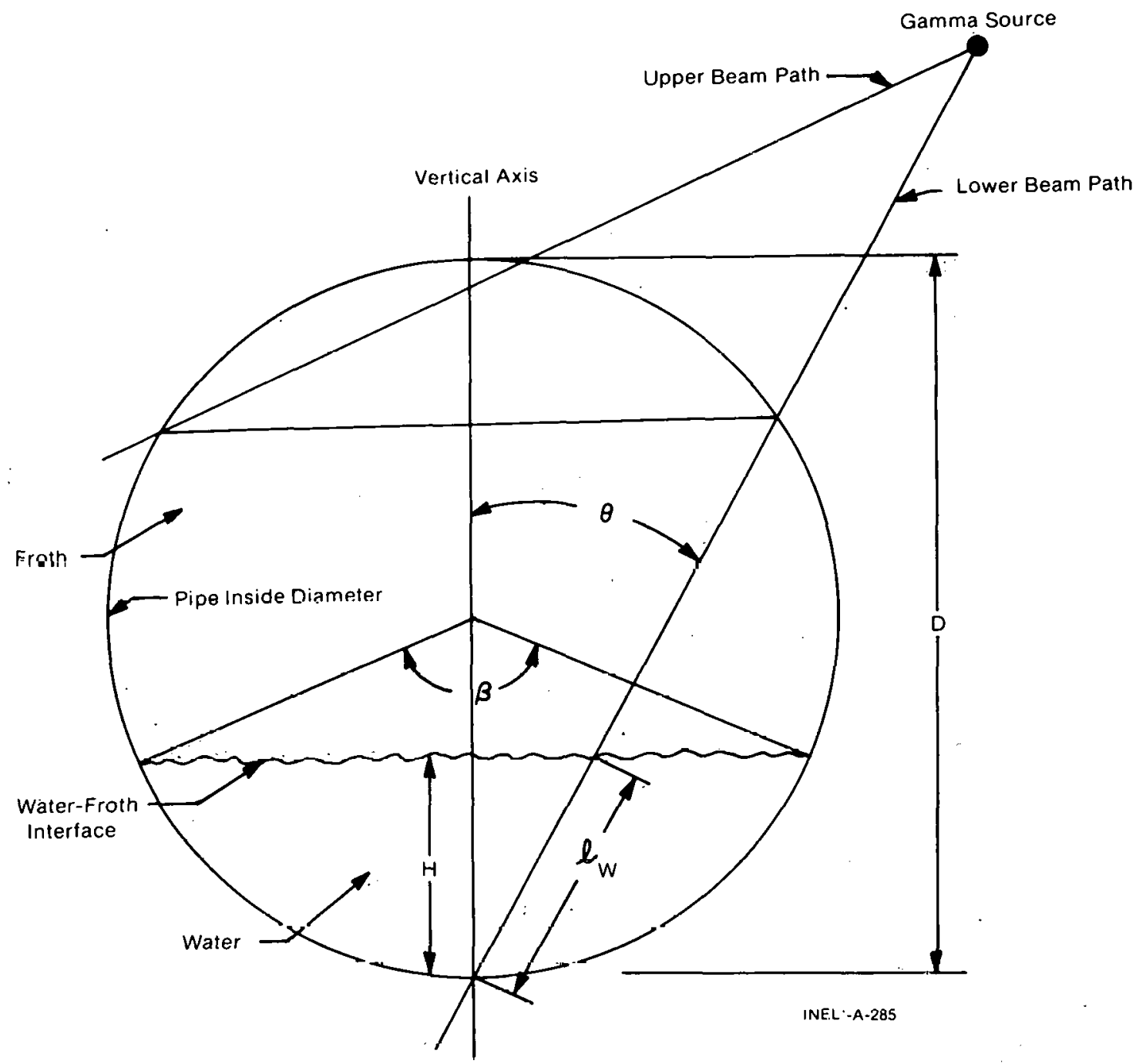

Flg. B-1 Geometry used for processing of density data obtained from two-beam gamma densitometers.

The average density measured by each individual gamma beam is obtained using the same equation as is used for the single-beam gamma densitometers. Values for the constants for the single-beam density measurements obtained with the two-beam gamma densitometers are presented in Table B-IV along with the constants for single-beam gamma densitometers.

In the Semiscale Mod-1 system, two-beam gamma densitometers provide added information which allows the calculation of a better average density than that obtained from a single beam. A mathematical model is used for processing the two-beam data to obtain the improved average density information. The processing method used is based on a froth-water model coupled with information from the two individual gamma beams and related beam path and piping cross-sectional geometry. The resulting information is recorded and reported under the density measurement identification ending with a " $\mathrm{C}$ ", for example, GB-21C. 
The use of the froth-water model for obtaining average density from a two-beam gamma densitometer is based on observations indicating that flow regimes in the Semiscale Mod-1 system can be modeled by a layer of water on the bottom of the pipe with a degree of froth on the surface. For homogeneous flow conditions such as all froth or all liquid the model remains valid. At any point in time slug flow is also modeled. The froth-water model does not model annular or inverted annular flows very well. However, these flows are not expected to exist for significant portions of a Semiscale Mod-1 system blowdown in horizontal piping. Density gradients from the top to the bottom of the pipe may exist showing no distinct location change from water to froth. This flow is neither totally homogeneous nor stratified, but the froth-water model does provide an adequate approximation of the average density characteristic of this flow pattern.

The average density obtained by using the gamma beam geometry shown in Figure B-1 and by applying the froth-water model is given by

$$
\bar{\rho}=\alpha_{f} \rho_{1}+\left(1-\alpha_{f}\right) \rho_{w} \mathrm{~kg} / \mathrm{m}^{3}
$$

where

$$
\begin{aligned}
& \bar{\rho}=\text { average cross-sectional density } \\
& \rho 1=\begin{array}{l}
\text { average density measured by the upper gamma beam } \\
\text { (measures the froth density) }
\end{array} \\
& \rho_{\mathrm{W}}=\text { density of liquid water (at local system conditions) } \\
& \alpha_{\mathrm{f}}=1+(1 / 2 \pi)(\sin \beta-\beta)=\text { froth fraction. }
\end{aligned}
$$

The angle which $\beta$ represents is shown in Figure B-1. Values for $\beta$ are obtained as follows:

$$
B=2 \cos ^{-1}(1-2 h)
$$

where

$$
\mathrm{h}=\frac{\mathrm{H}}{\mathrm{D}}=\cos ^{2} \theta \frac{\rho_{2}-\rho_{1}}{\rho_{\mathrm{w}}-\rho_{1}}
$$

where

$$
\begin{aligned}
& \mathrm{H}=\ell_{\mathrm{W}} \cos \theta\left(\ell_{\mathrm{W}} \text { and } \theta \text { are defined in Figure } \mathrm{B}-1\right) \\
& \mathrm{D}=\text { piping inside diameter } \\
& \rho_{2}=\text { the average density measured by the lower gamma beam. }
\end{aligned}
$$


Average density is not calculated using the two-beam froth-water model when the angle $\theta$ is not favorable due to system hardware restrictions in positioning the source. The froth-water model requires separate density sampling in both the upper portions of the piping cross section. 
APPENDIX C

SELECTED DATA WITH ESTIMATED TOTAL ERROR

BANDS FROM SEMISCALE MOD-1 TEST S-28-6 


\section{THIS PAGE \\ WAS INTENTIONALLY \\ LEFT BLANK}




\title{
APPENDIX C
}

\section{SELECंTED DATA WITH ESTIMATED TOTAL ERROR}

\author{
BANDS FROM SEMISCALE MOD-1 TEST S-28-6
}

Analysis has been performed on selected data from Test S-28-6 to provide a guide to the uncertainty associated with data measurements in the Semiscale Mod-1 system. The end result of the analysis is presented as error bands about the measured data which represent a $95 \%$ confidence level.

The error bands are obtained by combining uncertainties obtained from analysis of the data itself (random error) and engineering analysis of the measurement system (engineering error). The procedure by which error bands were established for the data presented in this appendix is described in the following paragraphs.

The data trace under analysis was empirically fitted with a linear difference equation, which was subject to a white noise input at each sampling time point. The objective of the empirical fitting procedure was to characterize the white noise, which was taken to represent the random error. The procedures for fitting the difference equation are discussed in depth in Reference C-1. A data trace was often segmented and different equations were fitted to each segment with statistical correlations between successive observations accounted for by the fitting procedure. The white noise input was assumed to arise from a normally distributed population. The standard deviation of the white noise, as found during the fitting procedures, was taken as an estimate of the random error standard deviation and is shown in Table C-I. The data traces of the error band analysis are shown in Figures C-1 through $\mathrm{C}-45$.

\section{TABLE C-I}

RANDOM ERROR VARIANCE (TEST S-28-6)

\begin{tabular}{|c|c|c|c|c|}
\hline Measurement & $\begin{array}{c}\text { Random Error } \\
\text { Variance } \\
\sigma_{\mathrm{R}} \\
\end{array}$ & $\begin{array}{l}\text { Period of } \\
\text { Application } \\
\text { (s) }\end{array}$ & Figure & Comments \\
\hline TFB-23 & $\begin{array}{r}11.439 \\
0.991\end{array}$ & $\begin{array}{c}0 \text { to } 75 \\
75 \text { to } 600\end{array}$ & $C-1$ & \\
\hline TFV-ANN-35A & $\begin{array}{r}1.2 .636 \\
0.786\end{array}$ & $\begin{array}{c}0 \text { to } 71 \\
71 \text { to } 600\end{array}$ & $C-2$ & \\
\hline TFV-LP-2 & $\begin{array}{r}25.603 \\
0.242\end{array}$ & $\begin{array}{rll}0 & \text { to } & 69 \\
69 & \text { to } & 600\end{array}$ & $C-3$ & \\
\hline
\end{tabular}


TABLE C-I (continued)

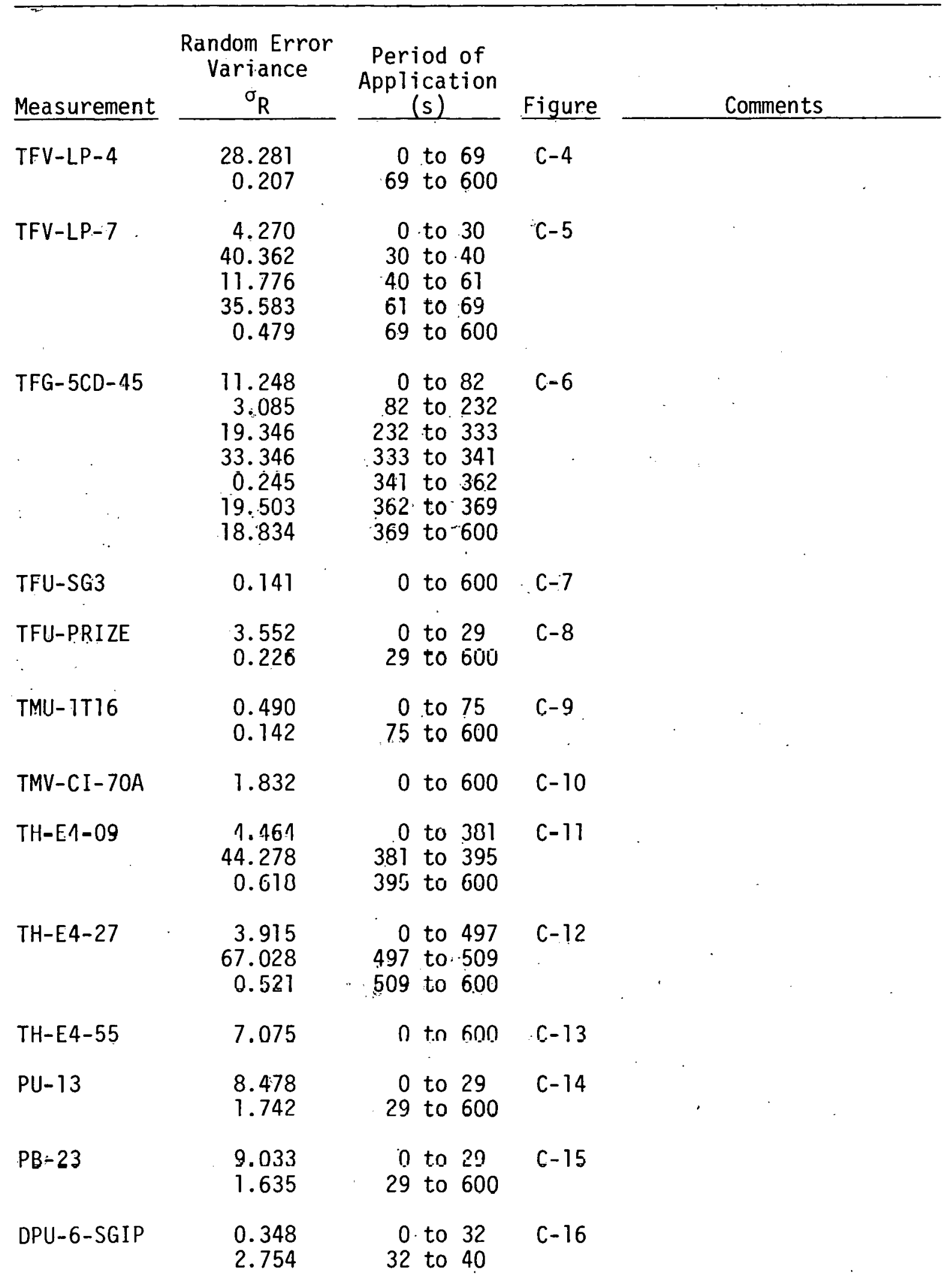


TABLE C-I (continued)

\begin{tabular}{|c|c|c|c|c|}
\hline Measurement & $\begin{array}{c}\text { Random Error } \\
\text { Variance } \\
\sigma_{\mathrm{R}} \\
\end{array}$ & $\begin{array}{c}\text { Period of } \\
\text { Application } \\
\text { (s) } \\
\end{array}$ & Figure & Comments \\
\hline $\begin{array}{l}\text { DPU-6-SGIP } \\
\text { (continued) }\end{array}$ & $\begin{array}{l}0.281 \\
0.084\end{array}$ & $\begin{array}{l}40 \text { to } 70 \\
70 \text { to } 600\end{array}$ & & \\
\hline DPU-SGOP-7 & $\begin{array}{l}0.908 \\
0.059\end{array}$ & $\begin{aligned} 0 & \text { to } 75 \\
75 & \text { to } 600\end{aligned}$ & $c-17$ & . \\
\hline DPU-7-10 & $\begin{array}{l}0.159 \\
0.053\end{array}$ & $\begin{array}{rrl}0 & \text { to } & 29 \\
29 & \text { to } & 600\end{array}$ & $C-18$ & \\
\hline DPU-12-10 & $\begin{array}{l}0.633 \\
0.119\end{array}$ & $\begin{array}{c}0 \text { to } 41 \\
41 \text { to } 600\end{array}$ & $C-19$ & \\
\hline DPU-12-15 & $\begin{array}{l}0.188 \\
0.037\end{array}$ & $\begin{array}{c}0 \text { to } 71 \\
71 \text { to } 600\end{array}$ & $C-20$ & \\
\hline FTU-1 & $\begin{array}{r}122.139 \\
38.099\end{array}$ & $\begin{array}{rll}0 & \text { to } & 67 \\
67 & \text { to } & 600\end{array}$ & $C-21$ & \\
\hline FTU- 15 & $\begin{array}{l}24.299 \\
29.946 \\
38.026 \\
61.669 \\
16.553\end{array}$ & $\begin{array}{r}0 \text { to } 59 \\
59 \text { to } 70 \\
70 \text { to } 91 \\
91 \text { to } 99 \\
99 \text { to } 600\end{array}$ & $C-22$ & \\
\hline FTU-HPIS & $\begin{array}{l}0.405 \\
0.179\end{array}$ & $\begin{array}{r}0 \text { to } 101 \\
101 \text { to } 600\end{array}$ & $C-23$ & 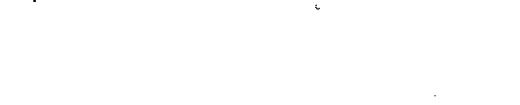 \\
\hline FTU-LPIS & $\begin{array}{l}0.937 \\
0.634 \\
0.209 \\
0.006\end{array}$ & $\begin{array}{l}0 \text { to } 18 \\
18 \text { to } 26 \\
26 \text { to } 67 \\
67 \text { to } 600\end{array}$ & $C-24$ & . \\
\hline FTU-ACC1 & 2.993 & 0 to 90 & $C-25$ & $\begin{array}{l}\text { Error bands are not pre- } \\
\text { sented from } t=90 \text { to } \\
t=600 \mathrm{~s}[\mathrm{a}] .\end{array}$ \\
\hline FTV $-40 A$ & $\begin{array}{l}7.841 \\
1.489\end{array}$ & 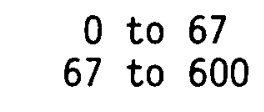 & $C-26$ & . \\
\hline FTV-40M & $\begin{array}{l}7.479 \\
0.961\end{array}$ & 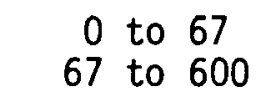 & $C-27$ & . \\
\hline GU-1T & $\begin{array}{l}0.839 \\
0.318\end{array}$ & $\begin{aligned} 0 & \text { to } 29 \\
29 & \text { to } 600\end{aligned}$ & $C-28$ & \\
\hline
\end{tabular}


TABLE C-I (continued)

\begin{tabular}{|c|c|c|c|c|c|}
\hline Measurement & $\begin{array}{c}\text { Random Error } \\
\text { Variance } \\
{ }^{\sigma_{R}} \\
\end{array}$ & $\begin{array}{l}\text { Period of } \\
\text { Application } \\
\text { (s) } \\
\end{array}$ & Figure & Comments & \\
\hline GU-1B & $\begin{array}{l}3.384 \\
0.677 \\
0.925 \\
1.394\end{array}$ & $\begin{array}{r}0 \text { to } 18 \\
18 \text { to } 61 \\
61 \text { to } 254 \\
254 \text { to } 600\end{array}$ & $C-29$ & & \\
\hline GU-1C & 0.559 & 0 to 600 & $C-30$ & & . \\
\hline GII-1NVR & $\begin{array}{l}1.644 \\
0.284\end{array}$ & 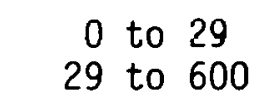 & $C-31$ & - & \\
\hline$G U-1.5 T$ & $\begin{array}{l}9.991 \\
2.394 \\
6.669 \\
0.606\end{array}$ & $\begin{array}{r}0 \text { to } 24 \\
24 \text { to } 59 \\
59 \text { to } 67 \\
67 \text { to } 600\end{array}$ & $c-32$ & & \\
\hline$G U-7.5 B$ & $\begin{array}{l}7.545 \\
1.690 \\
9.545 \\
0.187 \\
1.996 \\
1.118\end{array}$ & $\begin{array}{r}0 \text { to } 23 \\
23 \text { to } 59 \\
59 \text { to } 70 \\
70 \text { to } 91 \\
91 \text { to } 98 \\
98 \text { to } 600\end{array}$ & $C-33$ & & \\
\hline GU- $15 \mathrm{C}$ & $\begin{array}{l}7.292 \\
1.769 \\
1.203 \\
0.675\end{array}$ & $\begin{array}{r}0 \text { to } 24 \\
24 \text { to } 59 \\
59 \text { to } 70 \\
10 \text { to } 600\end{array}$ & $C-34$ & & . \\
\hline$G B-23 V R$ & $\begin{array}{l}8.358 \\
1.065\end{array}$ & 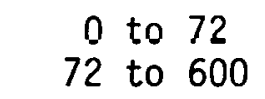 & $C-35$ & & \\
\hline $\mathrm{GV}-\mathrm{COR}-150 \mathrm{HZ}$ & $\begin{array}{r}1.708 \\
5.777 \\
15.801 \\
1.447\end{array}$ & $\begin{array}{r}0 \text { to } 85 \\
85 \text { to } 127 \\
127 \text { to } 305 \\
305 \text { to } 600\end{array}$ & $c-36$ & & \\
\hline GVLP-165HZ & $\begin{array}{l}5.744 \\
0.461 \\
1.841\end{array}$ & $\begin{array}{l}0 \text { to } 19 \\
19 \text { to } 61 \\
61 \text { to } 600\end{array}$ & $C-37$ & & \\
\hline GVLP- $172 \mathrm{HZ}$ & $\begin{array}{l}4.258 \\
5.720 \\
1.506 \\
0.437\end{array}$ & $\begin{array}{r}0 \text { to } 61 \\
61 \text { to } 69 \\
69 \text { to } 751 \\
151 \text { to } 600\end{array}$ & $C-38$ & . & \\
\hline
\end{tabular}


TABLE C-I (continued)

\begin{tabular}{|c|c|c|c|c|}
\hline Measurement & $\begin{array}{c}\text { Random Error } \\
\text { Variance } \\
\sigma_{\mathrm{R}} \\
\end{array}$ & $\begin{array}{l}\text { Period of } \\
\text { Application } \\
\text { (s) } \\
\end{array}$ & Figure & Comments \\
\hline $\begin{array}{l}\text { FTU-1, } \\
\text { GU-1C }\end{array}$ & 0.772 & 0 to 42 & $C-39$ & $\begin{array}{l}\text { Only short-term (0 to } 42 \mathrm{~s}) \\
\text { error bands reported. }\end{array}$ \\
\hline $\begin{array}{l}\text { FDU-5, } \\
\text { GU-5VR }\end{array}$ & $\begin{array}{l}0.533 \\
0.161\end{array}$ & $\begin{array}{r}0 \text { to } 29 \\
29 \text { to } 42\end{array}$ & $C-40$ & $\begin{array}{l}\text { Only short-term (0 to } 42 \mathrm{~s}) \\
\text { error bands reported. }\end{array}$ \\
\hline $\begin{array}{l}\text { FTU-13, } \\
\text { GU-13VR }\end{array}$ & 1.490 & 0 to 42 & $C-41$ & $\begin{array}{l}\text { Onty short-term (0 to } 42 \mathrm{~s}) \\
\text { error bands reported. }\end{array}$ \\
\hline $\begin{array}{l}\text { FTU-15, } \\
\text { GU-15C }\end{array}$ & 1.560 & 0 to 42 & $C-42$ & $\begin{array}{l}\text { Only short-term ( } 0 \text { to } 42 \mathrm{~s} \text { ) } \\
\text { error bands reported. }\end{array}$ \\
\hline $\begin{array}{l}\text { FDB-21, } \\
\text { GB-21C }\end{array}$ & 2.803 & 0 to 42 & $C-43$ & $\begin{array}{l}\text { Only short-term ( } 0 \text { to } 42 \mathrm{~s} \text { ) } \\
\text { error bands reported. }\end{array}$ \\
\hline $\begin{array}{l}\text { FTB-21, } \\
\text { GB-21C }\end{array}$ & 3.426 & 0 to 42 & $C-44$ & $\begin{array}{l}\text { Only short-term ( } 0 \text { to } 42 \mathrm{~s} \text { ) } \\
\text { error bands reported. }\end{array}$ \\
\hline $\begin{array}{l}\text { FTV-CORE-IN, } \\
\text { GV-COR- } 150 \mathrm{HZ}\end{array}$ & $\begin{array}{l}0.497 \\
1.465 \\
5.486\end{array}$ & $\begin{array}{r}0 \text { to } 123 \\
123 \text { to } 205 \\
205 \text { to } 600\end{array}$ & $C-45$ & \\
\hline $\begin{array}{l}\text { [a] Error b } \\
\text { constan } \\
\text { tion, } \\
\text { in meas }\end{array}$ & $\begin{array}{l}\text { ands are not } \\
t \text { reading due } \\
\text { (b) sensor dr } \\
\text { urement canno }\end{array}$ & $\begin{array}{l}\text { represented in } \\
\text { to one of the } \\
\text { opout, or (c) } \\
\text { t be modeled }\end{array}$ & $\begin{array}{l}\text { this } r \\
\text { follow } \\
\text { sensor } \\
\text { nder the }\end{array}$ & $\begin{array}{l}\text { gion. The sensor gave a } \\
\text { ng: (a) sensor satura- } \\
\text { eadband. Random error } \\
\text { se conditions. }\end{array}$ \\
\hline
\end{tabular}




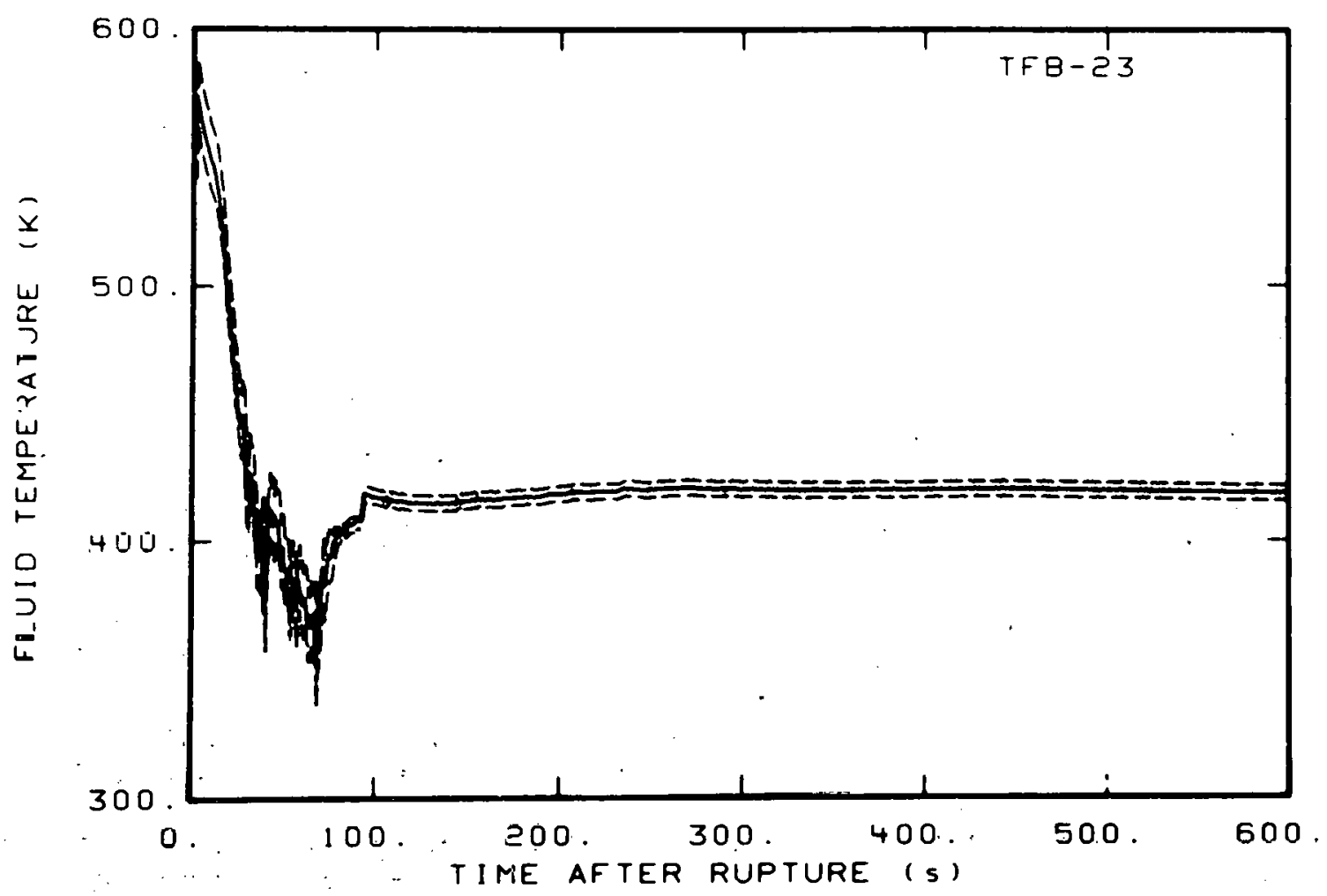

Fig. C-1 Fluid temperature in broken loop (TFB-23).

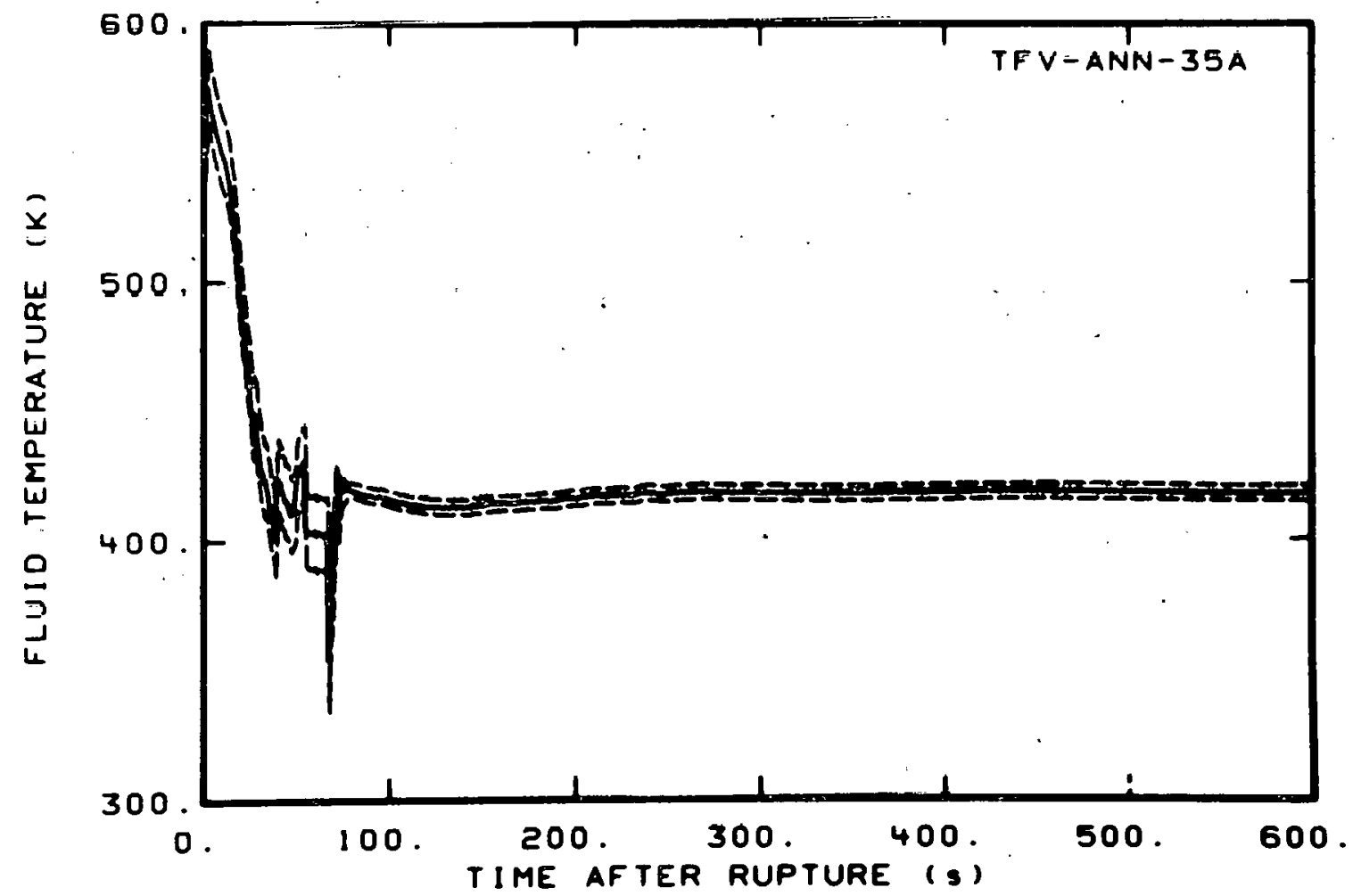

Fig. C-2 Fluid temperature in downcomer annulus (TFV-ANN-35A). 


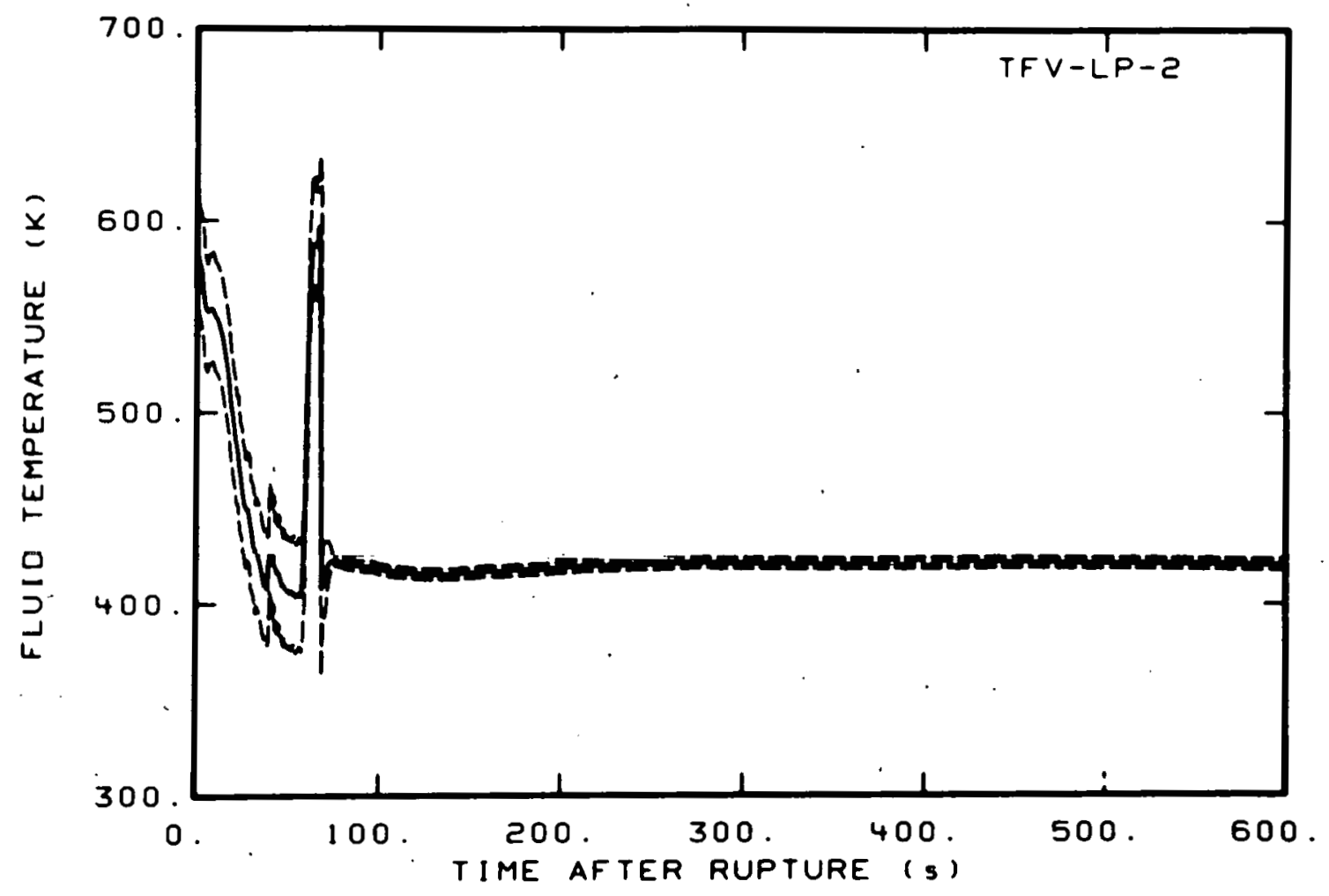

Fig. C-3 Fluid temperature in lower plenum (TFV-LP-2).

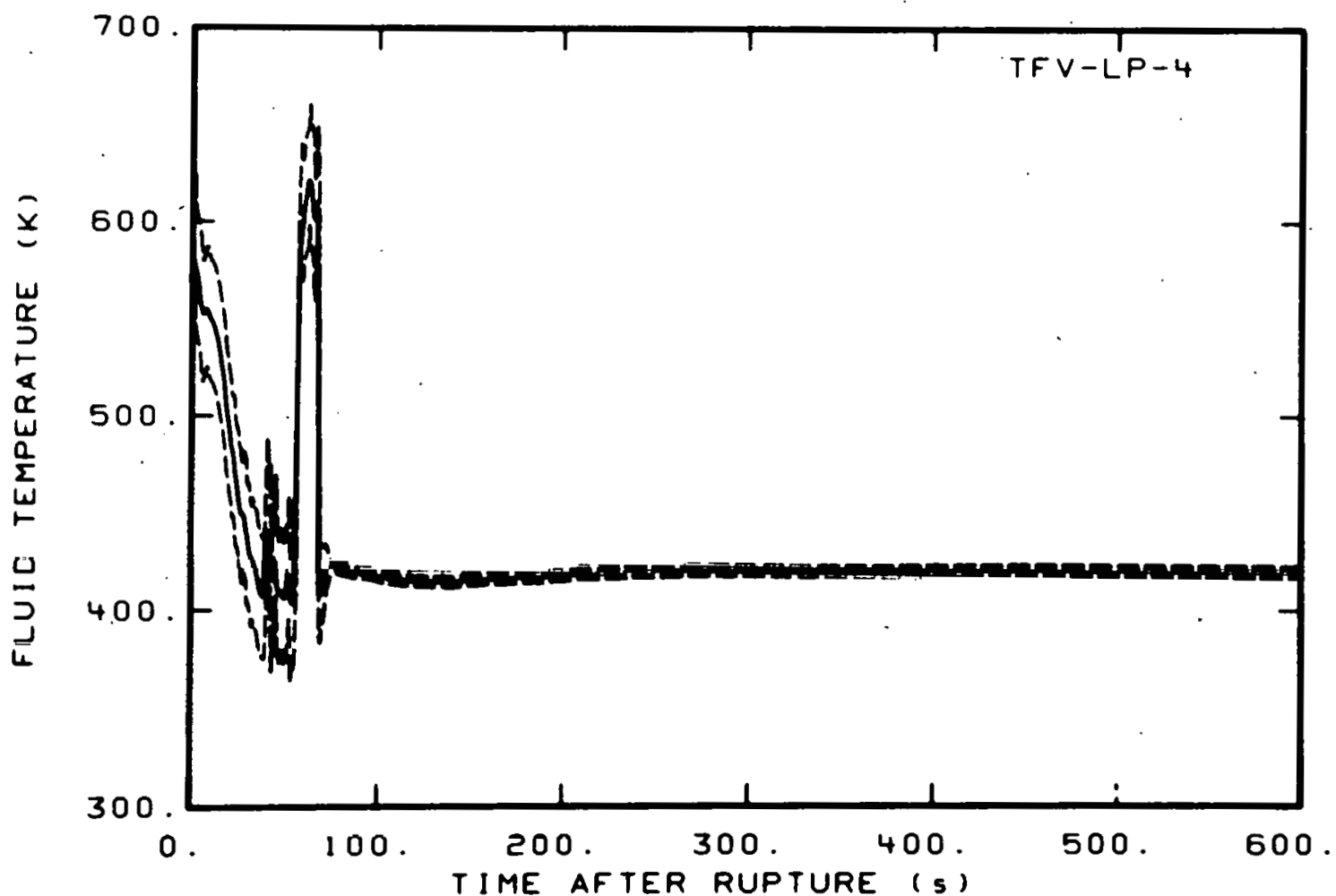

Fig. C.-4 Fluid temperature in lower plenum (TFV-LP-4). 


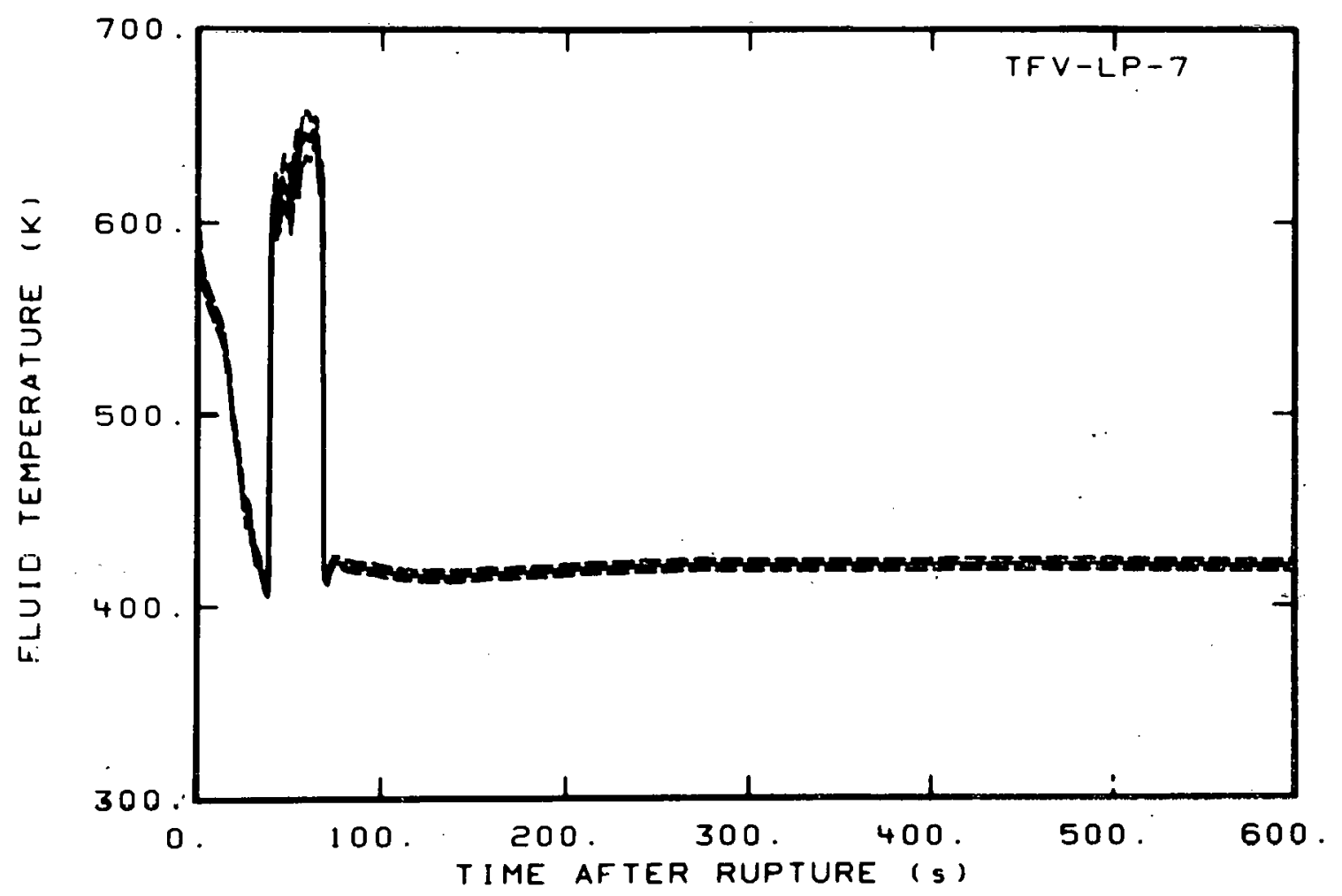

Fig. C-5 Fluid temperature in lower plenum (TFV-LP-7).

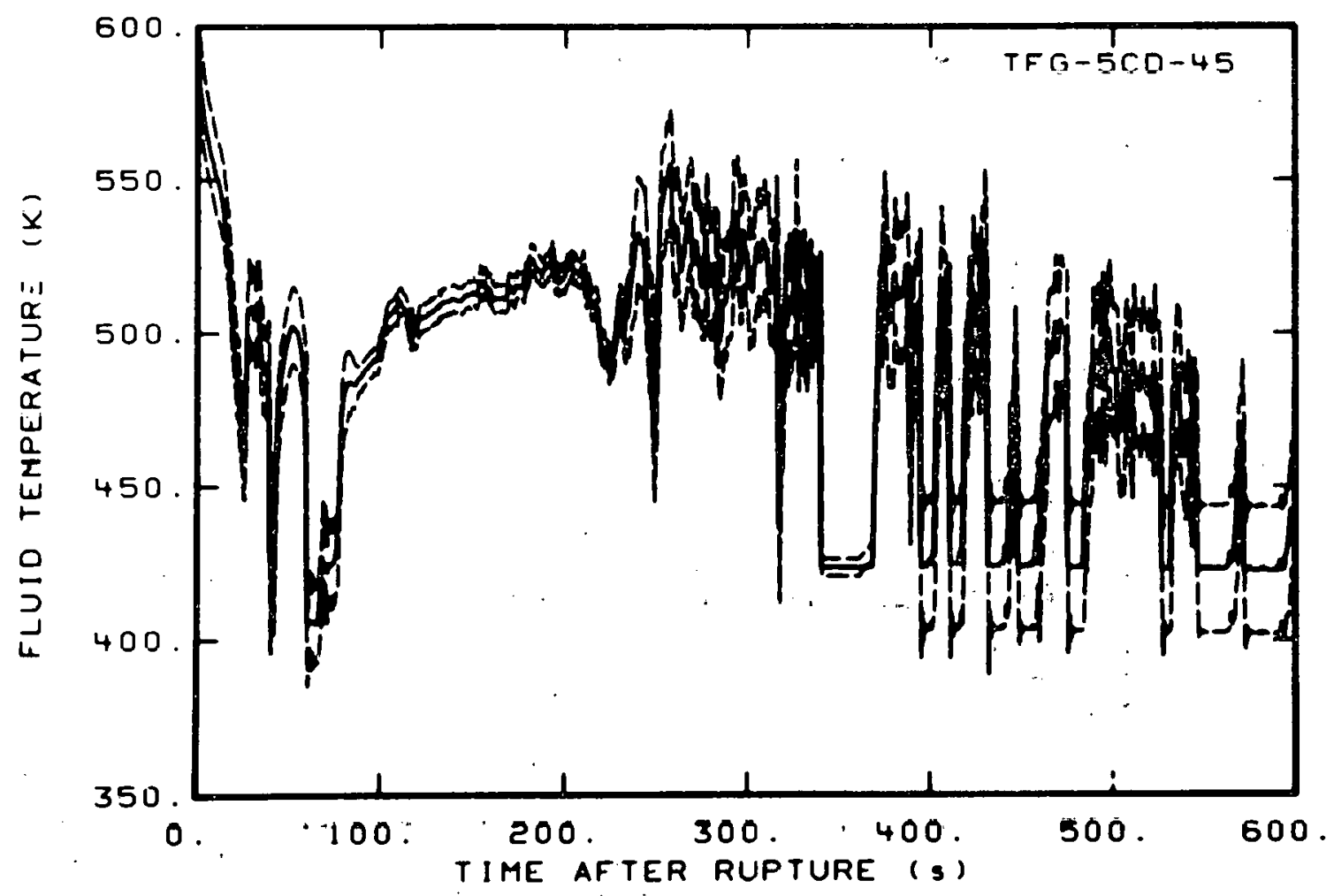

Fig. C-6 Fluid temperature in core, Grid Spacer 5 (TFG-5CD-45). 


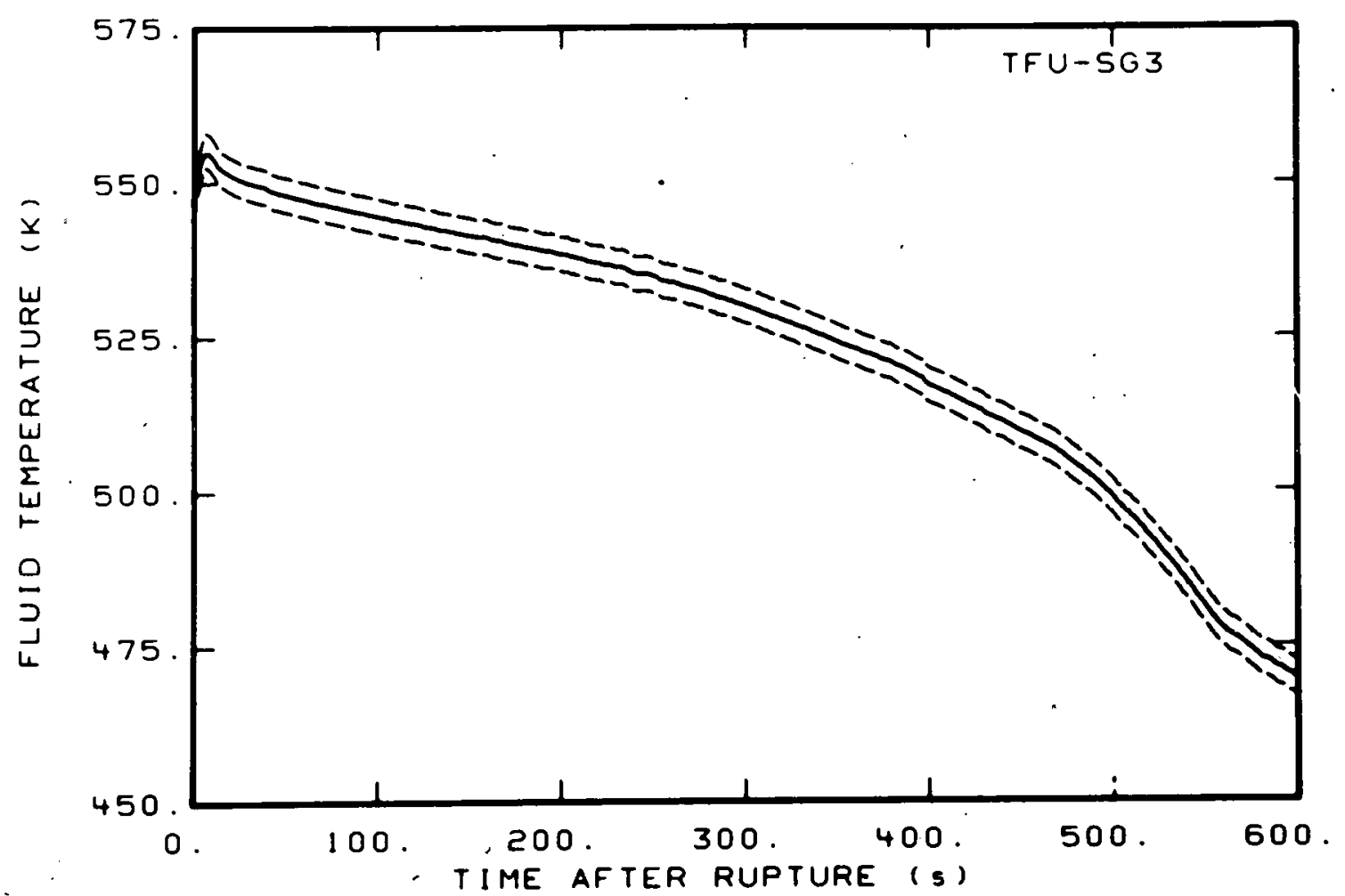

Fig. C-7 Fluid temperature in steam generator, secondary side (TFU-SG3).

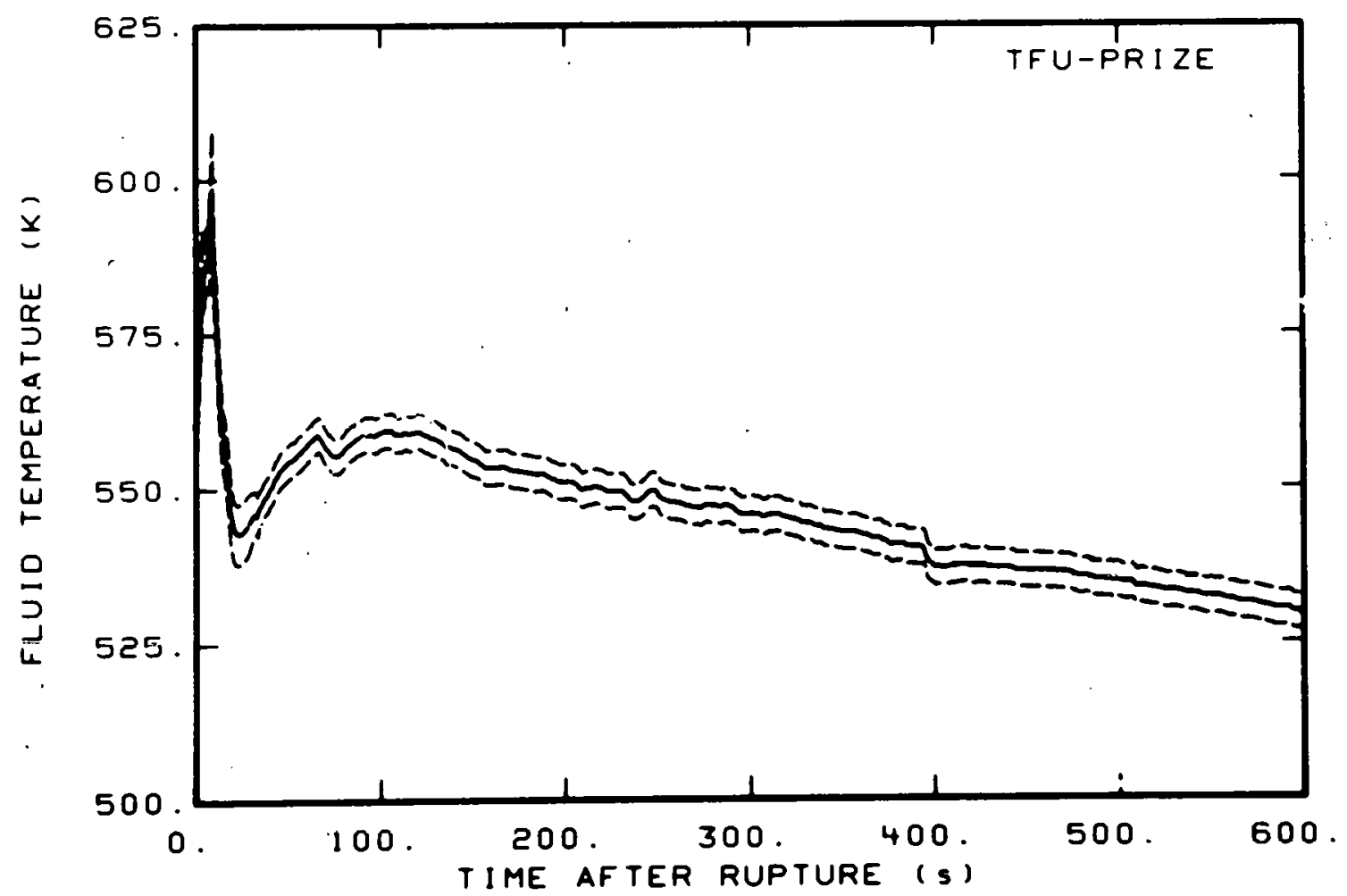

Fig. C-8 Fluid temperature in pressurizer surge line (TFU-PRIZE). 


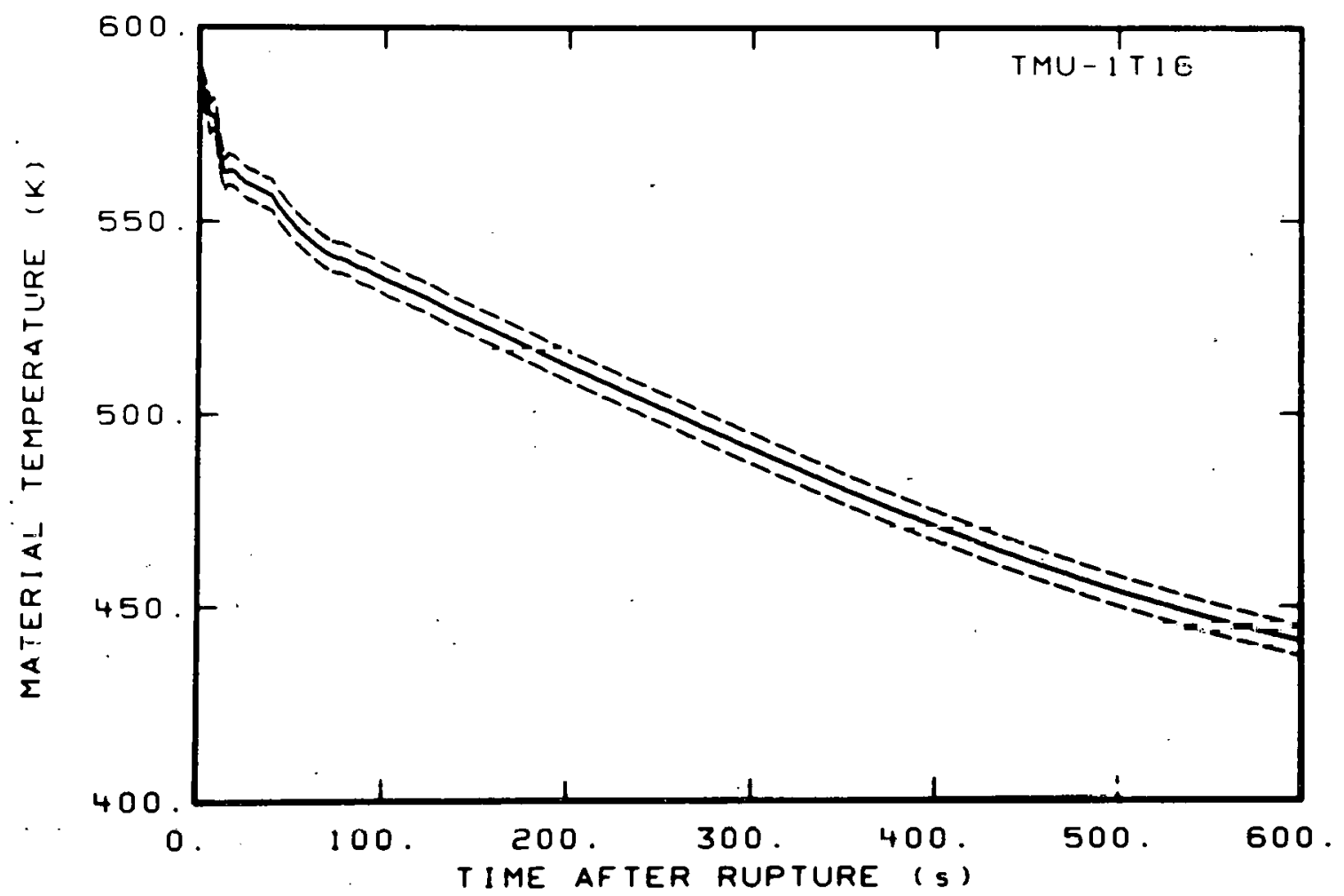

Fig. C-9 Material temperature in intact loop. (TMU-1T16).

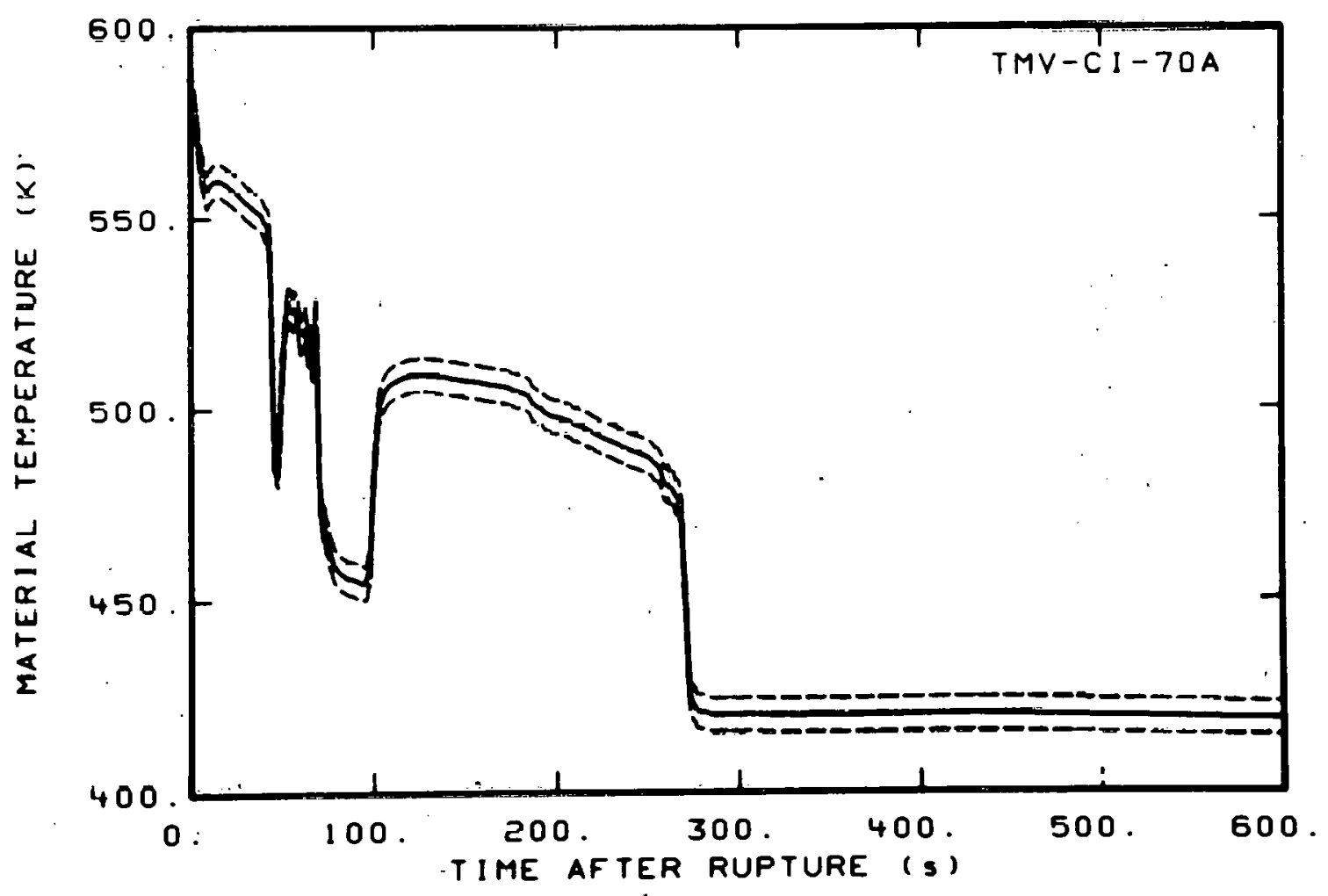

Fig. C-10 Material temperature in vessel filler (TMV-CI-70A). 


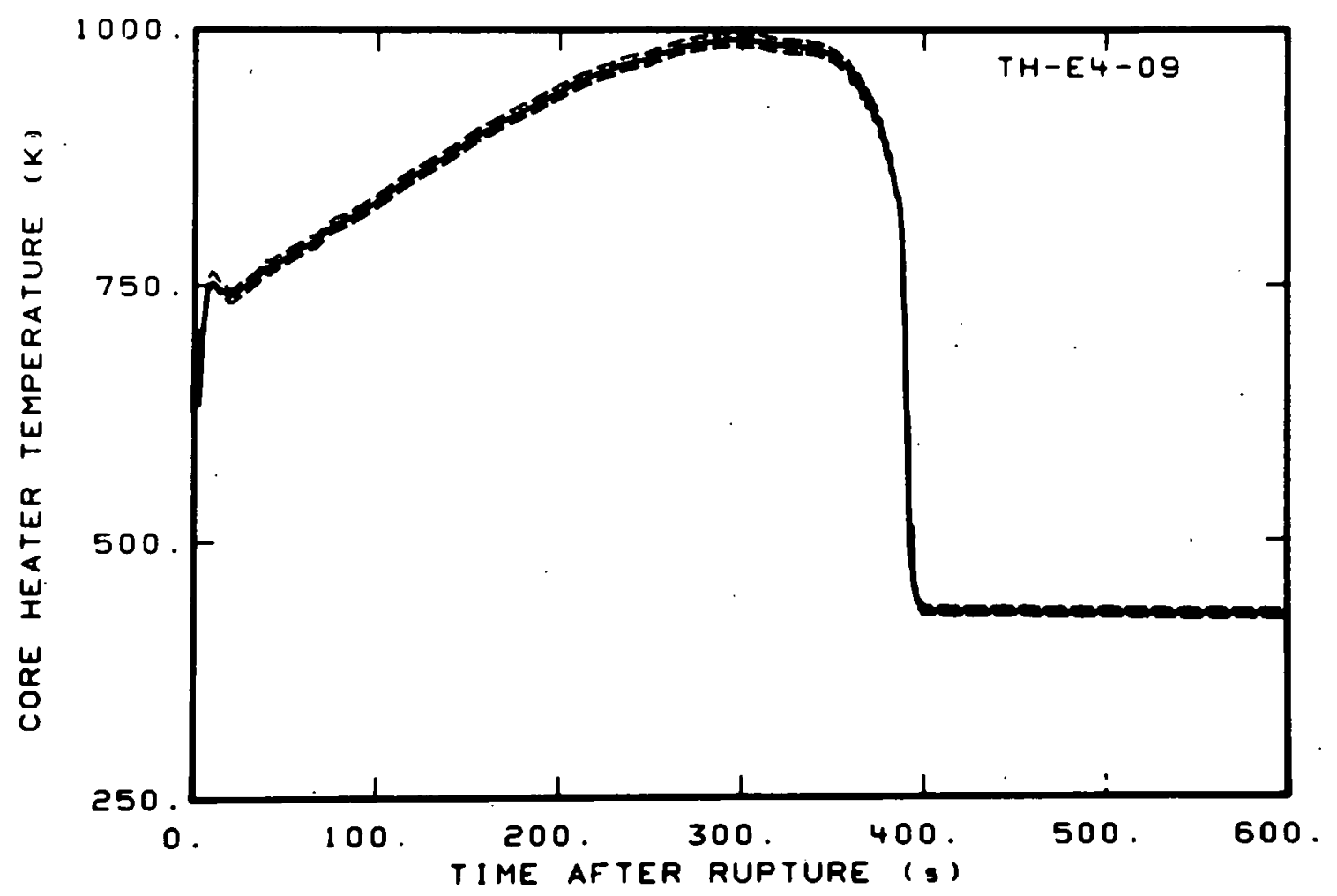

Fig. C-11 Core heater temperature, Rod E-4 (TH-E4-09).

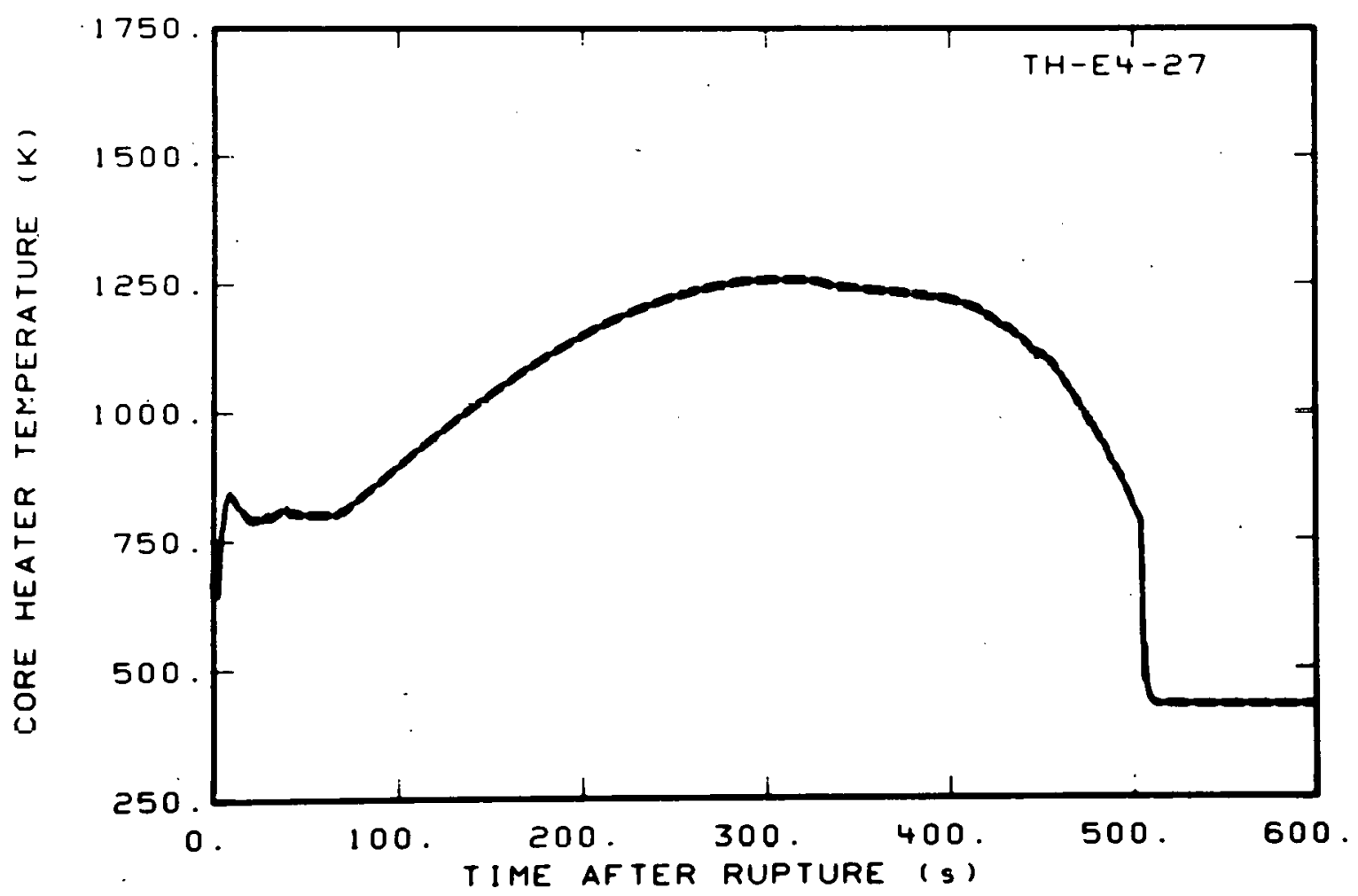

Fig. C-12 Core heater temperature, Rod E-4 (TH-E4-27). 


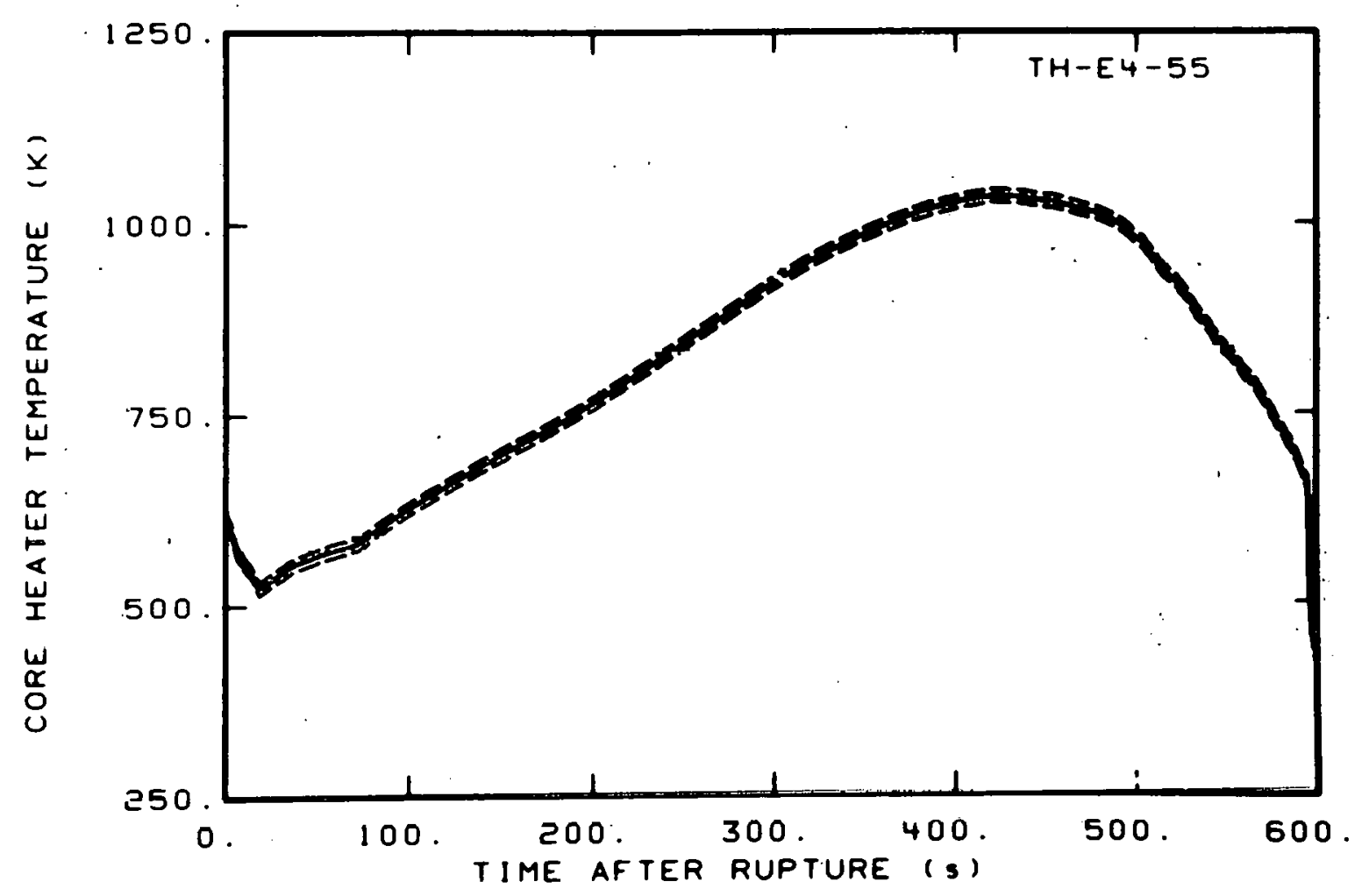

Fig. C-13 Core heater temperature, Rod E-4 (TH-E4-55).

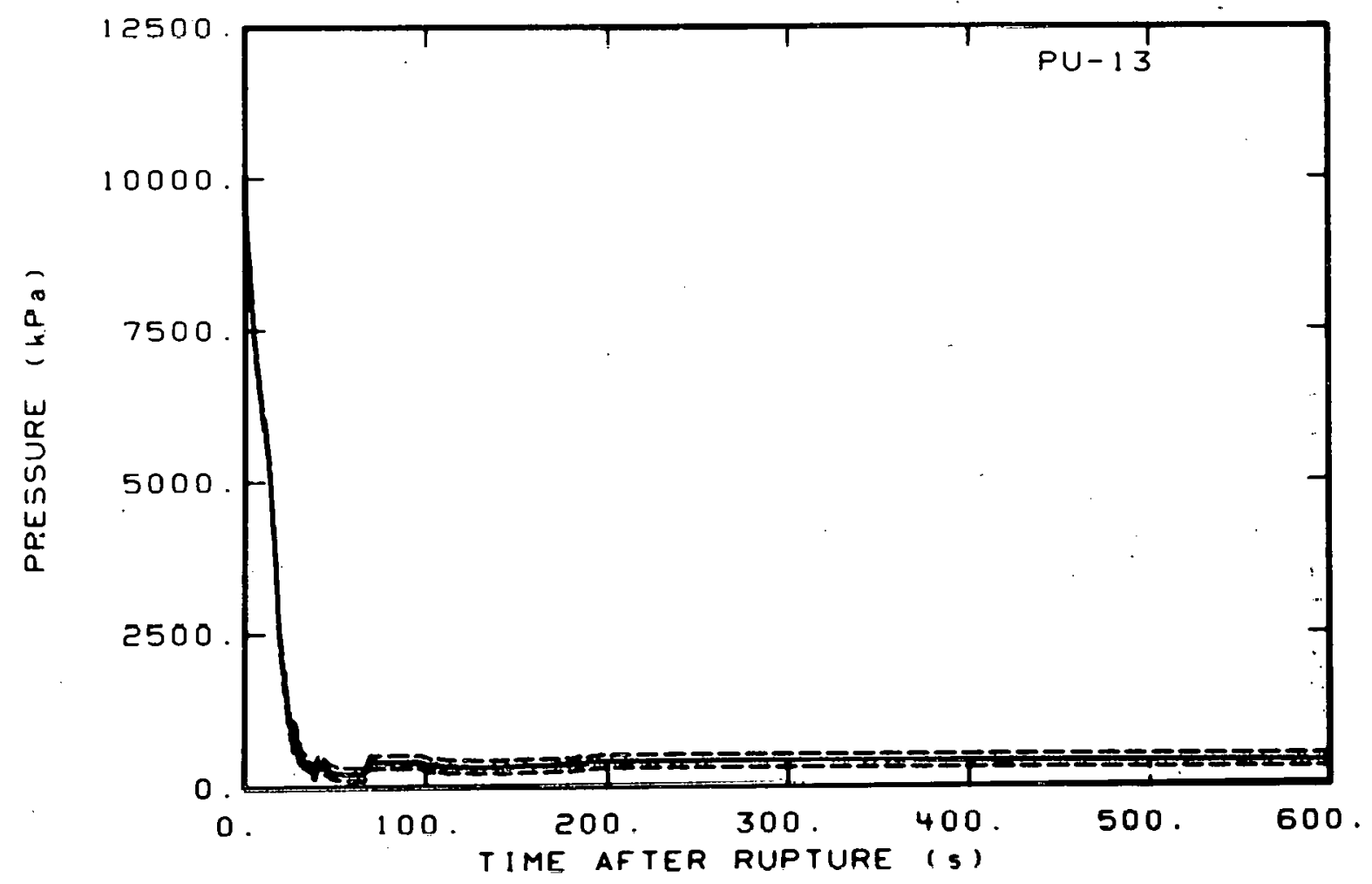

Fig. C-14 Pressure in intact 1oop, Spool 13.(PU-13). 


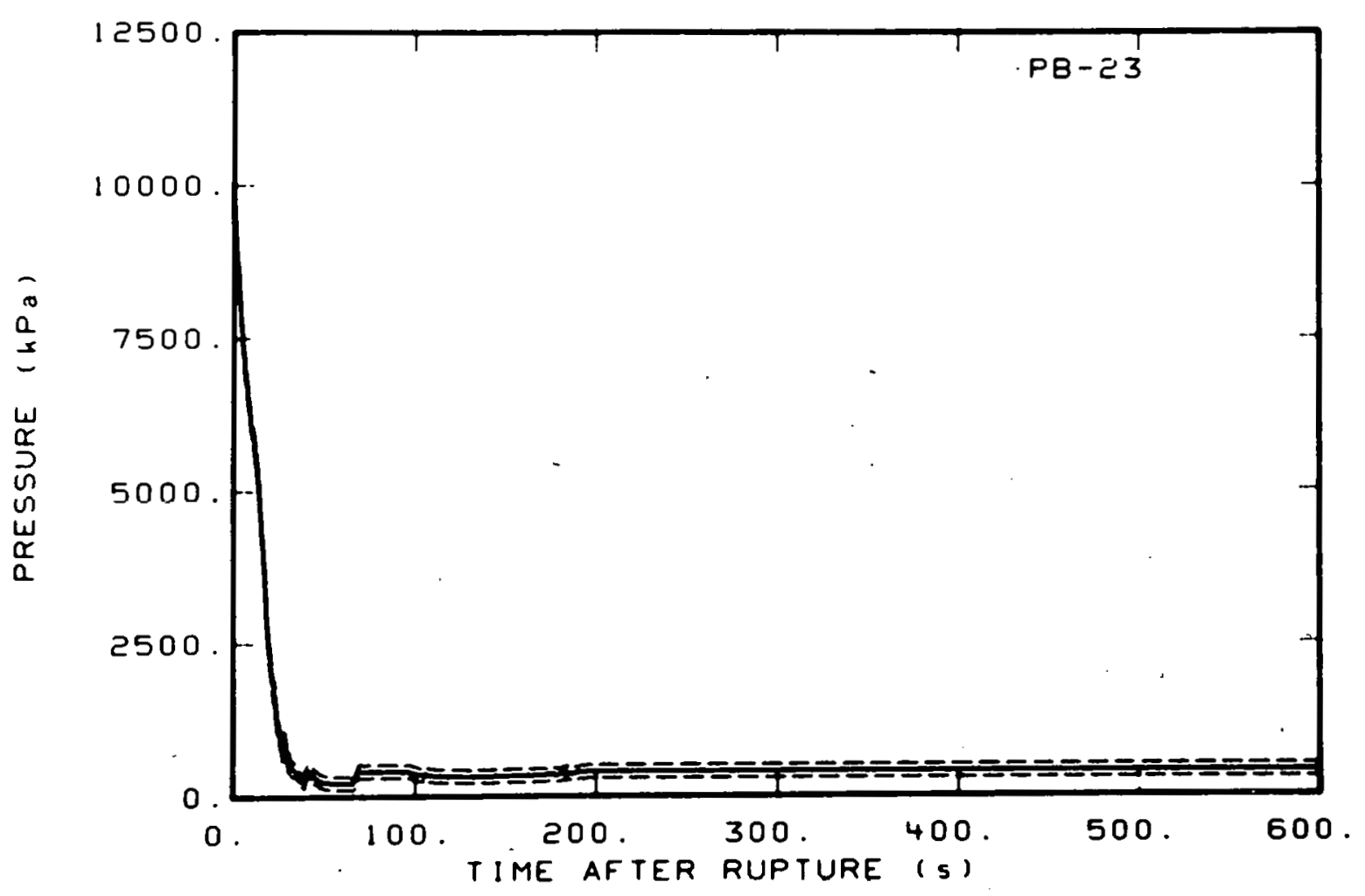

Fig. C-15 Pressure in broken loop, Spool 23 (PB-23).

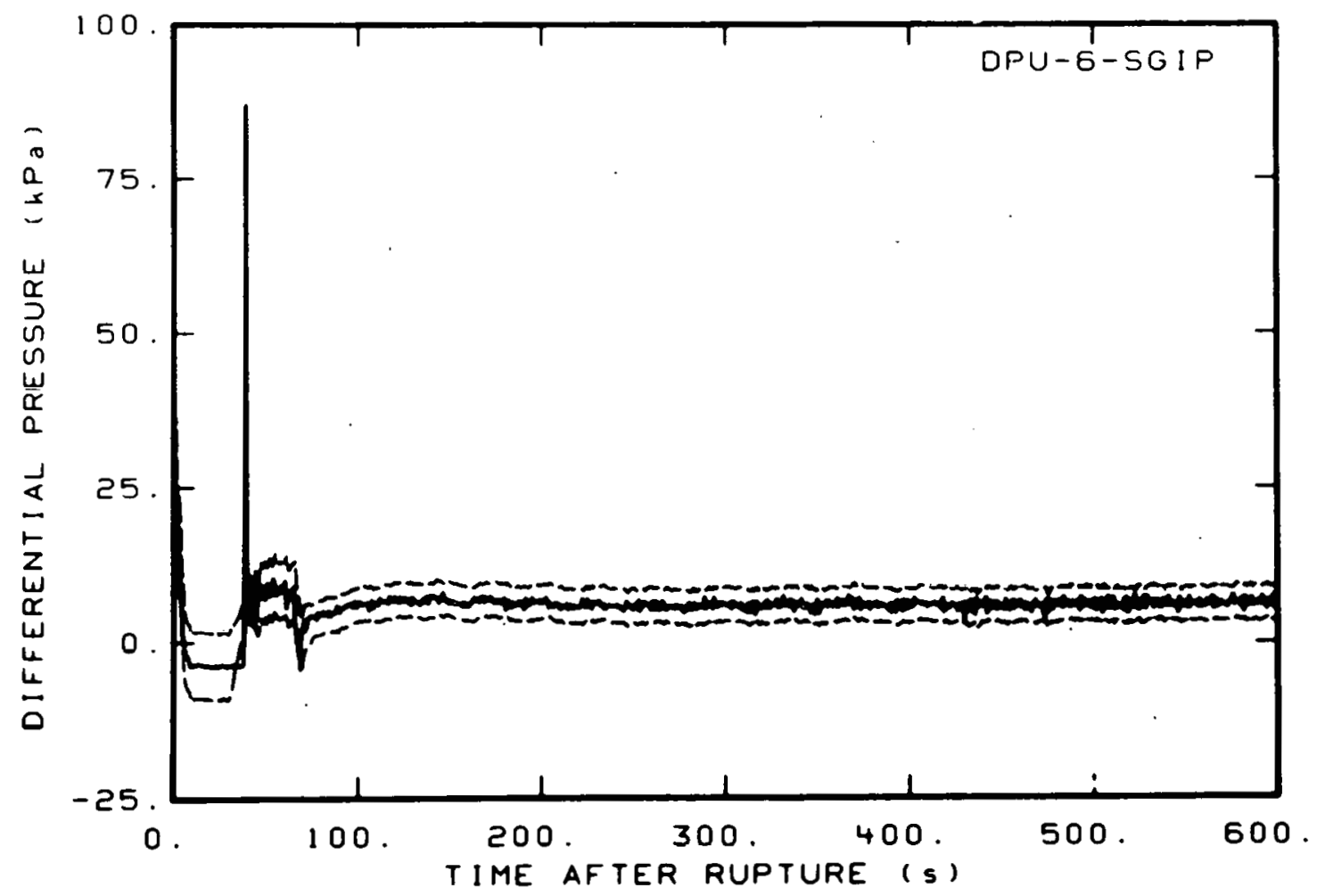

Fig. C-16 Differential pressure in intact loop (DPU-6-SGIP). 


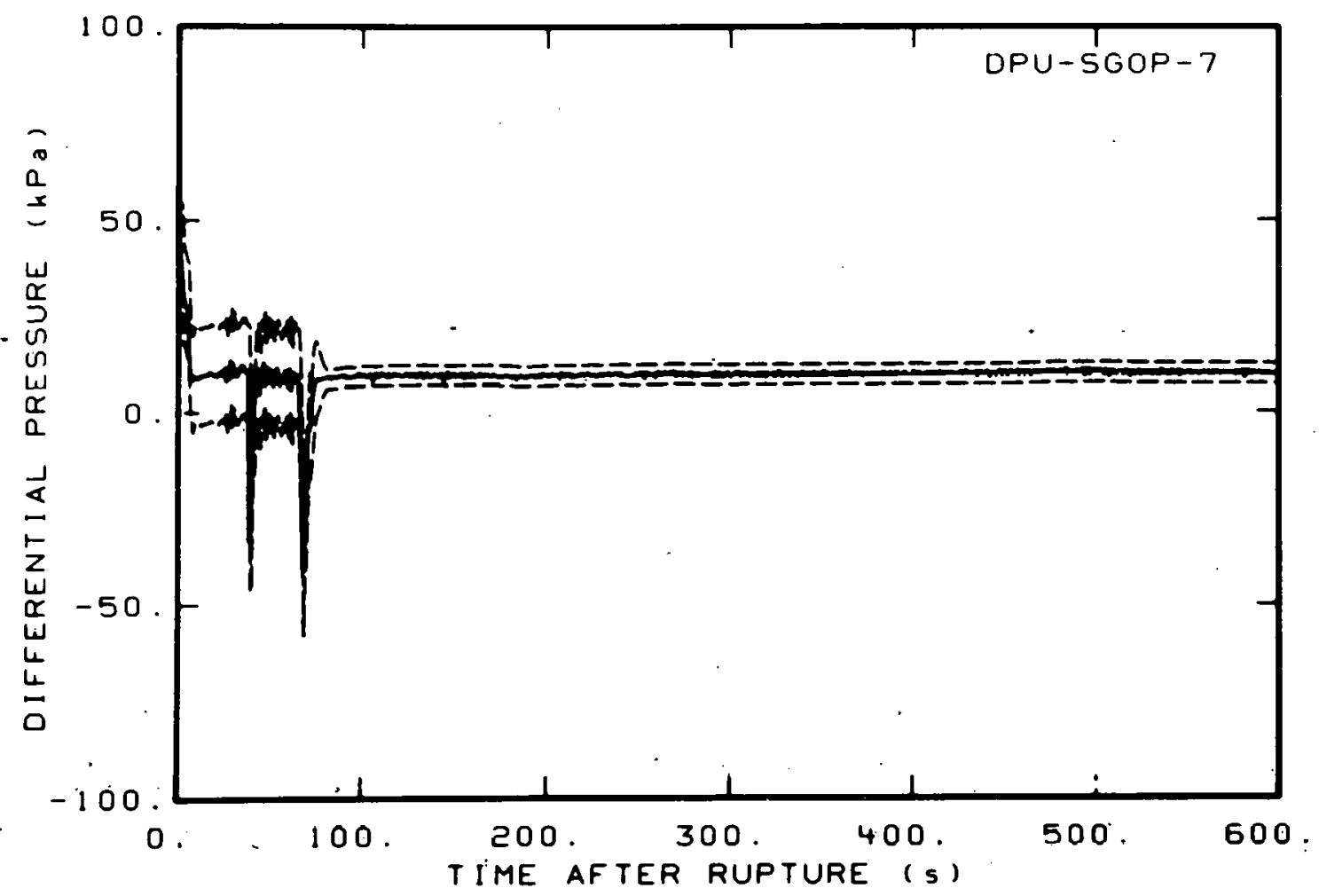

Fig. C-17 Differential pressure in intact loop (DPU-SGOP-7).

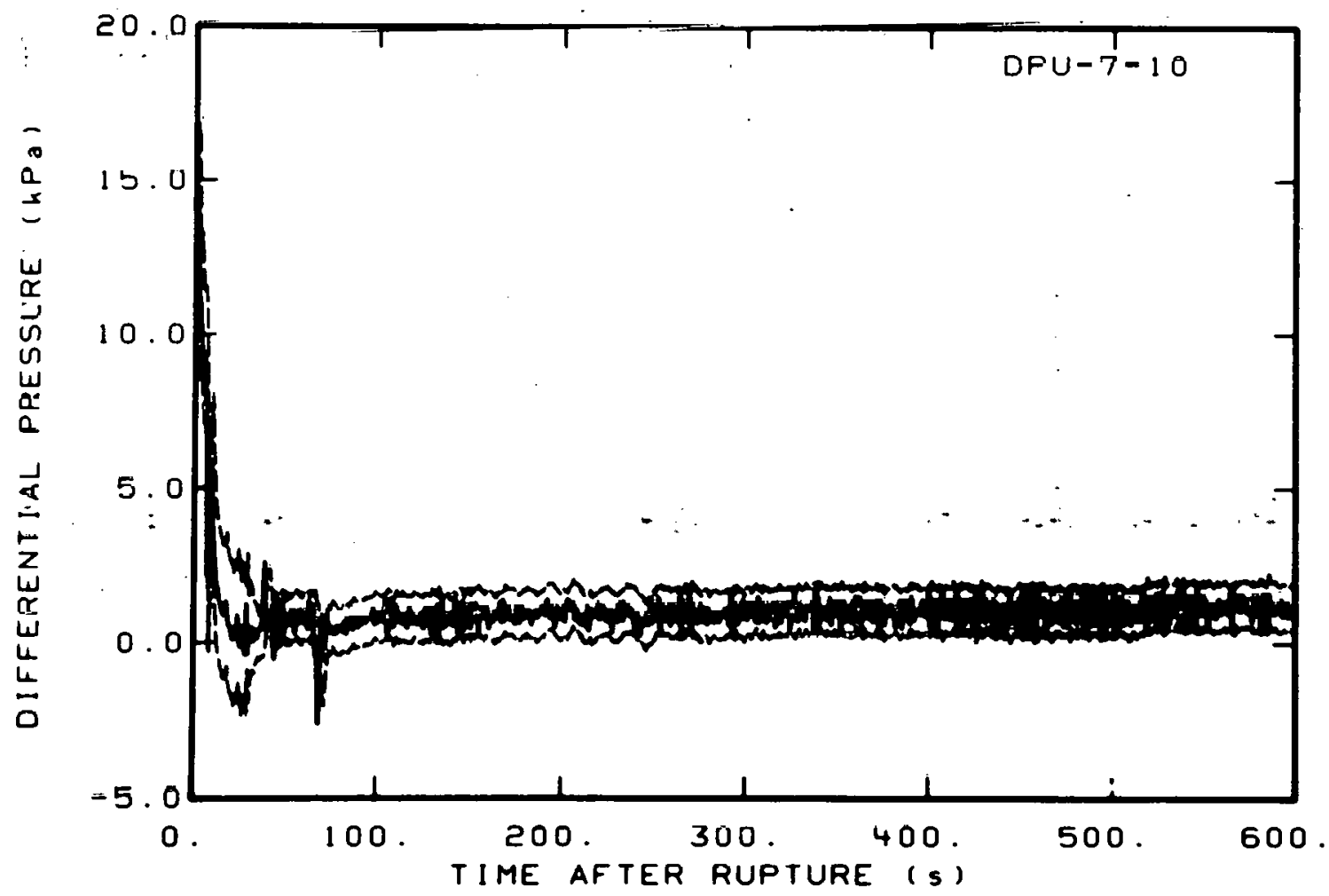

Fig. C-18 Differential pressure in intact 10op (DPU-7-10). 


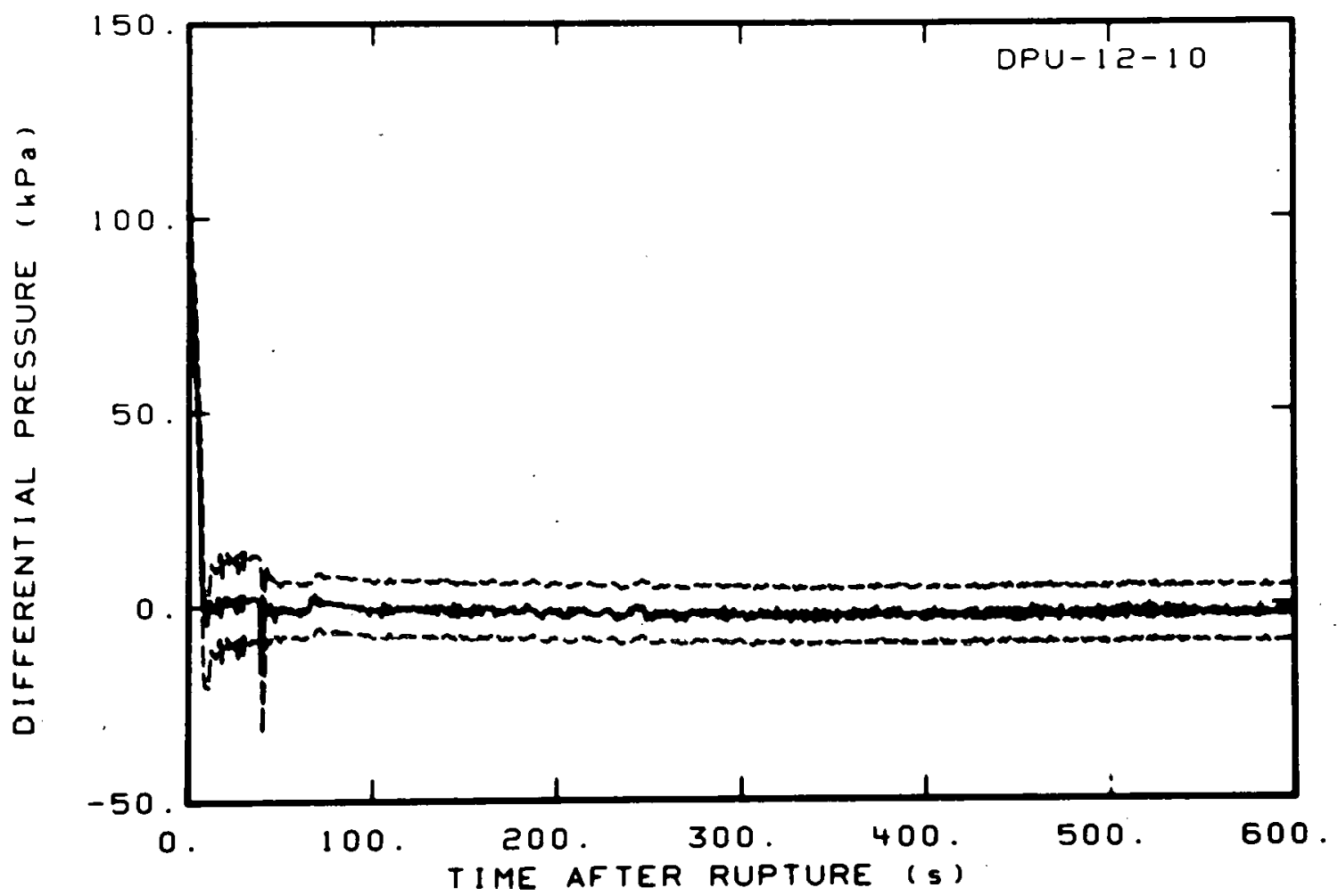

Fig. C-19 Differential pressure in intact 1oop (DPU-12-10).

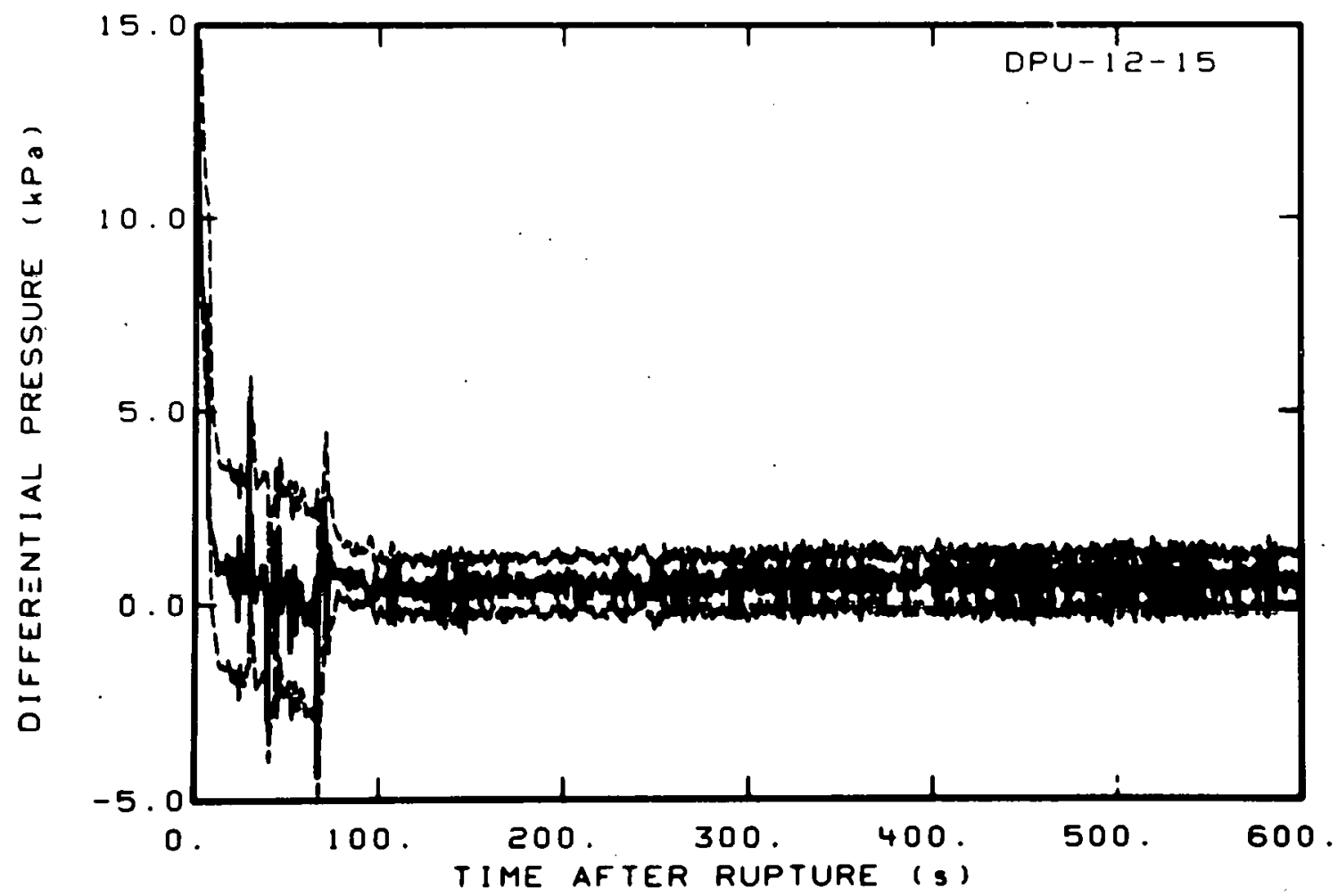

Fig. C-20 Differential pressure in intact loop (DPU-12-15). 


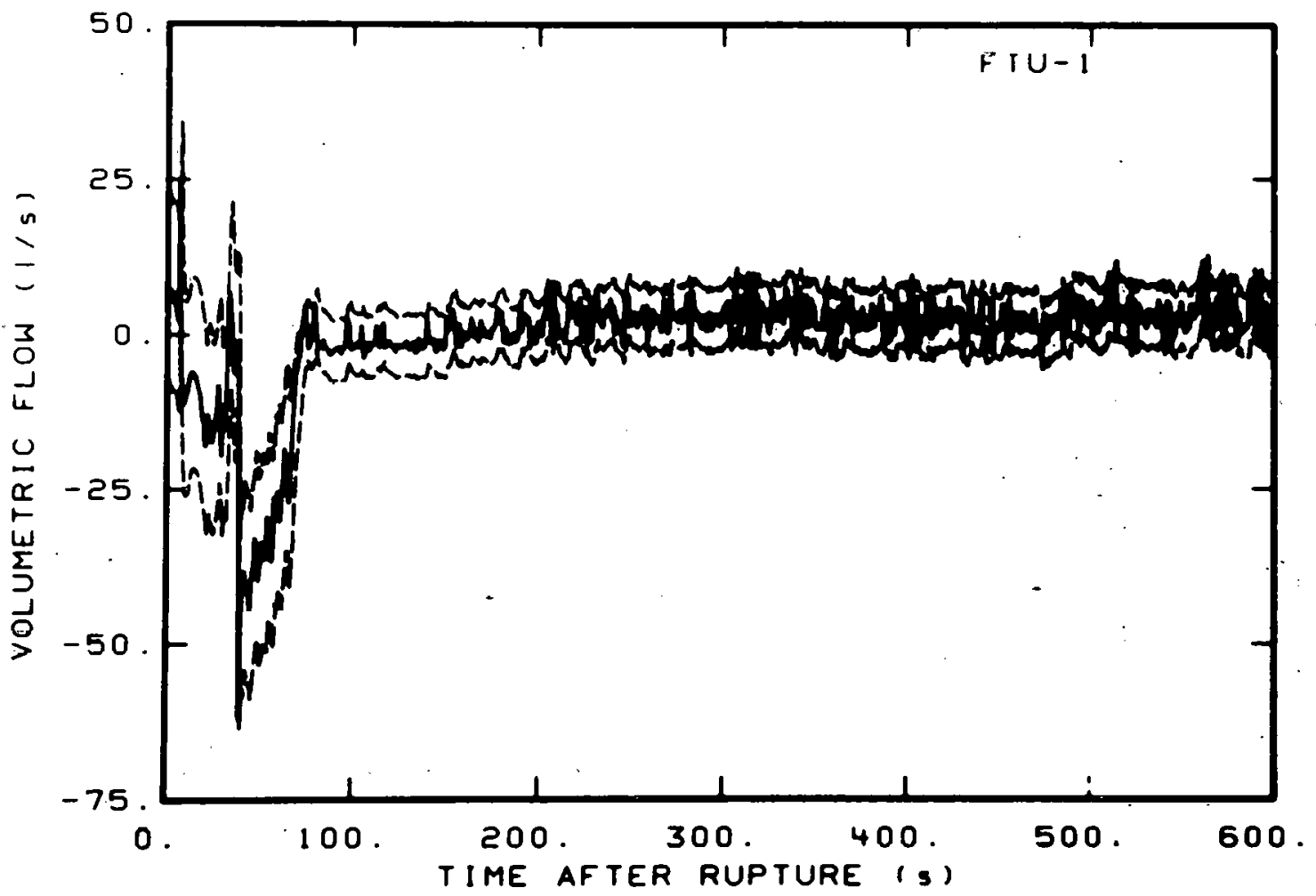

Fig. C-21 Volumetric flow in intact loop (FTU-1).

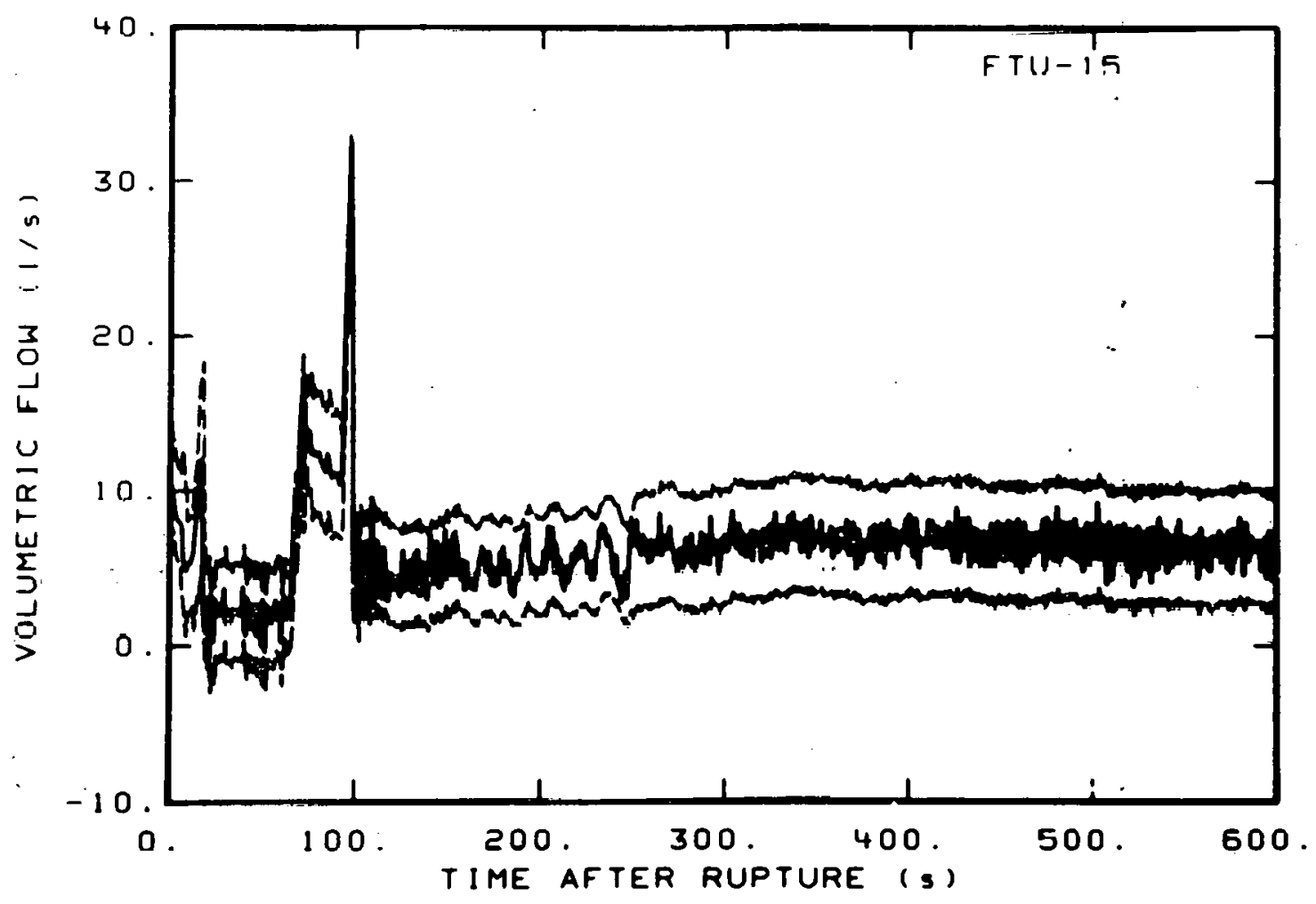

Fig. C-22 Volumetric flow in intact loop (FTU-15). 


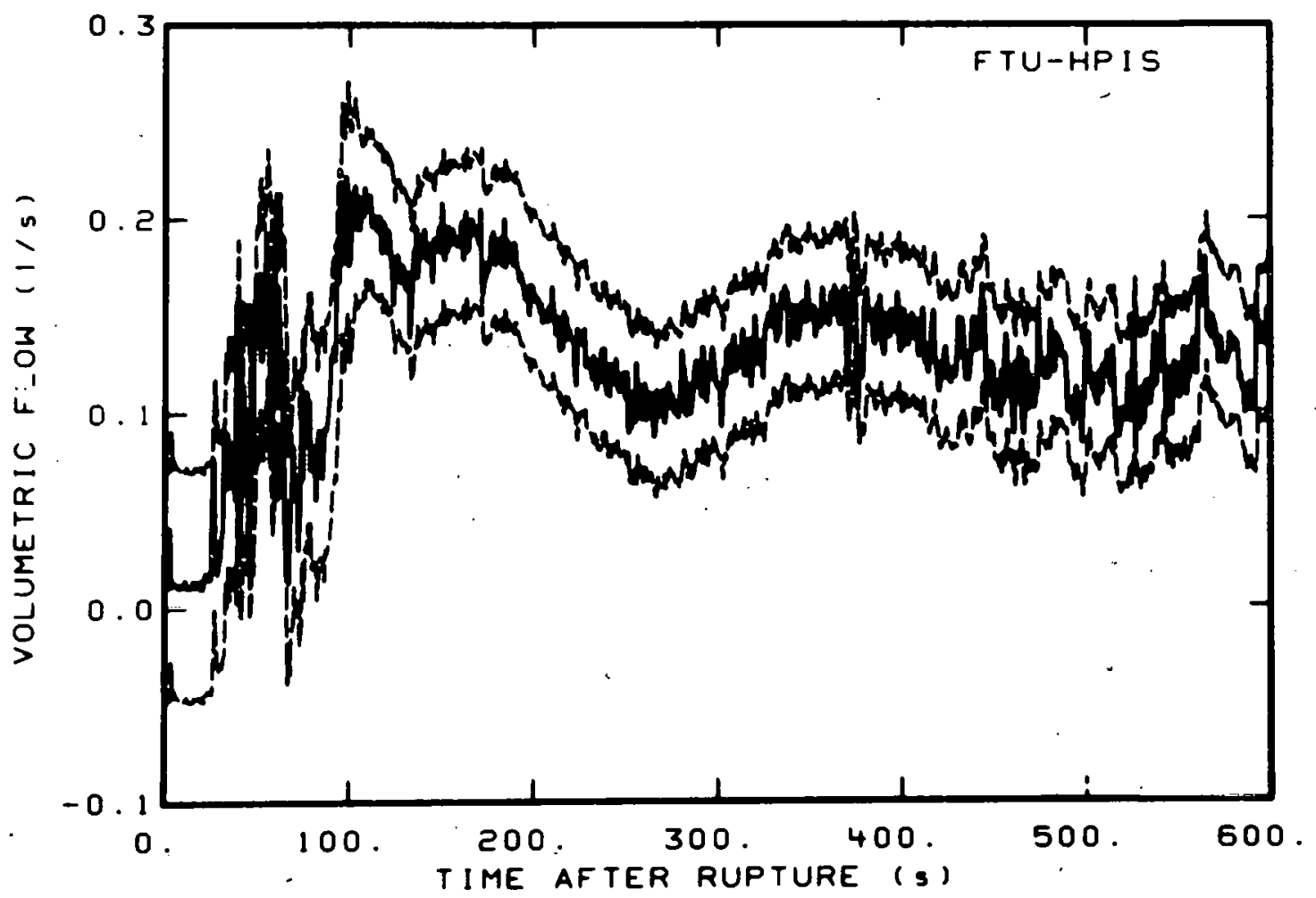

Fig. C-23 Volumetric flow in intact loop high pressure injection line (FTU-HPIS).

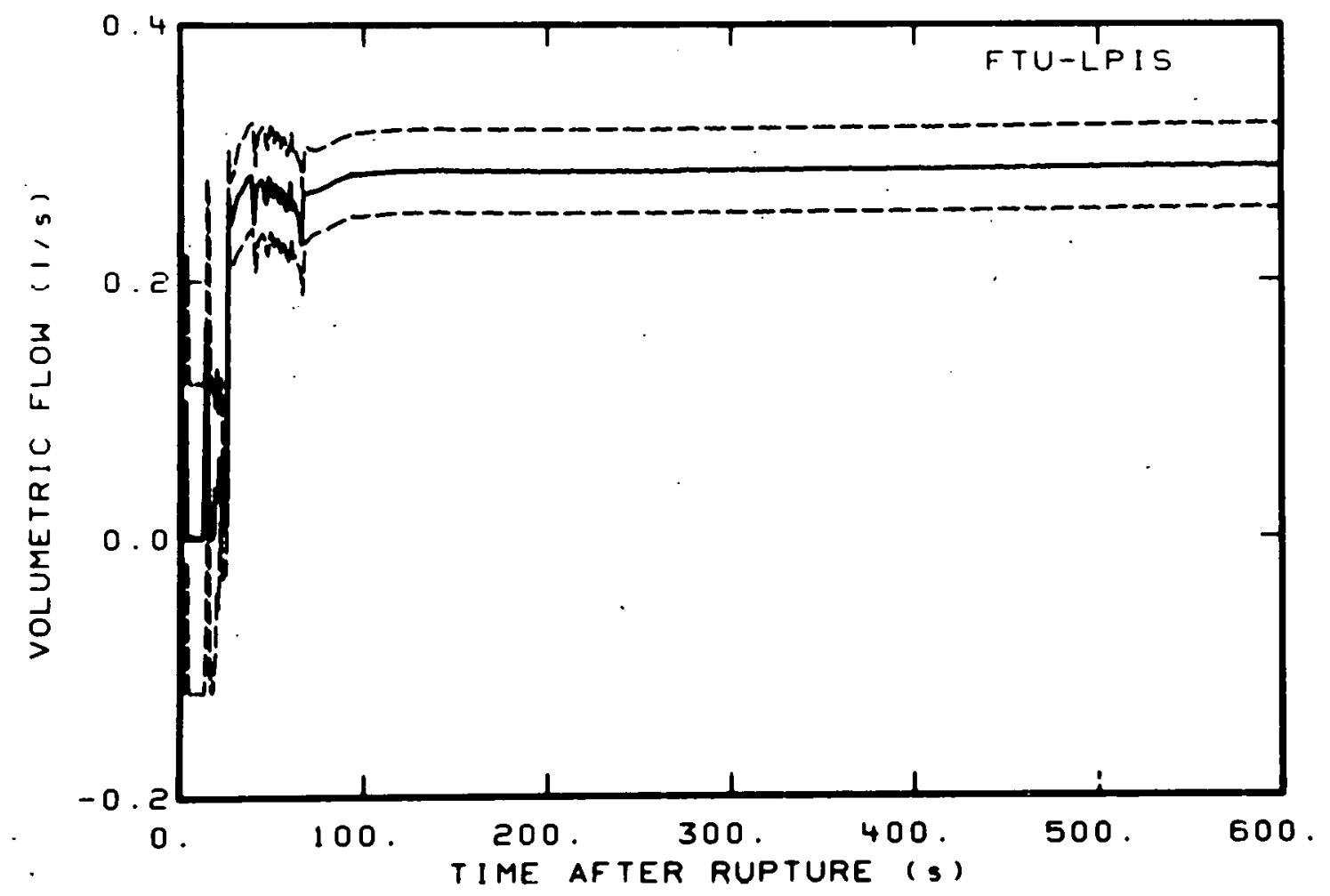

Fig. C-24 Volumetric flow in intact loop low pressure injection line (FTU-LPIS). 


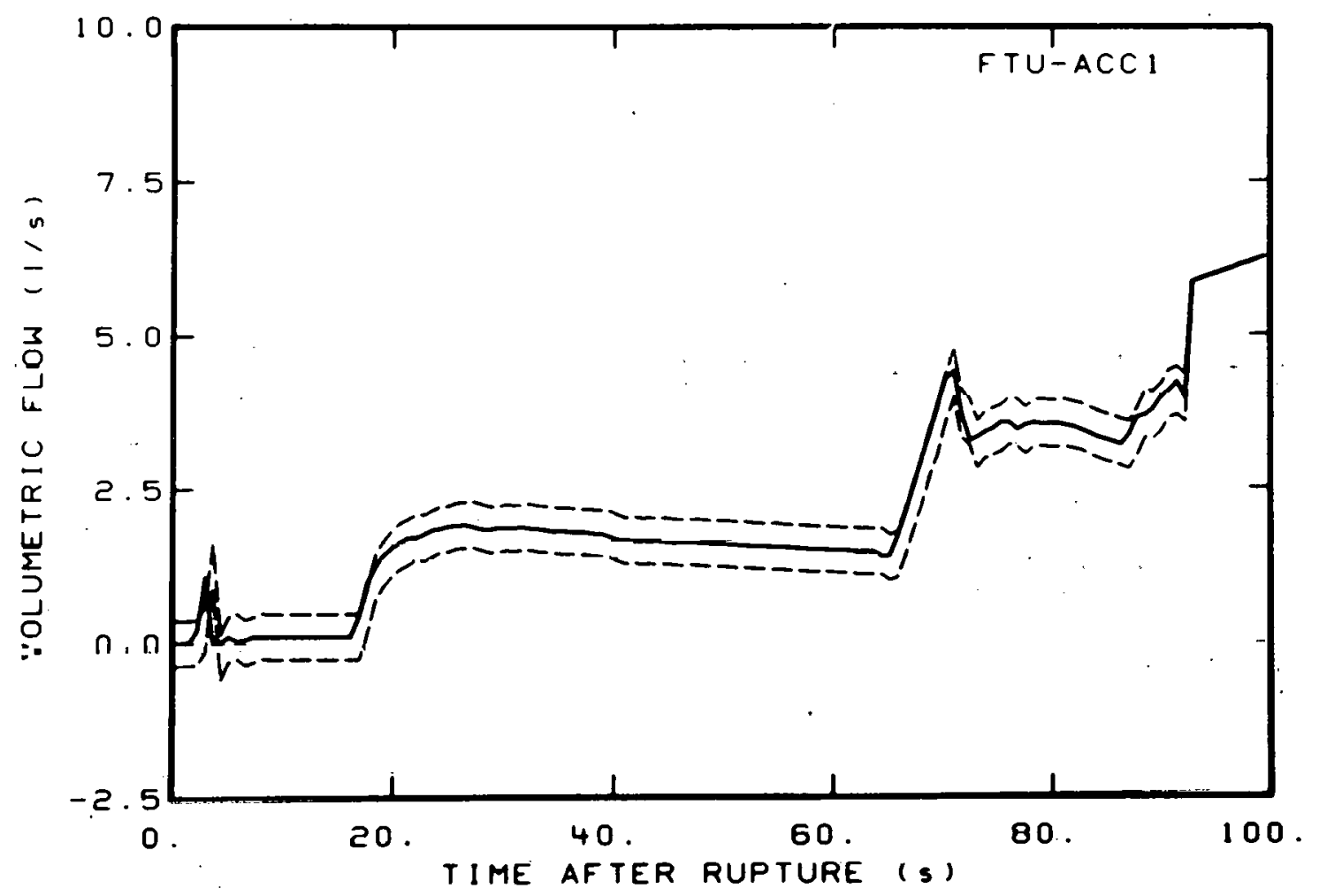

Fig. C-25 Volumetric flow in intact loop accumulator discharge line (FTU-ACCI).

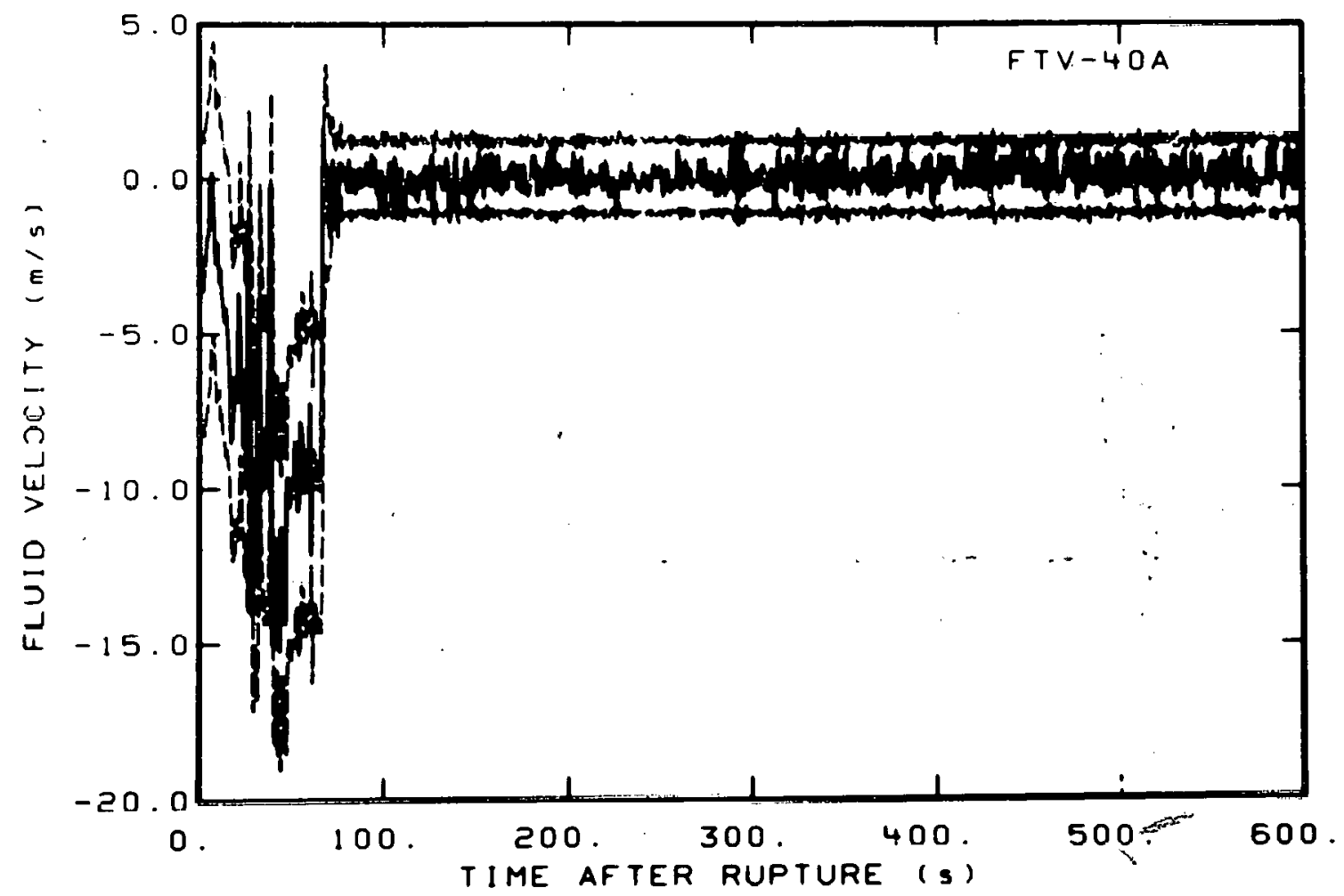

Fig. C-26 Fluid velocity in vessel (FTV-40A). 


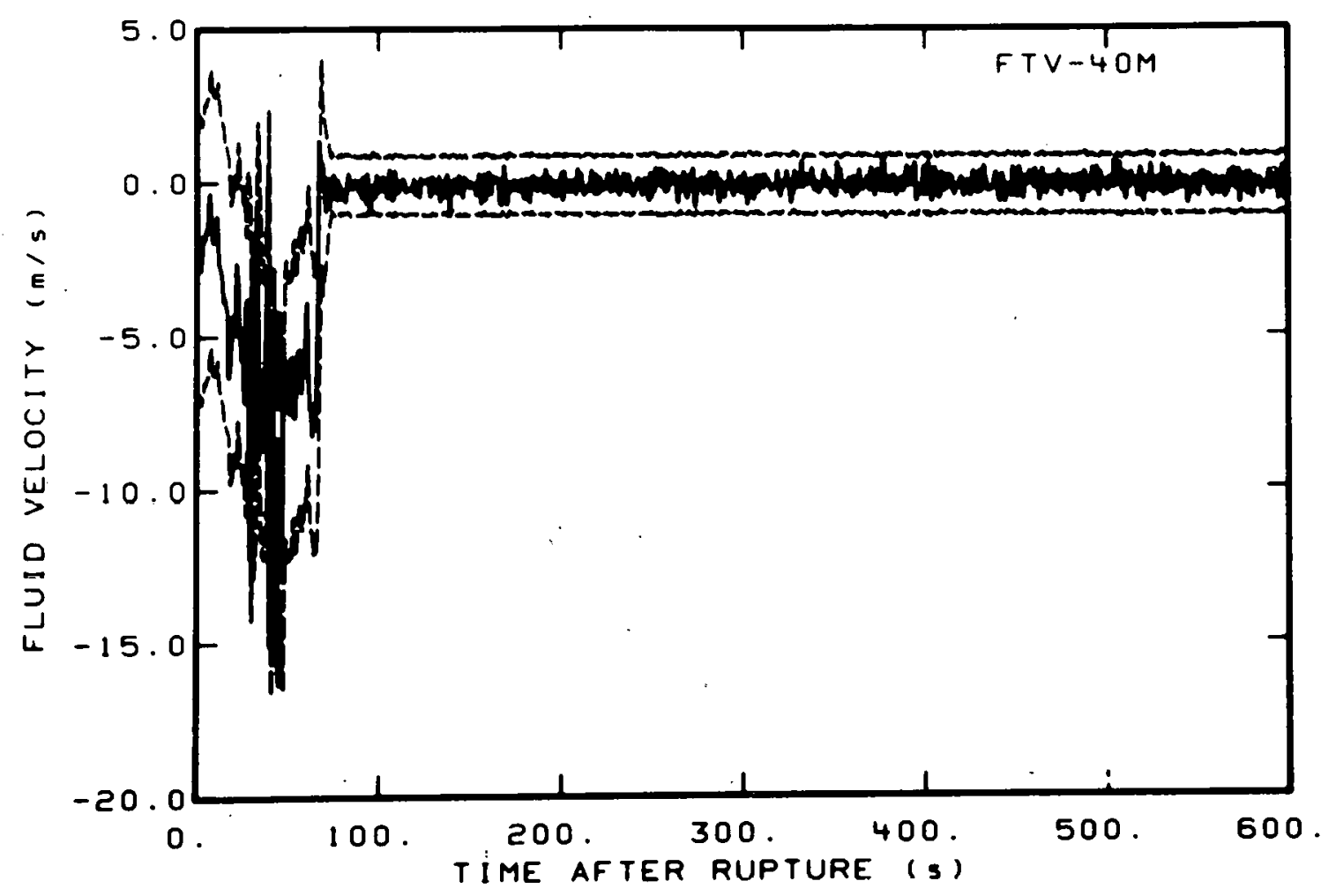

Fig. C-27 Fluid velocity in vessel (FTV-40M).

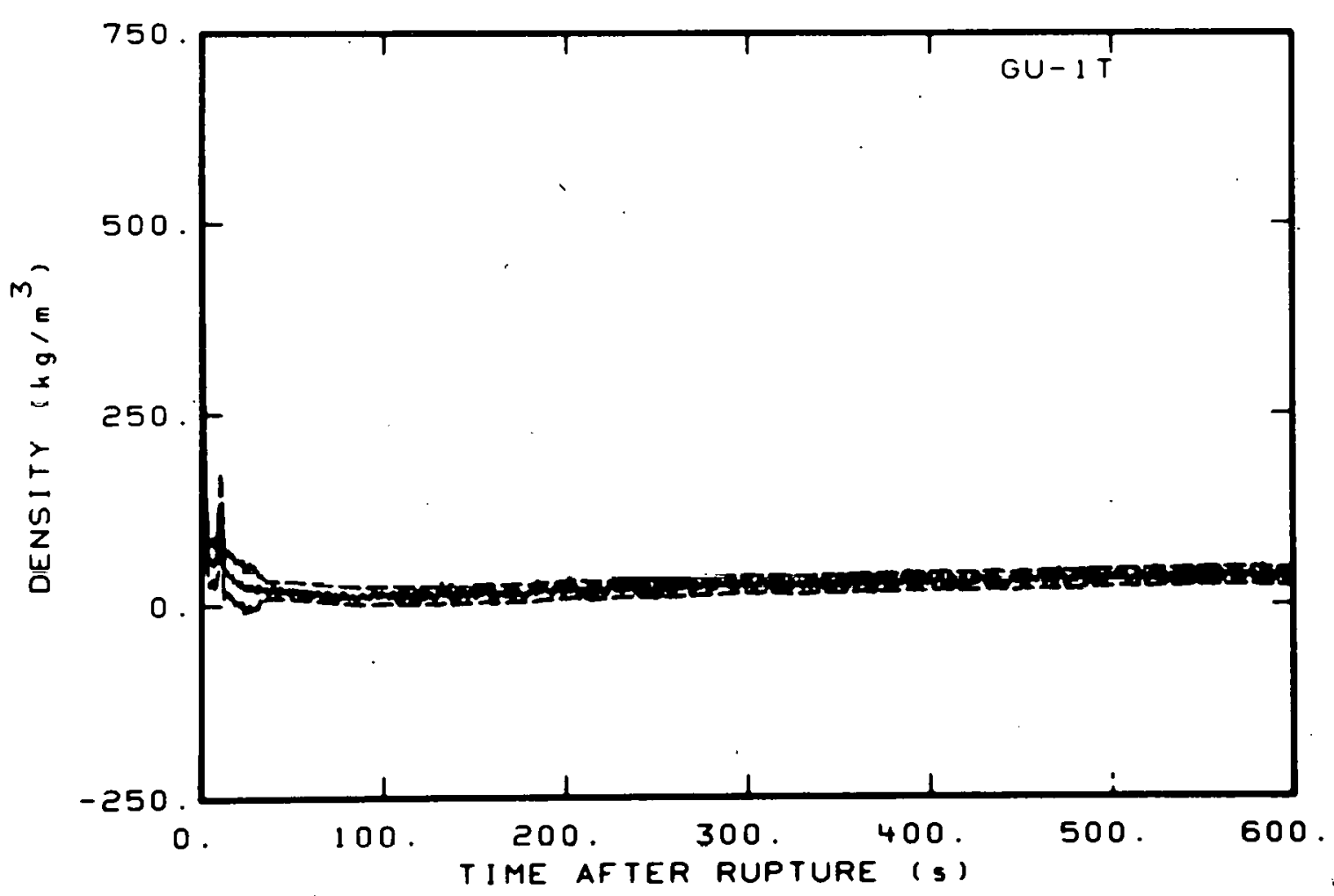

Fig. C-28 Density in intact loop (GU-1T). 


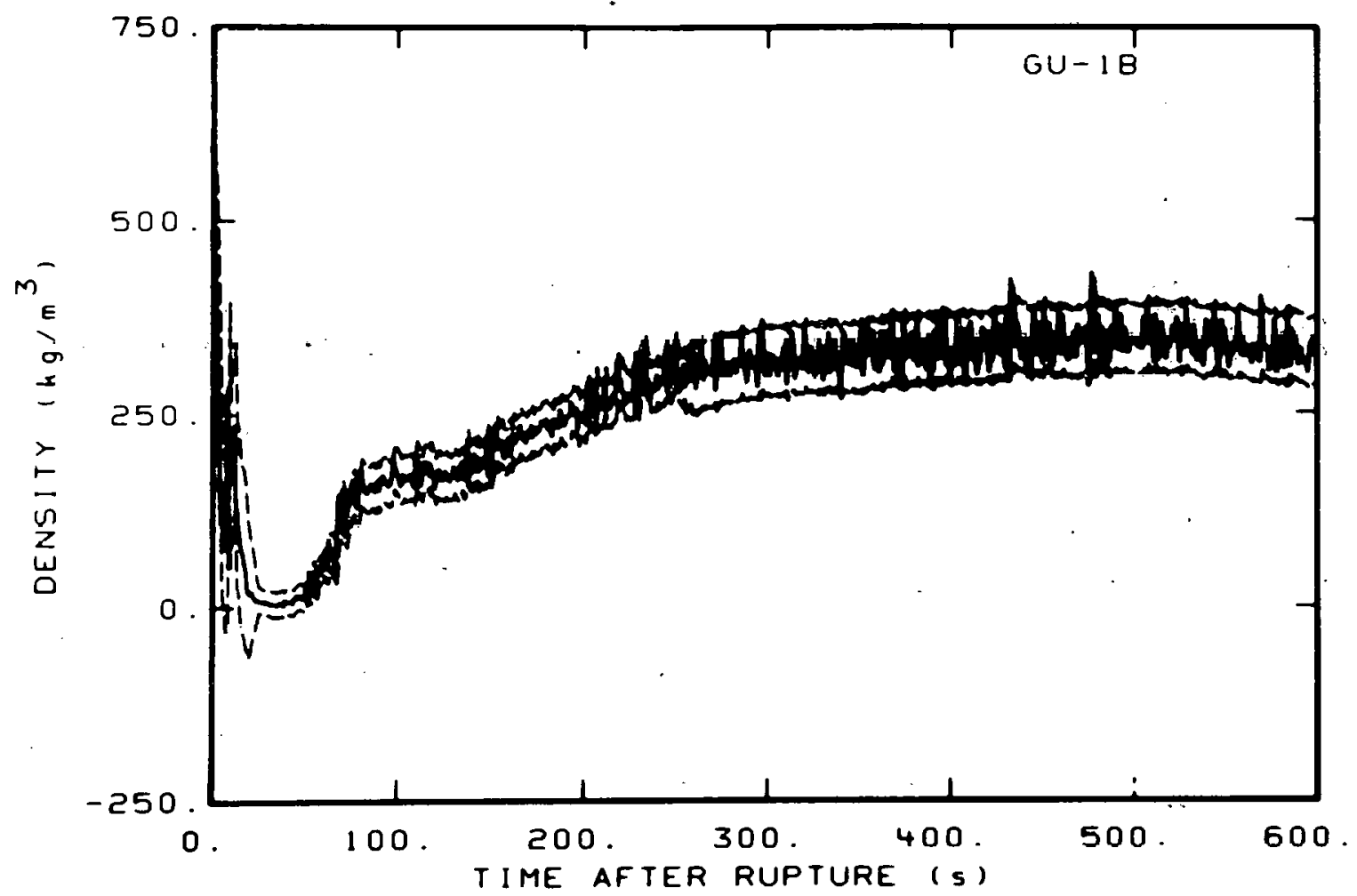

Fig. C-29 Density in intact loop (GU-1B).

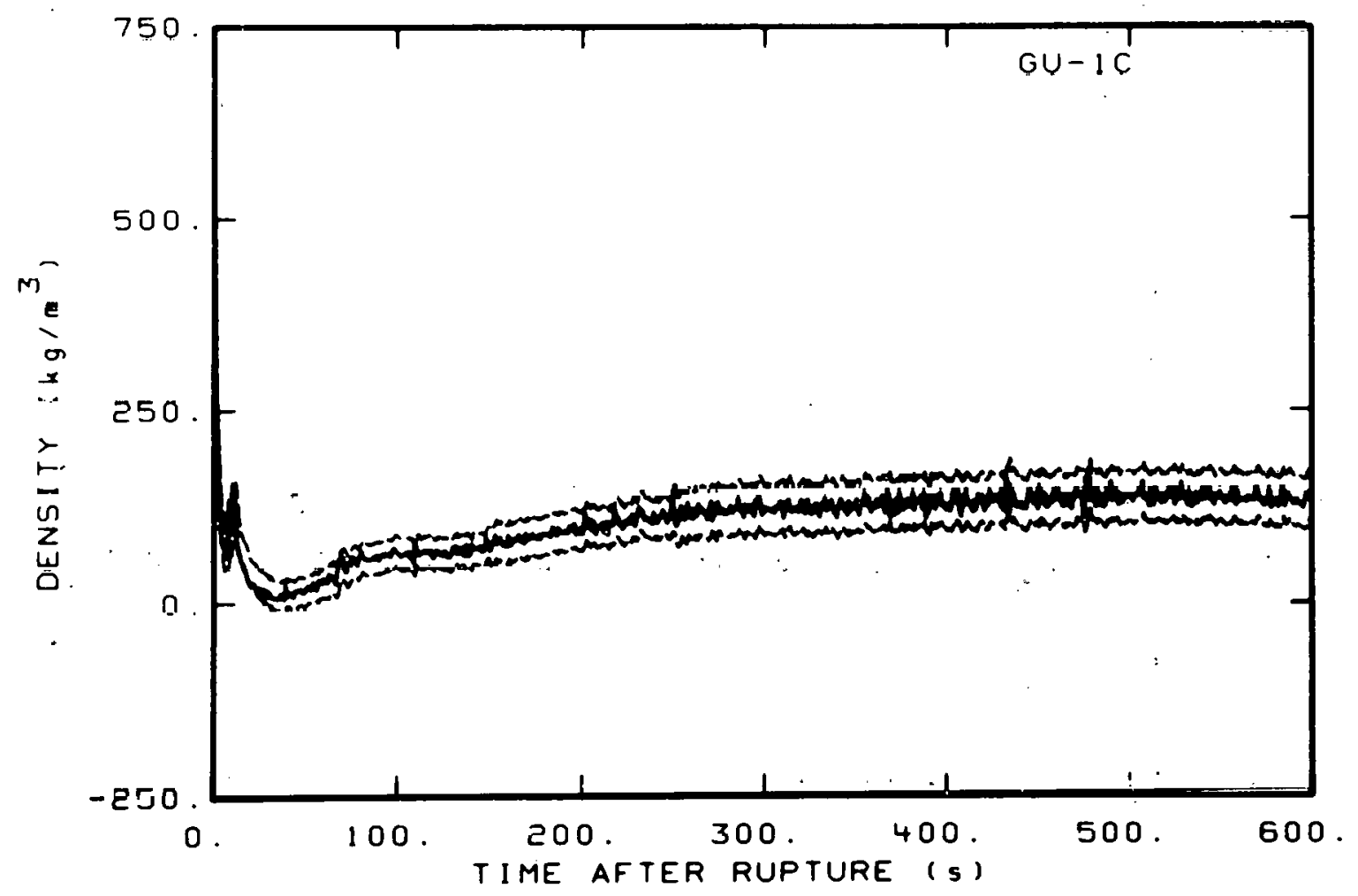

Fig. C-30 Density in intact 100p (GU-1C). 


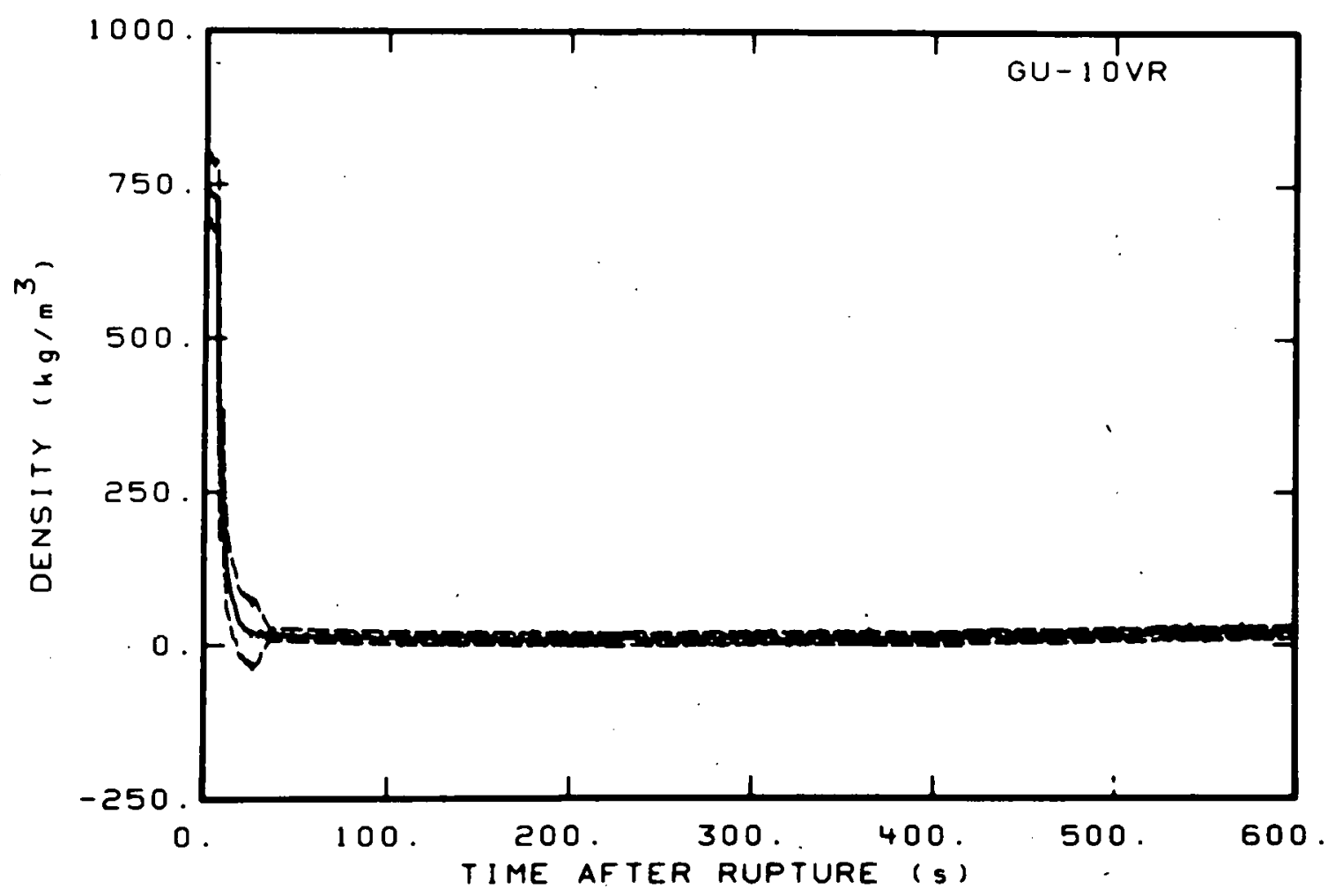

Fig. C-31 Density in intact loop (GU-10VR).

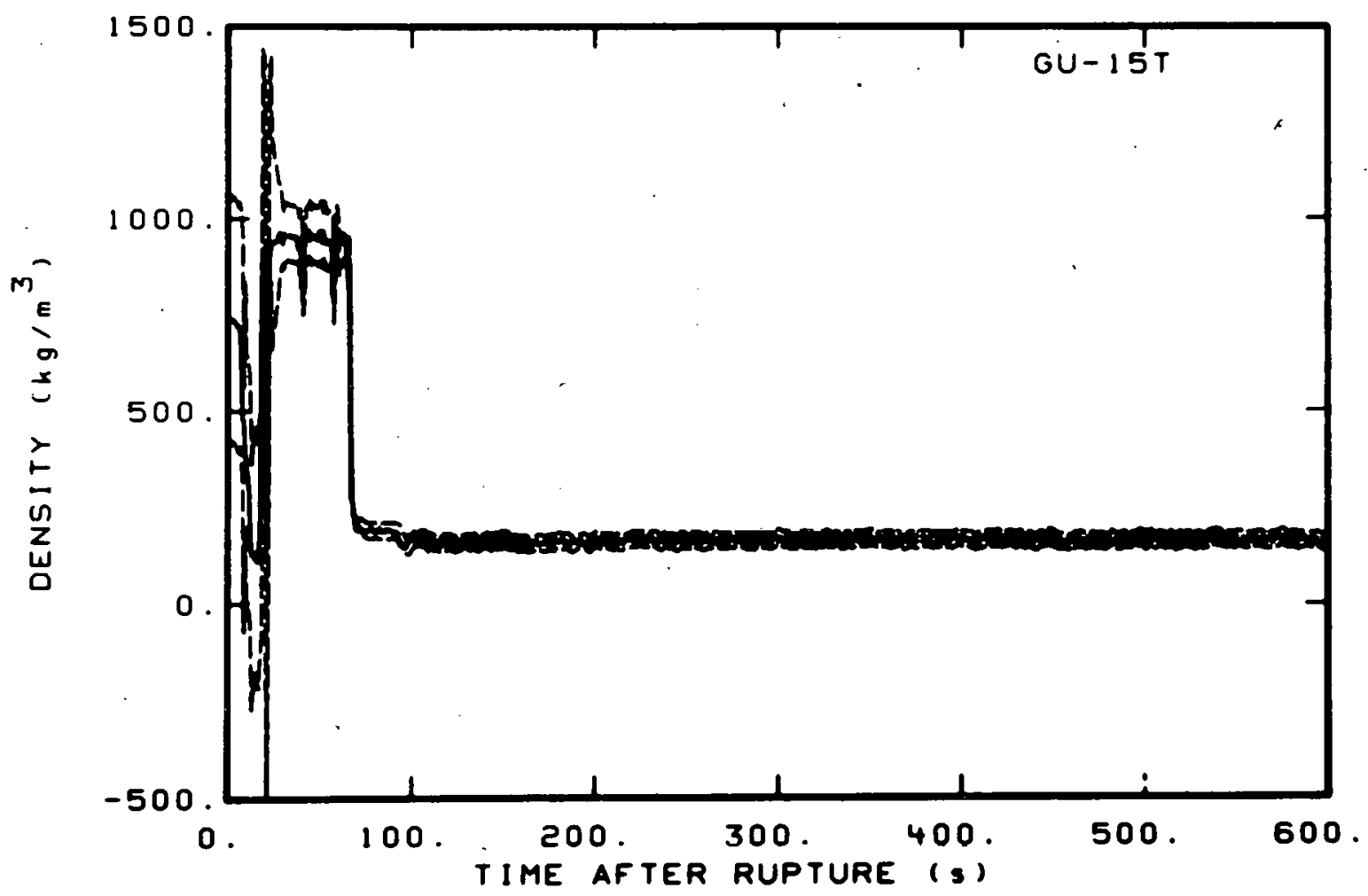

Fig. C-32. Density in intact loop (GU-15T). 


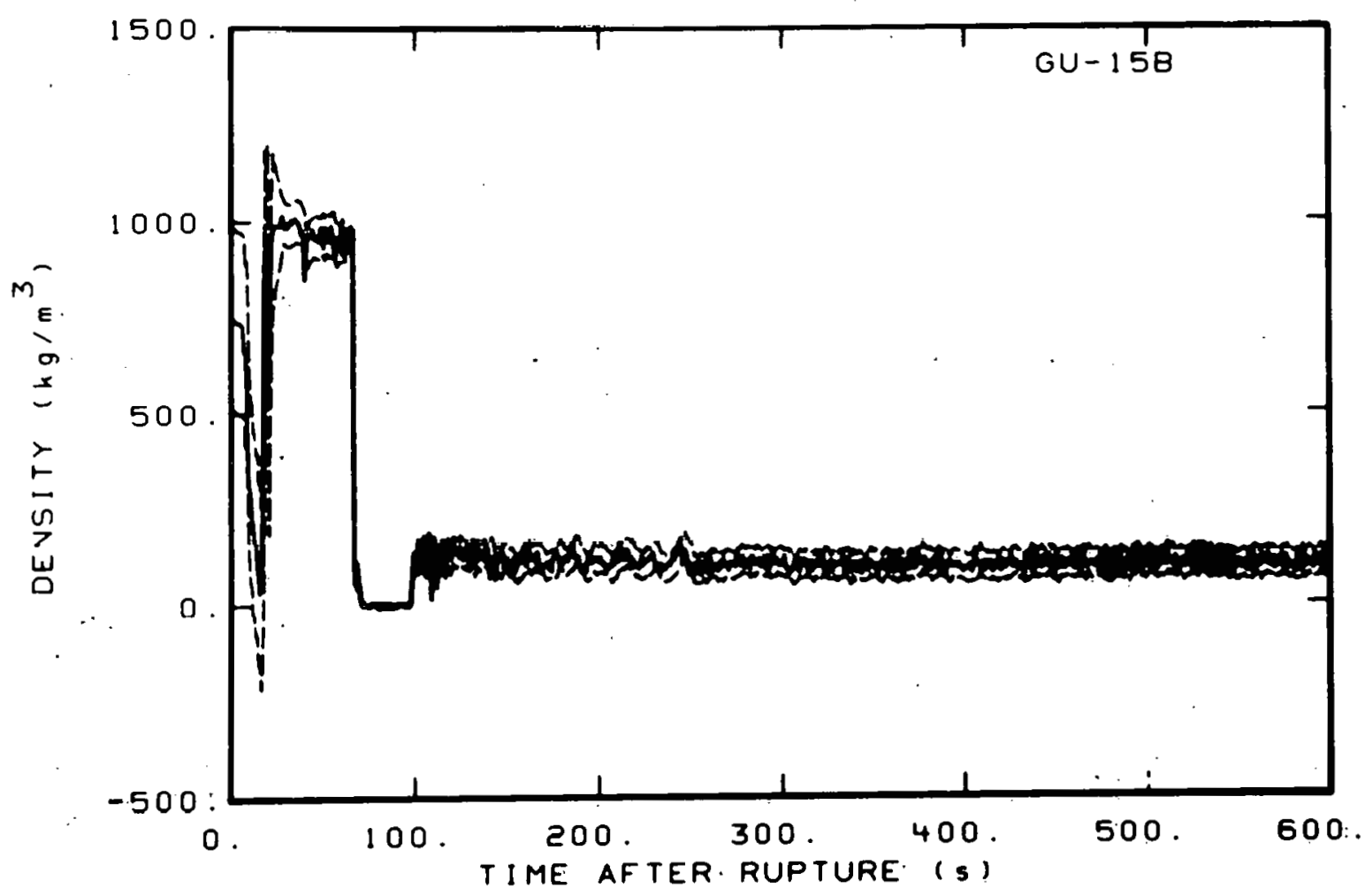

Fig. C-33 Density in intact loop (GU-15B).

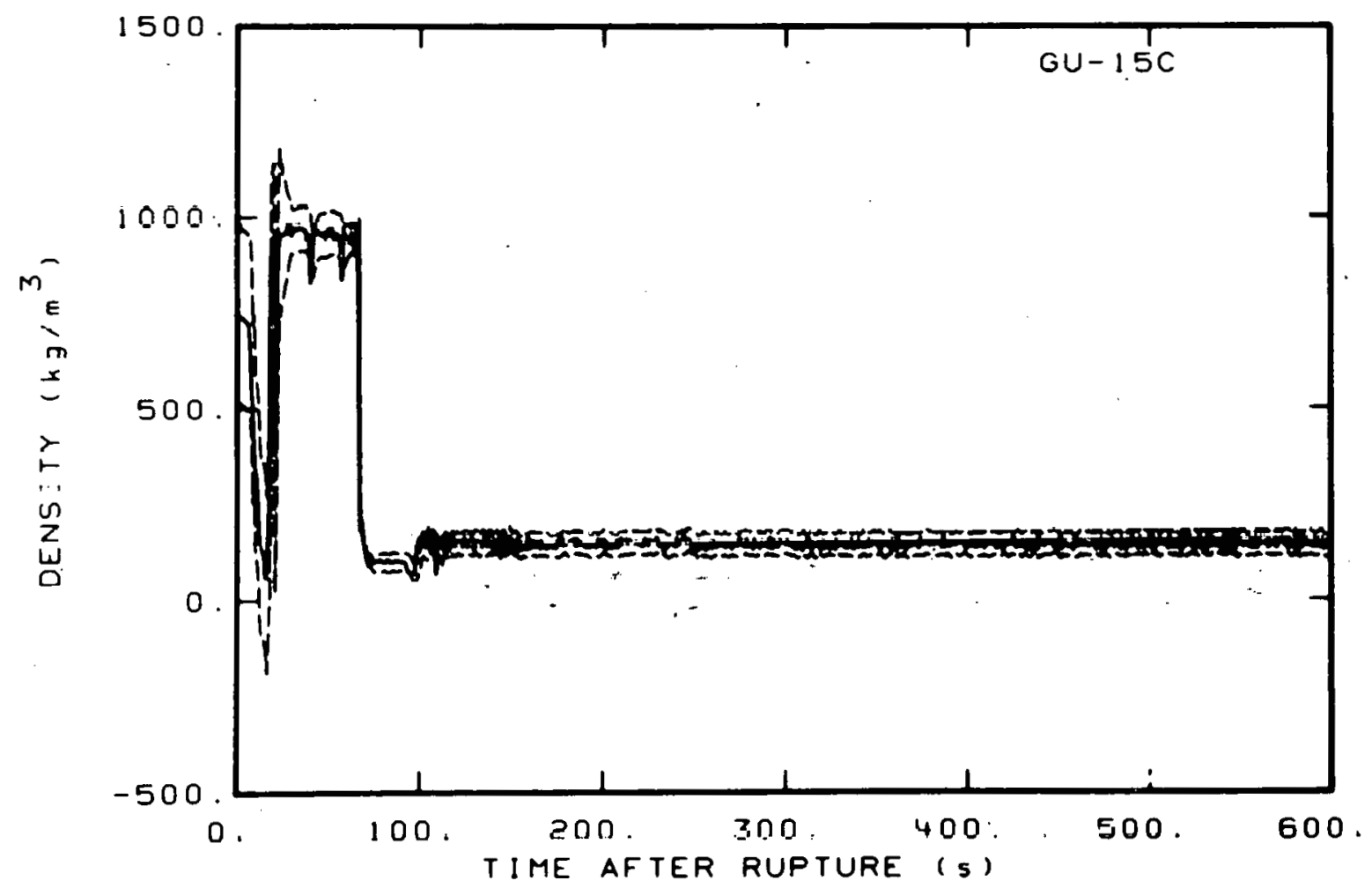

Fig. C-34 Density in intact loop (GU-15C). 


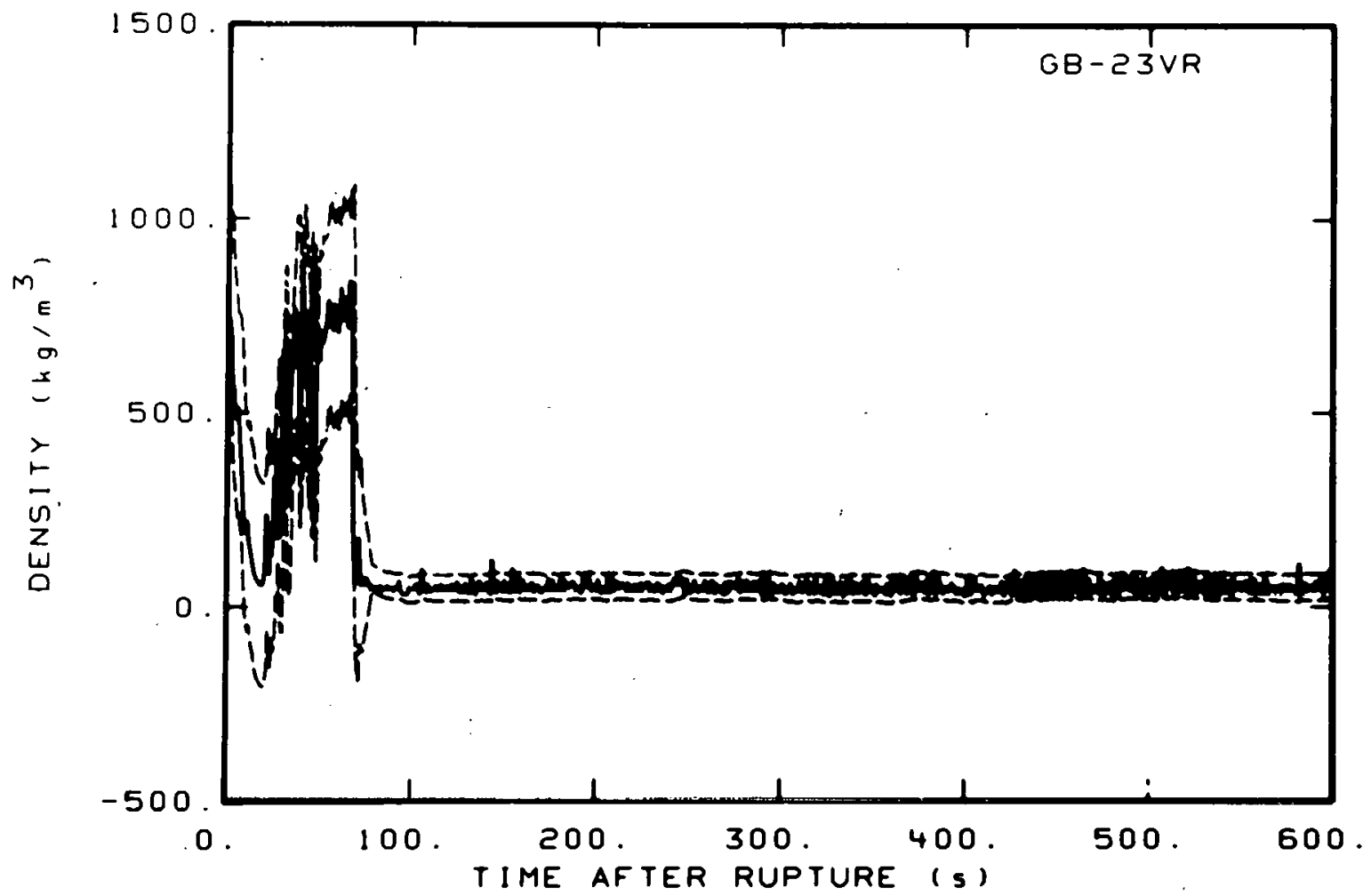

Fig. C-35 Density in broken loop (GB-23VR).

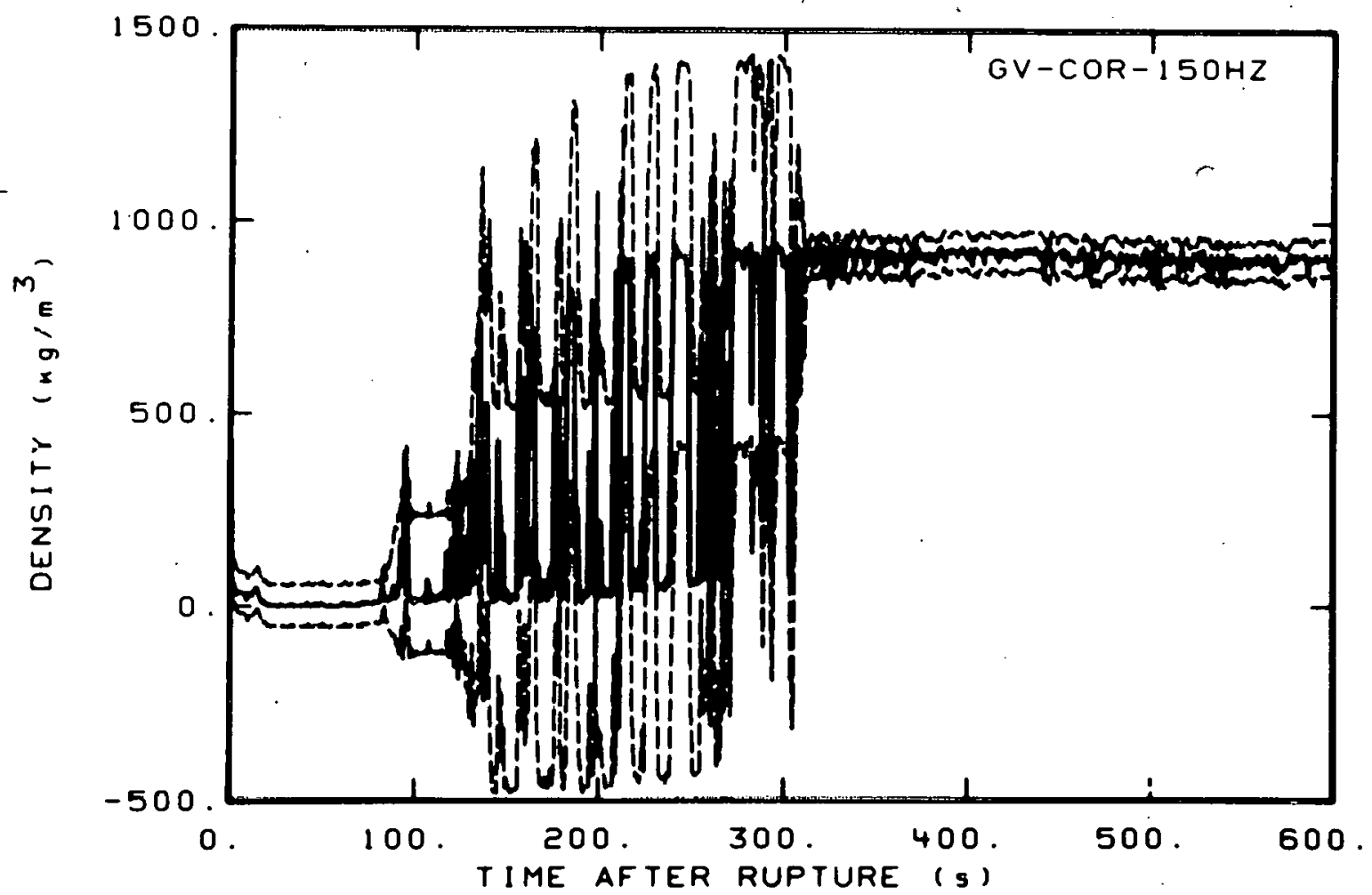

Fig. C-36 Density in vessel (GV-COR-150HZ). 


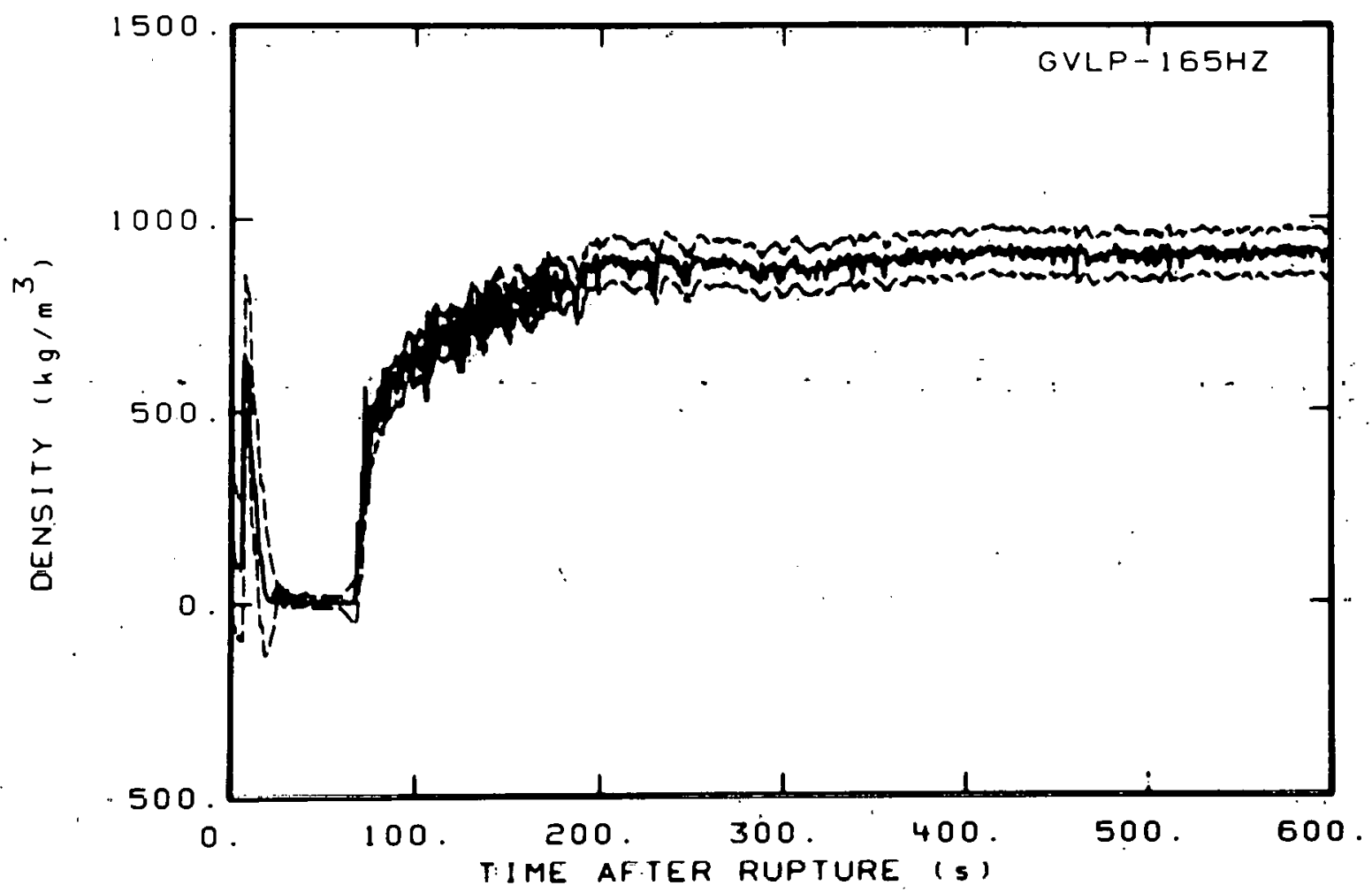

Fig. C-37 Density in vessel (GVLP-165HZ).

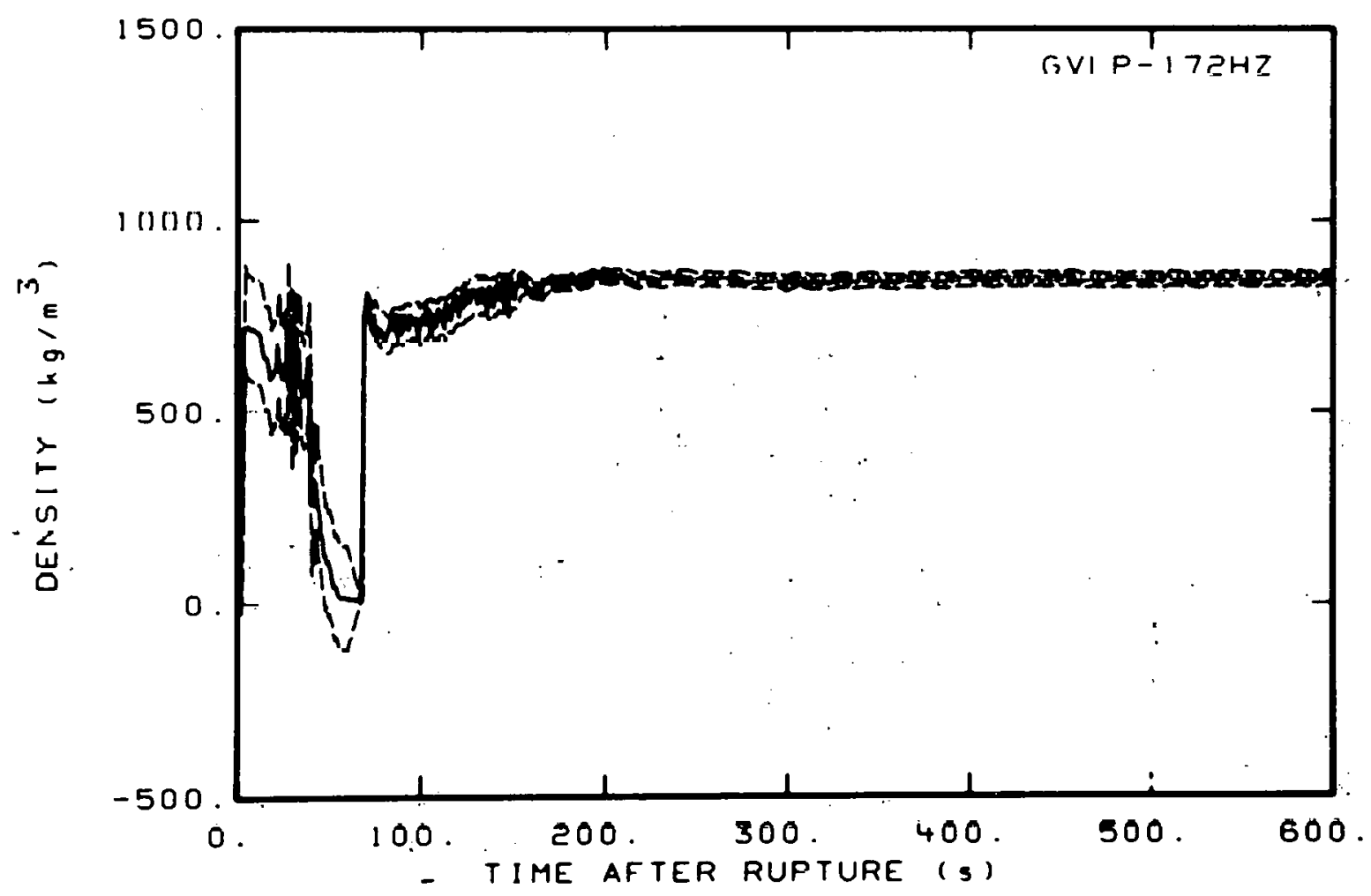

Fig. C-38 Density in vessel (GVLP-172HZ). 


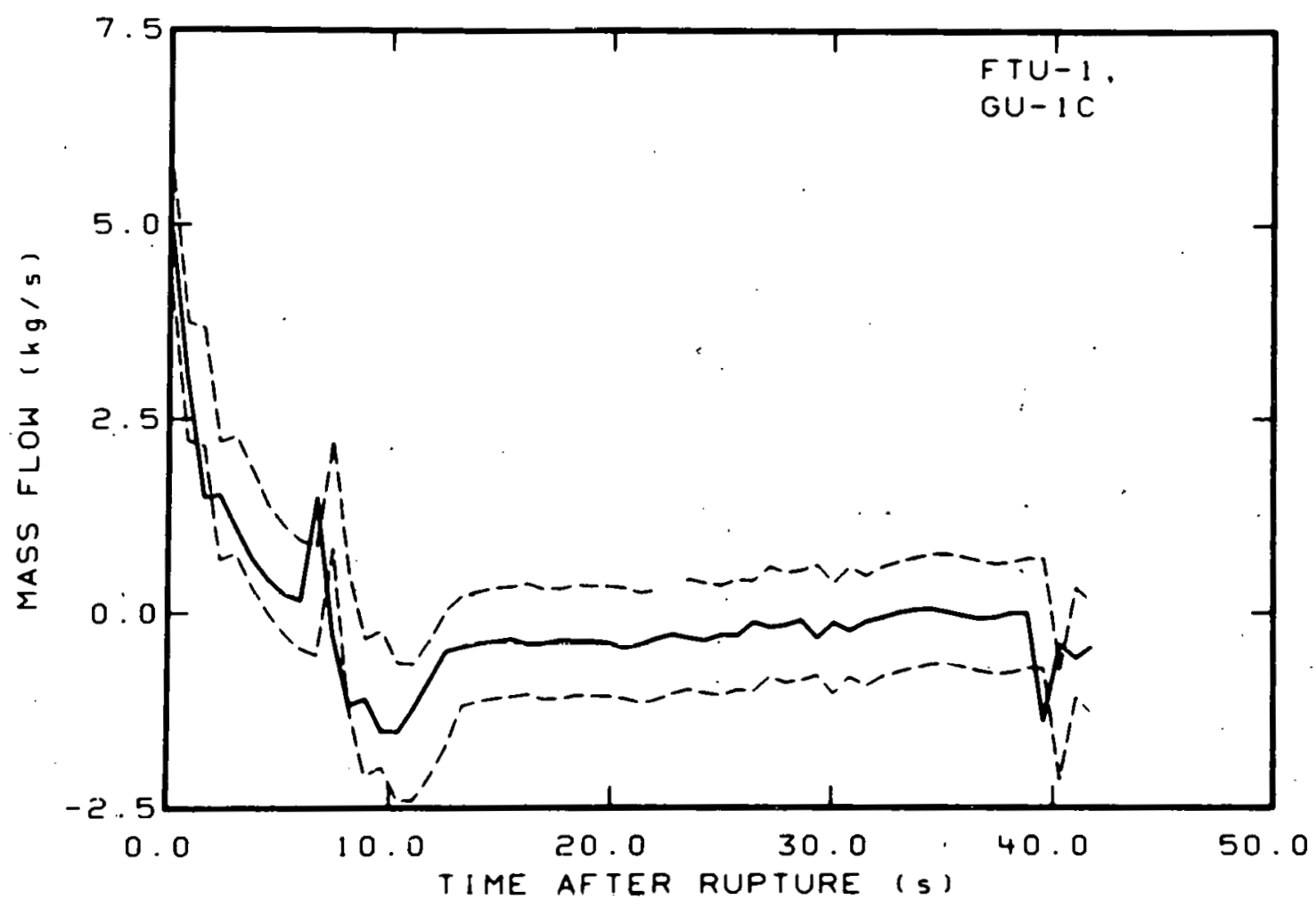

Fig. C-39 Mass fiow in intact loop (FTU-l, GU-1C).

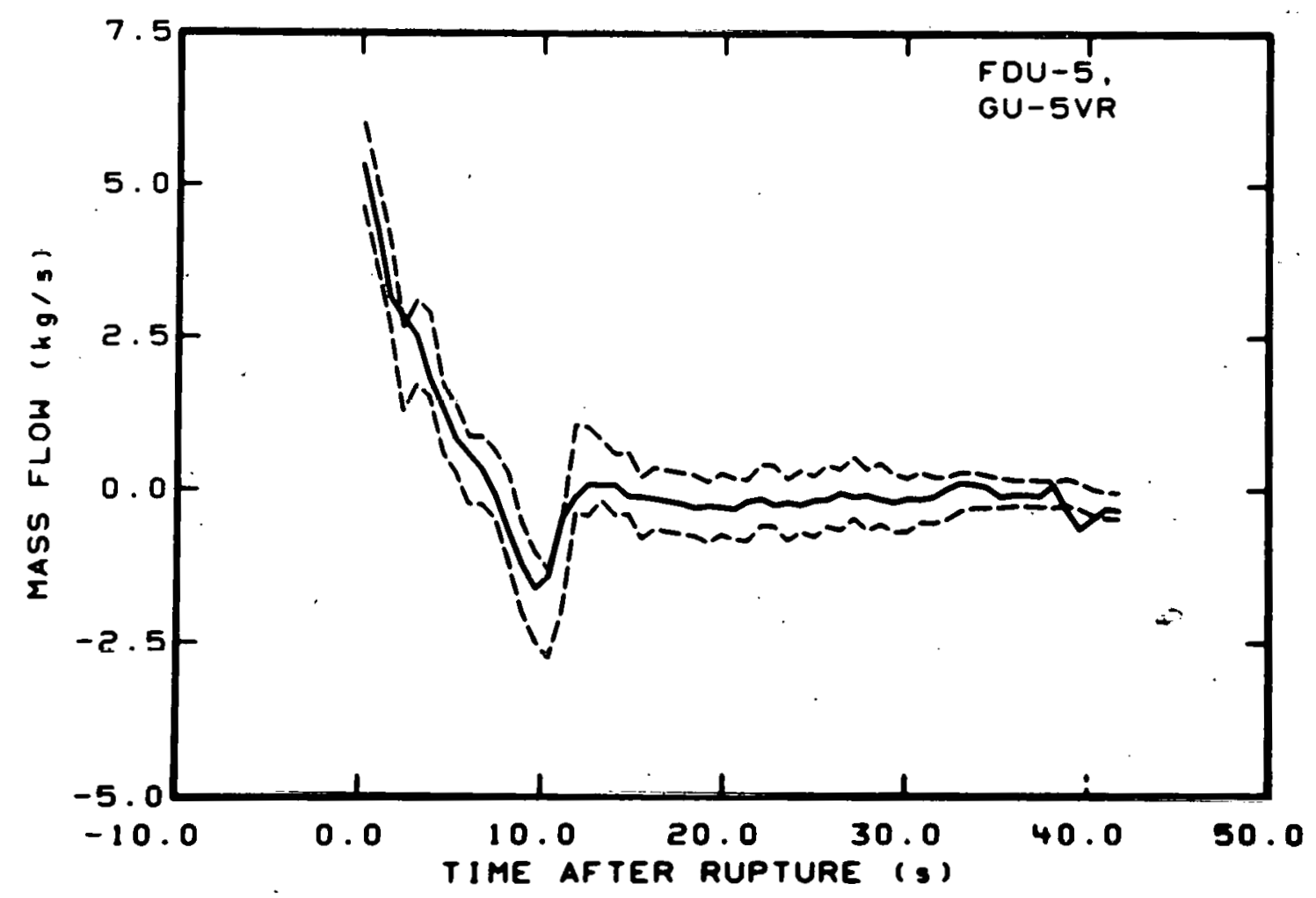

Fig. C-40 Mass flow in intact loop (FDU-5, GU-5VR). 


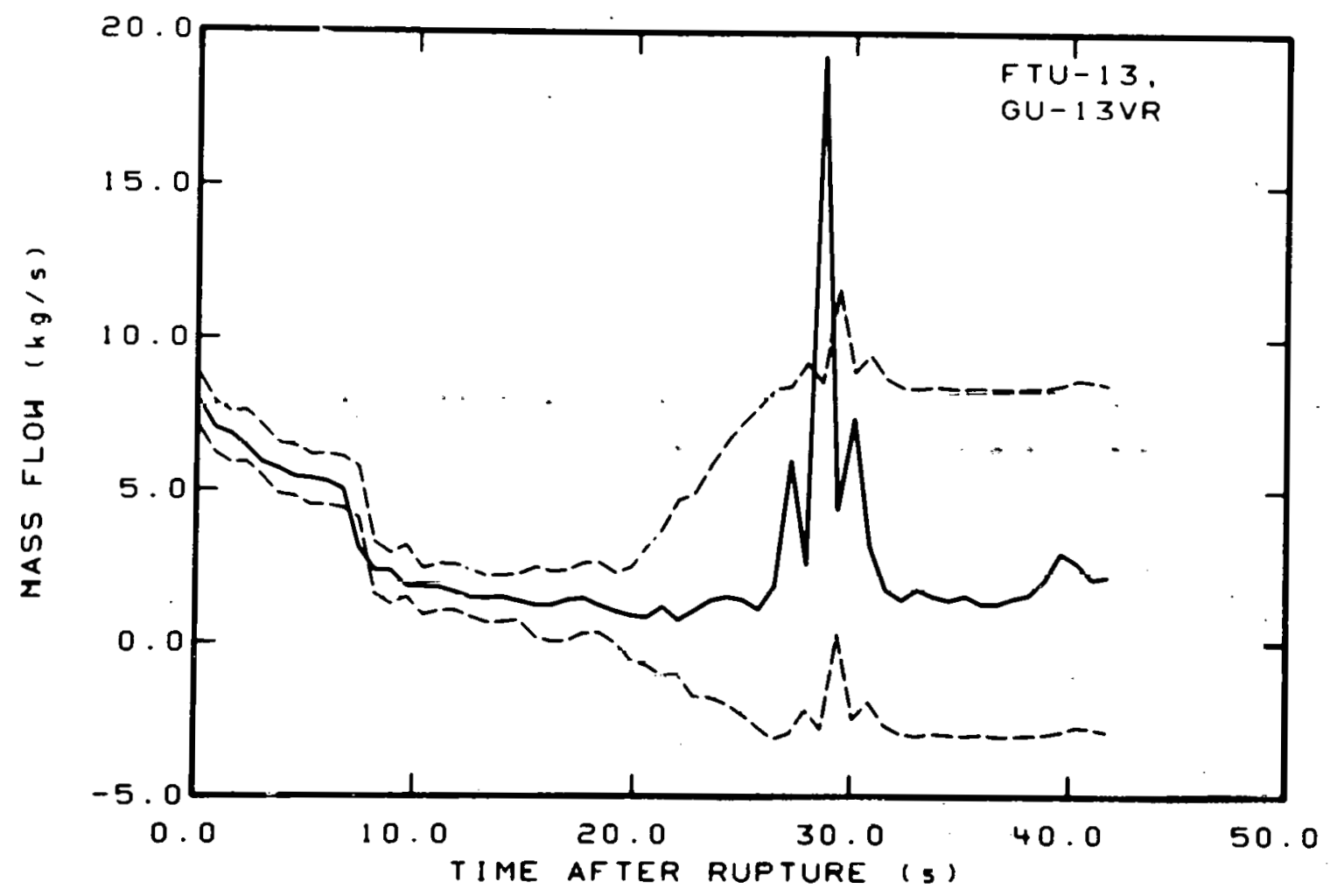

Fig. C-41 Mass flow in intact loop (FTU-13, GU-13VR).

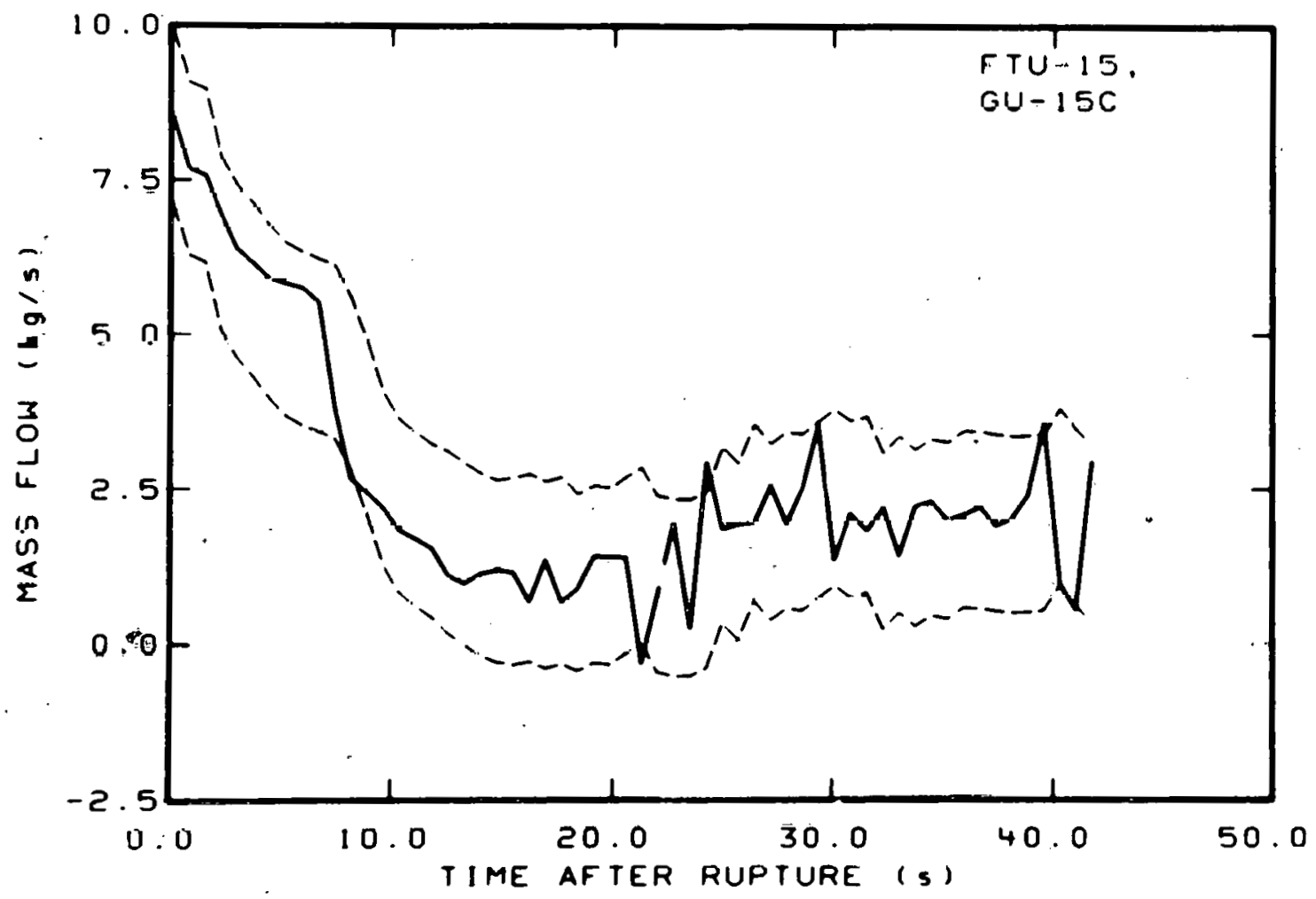

Fig. C-42 Mass flow in intact loop (FTU-15, GU-15C). 


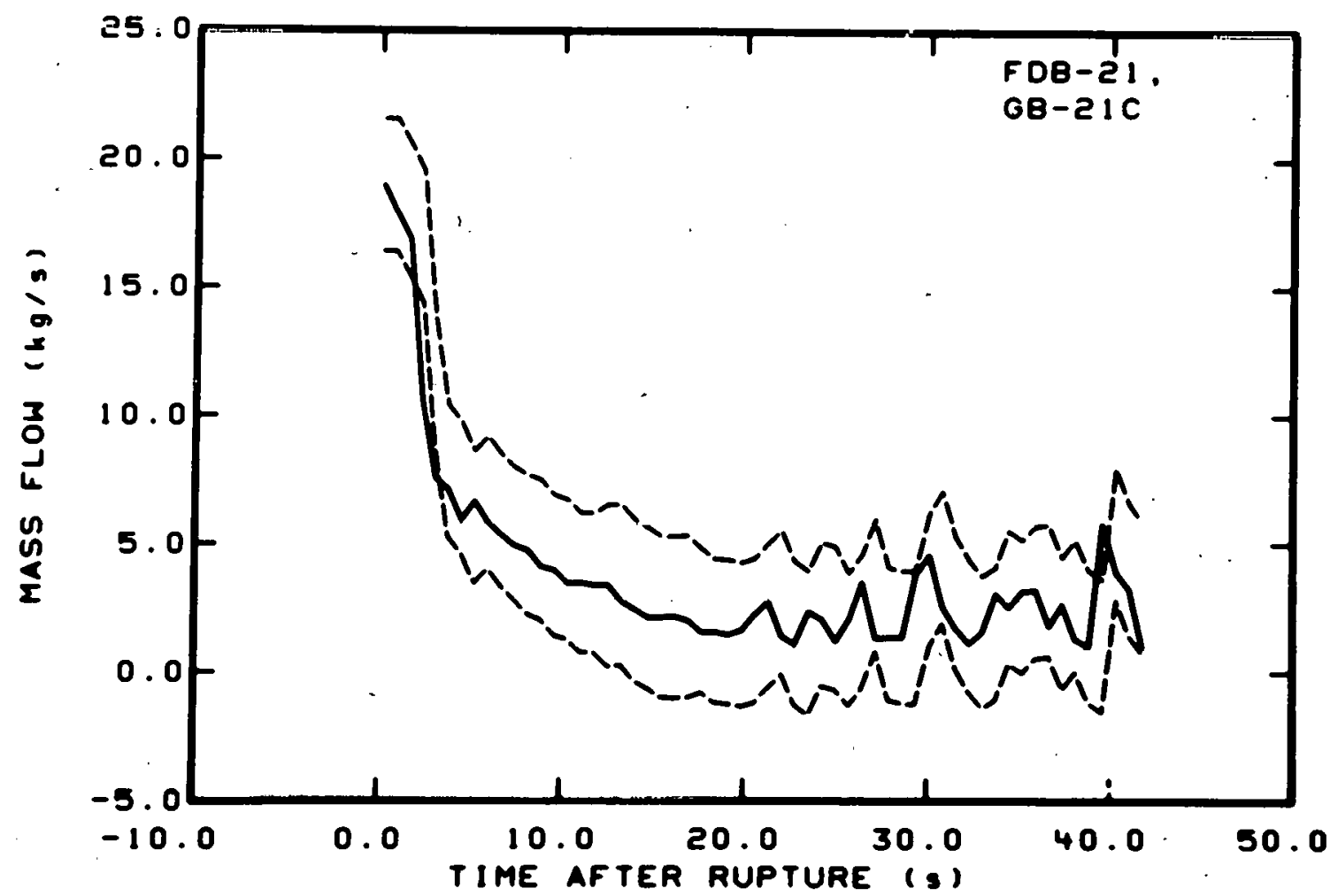

Fig. C-43 Mass flow in broken loop (FDB-21, GB-21C).

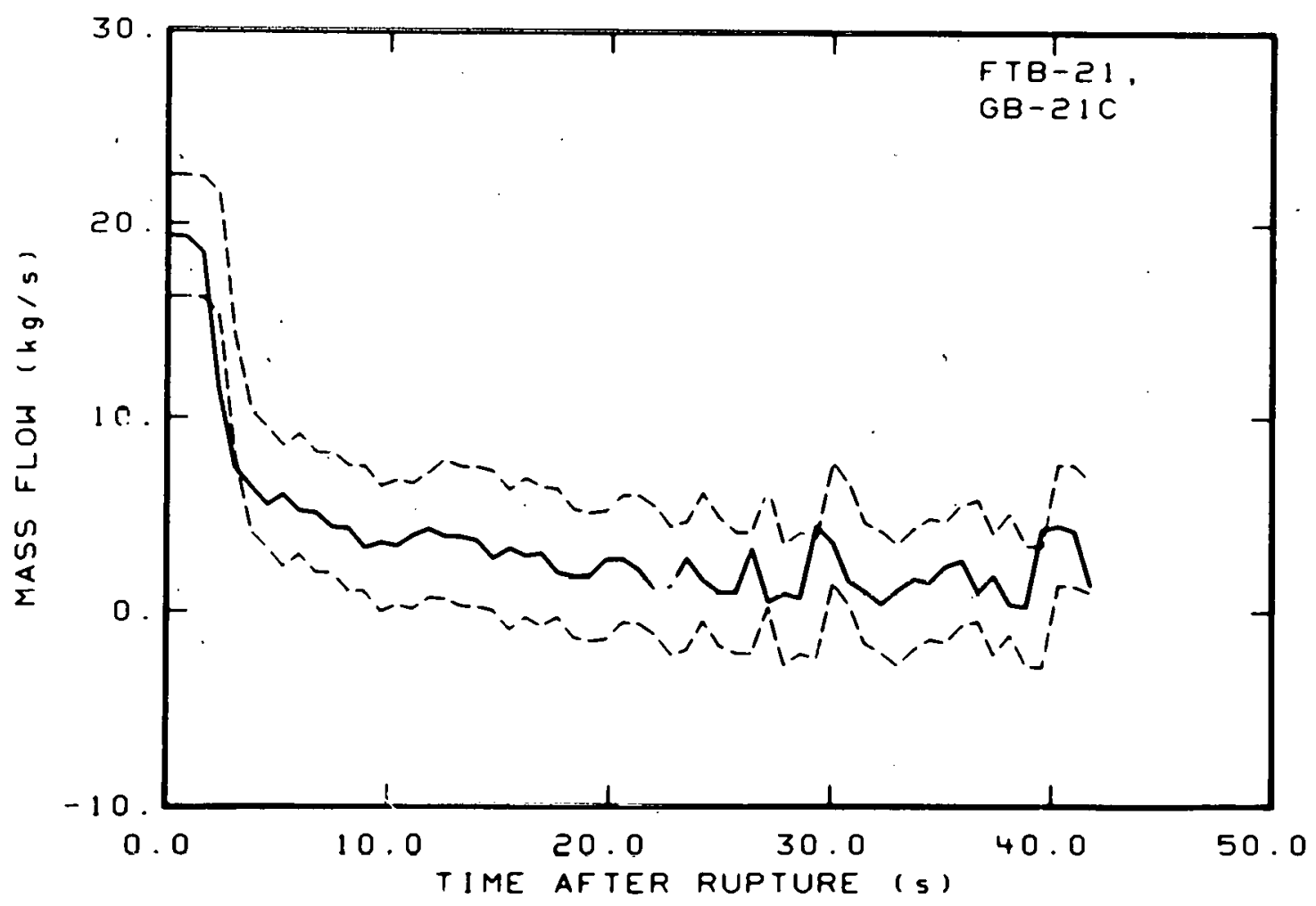

Fig. C-44 Mass flow in broken loop (FTB-21, GB-21C). 


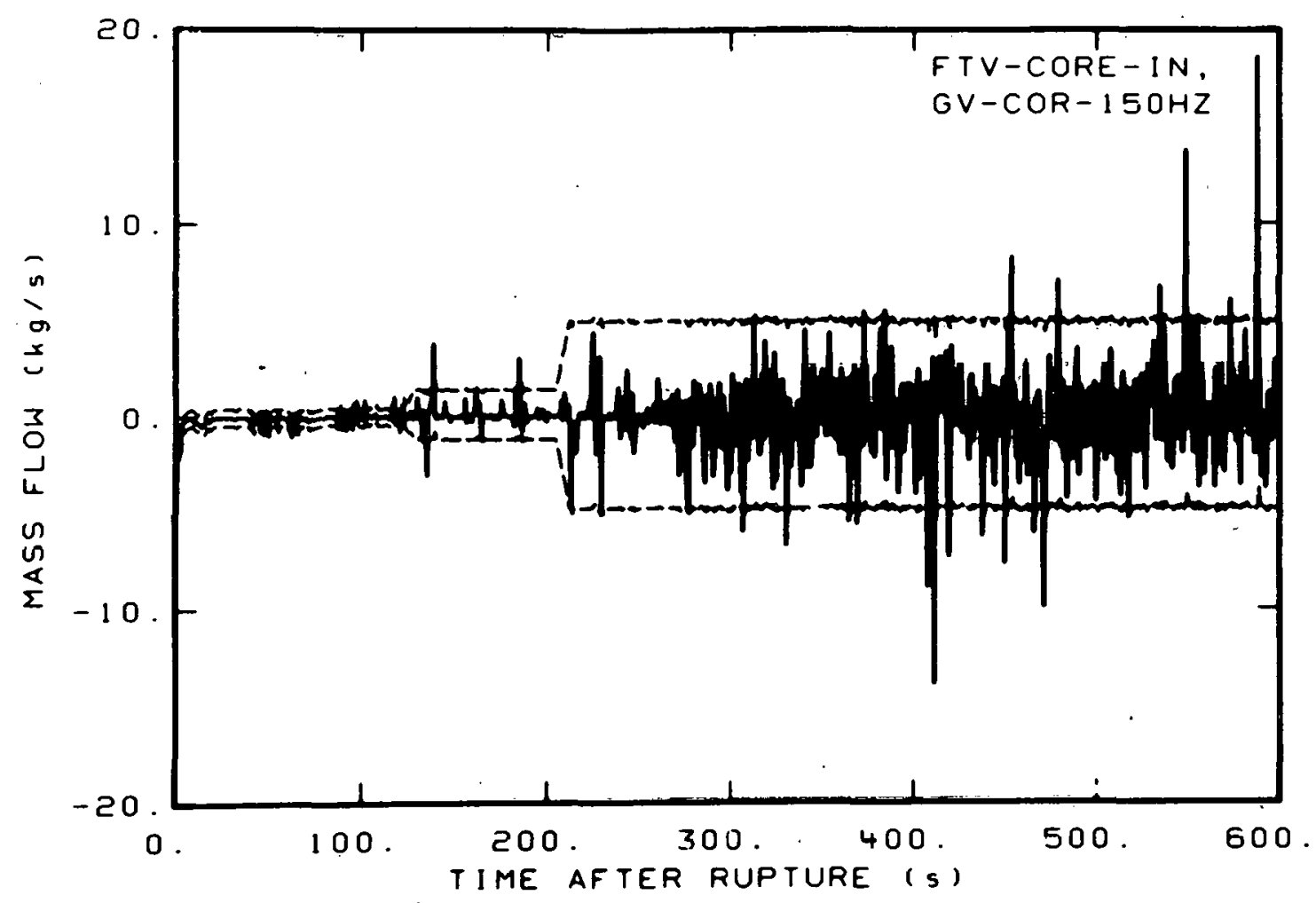

Fig. C-45 Mass flow in vessel (FTV-CORE-IN, GV-COR-150HZ).

Other errors in the data exist because of such factors as variability in installation procedures and techniques, calibration errors, variability in materials, and temperature and pressure sensitivitics. These errors and the procedures for estimating them are discussed in Reference C-2. They are referred to as engineering errors and the estimates are largely subjective. Because of the continuing effort to improve the accuracy of the measured data, such as through the use of better transducers, better signal conditioning and processing equipment, and better calibration and installation techniques, the engineering errors for data trom most of the transducer systems have changed from those published in Reference C-2. Table C-II provides a summary of engineering error values obtained from current analysis techniques as applied to the data presented herein.

In addition to the normal hardware and installation related sources of engineering error, a significant measurement uncertainty results when the current transducer systems are subjected to separated two-phase flow regimes during the course of the blowdown transient. $\Lambda$ ccordingly, for those data affected (fluid density, momentum flux, volumetric flow, and mass flow), which are presented in this appendix, a more extensive assessment was conducted for additional engineering error due to flow regime effects. Table C-III identifies the data analyzed and the period in the blowdown process for which flow regime errors were included as a part of the total engineering error. The time of occurrence of separated two-phase flow and the resulting effect on the uncertainty of the data were evaluated by considering, on an individual basis, each detector output with reference to indications by other auxiliary measurements. 


\section{TABLE C-II}

GENERAL MEASUREMENT ENGINEERING ERROR SOURCES AND ERROR VALUES (TEST S-28-6)

\begin{tabular}{|c|c|c|c|c|}
\hline $\begin{array}{c}\text { Measurement } \\
\text { Category }\end{array}$ & Error Sources & Error Value & \multicolumn{2}{|c|}{ Expected Error Value } \\
\hline \multirow[t]{4}{*}{$\begin{array}{l}\text { Fluid } \\
\text { Temperature }\end{array}$} & $\begin{array}{l}\text { Changes in homogeneity of the } \\
\text { thermocouple wire due to cold } \\
\text { working }\end{array}$ & $\pm 1.11 \mathrm{~K}$ & \multirow{4}{*}{ $\pm 2.8 k^{[a]}$} & \\
\hline & $\begin{array}{l}\text { Data interpretation from } \\
\text { standard reference tables }\end{array}$ & $\pm 1.11 \mathrm{~K}$ & & \\
\hline & $\begin{array}{l}\text { General data acquisition } \\
\text { processing }\end{array}$ & $\pm 2.50 \mathrm{~K}$ & & \\
\hline & Thermal aging of the thermocouples & $\pm 0.28 \mathrm{~K}$ & & \\
\hline \multirow[t]{5}{*}{$\begin{array}{l}\text { Material } \\
\text { Temperature }\end{array}$} & $\begin{array}{l}\text { Changes in homogeneity of the } \\
\text { thermocouple wire due to cold } \\
\text { working }\end{array}$ & $\pm 1.11 \mathrm{~K}$ & \multirow{5}{*}{ $\pm 3.9 \mathrm{~K}$} & . \\
\hline & Thermocouple radial position & $\pm 2.78 \mathrm{~K}$ & & \\
\hline & $\begin{array}{l}\text { Data interpretation from standard } \\
\text { reference tables }\end{array}$ & $\pm 1.11 \mathrm{~K}$ & & \\
\hline & $\begin{array}{l}\text { General data acquisition and } \\
\text { processing }\end{array}$ & $\pm 2.50 \mathrm{~K}$ & & \\
\hline & Thermal aging of the thermocouples & $\pm 0.28 \mathrm{~K}$ & & \\
\hline
\end{tabular}




\begin{tabular}{|c|c|c|c|}
\hline $\begin{array}{c}\text { Measurement } \\
\text { Category }\end{array}$ & Error Sources & Error Value & Expected Error Value \\
\hline \multirow[t]{4}{*}{ Pressure } & Entrance effects & $\begin{array}{l}+0.3 \% \text { of transducer } \\
\text { full scale }\end{array}$ & \\
\hline & Calibration & $\begin{array}{l}+0.26 \% \text { of transducer } \\
\text { full scale }\end{array}$ & \\
\hline & Temperature sensitivity & $\begin{array}{l}+0.13 \% \text { of transduzer } \\
\text { ful1 scale }\end{array}$ & \\
\hline & $\begin{array}{l}\text { General data acquisition and } \\
\text { processing }\end{array}$ & $\begin{array}{l}+0.1 \% \text { of system full } \\
\text { scale }\end{array}$ & '- \\
\hline \multirow[t]{6}{*}{$\begin{array}{l}\text { Differential } \\
\text { Pressure }\end{array}$} & Installation & $\begin{array}{l}+0.3 \% \text { of transducar } \\
\text { fuil scale }\end{array}$ & \\
\hline & $\begin{array}{l}\text { Calibration } \\
\text { Transducer ranges }-4.96 \text { through } \\
+199.26 \mathrm{kPa}\end{array}$ & $\begin{array}{l}+\left[(0.05)-(0.5 \mathrm{R}: F S)^{2}\right]^{1 / 2} \% \\
\text { of transducer full scale }\end{array}$ & \\
\hline & $\begin{array}{l}\text { Transducer ranges } \pm 344.74, \\
\pm 689.47, \pm 344 ? \mathrm{kPa}\end{array}$ & $\begin{array}{l}+\left[(0.03)+(0.5 \mathrm{R} ; \mathrm{FS})^{2}\right]^{1 / 2} \% \\
\text { of full scale }\end{array}$ & $\pm 2 \%$ of transducer \\
\hline & $\begin{array}{l}\text { Transducer ranges } \pm 6894, \\
\pm 10342 \mathrm{kPa}\end{array}$ & $\begin{array}{l} \pm\left[(0.02)+(0.5 \mathrm{R} ; \mathrm{FS})^{2}\right]^{1 / 2} \% \\
\text { of full scale }\end{array}$ & full sca \\
\hline & & where & \\
\hline &. & $\begin{aligned} R & =\text { transducer rezding } \\
& (\mathrm{kPa}) \\
\mathrm{FS}= & \text { trans Jucer range full } \\
& \text { scale }(\mathrm{kPa})\end{aligned}$ & \\
\hline
\end{tabular}




\begin{tabular}{|c|c|c|c|}
\hline $\begin{array}{l}\text { Measurement } \\
\text { Category }\end{array}$ & Error Sources & Error Value & Expected Error Value \\
\hline \multirow[t]{3}{*}{$\begin{array}{l}\text { Differential } \\
\text { Pressure } \\
\text { (continued) }\end{array}$} & Temperature sensitivity & $\begin{array}{l}+0.5 \% \text { of transducer } \\
\text { full scale }\end{array}$ & \\
\hline & $\begin{array}{l}\text { General data acquisition and } \\
\text { processing }\end{array}$ & $\begin{array}{l} \pm 0.1 \% \text { of system full } \\
\text { scale }\end{array}$ & $\begin{array}{l}+2 \% \text { of transducer } \\
\text { full scale } \mathrm{e}^{[\mathrm{c}]}\end{array}$ \\
\hline & Air entrapment & $\pm 0.069 \mathrm{kPa}$ & \\
\hline \multirow{4}{*}{$\begin{array}{l}\text { Fluid Velocity } \\
\text { ipoint velocities } \\
\text { measured with } \\
\text {-urboprobes) }\end{array}$} & Installation & $\begin{array}{l}+0.8 \% \text { of transducer } \\
\text { full scale }\end{array}$ & . \\
\hline & Calibration & $\begin{array}{l}+5 \omega \\
\text { full of transducer } \\
\text { scale }\end{array}$ & $\begin{array}{l} \pm[0.5825+ \\
\left.(0.008 R)^{2}\right] 1 / 2_{\mathrm{m} / \mathrm{s}}\end{array}$ \\
\hline & $\begin{array}{l}\text { Data acquisition and processing } \\
\text { frequency conversion }\end{array}$ & $\begin{array}{l}+0.25 \% \text { of transducer } \\
\text { full scale }\end{array}$ & \\
\hline & General & $\begin{array}{l}+0.1 \% \text { of system fuil } \\
\text { scale }\end{array}$ & $\begin{aligned} R= & \text { transducer read- } \\
& \text { ing }(\mathrm{m} / \mathrm{s})\end{aligned}$ \\
\hline \multirow[t]{3}{*}{ Density } & Calibration & $\pm 1.0 \%$ of reading $\left(\mathrm{kg} / \mathrm{m}^{3}\right)$ & \\
\hline & Detector system error & $+2.1 \mathrm{~kg} / \mathrm{m}^{3}$ & [d] \\
\hline & $\begin{array}{l}\text { General data acquisition and } \\
\text { processing }\end{array}$ & $\pm 1.6 \mathrm{~kg} / \mathrm{m}^{3}$ & \\
\hline
\end{tabular}


TABLE :-I (continued)

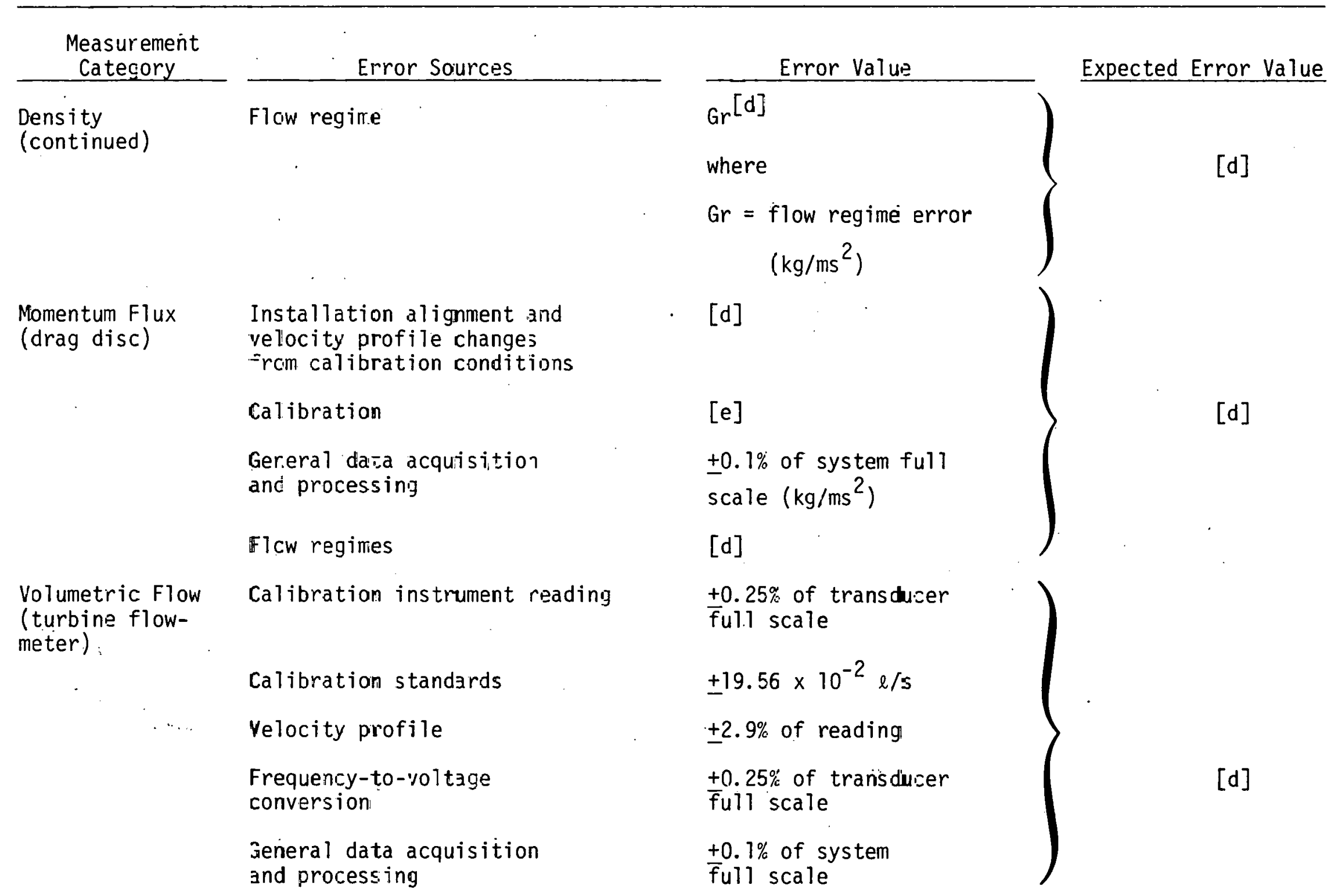


TABLE C-II (continued)

\begin{tabular}{|c|c|c|c|}
\hline $\begin{array}{c}\text { Measurement } \\
\text { Category }\end{array}$ & Error Sources & Error Value & Expected Error Value \\
\hline \multirow{2}{*}{$\begin{array}{l}\text { Volumetric Flow } \\
\text { (turbine flow- } \\
\text { meter) (continued) }\end{array}$} & Dead bands & $\begin{array}{l}+5 \% \text { of transducer } \\
\text { full scale }\end{array}$ & [d] \\
\hline & Flow regimes & [d] & \\
\hline $\begin{array}{l}\text { Mass Flow Rate } \\
\text { (from volumetric } \\
\text { flow and density } \\
\text { data) }\end{array}$ & $\begin{array}{l}\text { Combined results from } \\
\text { individual error sources } \\
\text { for volumetric flow and density } \\
\text { data }[\mathrm{f}]\end{array}$ & [d] & [d] \\
\hline $\begin{array}{l}\text { Mass Flow Rate } \\
\text { ifrom momentum } \\
\text { flux and density } \\
\text { data) }\end{array}$ & $\begin{array}{l}\text { Combined results from } \\
\text { individual error sources for } \\
\text { momentum flux and density data }[f]\end{array}$ & [d] & [d] \\
\hline
\end{tabular}

[a] This value is no longer valid after thermocouple dryout occurs.

[b] Value for transducers with $20684 \mathrm{kPa}$ full-scale ranges.

[c] Value is based on observed system performance. It is more conservative than that obtained from the statistical summation of the identified engineering errors.

[d] Error value is time and flow regime dependent.

[e] Dependent on transducer full-scale range reading.

[f] The general method for combining volumetric flow or momentum flux with density data to obtain mass flow rate and the resulting errors in the data are explained in Reference C-2. 
TABLE C-III

TIME PERIODS WHEN FLOW REGIME ERRORS WERE APPLIED (TEST S-28-6)

\begin{tabular}{|c|c|c|}
\hline $\begin{array}{c}\text { Tränsducer } \\
\text { Identification }\end{array}$ & $\begin{array}{l}\text { Time during which } \\
\text { Flow Regime Errors } \\
\text { were Applied } \\
\text { (s) }\end{array}$ & Figure Number \\
\hline GU-1C & $\begin{array}{l}2 \text { to } 18 \\
\text { and } \\
80 \text { to } 600\end{array}$ & $C-30$ \\
\hline GU-10VR & 8 to 20 & $c-31$ \\
\hline GU- $15 \mathrm{C}$ & $\begin{array}{c}6 \text { to } 20 \\
\text { and } \\
\text { fir tio } 600\end{array}$ & $C-34$ \\
\hline FDU- 1, GU- IC & $\begin{array}{l}? \text { tho } 78 \\
\text { and } \\
80 \text { tu } 600\end{array}$ & $C-33$ \\
\hline IDU-5, GU-5VR & $\begin{array}{l}2 \text { to } 18 \\
\text { and } \\
80 \text { to } 600\end{array}$ & $C-40$ \\
\hline FTU-13, GU-13VR & 15 to 28 & $C-41$ \\
\hline FTU-15, GU-15C & $\begin{array}{l}6 \text { to } 20 \\
\text { and } \\
66 \text { to } 600\end{array}$ & $C-42$ \\
\hline FTB-21; GB-21C & $\begin{array}{l}2 \text { to } 20 \\
\text { and } \\
66 \text { to } 600\end{array}$ & $C-44$ \\
\hline
\end{tabular}

The gamma densitometer density measurement data are affected by two phasc separated flow regimes. The resulting transducer output is a measurement of the average attenuation of the gamma beam through the measured medium. The beam attenuation, in turn, is interpreted through physical relationship to be a mcasurc of the average density along the beam path. When stratified type flow was considered present, the gamma beam attenuation was considered to be a result of a liquid layer and steam at system conditions. With this assumption and the system geometry, a void fraction was calculated and a new "effective" average density was calculated. The difference betwecn the average density based on the assumption of homngeneous conditions and the averagc density for stratified conditions was considered to be the error. 
Momentum flux measurement uncertainties for two-phase flow regimes present the most difficult engineering evaluation problems. The drag target and arm location, degree of flow stratification, transducer temperature sensitivity, and slip ratios all combine to produce possible flow regime errors ranging from a small fraction of the transducer output value to multiples of it. Therefore, the error values were obtained, where possible, through use of the observed discrepancies between the momentum flux and turbine flowmeter data in combination with system pressure measurements and the analysis of system fluid density measurements.

The flow regime errors of the turbine flowmeter were estimated by calculating a void fraction and the cross-sectional liquid and steam flow areas for stratified flow. This calculation was accomplished using methods similar to those used to calculate the average density for stratified flows. A simple model was used to equate the forces on the turbine with the assumption of a known void fraction, stratified flow, known component densities, and slip ratio greater than unity. This process provided phase velocities. With the phase densities, velocities, and void fraction, a volumetric flow rate could be calculated. The difference between this value and the measured value was considered to be the error.

The overall standard deviation of a data point is taken as the root mean of the sum of the random error variation and the total engineering error variance; that is,

$$
\sigma_{0}=\sqrt{\sigma_{R}^{2}+\sigma_{E}^{2}}
$$

where

$$
\begin{aligned}
& \sigma_{\mathrm{O}}=\text { overall standard deviation of a data point } \\
& \sigma_{\mathrm{R}}=\text { random error standard deviation } \\
& \sigma_{\mathrm{E}}=\text { engineering error standard deviation. }
\end{aligned}
$$

The error bands for the data are computed about the value given by the fitted difference equation $y_{i}$ at time point, $i$; that is,

$$
\text { error band }=y_{i} \pm 1.960_{0}
$$

With due regard to the fact that $\sigma_{\mathrm{E}}$ has been estimated subjectively, the error band may be interpreted as an approximate $95 \%$ confidence interval within which any true value of the measured variahle is consistent with the data.

On certain occasions, the symmetrical error band given by Equation (C-2) is not appropriate. On those occasions, asymmetrical error bands were computed. (That is, with the width being greater on one side of $y_{i}$ than on the other.) 
Finally, the original data trace, along with its error band from Equation (C-2), was input to a computer plot package. The resulting plot contained the actual data trace surrounded by an error band derived both from random error and engineering error considerations. The indicated error bands after thermocouple dryout occurred for the fluid temperature measurements should be ignored. Error bands indicated during periods when detector or data acquisition system saturation occurred should also be ignored. Error bands

for these segments of the data were not obtained and bands only appear because of limitations in the plotting package.

\section{$\underline{\text { REFERENCES }}$}

C-1. G. E. P. Box and B. M. Jenkins, Time Series Analysis - Forecasting and Control, San Francicco: Holden Day, 1970.

C-2. E. M. Feldman and S. A. Naff, Error Analysis for 1-1/2-Loop Semiscale System. Isothermal Test Data, ANCR-1 188 (May 1975). 
DISTRIBUTION RECORD FOR TREE-NUREG-1153

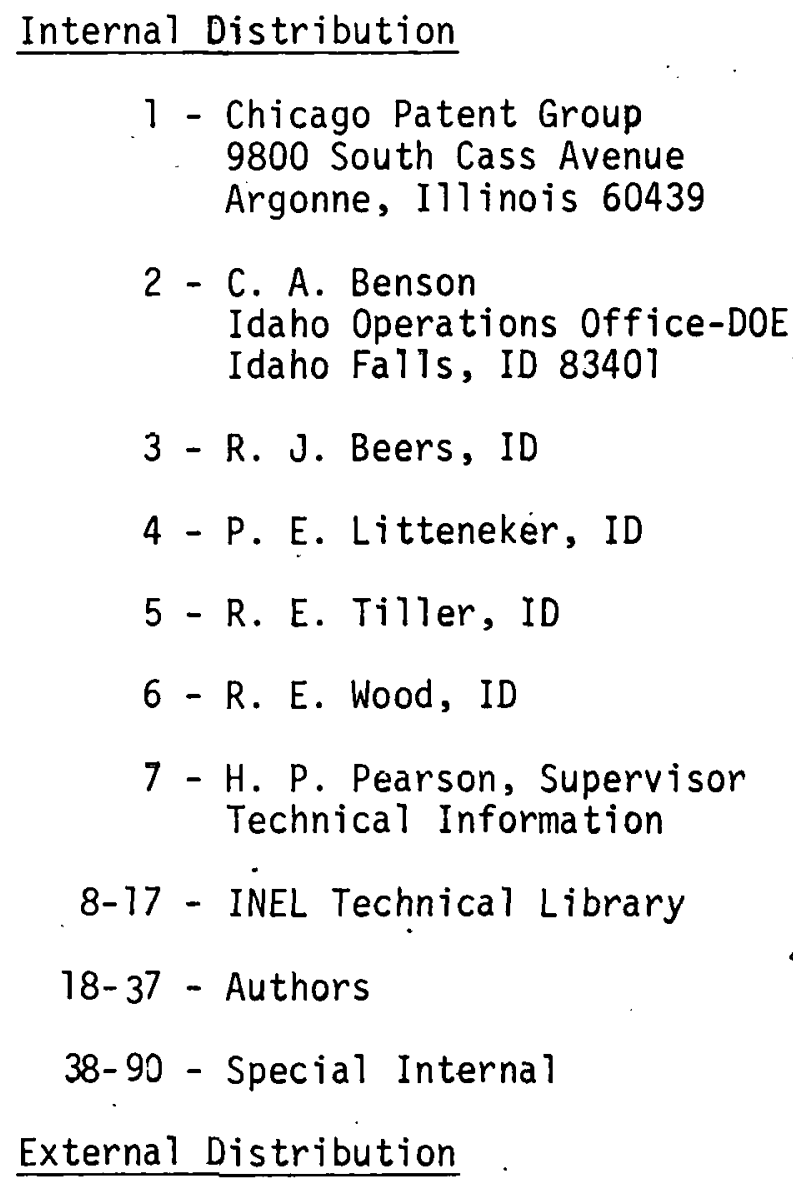

95-398 - Distribution under NRC-2, Water Reactor Safety Research Systems Engineering 